\title{
New Biomimetic Analogues of Functional [2Fe-2S] Proteins
}

Dissertation

zur Erlangung des Doktorgrades

der Mathematisch-Naturwissenschaftlichen Fakultäten der Georg-August-Universität zu Göttingen

\author{
vorgelegt von \\ Diplom-Chemiker \\ Hans Joachim Ballmann \\ aus Rothenburg o.d. T.
}

Göttingen 2008 
D7

Referent: Prof. Dr. Franc Meyer

Korreferent: Prof. Dr. Dietmar Stalke

Tag der mündlichen Prüfung: 29.10.2008 




\section{Abstract}

Iron-sulfur proteins are ubiquitous natural cofactors of prime importance in biological systems. While electron transfer is regarded as their main role, iron-sulfur clusters also feature increasingly recognized new functionalities, e.g. in catalysis, sensing of small molecules, radical-based processes and gene regulation. Accordingly, iron-sulfur proteins are nowadays viewed as nature's modular multipurpose structures, involved in crucial biological processes - most likely since the beginning of terrestrial life. The understanding of their structural and electronic properties has benefited significantly from investigations on synthetic model compounds over the last decades. A variety of synthetic challenges however remained to inorganic chemists even for the smallest $[2 \mathrm{Fe}-2 \mathrm{~S}]$ clusters. In the present work, novel biomimetic $[2 \mathrm{Fe}-2 \mathrm{~S}]$ clusters were synthesized and comprehensively examined in order to gain further insights into the fundamental characteristics of their biological counterparts. At first, a ligand exchange pathway starting from a homoleptic indolate-ligated [2Fe-2S] precursor was developed affording the thiophenolate-coordinated ferredoxin analogues via a convenient experimental procedure. In addition to various differently substituted thiophenols, heteroaromatic thiols and chelating biphenols were successfully applied as reagents in the latter exchange reaction, indicating that the conversion is of general use in synthetic $[2 \mathrm{Fe}-2 \mathrm{~S}]$ chemistry. Ligand effects on prominent spectroscopic characteristics of all-thiolate-ligated clusters were studied by introduction of electron-withdrawing and electron-donating substituents into chelating dithiobiphenyl-based ligand scaffolds. The anticipated ligand-mediated control over the redox potentials of those cluster compounds has been ascertained by electrochemical measurements. In order to provide models for the interaction of additional donor atoms with the iron atoms in biological [2Fe-2S] sites, a series of synthetic clusters with terminal thiophenolate ligands and tethered ether or thioether moieties has been prepared. Secondary interactions do occur in those clusters if the additional Lewis-bases are suitably positioned in proximity to the cluster core. Significant structural distortions of the usually rigid cluster core geometries were observed with the iron atoms approaching trigonal bipyramidal coordination polyhedra. The detected effects are clearly more pronounced for thioether compared to ether donor groups. DFT calculations are in agreement with the experimental implications. The potential relevance of these findings for biological iron-sulfur sites, e.g. for the unique arginine-ligated $[2 \mathrm{Fe}-2 \mathrm{~S}]$ cluster in biotin synthase is considered. Beyond those studies, the synthesis of a model compound for mixed-valent [2Fe-2S] ferredoxins is generally considered as pending task to be achieved by synthetic iron-sulfur chemists. In order to tackle this challenge, $\{\mathrm{N}\}$ homoleptic clusters with terminal dipyrromethanate and 1,2-benzene-bis-benzimidazolate 
coordination were synthesized and examined with respect to one-electron reduction. The chelating nature of those terminal ligands imparts a relatively high stability that permitted the coulometric generation and EPR characterization of a $[2 \mathrm{Fe}-2 \mathrm{~S}]^{+}$species ligated by 1,2-benzene-bis-benzimidazolate. Finally, asymmetrically coordinate [2Fe-2S] clusters were approached synthetically. After an extensive ligand screening, a chelating diskatyl- $\left\{\mathrm{N}_{2}\right\}$ ligand was discovered that allows the isolation of a heteroleptic $\left\{\mathrm{N}_{2} \mathrm{Cl}_{2}\right\}$-ligated cluster with both exchangeable chlorine substituents located on the same iron atom. A first accurate $\left\{\mathrm{N}_{2} \mathrm{~S}_{2}\right\}$-coordinate analogue of Rieske-type clusters could then be synthesized by consecutive replacement of both remaining halides for the chelating $o$-xylen- $\alpha, \alpha^{\prime}$-dithiolate. This Rieske-type cluster accurately emulates structural and spectroscopic features (inter alia the typical Mössbauer parameters) of the natural protein sites, including the characteristic low $g_{\mathrm{av}}$ value in the EPR spectra of the reduced $[2 \mathrm{Fe}-2 \mathrm{~S}]^{+}$species. 


\section{Table of Contents}

1 General Introduction 1

1.1 Iron-Sulfur Enzymes as Fundamental Active Sites in Nature . . . . . . . . 2

1.2 Canonical Iron-Sulfur Clusters . . . . . . . . . . . . . . . . . . . 2

1.3 Cluster Assembly, Biosynthesis and Cluster Conversions . . . . . . . . . . . 4

1.4 Functional Properties of Iron-Sulfur Sites . . . . . . . . . . . . . . . . . 4

1.5 Biological Relevant $[2 \mathrm{Fe}-2 \mathrm{~S}]$ Protein Variants . . . . . . . . . . . . . 5

1.6 Synthetic $[2 \mathrm{Fe}-2 \mathrm{~S}]$ Cluster Analogues . . . . . . . . . . . . . . . . . . . 10

1.7 Contemporary Biomimetic [2Fe-2S] Chemistry: Focus of this Contribution 12

2 A Convenient Ligand Exchange Pathway to [2Fe-2S] Ferredoxin $\begin{array}{ll}\text { Analogues } & 13\end{array}$

2.1 Introduction . . . . . . . . . . . . . . . . . . . . . 14

2.2 Synthesis and Characterization of $\{\mathrm{N}\}$-Ligated $[2 \mathrm{Fe}-2 \mathrm{~S}]$ Precursors $\ldots . . .14$

2.3 Ligand Exchange Reactivity of $\left.\left(\mathrm{NEt}_{4}\right)_{2}\left[\mathrm{Fe}_{2} \mathrm{~S}_{2} \text { (indolate) }\right)_{4}\right]$. . . . . . . 16

2.4 Selected Examples, Scope of the ligand Exchange Reaction and Limitations 17

2.5 Conclusions . . . . . . . . . . . . . . . . . . . . . . . . 22

3 Ligand Effects on Prominent Spectroscopic Properties of [2Fe-2S] $\begin{array}{ll}\text { Clusters } & 23\end{array}$

3.1 Introduction . . . . . . . . . . . . . . . . . . . . . . 24

3.2 Ligand Synthesis . . . . . . . . . . . . . . . . . 24

3.3 Cluster Synthesis and Structural Characterization . . . . . . . . . . 26

3.4 Spectroscopy in Solution . . . . . . . . . . . . . . . . . 29

3.5 Spectroscopy in the Solid State . . . . . . . . . . . . . . . 32

3.6 Conclusions . . . . . . . . . . . . . . . . . . . . . . . . . . . . 34

4 The Quest for Neutral [2Fe-2S] Clusters 35 


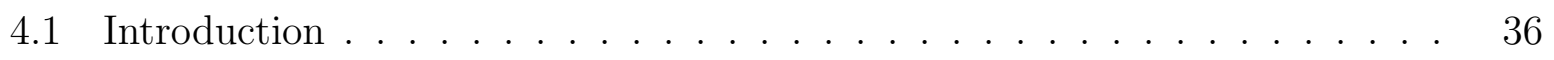

4.2 Attempts to Synthesize Zwitterionic Ferredoxin Analogues . . . . . . . 36

4.3 On the Application of Redox-non-innocent Dithiolenes in Fe/S Chemistry . 37

4.4 Attempts to Synthesize Analogues of Cluster 3 . . . . . . . . . . . 39

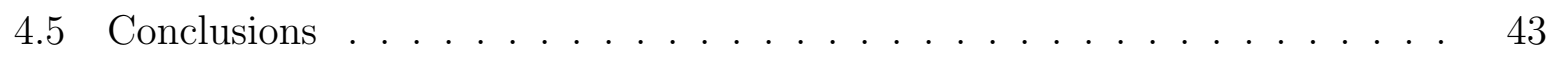

5 Secondary Bonding Interactions in Biomimetic $[2 \mathrm{Fe}-2 \mathrm{~S}]$ Clusters $\quad 45$

5.1 Introduction . . . . . . . . . . . . . . . . . . . . . . 46

5.2 Ligand Synthesis . . . . . . . . . . . . . . . . . . . . . . 47

5.3 Cluster Synthesis and Structural Characterization . . . . . . . . . . . . 47

5.4 Spectroscopy and Magnetic Properties in the Solid State . . . . . . . . 54

5.5 Spectroscopy in Solution . . . . . . . . . . . . . . . . . 57

5.6 DFT Calculations . . . . . . . . . . . . . . . . . . 60

5.7 Conclusions . . . . . . . . . . . . . . . . . . . . . . 62

6 Switching the Spin State in $\left\{\mathrm{S}_{4} \mathrm{X}_{2}\right\}$-Coordinated Iron(III) Complexes by Variation of $\mathrm{X}=\mathrm{N}, \mathrm{O}, \mathrm{P}, \mathrm{S} \quad 63$

6.1 Introduction . . . . . . . . . . . . . . . . . . . . . 64

6.2 Synthesis and Structural Characterization _. . . . . . . . . . . 64

6.3 Spectroscopy and Magnetic Properties in the Solid State . . . . . . . . 66

6.4 Spectroscopy in Solution . . . . . . . . . . . . . . . . . . 69

6.5 DFT Calculations . . . . . . . . . . . . . . . . 70

6.6 Conclusions . . . . . . . . . . . . . . . . . . . . . . 74

7 Synthetic $[2 \mathrm{Fe}-2 \mathrm{~S}]$ Clusters with Chelating N-Donor Capping Ligands 75

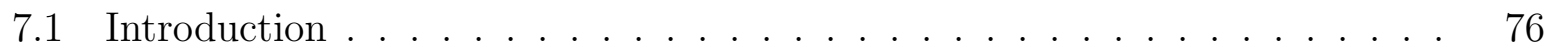

7.2 Dipyrromethane-Coordinate $[2 \mathrm{Fe}-2 \mathrm{~S}]$ Cluster Compounds . . . . . . . . . 77

7.2 .1 Synthesis and Structural Characterisation . . . . . . . . . 77

7.2 .2 Spectroscopy in the Solid State . . . . . . . . . . . . . 79

7.2.3 Spectroscopic and Electrochemical Characterization in Solution . . 80

7.3 [2Fe-2S] Clusters Coordinated by 1,2-Benzene-Bis-Benzimidazolate $\ldots . \quad 82$

7.3.1 Synthesis and Structural Characterization . . . . . . . . . 82

7.3 .2 Spectroscopy in Solution . . . . . . . . . . . . . . . 83

7.3 .3 Spectroscopy in the Solid State . . . . . . . . . . . . . 85 
7.3.4 Electrochemistry and Constant Potential Coulometry . . . . . . . 86

7.3.5 Spectroscopy on the Mixed-Valent $[2 \mathrm{Fe}-2 \mathrm{~S}]^{+}$Cluster . . . . . . . . 89

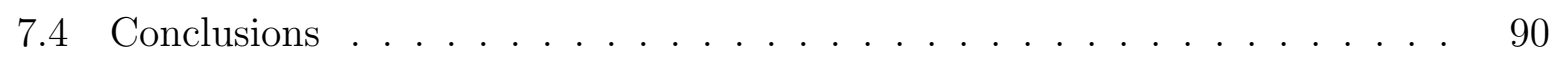

8 A Synthetic Analogue of Rieske-Type [2Fe-2S] Clusters $\quad 91$

8.1 Introduction . . . . . . . . . . . . . . . . . . . 92

8.2 Cluster Synthesis and Structural Characterization . . . . . . . . . . . 92

8.3 Spectroscopy in the Solid State . . . . . . . . . . . . . . . 97

8.4 Spectroscopy in Solution . . . . . . . . . . . . . . . . 99

8.5 Electrochemistry and Generation of the Reduced Rieske Analogue . . . . . 101

8.6 DFT Calculations . . . . . . . . . . . . . . . . . . . . . . . . 104

8.7 Conclusions . . . . . . . . . . . . . . . . . . . 106

9 Experimental Section $\quad 107$

9.1 General Considerations . . . . . . . . . . . . . . . 107

9.2 Synthesis of Ligands and Ligand Precursors _ . . . . . . . . . . . . . . . 109

9.3 Synthesis of Iron Complexes and Cluster Compounds . . . . . . . . . . . . 117

9.4 DFT Calculations . . . . . . . . . . . . . . . . . . . . 135

9.5 Mössbauer Experiments _. . . . . . . . . . . . . . . . . . 137

9.6 Magnetic Susceptibility Measurements . . . . . . . . . . . . . . 138

9.7 Electrochemical Measurements . . . . . . . . . . . . . . . . . 139

10 Crystallography $\quad 141$

$\begin{array}{ll}\text { Appendix A } & 153\end{array}$

$\begin{array}{ll}\text { Appendix B } & 155\end{array}$

$\begin{array}{ll}\text { Appendix C } & 163\end{array}$

$\begin{array}{lr}\text { Bibliography } & 171\end{array}$

$\begin{array}{ll}\text { Structures of Free Ligands } & 187\end{array}$

$\begin{array}{ll}\text { Structures of Complexes and Clusters } & 189\end{array}$

$\begin{array}{ll}\text { List of Abbreviations } & 195\end{array}$ 



\section{Chapter 1}

\section{General Introduction}

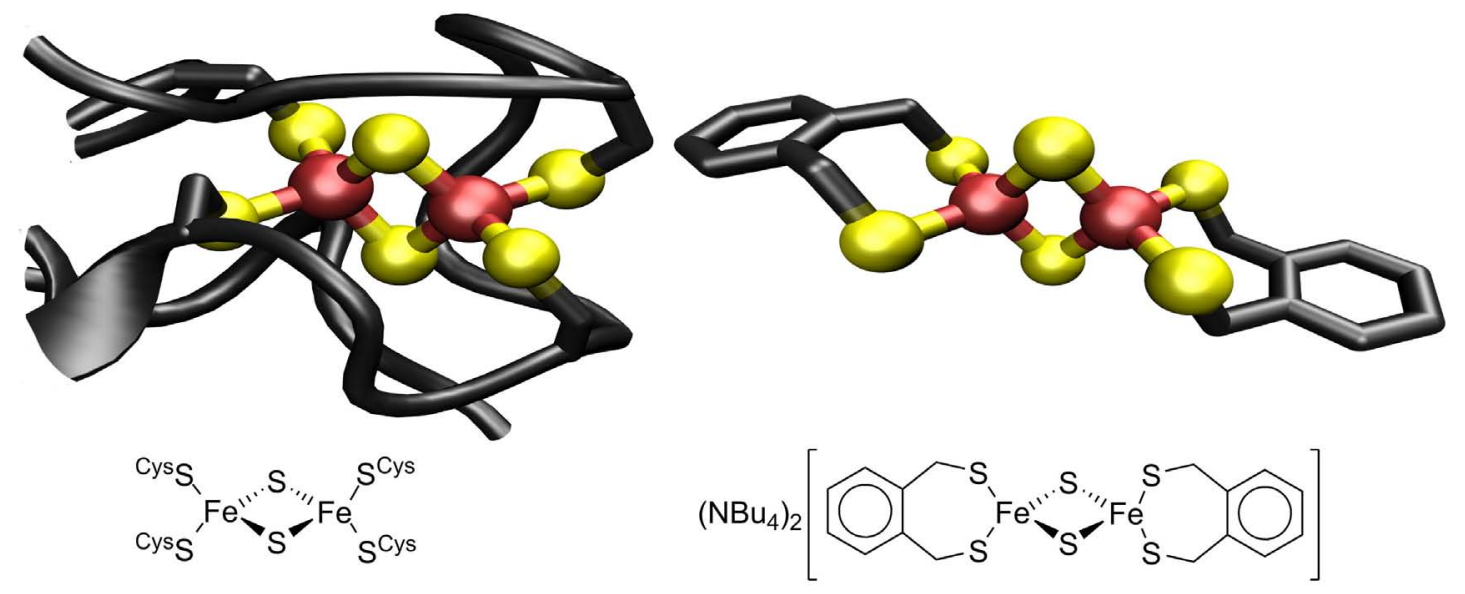

\section{Abstract}

The role of protein-bound iron-sulfur clusters as fundamental active sites in nature is discussed and a general introduction covering a brief description of the classical iron-sulfur systems, cluster biosynthesis and their main functions is given. Thereafter, the focus is centered on $[2 \mathrm{Fe}-2 \mathrm{~S}]$ enzymes with a detailed description of their six distinctive biologically relevant coordination environments identified so far. A selection of literature-known highlights in synthetic [2Fe-2S] chemistry is provided and the current focus of this area explained. Results presented in this contribution are outlined. 


\subsection{Iron-Sulfur Enzymes as Fundamental Active Sites in Nature}

Iron-sulfur proteins were initially discovered in 1960 by EPR spectroscopy on a mitochondrial $[2 \mathrm{Fe}-2 \mathrm{~S}]$ ferredoxin. ${ }^{[1]}$ Within a decade, a diversity of related iron-sulfur active sites was identified and subsequently isolated. ${ }^{[2-4]}$ Those early noticed clusters were rapidly characterized as cysteine-ligated iron complexes with incorporated acid-labile inorganic sulfides. ${ }^{[5]}$ That was the onset point of an enduring and exponentially increasing research activity in this area. ${ }^{[6-8]}$ Nowadays, iron-sulfur proteins are considered as ubiquitous and essential factors of living matter. ${ }^{[9]}$ Moreover, their participation as one of nature's first catalysts in the early development of life on earth is anticipated. ${ }^{[10]}$ Although iron-sulfur clusters are intrinsically sensitive to oxygen and therefore do require a protective protein surrounding, formation of the cluster compounds by spontaneous self-assembly assumingly was possible under the geochemical conditions present at that time on our planet. A vulcanic, sulfur-rich environment and a reducing atmosphere with soluble ferrous iron present in sufficient amounts provides a chemically rational situation for this hypothesis. ${ }^{[11]}$ Throughout the years of evolution, the entire variety of iron sulfur clusters could have been developed and then fine tuned with respect to their particular function by partial mutation of the cysteine residues, incorporation of other metals (e.g. molybdenum ${ }^{[12,13]}$ or nickel $^{[14,15]}$ ) or partial substitution with carbon monoxide and cyanide (e.g. in hydrogenases ${ }^{[16]}$ ). General overviews, covering the most prominent yet known active sites that contain iron-sulfur building blocks as a part of the active site were previously reported in the literature. ${ }^{[9,17-19]}$ Thus, only a brief introduction to the classical or so-called canonical iron-sulfur clusters that exclusively contain cysteine-coordinate iron and inorganic sulfide is presented in the following.

\subsection{Canonical Iron-Sulfur Clusters}

So far, canonical clusters relevant in biological systems were discovered with nuclearities one, two, three, four and eight. With exception of the $[8 \mathrm{Fe}-7 \mathrm{~S}]$ cluster, ${ }^{[20]}$ the so-called $\mathrm{P}^{\mathrm{N}}$-Cluster that is found exclusively in nitrogenase, ${ }^{[13]}$ the other four species represent the most stable and widespread iron-sulfur active sites in nature (Scheme 1.1). ${ }^{[19]}$ The simplest cluster, the rubredoxin $(\mathrm{Rd})^{[21]}$ actually contains only one iron centre and is deficient in acid-labile sulfide and thus sometimes not regarded as member of the iron-sulfur cluster family in strict interpretations (Note: By definition, metal-metal interactions are crucial features for cluster compounds - thus the term cluster is as well incorrect for some of the other iron-sulfur systems). From a chemical point of view however, analogues of the latter Rd-complexes can be converted to the sulfur-bridged species by reaction with elemental sulfur $^{[22]}$ and therefore be judged as common parent fragment of all iron-sulfur systems. Biologically, rubredoxins occur in the ferric $(S=5 / 2)$ and ferrous $(S=2)$ oxidation state, as - in our current understanding - these oxidation state are the only ones generally accessible by iron-sulfur clusters. Accordingly, the simplest sulfide-bridged cluster, the 


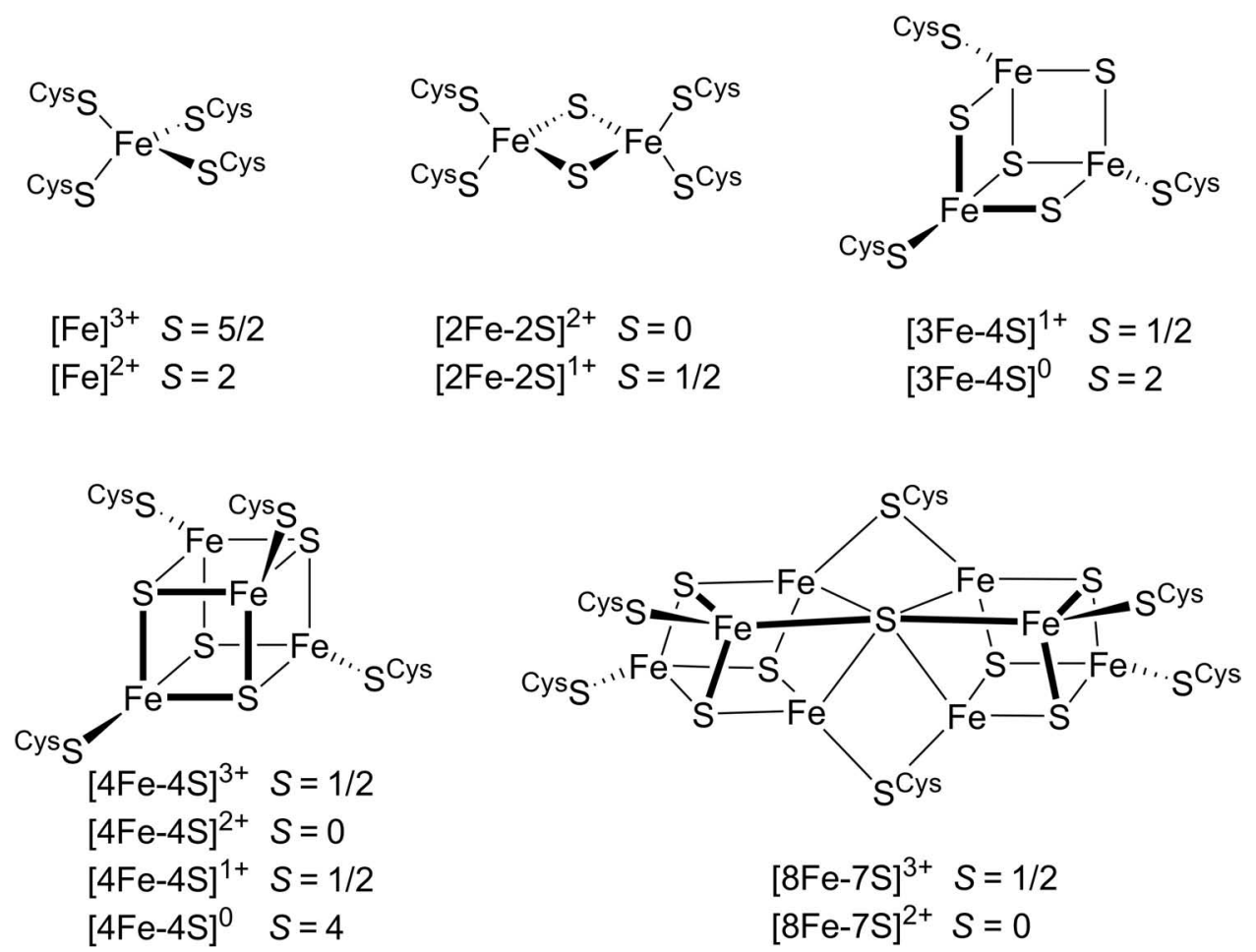

Scheme 1.1: Canonical iron-sulfur frameworks.

$[2 \mathrm{Fe}-2 \mathrm{~S}]$ ferredoxin $(\mathrm{Fd})^{[23]}$ in principal can adopt the all-ferric $(S=0)$, the mixed-valent ferric-ferrous $(S=1 / 2)$ and the all-ferrous state $(S=0)$, with the last so-called superreduced state observed only electrochemically ${ }^{[24]}$ and most likely not relevant in nature (therefore omitted in Scheme 1.1). Although neither the $[2 \mathrm{Fe}-2 \mathrm{~S}]^{1+}$ cluster itself (compared to $[4 \mathrm{Fe}-4 \mathrm{~S}]^{2+}$ ), nor it's mixed-valent oxidation state (compared to $[2 \mathrm{Fe}-2 \mathrm{~S}]^{2+}$ ) represents the most stable iron-sulfur cluster (in vivo as well as in vitro), that system was the first one to be detected by BEINERT and SANDS in 1960. ${ }^{[1]}$ The one iron extended $[3 \mathrm{Fe}-4 \mathrm{~S}]$ clusters are presumably the most unstable systems with respect to their kinetic lability. In vivo, four spin states $(S=1 / 2$ and $S=2$ are the two commonly observed spin multipicities ${ }^{[19]}$ ) were detected for the $C_{3}$-symmetric cuboidal form that is shown in Scheme 1.1. ${ }^{[25]}$ Rearrangement to a linear geometry can take place in non-physiological $\mathrm{pH}$ ranges $(\mathrm{pH} \simeq 9)$ or in presence of excess urea. ${ }^{[26-28]}$ Synthetically, both [3Fe-4S] cluster geometries are feasible, ${ }^{[22,29,30]}$ the cuboidal constitution however requires a carefully designed prearranged ligand scaffold. ${ }^{[31,32]}$ Formal addition of a further iron atom, that then occupies the remaining free corner of the cubus, affords the relatively stable and most widespread $[4 \mathrm{Fe}-4 \mathrm{~S}]$ ferredoxins. ${ }^{[33]}$ In biological systems, those clusters were observed in almost all theoretically accessible oxidation states with exception of the all-ferric system $[4 \mathrm{Fe}-4 \mathrm{~S}]^{4+}\left(S=0\right.$ in synthetic $\{\mathrm{N}\}$-ligated model compounds $\left.{ }^{[34]}\right)$. The couple $[4 \mathrm{Fe}-4 \mathrm{~S}]^{1+}$ $(S=1 / 2) /[4 \mathrm{Fe}-4 \mathrm{~S}]^{2+}(S=0)$ and the couple $[4 \mathrm{Fe}-4 \mathrm{~S}]^{2+}(S=0) /[4 \mathrm{Fe}-4 \mathrm{~S}]^{3+}(S=1 / 2)$ is commonly found in normal and so-called high-potential iron-sulfur proteins ${ }^{[35]}$ (HiPIP's), respectively. The all-ferrous $[4 \mathrm{Fe}-4 \mathrm{~S}]^{0}(S=4)$ from was so far only observed in nitrogenase bacteria of Azetobacter vinelandii under strongly reducing conditions. ${ }^{[36]}$ Nevertheless, a 
relevance of this cluster in the catalytic conversion of dinitrogen to ammonia is discussed and a two-electron oxidation of the $[8 \mathrm{Fe}-7 \mathrm{~S}]^{2+}(S=0) \mathrm{P}^{\mathrm{N}}$-Cluster proposed. ${ }^{[20]}$ Although the spin ground states of all the canonical iron-sulfur clusters were given throughout the text for completeness, theoretical explanations for those configurations are not provided herein since they were previously summarized in an excellent Science article. ${ }^{[9]}$

\subsection{Cluster Assembly, Biosynthesis and Cluster Conversions}

Approximately thirty years ago, it was demonstrated that iron-sulfur clusters can be assembled by reaction of an apoprotein with ferrous and sulfide salts. ${ }^{[37]}$ In the late $1990 \mathrm{~s}$, a small fully synthetic peptide (16 amino-acids) was utilized to obtain a [4Fe-4S] cluster by self-assembly at the peptide scaffold. ${ }^{[38]}$ From those experiments, it could be concluded that anaerobic conditions are generally required during cluster formation and that cysteine residues are by far favored over other amino-acid residue (e.g. histidine, serine or aspartic acid). Thus, the latter small peptide even dimerized to form the $[4 \mathrm{Fe}-4 \mathrm{~S}]$ ferredoxin analogue if only two cysteine sites were provided in the sequence. The biosynthesis of ironsulfur enzymes certainly is significantly more complicated since toxic ferrous salts as well as inorganic sulfides are virtually absent in living cells. ${ }^{[19,39]}$ Numerous machineries involved in the process were identified so far, ${ }^{[40,41]}$ but crucial mechanistic questions could not be elucidated yet. Cysteine clearly serves as a stable source of the bridging sulfur atoms, as cysteine desulfurases are essential for the procedure. ${ }^{[42,43]}$ The source of iron with respect to the identity of the iron-donating machinery is still doubtful, although frataxins were suggested as probable candidates. ${ }^{[44,45]}$ It remains also unclear if specific assembly proteins for each cluster are necessary or if clusters are build up from a common precursor (e.g. an initial $[2 \mathrm{Fe}-2 \mathrm{~S}]$ or $[4 \mathrm{Fe}-4 \mathrm{~S}]$ ferredoxin). The latter assumption seems to be reasonable since a variety of cluster transfer reactions, ${ }^{[46]}$ ligand swapping processes ${ }^{[4]}$ and cluster core transformations ${ }^{[4-51]}$ were observed already. In vivo as well as in vitro, $[2 \mathrm{Fe}-2 \mathrm{~S}]$ ferredoxins were shown to be convertible to $[4 \mathrm{Fe}-4 \mathrm{~S}]$ clusters by simple core dimerisation ${ }^{[52,53]}$ (in vivo a reversion of this process by oxidative cubane cleavage was observed as well ${ }^{[54]}$ ). Oxidative extrusion of one iron from the $[4 \mathrm{Fe}-4 \mathrm{~S}]$ cluster was also observed ${ }^{[48,49]}$ and would consequently provide a pathway to the kinetically labile $[4 \mathrm{Fe}-3 \mathrm{~S}]$ clusters (as mentioned above, geometric inter-conversions between their linear and cuboidal form are evident ${ }^{[26-28]}$ ). The latter clusters are believed to be transformable to most of the remaining clusters including heterometal-containing cluster species as well as asymmetrically substituted ones (e.g. aconitase). ${ }^{[55]}$

\subsection{Functional Properties of Iron-Sulfur Sites}

One-electron transfer processes from and to the actual catalytically active sites represent the classical and best studied functions, common to all canonical iron-sulfur clusters. With 
those few classical clusters at hand, electrochemical gradients defining the electron transfer pathways could be constructed by nature through arrangement of the different clusters (with distinct redox potentials) in appropriate distances. The biggest yet known multiple iron-sulfur chain was found in complex I of mammalian NADH dehydrogenase with eight clusters in a row. ${ }^{[56,57]}$ Taking all clusters together found so far in biological systems (including the hybrid cluster proteins ${ }^{[58-60]}$ and the HiPIP enzymes ${ }^{[35]}$ ) nature can cover a wide range of accessible redox potentials $\left(-600 \mathrm{mV}\right.$ to $+450 \mathrm{mV}$ vs. NHE). ${ }^{[61]}$ An accurate fine tuning within this range was readily achieved throughout evolution by modifications of the protein backbone that directly influences the redox potential of the system, e.g. by incorporation of the cluster in a hydrogen bridging framework ${ }^{[62]}$, by methionine S . S S contacts to the bridging cluster sulfides ${ }^{[63]}$ or by modulations of the proteins hydrophobicity. Besides those remarkable possibilities in controlling the electron transfer processes, the corresponding kinetics observed while switching the clusters oxidation states are exceptional as well. Due to electron delocalization ${ }^{[64,65]}$ over the iron and sulfur atoms of the cluster cores, almost constant core geometries are achieved and only minimal reorganizations are necessary in the course of the redox reactions. ${ }^{[66,67]}$ Thus, high-speed electron shifts are assumed to be possible or even essential in cases, where fast successive electron transfer, e.g. to unstable intermediates in the fixation of dinitrogen is required. ${ }^{[17]}$ In addition to the redox functionalities of nearly all clusters, some iron-sulfur sites are specialized for other reactivities, such as substrate activation, ${ }^{[68]}$ non-electron transfer catalysis, ${ }^{[69]}$ sensing of small molecules, ${ }^{[70-72]}$ regulatory functions via specific RNA binding ${ }^{[73]}$, DNA repair ${ }^{[74,75]}$ and SAM-dependent radical processes ${ }^{[76,77]}$ (and most likely other - still to discover - conversions). ${ }^{[8,17]}$

\subsection{Biological Relevant [2Fe-2S] Protein Variants}

Assuming that the broad introduction to the field of iron-sulfur clusters given above allows a fairly accurate positioning of the $[2 \mathrm{Fe}-2 \mathrm{~S}]$ ferredoxins in the entire research area and that provided references facilitate a more detailed exploration of the topic, the discussion will hereafter focus on the binuclear clusters only. Crystallographically, eleven different $[2 \mathrm{Fe}-2 \mathrm{~S}]$ protein folds and six ligand-distinct cluster coordination environments have been identified in the wild-type enzymes so far (Figure 1.1). ${ }^{[78]}$ 

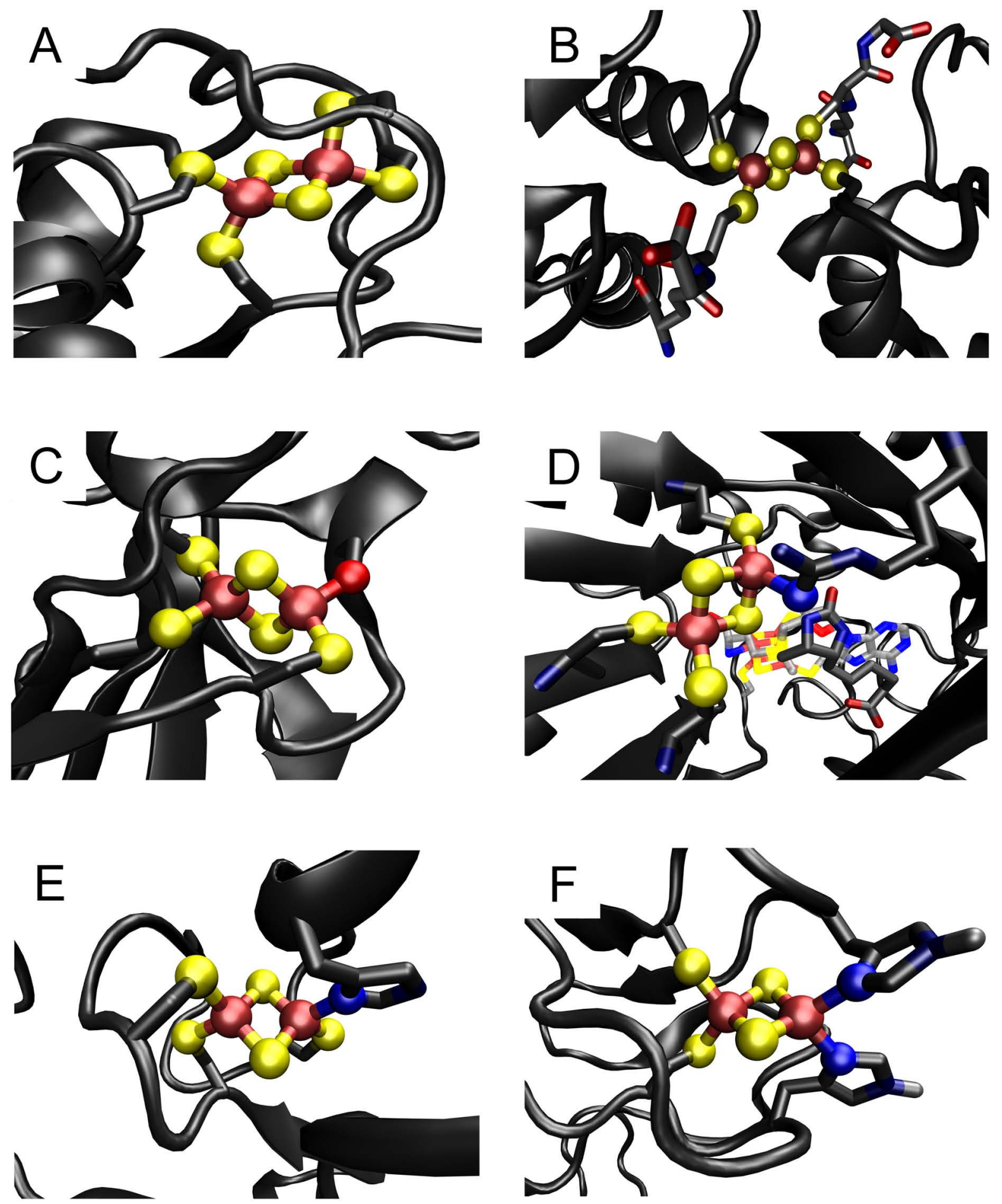

Figure 1.1: Illustration of the biologically relevant [2Fe-2S] variants in their six liganddistinct coordination environments. With exception of $C$, all other structures are nonmutated wild-type proteins characterized by $X$-ray diffraction. The serine-substituted mutant $C$ is added to the diagram as it can be seen as substitute for the so far not crystallized wild-type (Cys) ${ }_{3}$ Asp-ligated [2Fe-2S] ferredoxin from Pyrococcus furiosus with a related terminal $\left\{\mathrm{S}_{3} \mathrm{O}\right\}$-surrounded cluster core. 
The basic all-cysteine-ligated systems are most abundant in nature and found in plants, bacteria and vertebrates. ${ }^{[2,79]}$ Their most prominent task in plants is the distribution of electrons from the photo-reduced photosystem I to several metabolic routes including the NADPH production machinery (linkage between the "dark-reaction" and the "lightreaction"). ${ }^{[0,81]}$ Plant-type and bacterial ferredoxins are also involved in several crucial assimilation processes, while acting as electron carriers. ${ }^{[82]}$ The function of the so-called thioredoxin-like $[2 \mathrm{Fe}-2 \mathrm{~S}]$ family ${ }^{[83]}$ (all-cysteine-ligated as well) is widely obscure, but hints that those clusters are involved in nitrogen-fixation pathways were presented. ${ }^{[84]}$ Most mammalian and bacterial canonically coordinated ferredoxins are biochemically linked to cytochrome P450-monooxygenases ${ }^{[85,86]}$ or hydroxylases, ${ }^{[87]}$ again serving the required redox equivalents. Collectively, these proteins cover negative electrochemical potentials ranging from $-450 \mathrm{mV}$ to $-150 \mathrm{mV}$ vs. $\mathrm{NHE},{ }^{[61]}$ when switching between their all-ferric and their ferric-ferrous oxidation states (see Scheme 1.2). The oxidized form of the [2Fe-2S] cluster from Cyanobacter Anabaena PCC7119 is depicted in Figure 1.1-A as an example (Xray structure refined to $1.3 \AA$ resolution). ${ }^{[88]}$ The corresponding dithionite-reduced form (at $1.17 \AA$ resolution) was refined with a practically identical core geometry. ${ }^{[88]}$ In addi-

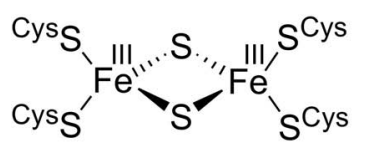

$[2 \mathrm{Fe}-2 \mathrm{~S}]^{2+} \mathrm{S}=0$
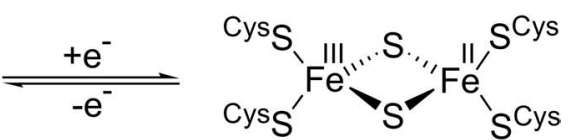

$[2 \mathrm{Fe}-2 \mathrm{~S}]^{1+} S=1 / 2$

Scheme 1.2: Illustration of the electron transfer process in [2Fe-2S] ferredoxins.

tion to the all-cysteine-ligated clusters, a structurally different, but also all- $\{\mathrm{S}\}$-coordinate $[2 \mathrm{Fe}-2 \mathrm{~S}]$ cluster of the thioredoxin superfamily was found in glutaredoxin $\mathrm{C} 1$ from Escherichia coli (Figure 1.1-B). ${ }^{[89]}$ Here, the bimetallic cluster bridges two protein subunits by ligation of two cysteines (one from each subunit) and two glutathiones in a symmetrical fashion, affording an $\alpha 2$ homodimeric protein. In the corresponding apo-enzyme, both cysteine residues are catalytically active in disulfide bond formation. Assumingly, this function is not maintained upon cluster binding during formation of the holo-enzyme. According to the current interpretations, this cluster is most likely involved in sensing the redox state of the cell. ${ }^{[90]}$ During conditions of oxidative stress, the cluster might be destroyed in order to re-activate the disulfide oxidoreductase function of the apo-enzyme. ${ }^{[91]}$ Another hypothesis implies that the apo-protein could serve as a template for cytosolic cluster assembly during [2Fe-2S] biosynthesis. Thus, glutaredoxins might be cluster carriers transferring the iron-sulfur cores to e.g. apo-ferredoxin proteins. ${ }^{[92]}$

Interestingly, only one wild-type protein with partial substitution of the cysteine sulfur donors for a smaller oxygen chalcogen donor was identified so far. ${ }^{[78]}$ In sulfide dehydrogenase from Pyrococcus furiosus the presence of an asymmetrically coordinated [2Fe- $2 \mathrm{~S}]$ cluster with $\operatorname{Asp}(\mathrm{Cys})_{3}$ ligand environment is evident from comparative sequence analysis and spectroscopic studies: ${ }^{[93]}$ An unusual positive redox potential $E_{1 / 2}=+80 \mathrm{mV}$ vs. NHE at $\mathrm{pH}=8$ and widely anisotropic EPR $g$-factors $\left(g_{1}=2.035, g_{2}=1.908, g_{3}=1.786\right)$ for the reduced enzyme $(S=1 / 2)$ were observed, reminiscent of the Rieske-type cluster, that are 
discussed below. Despite an involvement in the sulfur metabolism as a critical component, the function of this enzyme remains unclear. ${ }^{[93]}$ Detailed structural information based on $\mathrm{X}$-ray diffraction is currently not available. Thus it also remains obscure if the aspartic acid residue coordinates via one carboxylic oxygen atom or in a chelating mode. As a substitute for this $\left\{\mathrm{S}_{3} \mathrm{O}\right\}$-ligated cluster, the serine-substituted mutant of a thioredoxin-like [2Fe-2S] ferredoxin from Aquifex aeolicus is shown in Figure 1.1-C. ${ }^{[94]}$ Even so the latter cluster is biologically irrelevant, a unique phenomenon observed for the reduced cluster of this mutant should be noted here: In contrast to the generally detected antiferromagnetic coupling between the ferric and the ferrous ion with an $S=1 / 2$ ground state, ${ }^{[95]}$ a ferromagnetic coupling with an $S=9 / 2$ ground state is evident for this $\left\{\mathrm{S}_{3} \mathrm{O}\right\}$-coordinate system. ${ }^{[94]}$ Although several theoretical studies focusing on this experimental fact were presented, ${ }^{[96]}$ an insightful intuitive explanation for this curiosity has not been provided so far.

In 2004, another novel [2Fe-2S] cluster in a unique $\operatorname{Arg}(\mathrm{Cys})_{3}$ ligand environment was crystallized as a component of biotin synthase (Figure 1.1-D). ${ }^{[97]}$ Although adequate care in the interpretation of the protein's structural parameters should be taken, since refinement could only be performed to a resolution of $3.4 \AA$, an unusual arginine-coordination of the $[2 \mathrm{Fe}-2 \mathrm{~S}]$ core is apparently present. Most likely, a hydrogen bridge between an arginine-bound proton and one of the cluster's bridging sulfides truly exists, whereas the atypical large Fe $\cdots$ Fe separation $(>3.5 \AA$, regularly $<2.8 \AA$ ) probably is an artifact of refinement restraints (the latter structural interpretation is not based on literature documented assumptions, but on a private communication on a so far unpublished structure of the related $\operatorname{Arg}(\mathrm{Cys})_{3}$-ligated [2Fe-2S] cluster found in the radical SAM protein $\left.H y d E\right)$. In concert with an SAM-ligated [4Fe-4S] cluster, also embedded in the enzyme-scaffold, ${ }^{[98-100]}$ biotin synthase promotes the insertion of a sulfur atom into dethiobiotin (co-crystallized with the protein and recognizable in the background of Figure 1.1-D). ${ }^{[101]}$ According to the current mechanistic proposal (Scheme 1.3), the [2Fe-2S] cluster is partially destructed during this process by successive twofold radical attack and consequential extraction of one of its bridging sulfides that simultaneously is introduced into the biotin product. ${ }^{[76]}$ The fate of the residual mono- $\mu$-sulfido-bridged $[2 \mathrm{Fe}-1 \mathrm{~S}]$ cluster is not elucidated by now, loss of the cluster ("suicide-enzyme", "single-turnover enzyme", "[2Fe-2S] cluster as a substrate", etc.) however seems to be unlikely compared to cluster regeneration by subsequent sulfur re-insertion through yet unknown machineries, e.g. specialized cysteine desulfurases. ${ }^{[102]}$

Recently, a third $(\mathrm{Cys})_{3} \mathrm{X}_{1}$-coordinate $[2 \mathrm{Fe}-2 \mathrm{~S}]$ cluster with $\mathrm{X}=$ His was identified as active site in a mitochondrial membrane protein, named mitoNEET ("mito" for mitochondrial and "NEET" for a sequence motive Asn-Glu-Glu-Thr). Its structure has been ascertained by X-ray diffraction (refined to $1.8 \AA$ resolution, Figure 1.1-E). ${ }^{[103]}$ The protein's function is presently unknown, but recognition of specific drugs (e.g. plioglitazone) for type 2 diabetes treatments by the $\alpha 2$ homodimeric enzyme was shown. ${ }^{[104]}$ On the other hand, analogous (Cys) ${ }_{3}$ His cluster binding motives with nearly identical sequences were found in over hundred proteins in bacteria as well as in eukaryotes, suggesting a more wide-spread occurrence of this cluster and a more general electron transfer function. ${ }^{[103,105]}$ The redox potential and the EPR characteristics of mitoNEET were found accurately positioned in between those for the $\mathrm{Cys}_{4}$-ligated ferredoxins and the $(\mathrm{Cys})_{2}(\mathrm{His})_{2}$-ligated Rieske-type $[2 \mathrm{Fe}-2 \mathrm{~S}]$ clusters (discussed in the following paragraph). ${ }^{[106]}$ To the best of the author's 

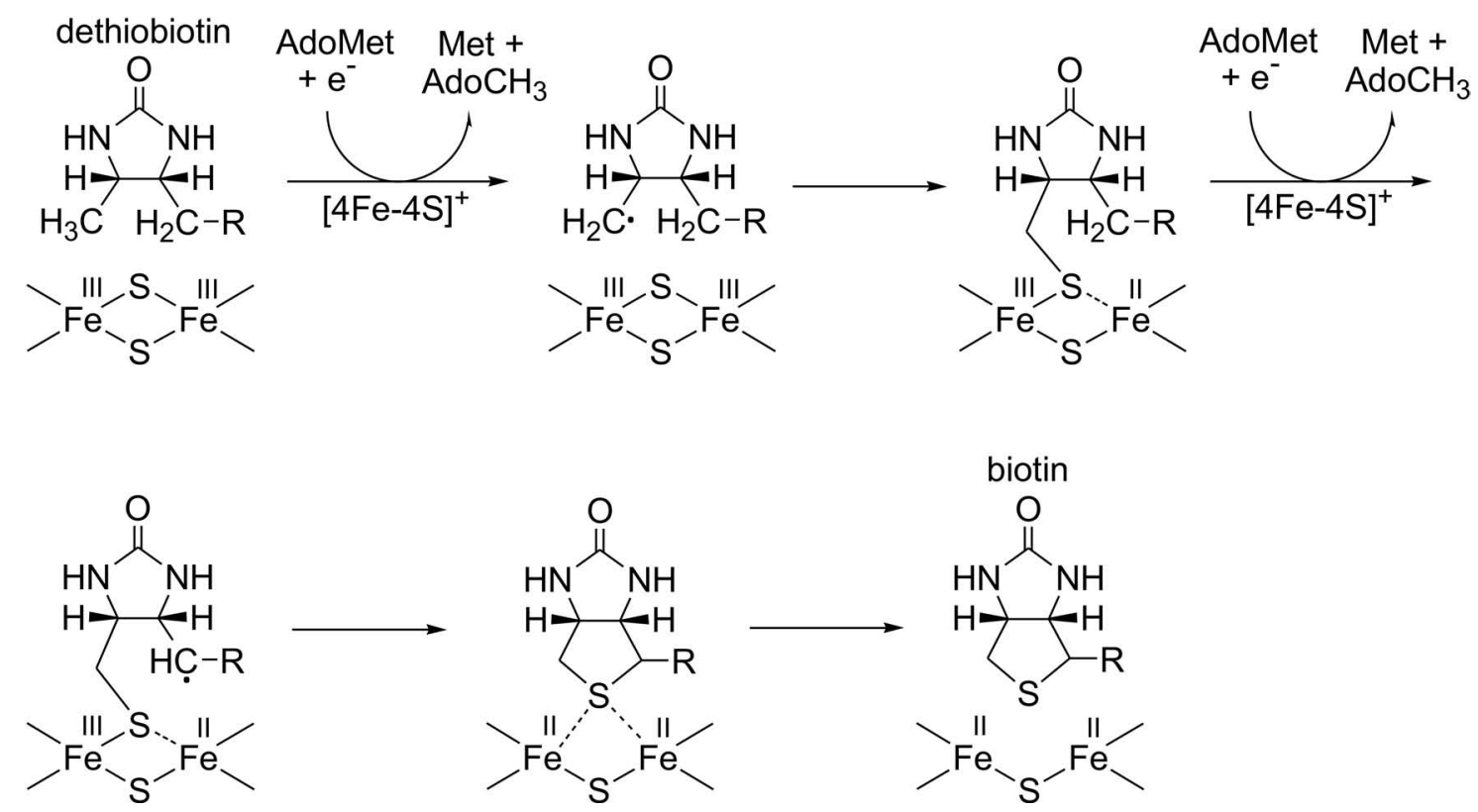

Scheme 1.3: Proposed mechanism for the formation of biotin form dethiobiotin promoted by the radical SAM enzyme biotin synthase.

knowledge, neither studies regarding the pH-dependence of mitoNEET's redox potential nor statements regarding the protonation state of the peripheral histidine nitrogens were hitherto presented in literature.

With exception of the all-cysteine-coordinate ferredoxins, the asymmetrically $(\mathrm{Cys})_{2}(\mathrm{His})_{2}-$ ligated Rieske-type clusters mentioned above represent the - so far - most intensively studied $[2 \mathrm{Fe}-2 \mathrm{~S}]$ proteins, ${ }^{[107,108]}$ discovered by RIESKE et al. already in 1964. ${ }^{[109]}$ Structurally they differ from the parent ferredoxins by an asymmetrical coordination environment, with one iron ligated by two (partially) protonated histidine residues and the other one ligated by two cysteine thiolates (Figure 1.1-F). ${ }^{[10]}$ Rieske proteins were initially identified as crucial subunits of the mitochondrial respiratory chain (cytochrome $b c_{1}$ unit $^{[111]}$ ) and the photosynthetic electron transfer complexes (cytochrome $b_{6} f$ unit $^{[112]}$ ), but subsequently found in oxygenases ${ }^{[13]}$ as well. In the former electron transfer machineries, Rieske sites act as primary electron acceptors during hydroquinone (ubihydroquinone or plastohydroquinone) oxidation. ${ }^{[114]}$ In both systems, the active Rieske core is located close to the protein surface with the cluster bound histidine $\varepsilon-\mathrm{NH}$ groups (peripheral imidazole nitrogens) exposed to the medium. ${ }^{[115]}$ In cytochrome $b c_{1}$ a movement of the catalytic Rieske domain was demonstrated by EPR spectroscopy on oriented samples involving three distinct states. ${ }^{[116,117]}$ In the ' $c_{1}$ positional state' and in the ' $b$ positional state' the exposed $\varepsilon-\mathrm{NH}$ protons are adequately arranged for hydrogen bonding to the heme $c_{1}$ complex and to the ubiquinone binding site in cytochrome $b$, respectively. According to current interpretations, comparable hydrogen bonding interactions with the environment or other subunits are absent in the third state, termed 'intermediate state'.[118, 119] Most likely, similar hydrogen bonding arrangements to e.g. plastohydroquinone also play a role in the Rieske domain integrated in cytochrome $b_{6} f,{ }^{[112,120]}$ a photosynthetic machinery performing the electron transfer be- 
tween both photosystems. For naphthalene 1,2-dioxygenase, ${ }^{[121]}$ a linkage between the mono-iron oxygenase component and the Rieske [2Fe-2S] protein via hydrogen bonding to an exposed single aspartate residue of the former protein subunit is evident from X-ray diffraction analysis (Scheme 1.4). ${ }^{[113,122]}$

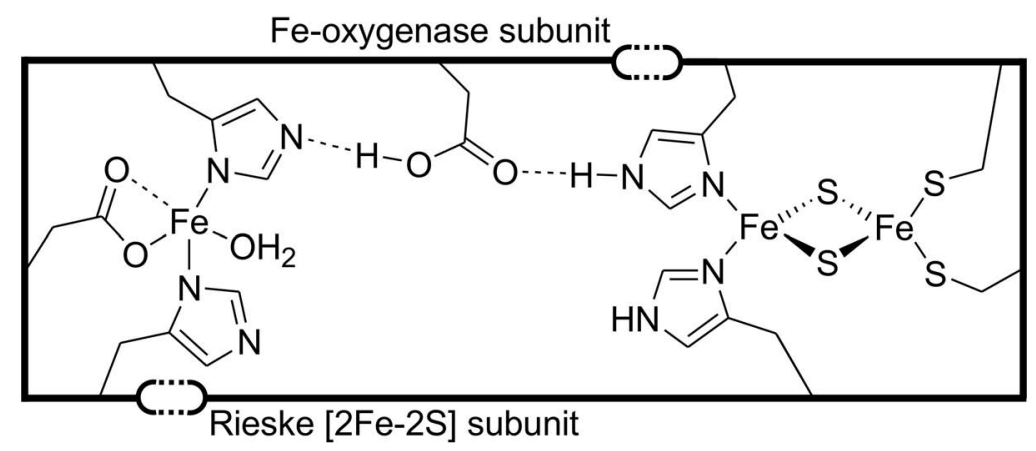

Scheme 1.4: Hydrogen bonding interaction between the Rieske and the mono-iron oxygenase subunit in naphthalene 1,2-dioxygenase.

Due to the (partial) protonation of the histidine residues, ${ }^{[120]}$ the incorporation of those histidines into hydrogen bonding frameworks ${ }^{[62]}$ and the proposed proton-assisted electron transfer mechanism, ${ }^{[123]}$ redox potentials of the biological Rieske sites (approximately $-100 \mathrm{mV}$ vs. NHE for oxygenase-type Rieske clusters, approximately $+250 \mathrm{mV}$ vs. NHE for mitochondrial and photosynthetic Rieske sites) are shifted to more positive values compared to the all-cysteine ferredoxins ${ }^{[23]}$ (approximately $-500 \mathrm{mV}$ to $-200 \mathrm{mV}$ vs. NHE). ${ }^{[24]}$ Protonation-deprotonation equilibria (studied by ${ }^{15} \mathrm{~N}$ NMR spectroscopy on isotopic labeled histidine residues ${ }^{[125]}$ ) result in a $\mathrm{pH}$-dependence of the redox potentials. The reduced $[2 \mathrm{Fe}-2 \mathrm{~S}]^{1+}$ Rieske cluster is valence-localized $(S=1 / 2$ ground state) with the ferrous iron ligated by the histidine residues. ${ }^{[126]}$ The corresponding EPR spectra exhibit wide $g$ anisotropies (e.g. $g_{1}=2.020, g_{2}=1.900, g_{3}=1.800$ for the reduced Rieske site in Thermus thermophilus) and low averaged $g$-values (commonly $\left.g_{\mathrm{av}} \simeq 1.91\right) .{ }^{[127]}$

\subsection{Synthetic $[2 \mathrm{Fe}-2 \mathrm{~S}]$ Cluster Analogues}

Especially in the beginning of the iron-sulfur protein research, biomimetic iron-sulfur chemistry has been an extremely valuable approach promoting a detailed understanding of the cluster's chemical properties. With exception of the $[8 \mathrm{Fe}-7 \mathrm{~S}] \mathrm{P}^{\mathrm{N}}-\mathrm{Cluster},{ }^{[128]}$ model compounds of all other canonical systems were synthesized shortly after their discovery in nature. In the models, cysteine residues are mostly mimicked by aliphatic and aromatic thiols. ${ }^{[129]}$ Although synthetic clusters are usually air- and moisture-sensitive, their basic characteristics essentially reflect the observations made for the aqueous and sporadically air-stable biological counterparts. Since the outstanding work on synthetic iron-sulfur clusters reported by HoLM and coworkers over the last thirty years is that wide-ranging, it can not be summarized herein, but it is referred to an excellent review article. ${ }^{[129]}$ As this contribution exclusively concentrates on synthetic $[2 \mathrm{Fe}-2 \mathrm{~S}]$ cluster analogues, a brief 
outline of the literature-known milestones (Scheme 1.5) in this subfield however appears indispensable at this moment: In 1973, the first biomimetic [2Fe-2S] cluster 1 with terminal chelating sulfur-ligand caps was synthesized starting from ferric chloride, elemental sulfur and the corresponding deprotonated xylyl- $\left\{\mathrm{S}_{2}\right\}$-ligand I. ${ }^{[130,131]}$ This preparation unambiguously demonstrated that $[2 \mathrm{Fe}-2 \mathrm{~S}]$ clusters are intrinsically stable even without a protective protein environment. A consecutive complete characterization of $\mathbf{1}$ has proven that basic features of the biological all-cysteine-ligated ferredoxins are properly mimicked by 1. ${ }^{[132-136]}$ The second milestone, the all-halide-ligated $\left[\mathrm{Fe}_{2} \mathrm{~S}_{2} \mathrm{Cl}_{4}\right]^{2-}$ system 2 was initially obtained by treatment of the xylyl- $\left\{\mathrm{S}_{2}\right\}$-ligated cluster $\mathbf{1}$ with benzoic chloride. ${ }^{[137]}$ Subsequently, a straight forward synthesis of $\mathbf{2}$, starting from monomeric ferric salts and hexamethyldisilathiane was developed affording the product in high yields. ${ }^{[138]}$ The latter compound was then used in salt metathesis reactions with deprotonated sulfur, oxygen and nitrogen ligands affording the corresponding homoleptic $\{\mathrm{S}\}-,\{\mathrm{O}\}$ - and $\{\mathrm{N}\}$-ligated cluster compounds (heteroleptic, but symmetrically $\left\{\mathrm{N}_{2} \mathrm{O}_{2}\right\}$ - and $\left\{\mathrm{N}_{2} \mathrm{~S}_{2}\right\}$-coordinate compounds were also obtained, when chelating $\{\mathrm{NO}\}$ - or $\{\mathrm{NS}\}$-ligands were applied). ${ }^{[129]}$ The first mixed-valent species have been generated and studied in situ by GIBSON and coworkers. Although this highly important development is hardly reflected by a specific cluster molecule, it certainly represents an equivalent breakthrough in synthetic [2Fe-2S] chemistry. ${ }^{[139,140]}$ In 2005, the first neutral [2Fe-2S] system 3, ${ }^{[34]}$ symmetrically ligated by two hexamethyldisilamides and two tetramethylthiourea molecules was synthesized by TATSUmi and coworkers. Although no principally new coordination environment was established with the preparation of $\mathbf{3}$, an improved solubility of this system in less polar solvents (e.g. toluene) assumingly allows novel reactivity studies with highly reactive reagents, that previously could not be conducted in polar MeCN or DMF solutions of the dianionic clusters.

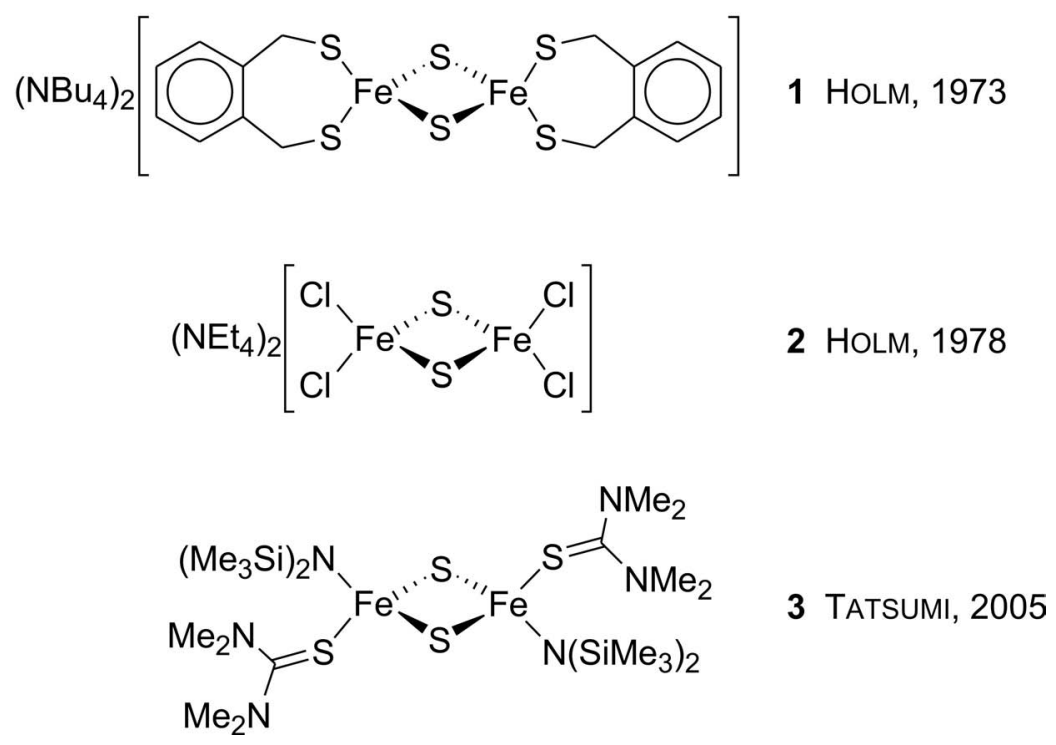

Scheme 1.5: Milestones in synthetic [2Fe-2S] cluster chemistry. 


\subsection{Contemporary Biomimetic [2Fe-2S] Chemistry: Focus of this Contribution}

As mentioned above, biomimetic [2Fe-2S] chemistry was by now particularly successful in reproducing the all-ferric ferredoxin-type cluster cores. Although several synthetic procedures affording those all-\{S\}-ligated cluster compounds are known, most preparations require copious recrystallization steps in order to isolate analytically pure products. However, a generally desired convenient pathway affording the thiophenolate-coordinate systems is available by now and presented in Chapter 2. Ligand effects on prominent spectroscopic characteristics of related $\{\mathrm{S}\}$-ligated clusters were studied by introduction of electron-withdrawing and electron-donating substituents into chelating dithiophenolate ligands (Chapter 3). As stated already, neutral [2Fe-2S] cluster compounds are expected to open up novel opportunities in reactivity studies due to their improved solubility in commonly unreactive solvents. Therefore, efforts to synthesize similar, but chelate-stabilized uncharged [2Fe-2S] systems were conducted and summarized in Chapter 4. Despite those synthetic developments, predominantly appealing to the bioinorganic iron-sulfur community, current $[2 \mathrm{Fe}-2 \mathrm{~S}]$ chemistry focuses on the examination of the protein's secondary interactions with the cluster cores, on mimicking radical reactions as observed in biotin synthase, on the isolation of a one-electron reduced synthetic [2Fe-2S] cluster and last but not least on the imitation of asymmetrical coordination environments as present in the biological (Cys) ${ }_{3} \mathrm{X}_{1}$-type and Rieske-type clusters. Interestingly, only very few studies concerning the interactions of the protein environment with the active sites by e.g. hydrogen bonding, S -.S contacts or other secondary bonding contacts were conducted so far. Thus, a detailed analysis with respect to possible secondary bonding interactions of neutral donor sites incorporated in ridig $\left\{\mathrm{S}_{2}\right\}$-ligand scaffolds was carried out and is presented in Chapter 5. Monomeric iron complexes, coordinated by the same ligands were initially isolated as side-products during the latter experiments. Their unusual magnetic properties are discussed in Chapter 6. Beyond those studies, the synthesis of a model compound for mixed-valent (ferric-ferrous) $[2 \mathrm{Fe}-2 \mathrm{~S}]$ clusters is generally considered as a pending task to be achieved by synthetic iron-sulfur chemists. Although generation of one-electron reduced species and their EPR-identification was presented in literature over twenty years ago, no stable isolable systems consent to crystallization and X-ray diffraction analysis are presently known. Efforts to obtain such species with terminal nitrogen coordination are presented in Chapter 7. Finally, asymmetrically coordinated $[2 \mathrm{Fe}-2 \mathrm{~S}]$ clusters were approached. After extensive ligand screening, a chelating $\left\{\mathrm{N}_{2}\right\}$-ligand was discovered tolerating the isolation of a heteroleptic $\left\{\mathrm{N}_{2} \mathrm{Cl}_{2}\right\}$-ligated cluster with both exchangeable halide substituents attached to the same iron atom. Subsequently, a first accurate $\left\{\mathrm{N}_{2} \mathrm{~S}_{2}\right\}$-coordinate analogue of Rieske-type clusters could be obtained by consecutive salt metathesis utilizing the latter $\left\{\mathrm{N}_{2} \mathrm{Cl}_{2}\right\}$-substituted intermediate (Chapter 8). 


\section{Chapter 2}

\section{A Convenient Ligand Exchange Pathway to [2Fe-2S] Ferredoxin Analogues}

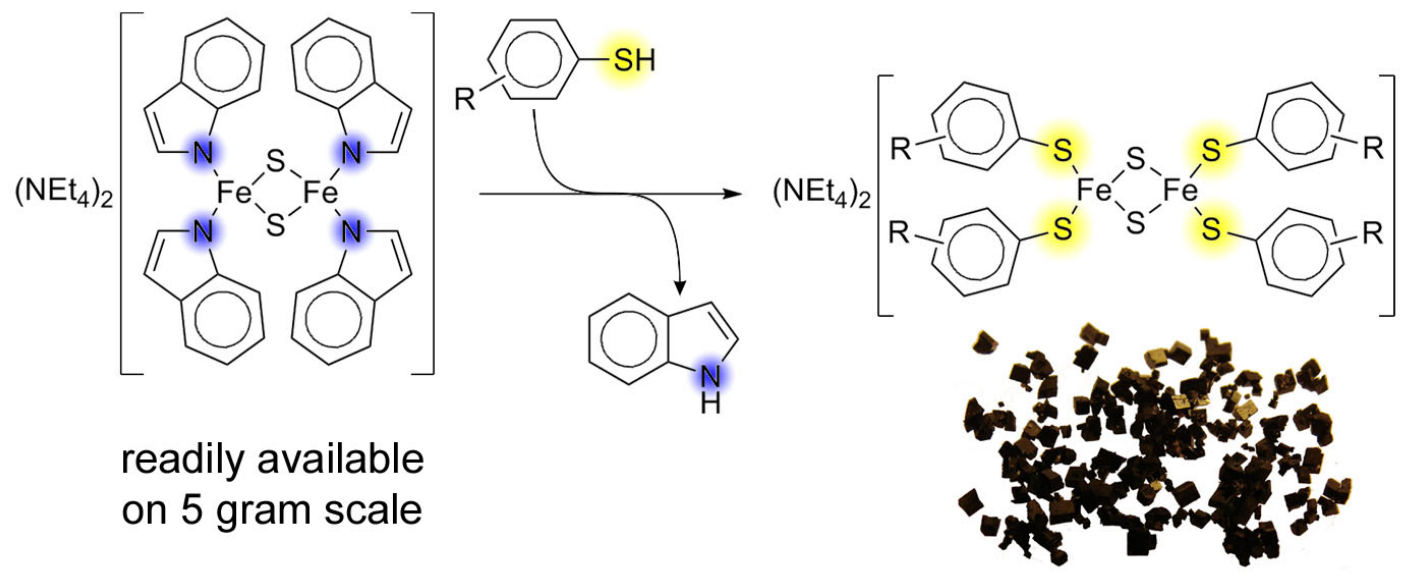

\section{Abstract}

The benzanellated analogues $\left.\left(\mathrm{NEt}_{4}\right)_{2}\left[\mathrm{Fe}_{2} \mathrm{~S}_{2} \text { (indolate }\right)_{4}\right] \mathbf{5}$ and $\left.\left(\mathrm{NEt}_{4}\right)_{2}\left[\mathrm{Fe}_{2} \mathrm{~S}_{2} \text { (carbazolate) }\right)_{4}\right]$ 6 of the previously reported $\left.\left(\mathrm{NEt}_{4}\right)_{2}\left[\mathrm{Fe}_{2} \mathrm{~S}_{2} \text { (pyrrolate) }\right)_{4}\right]$ cluster 4 were synthesized and comprehensively characterized. In contrast to $\mathbf{4}$ and $\mathbf{6}$, compound $\mathbf{5}$ can be applied as common precursor in convenient ligand exchange reactions with various thiophenols affording the thiophenolate-coordinate $[2 \mathrm{Fe}-2 \mathrm{~S}]$ clusters. Heteroaromatic thiols and chelating biphenols are suitable substrates in this conversion as well. 


\subsection{Introduction}

As mentioned in Chapter 1.6, the first synthetic [2Fe-2S] cluster $\mathbf{1}$ has been prepared in 1973 starting from elemental sulfur and a mononuclear iron precursor (generated in situ from ferric chloride and the dianion of the corresponding ligand I). Since this time, a few synthetic pathways to the all- $\{\mathrm{S}\}$-ligated clusters have been developed with the salt metathesis starting from 2 being the most prominent route. Although synthesis of $[2 \mathrm{Fe}-2 \mathrm{~S}]$ cluster compounds (in form of their crude products) is straight forward in some cases, isolation of pure target clusters often is quite inconvenient, frequently including several recrystallization steps. In consequence, a readily accessible library of synthetic [2Fe-2S] clusters covering a wide range of physical and chemical cluster properties (e.g. solubility, kinetic ligand lability, electrochemical potential, etc.) is missing. As rapidly available cluster families are required, inter alia for biomimetic reactivity studies, the exploration of alternative synthetic routes that allow a convenient isolation of entire cluster libraries is of general interest for iron-sulfur chemists. Thus, a convenient ligand exchange pathway - including an easy work-up procedure - to synthetic [2Fe-2S] ferredoxin analogues (starting from a novel indolate-coordinate $[2 \mathrm{Fe}-2 \mathrm{~S}]$ precursor) has been developed and is described in the following.

\subsection{Synthesis and Characterization of $\{\mathrm{N}\}$-Ligated [2Fe-2S] Precursors}

In the design of a suitable $[2 \mathrm{Fe}-2 \mathrm{~S}]$ precursor for ligand exchange reactions the following factors were considered as advantageous for an efficient conversion: (i) Terminal ligands should be monodentate instead of chelating in order to avoid highly negative reaction entropies, (ii) $p K_{\mathrm{a}}$ values of the free terminal ligands should be lower than the $p K_{\mathrm{a}}$ values of the free thiophenol-substrates in order to shift possible equilibria towards the thiophenolate-ligated $[2 \mathrm{Fe}-2 \mathrm{~S}]$ product species, (iii) decomposition of the $[2 \mathrm{Fe}-2 \mathrm{~S}]$ precursor to e.g. $[4 \mathrm{Fe}-4 \mathrm{~S}]$ clusters should be negligible on the timescale of the ligand exchange reaction. At a first glance, the literature-known pyrrolate-ligated species $\left.\left(\mathrm{NEt}_{4}\right)_{2}\left[\mathrm{Fe}_{2} \mathrm{~S}_{2} \text { (pyrrolate) }\right)_{4}\right] 4^{[141,142]}$ seemed to serve these requirements, in preliminary experiments however, compound 4 proved to be relatively unstable in (deuterated) MeCN solution (decomposition within hours with liberation of free pyrrole was observed in the proton NMR spectra at room temperature). Therefore the indolate and carbazolate derivatives $\left.\left(\mathrm{NEt}_{4}\right)_{2}\left[\mathrm{Fe}_{2} \mathrm{~S}_{2} \text { (indolate }\right)_{4}\right] \mathbf{5}$ and $\left.\left(\mathrm{NEt}_{4}\right)_{2}\left[\mathrm{Fe}_{2} \mathrm{~S}_{2} \text { (carbazolate) }\right)_{4}\right] 6$ were synthesized and explored (Scheme 2.1). Synthesis of $\mathbf{5}$ and $\mathbf{6}$ was carried out by standard salt metathesis reactions (starting from $\mathbf{2}^{[138]}$ ) in MeCN/THF mixtures with both target compounds precipitating during the course of the reactions. Subsequent filtration and rinsing with $\mathrm{Et}_{2} \mathrm{O}(\mathbf{5})$ or crystallization from $\mathrm{DMF} / \mathrm{Et}_{2} \mathrm{O}(\mathbf{6})$ afforded the pure products.

Both new complexes were characterized by ESI mass spectrometry, proton NMR spectroscopy, cyclic voltammetry, UV-Vis spectroscopy (Figure 2.1), magnetic susceptibility measurements and Mössbauer spectroscopy. Prominent spectroscopic and electrochemical data 


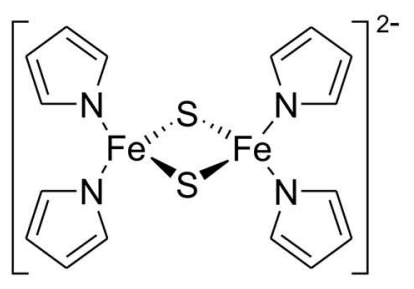

4

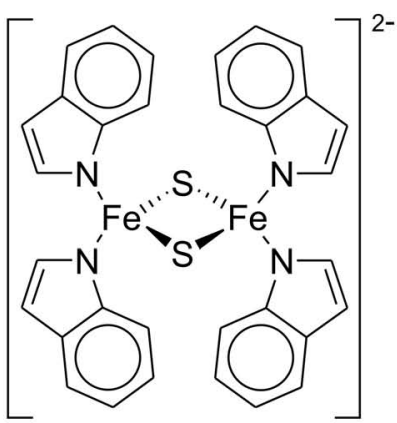

5

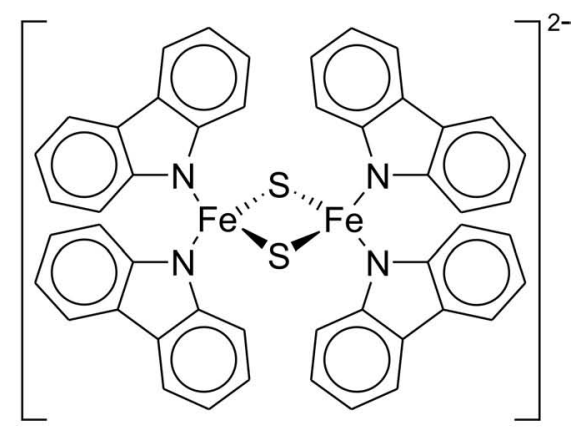

6

Scheme 2.1: Possible $\{N\}$-ligated [2Fe-2S] cluster precursors for ligand exchange reactions. $\mathrm{NEt}_{4}^{+}$cations were applied as counter ions.

are summarized in Table 2.1. Compared to 4 , the $\sigma$-electron donating effects of the terminal ligands increase in the row $\mathbf{4}<\mathbf{5}<\mathbf{6}$ and therefore increase the total electron density on the central $[2 \mathrm{Fe}-2 \mathrm{~S}]$ core in the same order. However, reverse $\pi$-electron withdrawing effects and an extended electron delocalization in the benzanellated systems complicate the interpretation of the observed spectroscopic properties. Nevertheless, prominent characteristics of 4-6 show discrete trends: (i) Transition energies for the visible absorptions, which were assigned previously to ligand-to-core charge transfer transitions, ${ }^{[144]}$ decrease in the order $\mathbf{4}>\mathbf{5}>\mathbf{6}$. Thus, a red-shift is observable with naked eye and quantified by UV-Vis spectroscopy (Figure 2.1). Solutions of 4 appear deep red with a maximum at $\simeq 490 \mathrm{~nm}$, whereas solutions of $\mathbf{5}$ are reddish purple (maximum at $520 \mathrm{~nm}$ ) and solutions of 6 are colored purple-blue (maximum at $548 \mathrm{~nm}$ ). (ii) Isomer shifts $\delta$ (obtained from spectral fits to zero-field Mössbauer data using Lorentzian line doublets) increase in the row 4 $(0.26 \mathrm{~mm} / \mathrm{s})<\mathbf{5}(0.27 \mathrm{~mm} / \mathrm{s})<\mathbf{6}(0.30 \mathrm{~mm} / \mathrm{s})$, reflecting an increasing d-electron density at the core positions. Interestingly, the corresponding quadrupole splittings $\Delta E_{\mathrm{Q}}$ for $\mathbf{5}$ and 6 are somewhat higher $(\simeq 0.6 \mathrm{~mm} / \mathrm{s})$ than that one determined for $\mathbf{4}(\simeq 0.5 \mathrm{~mm} / \mathrm{s})$. This trend probably originates from altered ligand orientations (caused by an increasing steric bulk) rather than from electronic effects. (iii) Cathodic peak potential increase in the order $\mathbf{4}<\mathbf{5}<\mathbf{6}$, assumingly due to the increasing electron delocalization over the expanding $\pi$-systems, favoring the reduction to the mixed-valent species.

In order to gain further insight into solution stabilities of $\mathbf{5}$ and $\mathbf{6}$ (considered as most important characteristics regarding the subsequent ligand exchange studies) proton NMR and ESI mass spectra were recorded. Compound 5 rapidly decomposes in DMSO- $\mathrm{d}_{6}$ by liberation of free indole, however diluted MeCN (solubility $\simeq 2 \mathrm{mg} / \mathrm{ml}$ ) or DMF (solubility $\simeq 6 \mathrm{mg} / \mathrm{ml}$ ) solutions of 5 are stable over night at room temperature as indicated by ESI mass spectrometry (the moderate solubility in MeCN and DMF prevents proton NMR studies in $\mathrm{MeCN}-\mathrm{d}_{3}$ or $\mathrm{DMF}-\mathrm{d}_{7}$ ). Therefore $\mathbf{5}$ seems to be suitable for further ligand exchange reactions if carried out in the latter solvents, whereas restricted solubility of 6 (moderately soluble in DMF and DMSO, insoluble in MeCN) renders it inconvenient for further studies, although solutions of $\mathbf{6}$ are stable in DMF and DMSO for days. 


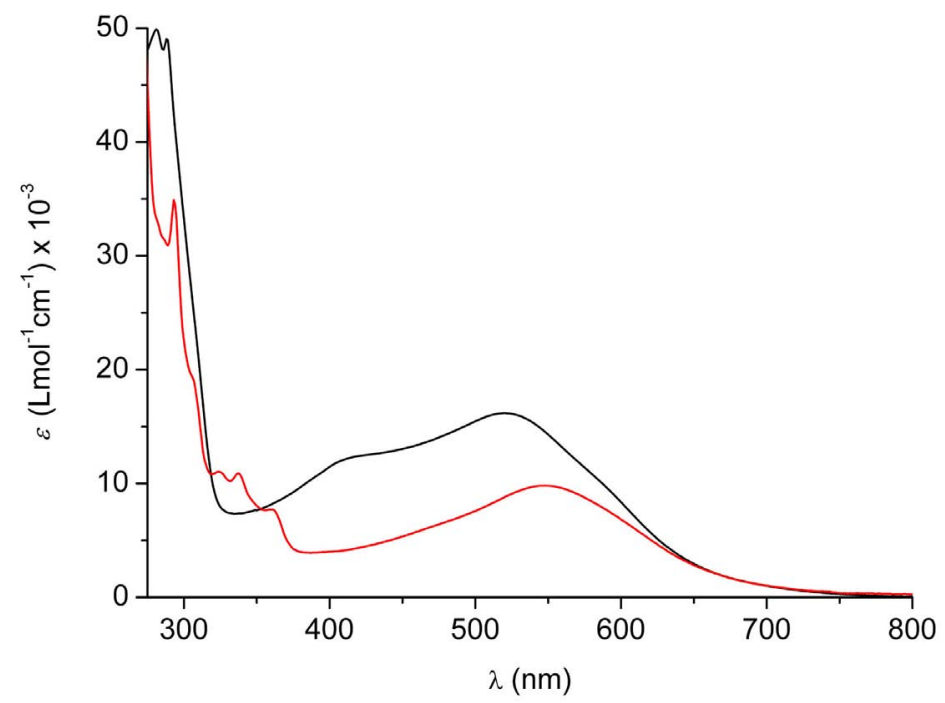

Figure 2.1: Electronic absorption spectra of $\mathbf{5}$ (black line) and $\mathbf{6}$ (red line) in DMF solution.

Table 2.1: Spectroscopic and electrochemical data for complexes 4-6.

\begin{tabular}{ccccc}
\hline compound & $\delta\left(\Delta E_{\mathrm{Q}}\right)[\mathrm{mm} / \mathrm{s}]$ & $\lambda_{\max }[\mathrm{nm}]\left(\varepsilon\left[\mathrm{M}^{-1} \mathrm{~cm}^{-1}\right]\right)$ & $J\left[\mathrm{~cm}^{-1}\right]^{\mathrm{e}}$ & $E_{1 / 2}[\mathrm{~V}]$ \\
\hline $\mathbf{4}^{[141,142]}$ & $0.26(0.49)^{\mathrm{a}}$ & $370(8330), 490(s h, 3290)$, & not & $-1.30^{\mathrm{f}}$ \\
& & $550(s h, 2480)^{\mathrm{c}}$ & reported & \\
$\mathbf{5}$ & $0.27(0.61)^{\mathrm{b}}$ & $281(\simeq 50000), 288(\simeq 50000)$, & -159 & $-1.24^{\mathrm{g}}$ \\
& & $410(\mathrm{sh}, 12225), 520(16200)^{\mathrm{d}}$ & & \\
$\mathbf{6}$ & $0.30(0.60)^{\mathrm{b}}$ & $293(\simeq 35000), 324(11000), 337$ & -199 & $-1.19^{\mathrm{g}}$ \\
& & $(10900), 360(7700), 548(9800)^{\mathrm{d}}$ & & \\
\hline
\end{tabular}

(a) ${ }^{57} \mathrm{Fe}$ Mössbauer parameters at $77 \mathrm{~K}$, relative to Fe metal at room temperature. (b) ${ }^{57} \mathrm{Fe}$ Mössbauer parameters at $80 \mathrm{~K}$, relative to Fe metal at room temperature. (c) Recorded in MeCN solution at room temperature. (d) Recorded in DMF solution at room temperature. (e) Values obtained from fits to SQUID data, see Chapter 9.6. (f) Cathodic peak potential of the irreversible process in DMF $/ 0.1 \mathrm{M} \mathrm{NBu}_{4} \mathrm{ClO}_{4}$ at a scan rate of $200 \mathrm{mV} / \mathrm{s}$ vs. $\mathrm{SCE}$ is $-1.28 \mathrm{~V}$, corresponding to $-1.30 \mathrm{~V}$ vs. the $\mathrm{Cp}_{2}^{*} \mathrm{Fe} / \mathrm{Cp}_{2}^{*} \mathrm{Fe}^{+}$ couple. ${ }^{[145,146]}(\mathrm{g})$ Cathodic peak potential of the irreversible process in DMF / 0.1 M NBu $4 \mathrm{PF}_{6}$ at a scan rate of $200 \mathrm{mV} / \mathrm{s}$ vs. the $\mathrm{Cp}_{2}^{*} \mathrm{Fe} / \mathrm{Cp}_{2}^{*} \mathrm{Fe}^{+}$couple.

\subsection{Ligand Exchange Reactivity of $\left(\mathrm{NEt}_{4}\right)_{2}\left[\mathrm{Fe}_{2} \mathrm{~S}_{2}\right.$ (in- dolate) $\left.)_{4}\right] 5$}

Substitution of the indolates in $\mathbf{5}$ by different thiophenolates is straight forward and was performed by simply mixing both reactants in MeCN (Scheme 2.2, bottom right). Stirring over night, removal of the solvent and separation of free indole by washing with THF / $\mathrm{Et}_{2} \mathrm{O}$ 
(1/3) affords the pure cluster products as black powders. Compared to the most prominent and frequently used synthetic approaches to [2Fe-2S] clusters (Scheme 2.2), this procedure involves several advantages: (i) No salt species are present, except the starting and the target material. This significantly simplifies the work-up procedure since separation of the product cluster salt can be rather cumbersome, involving numerous recrystallization steps if by-products are salts as well (e.g. alkali metal chlorides in the salt metathesis approach, Scheme 2.2, top right). ${ }^{[147]}$ (ii) In contrast to the previously reported ligand exchange strategy starting from $\left(\mathrm{NEt}_{4}\right)_{2}\left[\mathrm{Fe}_{2} \mathrm{~S}_{2}\left(\mathrm{~S}^{t} \mathrm{Bu}\right)_{4}\right]$ (Scheme 2.2, top left), no [4Fe-4S] side products are observed. ${ }^{[148]}$ (iii) Compared to the thiophenolate-to-thiophenolate exchange pathway (Scheme 2.2, top center), no large excess of the thiophenol is required (20 eq vs. $4.4 \mathrm{eq}$ ). ${ }^{[132]}$ This fact seems to be of minor importance, if cheap commercially available thiophenols are applied, but becomes crucial in cases of expensive or unavailable thiophenols, especially if synthesized prior to reaction in multi-step procedures. In this context it should be noted that both approaches starting from monomeric compounds (Scheme 2.2, bottom left and bottom center) also suffer from substantial loss of the free thiophenolate ligand. ${ }^{[22,135]}$ (iv) In contrast to the latter monomer-to-cluster pathways, only one single starting material is suitable for conversion to a variety of products. ${ }^{[149]}$ (v) The progress of those conversions can be monitored conveniently by the naked eye. Prior to conversion soluble amounts of 5 cause a reddish purple coloration of the reaction mixture with most of the starting material still undissolved in MeCN (suspension), whereas after complete reaction the deep red color of the products shows up with no insoluble material present any more (clear solution).

\subsection{Selected Examples, Scope of the Ligand Exchange Reaction and Limitations}

In order to probe the scope of the reaction, thiophenol, a few methyl-substituted thiophenols (varied with respect to the steric bulk) as well as the electron deficient parafluorthiophenol were tested as substrates. Applying the general protocol led to successful isolation of the corresponding $[2 \mathrm{Fe}-2 \mathrm{~S}]$ cluster compounds $\left(\mathrm{NEt}_{4}\right)_{2}\left[\mathrm{Fe}_{2} \mathrm{~S}_{2}(\mathrm{SPh})_{4}\right] \mathbf{7 a},{ }^{[135,150]}$ $\left(\mathrm{NEt}_{4}\right)_{2}\left[\mathrm{Fe}_{2} \mathrm{~S}_{2}\left(\mathrm{SC}_{6} \mathrm{H}_{4} \mathrm{Me}-4\right)_{4}\right] \quad \mathbf{7 b}{ }^{[135]}\left(\mathrm{NEt}_{4}\right)_{2}\left[\mathrm{Fe}_{2} \mathrm{~S}_{2}\left(\mathrm{SC}_{6} \mathrm{H}_{3} \mathrm{Me}_{2}-2,6\right)_{4}\right] \mathbf{7 c}, \quad\left(\mathrm{NEt}_{4}\right)_{2}\left[\mathrm{Fe}_{2} \mathrm{~S}_{2^{-}}\right.$ $\left.\left(\mathrm{SC}_{6} \mathrm{H}_{3} \mathrm{Me}_{2}-3,5\right)_{4}\right] \mathbf{7 d},\left(\mathrm{NEt}_{4}\right)_{2}\left[\mathrm{Fe}_{2} \mathrm{~S}_{2}\left(\mathrm{SC}_{6} \mathrm{H}_{3} \mathrm{Me}_{2}-2,4\right)_{4}\right]$ 7e and $\left(\mathrm{NEt}_{4}\right)_{2}\left[\mathrm{Fe}_{2} \mathrm{~S}_{2}\left(\mathrm{SC}_{6} \mathrm{H}_{4} \mathrm{~F}-4\right)_{4}\right]$ $\mathbf{7} \mathbf{f}^{[151]}$ without difficulties (Scheme 2.3).

Black crystals of $\mathbf{7 c}$ - $\mathbf{7 f}$ suitable for X-ray diffraction were obtained from MeCN $/ \mathrm{Et}_{2} \mathrm{O}$ solutions at room temperature. ORTEP diagrams of their molecular structures are shown in Figure 2.2 (molecular structures for clusters $\mathbf{7} \mathbf{a}^{[152]}$ and $\mathbf{7} \mathbf{b}^{[132]}$ have been reported earlier). Selected atom distances and bond angles for $\mathbf{7 c - 7 f}$ are listed in Table 2.2 (the corresponding values for $\mathbf{7 a}$ and $\mathbf{7 b}$ are provided in Appendix A). All compounds are sulfurbridged dimeric molecules in the solid state. The unit cells contain negatively charged $\left[\mathrm{Fe}_{2} \mathrm{~S}_{2}(\mathrm{SAr})_{4}\right]^{2-}$ ions which are well separated from the charge compensating $\mathrm{NEt}_{4}^{+}$cations. Except for $\mathbf{7 f}$ all complexes have crystallographically imposed inversion symmetry with one $(\mathbf{7 c}, \mathbf{7 d})$ or two half molecules $(\mathbf{7 e})$ in the asymmetric unit. The four-membered $\mathrm{Fe}_{2} \mathrm{~S}_{2}$ 


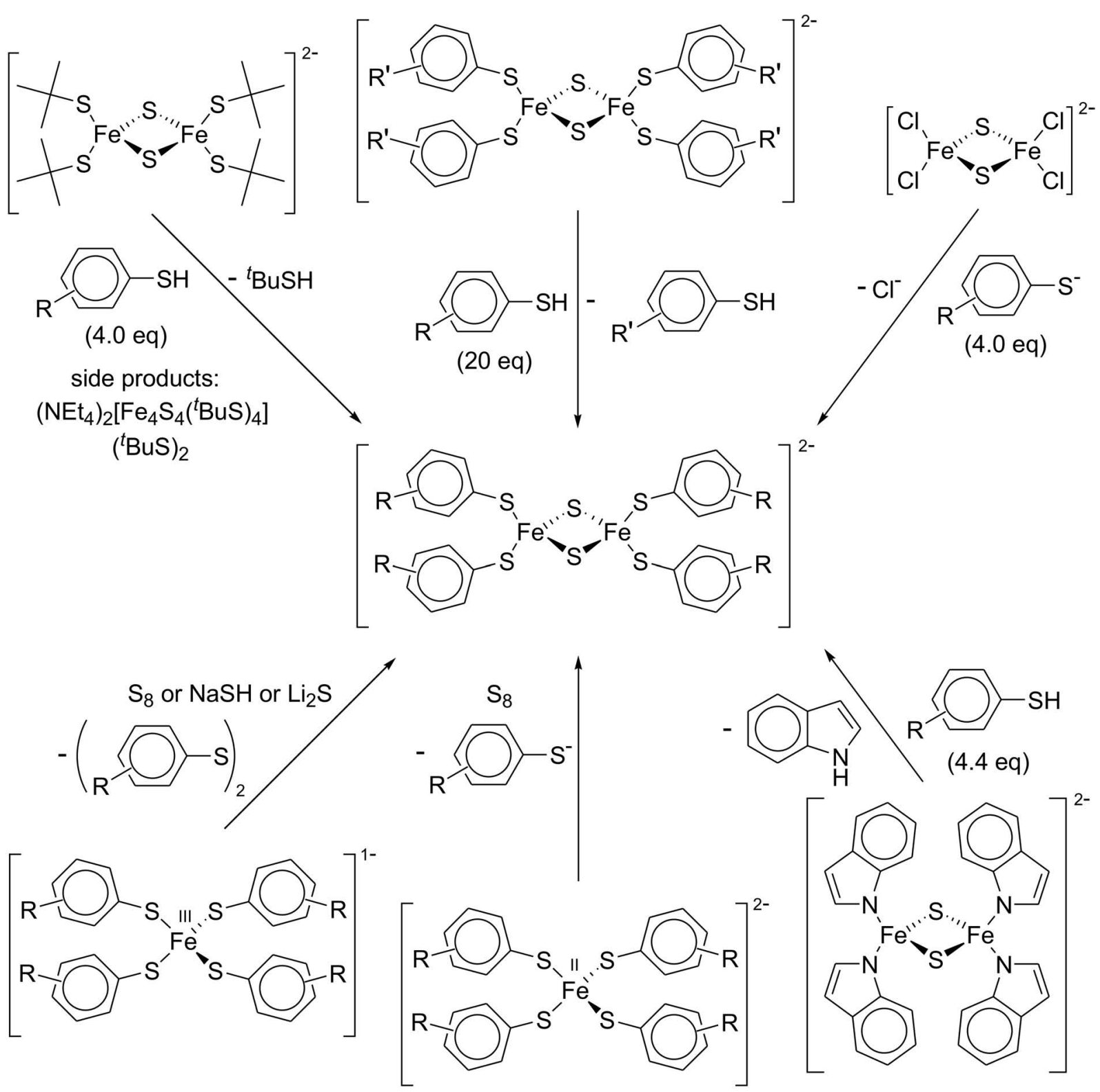

Scheme 2.2: Synthetic pathways to [2Fe-2S] clusters.

rings of $\mathbf{7 c - 7 e}$ are therefore perfectly planar and the sum of the Fe- $(\mu-\mathrm{S})-\mathrm{Fe}$ and $(\mu-\mathrm{S})-\mathrm{Fe}-$ $(\mu-\mathrm{S})$ angles is almost $360^{\circ}$. Although there is no crystallographically imposed symmetry in $7 \mathbf{f}$, the sum of the $\mathrm{Fe}-(\mu-\mathrm{S})-\mathrm{Fe}$ and $(\mu-\mathrm{S})-\mathrm{Fe}-(\mu-\mathrm{S})$ angles again is close to $360^{\circ}$. All iron atoms are four-coordinate (in a distorted tetrahedral geometry), with the two $\left\{\mathrm{FeS}_{4}\right\}$ tetrahedra sharing one edge. Regardless of the electron pushing or withdrawing character of the terminal ligands the distances between the iron and the bridging sulfur atoms differ only slightly among the series, but are approximately $0.1 \AA$ shorter than the Fe-SR bonds. The corresponding $(\mu-\mathrm{S})-\mathrm{Fe}-(\mu-\mathrm{S})$ and RS-Fe-SR angles show an analogous behaviour. Similar metric parameters were reported for $\left[\mathrm{Fe}_{2} \mathrm{~S}_{2}\left(\mathrm{SC}_{6} \mathrm{H}_{4} \mathrm{R}-4\right)_{4}\right]^{2-}\left(\mathrm{R}=\mathrm{H} \mathbf{7 a},{ }^{[152]} \mathrm{Me} \mathbf{7 b},{ }^{[132]}\right.$ $\left.\mathrm{Cl} \mathbf{7 g}{ }^{[153]}\right),\left[\mathrm{Fe}_{2} \mathrm{~S}_{2}\left(\mathrm{SC}_{6} \mathrm{H}_{2} \mathrm{Me}_{3}-2,4,6\right)_{4}\right]^{2-} \mathbf{7} \mathbf{h},{ }^{[154]}\left[\mathrm{Fe}_{2} \mathrm{~S}_{2}\left(\mathrm{SC}_{6} \mathrm{H}_{3}\left(\mathrm{NHC}(\mathrm{O})^{t} \mathrm{Bu}\right)_{2}-2,6\right)_{4}\right]^{2-} \mathbf{7 \mathbf { i } ^ { [ 1 5 5 } ]}$ 


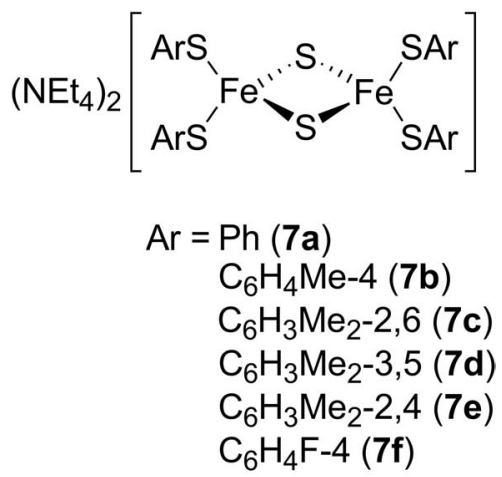

Scheme 2.3: Thiophenolate-coordinate [2Fe-2S] clusters synthesized starting from $\mathbf{5}$.

and $\left[\mathrm{Fe}_{2} \mathrm{~S}_{2}\left(\mathrm{C}_{6} \mathrm{H}_{4}-1,2-\left(\mathrm{CH}_{2} \mathrm{~S}\right)_{2}\right)_{2}\right]^{2-} \mathbf{1}^{[132]}$ (see Appendix A, structural drawings for $\mathbf{7 g}, \mathbf{7} \mathbf{h}$ and $7 \mathbf{i}$ are depicted on page 190). The average distances between the iron and the bridging $(2.20 \AA)$ and terminal sulfur atoms $(2.31 \AA)$ as well as the average $\left(\mu\right.$-S)-Fe- $(\mu-\mathrm{S})\left(104.4^{\circ}\right)$ and RS-Fe-SR $\left(108.6^{\circ}\right)$ angles of all those known compounds agree well with the values determined for 7c-7f. Only the RS-Fe-SR angle of $\left[\mathrm{Fe}_{2} \mathrm{~S}_{2}\left(\mathrm{SC}_{6} \mathrm{H}_{4} \mathrm{Cl}-4\right)_{4}\right]^{2-}\left(100.26(10)^{\circ}\right)$ is somewhat smaller than the mean value. The $\mathrm{Fe} \cdot \mathrm{Fe}$ separations for $\mathbf{7} \mathbf{c}-\mathbf{7 f}$ lie in the narrow range 2.69-2.72 $\mathrm{A}$ that is common to most $[2 \mathrm{Fe}-2 \mathrm{~S}]$ model complexes. Rhomb dimensions in synthetic $[2 \mathrm{Fe}-2 \mathrm{~S}]$ have been discussed earlier. ${ }^{[150]}$

Table 2.2: Selected bond distances $[\AA]$ and angles $\left[{ }^{\circ}\right]$ for $\mathbf{7 c}-\mathbf{7 f}$.

\begin{tabular}{ccccc}
\hline compound & $\mathbf{7 c}$ & $\mathbf{7 d}$ & $\mathbf{7 e}^{\mathrm{a}}$ & $\mathbf{7 f}^{\mathrm{a}}$ \\
\hline \multirow{2}{*}{$\mathrm{Fe} \cdots \mathrm{Fe}$} & $2.716(19)$ & $2.6996(10)$ & $2.7020(5) /$ & $2.6875(4) /$ \\
& $2.311(13) /$ & $2.3023(10) /$ & $2.3225(6)$ to & $2.2968(6)$ to \\
Fe-SR & $2.324(17)$ & $2.3279(9)$ & $2.3313(6)$ & $2.3196(6)$ \\
& $2.19(2) /$ & $2.2018(10) /$ & $2.1969(9)$ to & $2.1942(6)$ to \\
$\mathrm{Fe}-(\mu-\mathrm{S})$ & $2.196(13)$ & $2.2043(10)$ & $2.2158(5)$ & $2.2062(6)$ \\
& & & $110.367(17) /$ & $108.91(2)$ to \\
$\mathrm{RS}-\mathrm{Fe}-\mathrm{SR}$ & $105.3(6)$ & $110.52(4)$ & $112.159(15)$ & $111.28(2)$ \\
& & & $75.42(3) /$ & $75.195(19)$ to \\
$\mathrm{Fe}-(\mu-\mathrm{S})-\mathrm{Fe}$ & $76.6(2)$ & $75.57(3)$ & $76.02(3)$ & $75.672(19)$ \\
& & & $103.98(3) /$ & $104.18(2)$ to \\
$(\mu-\mathrm{S})-\mathrm{Fe}-(\mu-\mathrm{S})$ & $103.4(2)$ & $104.43(3)$ & $104.58(3)$ & $104.83(2)$ \\
\hline
\end{tabular}

(a) Two crystallographically independent molecules.

All complexes have been further characterized by UV-Vis spectroscopy, ${ }^{1} \mathrm{H}$ NMR spectroscopy, ESI MS spectrometry, elemental analysis or HRMS ESI spectrometry and cyclic voltammetry. Selected experimental data are summarized in Table 2.3 (additional data are provided in the experimental section, Chapter 9). Analytical data for the literature-known 

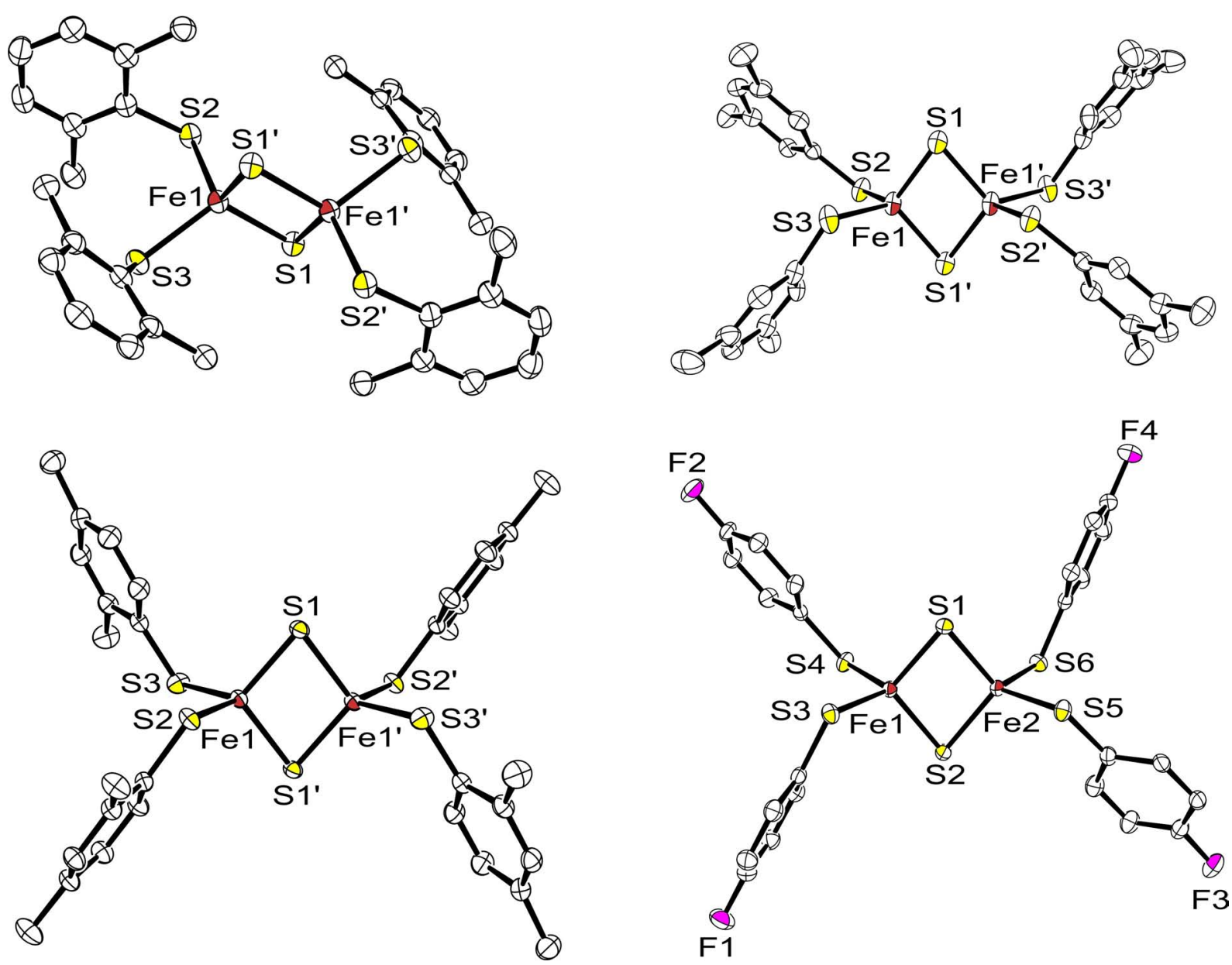

Figure 2.2: ORTEP plots (50\% probability thermal ellipsoids) of the molecular structures of the dianions of $\mathbf{7} \boldsymbol{c}-\boldsymbol{7} \boldsymbol{f}$. All hydrogen atoms have been omitted for clarity.

compound $\mathbf{7 a},{ }^{[150]} \mathbf{7} \mathbf{b}^{[132]}$ and $\mathbf{7} \mathbf{f}^{[151]}$ were found as previously reported and added to Table 2.3 for completeness.

Aliphatic thiols and aliphatic alcohols are unreactive in the above ligand exchange reaction, even if chelating derivatives (e.g. 1,2-phenylenedimethanethiol I) were used. Phenols however are suitable substrates with certain limitations. Acidic phenols (e.g. para-fluorphenol) assumingly protonate the bridging sulfides of $\mathbf{5}$ and therefore promote its' decomposition, whereas usual phenols can be applied. However, reactions proceed not as clean as observed for the corresponding thiophenols, with certain amounts of unidentified insoluble material forming as by-product. Nevertheless, chelating phenolic substrates react straight forward, as demonstrated by the preparation of $\left(\mathrm{NEt}_{4}\right)_{2}\left[\mathrm{Fe}_{2} \mathrm{~S}_{2}\left(1,1^{\prime}-\left(o-\mathrm{O}-\mathrm{C}_{6} \mathrm{H}_{2} \mathrm{Cl}_{2}\right)_{2}\right)_{2}\right]$ 8a. Single crystals of $\mathbf{8 a}$ suitable for X-ray diffraction were obtained after several days by cooling a saturated $\mathrm{MeCN}$ solution of the compound to $-20^{\circ} \mathrm{C}$ (Figure 2.3). Cluster 8a represents the third example of a crystallographically characterized $\{\mathrm{O}\}$-coordinate $[2 \mathrm{Fe}-2 \mathrm{~S}]$ system, besides the corresponding unsubstituted cluster $\left(\mathrm{NEt}_{4}\right)_{2}\left[\mathrm{Fe}_{2} \mathrm{~S}_{2}\left(1,1^{\prime}-\left(o-\mathrm{O}-\mathrm{C}_{6} \mathrm{H}_{4}\right)_{2}\right)_{2}\right] \mathbf{8} \mathbf{b}$ and the oxo-analogue of $\mathbf{7 b}-\left(\mathrm{NEt}_{4}\right)_{2}\left[\mathrm{Fe}_{2} \mathrm{~S}_{2}\left(\mathrm{OC}_{7} \mathrm{H}_{7}\right)_{4}\right] \mathbf{9}^{[141]}$ (structural drawings of $\mathbf{8 b}$ and 
Table 2.3: Mössbauer, UV-Vis and electrochemical data of complexes $\mathbf{7 a - 7 f}$.

\begin{tabular}{lcc}
\hline compound & $\lambda_{\max }[\mathrm{nm}]\left(\varepsilon\left[\mathrm{M}^{-1} \mathrm{~cm}^{-1}\right]\right)^{\mathrm{a}}$ & $E_{\mathrm{p}}^{\mathrm{c}}[\mathrm{V}]^{\mathrm{b}}$ \\
\hline $\mathbf{7 a}^{[150]}$ & $265(\simeq 41500), 330(21300), 481(12000)^{\mathrm{c}}$ & $-1.22^{\mathrm{c}}$ \\
$\mathbf{7 b}^{[132]}$ & $265(\simeq 42000), 335(20400), 488(12000)^{\mathrm{c}}$ & $-1.22^{\mathrm{c}}$ \\
$\mathbf{7} \mathbf{c}$ & $265(\simeq 40200), 287(32000), 333(13000), 418(10800)$ & -1.35 \\
$\mathbf{7 d}$ & $263(\simeq 40000), 332(20300), 473(7800)$ & -1.08 \\
$\mathbf{7 e}$ & $262(\simeq 44700), 340(21600), 432(13100)$ & -0.99 \\
$\mathbf{7} \mathbf{f}^{[151]}$ & $257(s h, \simeq 44200), 310(22400), 460(s h, 10400)^{\mathrm{c}}$ & $-1.25^{\mathrm{c}}$ \\
\hline
\end{tabular}

(a) Recorded in $\mathrm{MeCN}$ solution at room temperature.

(b) Cathodic peak potential recorded in $\mathrm{MeCN} / 0.1 \mathrm{M} \mathrm{NBu}_{4} \mathrm{PF}_{6}$ solution at a scan rate of $100 \mathrm{mV} / \mathrm{s}$ vs. the $\mathrm{Cp}_{2}^{*} \mathrm{Fe} / \mathrm{Cp}_{2}^{*} \mathrm{Fe}^{+}$couple. (c) Values are identical or - in case of different experimental conditions - in agreement with those reported in literature. ${ }^{[132,150,151]}$

$\mathbf{9}$ are depicted on page 191, selected structural parameters of $\mathbf{8} \mathbf{b}$ and $\mathbf{9}$ are provided in Appendix A). In this context it seems to be noteworthy that the related $3,3^{\prime}, 5,5^{\prime}$-tertbutyl-substituted derivative $\mathbf{8} \mathbf{c}$ could not be isolated in numerous attempts - via none of the synthetic routes outlined in Figure 2.2.

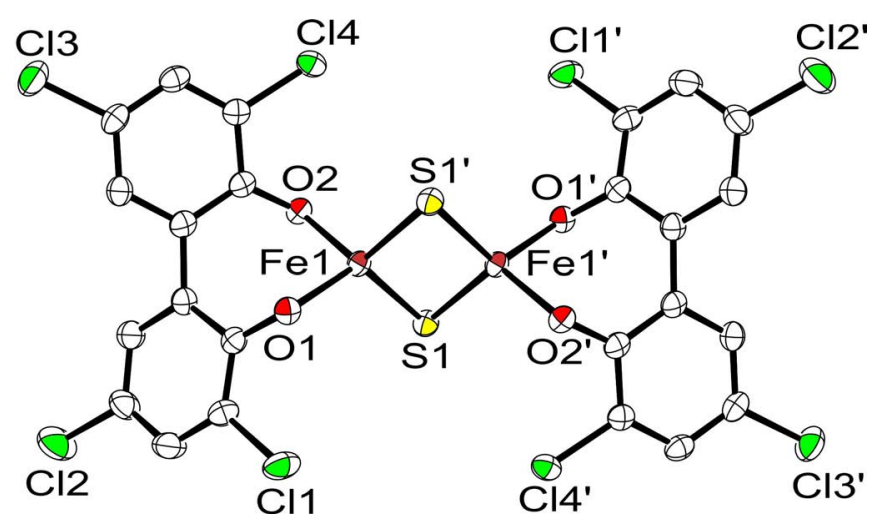

Figure 2.3: ORTEP plot (50\% probability thermal ellipsoids) of the molecular structure of the dianion of $\mathbf{8 a}$. All hydrogen atoms have been omitted for clarity. Two crystallographically independent molecules were found in the asymmetric unit. Equivalent atoms are generated by the $1-x+1,-y,-z$ and the $-x+1,-y+1,-z+1$ symmetry transformation. Selected atom distances $[\AA]$ and interatomic angles $\left[{ }^{\circ}\right]$ for the depicted molecule: Fe1‥Fe1' 2.6688(8), Fe1-O1 1.8992(17), Fe1-O2 1.9112(19), Fe1-S1 2.2129(7), Fe1-S1' 2.2073(8), O1-Fe1-O2 96.31(8), O1-Fe1-S1' 109.50(6), O2-Fe1-S1' 119.50(6), O1-Fe1-S1 115.12(6), O2-Fe1-S1 110.96(6), S1'-Fe1-S1 105.72(3). Selected atom distances [A] and interatomic angles $\left[{ }^{\circ}\right]$ for the second molecule: Fe2...Fe2' 2.7146(7), Fe2-O11 1.8976(18), Fe2-O12 1.8996(17), Fe2-S2 2.2207(7), Fe2-S2' 2.2179(7), O11-Fe2-O12 95.29(7), O11Fe2-S2' 113.79(6), O12-Fe2-S2' 115.99(6), O11-Fe2-S2 116.43(6), O12-Fe2-S2 111.13(6), S2-Fe2-S2' 104.59(2). 
Aromatic thiols containing heteroatoms proved to be reactive as well, as demonstrated by the preparation of the 2-mercaptothiophene derivative $\left(\mathrm{NEt}_{4}\right)_{2}\left[\mathrm{Fe}_{2} \mathrm{~S}_{2}\left(2-\mathrm{S}_{-} \mathrm{C}_{4} \mathrm{H}_{3} \mathrm{~S}\right)_{4}\right] \mathbf{1 0}$ (structural drawing depicted on page 191). Basic analytical data (NMR, MS, elemental analysis) for $\mathbf{8 a}$ and $\mathbf{1 0}$ are provided in the experimental section, electrochemical, zerofield Mössbauer and SQUID data are summarized in Table 2.4. Electrochemical potentials, magnetic coupling constants and isomer shifts for $\mathbf{1 0}$ and $\mathbf{8 a}$ are unexceptional (compare to data listed in Chapter 9.5 - 9.7). The quadrupole splitting for $\mathbf{8} \mathbf{a}$ is in the typical range for phenolate-coordinate $[2 \mathrm{Fe}-2 \mathrm{~S}]$ clusters and increased by $\simeq 0.3 \mathrm{~mm} / \mathrm{s}$ compared to the related 2,2'-dithiobiphenyl-ligated clusters (see Chapter 3). The quadrupole splitting for 10 indicates the absence of secondary bonding interactions between the thiophene-sulfurs and the iron atoms (see Chapter 5).

Table 2.4: Spectroscopic and electrochemical data for complexes $\mathbf{8 a}$ and $\mathbf{1 0 .}$

\begin{tabular}{ccccc}
\hline compound & $\delta\left(\Delta E_{\mathrm{Q}}\right)[\mathrm{mm} / \mathrm{s}]^{\mathrm{a}}$ & $\lambda_{\max }[\mathrm{nm}]\left(\varepsilon\left[\mathrm{M}^{-1} \mathrm{~cm}^{-1}\right]\right)$ & $J\left[\mathrm{~cm}^{-1}\right]^{\mathrm{b}}$ & $E_{\mathrm{p}}^{\mathrm{c}}[\mathrm{V}]^{\mathrm{c}}$ \\
\hline \multirow{3}{*}{$\mathbf{a}$} & $0.33(1.15)$ & $278(\simeq 26000), 315(\simeq 27000)$, & \\
& $413(9700), 492(s h, 4500)$, & -188 & $-1.12^{\mathrm{d}}$ \\
\multirow{4}{*}{$\mathbf{1 0}$} & $0.30(0.40)$ & $552(\operatorname{sh}, 2900)^{\mathrm{d}}$ & \\
& & $453(27000), 333(\simeq 35000)$, & & \\
& & $697(s h, 2500)^{\mathrm{e}}$ & -176 & $-1.05^{\mathrm{e}}$ \\
\hline
\end{tabular}

(a) ${ }^{57} \mathrm{Fe}$ Mössbauer parameters at $80 \mathrm{~K}$, relative to Fe metal at room temperature. (b) Values obtained from fits to SQUID data, see Chapter 9.6. (c) Cathodic peak potential recorded in presence of $\mathrm{NBu}_{4} \mathrm{PF}_{6}$ ( $0.1 \mathrm{~m}$ solution) at a scan rate of $100 \mathrm{mV} / \mathrm{s}$ vs. the $\mathrm{Cp}_{2}^{*} \mathrm{Fe} / \mathrm{Cp}_{2}^{*} \mathrm{Fe}^{+}$couple.(d) Recorded in MeCN solution at room temperature. (e) Recorded in DMF solution at room temperature.

\subsection{Conclusions}

Three homoleptic [2Fe-2S] cluster compounds - coordinated by the monodentate N-heteroaromatic amides pyrrolate, indolate and carbazolate - were examined with respect to their ligand exchange reactivity. The indolate-ligated cluster was discovered as a suitable precursor for the intended ligand exchange, affording a novel convenient pathway for the preparation of thiophenolate-coordinate $[2 \mathrm{Fe}-2 \mathrm{~S}]$ ferredoxin analogues. Six thiophenolate derivatives, 2-mercaptothiophene and a chelating biphenol were utilized as substrates in order to explore the scope of this conversion. No difficulties were encountered in the latter reactions, indicating that the reported method is of general use in synthetic $[2 \mathrm{Fe}-2 \mathrm{~S}]$ chemistry. 


\section{Chapter 3}

\section{Ligand Effects on Prominent Spectroscopic Properties of $[2 \mathrm{Fe}-2 \mathrm{~S}]$ Clusters}
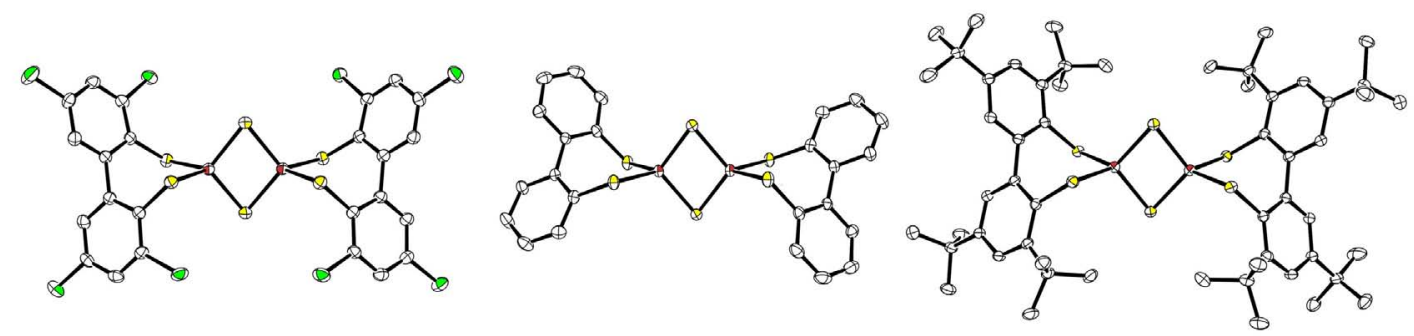

ELECTRON WITHDRAWING

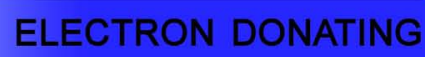

$\left(\mathrm{NEt}_{4}\right)_{2}\left[\mathrm{Fe}_{2} \mathrm{~S}_{2}\left(\mathrm{~S}_{2} \text {-biphenyl-R }\right)_{2}\right]$

$\mathrm{R}=\mathrm{Cl}, \mathrm{H},{ }^{t} \mathrm{Bu}$

\section{Abstract}

In order to examine electronic effects of coordinated thiolate ligands on the $[2 \mathrm{Fe}-2 \mathrm{~S}]$ cluster core, three chelating 2,2'-dithiobiphenyl ligands with electronically different substituents were prepared and coordinated to the cluster core. Spectroscopy in solution and in the solid state (including X-ray diffraction) was utilized to demonstrate that the different ligand environments indeed cause significant changes of the clusters' properties (e.g. the redox potentials). 


\subsection{Introduction}

Although various thiophenolate coordinated $[2 \mathrm{Fe}-2 \mathrm{~S}]$ clusters were reported in literature already, ${ }^{[150,151,154-156]}$ a systematic study with respect to the influence of the ligands electronic properties was not presented so far. ${ }^{[129]}$ As almost all of the literature-known thiophenolate ligated $[2 \mathrm{Fe}-2 \mathrm{~S}]$ clusters bear monodentate ligands, especially the examination of the redox potentials as a function of the ligand environment is difficult, due to the irreversibility of their reduction processes (formation of [4Fe-4S] clusters upon reduction). ${ }^{52,53]}$ One of the best studied clusters is the xylyl- $\left\{\mathrm{S}_{2}\right\}$-ligated ferredoxin analogue $\mathbf{1}$ with 7 -membered chelate rings (Scheme 1.5) that stabilizes the system to an extent that decomposition upon electrochemical reduction is negligible on the timescale of the cyclic voltammetry experiment. ${ }^{[32]}$ Assuming that the 7 -membered chelate rings in general afford relatively stable $[2 \mathrm{Fe}-2 \mathrm{~S}]$ clusters, it was intended to transfer this motive to the thiophenolatecoordinate clusters by application of $2,2^{\prime}$-dithiobiphenyl ligands. Thus, three biphenylbased ligands with electronically different substituents $\left(\mathrm{Cl}, \mathrm{H},{ }^{t} \mathrm{Bu}\right)$ were prepared and coordinated to the cluster core affording compounds 11a, 11b and 11c (Scheme 3.1). Ligand syntheses, cluster syntheses and spectroscopic characterizations of the clusters are described in detail in this chapter.

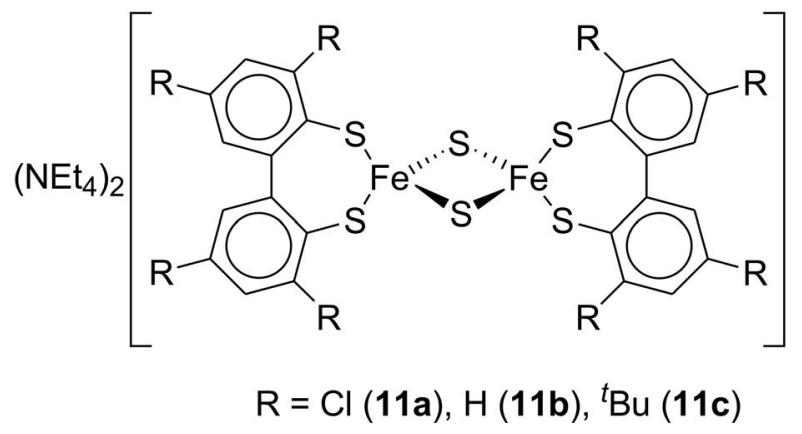

Scheme 3.1: [2Fe-2S] clusters 11a, 11b and 11c coordinated by the electronically different biphenyl-based ligands $\boldsymbol{V a}, \boldsymbol{V b}$ and $\boldsymbol{V} \boldsymbol{c}$.

\subsection{Ligand Synthesis}

In order to access the sought-after substituted 2,2'-dithiobiphenyl ligands via a MiyazakiNewman-Kwart rearrangement strategy ${ }^{[157]}$ (it was recently suggested to use this term instead of Newman-Kwart rearrangement, taking important studies of Miyazaki et al. ${ }^{[158]}$ on the mechanism of thione-to-thiol rearrangements into account), ${ }^{[159]}$ the biphenols IIaIIc serve as convenient starting material available commercially or according to literature methods. ${ }^{[160,161]}$ The corresponding dithiols could be obtained in a three step synthetic sequence (Scheme 3.2, preparation of $\mathbf{I I b}$ - Vb is literature-known and added to the scheme for completeness. ${ }^{[162]}$ The given yields for these compounds are those obtained in this work - literature yields are slightly different). After deprotonation with sodium hydride, the biphenolates were treated with dimethylcarbamothioic chloride. Although an excess 
of both base and dimethylcarbamothioic chloride was used, the reactions afforded the desired bis- $O$-thiocarbamate ester IIIa only in moderate yields of around $30 \%$, as an equal amount of mono- $O$-thiocarbamate ester remains. In case of IIIb and IIIc formation of the corresponding mono- $O$-thiocarbamate esters was observed as well, but yields of the desired products were somewhat higher. After purification, the neat compounds IIIaIIIc were heated to $320^{\circ} \mathrm{C}$ for up to $4 \mathrm{~h}$. At this temperature, a Miyazaki-Newman-Kwart rearrangement takes place to give the bis- $S$-thiocarbamate esters IVa-IVc in yields of 71 to $85 \%$. Formation of the free dithiols $\mathbf{V a}-\mathbf{V c}$ was achieved by reduction with $\mathrm{LiAlH}_{4}$ and subsequent acidic work-up. To prevent possible oxidation to internal disulfides, the work-up was carried out under inert dinitrogen atmosphere.
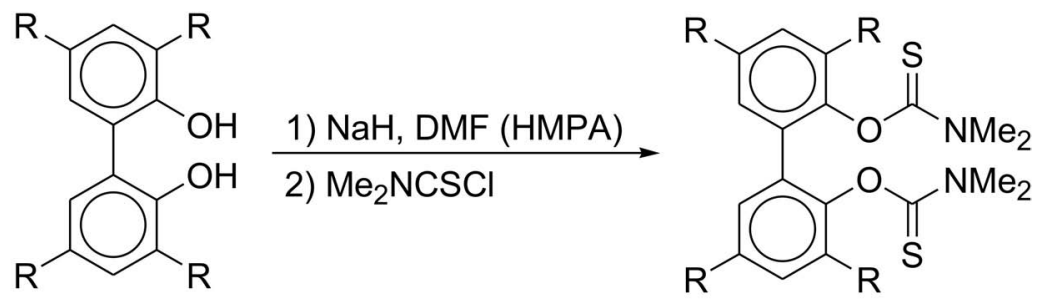

$$
\begin{aligned}
& \text { Ila }(R=C l) \\
& \text { Ilb }(R=H) \\
& \text { Ilc }\left(R={ }^{t} \mathrm{Bu}\right)
\end{aligned}
$$

$$
\begin{aligned}
& \text { IIIa }(R=C l) 32 \% \\
& \text { IIIb }(R=H) 80 \% \\
& \text { IIIc }\left(R={ }^{t} B u\right) 65 \%
\end{aligned}
$$

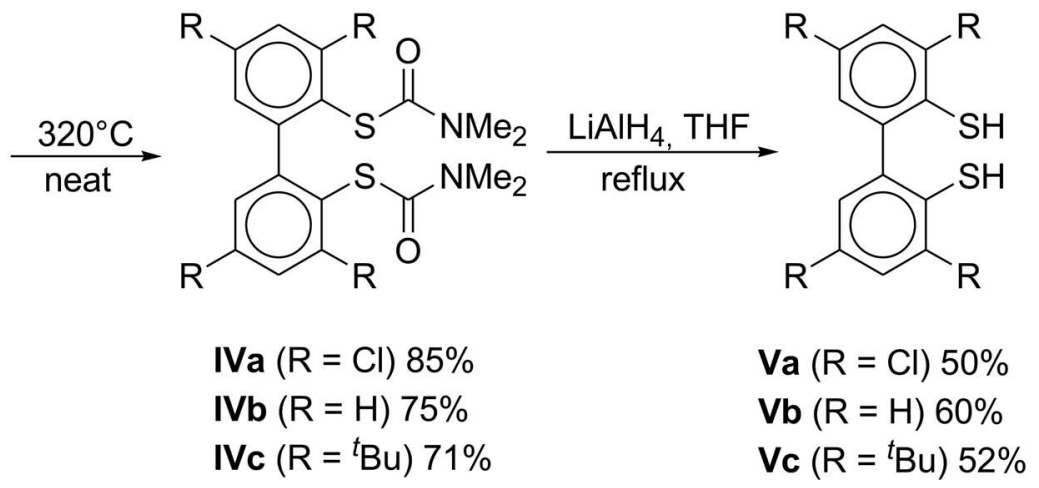

Scheme 3.2: Synthesis of substituted 2,2'-dithiobiphenyl ligands $\boldsymbol{V a}, \boldsymbol{V b}$ and $\boldsymbol{V} \boldsymbol{c}$.

In the ${ }^{1} \mathrm{H}$ and ${ }^{13} \mathrm{C}$ NMR spectra of the $O$-carbamate ester compounds IIIb and IIIc, two signals are found for the methyl groups attached to the carbamate nitrogen atoms. This indicates a slow rotation around the C-N bond, probably due to delocalization of the carbamate double bond. The corresponding spectrum of compound IIIa shows even four methyl signals, indicating that a barrier also exists for the rotation of the entire ester group around the aryl-O bond. At low temperature $\left(-60^{\circ} \mathrm{C}\right)$, three distinct sets of signals (i.e. 6 methyl signals) are observed for IIIa. The observations in the NMR spectra are in agreement with crystal structures of related compounds, ${ }^{[157]}$ where both the aryl-aryl and aryl $O$-carbamate ester groups are twisted nearly $90^{\circ}$ with respect to each other, resulting in two sterically favored (racemic) diastereomers: $\mathrm{A} C_{2}$-symmetric species with the same relative orientation of the ester groups, producing one set of NMR peaks, and a completely asymmetric species with opposite orientation of the ester groups, 
producing a split set of peaks with a 1:1 integral ratio. At $-60^{\circ} \mathrm{C}$, the signals of the split set slowly interconvert through rotation of the ary-aryl bond, while at room temperature most of them have coalesced, as expected for an energetic barrier of about $60 \mathrm{~kJ} / \mathrm{mol}$. ${ }^{[63]}$ The barriers for rotation of the ester group and the carbamate $\mathrm{C}-\mathrm{N}$ bond are much higher (both about $75 \mathrm{~kJ} / \mathrm{mol}$ ), so that separate, albeit broad, signals are still observable at room temperature. At closer inspection, three sets of peaks are also observed in the NMR spectra of IIIc, but with a very minor population (about $6 \%$ ) of the asymmetric isomer. None of the peaks shows dynamic exchange up to $50{ }^{\circ} \mathrm{C}$, presumably due to the steric bulk of the tert-butyl groups which locks the molecules in their individual conformations. After the Miyazaki-Newman-Kwart rearrangement, only one single signal is found for the N-bound methyl groups, which is in agreement with earlier reports for $S$-thiocarbamate esters. ${ }^{[164]}$ Also, the ${ }^{13} \mathrm{C}$ resonance of the thione carbon undergoes a large upfield shift from about 186 to about $166 \mathrm{ppm}$. Thus, formation of the bis- $S$-thiocarbamate esters IVaIVc can be monitored by NMR and IR (thione bands at $1530 \mathrm{~cm}^{-1}$ to $1550 \mathrm{~cm}^{-1}$ versus carbonyl bands at $\simeq 1670 \mathrm{~cm}^{-1}$ ) spectroscopy. As expected, bond rotations in the free dithiols Va-Vc are not hindered, which is evident from sharp ${ }^{1} \mathrm{H}$ NMR signals for all aromatic resonances. The coordination chemistry of $\mathbf{V a}$ and $\mathbf{V c}$ was examined by the preparation of their zinc complexes as described in Appendix B. Compound Va could be crystallized from THF / pentane to give single crystals suitable for X-ray diffraction. This is a rare example of a structurally characterized $2,2^{\prime}$-dithiobiphenyl derivative (Figure 3.1; at present $4,4^{\prime}$-diamino-2,2' $, 6,6^{\prime}$-tetrathio-1, $1^{\prime}$-biphenyl is the only other crystallographically characterized biphenyl derivative with two free thiol groups in $2,2^{\prime}$-position $\left.{ }^{[165]}\right)$. The $1,1^{\prime}-$ linked phenyl rings are found perpendicular to each other with a dihedral angle $\mathrm{C} 2$-C1$\mathrm{C} 1^{\prime}-\mathrm{C} 2^{\prime}$ of $90.61(19)^{\circ}$.

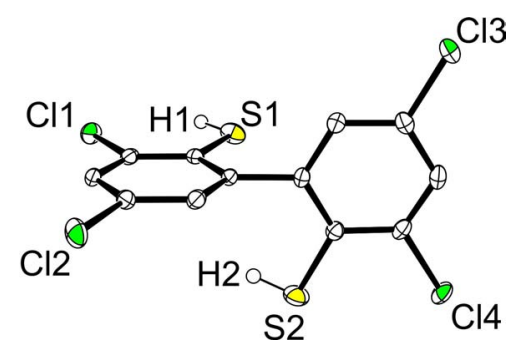

Figure 3.1: ORTEP plot (50\% probability thermal ellipsoids) of the molecular structure of Va. Selected atom distances [A] and angles [0]: S1-H1 1.02(4), S2-H2 1.02(4), S1-C2 1.7581(16), S2-C2' 1.7565(16), C1-C1' 1.498(2), H1-S1-C2 94.6(19), H2-S2-C2' 106(2), C2-C1-C1'-C2' 90.61(19).

\subsection{Cluster Synthesis and Structural Characterization}

Clusters $11 \mathbf{a}$ and 11c were synthesized using standard salt metathesis reactions, starting from $\left(\mathrm{NEt}_{4}\right)_{2}\left[\mathrm{Fe}_{2} \mathrm{~S}_{2} \mathrm{Cl}_{4}\right] \mathbf{2}$, whereas $\mathbf{1 1 b}$ was more conveniently obtained on a ligand exchange pathway (see Chapter 2), starting from the indolate coordinated cluster precursor $\left.\left(\mathrm{NEt}_{4}\right)_{2}\left[\mathrm{Fe}_{2} \mathrm{~S}_{2} \text { (indolate) }\right)_{4}\right] \mathbf{5}$ (the existance of $\mathbf{1 1 b}$ was previously mentioned in literature ${ }^{[139]}$ - a synthetic procedure and spectroscopic characteristics however were not reported). An 
alternative synthesis of 11a using the latter ligand exchange approach is possible as well and described in the experimental part (Chapter 9). Compound 11a and 11b were isolated in good yields (>50\%), 11c however only in $23 \%$, probably due to the sterically bulky tert-butyl ortho-substituents, disfavoring the coordination of two ligands to one cluster core. Single crystals suitable for X-ray diffraction were grown for all three compounds by slow diffusion of $\mathrm{Et}_{2} \mathrm{O}$ into MeCN solutions of the beforehand isolated and purified clusters (ORTEP plots depicted in Figure 3.2).
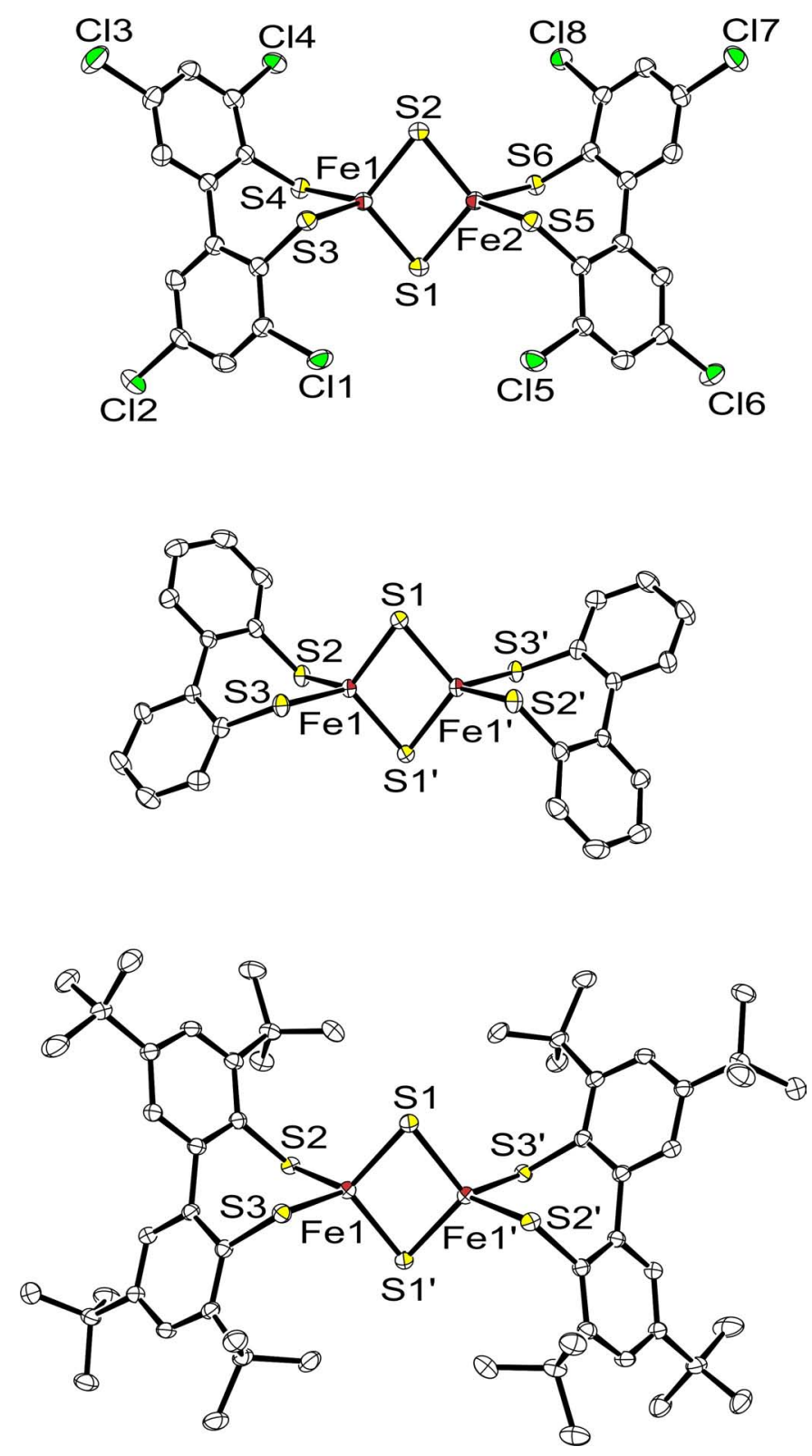

Figure 3.2: ORTEP plots (50\% probability thermal ellipsoids) of the molecular structures of $11 \boldsymbol{a}$ (top), $11 \mathrm{~b}$ (middle) and $11 \mathrm{c}$ (bottom). The NEt $\mathrm{t}_{4}^{+}$counter ions and all hydrogen atoms have been omitted for clarity. Prominent structural parameters are summarized in Table 3.1. 
All three cluster compounds crystallize as (pseudo-) $C_{2 \mathrm{~h}}$ symmetric molecules with the $C_{2^{-}}$ axis through both iron atoms and the perpendicular mirror plane through the bridging sulfides. Thus, one of the $2,2^{\prime}$-dithiobiphenyl ligands in each cluster is axial- $R$ and the other axial- $S$ configured. Most likely, these meso-compounds preferably crystallize with tetraethylammonium counter ions, whereas the $R R, S S$-pairs precipitate as powders or remain dissolved until internal racemisation to the $R S, S R$-meso-pairs took place. This seems not be the case if other counter ions are used. Accidentally, the dianion of 11a crystallized in $C_{1}$ symmetry with one $\mathrm{NEt}_{4}^{+}$and one $\mathrm{Cp}_{2} \mathrm{Co}^{+}$counter ion $\left(\mathbf{1 1 a}^{\prime}\right)$ during an unsuccessful reduction attempt (to the mixed-valent species using cobaltocene as reduc-

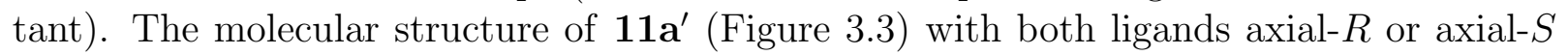
configured (independent $R R$ - and $S S$-enantiomers are present in the asymmetric unit, both inter-convertible by a mirror plane) supports the latter assumption regarding the favored crystallization of the meso-diastereomers of $11 \mathrm{a}-11 \mathrm{c}$ with two $\mathrm{NEt}_{4}^{+}$counter ions.

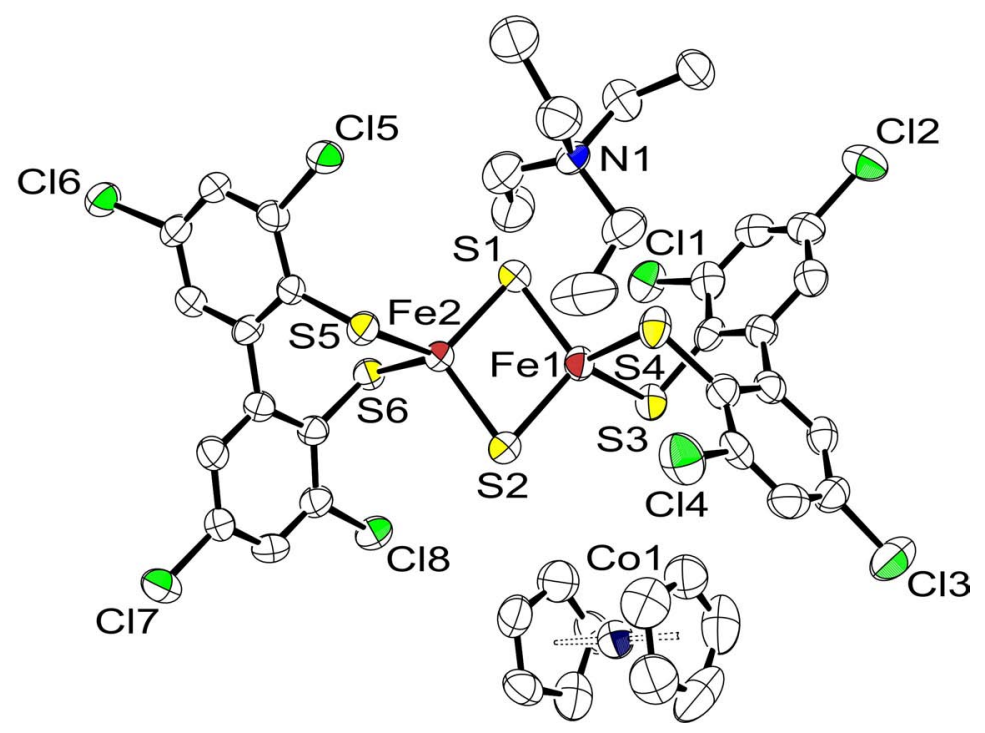

Figure 3.3: ORTEP plot (50\% probability thermal ellipsoids) of the molecular structure of $\left(\mathrm{NEt}_{4}\right)\left(\mathrm{Cp}_{2} \mathrm{Co}\right)\left[\mathrm{Fe}_{2} \mathrm{~S}_{2}\left(3,3^{\prime}, 5,5^{\prime} \text {-tetrachloro-1,1'-biphenyl-2,2'-dithiolate }\right)_{2}\right]\left(\mathbf{1 1} \mathbf{a}^{\prime}\right)$. All hydrogen atoms are omitted for sake of clarity. Selected atom distances [A] and interatomic angles [ ${ }^{\circ}$ : Fe1...Fe2 2.6936(14), Fe1-S1 2.187(2), Fe1-S2 2.2004(18), Fe1-S3 2.3111(19), Fe1-S4 2.318(2), Fe2-S1 2.1952(18), Fe2-S2 2.219(2), Fe2-S6 2.301(2), Fe2-S5 2.3239(18), S1-Fe1-S2 104.92(8), S1-Fe1-S3 114.20(7), S2-Fe1-S3 108.73(7), S1-Fe1-S4 109.09(8), S2-Fe1-S4 115.30(8), S3-Fe1-S4 104.89(7), S1-Fe2-S2 104.04(8), S1-Fe2-S6 109.23(7), S2-Fe2-S6 115.42(8), S1-Fe2-S5 115.13(8), S2-Fe2-S5 108.03(7), S6-Fe2-S5 105.33(7).

Although eight chlorine atoms in $\mathbf{1 1 a}$ or eight tert-butyl groups in $\mathbf{1 1} \mathbf{c}$ are expected to significantly influence the electronic structure of the central $[2 \mathrm{Fe}-2 \mathrm{~S}]$ core compared to the parent $\mathbf{1 1 b}$, only minor structural changes of the corresponding core geometries ${ }^{[150]}$ are observed (prominent atom distances and angles are summarized in Table 3.1). Thus, the Fe $\cdots$ Fe distances in $\mathbf{1 1 a}$ and $\mathbf{1 1} \mathbf{b}$ are qualitatively identical $(\simeq 2.67 \AA)$, while a slightly elongated inter-metallic distance is found in 11c $(\simeq 2.72 \AA)$. Accordingly the bond lengths to the bridging sulfides $(\simeq 2.21 \AA)$ and the angles at those sulfides Fe- $\left(\mu\right.$-S)-Fe $\left(\simeq 75.9^{\circ}\right)$ in 
11c are increased to some extent. Ligand bite angles RS-Fe-SR, distances to the terminal thiolates Fe-SR and geometric distortions at the iron sites (semi-quantified by $\tau_{4}$ values) ${ }^{[166]}$ are in a similar range for all three clusters.

Table 3.1: Selected structural parameters for clusters 11a-11c. Interatomic distances are given in $[\AA]$ ] and angles in $\left[{ }^{\circ}\right]$.

\begin{tabular}{cccc}
\hline compound & $\mathbf{1 1 a}$ & $\mathbf{1 1 b}$ & $\mathbf{1 1} \mathbf{c}$ \\
\hline Fe $\cdots$ Fe & $2.6748(7)$ & $2.6722(4)$ & $2.7212(5)$ \\
& $2.3080(8)$ & $2.2864(4)$ & $2.3002(5)$ \\
Fe-SR & $2.3051(7)$ & $2.2989(4)$ & $2.2983(5)$ \\
& $2.3121(8)$ & $2.2864(4)$ & $2.3002(5)$ \\
& $2.3112(8)$ & $2.2989(4)$ & $2.2983(5)$ \\
& $2.1964(7)$ & $2.2094(5)$ & $2.2113(5)$ \\
Fe- $(\mu-\mathrm{S})$ & $2.1925(7)$ & $2.1969(5)$ & $2.2152(5)$ \\
& $2.2010(7)$ & $2.2094(5)$ & $2.2113(5)$ \\
$\mathrm{Fe}-(\mu-\mathrm{S})-\mathrm{Fe}$ & $2.2037(7)$ & $2.1969(5)$ & $2.2152(5)$ \\
& $74.93(3)$ & $74.665(15)$ & $75.866(16)$ \\
$\mathrm{RS}-\mathrm{Fe}-\mathrm{SR}$ & $74.95(2)$ & $74.665(15)$ & $75.866(16)$ \\
& $103.51(3)$ & $103.684(16)$ & $104.735(18)$ \\
$\tau_{4}$ & $105.55(3)$ & $103.684(16)$ & $104.735(18)$ \\
& 0.874 & 0.878 & 0.884 \\
& 0.895 & 0.878 & 0.884 \\
\hline
\end{tabular}

\subsection{Spectroscopy in Solution}

In order to elucidate whether the ligand substitutions indeed do effect the electronic properties of the cluster cores, regardless of their rigid geometry, all new complexes were characterized in solution by several spectroscopic methods. Positive and negative ion ESI mass spectrometry proved to be a valuable analytical tool to confirm formation and intactness of the anticipated complexes since spectra of MeCN solutions show dominant signals for $\left[\mathrm{Fe}_{2} \mathrm{~S}_{2} \mathrm{~L}_{2}\left(\mathrm{NEt}_{4}\right)_{3}\right]^{+}$and $\left[\mathrm{Fe}_{2} \mathrm{~S}_{2} \mathrm{~L}_{2}\left(\mathrm{NEt}_{4}\right)\right]^{-}$, respectively (the positive ion ESI spectrum of 11a is shown in Figure 3.4 as an example). The absence of possible diamagnetic impurities (e.g. free ligands) was proven by proton NMR spectroscopy in $\mathrm{MeCN}^{-\mathrm{d}_{3}}$ at room temperature. Due to the strong antiferromagnetic coupling between both irons (as generally observed in all synthetic [2Fe-2S] cluster reported so far), reasonable resolved, but broadened signals were recorded for $\mathbf{1 1 a - 1 1 c}$, with dominant $\mathrm{NEt}_{4}^{+}$resonances in the aliphatic regime and characteristic signals for the aromatic 4,4',6,6'-protons at $\simeq 9$ and $\simeq 10 \mathrm{ppm}$. In contrast to those lucid NMR spectra, interpretation of the UV-Vis absorptions in MeCN solution is challenging. Each complex exhibits three distinct absorption bands at $\simeq 257$ - 
$260 \mathrm{~nm}, \simeq 420-450 \mathrm{~nm}$ and $\simeq 520-550 \mathrm{~nm}$ (for 11b and 11c additional bands or shoulders are observed at $336 \mathrm{~nm}$ and $383 \mathrm{~nm}$, respectively). Theoretically, electron donating substituents as the tert-butyl moieties in 11c are expected to lower the transition energies for the main visible bands, which previously were assigned to thiophenolate-to-core charge transfer transitions. ${ }^{[150]}$ Indeed, a red-shift of those absorptions is apparent for compound $\mathbf{1 1 c}$ relative to the unsubstituted cluster $\mathbf{1 1 b}$. However, the chlorine substituted cluster 11a deviates form the expected behavior, to some extent. Its energetically lowest absorption at $523 \mathrm{~nm}$ matches the theoretical assumptions and indeed is blue-shifted compared to 11b, whereas both energetically higher bands do not shift or even shift to lower energies compared to 11b. A possible explanation for this observation is that the latter absorption bands partially correspond to charge transfer transitions between the bridging inorganic sulfides and the iron atoms as a reverse trend would be expected for these transition energies.

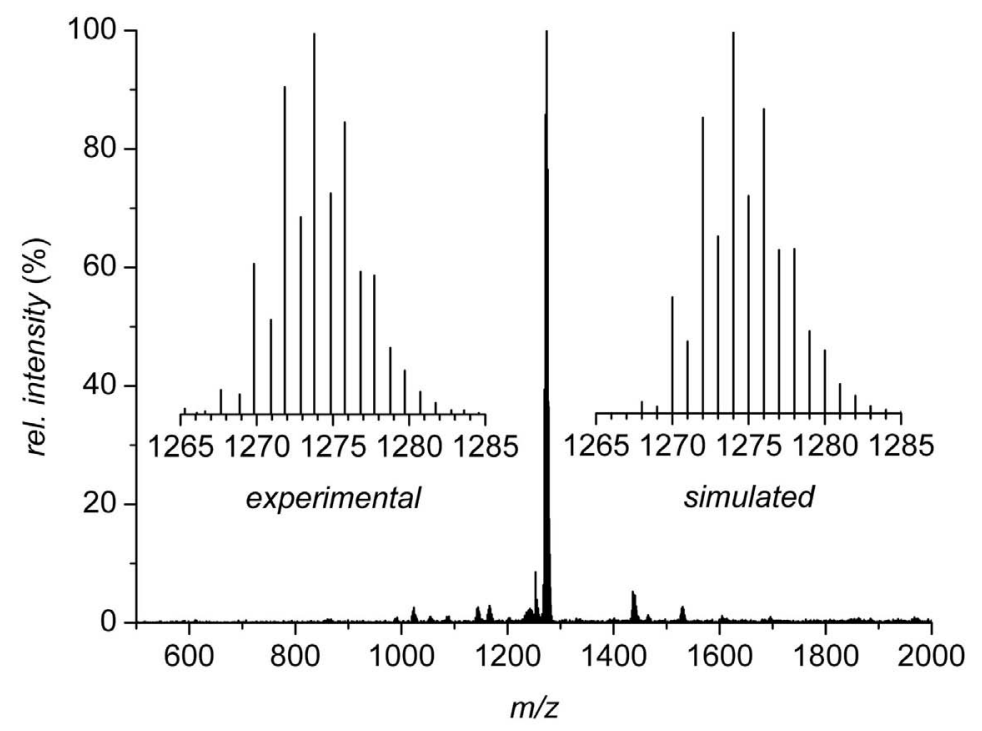

Figure 3.4: Positive ESI-MS spectrum of 11a in MeCN solution. The insets show the experimental and expected isotopic distribution pattern for $\left[\mathrm{M}+\mathrm{NEt}_{4}\right]^{+}$.

In order to probe the electronic structure of all clusters, electrochemical studies were conducted (i.e. cyclic voltammetry in MeCN solution at room temperature, utilizing the $\mathrm{Cp}_{2}^{*} \mathrm{Fe} / \mathrm{Cp}_{2}^{*} \mathrm{Fe}^{+}$couple as internal standard). Assuming that electron withdrawing ligand substituents lower the electron density at the cluster core and electron donating ligand substituents higher the electron density at the core position, a decreasing redox potential was predicted in the row $\mathrm{E}_{1 / 2}(\mathbf{1 1} \mathbf{a})>\mathrm{E}_{1 / 2}(\mathbf{1 1} \mathbf{b})>\mathrm{E}_{1 / 2}(\mathbf{1 1} \mathbf{c})$. As anticipated, the electron poor cluster core in $\mathbf{1 1 a}$ readily accepts a further electron and consequently converts to the mixed-valent state at a relatively high potential of $-0.96 \mathrm{~V}$, whereas the corresponding halfwave potential for the parent compound $\mathbf{1 1 b}$ is found at a lower potential $\left(\mathrm{E}_{1 / 2}=-1.14 \mathrm{~V}\right)$. As a result of the electron rich cluster core in $\mathbf{1 1 c}$, generated by the overall eight $\sigma$-electron donating tert-butyl substituents, its reduction wave is shifted significantly to a more negative region (compared to $\mathbf{1 1 b}$ ) and found at $-1.43 \mathrm{~V}$ (Figure 3.6). 


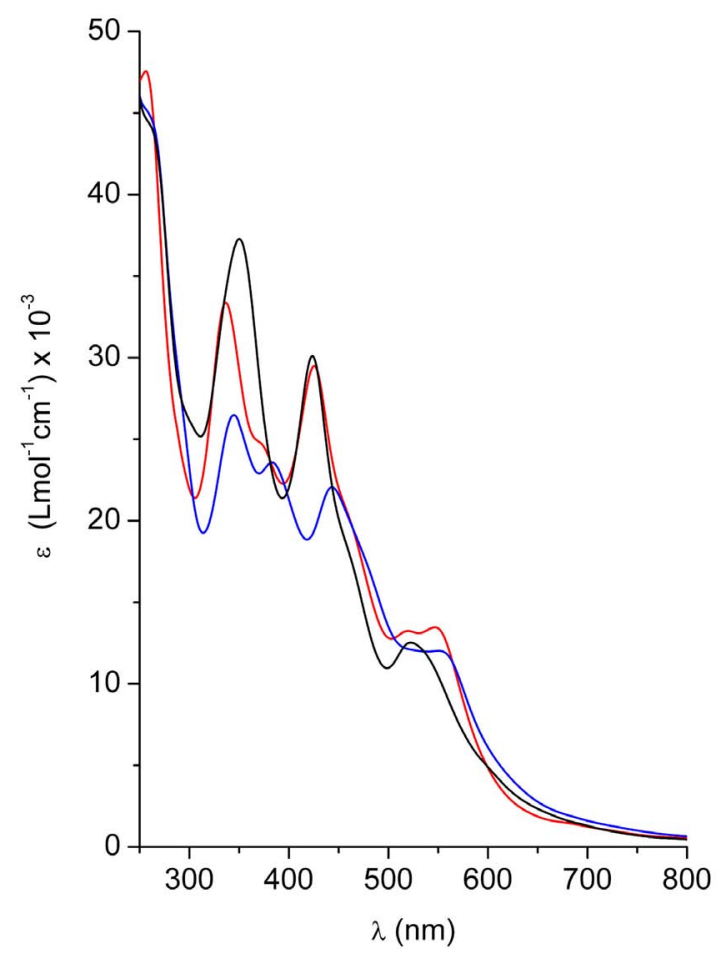

Figure 3.5: Electronic absorption spectra of $\mathbf{1 1 a}$ (black line), $\mathbf{1 1 b}$ (red line) and $\mathbf{1 1 c}$ (blue line) in $M e C N$ solution.

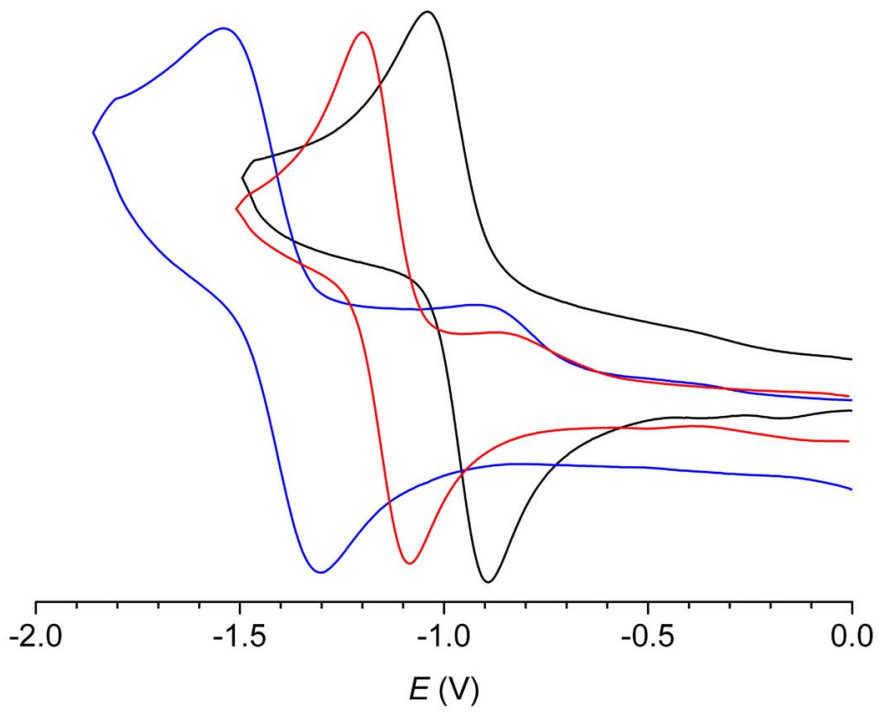

Figure 3.6: Cyclic voltammograms of $\mathbf{1 1 a}$ (black line), $\mathbf{1 1 b}$ (red line) and 11c (blue line)

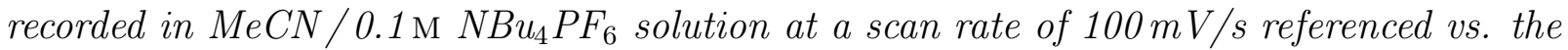
$\mathrm{Cp}_{2}^{*} \mathrm{Fe} / \mathrm{Cp}_{2}^{*} \mathrm{Fe}^{+}$couple. 


\subsection{Spectroscopy in the Solid State}

In order to gain further insight into the electronic structure of the cluster cores, compounds 11a-11c were additionally characterized by zero-field Mössbauer spectroscopy and temperature dependent magnetic susceptibility measurements. Spectral fits to the Mössbauer data were obtained by using Lorentzian line doublets with isomer shifts $\delta$ and quadrupole splittings $\Delta E_{\mathrm{Q}}$ summarized in Table 3.2 (the spectrum of $\mathbf{1 1} \mathbf{b}$ is shown in Figure 3.7 as an example). Unexpectedly, neither $\delta$-values nor $\Delta E_{\mathrm{Q}}$-values show a clear trend while going form electron-withdrawing ligand substituents to electron-donating groups. Instead, isomer shifts are positioned in a range of $\delta=0.30 \pm 0.02 \mathrm{~mm} / \mathrm{s}$ and quadrupole splittings found in a range of $\Delta E_{\mathrm{Q}}=0.82 \pm 0.04 \mathrm{~mm} / \mathrm{s}$ for all three cluster compounds. Thus, isomer shifts only confirm the ferric oxidation states $s=2.825 \pm 0.05$ (calculated from the empirical correlation $\delta=1.43-0.40 s$ between oxidation state $s$ and $\delta$ applicable for tetrahedral $\left\{\mathrm{FeS}_{4}\right\}$ sites $)^{[167]}$, but do not reflect the electronic influences of the distinctive ligand environments on the iron-sulfur cores. Compared to $11 \mathbf{a}\left(\Delta E_{\mathrm{Q}}=0.77 \mathrm{~mm} / \mathrm{s}\right)$ and 11c $\left(\Delta E_{\mathrm{Q}}=0.83 \mathrm{~mm} / \mathrm{s}\right), \mathbf{1 1 b}\left(\Delta E_{\mathrm{Q}}=0.86 \mathrm{~mm} / \mathrm{s}\right)$ exhibits the largest quadrupole splitting of the three clusters reported herein. Currently, no persuasive explanation for this finding can be provided, but an involvement of solid state effects might play a role. In this context it seems to be noteworthy that each angle C-S-Fe in $11 \mathbf{a}$ and $11 \mathbf{c}$ is found in the range $100 \pm 3^{\circ}$, whereas only one angle C-S-Fe in $\mathbf{1 1 b}$ is found in this range and the other widened to $\simeq 109^{\circ}$. As the latter geometric parameters are expected to directly control the irons' delectron delocalisation over the ligand systems, ${ }^{[155]}$ an indirect influence on the corresponding isomer shifts and a direct influence on the corresponding quadrupole splittings seems to be possible. Dynamic processes in solution (interconversion of the ligand-diastereomers is discussed above) however would certainly overtop those effects and consequently cause the substitution-dependent properties of $\mathbf{1 1 a - 1 1 c}$ as observed in solution.

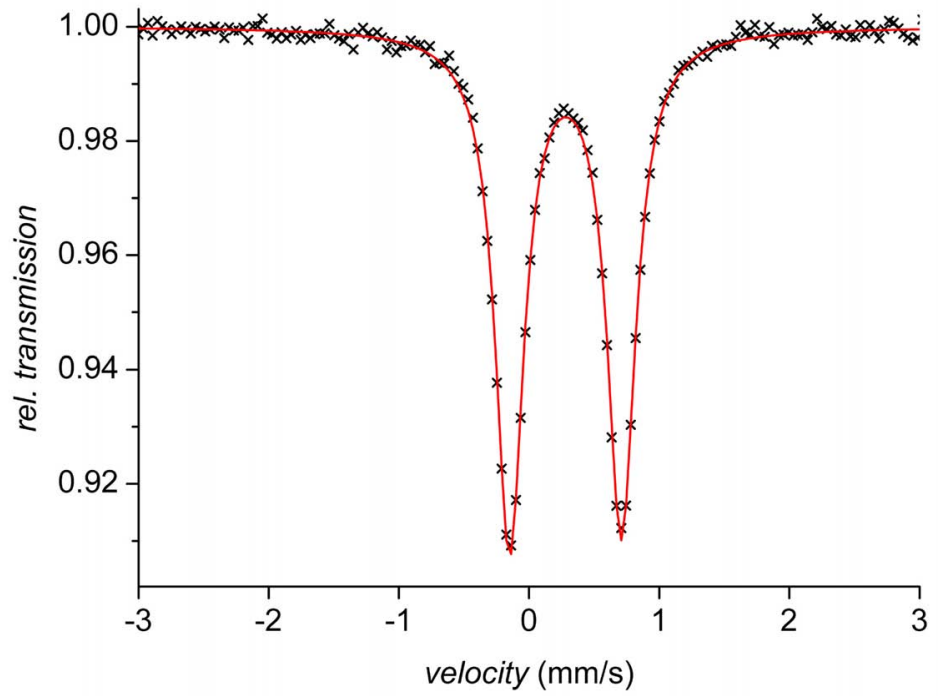

Figure 3.7: Zero-field Mössbauer spectrum of $\mathbf{1 1 b}$ recorded at $80 \mathrm{~K}$. The red curve is fitted to the experimental values (crosses) with $\delta=0.28 \mathrm{~mm} / \mathrm{s}$ and $\Delta E_{Q}=0.86 \mathrm{~mm} / \mathrm{s}$. 
Table 3.2: Spectroscopical and electrochemical data for 11a-11c.

\begin{tabular}{|c|c|c|c|c|}
\hline compound & $\delta\left(\Delta E_{\mathrm{Q}}\right)[\mathrm{mm} / \mathrm{s}]^{\mathrm{a}}$ & $\lambda_{\max }[\mathrm{nm}]\left(\varepsilon\left[\mathrm{M}^{-1} \mathrm{~cm}^{-1}\right]\right)^{\mathrm{b}}$ & $J\left[\mathrm{~cm}^{-1}\right]^{\mathrm{c}}$ & $E_{1 / 2}[\mathrm{~V}]^{\mathrm{d}}$ \\
\hline $11 a$ & $0.28(0.77)$ & $\begin{array}{c}260(s h, \simeq 34000), 350 \\
(\simeq 25000), 424(17800), \\
523(6050)\end{array}$ & -177 & -0.96 \\
\hline $11 b$ & $0.28(0.86)$ & $\begin{array}{c}257(\simeq 47500), 336(\simeq 33400) \\
425(29500), 520(13250) \\
547(13500)\end{array}$ & -158 & -1.14 \\
\hline $11 c$ & $0.29(0.83)$ & $\begin{array}{c}260(s h, \simeq 45000), 345(26500) \\
383(23600), 443(22000) \\
550(s h, 12000)\end{array}$ & -141 & $-1.43^{\mathrm{e}}$ \\
\hline
\end{tabular}

(a) ${ }^{57} \mathrm{Fe}$ Mössbauer parameters at $80 \mathrm{~K}$, relative to Fe metal at room temperature. (b) Recorded in MeCN solution at room temperature. (c) Values obtained from fits to SQUID data, see Chapter 9.6. (d) Halfwave potential of the (quasi)-reversible process in $\mathrm{MeCN} / 0.1 \mathrm{M} \mathrm{NBu}_{4} \mathrm{PF}_{6}$ at a scan rate of $100 \mathrm{mV} / \mathrm{s}$ vs. the $\mathrm{Cp}_{2}^{*} \mathrm{Fe} / \mathrm{Cp}_{2}^{*} \mathrm{Fe}^{+}$couple.(e) Potential determined in $\mathrm{MeCN} / 0.1 \mathrm{M} \mathrm{NBu}{ }_{4} \mathrm{PF}_{6}$ solution at a scan rate of $100 \mathrm{mV} / \mathrm{s}$ vs. the $\mathrm{Cp}_{2} \mathrm{Fe} / \mathrm{Cp}_{2} \mathrm{Fe}^{+}$couple is $-1.94 \mathrm{~V}$, corresponding to $-1.43 \mathrm{~V}$ vs. the $\mathrm{Cp}_{2}^{*} \mathrm{Fe} / \mathrm{Cp}_{2}^{*} \mathrm{Fe}^{+}$ couple. ${ }^{[145,146]}$

Magnetic susceptibility measurements for compounds $\mathbf{1 1 a - 1 1 c}$ were carried out at $0.5 \mathrm{~T}$ from $2 \mathrm{~K}$ to $295 \mathrm{~K}$. Magnetic moments $\mu_{\mathrm{eff}}$ at room temperature are in the range $1.9-2.7 \mu_{\mathrm{B}}$ and decrease towards zero upon cooling, indicating significant antiferromagnetic coupling as is commonly observed for the $\mathrm{Fe}_{2} \mathrm{~S}_{2}$ core $(S=0$ ground state). Coupling constants $J$ were determined using a fitting procedure to the appropriate Heisenberg spin Hamiltonian for isotropic exchange coupling and are summarized in Table 3.2 (details are provided in Chapter 9.6). In contrast to the Mössbauer parameters $\delta$ and $\Delta E_{\mathrm{Q}}$, coupling constants $J$ obtained from the SQUID data clearly show a considerable trend, i.e. values are decreasing in the row $J(\mathbf{1 1 a})>J(\mathbf{1 1} \mathbf{b})>J(\mathbf{1 1} \mathbf{c})$. As these parameters are expected to directly correlate with the Fe. $\cdots$ Fe separations and the Fe- $(\mu-\mathrm{S})$-Fe angles, a distinct trend $J(\mathbf{1 1} \mathbf{a}) \simeq J(\mathbf{1 1} \mathbf{b})>J(\mathbf{1 1} \mathbf{c})$ would have been predicted on the basis of the molecular structures, as nearly identical core geometries for $\mathbf{1 1 a}$ and $\mathbf{1 1} \mathbf{b}$ are evident from X-ray diffraction analysis. Thus, results from both spectroscopic techniques that require solid state samples - Mössbauer and magnetic susceptibility measurements - disagree with common expectations. As powdered samples were used in the latter experiments, the simplest explanation for these observations arises from a contribution of $R R, S S$-configured forms in addition to the $R S, S R$-diastereomers of 11a-11c. Since crystallization of the $R S, S R$-meso-pairs is persistently accompanied by the precipitation of some powdered material and crystallization of the $R R, S S$-pairs of 11a-11c is unfeasible in case of the $\mathrm{NEt}_{4}^{+}$salts, neither an experimental separation nor a theoretical analysis of the expected spectroscopic properties of these $R R, S S$-pairs could be performed (manual separation of crystals from powdered material is not considered as an appropriate experimental technique and was not carried out). In this context, it seems to be noteworthy that the mixed $\mathrm{NEt}_{4}^{+}-\mathrm{Cp}_{2} \mathrm{Co}^{+}$salt of 11a $\left(R R, S S \mathbf{- 1 1} \mathbf{a}^{\prime}\right)$ exhibits an elongated Fe $\cdots$ Fe separation of 2.6936(14) $\AA$ (compare to 
11a: $d(\mathrm{Fe} \cdots \mathrm{Fe})=2.6748(7) \AA)$, whereas a shortened one would fit to the observed trend in coupling constants $J$. Also line width parameters from the Mössbauer spectra do not indicate the presence of additional cluster species (as the $R R, S S$-forms) with significantly different coordination environments at the iron atoms.

\subsection{Conclusions}

In summary, three electronically different dithiolates were applied as terminal ligands on $[2 \mathrm{Fe}-2 \mathrm{~S}]^{2+}$ cores. Upon coordination, significant changes of the clusters' properties have been detected. Although interpretation of the acquired Mössbauer and SQUID data is speculative to some extent, the observed characteristics in solution reflect the expected trend: Electron-withdrawing ligand substituents lower the total electron density at the cluster cores and consequently shift the corresponding have-wave potentials for the $[2 \mathrm{Fe}-2 \mathrm{~S}]^{2+} /[2 \mathrm{Fe}-2 \mathrm{~S}]^{+}$couple to a more positive region. Reduction of those systems becomes facilitated compared to systems with electron-donating ligand substituents. As the isolation of mixed-valent $[2 \mathrm{Fe}-2 \mathrm{~S}]^{+}$ferredoxin-analogues is generally considered as pending task to be achieved by synthetic bioinorganic chemists, future efforts will focus on the preparative chemical reduction of electron-deficient all- $\{\mathrm{S}\}$-ligated clusters. Thus, the synthesis of a per-fluorinated 2,2'-dithio-1,1'-biphenyl derivative and its coordination to the cluster core is planed as well in order to position the clusters redox potential as suitable as possible for the latter challenge. 


\section{Chapter 4}

\section{The Quest for Neutral [2Fe-2S] Clusters}

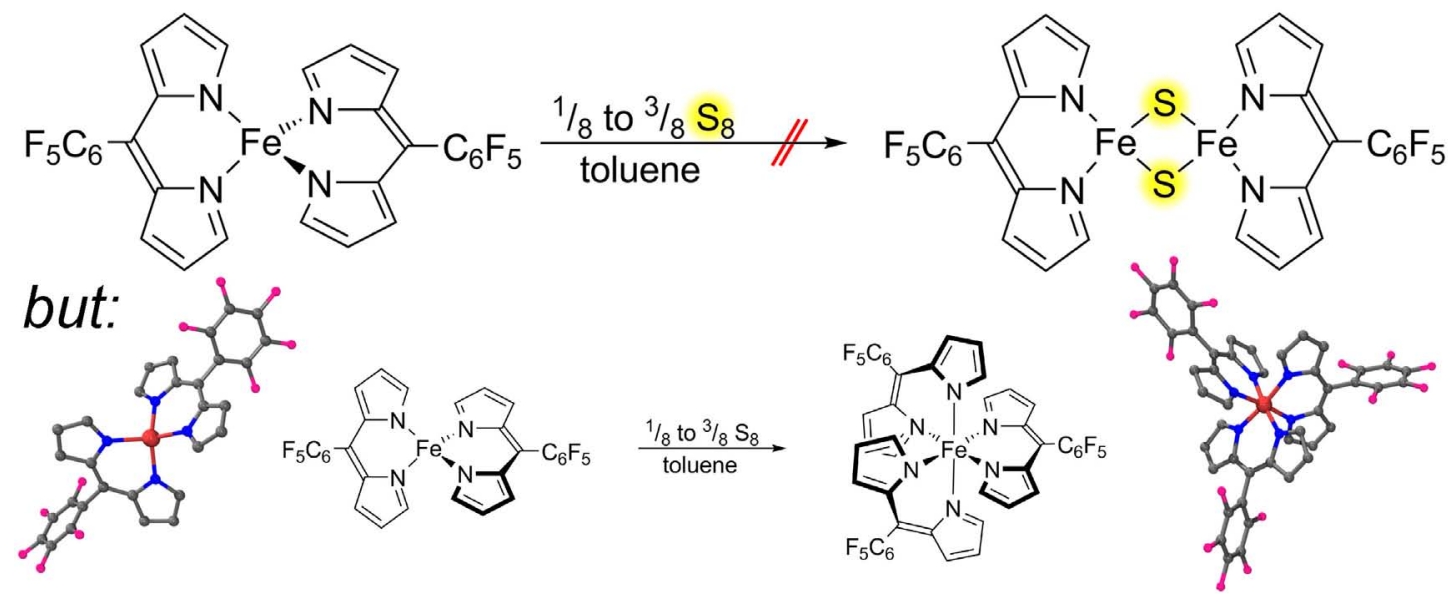

\section{Abstract}

Three different strategies towards neutral [2Fe-2S] cluster compounds utilizing a zwitterionic, a dithiolene and a monoanionic dipyrrine ligand are discussed in this chapter. Unfortunately, the experimental realization of those approaches afforded monomeric or insoluble compounds instead of the intended neutral [2Fe-2S] clusters. Nevertheless, these findings are documented herein, in order to facilitate future efforts in uncovering (novel) pathways to neutral cluster compounds. 


\subsection{Introduction}

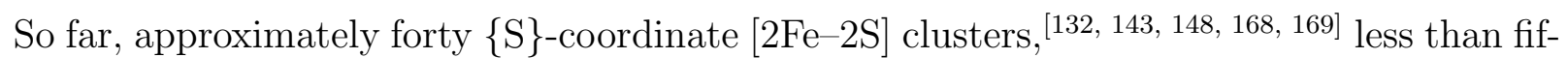
teen $\{\mathrm{N}\}$-coordinate ${ }^{[141,142,170,171]}$ and a few $\{\mathrm{O}\}-{ }_{-}^{[141,172-174]}$ mixed- ${ }^{[171]}$ or halide-coordinate $^{[137]}[2 \mathrm{Fe}-2 \mathrm{~S}]$ species have been reported in literature. ${ }^{[129]}$ All of them have dicationic all-ferric cores ligated by four negatively charged donor atoms with the only exception (reported by TATSUmi et al. ${ }^{[34]}$ ) shown in Scheme 4.1 (the formally all-ferrous $\mathrm{Fe}_{2} \mathrm{~S}_{2}(\mathrm{CO})_{6}$ system reported by HIEBER et al. ${ }^{[175,176]}$ differs significantly from the usual $[2 \mathrm{Fe}-2 \mathrm{~S}]$ core geometries, ${ }^{[177]}$ most likely due to a bonding Fe-Fe interaction - therefore it was not considered as neutral $[2 \mathrm{Fe}-2 \mathrm{~S}]$ cluster in the above sense).

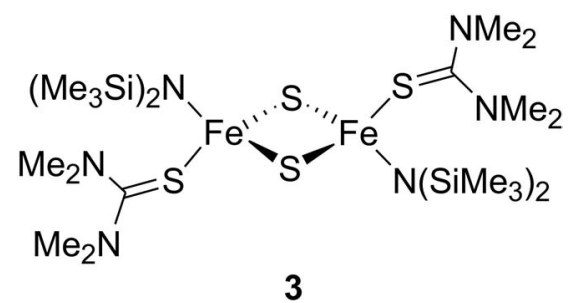

Scheme 4.1: Neutral [2Fe-2S] cluster 3 reported by TATSUmi and co-workers.

A drastic change of physiochemical properties, as reported for $3^{[34]}$ is generally expected for neutral clusters of this type compared to the usual ionic ones. An improved solubility of such neutral compounds in normally unreactive solvents (e.g. benzene or THF) is anticipated as main advantage. This would significantly widen the scope for reactivity studies, as e.g. powerful reductants like $\mathrm{KC}_{8}$ or $\mathrm{NaC}_{10} \mathrm{H}_{8}$ could be used in the latter solvents. At this point it should be mentioned that reduction of neutral compounds commonly is facilitated over the addition of extra-electrons to negatively charged species. Thus, chelate-stabilized analogues of $\mathbf{3}$ would be desired starting materials in the search for isolable mixed-valent [2Fe-2S] clusters (unfortunately, 3 exhibits two irreversible cathodic peaks in the cyclic voltammogram, as often observed for systems with monodentate terminal ligands).

\subsection{Attempts to Synthesize Zwitterionic Ferredoxin Analogues}

In the first approach towards a neutral [2Fe-2S] clusters, a dipolar ligand with the counter ions embedded in the organic backbone was designed. The potentially chelating dithiol $\mathbf{X}$ with two anionic sulfur donor atoms and an incorporated, but peripheral cationic tetraalkylammonium moiety was considered suitable since its coordination would certainly result in an unshielded central cluster core (Scheme 4.2). Synthesis of the desired ligand was straight forward affording its hexafluorophosphonium salt on a $5 \mathrm{~g}$ scale (preparation of VII and VIII was inspired by synthetic procedures reported for related compounds ${ }^{[178,179]}$ ). Deprotonation with potassium hydride (or $n$-BuLi) and subsequent salt metathesis with $\left(\mathrm{NEt}_{4}\right)_{2}\left[\mathrm{Fe}_{2} \mathrm{~S}_{2} \mathrm{Cl}_{4}\right] \mathbf{2}^{[138]}$ resulted in the precipitation of a dark brown powder, insoluble in 
all common organic solvents. Thus, it remains unclear whether cluster $\mathbf{1 2}$ or any kind of polymeric species was formed. No further affords to clarify this concern were made since the material (even if it is cluster 12) is useless for whichever reactivity, due to its insolubility.<smiles>CN(CCO)CCO[Mg]</smiles>
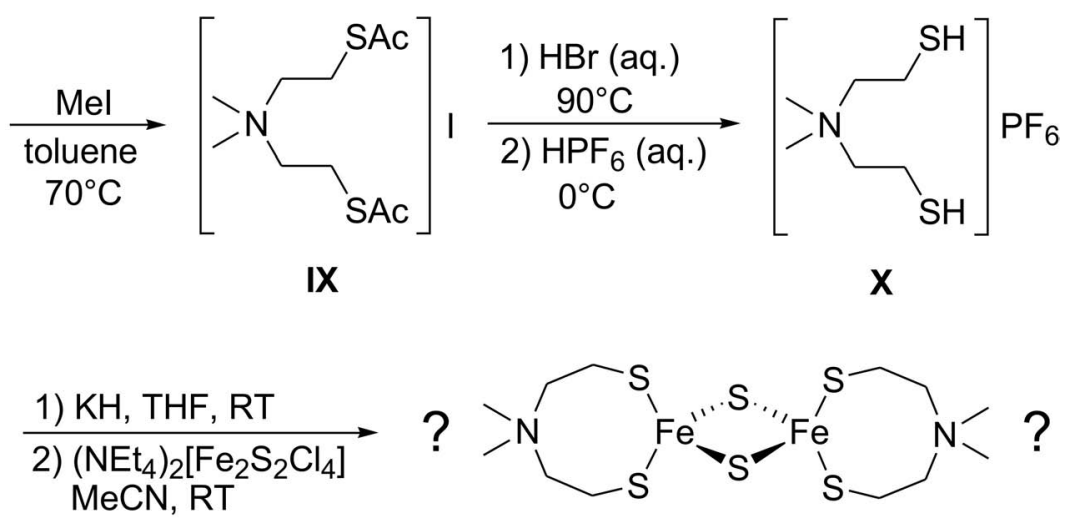

12

Scheme 4.2: Examined synthetic approach to the zwitterionic [2Fe-2S] cluster 12.

\subsection{On the Application of Redox-non-innocent Di- thiolenes in Fe/S Chemistry}

In 1966 SCHRAUZER et al. examined the reaction of 1,2-diphenylethylen-1,2-dithiol (regularly abbreviated by "S-S,Ph") with $\mathrm{Fe}(\mathrm{CO})_{5}$ or $\mathrm{Fe}_{3}(\mathrm{CO})_{12}$ and reported on a compound with $\mathrm{Fe}_{2} \mathrm{~S}_{2}(\mathrm{~S}-\mathrm{S}, \mathrm{Ph})_{2}$ stochiometry. ${ }^{[180]}$ Three years later, A. L. BALCH mentioned, that this compound might be a neutral $[4 \mathrm{Fe}-4 \mathrm{~S}]$ cluster. ${ }^{[181]}$ Assuming that the compound could as well be a neutral [2Fe-2S] cluster, the original procedure of SCHRAUzER et al. was reproduced, affording the described compound as a dark purple-blue powder. Unfortunately, this solid again is insoluble in all organic solvents (slightly blue "solutions" can be obtained by stirring the compound over $\mathrm{CS}_{2}$ for $24 \mathrm{~h}$; concentrations however are insufficient for any spectroscopic characterization). Reduction of a methanolic suspension of this material with hydrazine and precipitation of the soluble reduced species with $\mathrm{Ph}_{4} \mathrm{PCl}$ afforded the $[4 \mathrm{Fe}-4 \mathrm{~S}]$ cluster $\left(\mathrm{Ph}_{4} \mathrm{P}\right)_{2}\left[\mathrm{Fe}_{4} \mathrm{~S}_{4}(\mathrm{~S}-\mathrm{S}, \mathrm{Ph})_{4}\right] \mathbf{1 3}$ (identified by X-ray diffraction, Figure 4.1). Therefore it is assumed that the starting material actually was the corresponding neutral [4Fe-4S] cluster. Although dimerisation during reduction would be a possible scenario as well, existence of the $[4 \mathrm{Fe}-4 \mathrm{~S}]$ species is more likely since a similar literature-known compound, i.e. $\left[\mathrm{Fe}_{4} \mathrm{~S}_{4} \mathrm{Cp}_{2}^{*}(\mathrm{~S}-\mathrm{S}, \mathrm{Ph})_{2}\right]$ was prepared accordingly and characterized by X-ray 
diffraction. ${ }^{[182-184]}$ Again, no further experiments with respect to the true nuclearity of the neutral compound were conducted, due to its restricted solubility and therefore limited suitability in subsequent reactivity studies.

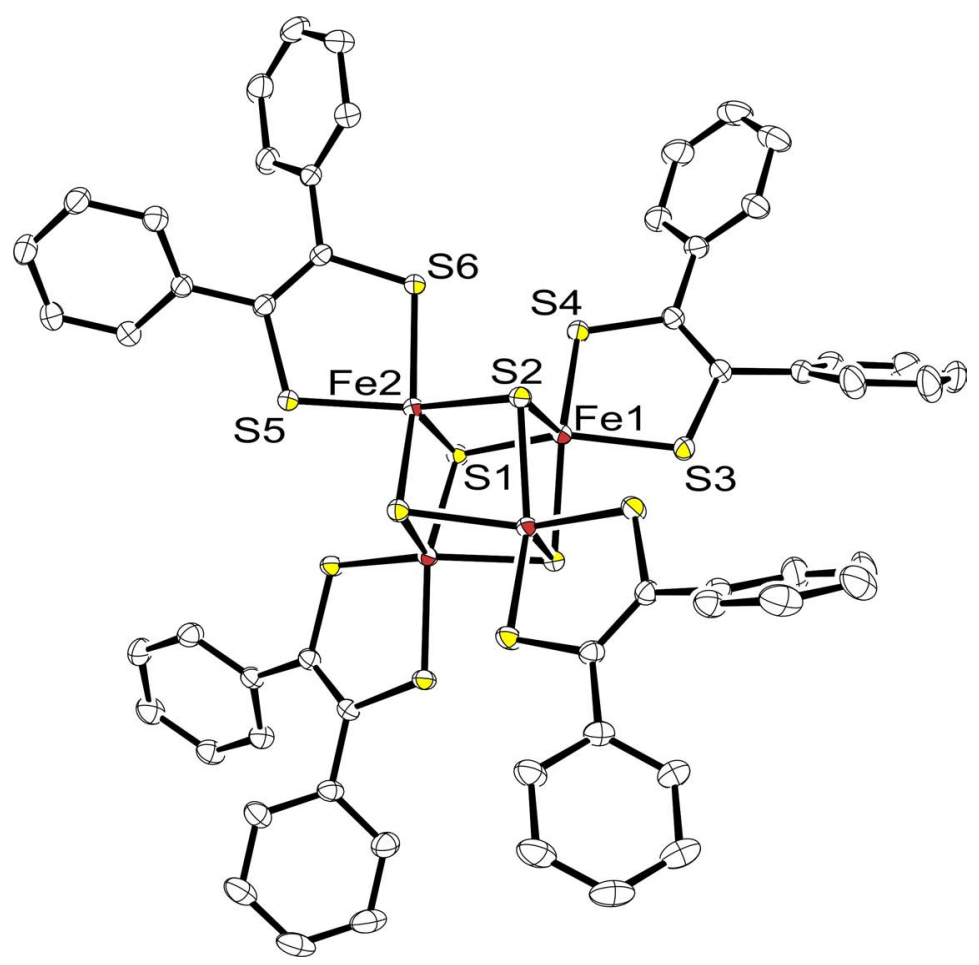

Figure 4.1: ORTEP plot (50\% probability thermal ellipsoids) of the molecular structure of 13. Only atoms of one asymmetric unit are labeled, equivalent atoms are generated by the $1-x+2, y-z+3 / 2$ symmetry transformation. The $P P h_{4}^{+}$counter ions and all hydrogen atoms have been omitted for clarity. Selected atom distances [A] and interatomic angles [0]: Fe1-S2 2.1455(6), Fe1-S3 2.1837(6), Fe1-S4 2.1901(6), Fe1-S1 2.2439(6),

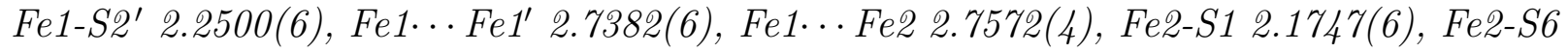
2.1930(6), Fe2-S5 2.1997(6), Fe2-S1' 2.2481(6), Fe2-S2 2.2484(6), Fe2-Fe2' 2.7066(6), S1-Fe2' 2.2481(6), S2-Fe1' 2.2500(6), S2-Fe1-S3 111.62(2), S2-Fe1-S4 104.87(2), S3Fe1-S4 88.02(2), S2-Fe1-S1 102.83(2), S3-Fe1-S1 144.95(3), S4-Fe1-S1 89.30(2), S2-Fe1$S_{2 \prime} 102.51(2), S 3-F e 1-S 2^{\prime}$ 86.96(2), S4-Fe1-S2' 152.09(3), S1-Fe1-S2' 79.36(2), S1-Fe2S6 105.79(2), S1-Fe2-S5 113.67(2), S6-Fe2-S5 87.52(2), S1-Fe2-S1' 103.99(2), S6-Fe2S1' 149.67(3), S5-Fe2-S1' 85.73(2), S1-Fe2-S2 101.75(2), S6-Fe2-S2 89.17(2), S5-Fe2-S2 143.96(2), S1'-Fe2-S2 79.31(2), Fe2-S1-Fe1 77.20(2), Fe2-S1-Fe2' 75.44(2), Fe1-S1-Fe2' 91.66(2), Fe1-S2-Fe2 77.69(2), Fe1-S2-Fe1' 77.02(2), Fe2-S2-Fe1' 91.49(2).

In this context it seems to be noteworthy that a dianionic [2Fe-2S] cluster coordinated by capping benzenedithiolates was reported by NAKAMURA et al. with insufficient characterization data (especially no X-ray diffraction and no Mössbauer data were provided). ${ }^{[185]}$ Several attempts to reproduce the experimental procedure failed (as well as numerous modifications of the original protocol and approaches on the standard salt metathesis route and the indolate-ligand exchange pathway). The existence of this compound is doubtful. 
All- $\{\mathrm{S}\}$-coordinate clusters with terminal six-membered chelate rings, however, seem to be stable, although only a single example ligated by the uncommon $\mathrm{S}_{5}^{2-}$-ligand - namely $\left(\mathrm{PPh}_{4}\right)_{2}\left[\mathrm{Fe}_{2} \mathrm{~S}_{2}\left(\mathrm{~S}_{5}\right)_{2}\right] \mathbf{1 4}$ - has been reported (selected structural parameters are listed in Appendix A). ${ }^{[169]}$

\subsection{Attempts to Synthesize Analogues of Cluster 3}

As mentioned above, cluster 3 exhibits the desired properties of neutral $[2 \mathrm{Fe}-2 \mathrm{~S}]$ clusters with respect to its solubility, although it is certainly unsuitable for chemical reduction. Unfortunately, no detailed experimental procedure for the synthesis of $\mathbf{3}$ is provided in literature, ${ }^{[34]}$ but it is mentioned in the manuscript that addition of sulfur to a toluene solution of $\left\{\mathrm{Fe}\left[\mathrm{N}\left(\mathrm{SiMe}_{3}\right)_{2}\right]\right\}_{2} \mathbf{1 5}^{[186,187]}$ in the presence of tetramethylthiourea affords $\mathbf{3}$ (Scheme 4.3).
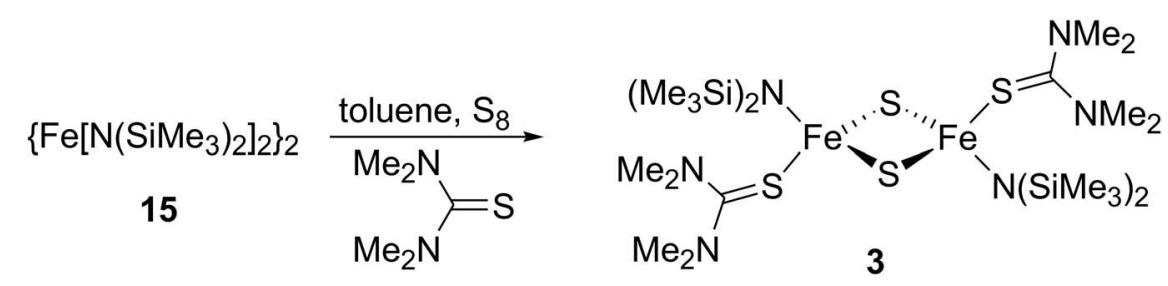

Scheme 4.3: Synthesis of 3 .

Thus, a solution of sulfur (sublimed prior to use) in toluene was added dropwise to a cold toluene solution of $\left\{\mathrm{Fe}\left[\mathrm{N}\left(\mathrm{SiMe}_{3}\right)_{2}\right]\right\}_{2} \mathbf{1 5}$ and tetramethylthiourea. Subsequent warming to room temperature (cyclic voltammetry of $\mathbf{3}$ was performed at ambient temperature, indicating that the compound is stable under these conditions ${ }^{[34]}$ ) and removal of the solvent afforded a dark brown oily residue. Proton NMR analysis of this material revealed numerous non-assignable aliphatic singlets (no reference NMR data reported), that do not significantly change in intensity upon changing the reaction stochiometry (equivalents of added sulfur and present tetramethylthiourea were varied). Crystallization efforts from various solvent mixtures afforded oily precipitates in most cases, with a handful tiny crystals (insufficient in size for X-ray diffraction) sometimes present in those oils. Thus, it remains unclear, whether compound $\mathbf{3}$ is feasible in reasonable yields (or at least reasonable amounts), although its' existence was unambiguously demonstrated by X-ray diffraction. ${ }^{[34]}$ In order to avoid the highly reactive iron precursor and the commonly unselective elemental sulfur as reagent, attempts to obtain $\mathbf{1 7}$ - the iodine-substituted analogue of $\mathbf{3}$ - were conducted. Disappointingly, the defined and stable one-to-one adduct of tetramethylthiourea and iron(III)-iodide $\mathbf{1 6}^{[188]}$ was not reactive towards the chosen sulfur sources $\mathrm{Li}_{2} \mathrm{~S}$ and $\mathrm{Na}_{2} \mathrm{~S}$ (Scheme 4.4, hexamethyldisilathiane was not tested as sulfur-transferring agent, due to the inadequate difference in bond enthalpies, i.e. Si-I vs. Si-S).

Bearing in mind that only one hexamethyldisilazane is lost during the synthesis of $\mathbf{3}$ and that substitution of the remaining terminal ligands in $\mathbf{3}$ for a chelating capping ligand would probably result in a superior cluster stability, analogues of compound $\mathbf{1 8}^{[189]}$ (Scheme 4.5) 


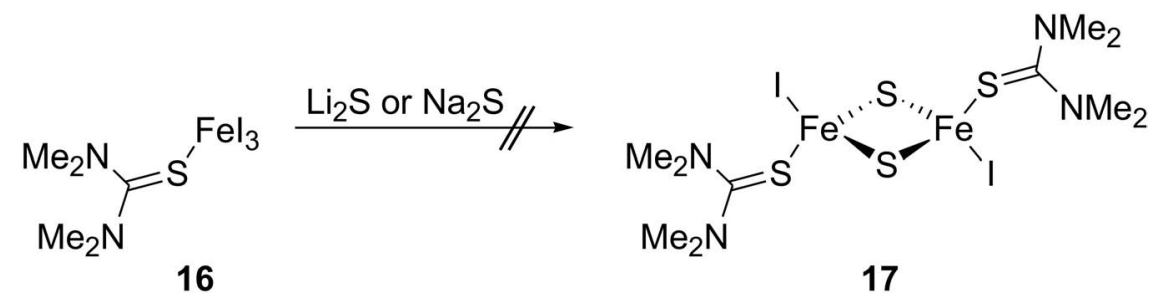

Scheme 4.4: Failed synthesis of $\mathbf{1 7}$.

were considered as suitable starting materials in a sulfur-incorporating oxidative dimerisation to the corresponding neutral $[2 \mathrm{Fe}-2 \mathrm{~S}]$ clusters.

HoLlAND et al. reported that compound $\mathbf{1 8}^{[190]}$ readily forms the mono- $\mu$-sulfido bridged species 19 (rationally synthesized through reaction of sulfur with the related dinitrogenbridged NacNac-coordinate iron(I) precursor ${ }^{[191]}$ ) and that a reproducible synthesis for the corresponding bis- $\mu$-sulfido bridged species $\mathbf{2 0}$ is not available so far, although serendipitously obtained single crystals suitable for X-ray diffraction revealed the existence of this species (unpublished results, private communication). Synthetic approaches starting from $\mathbf{1 9}^{[190]}$ or $\mathbf{2 1}^{[189]}$ (synthesis of the required ligand XI is described in literature ${ }^{[192]}$ ) according to Scheme 4.6 however were unsuccessful with respect to the isolation of the neutral [2Fe-2S] cluster 20. Taking into account that dipyrromethane ligands were successfully applied in the synthesis of dianionic $[2 \mathrm{Fe}-2 \mathrm{~S}]$ clusters, ${ }^{[170]}$ their oxidized dipyrrine derivatives seemed to be suitable for stabilizing neutral type-20 clusters. Moreover, an approach via an analogue of the 3-coordinate compound 18 and subsequent oxidation with elemental sulfur appeared reasonable, as a diminished sterical demand of the dipyrrine moiety was expected to facilitate the latter conversion. Unfortunately, the desired three-coordinate complex 22 was neither obtained through conversion according to Scheme 4.5, nor through transference of the reaction into a toluene solution at room temperature (Scheme 4.7).

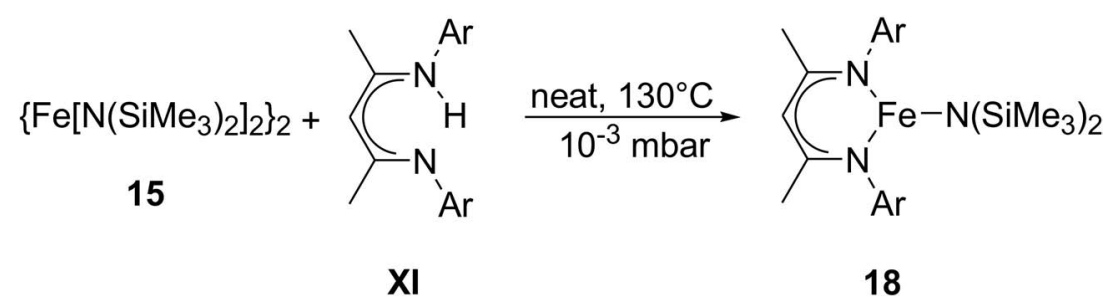

Scheme 4.5: Solvent-free synthesis of 18 (Ar=2,6-di-iso-propylphenyl).

Instead, large green block-shaped crystals of the bis-substituted complex $\mathbf{2 3}$ were isolated in good yields (with respect to the dipyrrine starting material) from the latter experiment (heating both reactants in vacuum without solvent led to a black oily mixture of unidentified compounds, as indicated by ${ }^{19} \mathrm{~F}$ NMR spectroscopy). Single crystals (beautiful green colored, twinkling in all spectral colors) of $\mathbf{2 3}$ suitable for X-ray diffraction were obtained by cooling a saturated benzene/pentane or toluene/pentane solution (both intensively red colored) of the complex to $-30{ }^{\circ} \mathrm{C}$ (Figure 4.2). Most likely, 22 is an intermediate in the formation of $\mathbf{2 3}$, but by far more reactive towards the free ligand $\mathbf{X I I}{ }^{[193,194]}$ than 
<smiles></smiles>

19<smiles></smiles>

21

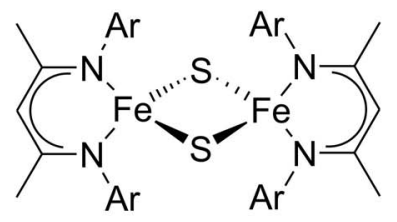

20

Scheme 4.6: Failed syntheses of 20 (Ar=2,6-di-iso-propylphenyl).

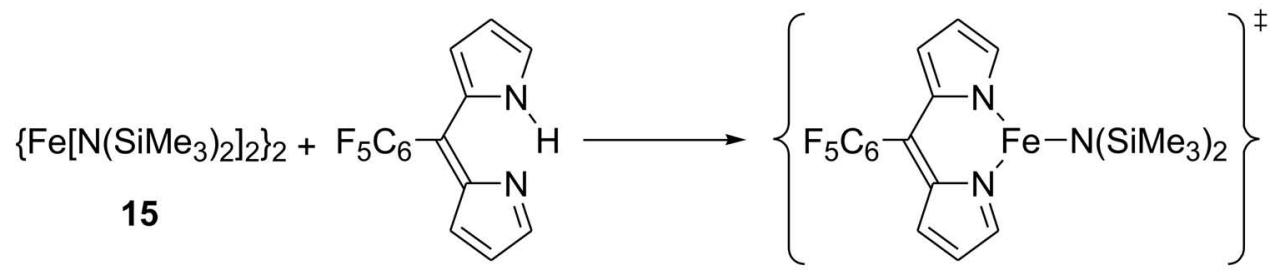

XII

22

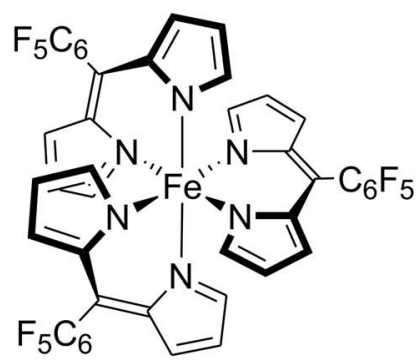

$\frac{1 / 8 \text { to } 3 / 8 S_{8}}{\text { or } \mathrm{O}_{2} \text { (air) }}$

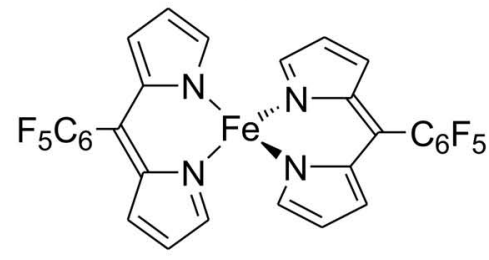

23

24

Scheme 4.7: Synthesis of 23 and 24.

$\left\{\mathrm{Fe}\left[\mathrm{N}\left(\mathrm{SiMe}_{3}\right)_{2}\right]_{2}\right\}_{2} \mathbf{1 5}$, even at low temperatures and under dropwise addition of a highly diluted solution of the dipyrrine.

Nevertheless, elemental sulfur was added to 23 assuming that oxidative dimerisation to the neutral $[2 \mathrm{Fe}-2 \mathrm{~S}]$ cluster might proceed under elimination of one $\left\{\mathrm{N}_{2}\right\}$-ligand (compare to the elimination of $\mathbf{I}$ during the first synthesis of $\left.\mathbf{1}^{[131]}\right)$. However, inspection of the crude product of this reaction by proton and fluorine NMR spectroscopy revealed that the obtained material was identical with product $\mathbf{2 4}$ arising from aerial oxidation (observed accidentally by exposure of an NMR sample of 23 to air). Based on the molecular structure obtained from low-quality single crystals by X-ray diffraction (collected data were 
insufficient for appropriate refinement) and the NMR data, compound $\mathbf{2 4}$ was identified as octahedral tris-substituted iron (III) complex (Figure 4.3, synthesis of this compound - starting from $\mathrm{FeCl}_{3}$ and XII - was previously reported in literature ${ }^{[195]}$ ).

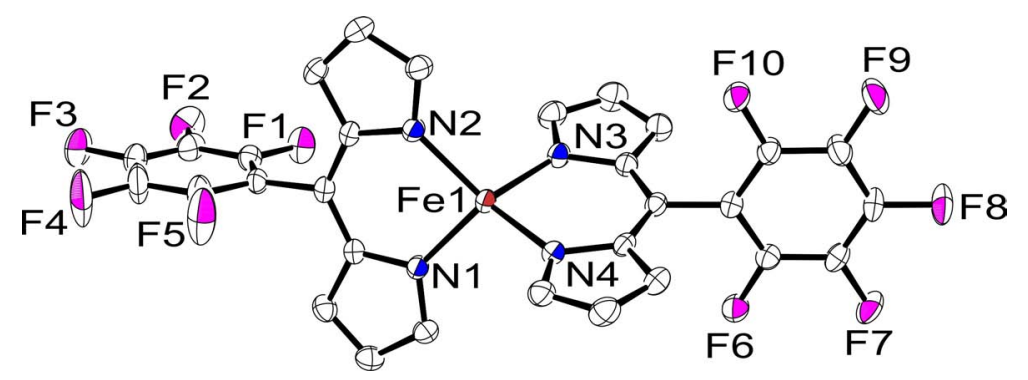

Figure 4.2: ORTEP plot (50\% probability thermal ellipsoids) of the molecular structure of 23. Hydrogen atoms and encapsulated benzene molecules have been omitted for clarity. Selected atom distances [A] and interatomic angles [०]: Fe1-N1 2.0258(12), Fe1N2 2.0283(12), Fe1-N4 2.0295(13), Fe1-N3 2.0347(14), N1-Fe1-N2 91.55(5), N1-Fe1-N4 107.77(5), N2-Fe1-N4 136.36(5), N1-Fe1-N3 128.68(6), N2-Fe1-N3 105.64(5), N4-Fe1-N3 92.38(5).

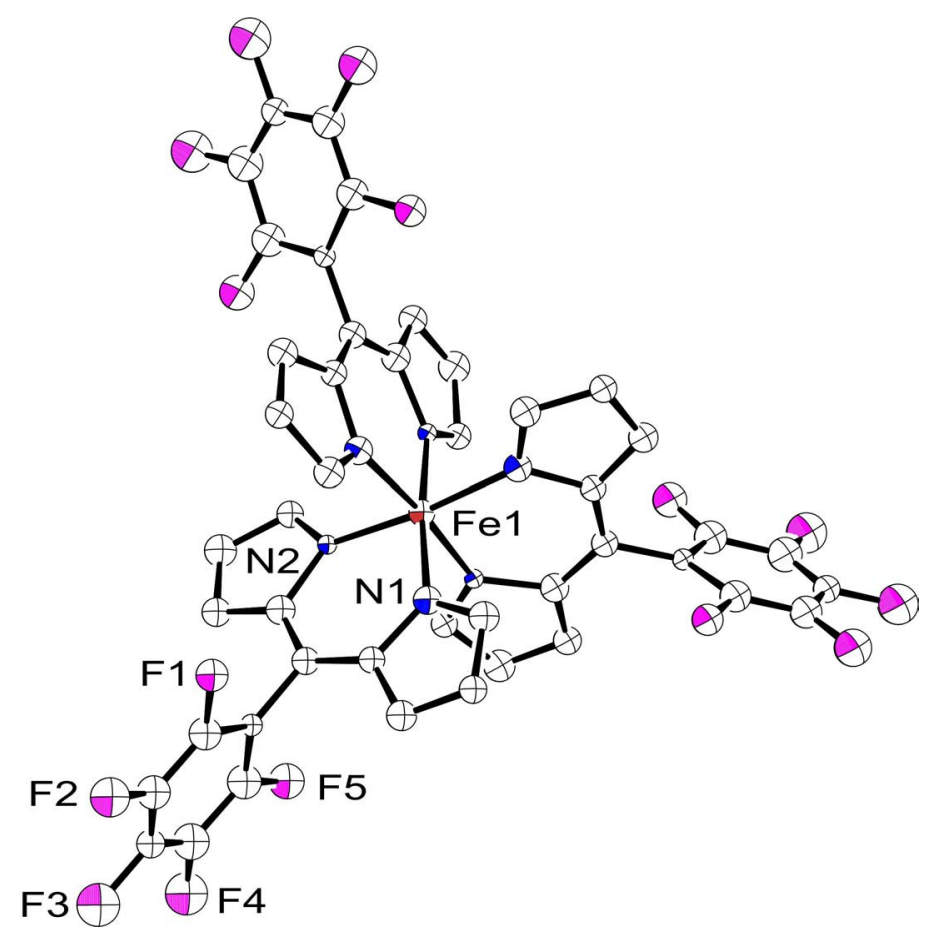

Figure 4.3: ORTEP plot of the molecular structure of 24. Only atoms of one asymmetric unit are labeled, equivalent atoms are generated by the $1-y+1, x-y, z$ and the $-x+$ $y+1,-x+1, z$ symmetry transformation. Hydrogen atoms have been omitted for clarity. Note: Quality of single crystals and collected crystallographic data were insufficient for refinement. Therefore only a picture is shown as an "educated guess" for the molecular structure of $\mathbf{2 4}$. Further interpretation or analysis is inappropriate. 


\subsection{Conclusions}

Discrete approaches towards neutral [2Fe-2S] cluster were examined utilizing a zwitterionic, a dithiolene and a monoanionic dipyrrine ligand. In the case of the zwitterionic and the dithiolene ligand, insoluble iron-containing solids were isolated, but only identified in the latter case as a $[4 \mathrm{Fe}-4 \mathrm{~S}]$ cluster. Both strategies are unsuitable for the isolation of the desired neutral [2Fe-2S] cluster compounds. In contrast, reactions of (low-coordinate) ferrous iron complexes with elemental sulfur definitely can afford sulfur-bridged dinuclear compounds with high solubility, but products strongly depend on the ligand environment (NacNac-coordinate $[2 \mathrm{Fe}-1 \mathrm{~S}]$ vs. $\left(\mathrm{Me}_{3} \mathrm{Si}\right)_{2} \mathrm{~N}$-coordinate $[2 \mathrm{Fe}-2 \mathrm{~S}]$ systems). Thus, sterically bulky substitution in both dipyrrine $\alpha$-positions might afford three-coordinate type-22 intermediates, that possible prefer oxidative sulfur-incorporation (to form the $[2 \mathrm{Fe}-1 \mathrm{~S}]$ or [2Fe-2S] compounds) over formation of octahedral complexes (as observed here for the unsubstituted dipyrrine ligands). 


\section{Chapter 5}

\section{Secondary Bonding Interactions in Biomimetic [2Fe-2S] Clusters}

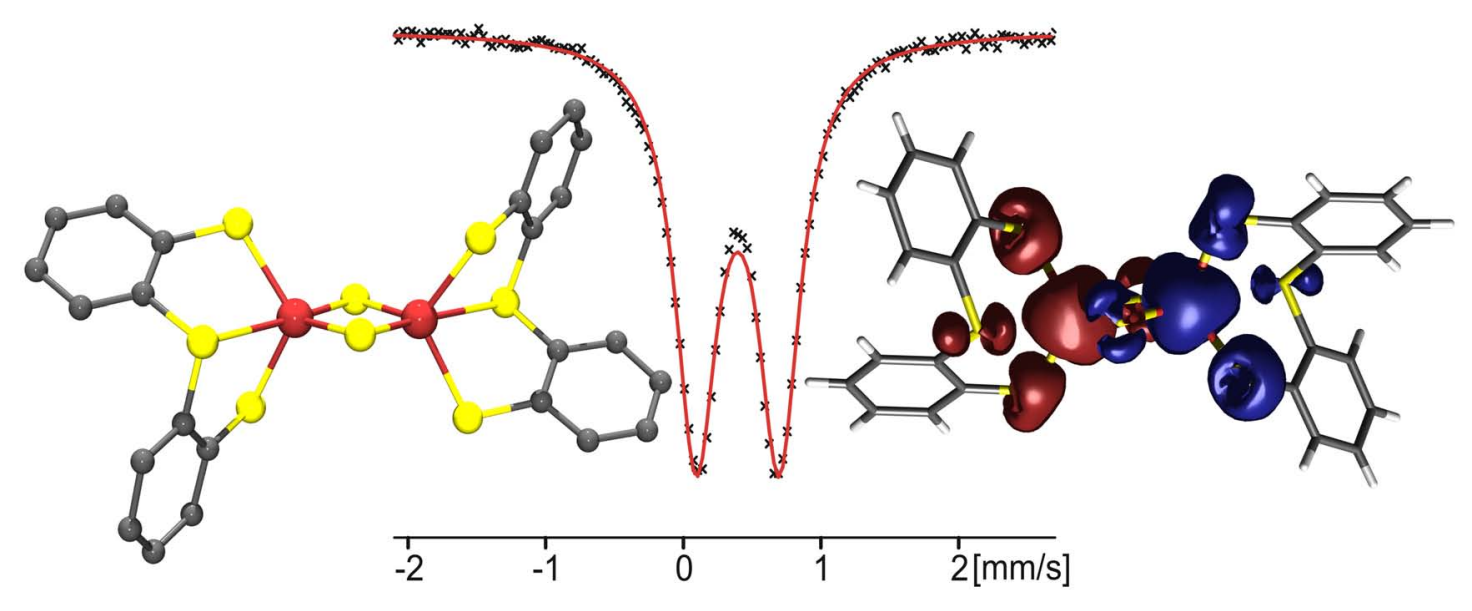

\section{Abstract}

A series of synthetic $[2 \mathrm{Fe}-2 \mathrm{~S}]$ complexes with terminal thiophenolate ligands and tethered ether or thioether moieties has been prepared and investigated in order to provide models for the interaction of additional donor atoms with the Fe atoms in biological $[2 \mathrm{Fe}-2 \mathrm{~S}]$ clusters. Structural consequences of the secondary bonding interactions were analyzed in detail, and effects on the spectroscopic and electronic properties probed by UV-Vis, Mössbauer, and ${ }^{1} \mathrm{H}$ NMR spectroscopy, as well by SQUID measurements and cyclic voltammetry. The potential relevance of the findings for biological [2Fe-2S] sites is considered. 


\subsection{Introduction}

The recent crystallographic analysis of biotin synthase revealed a unique coordination environment of the enzymes' [2Fe-2S] cluster, with three terminal cysteine-S ligands and an unprecedented terminal arginine- $\mathrm{N}(d(\mathrm{Fe}-\mathrm{N})=2.40 \AA)$ that causes a noticeable distortion of the local cluster symmetry (Scheme 5.1). ${ }^{[97]}$ Interestingly, a second N-atom of the arginine residue appears to be located relatively close to the $\mathrm{Fe}$ at $d(\mathrm{Fe} \cdots \mathrm{N})=3.07 \AA$, suggesting that secondary bonding interactions, a possible bidentate coordination or most likely a hydrogen-bridging interaction to one of the $\mu$-sulfides of the cluster core might play a role. While the arginine residue does not seem to be essential for the catalytic reaction of biotin synthase, ${ }^{[196]}$ the biological relevance of this very unusual cluster coordination remains to be elucidated. One should note that arginine is a very rare ligand in metallobiosites, ${ }^{[197]}$ although guanidine-metal interactions are quite flexible and may comprise syn, anti and chelating coordination.

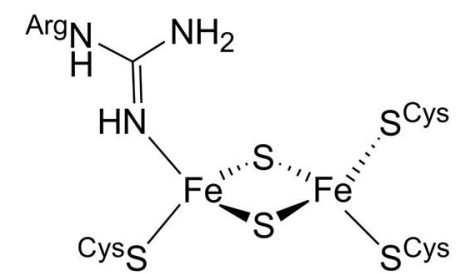

[2Fe-2S] site of Biotin Synthase

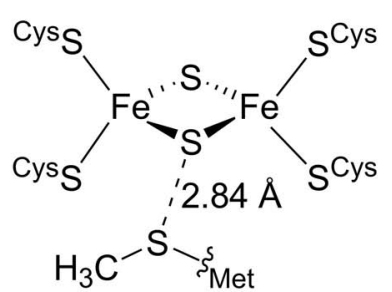

reduced form of the $[2 \mathrm{Fe}-2 \mathrm{~S}]$ ferredoxin from Rhodobacter capsulatus

Scheme 5.1: Selected natural [2Fe-2S] sites.

In another new turn in biological [2Fe-2S] cluster chemistry, considerable conformational differences have recently been reported for a $[2 \mathrm{Fe}-2 \mathrm{~S}]$ ferredoxin from Rhodobacter capsulatus in its oxidized and reduced forms. ${ }^{[63]}$ Upon reduction the $[2 \mathrm{Fe}-2 \mathrm{~S}]$ core switches from a planar to a distorted lozenge geometry, and the movement of a methionine side chain results in the methionine-S $\delta$ atom approaching a bridging sulfide of the cluster at less than $2.9 \AA$ (Scheme 5.1). The functional significance of these changes is still unclear, but it has been speculated that the proximity of the electron-rich thioether-S may contribute to controlling the redox potential of the cluster by modulating the overall electrostatic environment. In the context of those new developments in iron-sulfur cluster chemistry,it was realized that geometric distortions and consequences of secondary bonding interactions have only been scarcely addressed for synthetic [2Fe-2S] complexes. ${ }^{[155]}$ HoLm and co-workers had previously studied the occurrence of secondary bonding interactions in $[4 \mathrm{Fe}-4 \mathrm{~S}]$ clusters, ${ }^{[144]}$ where the terminal thiolate ligands contained potentially coordinating ortho-substituents, $\left[\mathrm{Fe}_{4} \mathrm{~S}_{4}\left(\mathrm{SC}_{6} \mathrm{H}_{4}-\mathrm{o}-\mathrm{X}\right)_{4}\right]^{2-}$ with $\mathrm{X}=\mathrm{OH}, \mathrm{OMe}, \mathrm{NH}_{2}(\mathrm{NH} \cdots \mathrm{S}$ hydrogen bonding interactions in synthetic $[2 \mathrm{Fe}-2 \mathrm{~S}]$ clusters were reported in literature as well $\left.{ }^{[198]}\right)$. Indeed, unique Fe-site chemistry during catalytic turnover has recently been demonstrated for the $[4 \mathrm{Fe}-4 \mathrm{~S}]$ cluster in ferredoxin:thioredoxin reductase (FTR), which involves interaction of a disulfide with one Fe, followed by breaking of the disulfide bond and five-coordination of that unique Fe site with two cysteinate ligands. ${ }^{[199]}$ In order to 
assess whether such interactions are feasible in syntethic [2Fe-2S] systems and to evaluate possible effects on spectroscopic and electronic properties of the cluster, a series of synthetic $[2 \mathrm{Fe}-2 \mathrm{~S}]$ clusters coordinated by thiophenolate derivatives bearing additional donor sites has been examined. Some particularly preorganized chelate ligands have been employed to enforce additional bonding interactions, and DFT calculations have been carried out to corroborate the structural and spectroscopic findings.

\section{$5.2 \quad$ Ligand Synthesis}

Two of the three monodentate thiophenols utilized in the present study are commercially available, namely 2-ethyl-thiophenol $\mathbf{X I I I}^{\mathbf{C}}$ and 2-metoxy-thiophenol $\mathbf{X I I I}^{\mathbf{O}}$. The third one, 2-mercaptomethyl-thiophenol $\mathbf{X I I I}^{\mathrm{S}}$ was synthesized via mono-methylation of ironcoordinate 1,2-benzene-dithiolate and subsequent acid hydrolysis of the complex according to literature methods. ${ }^{[200-202]}$ The chelating ligands $2,2^{\prime}$-oxydibenzenethiol $\mathbf{X I V} \mathbf{O}^{\mathbf{O}}$ [203] and $2,2^{\prime}$-thiodibenzenethiol $\mathbf{X I V}^{\mathbf{S}}{ }^{\mathbf{2} 204]}$ were synthesized according to published procedures as well, starting from diphenylether and diphenylthioether, respectively. In both cases, TMEDA-assisted twofold ortho-lithiation, prior to reaction with elemental sulfur and reductive cleavage of the generated polysulfide species with $\mathrm{LiAlH}_{4}$ afforded the air-sensitive ligands in one-pot reactions. The third chelating ligand $-2,2^{\prime}$-methylenedibenzenethiol $\mathbf{X I V}^{\mathbf{C}}$ - was previously unknown in literature and synthesized in a five step procedure according to Scheme 5.2. Condensation of 2,4-di-tert-butylphenol XV with para-formaldehyde by modified literature methods resulted in the formation of $6,6^{\prime}$-methylenebis(2, 4-di-tert-butylphenol) XVI. ${ }^{[205]}$ Subsequent stepwise removal of all tert-butyl protective groups by modifications of previously reported procedures afforded the required unsubstituted 2,2'-methylenediphenol XVII. ${ }^{[206,207]}$ Preparation of the bis- $O$-thiocarbamate ester XVIII and separation from the undesired mono- $O$-thiocarbamate ester was straight forward ( $74 \%$ yield). Rearrangement to the bis- $S$-carbamate ester XIX and reductive carbamate cleavage using the standard protocol for Miyazaki-Newman-Kwart rearrangements afforded the free dithiol $\mathbf{X I V} \mathbf{C}^{\mathbf{C}}$ (a crystallographically characterized tert-butyl-substituted derivative of this ligand was previously reported without explicit experimental procedure, ${ }^{[208]}$ the coordination chemistry of $\mathbf{X I V}^{\mathbf{C}}$ was examined by the preparation of a $\left\{\mathrm{N}_{2} \mathrm{~S}_{2}\right\}$-ligated zinc complex as described in Appendix B).

\subsection{Cluster Synthesis and Structural Characterization}

With the latter monodentate ligands at hand, a series of new [2Fe-2S] clusters has been synthesized by means of standard salt metathesis reactions starting from the readily available $\left(\mathrm{NEt}_{4}\right)_{2}\left[\mathrm{Fe}_{2} \mathrm{~S}_{2} \mathrm{Cl}_{4}\right] \mathbf{2}$ (Scheme 5.3). Complexes $\mathbf{2 5}^{\mathrm{C}}, \mathbf{2 5}^{\mathrm{O}}$ and $\mathbf{2 5}$ were obtained in moderate to good yields, and crystalline material could be obtained by diffusion of $\mathrm{Et}_{2} \mathrm{O}$ into DMF solutions $\left(\mathbf{2 5}^{\mathrm{O}}, \mathbf{2 5 ^ { \mathrm { S } }}\right)$ or by slowly cooling a saturated MeCN solution from

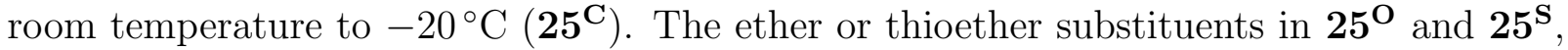
respectively, were anticipated to potentially interact with the Fe centers, and the alkyl- 
<smiles>CC(C)(C)c1ccc(O)c(C(C)(C)C)c1</smiles><smiles>CC(C)(C)c1cc(Cc2cc(C(C)(C)C)cc(C(C)(C)C)c2O)c(O)c(C(C)(C)C)c1</smiles>

XVI<smiles>Oc1ccccc1Cc1ccccc1O</smiles>

XVII<smiles>CN(C)C(=O)Sc1ccccc1Cc1ccccc1SC(=O)N(C)C</smiles>

XIX
1) $\mathrm{NaH}, \mathrm{DMF}$,

HMPA

2) $\mathrm{Me}_{2} \mathrm{NCSCl}$, $80^{\circ} \mathrm{C}$<smiles>CN(C)C(=S)Oc1ccccc1Cc1ccccc1OC(=S)N(C)C</smiles>

XVIII

1) $\mathrm{AlCl}_{3}, \mathrm{MeNO}_{2}$, toluene, $0^{\circ} \mathrm{C}$

2) $\mathrm{AlCl}_{3}$, toluene, $50^{\circ} \mathrm{C}$

Scheme 5.2: Synthesis of ligand $\boldsymbol{X I V ^ { C }}$.

substituted $25^{\mathrm{C}}$ was prepared to allow accurate structural comparison with an analogous system that lacks the additional donor groups. Molecular structures of $25^{\mathrm{C}}, \mathbf{2 5 ^ { \mathrm { O } }}$, and $25^{\mathrm{S}}$ are quite similar, and the anions of all three complexes are shown in Figure 5.1 for comparison. Selected structural parameters are listed in Table 5.1.<smiles>[X]c1ccccc1S</smiles>

XIIIC $\left(X=\mathrm{CH}_{2} \mathrm{Me}\right)$

$\mathrm{XIII}^{\mathrm{O}}(\mathrm{X}=\mathrm{OMe})$

$X_{I I} \mathrm{~S}$ (X=SMe)<smiles>[X]c1ccccc1SO[Sn]Sc1ccccc1Sc1ccccc1[X]</smiles>

$25^{\mathrm{C}}\left(\mathrm{X}=\mathrm{CH}_{2} \mathrm{Me}\right)$

$25^{\circ}$ (X=OMe)

$25^{S}$ (X=SMe)

Scheme 5.3: Synthesis of complexes $25^{C}, 25^{\circ}$ and $25^{S}$. 
In all cases, the tetraethylammonium cations are well separated from the $[2 \mathrm{Fe}-2 \mathrm{~S}]$ dianions. Compound $25^{\mathrm{O}}$ crystallizes in the monoclinic space group $P 2_{1} / \mathrm{c}$ with four formula units per unit cell. The asymmetric unit contains two crystallographically independent anionfragments, and each $[2 \mathrm{Fe}-2 \mathrm{~S}]$ dianion consists of two fragments as a centrosymmetric dimer with crystallographically imposed $C_{\mathrm{i}}$ symmetry. $25^{\mathrm{S}}$ crystallizes in the monoclinic space group $P 2_{1} / \mathrm{n}$ with two molecules per unit cell and also features crystallographically imposed $C_{\mathrm{i}}$ symmetry. The cores of both $25^{\mathrm{O}}$ and $25^{\mathrm{S}}$ are close to effective $C_{2 \mathrm{~h}}$ symmetry due to the only marginal differences between the Fe1-S2 and Fe1-S3 bond lengths. The alkyl derivate $\mathbf{2 5}^{\mathrm{C}}$ crystallizes in the monoclinic space group $C 2 / \mathrm{c}$ with four formula units and eight MeCN molecules per unit cell. In contrast to $25^{\mathrm{O}}$ and $25^{\mathrm{S}}$, the anions of $25^{\mathrm{C}}$ are perfectly $C_{2}$-symmetric molecules, with the $C_{2}$-axis along Fe1 and Fe2. Bond lengths Fe1-S1 and Fe1-S6 and all bond lengths between the iron atoms and the terminal thiophenolate sulfur atoms are almost identical for $\mathbf{2 5}^{\mathrm{C}}$, but in this case differences in the angles S2-Fe1-S3 and $\mathrm{S} 4-\mathrm{Fe} 2-\mathrm{S} 5$ cause deviations from an effective $C_{2 \mathrm{~h}}$-symmetry. The Fe $\cdots$ Fe distances of $25^{\mathrm{O}}, \mathbf{2 5 ^ { \mathrm { S } }}$ and $25^{\mathrm{C}}$ resemble those of the other $\left[\mathrm{Fe}_{2} \mathrm{~S}_{2}(\mathrm{SR})_{4}\right]^{2-}$ clusters with terminal thiophenolate derivatives that have been characterized to date (around 2.67-2.70 $\mathrm{A}$ ). All type 25 complexes contain symmetric $\left(\mathrm{Fe}_{2} \mathrm{~S}_{2}\right)^{2+}$ cores with a perfectly planar structure (dihedral angles Fe1-S1-Fe2-S6 $\left.=0^{\circ}\right)$. Distances Fe-SR and Fe- $(\mu-\mathrm{S})$ as well as angles RSFe-SR and $(\mu-\mathrm{S})-\mathrm{Fe}-(\mu-\mathrm{S})$ are in the usual range (Table 5.1). 

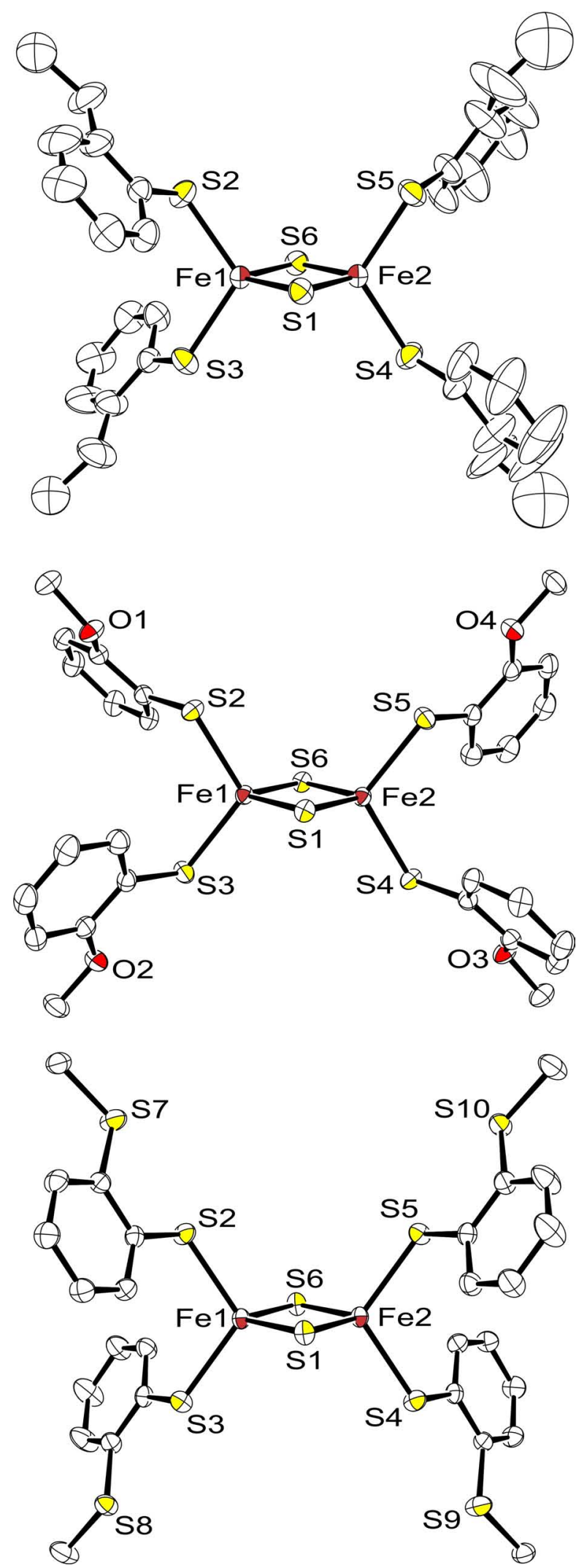

Figure 5.1: ORTEP plot (50\% probability thermal ellipsoids) of the molecular structures of the dianions of $25^{C}$ (top), $25^{O}$ (middle), and $25^{S}$ (bottom). All hydrogen atoms have been omitted for clarity. 


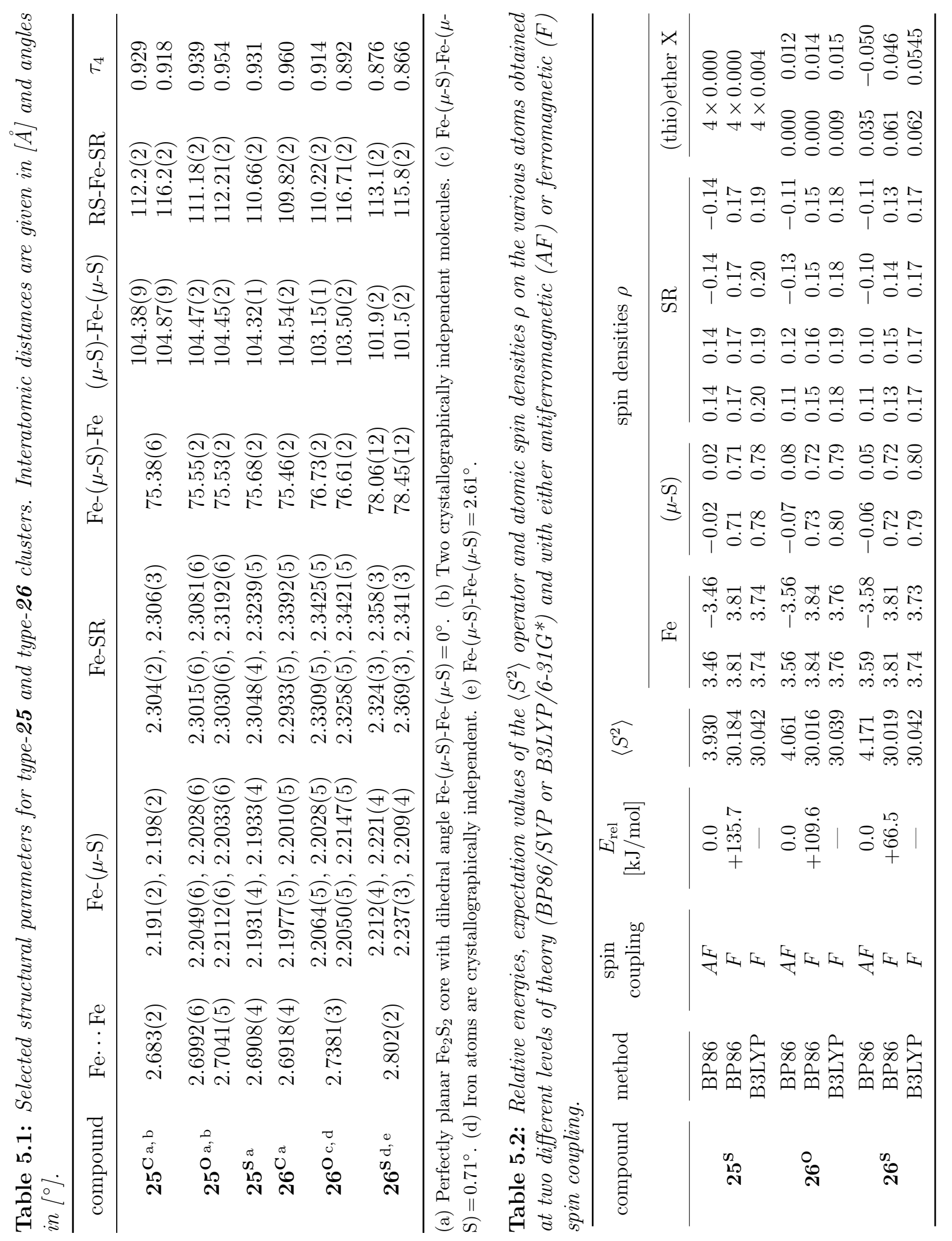


It is obvious from the X-ray structural analyses of $25^{\mathrm{O}}$ and $25^{\mathrm{S}}$ that no interaction between the Fe atoms and the ether or thioether groups occurs in the solid state, and both molecules adopt conformations very similar to that found for the alkyl analogue $\mathbf{2 5}^{\mathrm{C}}$. The substituents do not induce any significant structural distortion, as evaluated by the $\tau_{4}$-values (Table 5.1).[166, 209] In order to enforce secondary interactions with the ether or thioether moiety in a more rigid chelate situation, the related systems $26^{\mathrm{C}}, \mathbf{2 6}^{\mathrm{O}}$ and $\mathbf{2 6}^{\mathrm{S}}$ were synthesized starting from the tethered bis(benzenethiolato) ligands (Scheme 5.4). Here the yield decreased in the order $\mathbf{2 6}^{\mathrm{C}}>\mathbf{2 6}^{\mathrm{O}}>\mathbf{2 6}^{\mathrm{S}}$ due to the formation of significant amounts of mononuclear complexes (see Chapter 6). It should be noted that these mononuclear compounds become the preferred products with increasing donor strength of the potentially tridentate ligands, and no type $\mathbf{2 6}[2 \mathrm{Fe}-2 \mathrm{~S}]$ cluster could be isolated for the related systems with amine- or phosphine-based linkers $(\mathrm{X}=\mathrm{NMe}, \mathrm{PPh}) .{ }^{[210,211]}$

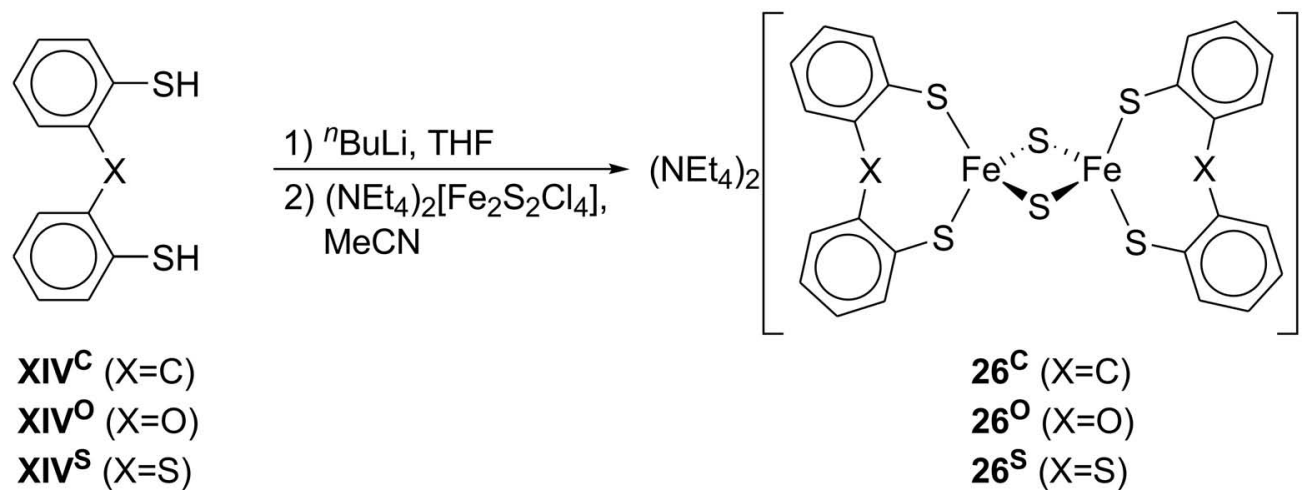

Scheme 5.4: Synthesis of complexes $2 \boldsymbol{6}^{C}, \mathbf{2 6}^{O}$ and $2 \boldsymbol{6}^{S}$.

Single crystals suitable for X-ray analysis were obtained by diffusion of diethyl ether into a saturated solution of the complex in $\mathrm{MeCN}\left(\mathbf{2 6}^{\mathbf{C}}\right)$ or by slow diffusion of diethyl ether into DMF solutions $\left(\mathbf{2 6}^{\mathrm{O}}\right.$ and $\left.\mathbf{2 6}^{\mathrm{S}}\right)$. Molecular structures of the anions of $\mathbf{2 6}^{\mathrm{C}}, \mathbf{2 6}^{\mathrm{O}}$ and $\mathbf{2 6}^{\mathrm{S}}$ are depicted in Figure 5.2, and selected structural parameters are included in Table 5.1. Clusters $\mathbf{2 6}^{\mathbf{C}}$ and $\mathbf{2 6}^{\mathbf{O}}$ crystallize in the monoclinic space group $P 2_{1} / \mathrm{c}$ with two or four formula units per unit cell, respectively, while $\mathbf{2 6}^{\mathrm{S}}$ crystallizes in $P 2_{1}$ with two molecules

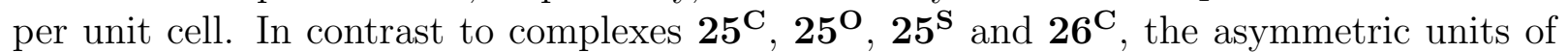
$\mathbf{2 6}^{\mathrm{O}}$ and $26^{\mathrm{S}}$ each contain one complete dianion and two well-separated tetraethylammonium cations. In both latter cases the point group symmetries of the clusters are reduced from apparent $C_{2 \mathrm{~h}}$ (with the horizontal mirror planes through Fe1, Fe2, S1, S2, X1 and X2 (X = ether-O or thioether-S atoms) and the perpendicular $C_{2}$ axes though the centroids of the $\mathrm{Fe}_{2} \mathrm{~S}_{2}$-cores $)$. Bond lengths $\mathrm{Fe}-\mathrm{SR}$ and $\mathrm{Fe}-(\mu-\mathrm{S})$ are not drastically different from those of other $[2 \mathrm{Fe}-2 \mathrm{~S}]$ clusters coordinated by thiophenolate derivatives (summarized in Table 5.1), but a slight bond elongation is discernible for $\mathbf{2 6}^{\mathrm{S}}$. Differences are more significant for the Fe...Fe separations and the angles $(\mu-\mathrm{S})-\mathrm{Fe}-(\mu-\mathrm{S})$. While the elongation of the $\mathrm{Fe} \cdot \mathrm{F}$ Fe distance by approximately $4 \mathrm{pm}$ is still moderate in $\mathbf{2 6}^{\mathbf{O}}(2.738(1) \AA$ versus 2.683 $2.704 \AA$ for type $\mathbf{2 5}$ complexes and $\left.\mathbf{2 6}^{\mathbf{C}}\right)$, it is much more pronounced for $\mathbf{2 6}^{\mathbf{S}}(2.802(2) \AA)$. This goes along with a decrease in the $(\mu-\mathrm{S})-\mathrm{Fe}-(\mu-\mathrm{S})$ angles and a corresponding increase of the $\mathrm{Fe}-(\mu-\mathrm{S})-\mathrm{Fe}$ angles, as well as some distortion of the $\left(\mathrm{Fe}_{2} \mathrm{~S}_{2}\right)^{2+}$ cores away 
from planarity (dihedral angles Fe1-S1-Fe2-S2 are $0.71^{\circ}$ for $\mathbf{2 6}^{\mathbf{O}}$ and $2.61^{\circ}$ for $\mathbf{2 6}^{\mathbf{S}}$ ). It is interesting to note that $[2 \mathrm{Fe}-2 \mathrm{~S}]$ clusters in proteins also tend to have longer $\mathrm{Fe} \cdots \mathrm{Fe}$ than typical synthetic $\left[\mathrm{Fe}_{2} \mathrm{~S}_{2}\left(\mathrm{SR}_{4}\right)\right]^{2-}$ complexes such as the above type $\mathbf{2 5}$ systems, e.g., $d(\mathrm{Fe} \cdots \mathrm{Fe})=2.733(7) \AA$ in the oxidized from of a green algae ferredoxin. ${ }^{[212]}$
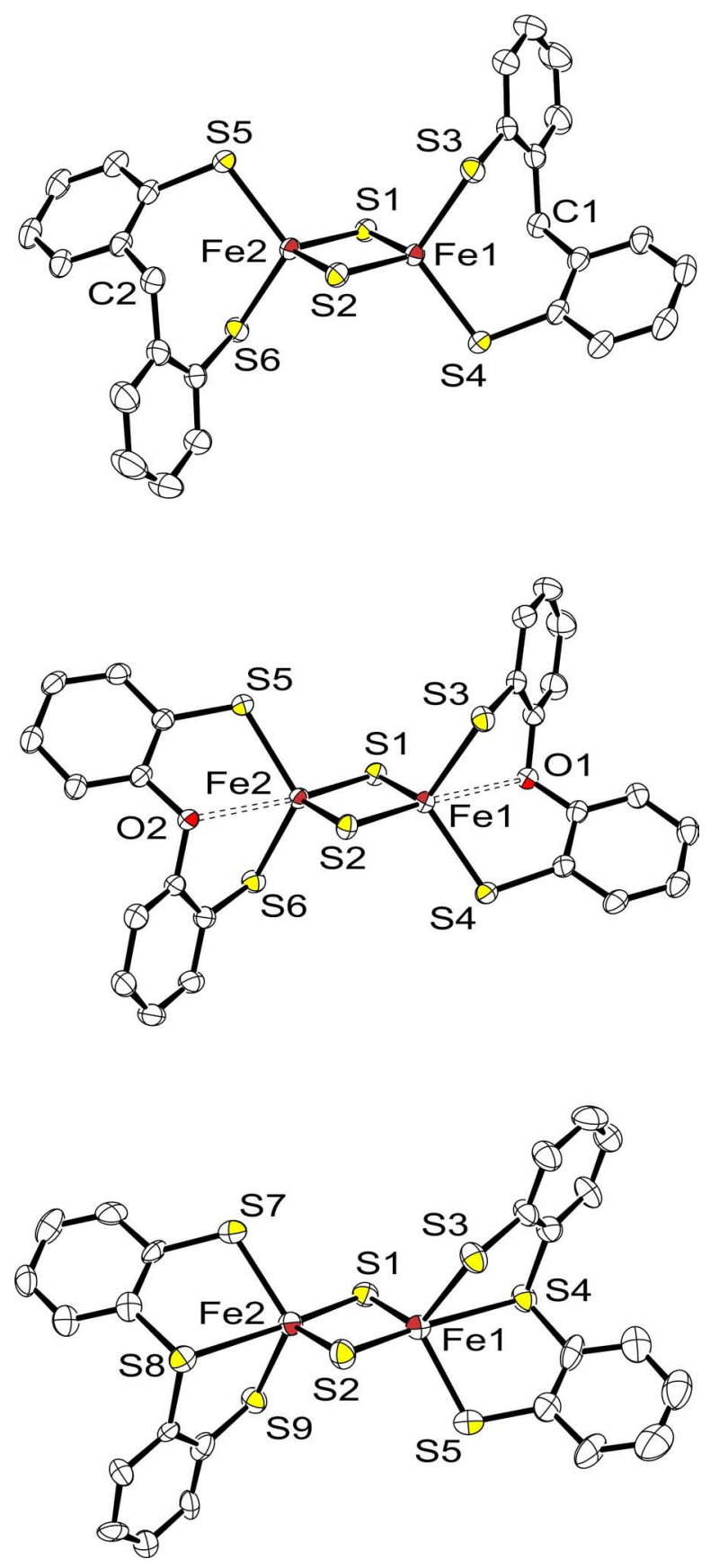

Figure 5.2: ORTEP plots (50\% probability thermal ellipsoids) of the molecular structures of the dianions of $\mathbf{2 6}^{\boldsymbol{C}}$ (top), $\mathbf{2 6}^{\boldsymbol{O}}$ (middle) and $\mathbf{2 6}^{\boldsymbol{S}}$ (bottom). All hydrogen atoms have been omitted for clarity. 
Inspection of the $\tau_{4}$ values reveals an increasing deviation from tetrahedral geometry for the $\left\{\mathrm{FeS}_{4}\right\}$ in the order $\mathbf{2 6}^{\mathbf{C}}\left(\tau_{4}=0.960\right)<\mathbf{2 6}^{\mathbf{O}}\left(\tau_{4}=0.914 / 0.892\right)<\mathbf{2 6}^{\mathbf{S}}\left(\tau_{4}=0.876 / 0.866\right)$, signifying involvement of the additional ether or thioether donor in metal coordination and a gradual transition to trigonal bipyramidal iron environment within this series of complexes. For $26^{\mathrm{S}}$ a $\tau_{4}$ value close to the theoretical value of 0.85 for an ideal trigonal bipyramid is observed, with the additional donor site in an axial position. The distances $\mathrm{Fe} \cdots \mathrm{X}\left(\mathrm{X}=\mathrm{CH}_{2}, \mathrm{O}, \mathrm{S}\right)$ decrease in the order $\mathbf{2 6}^{\mathbf{C}}[d(\mathrm{Fe} \cdots \mathrm{C})=3.335(2)]>$ $\mathbf{2 6}^{\mathrm{O}}[d(\mathrm{Fe} \cdots \mathrm{O})=2.813(2) / 2.679(2)] \simeq \mathbf{2 6}^{\mathrm{S}}[d(\mathrm{Fe} \cdots \mathrm{S})=2.914(4) / 2.777(4)]$, which is accompanied by decreasing distances between the iron atoms and the equatorial planes (which for $\mathbf{2 6}^{\mathbf{S}}$ are given by S1/S3/S5 and S2/S7/S9) in the series $\mathbf{2 6}^{\mathbf{C}}[0.6798(2) \AA]>$ $\mathbf{2 6}^{\mathbf{O}}[0.5811(2) / 0.5110(2) \AA]>\mathbf{2 6}^{\mathbf{S}}(0.403(2) / 0.375(2) \AA]$. The approach of the additional donor atoms in $26^{\mathrm{O}}$ and $26^{\mathrm{S}}$ causes a significant "out-of-plane distortion" compared to $\mathbf{2 6}^{\mathbf{C}}$ (Figure 5.3). This distortion can be quantified by comparing the angles between the planes through RS-Fe-SR and the planes perpendicular to the $\mathrm{Fe}_{2} \mathrm{~S}_{2}$-diamond (constructed from the centroid of the $\mathrm{Fe}_{2} \mathrm{~S}_{2}$-core and the vector through the bridging sulfides as normal of the plane; see Figure 5.3). These angles increase in the row $\mathbf{2 6}^{\mathbf{C}}\left(3.51(1)^{\circ}\right)$ $<\mathbf{2 6}^{\mathbf{O}}\left(13.52(3)^{\circ}\right)<\mathbf{2 6}^{\mathbf{S}}\left(23.79(11)^{\circ}\right)$, whereas type $\mathbf{2 5}$ complexes are only slightly distorted. Taken together, the structural features strongly suggest an increase in coordination number for the Fe atoms and significant structural distortion of the $\left[\mathrm{Fe}_{2} \mathrm{~S}_{2}(\mathrm{SR})_{4}\right]^{2-}$ cores in $26^{\mathrm{O}}$ and $26^{\mathrm{S}}$ due to secondary bonding interactions with the ether-O or thioether-S atoms, respectively, in particular in the latter case. In order to probe the nature of these interactions and consequences for electronic structures of the [2Fe-2S] clusters, detailed spectroscopic and DFT studies have been performed.

\subsection{Spectroscopy and Magnetic Properties in the Solid State}

Zero-field Mössbauer spectra for all clusters have been recorded at $80 \mathrm{~K}$. Spectral fits to the data were obtained by using Lorentzian line doublets with isomer shifts $\delta$ and quadrupole splittings $\Delta E_{\mathrm{Q}}$ summarized in Table 5.3. It should be noted that Mössbauer data for synthetic $[2 \mathrm{Fe}-2 \mathrm{~S}]$ compounds with purely thiolato terminal ligation are still quite scarce. ${ }^{[129]}$ Mössbauer spectra of $\mathbf{2 5}^{\mathrm{S}}$ and $\mathbf{2 6}^{\mathrm{S}}$ are representative examples for type $\mathbf{2 5}$ and type 26 cluster compounds and are depicted in Figure 5.4. All six compounds exhibit isomer shifts $\delta$ in the range $0.29-0.39 \mathrm{~mm} / \mathrm{s}$, which is typical for high-spin ferric ions. Whereas $\delta$ parameters for type $\mathbf{2 5}$ systems and $\mathbf{2 6}^{\mathbf{C}}$ are comparable to those of parent $\left[\mathrm{Fe}_{2} \mathrm{~S}_{2}(\mathrm{SPh})_{4}\right]^{2-} \mathbf{7 a}$ and the related $\left[\mathrm{Fe}_{2} \mathrm{~S}_{2}\left(\mathrm{~S}_{2-O}-\mathrm{xyl}\right)_{2}\right]^{2-} \mathbf{1}$, values for type $\mathbf{2 6}$ complexes are clearly increasing in the order $\mathbf{2 6}^{\mathrm{C}}<\mathbf{2 6}^{\mathrm{O}}<\mathbf{2 6}^{\mathrm{S}}$. Isomer shifts have been empirically related to the oxidation state $s$ of the iron atoms according to $\delta=1.43-0.40 s$ (correlation found for tetrahedral $\left\{\mathrm{FeS}_{4}\right\}$ sites at $77 \mathrm{~K}$ by linear regression analysis). ${ }^{[167]}$ Applying this equation to $\mathbf{2 5}^{\mathrm{C}}, \mathbf{2 5 ^ { \mathrm { O } } , \mathbf { 2 5 }} \mathbf{5}^{\mathrm{S}}$ and $\mathbf{2 6}^{\mathrm{C}}$ reveals formal oxidation states $s$ between 2.825 and 2.850 since the coordinated electron-donating thiophenolates increase the electron densities at the iron sites. Significantly lower values are found for $\mathbf{2 6}^{\mathbf{O}}(s=2.78)$ and $\mathbf{2 6}^{\mathbf{S}}(s=2.60)$, however, suggesting that additional interactions between the ether-O or thioether-S and 

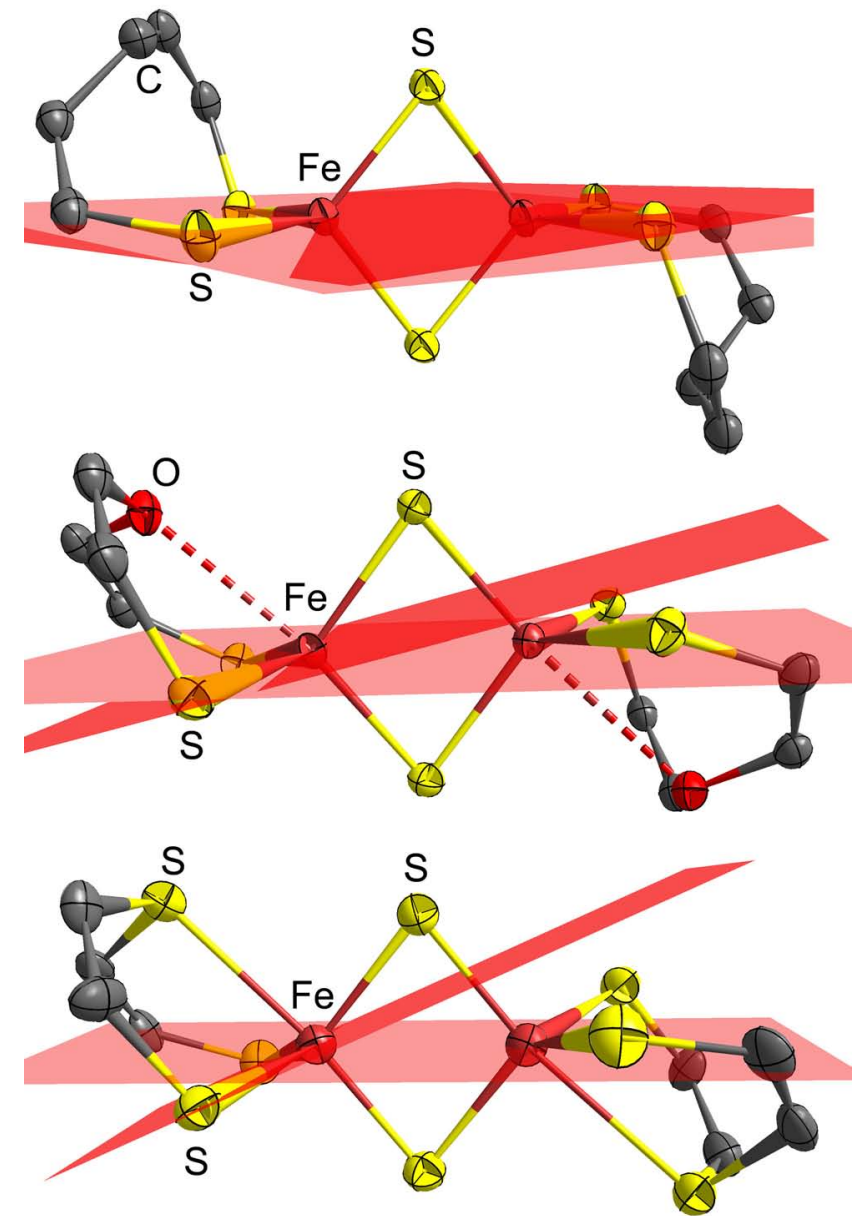

Figure 5.3: Illustration of increasing "out-of-plane distortion" in the order $\mathbf{2 6}^{C}$ (top), $\mathbf{2 6}^{\boldsymbol{O}}$ (middle) and $\mathbf{2 6}^{S}$ (bottom). Counterions, protons and peripheral aromatic carbons are omitted for clarity.

the iron atoms are present, thus further increasing the electron densities at the ferric ions. Hence the above equation seems to be invalid for $\mathbf{2 6}^{\mathrm{O}}$ and $\mathbf{2 6}^{\mathrm{S}}$, due to the presence of $\left\{\mathrm{FeS}_{4} \mathrm{O}\right\}$ or $\left\{\mathrm{FeS}_{5}\right\}$ motives rather than tetrahedral $\left\{\mathrm{FeS}_{4}\right\}$. Quadrupole splittings $\Delta E_{\mathrm{Q}}$ are similar in the series $25^{\mathrm{C}}(0.44 \mathrm{~mm} / \mathrm{s}), \mathbf{2 5}^{\mathrm{O}}(0.42 \mathrm{~mm} / \mathrm{s})$, and $25^{\mathrm{S}}(0.44 \mathrm{~mm} / \mathrm{s})$ and in the series $\mathbf{2 6}^{\mathbf{C}}(0.56 \mathrm{~mm} / \mathrm{s}), \mathbf{2 6}^{\mathbf{O}}(0.56 \mathrm{~mm} / \mathrm{s})$ and $\mathbf{2 6}^{\mathbf{S}}(0.61 \mathrm{~mm} / \mathrm{s})$. Values for type 25 complexes are comparable to those reported previously for synthetic $\{\mathrm{S}\}$-coordinated $[2 \mathrm{Fe}-2 \mathrm{~S}]$ clusters $\left(\left[\mathrm{Fe}_{2} \mathrm{~S}_{2}\left(\mathrm{~S}_{2}-O-\mathrm{xyl}\right)_{2}\right]^{2-} \mathbf{1}: \delta=0.28 \mathrm{~mm} / \mathrm{s}, \Delta E_{\mathrm{Q}}=0.36 \mathrm{~mm} / \mathrm{s}\right.$, recorded at $4.2 \mathrm{~K} ;\left[\mathrm{Fe}_{2} \mathrm{~S}_{2}(\mathrm{SPh})_{4}\right]^{2-} \mathbf{7 a}: \delta=0.28 \mathrm{~mm} / \mathrm{s}, \Delta E_{\mathrm{Q}}=0.32 \mathrm{~mm} / \mathrm{s}$, recorded at $\left.77 \mathrm{~K}\right)^{[134]}$, whereas quadrupole splittings for type $\mathbf{2 6}$ complexes are augmented by $\geq 0.2 \mathrm{~mm} / \mathrm{s}$ compared to $\mathbf{1}$ and $\mathbf{7 a}$. It is interesting to note that oxidized ferredoxins exhibit quite large quadrupole splittings $\Delta E_{\mathrm{Q}}$ in the range $0.6 \mathrm{~mm} / \mathrm{s}-0.8 \mathrm{~mm} / \mathrm{s},{ }^{[140]}$ which is significantly larger than for previously synthesized [2Fe-2S] model systems but similar to $\Delta E_{\mathrm{Q}}$ values of the distorted type 26 clusters (spinach $F d_{\mathrm{ox}}: \delta=0.22 \mathrm{~mm} / \mathrm{s}, \Delta E_{\mathrm{Q}}=0.65 \mathrm{~mm} / \mathrm{s}^{[134]}$ and IscA1: $\left.\delta=0.27 \mathrm{~mm} / \mathrm{s}, \Delta E_{\mathrm{Q}}=0.57 \mathrm{~mm} / \mathrm{s}^{[213]}\right)$. 
Table 5.3: Spectroscopic, magnetic and electrochemical data for the new complexes.

\begin{tabular}{ccccc}
\hline compound & $\delta\left(\Delta E_{\mathrm{Q}}\right)[\mathrm{mm} / \mathrm{s}]^{\mathrm{a}}$ & $\lambda_{\max }[\mathrm{nm}]\left(\varepsilon\left[\mathrm{M}^{-1} \mathrm{~cm}^{-1}\right]\right)^{\mathrm{b}}$ & $J\left[\mathrm{~cm}^{-1}\right]^{\mathrm{c}}$ & $E_{\mathrm{p}}^{\mathrm{c}}[\mathrm{V}]^{\mathrm{d}}$ \\
\hline $\mathbf{1}^{[134]}$ & $0.28(0.36)^{\mathrm{e}}$ & $\begin{array}{c}294(14500), 338(16200), 414 \\
(11000), \simeq 455(s h, 9200),\end{array}$ & $-149 \pm 8$ & $-1.51^{\mathrm{f}}$ \\
& & $590(4800)$ & \\
$\mathbf{7 a}^{[134]}$ & $0.28(0.32)^{\mathrm{g}}$ & $333(19500), 490(11200)$ & $\mathrm{n} / \mathrm{a}$ & $-1.11^{\mathrm{h}}$ \\
$\mathbf{2 5}^{\mathbf{C}}$ & $0.30(0.44)$ & $331(29000), 476(15000)$ & -197 & -1.24 \\
$\mathbf{2 5}^{\mathbf{O}}$ & $0.29(0.42)$ & $296(s h, 58000), 336(33000)$, & -180 & -1.32 \\
& $0.29(0.44)$ & $307(50000), 350(s h, 27000)$, & -181 & -1.14 \\
$\mathbf{2 5}^{\mathbf{S}}$ & $0.30(0.56)$ & $291(16000), 352(20500), 444$ & & -204 \\
$\mathbf{2 6}^{\mathbf{C}}$ & $0.32(0.56)$ & $329(19000), 486(9000)$ & -158 & -1.48 \\
$\mathbf{2 6}^{\mathbf{O}}$ & $0.39(0.61)$ & $288(59000), 322(s h, 37000)$, & -126 & -0.99 \\
$\mathbf{2 6}^{\mathbf{S}}$ & & $475(11000)$ & & \\
\hline
\end{tabular}

(a) ${ }^{57} \mathrm{Fe}$ Mössbauer parameters at $80 \mathrm{~K}$, relative to Fe metal at room temperature. (b) Recorded in DMF solution at room temperature. (c) values obtained from simulation of SQUID data, see Chapter 9.6. (d) Cathodic peak potentials in $\mathrm{DMF} / 0.1 \mathrm{M} \mathrm{NBu}_{4} \mathrm{PF}_{6}$ at a scan rate of $100 \mathrm{mV} / \mathrm{s}$ vs. the $\mathrm{Cp}_{2}^{*} \mathrm{Fe} / \mathrm{Cp}_{2}^{*} \mathrm{Fe}^{+}$ couple. (e) Recorded at $4.2 \mathrm{~K}$. (f) Half-wave potential $E_{1 / 2}$ of the reversible process in DMF vs. SCE is $-1.09 \mathrm{~V}$, corresponding to $-1.11 \mathrm{~V}$ vs. the $\mathrm{Cp}_{2}^{*} \mathrm{Fe} / \mathrm{Cp}_{2}^{*} \mathrm{Fe}^{+}$couple. ${ }^{[145,146]}(\mathrm{g})$ Recorded at $77 \mathrm{~K}(\mathrm{~h})$ Half-wave potential $E_{1 / 2}$ in DMF vs. SCE is $-1.49 \mathrm{~V}$, corresponding to $-1.51 \mathrm{~V}$ vs. the $\mathrm{Cp}_{2}^{*} \mathrm{Fe} / \mathrm{Cp}_{2}^{*} \mathrm{Fe}^{+}$ couple. ${ }^{[145,146]}$

Magnetic susceptibility measurements for all new complexes were carried out at $1 \mathrm{~T}$ from $2 \mathrm{~K}$ to $290 \mathrm{~K}$. Magnetic moments $\mu_{\text {eff }}$ at room temperature are in the range $1.7-2.6 \mu_{\mathrm{B}}$, i.e., much lower than expected for two uncoupled ferric $(S=5 / 2)$ ions, and they rapidly decrease upon lowering the temperature. This behavior is in accordance with significant antiferromagnetic coupling between the two ferric ions to give an $S=0$ ground state, as is usually observed for [2Fe-2S] clusters. Coupling constants $J$ (Table 5.3, Chapter 9.6) were determined by using a fitting procedure to the appropriate Heisenberg spin Hamiltonian for isotropic exchange coupling and Zeeman interaction:

$$
\mathcal{H}=-2 J \overrightarrow{S_{1}} \cdot \overrightarrow{S_{2}}+g \mu_{B}\left(\overrightarrow{S_{1}}+\overrightarrow{S_{2}}\right) \cdot \vec{B}
$$

For type 25 complexes the coupling is very strong $\left(J \simeq-180 \mathrm{~cm}^{-1}\right)$ and is slightly higher than those observed for, e.g., dipyrromethane coordinated clusters (see Chapter 7) with terminal $\left\{\mathrm{N}_{2}\right\}$ ligation $\left(J \simeq-170 \mathrm{~cm}^{-1}\right) .{ }^{[170]}$ Complex $\mathbf{2 6}^{\mathbf{C}}$ exhibits the highest antiferromagnetic exchange constant $J=-204 \mathrm{~cm}^{-1}$ reported so far for synthetic [2Fe-2S] clusters. The lower $J$ value for compound $\mathbf{2 6}^{\mathbf{O}}\left(J=-158 \mathrm{~cm}^{-1}\right)$ is comparable to that of $\left(\mathrm{NBu}_{4}\right)_{2}\left[\mathrm{Fe}_{2} \mathrm{~S}_{2}\left(\mathrm{~S}_{2}-O-\mathrm{xyl}\right)_{2}\right] \mathbf{1}\left(J \simeq-150 \mathrm{~cm}^{-1}\right),{ }^{[134]}$ whereas $\mathbf{2 6}^{\mathrm{S}}\left(J=-126 \mathrm{~cm}^{-1}\right)$ exhibits the weakest antiferromagnetic coupling reported for synthetic [2Fe-2S] clusters until now. 

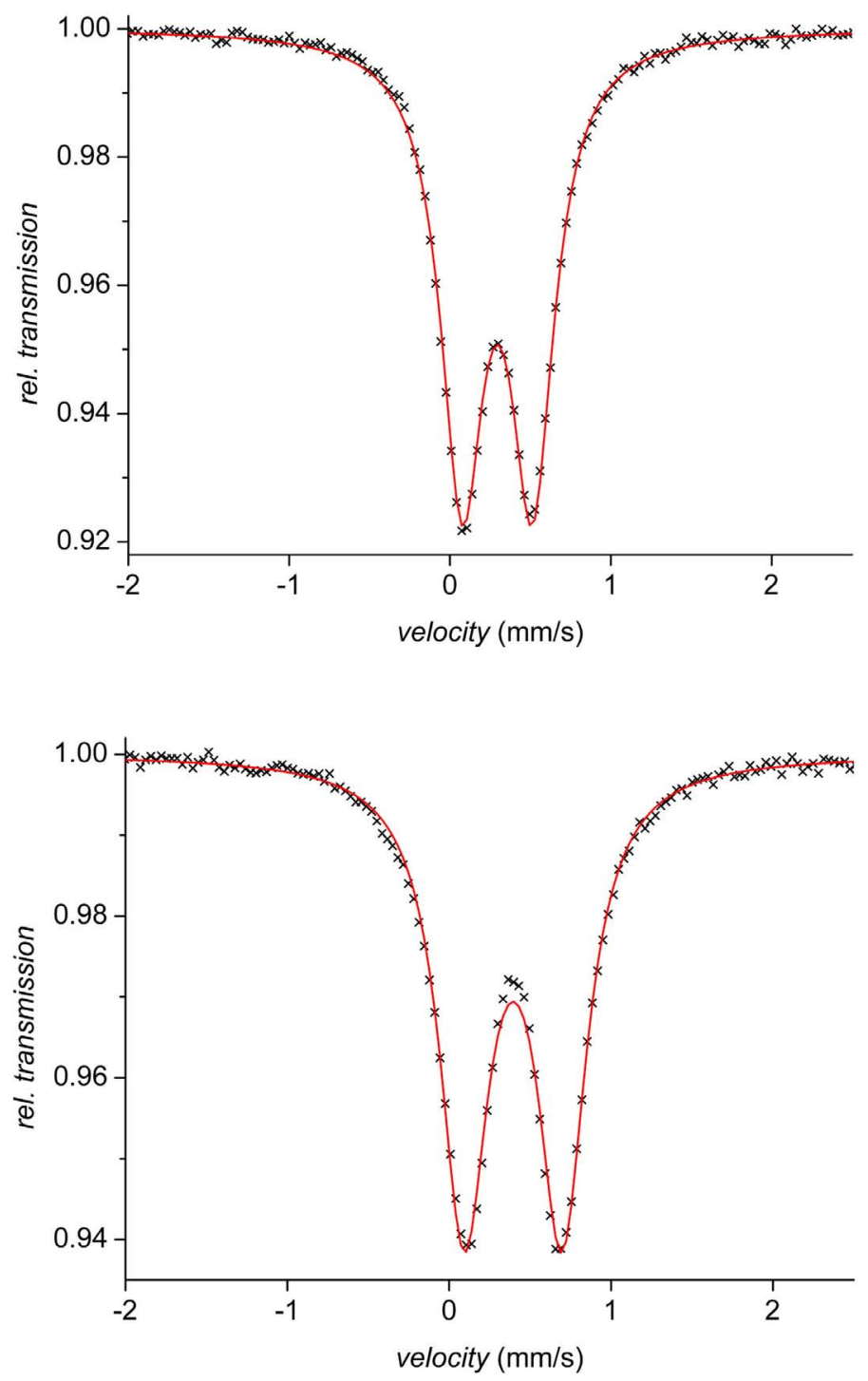

Figure 5.4: Zero-field Mössbauer spectra of $25^{S}$ (top) and $26^{S}$ (bottom) at $80 \mathrm{~K}$. The solid lines are Lorentzian doublets fitted to the experimental values (crosses).

It is likely that the decrease in antiferromagnetic coupling in the sequence $\mathbf{2 6}^{\mathrm{C}}<\mathbf{2 6}^{\mathrm{O}}<$ $\mathbf{2 6}^{\mathrm{S}}$ is caused by the widening of the Fe- $(\mu-\mathrm{S})-\mathrm{Fe}$ angles and the increasing Fe $\cdots$ Fe distance. A coupling constant of $-183 \mathrm{~cm}^{-1}$ was reported for spinach $F d_{\mathrm{ox}}{ }^{[214]}$

\subsection{Spectroscopy in Solution}

All new complexes were characterized by NMR, cyclic voltammetry and UV-Vis spectroscopy in order to clarify whether the situation observed in the solid state is preserved in solution and whether secondary interactions are present or absent in polar solvents. Electronic absorption spectra in DMF solution are shown in Figure 5.5a for clusters $\mathbf{2 5}^{\mathrm{C}}$, 


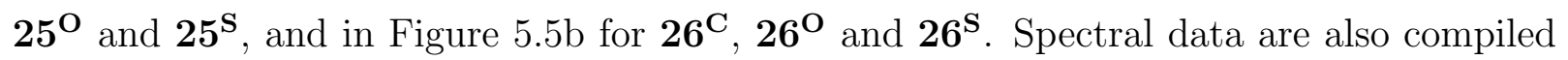
in Table 5.3.
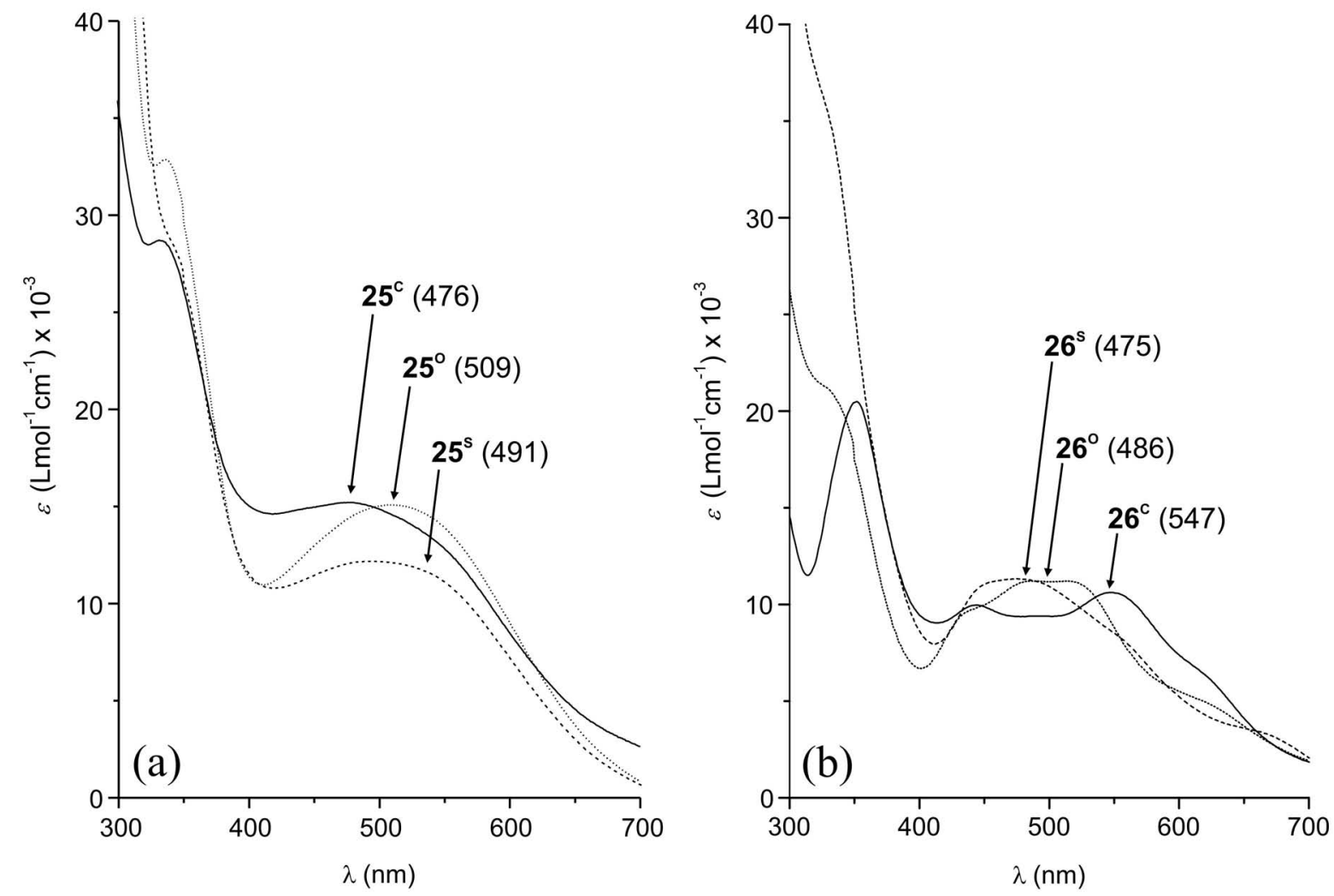

Figure 5.5: (a) Absorption spectra of $\left(\mathrm{NEt}_{4}\right)_{2}\left[\mathrm{Fe}_{2} \mathrm{~S}_{2}\left(\mathrm{SC}_{6} \mathrm{H}_{4} \mathrm{-O}-\mathrm{X}\right)_{4}\right], \mathrm{X}=\mathrm{CH}_{2} \mathrm{Me}\left(\mathbf{2 5}^{\mathrm{C}}\right)$, $O M e$ (25) $\mathbf{2}^{\mathrm{O}}$, SMe (25) in DMF; (b) Absorption spectra of $\left(\mathrm{NEt}_{4}\right)_{2}\left[\mathrm{Fe}_{2} \mathrm{~S}_{2}\left(\mathrm{SC}_{6} \mathrm{H}_{4}-\mathrm{X}\right.\right.$ $\left.\mathrm{C}_{6} \mathrm{H}_{4} \mathrm{~S}\right)_{2}$ ], $\mathrm{X}=\mathrm{CH}_{2}\left(\mathbf{2 6}^{\mathrm{C}}\right), \mathrm{O}\left(\mathbf{2 6}^{\boldsymbol{O}}\right), S\left(\mathbf{2 6}^{\mathrm{S}}\right)$ in DMF (wavelengths of visible band maxima are given in parentheses).

Compared to $\left(\mathrm{NEt}_{4}\right)_{2}\left[\mathrm{Fe}_{2} \mathrm{~S}_{2}\left(\mathrm{SC}_{6} \mathrm{H}_{4}-\mathrm{o}-\mathrm{Et}\right)_{4}\right]\left(\mathbf{2 5}^{\mathrm{C}}\right)$, the $\pi$-electron donating methoxy $\left(\mathbf{2 5}^{\mathrm{O}}\right)$ and thiomethyl substituents $\left(\mathbf{2 5}^{\mathrm{S}}\right)$ are expected to lower the energies for the visible absorptions, which were assigned previously to thiophenolate-to-core charge transfer transitions. ${ }^{[144]}$ Indeed a red-shift by $15 \mathrm{~nm}(\mathbf{2 5})$ or $33 \mathrm{~nm}\left(\mathbf{2 5}^{\mathbf{O}}\right)$ relative to $\mathbf{2 5}^{\mathrm{C}}$ is observed. Any additional interaction of the ether or thioether functions with the iron atoms of the $[2 \mathrm{Fe}-2 \mathrm{~S}]$ core should decrease the substituent's electron donating ability towards the benzenethiolate but increase the electron density at the iron atoms, resulting in a blue shift of the ligand-to-metal charge transfer bands. Such trends have also been discussed for [4Fe $-4 \mathrm{~S}]$ clusters with substituted thiophenolate ligands and potential secondary bonding interactions. ${ }^{[144]}$ Therefore the observed spectral shifts for $25^{\mathrm{O}}$ and $25^{\mathrm{S}}$ implicate that no chelate rings are formed in DMF solution, similar to the situation in the solid state. Consistent with these observations, the ${ }^{1} \mathrm{H}$ NMR spectra of $25^{\mathrm{O}}$ and $25^{\mathrm{S}}$ in DMSO-d $\mathrm{d}_{6}$ show relatively sharp resonances for the methyl groups that are only slightly shifted with respect to the resonances for the free ligand, whereas secondary bonding interactions with the iron atoms should significantly broaden these signals. In contrast to type $\mathbf{2 5}$ complexes, a blue 
shift of the ligand-to-metal charge transfer bands is observed for $26^{\mathbf{O}}$ and $26^{\mathrm{S}}$ relative to $\mathbf{2 6}^{\mathrm{C}}$. Since the trend in solution optical properties is in accordance with what is expected from the solid-state structures, it can be assumed that secondary bonding interactions are also present in solution for $\mathbf{2 6}^{\mathbf{O}}$ and $\mathbf{2 6}^{\mathrm{S}}$. The ${ }^{1} \mathrm{H}$ NMR spectrum for $\mathbf{2 6}^{\mathbf{O}}$ in deuterated DMSO is shown in Figure 5.6 as an example. Reasonably resolved spectra are obtained because of the strong antiferromagnetic coupling between the two ferric ions $(S=0$ ground state), and all resonances appear as broad singlets. In addition to signals for the tetraethylammonium cations, isotropically shifted signals for the aromatic protons are observed.

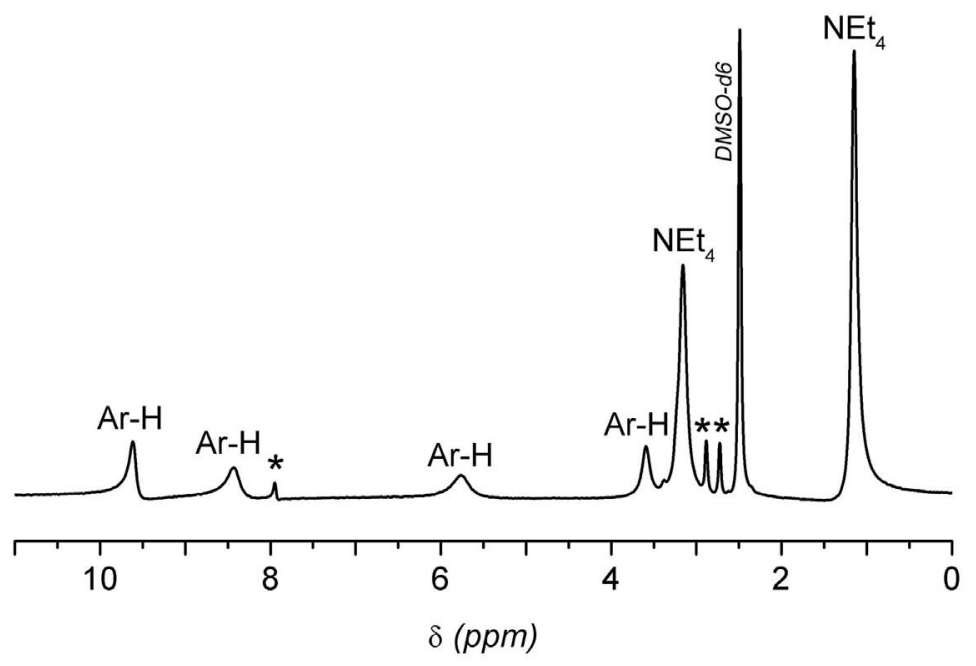

Figure 5.6: ${ }^{1} \mathrm{H}$ NMR spectrum $\left(500 \mathrm{MHz}, 25^{\circ} \mathrm{C}\right)$ of $\mathbf{2 6}^{\boldsymbol{O}}$ recorded in DMSO-d (residual $D M F$ signals are marked by $*$ ).

Redox properties of all clusters have been examined by cyclic voltammetry in DMF / $0.1 \mathrm{M}$ $\mathrm{NBu}_{4} \mathrm{PF}_{6}$ at room temperature. The coumpounds $25^{\mathrm{C}}, \mathbf{2 5 ^ { \mathrm { O } }}$ and $25^{\mathrm{S}}$ all exhibit an irreversible reduction process with cathodic peak potentials around $-1.2 \mathrm{~V}$ vs. the $\mathrm{Cp}_{2}^{*} \mathrm{Fe} / \mathrm{Cp}_{2}^{*} \mathrm{Fe}^{+}$ couple at scan rate $100 \mathrm{mV} / \mathrm{s}$ (Table 5.3) followed by a second irreversible process at even lower potentials. The first reduction is assigned to formation of the mixed-valent $\mathrm{Fe}^{\mathrm{II}} \mathrm{Fe}^{\mathrm{III}}$ species, but these are not stable since the cathodic peak and the anodic response in the reverse scan are separated by more than $600 \mathrm{mV}$ at a scan rate of $100 \mathrm{mV} / \mathrm{s}$. It is interesting to note, however, that reduction of $25^{\mathrm{S}}\left(E_{\mathrm{p}}^{\mathrm{c}}=-1.14 \mathrm{~V}\right)$ seems to be more facile than reduction of $25^{\mathrm{O}}\left(E_{\mathrm{p}}^{\mathrm{c}}=-1.32 \mathrm{~V}\right)$, which is presumably due to a higher degree of electron delocalization in the thioether derivative. This observation is in accordance with the optical spectra, from which it was concluded that the $p$-OMe group in $25^{\mathrm{O}}$ transfers more electron density towards the $\left\{\mathrm{Fe}_{2} \mathrm{~S}_{2}\right\}$ core than the $p$-SMe group in $\mathbf{2 5}^{\mathrm{S}}$. Electrochemical measurements for $\mathbf{2 6}^{\mathrm{C}}, \mathbf{2 6}^{\mathrm{O}}$ and $\mathbf{2 6}^{\mathrm{S}}$ under identical conditions revealed two sequential reduction processes with broadened anodic reverse peaks (Table 5.3). Again the thioether derivative $26^{\mathrm{S}}$ is easier to reduce than the ether analogue $26^{\mathrm{O}}$, and furthermore $26^{\mathrm{S}}$ has the lowest $E_{\mathrm{p}}^{\mathrm{c}}(-0.99 \mathrm{~V})$ of all complexes studied here. 


\subsection{DFT Calculations}

In order to corroborate the conclusions from structural and spectroscopic findings and to gain insight into the nature of the secondary bonding interactions in $\mathbf{2 6}^{\mathbf{O}}$ and $\mathbf{2 6}^{\mathrm{S}}$, DFT calculations were performed for complexes $25^{\mathrm{S}}, \mathbf{2 6}^{\mathrm{O}}$ and $26^{\mathrm{S}}$. The pure BP86 functional (which for open-shell systems usually favors the low-spin state) has been used for both the antiferromagnetically coupled ${ }^{1} \mathrm{X}$ as well as the ferromagnetically coupled ${ }^{11} \mathrm{X}$ states, and the hybrid B3LYP functional (which usually predicts the high-spin state) has been tested for the ferromagnetically coupled state for comparison (technical details are provided in Chapter 9.4). In accordance with the experimental findings, the BP86 results confirm that the singlet state is lower in energy (by 136, 110 and $66 \mathrm{~kJ} / \mathrm{mol}$ for $\mathbf{2 5}^{\mathbf{S}}, \mathbf{2 6}^{\mathbf{O}}$ and $\mathbf{2 6}^{\mathbf{S}}$, respectively) than the high-spin state for all three models (Table 5.2). Calculated spin densities on the ether-O and thioether-S atoms are considered for evaluating the secondary interactions in $\mathbf{2 6 ^ { \mathrm { O } }}$ and $\mathbf{2 6 ^ { \mathrm { S } }}$, in comparison to $\mathbf{2 5 ^ { \mathrm { S } }}$ where no such interaction is present. The results collected in Table 5.2 show that there is no spin density on the pendent thioether groups for the $25^{\mathrm{S}}$ model, which confirms the expectation that there is no bonding interaction between those atoms. This is also validated by the atoms-in-molecules (AIM) analysis, which cannot detect any Fe-thioether bond in $\mathbf{2 5}^{\mathrm{S}}$.

Table 5.4: Calculated eigenvalues of the field gradient tensor for the singlet states of ${ }^{25}{ }^{S}, \mathbf{2 6}^{\boldsymbol{O}}$ and $\mathbf{2 6}^{S}$ at the BP86/SVP level of theory, and calculated and experimental $\Delta E_{Q}$ values.

\begin{tabular}{cccc}
\hline compound & calculated $\mathrm{EFG}^{\mathrm{a}}$ & $\begin{array}{c}\Delta E_{\mathrm{Q}} \text { calculated } \\
{[\mathrm{mm} / \mathrm{s}]^{\mathrm{b}}}\end{array}$ & $\begin{array}{c}\Delta E_{\mathrm{Q}} \text { experimental } \\
{[\mathrm{mm} / \mathrm{s}]^{\mathrm{c}}}\end{array}$ \\
\hline \multirow{2}{*}{$\mathbf{5 5}^{\mathbf{S}}$} & $0.169 /-0.0487 /-0.120(\mathrm{Fe} 1)$ & 0.28 & 0.44 \\
& $0.168 /-0.0464 /-0.121(\mathrm{Fe} 2)$ & 0.28 & 0.56 \\
$\mathbf{2 6}^{\mathbf{O}}$ & $0.263 /-0.00184 /-0.261(\mathrm{Fe} 1)$ & 0.49 & \\
& $0.266 /-0.0858 /-0.180(\mathrm{Fe} 2)$ & 0.44 & 0.61 \\
$\mathbf{2} \mathbf{2 6}^{\mathbf{S}}$ & $0.330 /-0.110 /-0.220(\mathrm{Fe} 1)$ & 0.54 & \\
\hline
\end{tabular}

(a) The three eigenvalues of the field gradient tensor given in atomic units $\left(1\right.$ a.u. $\left.=9 \cdot 72 \cdot 10^{21} \mathrm{~V} / \mathrm{m}^{2}\right)$. (b) $\Delta E_{\mathrm{Q}}$ calculated according to $\Delta E_{\mathrm{Q}}=\frac{1}{2} e Q V_{z z} \cdot\left(1+\eta^{2} / 3\right)^{1 / 2}$, where the quadrupole moment $Q$ is 0.16 barn $\left(0.16 \cdot 10^{-28} \mathrm{~m}^{2}\right)$ for ${ }^{57} \mathrm{Fe}, V_{z z}$ is the main value of the EFG, $\eta=\left(V_{x x}-V_{y y}\right) / V_{z z}$ (with $\left|V_{x x}\right|<\left|V_{y y}\right|<$ $\left.\left|V_{z z}\right|\right)$ and $1 \mathrm{~mm} / \mathrm{s}=4.8075 \cdot 10^{-8} \mathrm{eV}$. (c) Data from Table 5.3.

On the other hand, for the $2 \mathbf{6}^{\mathrm{S}}$ model, significant spin density is found on the two thioether$\mathrm{S}$ atoms (Figure 5.7), and non-negligible spin density is also found on the ether-O atoms of the $\mathbf{2 6}^{\mathbf{O}}$ model. While the spin density on the thioether-S atoms $(\simeq 0.04 e)$ is much lower than that on the thiolate $(0.10 e)$ atoms, suggesting that the thioether bonds are weaker than the bonds to the other two groups, the density is still large enough to indicate a connection between the ferric ions and the thioether-S. This is also confirmed by the AIM analysis, which clearly detects a bond between the Fe ions and the thioether groups. The electronic density in the middle of these bonds (at the bond critical point) amounts 

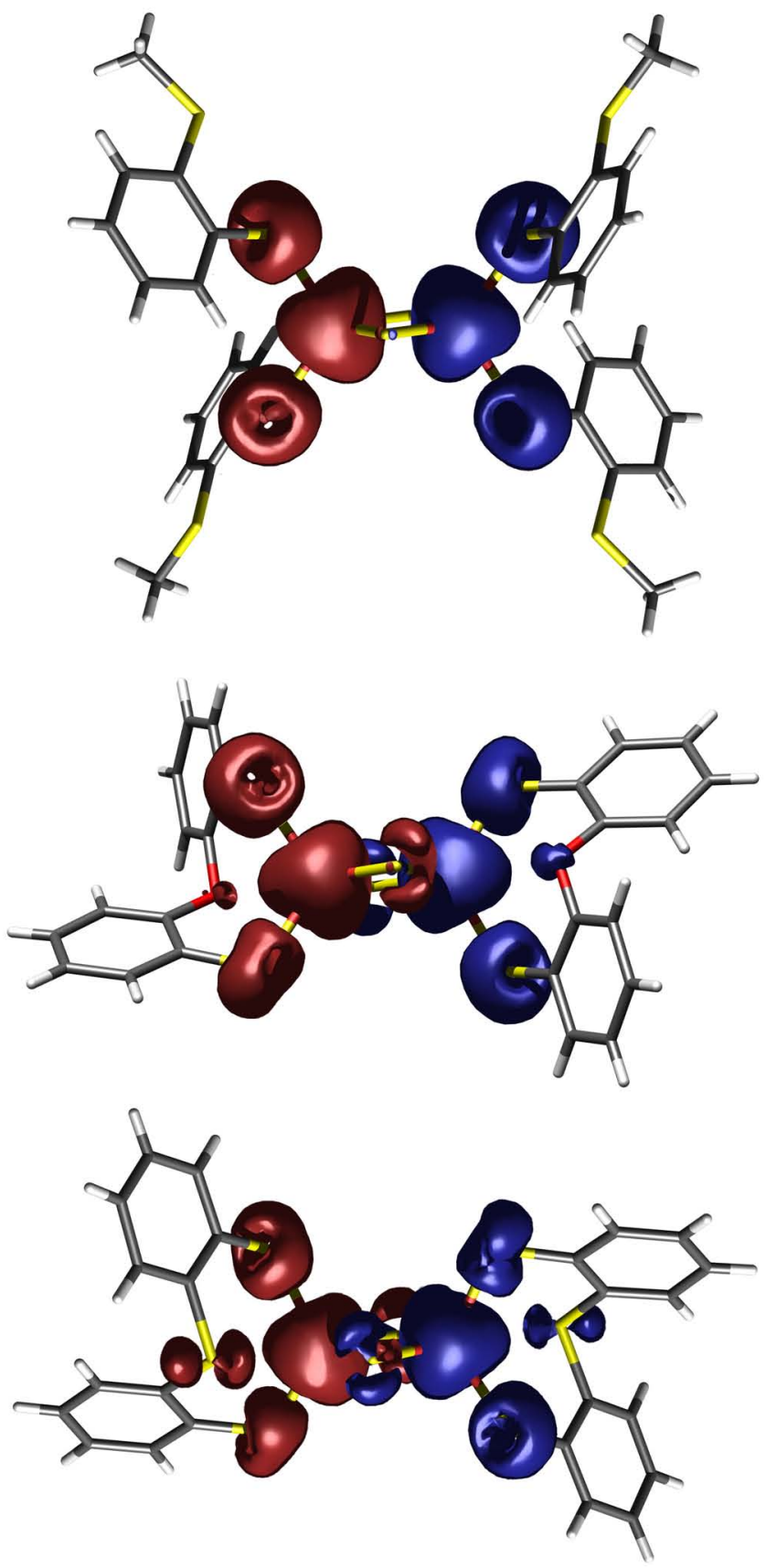

Figure 5.7: Spin densities (0.0035 a.u. level) for the $25^{S}$ (top), $26^{O}$ (middle) and ${ }_{26}{ }^{S}$ (bottom) models, calculated at the BP86/def2-SVP level.

to $0.03 e$, which again is slightly lower than that of the Fe-sulfide and Fe-thiolate bonds (0.09 and $0.07 e$, respectively). For the $\mathbf{2 6}^{\mathbf{O}}$ model, the spin density on the ether-O atoms (0.01e) is appreciably smaller than on the thioether atoms in the $\mathbf{2 6} \mathbf{S}^{\mathbf{S}}$ model, but still significant. Likewise, the AIM analysis identifies a bond between the Fe ions and the $\mathrm{O}$ atoms, with an electronic density $(0.02 e)$ that is slightly lower than for the $\mathbf{2 6}^{\mathbf{S}}$ model. Thus, the calculations unambiguously confirm the existence of a Fe-thioether interaction 
in the $\mathbf{2 6}^{\mathrm{S}}$ model, albeit this is a relatively weak bond, and an even weaker bond in the $\mathbf{2 6}^{\mathbf{O}}$ model. In order to rationalize the trend in the quadrupole splittings $\Delta E_{\mathrm{Q}}$ observed in the Mössbauer spectra, eigenvalues of the electric field gradient (EFG) tensor have been calculated for the singlet states of the $25^{\mathrm{S}}, \mathbf{2 6}^{\mathrm{O}}$ and $\mathbf{2 6}^{\mathrm{S}}$ models. Quadrupole splittings $\Delta E_{\mathrm{Q}}$ derived from those values are compared with experimental data in Table 5.4. While the calculated values appear to be systematically too low by $\simeq 0.12 \mathrm{~mm} / \mathrm{s}$, the overall agreement with experimental values is quite satisfying, and most importantly the trend for $\Delta E_{\mathrm{Q}}\left(25^{\mathrm{S}}<\mathbf{2 6}^{\mathrm{O}}<\mathbf{2 6}^{\mathrm{S}}\right)$ is almost quantitatively reproduced.

\subsection{Conclusions}

Secondary interactions between the ferric ions and added ether or thioether moieties do occur in oxidized [2Fe-2S] clusters if the additional $\mathrm{O}$ or $\mathrm{S}$ donor atoms are suitably positioned in proximity to the cluster core. In the case of $[2 \mathrm{Fe}-2 \mathrm{~S}]$ clusters with capping thiophenolate ligands this situation has to be enforced by a confined chelate arrangement since no bonding interaction is observed when the tethered ether or thioether groups are free to rotate away from the metal. Due to the secondary interaction, which is clearly more pronounced for a thioether-S compared to an ether-O, the Fe atoms approach a trigonal bipyramidal coordination geometry with the additional donor atom and one of the bridging sulfides in apical positions. This gives rise to significant structural distortion of the cluster core with increasing Fe $\cdots$ Fe distances and widened Fe- $(\mu-\mathrm{S})$-Fe angles, which is reflected by marked changes in the spectroscopic and magnetic properties, in particular a distinct decrease in antiferromagnetic coupling and an increase in the Mössbauer quadrupole splitting. Considerable spin density is found on the fifth donor atom, and reduction is facilitated for the system with additional thioether-Fe bonds. Taken together, these findings show that secondary bonding interactions can modulate the electronic properties of biological [2Fe-2S] clusters, which may well play a role for, e.g., the unique [2Fe-2S] cluster in biotin synthase with its unusual (and potentially chelating) arginine ligand. 


\section{Chapter 6}

\section{Switching the Spin State in $\left\{\mathrm{S}_{4} \mathrm{X}_{2}\right\}$ - Coordinated Iron(III) Complexes by Variation of $\mathrm{X}=\mathrm{N}, \mathrm{O}, \mathrm{P}, \mathrm{S}$}

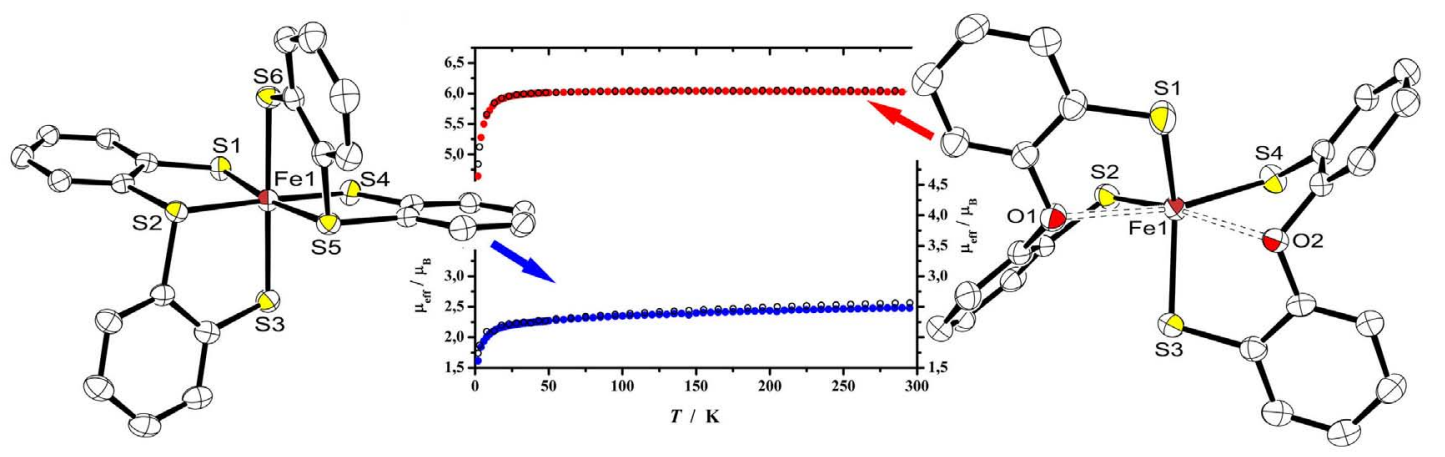

Low Spin $\left(S=\frac{1}{2}\right) \stackrel{X=S, P P h}{\longrightarrow}\left[\left(S_{4} X_{2}\right) F e^{\prime \prime \prime}\right]^{-} \stackrel{x=0, N M e}{\longrightarrow}$ High Spin $\left(S=\frac{5}{2}\right)$

\section{Abstract}

A series of $\left\{\mathrm{S}_{4} \mathrm{X}_{2}\right\}$-coordinate iron complexes $\left(\mathrm{NEt}_{4}\right)\left[\left(1,1^{\prime}-\mathrm{X}-\left(o-\mathrm{C}_{6} \mathrm{H}_{4} \mathrm{~S}\right)_{2}\right)_{2} \mathrm{Fe}\right](\mathrm{X}=\mathrm{NMe}$, $\mathrm{O}, \mathrm{PPh}, \mathrm{S})$ was prepared and comprehensively characterized. A correlation between the experimental spin state and the tethered neutral donor atom $(S=5 / 2$ for $\mathrm{X}=\mathrm{NMe}, \mathrm{O}$ and $S=1 / 2$ for $\mathrm{X}=\mathrm{PPh}, \mathrm{S}$ ) is evident from magnetic susceptibility measurements. In contrast to the low spin complexes, incomplete spin relaxation is observed for both high spin complexes, as indicated by broadened Mössbauer absorptions at $80 \mathrm{~K}$ (magnetic subspectra detected at $7 \mathrm{~K}$ ). DFT calculations agree well with the experimental findings. 


\subsection{Introduction}

Compounds $\left(\mathrm{NEt}_{4}\right)\left[\left(1,1^{\prime}-\mathrm{X}-\left(o-\mathrm{C}_{6} \mathrm{H}_{4} \mathrm{~S}\right)_{2}\right)_{2} \mathrm{Fe}\right] 27(\mathrm{X}=\mathrm{NMe}, \mathrm{O}, \mathrm{PPh}, \mathrm{S})$ were initially observed as byproducts in the synthesis of the [2Fe-2S] clusters 26 (see Chapter 5), ${ }^{[143]}$ where the potentially tridentate bis-(benzenethiols) ligands $1,1^{\prime}-\mathrm{X}-\left(o-\mathrm{C}_{6} \mathrm{H}_{4} \mathrm{SH}\right)_{2}\left(\mathrm{X}=\mathrm{NMe},{ }^{[210]} \mathrm{O},{ }^{[203]}\right.$ $\left.\mathrm{PPh},{ }^{[211]} \mathrm{S}^{[204]}\right) \mathbf{X I V}$ were applied as capping terminal chelates. Separation of these monomeric compounds $\mathbf{2 7}$ from the [2Fe-2S] cluster species could not be performed by a standard protocol since solubilities of $\mathbf{2 7}$ are strongly dependent on the tethered donorfunctionality: Complexes $27^{\mathrm{N}}(\mathrm{X}=\mathrm{NMe})$ and $27^{\mathrm{O}}(\mathrm{X}=\mathrm{O})$ are readily soluble in MeCN forming intensive green-blue solutions, whereas $27^{\mathrm{P}}(\mathrm{X}=\mathrm{PPh})$ and $27^{\mathrm{S}}(\mathrm{X}=\mathrm{S})$ are only sparingly soluble in MeCN, but dissolve readily in DMF forming intensive purple-red solutions. As convincing explanations for this observation were missing a priori, a more detailed study of these complexes and their properties was conducted.

\subsection{Synthesis and Structural Characterization}

In order to facilitate isolation and purification of complexes $\mathbf{2 7}$, a rational synthesis for the complete series was developed utilizing standard salt metathesis reactions starting from $\left(\mathrm{NEt}_{4}\right)\left[\mathrm{FeCl}_{4}\right] \mathbf{2 8}^{[215]}$ (Scheme 6.1). During the course of those reactions, crude complexes 27 precipitated from the reaction media (THF) as main products and were subsequently

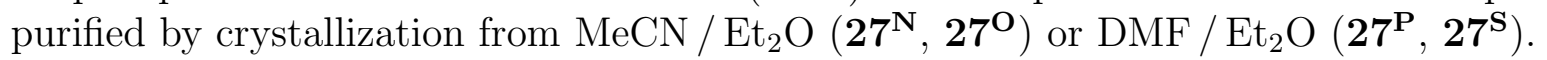
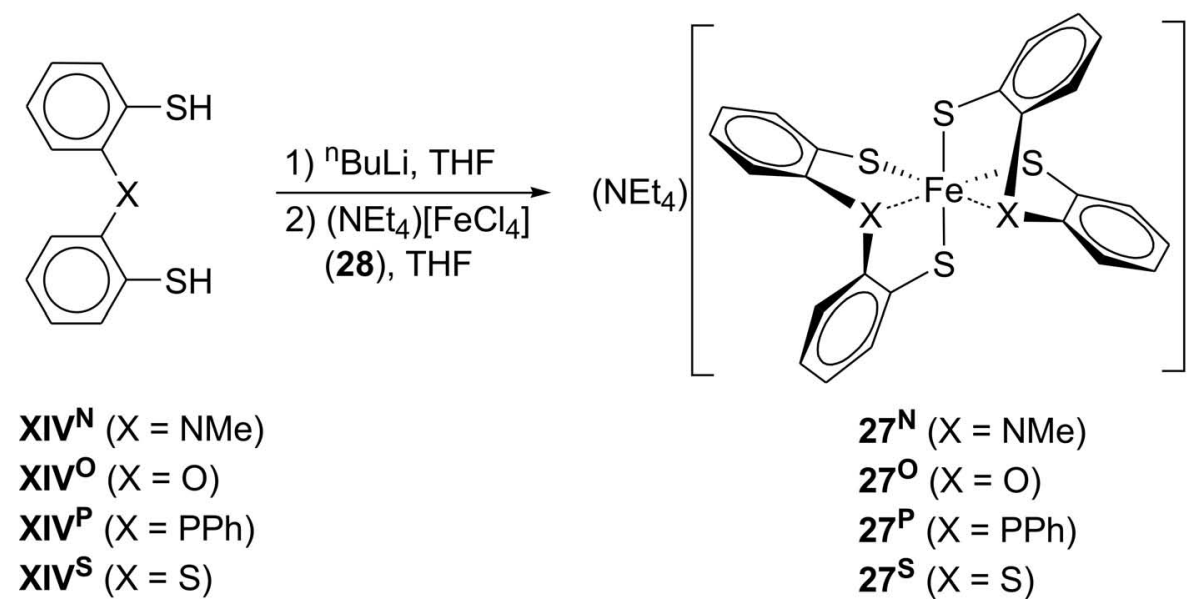

Scheme 6.1: Synthesis of complexes 27.

Single crystals suitable for X-ray diffraction were obtained by slow diffusion of $\mathrm{Et}_{2} \mathrm{O}$ into MeCN or DMF solutions of $\mathbf{2 7}$ (Figure 6.1 and Figure 6.2). As expected, molecular structures of $27^{\mathrm{P}}$ and $\mathbf{2 7}$ are almost ideally octahedral with the weaker phosphine and thioether donors (compared to the monoanionic thiophenolates) cis to each other. Interestingly, this is not the case for $\mathbf{2 7 ^ { \mathrm { N } }}$ and $\mathbf{2 7 ^ { \mathrm { O } }}$ - their structures are found in an intermediate geometry between tetrahedral and octahedral with both additional donor sites orientated again in a 
(pseudo-) cis fashion. Their structural distortions are intuitively rationalized by comparison of the Fe-S and Fe-X distances as well as the S-Fe-S and X-Fe-X angles (Table 6.1). For $27^{\mathrm{P}}$ and $27^{\mathrm{S}}$ all bond distances to the iron centers are in a range of $2.26 \pm 0.05 \AA$, whereas distances for the iron-thiolate bonds in $27^{\mathrm{N}}$ and $27^{\mathrm{O}}$ are significant enlarged by approximately $0.1 \AA$ compared to those in $27^{\mathbf{P}}$ and $27^{\mathrm{S}}$. The additional nitrogen and oxygen donor sites in $27^{\mathbf{N}}$ and $27^{\mathrm{O}}$ occupy positions in typical distances for secondary bonding interactions $\left(\mathrm{Fe} 1-\mathrm{N} 1=2.4232(17)\right.$ and $\mathrm{Fe} 1-\mathrm{N} 2=2.4581(17)$ for $\mathbf{2 7}^{\mathbf{N}}, \mathrm{Fe} 1-\mathrm{O} 1=2.5696(41)$ and $\mathrm{Fe} 1-\mathrm{O} 2=2.6341(35)$ for $\left.\mathbf{2 7 ^ { \mathrm { O } }}\right)$.
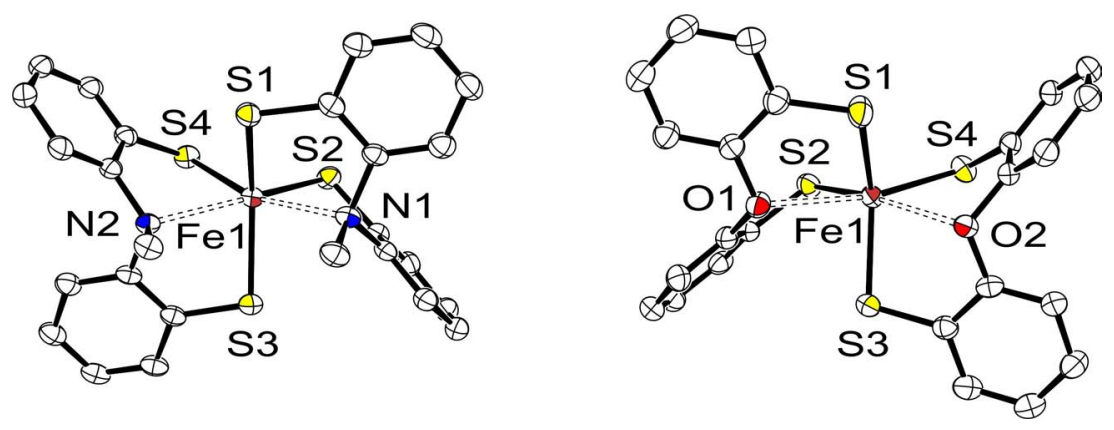

Figure 6.1: ORTEP plot (50\% probability thermal ellipsoids) of the molecular structures of the anions of $\mathbf{2}^{\mathbf{N}}$ (left) and $\mathbf{2}^{\mathbf{O}}$ (right). All hydrogen atoms and counter ions have been omitted for clarity reasons. Selected interatomic distances and angels are summarized in Table 6.1.
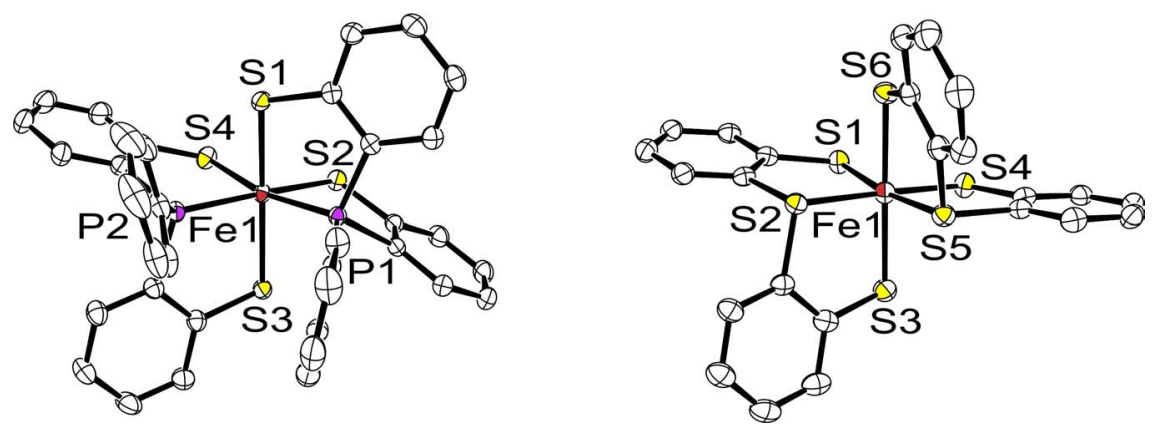

Figure 6.2: ORTEP plot (50\% probability thermal ellipsoids) of the molecular structures of the anions of $\mathbf{2}^{\boldsymbol{P}}$ (left) and $\mathbf{2}^{\boldsymbol{S}}$ (right). All hydrogen atoms and counter ions have been omitted for clarity reasons. Selected interatomic distances and angels are summarized in Table 6.1.

A qualitative interpretation of the angles S-Fe-S is obvious for $27^{\mathrm{S}}$ only, where one angle is found close to $180^{\circ}$ and the remaining five close to $90^{\circ}$, as expected for a cis-configured

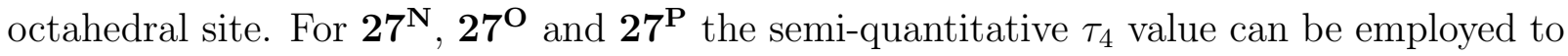
estimate their internal geometric distortion. This parameter has been proposed as a simple geometry index to quantify the distortion from tetrahedral geometries $\left(\tau_{4}=1\right)$ and is calculated by $\tau_{4}=1 / 141^{\circ} \cdot\left(360^{\circ}-\alpha-\beta\right)$, with $\alpha$ and $\beta$ defined as the two largest angles (ligand)(metal)-(ligand) in a four-coordinate complex. ${ }^{[166]}$ This concept is readily transferred and 
valid for octahedral complexes as well if two of the six coordinating atoms are distinguishable from the remaining donor atoms. Angles for the latter two donor sites are simply disregarded in those cases. Thus, $\tau_{4}$ values are expected to be zero for trans-configured octahedral geometries, whereas perfectly cis-configured sites are supposed to exhibit $\tau_{4}$ values of approximately 0.638. Applying this methodology indeed reveals intermediate geometries (between tetrahedral and octahedral structures) for $27^{\mathbf{N}}\left(\tau_{4}=0.785\right)$ and $\mathbf{2} 7^{\mathrm{O}}$ $\left(\tau_{4}=0.834\right)$, and confirms an octahedral coordination environment for $27^{\mathbf{P}}\left(\tau_{4}=0.654\right)$ and $27^{\mathrm{S}}\left(\tau_{4}=0.624\right)$.

Table 6.1: Selected structural parameters for complexes 27. Interatomic distances are given in $[\AA]$ and angles in $\left[^{\circ}\right]$ ( $S=$ thiophenolate sulfur atoms).

\begin{tabular}{ccccc}
\hline compound & $\mathbf{2 7}^{\mathbf{N}}$ & $\mathbf{2 7}^{\mathbf{O}}$ & $\mathbf{2 7}^{\mathbf{P}}$ & $\mathbf{2 7}^{\mathbf{S}}$ \\
\hline \multirow{2}{*}{$\mathrm{Fe}-\mathrm{S}$} & $2.3635(6)$ & $2.3572(17)$ & $2.2831(4)$ & $2.2288(9)$ \\
& $2.3756(6)$ & $2.3603(16)$ & $2.2860(4)$ & $2.2605(9)$ \\
& $2.3775(6)$ & $2.3747(16)$ & $2.2948(4)$ & $2.2743(9)$ \\
$\mathrm{Fe}-\mathrm{X}$ & $2.3884(6)$ & $2.3749(16)$ & $2.3217(4)$ & $2.3199(9)$ \\
& $2.4232(17)$ & $2.6341(35)$ & $2.2101(4)$ & $2.2472(9)$ \\
& $2.4581(17)$ & $2.5696(41)$ & $2.2117(4)$ & $2.2784(9)$ \\
$\mathrm{S}-\mathrm{Fe}-\mathrm{S}$ & $139.03(2)$ & $130.26(7)$ & $167.628(16)$ & $173.23(4)$ \\
& $110.23(2)$ & $112.17(7)$ & $100.063(15)$ & $85.71(3)$ \\
& $99.28(2)$ & $101.13(6)$ & $100.098(15)$ & $98.84(3)$ \\
$\mathrm{X}-\mathrm{Fe}-\mathrm{X}$ & $99.38(2)$ & $100.39(6)$ & $89.753(15)$ & $87.68(3)$ \\
$\tau_{4}$ & $109.97(2)$ & $110.07(6)$ & $88.198(14)$ & $87.30(3)$ \\
& $87.45(2)$ & $98.81(6)$ & $85.812(15)$ & $95.66(3)$ \\
& $121.68(5)$ & $116.857(113)$ & $108.183(15)$ & $93.36(3)$ \\
\hline
\end{tabular}

\subsection{Spectroscopy and Magnetic Properties in the Solid State}

Magnetic susceptibility measurements for all new complexes were carried out at $0.2 \mathrm{~T}$ and $0.5 \mathrm{~T}$ from $2 \mathrm{~K}$ to $295 \mathrm{~K}$. Magnetic moments $\mu_{\text {eff }}$ are almost constant over the complete temperature range for all complexes, but significantly different in magnitude (Figure 6.3). For $27^{\mathrm{N}}$ and $27^{\mathrm{O}}$ an effective moment of $\simeq 6.0 \mu_{\mathrm{B}}$ is found, indicating the presence of high spin ferric $(S=5 / 2)$ central ions. In contrast, $\mu_{\text {eff }}$ values of $\simeq 2.0 \mu_{\mathrm{B}}$ and $\simeq 2.2-2.5 \mu_{\mathrm{B}}$ are observed for $27^{\mathbf{P}}$ and $27^{\mathrm{S}}$, respectively. Thus, low spin configured ferric $(S=1 / 2)$ ions are present in these compounds. No dependence of the magnetic susceptibilities on the applied magnetic field was found for any of the complexes 27.

Zero-field Mössbauer spectra for all complexes have been recorded at room temperature, $80 \mathrm{~K}, 20 \mathrm{~K}$ and $7 \mathrm{~K}$ and fitted by using Lorentzian line doublets. Isomer shifts $\delta$ and 

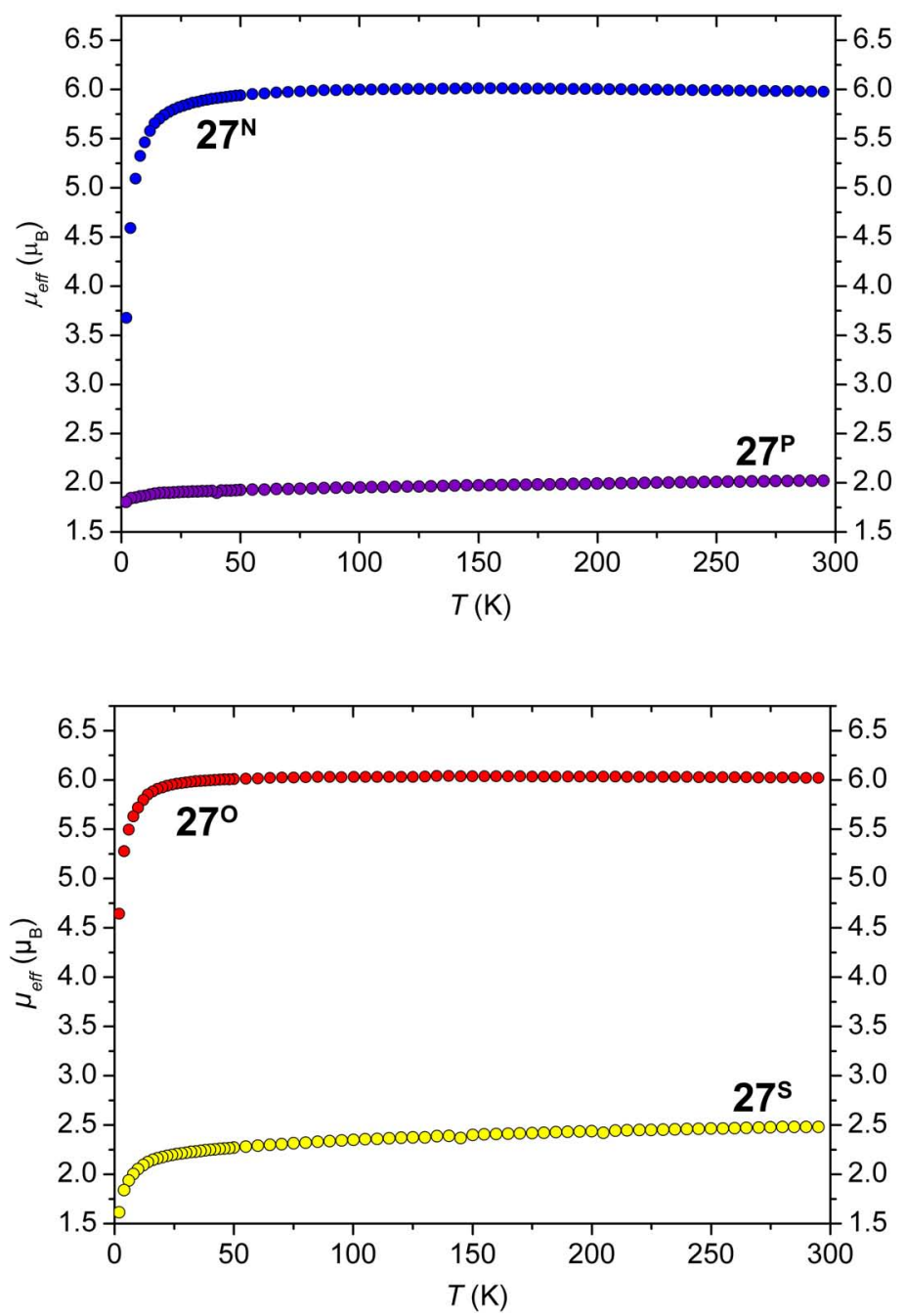

Figure 6.3: Plots of $\mu_{\text {eff }}$ vs. temperature for $\mathbf{2}^{N}$ (blue), $\mathbf{2 7}^{\mathbf{O}}$ (red), $\mathbf{2}^{\boldsymbol{P}}$ (violet) and ${ }^{2} \gamma^{S}$ (yellow) at a magnetic field $B=0.2 T$.

quadrupole splittings $\Delta E_{\mathrm{Q}}$ (determined at $80 \mathrm{~K}$ ) are summarized in Table 6.2. Significant differences between the collected data for the high spin complexes $\left(27^{\mathrm{N}}\right.$ and $\left.27^{\mathrm{O}}\right)$ and the low spin complexes $\left(\mathbf{2 7 ^ { \mathrm { P } }}\right.$ and $\left.\mathbf{2 7 ^ { \mathrm { S } }}\right)$ are evident over the entire temperature range. In case of $27^{\mathrm{P}}$ and $27^{\mathrm{S}}$ (spectra shown in Appendix C), comparatively sharp quadrupole doublets ( $\Gamma \simeq 0.3 \mathrm{~mm} / \mathrm{s}$ ) were obtained form $80 \mathrm{~K}$ to $7 \mathrm{~K}$ and even at room temperature, indicating a usual spin relaxation behavior (fast and therefore unresolved on the timescale of the Mössbauer experiment) for both complexes. Isomer shifts $\left(\delta=0.25 \mathrm{~mm} / \mathrm{s}\right.$ for $27^{\mathbf{P}}, \delta=0.32 \mathrm{~mm} / \mathrm{s}$

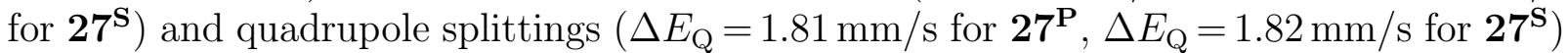
at $80 \mathrm{~K}$ are unexceptional for low spin ferric ions in an octahedral coordination environment. However, additional signals were detected in the Mössbauer spectra of $\mathbf{2 7 ^ { \mathrm { P } }}$ and $\mathbf{2 7 ^ { \mathrm { S } }}$, assumingly due to co-crystallized impurities. In the case of $27^{\mathbf{P}}$, temperature-independent shoulders close to the baseline - distal to the dominant quadrupole absorption of the complex were observed and fitted with $\delta=0.11 \mathrm{~mm} / \mathrm{s}, \Delta E_{\mathrm{Q}}=3.17 \mathrm{~mm} / \mathrm{s}$. For $27^{\mathrm{S}}$, mi- 
nor broadened absorptions $(<10 \%, \delta \simeq 0.38 \mathrm{~mm} / \mathrm{s})$ needed to be included in the fitting procedure for those spectra recorded at room temperature, $80 \mathrm{~K}$ and $20 \mathrm{~K}$ - this signal however disappeared at $7 \mathrm{~K}$ (identical sample measured, decomposition of the sample can be excluded as a reason for this phenomenon - alternating measurements at RT and $7 \mathrm{~K}$ afforded reproducible results with the latter signal appearing at RT and disappearing at $7 \mathrm{~K})$. Interestingly, a temperature-dependence of the quadrupole splitting was observed in the case of $27^{\mathrm{S}}$, i.e. $\Delta E_{\mathrm{Q}}$ is increasing upon cooling from $1.16 \mathrm{~mm} / \mathrm{s}$ (RT) to $1.81 \mathrm{~mm} / \mathrm{s}$ $(80 \mathrm{~K})$ and finally to $1.97 \mathrm{~mm} / \mathrm{s}(7 \mathrm{~K})$. Corresponding isomer shifts $\delta$ increase as well from $0.22 \mathrm{~mm} / \mathrm{s}(\mathrm{RT})$ to $0.36 \mathrm{~mm} / \mathrm{s}(7 \mathrm{~K})$. While the latter temperature-dependence of $\delta$ is quite common and also evident for $27^{\mathrm{P}}$, quadrupole splittings are commonly constant vs. temperature. Zero-field Mössbauer spectra for $27^{\mathrm{N}}$ and $27^{\mathrm{O}}$ are relatively uncommon, with unusually broad quadrupole doublets $\left(\delta=0.34 \mathrm{~mm} / \mathrm{s}\right.$ for $27^{\mathrm{N}}, \delta=0.27 \mathrm{~mm} / \mathrm{s}$ for $27^{\mathrm{O}}$, values for $\Delta E_{\mathrm{Q}}$ not fitted in both cases) detectable at room temperature $(\Gamma>0.7 \mathrm{~mm} / \mathrm{s})$. The spectra of $\mathbf{2 7 ^ { \mathbf { N } }}$ at room temperature, $80 \mathrm{~K}, 20 \mathrm{~K}$ and $7 \mathrm{~K}$ are collected in Figure 6.4. Upon cooling from RT to $20 \mathrm{~K}$, the quadrupole doublet coalesces to a central broad singlet. The spectrum at $80 \mathrm{~K}$ represents an intermediate situation. As only the quadrupole doublet could be included in the fitting procedure, considerable discrepancies between the experimental and the fitted curves are unavoidable in this case. The important area around the signal pivot however is well reproduced with $\Delta E_{\mathrm{Q}}=0.70 \mathrm{~mm} / \mathrm{s}$ (manually fitted by trialand-error procedure). At $7 \mathrm{~K}$ spin relaxation is slowed down further - changes in the electric and magnetic fields of the involved electron configurations are no longer distinguishable in the Mössbauer experiment. Therefore Zeeman-splitted magnetic subspectra are observed at this temperature (usually, Zeeman splittings are only detectable for particular polymeric materials or if external $B$-fields are applied perpendicular to the $\gamma$-ray). This sextet was fitted with $\delta=0.47 \mathrm{~mm} / \mathrm{s}, \Delta E_{\mathrm{Q}}=0.49 \mathrm{~mm} / \mathrm{s}$ and $B=40.4 \mathrm{~T}$. A similar phenomenon is evident for $27^{\mathrm{O}}$ as well. In this case, however, an additional quadrupole doublet is present in the spectra (at all temperatures), which increases in intensity upon cooling (spectra at $\mathrm{RT}, 80 \mathrm{~K}$ and $7 \mathrm{~K}$ are depicted in Appendix C). Although independently prepared samples afforded reproducible results, this signal most likely accounts to an impurity and does not reflect an intrinsic property of $27^{\mathrm{O}}$ (the origin of this doublet, however, remains to be clarified).

Solid state EPR measurements indicate considerable differences between both high spin configured and both low spin configured complexes. In addition to the main signal at $\simeq 350 \mathrm{mT}$ (detected for all complexes), intense low-field signals at $\simeq 80 \mathrm{G}$ were observed for $27^{\mathrm{N}}$ and $27^{\mathrm{O}}$ (spectra for all complexes $\mathbf{2 7}$ provided in Appendix C). Those signals might either originate form considerable large zero-field splitting parameters in both high spin complexes or correspond to higher aggregated forms of the latter compounds (compare: half-field signals in dimeric complexes). EPR Spectra in frozen solution might clarify this concern and need to be recorded yet. As additional spectroscopic techniques for the required temperature range $(20 \mathrm{~K}$ to $7 \mathrm{~K})$ are rare, it remains doubtful if a more detailed picture of the latter findings can be obtained. 

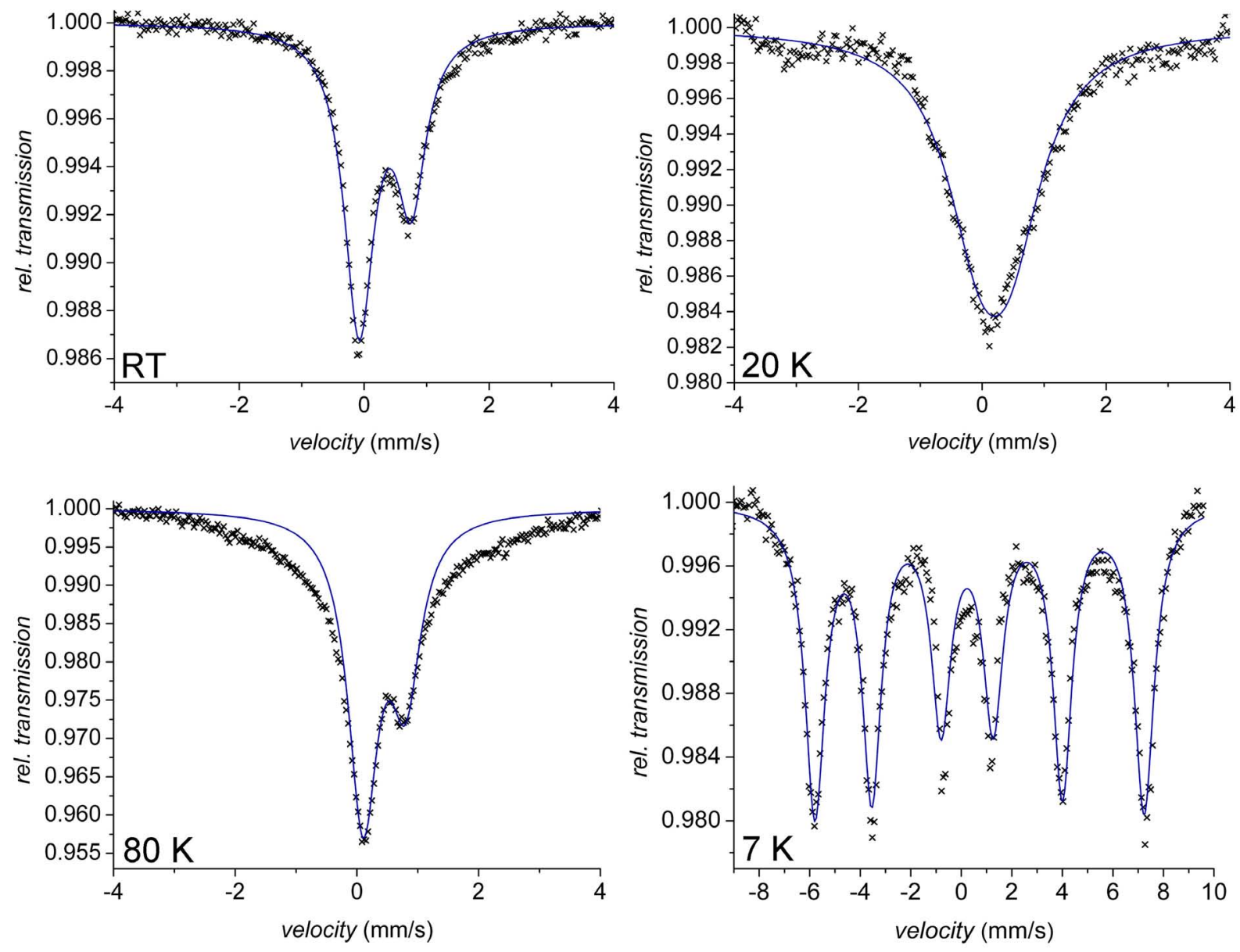

Figure 6.4: Zero-field Mössbauer spectra of $\mathbf{2}^{\boldsymbol{N}}$ recorded at $R T, 80 \mathrm{~K}, 20 \mathrm{~K}$ and $7 \mathrm{~K}$.

\subsection{Spectroscopy in Solution}

All new complexes were studied in solution by ESI mass spectrometry, cyclic voltammetry, UV-Vis and ${ }^{1} \mathrm{H}$ NMR spectroscopy. ESI mass spectroscopy in MeCN indicates, that all four complexes $\mathbf{2 7}$ are preserved in solution as mononuclear coordination compounds, with prominent peaks for $\left[\mathrm{M}+\mathrm{NEt}_{4}\right]^{+}(\mathrm{ESI}(+)-\mathrm{MS})$ and $\left[\mathrm{M}-\mathrm{NEt}_{4}\right]^{-}(\mathrm{ESI}(-)-\mathrm{MS})$. Proton NMR spectra at room temperature could only be recorded for $27^{\mathbf{P}}$ and $\mathbf{2 7}{ }^{\mathrm{S}}$ suggesting that these complexes retain their low spin configuration in solution, whereas samples of $27^{\mathrm{N}}$ and $27^{\mathrm{O}}$ gave broad baseline modulations, due to their $S=5 / 2$ ground state. Taking the observations from ESI-MS and NMR analysis together, one can conclude that complexes $\mathbf{2 7}$ are stable in solution preserving their spin state as it was observed for the corresponding solid state samples. In order to clarify whether the magnetic properties of $\mathbf{2 7}$ are temperature dependent in solution, UV-Vis spectra for all complexes were recorded in EtCN solution at $-80^{\circ} \mathrm{C}, 0^{\circ} \mathrm{C}$ and $+80^{\circ} \mathrm{C}$ (spectra are shown in Appendix $\mathrm{C}$ ). Broadened absorption bands were observed in all cases at $+80^{\circ} \mathrm{C}$, but no significant shift of any transition occurred, implying the absence of spin crossover activity in solution. Electro- 
chemical analysis by cyclic voltammetry was carried out in MeCN or DMF solution using the $\mathrm{Cp}_{2}^{*} \mathrm{Fe} / \mathrm{Cp}_{2}^{*} \mathrm{Fe}^{+}$couple as internal reference. As expected, a (quasi-)reversible peak in the cathodic regime (measured from zero to minus two volts) is observed for all complexes $\mathbf{2 7}$ and assigned to formation of the corresponding ferrous forms of complexes $\mathbf{2 7}$ (electrochemical potentials are summarized together with other spectroscopic data in Table 6.2). As a general rule, reduction from ferric to ferrous complexes is favored (found at higher potentials) for electron poor ferric ions. Thus, measured potentials indicated an increasing electron density on the iron centers in the order $27^{\mathrm{O}}<27^{\mathrm{N}}<27^{\mathrm{S}}<27^{\mathrm{P}}$. This trend is qualitatively reproduced by the distances of the electron donating $\mathrm{X}$-atoms to the central iron, decreasing in that order with $\mathrm{Fe}-\mathrm{O}=2.6341(35), 2.5696(41)>\mathrm{Fe}-\mathrm{N}=2.4232(17)$, $2.4581(17)>$ Fe-S $=2.2472(9), 2.2784(9)>$ Fe-P $=2.2101(4), 2.2117(4)$.

Table 6.2: Spectroscopic and electrochemical data for complexes 27.

\begin{tabular}{|c|c|c|c|}
\hline compound & $\delta\left(\Delta E_{\mathrm{Q}}\right)[\mathrm{mm} / \mathrm{s}]^{\mathrm{a}}$ & $\lambda_{\max }[\mathrm{nm}]\left(\varepsilon\left[\mathrm{M}^{-1} \mathrm{~cm}^{-1}\right]\right)^{\mathrm{b}}$ & $E_{1 / 2}[\mathrm{~V}]$ \\
\hline $27^{\mathrm{N}}$ & $0.45(0.70)$ & $340(s h, 15000), 580(5150)$ & $-1.30^{\mathrm{c}}$ \\
\hline $27^{\mathrm{O}}$ & $0.35(0.83)$ & $359(12500), 603(7920)$ & $-0.95^{\mathrm{c}}$ \\
\hline $27^{\mathrm{P}}$ & $0.25(1.81)$ & $579(1730), 753(1550)$ & $-1.64^{\mathrm{d}}$ \\
\hline $27^{\mathrm{S}}$ & $0.32(1.82)$ & $504(1580), 842(940)$ & $-1.37^{\mathrm{d}}$ \\
\hline
\end{tabular}

(a) ${ }^{57} \mathrm{Fe}$ Mössbauer parameters at $80 \mathrm{~K}$, relative to Fe metal at room temperature. (b) Recorded in DMF solution at room temperature. (c) Potentials in $\mathrm{MeCN} / 0.1 \mathrm{M} \mathrm{NBu} \mathrm{PF}_{6}$ at a scan rate of $100 \mathrm{mV} / \mathrm{s}$ vs. the $\mathrm{Cp}_{2}^{*} \mathrm{Fe} / \mathrm{Cp}_{2}^{*} \mathrm{Fe}^{+}$couple. (d) Potentials in $\mathrm{DMF} / 0.1 \mathrm{M} \mathrm{NBu} \mathrm{NF}_{6}$ at a scan rate of $100 \mathrm{mV} / \mathrm{s}$ vs. the $\mathrm{Cp}_{2}^{*} \mathrm{Fe} / \mathrm{Cp}_{2}^{*} \mathrm{Fe}^{+}$couple.

\subsection{DFT Calculations}

In order to corroborate the conclusions from structural and spectroscopic findings and to elucidate the influence of the different tethered donor groups $\mathrm{X}$ on the spin state of the corresponding complexes 27, DFT calculations were performed, using the BP86 functional. All complexes $\mathbf{2 7}$ were studied in both the singlet and sextet spin states for sake of comparison. In accordance with the experimental findings, the BP86 results confirm that the singlet state is lower in energy by 265 and $199 \mathrm{~kJ} / \mathrm{mol}$ for $\mathbf{2} 7^{\mathbf{P}}$ and $\mathbf{2} 7^{\mathrm{S}}$, respectively, whereas the sextet state is energetically favored for $\mathbf{2 7}^{\mathbf{N}}$ and $27^{\mathbf{O}}$ by 48 and $91 \mathrm{~kJ} / \mathrm{mol}$, respectively (Table 6.3).

Spin densities $\rho$ on all atoms were calculated for all complexes $\mathbf{2 7}$ for either the high spin or low spin states. For all sextet states a significant delocalization of the overall five electrons on the thiolates (approximately 0.17-0.26 electrons on each sulfur atom) is observed. In contrast, unpaired electron densities are found localized on the central iron for all low spin configurations. Interestingly, virtually no spin density on the neutral donor atoms $\mathrm{X}$ is found in all cases. In this context it should be noted that $\rho$ values on $\mathrm{X}$ are of opposite 
Table 6.3: Relative energies and atomic spin densities $\rho$ on the various atoms obtained at the BP86/SVP level of theory for either the high spin (HS) or low spin (LS) state of $27(X=N, O, P, S)$ utilizing the corresponding atom coordinates obtained from X-ray diffraction.

\begin{tabular}{|c|c|c|c|c|c|c|c|c|c|}
\hline \multirow[t]{2}{*}{ compound } & \multirow{2}{*}{$\begin{array}{l}\text { spin } \\
\text { state }\end{array}$} & \multirow{2}{*}{$\begin{array}{c}E_{\text {rel }} \\
{[\mathrm{kJ} / \mathrm{mol}]}\end{array}$} & \multicolumn{7}{|c|}{ spin densities $\rho$} \\
\hline & & & $\mathrm{Fe}$ & & S (thi & olate) & & $y$ & \\
\hline $27^{\mathrm{N}}$ & $\begin{array}{l}H S \\
L S\end{array}$ & $\begin{array}{c}0.0 \\
+48\end{array}$ & $\begin{array}{l}3.8858 \\
1.1037\end{array}$ & $\begin{array}{c}0.2317 \\
-0.0462\end{array}$ & $\begin{array}{c}0.2315 \\
-0.0465\end{array}$ & $\begin{array}{l}0.1787 \\
<0.01\end{array}$ & $\begin{array}{l}0.1721 \\
<0.01\end{array}$ & $\begin{array}{l}0.0443 \\
<0.01\end{array}$ & $\begin{array}{l}0.0438 \\
<0.01\end{array}$ \\
\hline $27^{\mathrm{O}}$ & $\begin{array}{l}H S \\
L S\end{array}$ & $\begin{array}{c}0.0 \\
+91\end{array}$ & $\begin{array}{l}3.9102 \\
1.1903\end{array}$ & $\begin{array}{c}0.2061 \\
-0.0203\end{array}$ & $\begin{array}{c}0.2035 \\
-0.0384\end{array}$ & $\begin{array}{c}0.2252 \\
-0.0318\end{array}$ & $\begin{array}{c}0.2317 \\
-0.0525\end{array}$ & $\begin{array}{l}0.0149 \\
<0.01\end{array}$ & $\begin{array}{l}0.0194 \\
<0.01\end{array}$ \\
\hline $27^{\mathrm{P}}$ & $\begin{array}{l}H S \\
L S\end{array}$ & $\begin{array}{c}+265 \\
0.0\end{array}$ & $\begin{array}{l}3.4012 \\
0.9555\end{array}$ & $\begin{array}{l}0.2458 \\
0.0187\end{array}$ & $\begin{array}{l}0.2486 \\
0.0419\end{array}$ & $\begin{array}{l}0.2645 \\
0.0268\end{array}$ & $\begin{array}{l}0.2311 \\
<0.01\end{array}$ & $\begin{array}{c}0.0723 \\
-0.0535\end{array}$ & $\begin{array}{c}0.0670 \\
-0.0511\end{array}$ \\
\hline $27^{\mathrm{S}}$ & $\begin{array}{l}H S \\
L S\end{array}$ & $\begin{array}{c}+199 \\
0.0\end{array}$ & $\begin{array}{l}3.6331 \\
0.9306\end{array}$ & $\begin{array}{l}0.2445 \\
0.0347\end{array}$ & $\begin{array}{l}0.2581 \\
0.0296\end{array}$ & $\begin{array}{c}0.2356 \\
-0.0139\end{array}$ & $\begin{array}{l}0.2202 \\
<0.01\end{array}$ & $\begin{array}{c}0.0890 \\
-0.0130\end{array}$ & $\begin{array}{c}0.0832 \\
-0.0169\end{array}$ \\
\hline
\end{tabular}

sign for singlet $27^{\mathrm{P}}$ and $27^{\mathrm{S}}$ (even so their magnitude is negligible), indicating a bonding $\mathrm{Fe}-\mathrm{X}$ interaction. In order to gain further insight into the nature of this interaction, total d-electron densities were analyzed for all complexes (Table 6.4). Obviously, population of d-shells plays a minor role for all thiolate atoms since corresponding density values are diminutive in a range of $0.063 \pm 0.01$ for all complexes in both multiplicities. However, this is not the case for the thioether sulfurs in $27^{\mathrm{S}}$ and the phosphor atoms in $27^{\mathbf{P}}$, each accepting significant d-electron density in either the singlet or sextet state. As expected, d-electron densities on the nitrogen $\left(27^{\mathrm{N}}\right)$ or oxygen $\left(27^{\mathrm{O}}\right)$ atoms are negligible. Thus, results from the DFT calculations suggest that complexes $\mathbf{2 7}$ prefer singlet configurations in cases of the tethered $\pi$-acceptor atoms $\mathrm{P}$ or $\mathrm{S}$ (in $27^{\mathrm{P}}$ or $27^{\mathrm{S}}$, respectively) and sextet configurations in cases of the tethered purely $\sigma$-donating atoms $\mathrm{N}$ or $\mathrm{O}$ (in $27^{\mathbf{N}}$ or $27^{\mathrm{O}}$, respectively). Qualitatively identical results are obtained, when all calculations were performed on optimized geometries.

In order to address the question whether any of the complexes might be in close proximity to a spin crossover situation, at least two issues, the energy difference between both multiplicities and the corresponding activation barrier should be taken into account. The first aspect - the energy difference - was already discussed and summarized in Table 6.3: Both

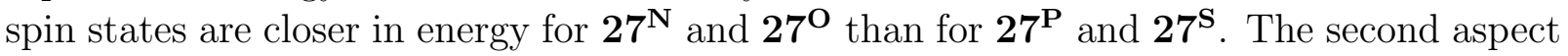
- the activation barrier - can be estimated from the structural rearrangements necessary when switching between both multiplicities. Therefore, experimental structures (from Xray diffraction) and optimized structures for the singlet and sextet states were aligned and superimposed as shown in Figure 6.5. It is obvious from this illustration that significant structural changes would be expected for $27^{\mathrm{O}}$ (and $27^{\mathrm{S}}$ ). Thus, modifications on $27^{\mathrm{N}}$ (e.g. by substituents in para-position to the thiolates) are most promising, when trying to obtain type 27 complexes with spin crossover characteristics. In order to rationalize the trend in the quadrupole splittings $\Delta E_{\mathrm{Q}}$ observed in the Mössbauer spectra, eigenvalues of the electric field gradient (EFG) tensor have been calculated for the singlet and sextet states 
Table 6.4: Atomic populations from total densities in $n(d)$-shells on the various atoms obtained at the BP86/SVP level of theory for either the high spin (HS) or low spin (LS) state of $\mathbf{2 7}(X=N, O, P, S)$ utilizing the corresponding atom coordinates obtained from $X$-ray diffraction.

\begin{tabular}{|c|c|c|c|c|c|c|c|c|}
\hline \multirow[t]{2}{*}{ compound } & \multirow{2}{*}{$\begin{array}{l}\text { spin } \\
\text { state }\end{array}$} & \multicolumn{7}{|c|}{ total electron densities in $n(d)$-shells } \\
\hline & & $\mathrm{Fe}$ & & S (thi & late) & & & \\
\hline \multirow{2}{*}{$27^{\mathrm{N}}$} & $H S$ & 6.18203 & 0.05685 & 0.05598 & 0.05763 & 0.05881 & 0.02812 & 0.02832 \\
\hline & $L S$ & 6.46832 & 0.05876 & 0.05769 & 0.05960 & 0.06089 & 0.02823 & 0.02843 \\
\hline \multirow{2}{*}{$27^{\mathrm{O}}$} & $H S$ & 6.20152 & 0.05632 & 0.05757 & 0.05534 & 0.05443 & 0.01605 & 0.01592 \\
\hline & $L S$ & 6.47204 & 0.05827 & 0.05963 & 0.05796 & 0.05712 & 0.01599 & 0.01587 \\
\hline \multirow{2}{*}{$27^{\mathrm{P}}$} & $H S$ & 6.35396 & 0.06474 & 0.06740 & 0.06301 & 0.06901 & 0.24268 & 0.25075 \\
\hline & $L S$ & 6.62901 & 0.06742 & 0.07020 & 0.06528 & 0.07172 & 0.25398 & 0.25956 \\
\hline \multirow{2}{*}{$27^{\mathrm{S}}$} & $H S$ & 6.32963 & 0.06575 & 0.06741 & 0.06794 & 0.06583 & 0.12422 & 0.12769 \\
\hline & $L S$ & 6.64275 & 0.07038 & 0.07265 & 0.06943 & 0.06672 & 0.12947 & 0.13330 \\
\hline
\end{tabular}

of compounds 27. Quadrupole splittings $\Delta E_{\mathrm{Q}}$ derived from those values are compared with the experimental data in Table 6.5 (As data at RT interfere with thermal background noise and data below $\simeq 40 \mathrm{~K}$ are disturbed by signal coalescence in the case of $27^{\mathbf{N}}$ and

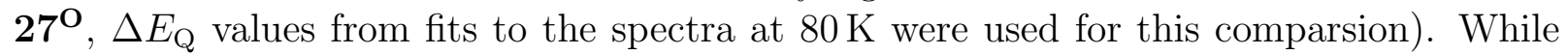
the calculated $\Delta E_{\mathrm{Q}}$ values for $L S-\mathbf{2} 7^{\mathrm{P}}$ and $L S-\mathbf{2 7 ^ { \mathrm { S } }}$ appear to be systematically too low by $0.50 \mathrm{~mm} / \mathrm{s}$ and $0.65 \mathrm{~mm} / \mathrm{s}$, a satisfying agreement between the calculated and experimental quadrupole splittings is observed for both high spin complexes. Most importantly, the trend $\Delta E_{\mathrm{Q}}(\mathbf{2 7}, H S)<\Delta E_{\mathrm{Q}}(\mathbf{2 7}, L S)$ is reproduced at the applied BP86/SVP level of theory.

Table 6.5: Calculated eigenvalues of the field gradient tensor for the singlet and sextet states of 27 at the BP86/SVP level of theory, and calculated and experimental $\Delta E_{Q}$ values.

\begin{tabular}{|c|c|c|c|c|}
\hline compound & calculated $\mathrm{EFG}^{\mathrm{a}}$ & $\begin{array}{c}\Delta E_{\mathrm{Q}} \text { calculated } \\
{[\mathrm{mm} / \mathrm{s}]^{\mathrm{b}}}\end{array}$ & $\begin{array}{l}\text { experimental } \\
\text { spin state }\end{array}$ & $\begin{array}{c}\Delta E_{\mathrm{Q}} \text { experimental } \\
{[\mathrm{mm} / \mathrm{s}]^{\mathrm{c}}} \\
\end{array}$ \\
\hline $27^{\mathrm{N}}$ & $\begin{array}{c}0.159 / 0.163 /-0.323(H S) \\
-0.000936 /-0.0790 / 0.0800(L S)\end{array}$ & $\begin{array}{l}0.52(H S) \\
0.15(L S)\end{array}$ & $H S$ & 0.70 \\
\hline $27^{\mathrm{O}}$ & $\begin{array}{c}0.231 / 0.262 /-0.492(H S) \\
0.00352 / 0.0292 /-0.0327(L S)\end{array}$ & $\begin{array}{l}0.80(H S) \\
0.06(L S)\end{array}$ & $H S$ & 0.83 \\
\hline $27^{\mathrm{P}}$ & $\begin{array}{l}0.0349 / 0.196 /-0.231(H S) \\
-0.306 /-0.501 / 0.807(L S)\end{array}$ & $\begin{array}{l}0.40(H S) \\
1.32(L S)\end{array}$ & $L S$ & 1.81 \\
\hline $27^{\mathrm{S}}$ & $\begin{array}{c}-0.0736 /-0.194 / 0.268(H S) \\
-0.119 /-0.552 / 0.671(L S)\end{array}$ & $\begin{array}{l}0.45(H S) \\
1.16(L S)\end{array}$ & $L S$ & 1.82 \\
\hline
\end{tabular}

(a) the three eigenvalues of the field gradient tensor given in atomic units $\left(1\right.$ a.u. $\left.=9 \cdot 72 \cdot 10^{21} \mathrm{~V} / \mathrm{m}^{2}\right)$. (b) $\Delta E_{\mathrm{Q}}$ calculated according to $\Delta E_{\mathrm{Q}}=\frac{1}{2} e Q V_{z z} \cdot\left(1+\eta^{2} / 3\right)^{1 / 2}$, where the quadrupole moment $Q$ is 0.16 barn $\left(0.16 \cdot 10^{-28} \mathrm{~m}^{2}\right)$ for ${ }^{57} \mathrm{Fe}, V_{z z}$ is the main value of the EFG, $\eta=\left(V_{x x}-V_{y y}\right) / V_{z z}$ (with $\left|V_{x x}\right|<\left|V_{y y}\right|<$ $\left.\left|V_{z z}\right|\right)$ and $1 \mathrm{~mm} / \mathrm{s}=4.8075 \cdot 10^{-8} \mathrm{eV}$. (c) data from Table 6.2 . 

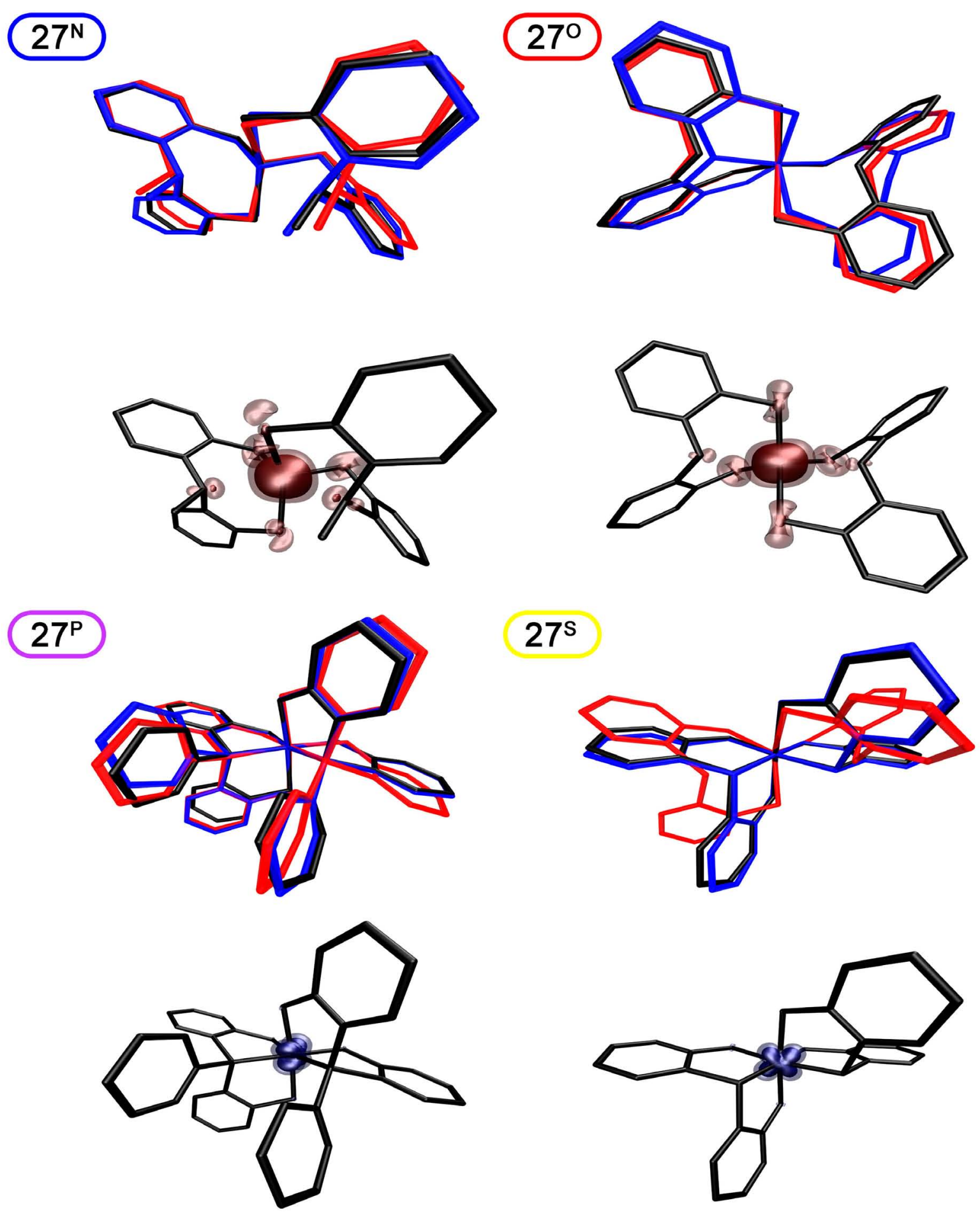

Figure 6.5: Superposition of experimental structures (black) and optimized structures for high spin (red) and low spin (blue) states for $\mathbf{2}^{\boldsymbol{N}}, \mathfrak{2}^{\boldsymbol{O}}, \mathbf{2}^{\boldsymbol{P}}$ and $\mathfrak{2}^{\boldsymbol{S}}$ together with spin densities (opaque: 0.025 a.u. level, translucent: $0.00^{75}$ a.u. level) for the sextet (27 ${ }^{N}$ and

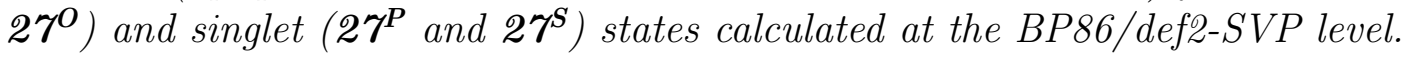




\subsection{Conclusions}

In summary, unexpected structural and magnetic characteristics of the readily synthesized mononuclear ferric complexes $\left(\mathrm{NEt}_{4}\right)\left[\left(1,1^{\prime}-\mathrm{X}-\left(o-\mathrm{C}_{6} \mathrm{H}_{4} \mathrm{~S}\right)_{2}\right)_{2} \mathrm{Fe}\right] 27(\mathrm{X}=\mathrm{NMe}, \mathrm{O}, \mathrm{PPh}, \mathrm{S})$ were illuminated to some detail. $S=5 / 2$ spin states for $27^{\mathrm{P}}$ and $27^{\mathrm{S}}$ and $S=1 / 2$ spin states for $27^{\mathrm{N}}$ and $27^{\mathrm{O}}$ were unambiguously manifested for the solid samples of $\mathbf{2 7}$ by magnetic susceptibility measurements. DFT calculations revealed that d-electron shells of the phosphine and thioether moieties in $27^{\mathrm{P}}$ and $27^{\mathrm{S}}$ are in conjugation with the dorbitals of the central ferric ion. In contrast to $27^{\mathrm{N}}$ and $27^{\mathrm{O}}$, low spin configurations are energetically favored for the latter systems. Akin to $\pi$-acceptor ligands that usually enforce low spin configurations, the d-acceptor character of the third row elements phosphor and sulfur might account for the established $S=1 / 2$ ground state in $27^{\mathbf{P}}$ and $27^{\mathrm{S}}$. The observed incomplete spin relaxation features of $27^{\mathrm{N}}$ and $27^{\mathrm{O}}$ however remain curious to some extent, especially as no drastic changes in the SQUID data were recorded in the crucial temperature range from $80 \mathrm{~K}$ to $7 \mathrm{~K}$. 


\section{Chapter 7}

\section{Synthetic [2Fe-2S] Clusters with Chelating N-Donor Capping Ligands}

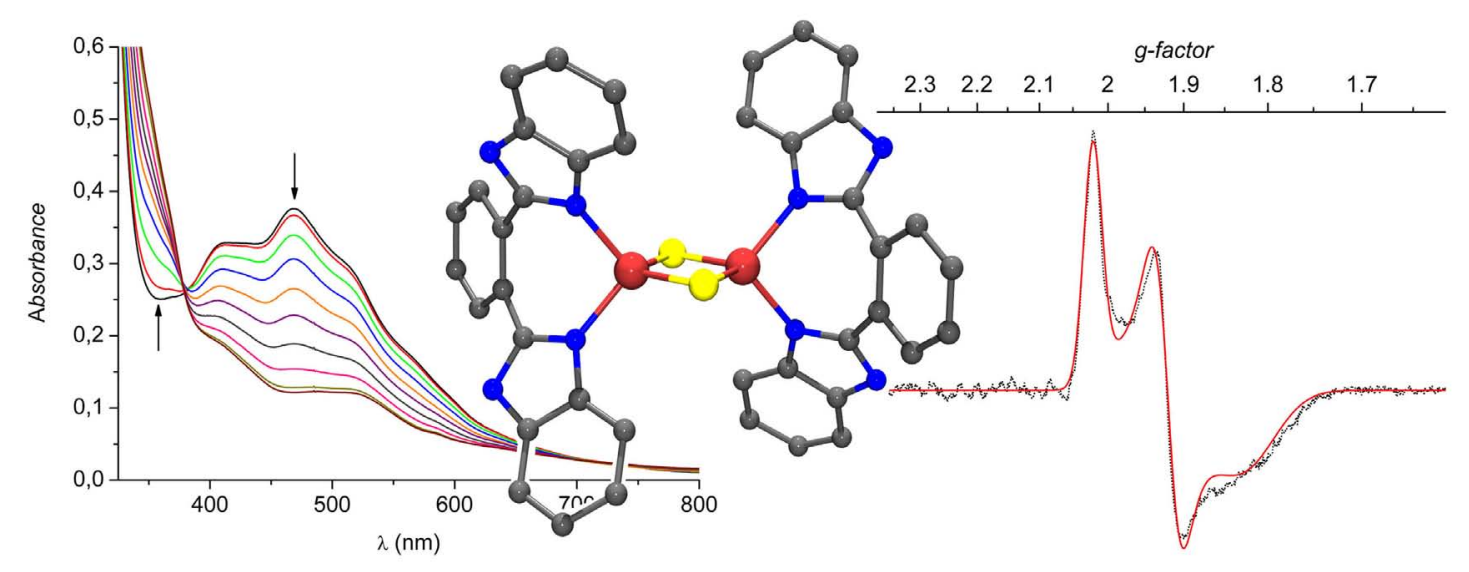

\section{Abstract}

Five new $[2 \mathrm{Fe}-2 \mathrm{~S}]^{2+}$ clusters with different 1,1'-dipyrromethane and 1,2-bis-benzimidazolebenzene derivatives as chelating terminal ligands have been prepared and fully characterized, including X-ray diffraction analyses. These systems represent rare examples of synthetic $[2 \mathrm{Fe}-2 \mathrm{~S}]$ complexes with $\{\mathrm{N}\}$-donor capping ligands. While geometric parameters as well as spectroscopic and electrochemical characteristics of the new complexes were found as anticipated, the chelating nature of the terminal ligands imparts a relatively high stability that permitted the generation and EPR characterisation of a $[2 \mathrm{Fe}-2 \mathrm{~S}]^{+}$species ligated by benzene-1,2-bis-benzimidazolate. 


\subsection{Introduction}

Proteins, including a $[2 \mathrm{Fe}-2 \mathrm{~S}]$ moiety with terminal $\{\mathrm{N}\}$-ligation (His, Arg) are increasingly recognized in biological systems due to their unique functions . [17, 216-221] The Rieske center - with two imidazole ligands from histidine residues bound to one iron atom of the cluster core - probably represents the most prominent example. Interestingly, no accurate structural analogue of the Rieske-type [2Fe-2S] clusters with mixed $\left\{\mathrm{N}_{2} / \mathrm{S}_{2}\right\}$ ligation has been reported prior to the present contribution (see Chapter 8). In this context it should be noted that only very few synthetic $[2 \mathrm{Fe}-2 \mathrm{~S}]$ clusters with terminal $\{\mathrm{N}\}$-donor ligands are known altogether, ${ }^{[34,141,142,171,222]}$ with $4^{[141,142]}$ and the more recently reported $\mathbf{3}^{[34]}$ (Scheme 7.1) being the only examples that have been characterized crystallographically.

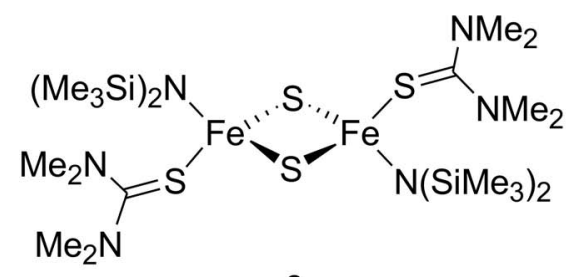

3

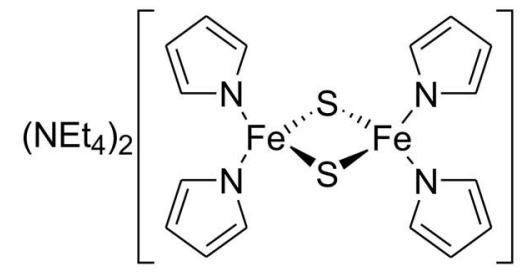

4

Scheme 7.1: Rare examples of (partially) $\{N\}$-coordinate [2Fe-2S] model compounds.

This chapter describes the synthesis and structural characterization of a series of new [2Fe-2S] clusters with bidentate dipyrromethane ${ }^{[194,223-225]}$ and 1,2-bis-benzimidazole-benzene $^{[226]}$ capping ligands (Scheme 7.2). Due to the chelating nature of these ligands, their complexes exhibit comparatively high stabilities. The availability of the new complexes and the examination of their reactivity finally paved the way for the synthesis of the asymmetrically coordinated model compound for the Rieske site.

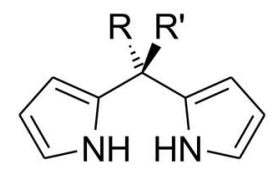

$$
\begin{aligned}
& X X a, R=R^{\prime}=M e \\
& \mathbf{X X b}, \mathrm{R}=\mathrm{R}^{\prime}=\mathrm{Ph} \\
& \mathbf{X X c},-\left[\mathrm{R} \mathrm{R}^{\prime}\right]-=-\left[\left(\mathrm{CH}_{2}\right)_{5}\right]-
\end{aligned}
$$

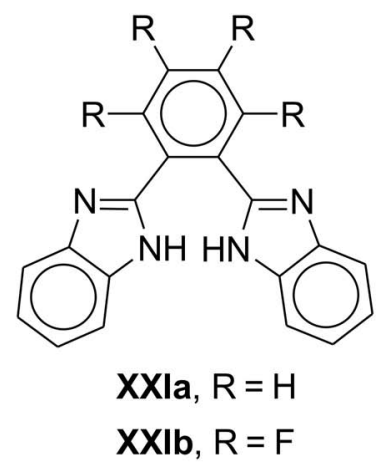

Scheme 7.2: Utilized dipyrromethane and 1,2-bis-benzimidazole-benzene derivatives. 


\subsection{Dipyrromethane-Coordinate $[2 \mathrm{Fe}-2 \mathrm{~S}]$ Cluster Compounds}

\subsubsection{Synthesis and Structural Characterisation}

Deprotonated dipyrromethane derivatives XXa-XXc ${ }^{[194,223-225]}$ have been applied as bidentate $\{\mathrm{N}\}$-donor ligands in salt metathesis reactions with $\left(\mathrm{NEt}_{4}\right)_{2}\left[\mathrm{Fe}_{2} \mathrm{~S}_{2} \mathrm{Cl}_{4}\right] \mathbf{2}^{[138]}$ to afford dark brown solutions of the respective iron-sulfur clusters $\left(\mathrm{NEt}_{4}\right)_{2}\left[\mathrm{Fe}_{2} \mathrm{~S}_{2}\left\{\mathrm{~N}_{2}\right\}_{2}\right] \mathbf{2 9} \mathbf{a}$ 29c (Scheme 7.3), which precipitate from saturated MeCN or DMF solutions upon cooling.

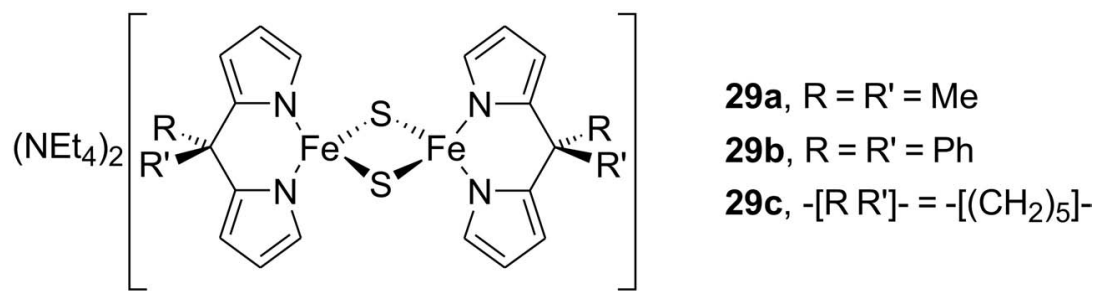

Scheme 7.3: Dipyrromethane-coordinate clusters 29a, 29b and 29c.

Positive ion ESI mass spectrometry proved to be a valuable analytical tool to confirm formation of the anticipated complexes since spectra of MeCN solutions show dominant signals for the ions $\left[\mathrm{M}+\mathrm{NEt}_{4}\right]^{+}$comprising one additional $\mathrm{NEt}_{4}^{+}$counter cation, as well as further signals for $\left[2 \mathrm{M}+\mathrm{NEt}_{4}\right]^{+}$. All complexes could be obtained as black crystals suitable for X-ray analysis from MeCN $(29 b)$ or DMF $(29 a, 29 c)$ solutions. The crystal structures of 29a-29c consist of discrete cations and anions. The molecular structures of the anions are depicted in Figure 7.1, selected atom distances and bond angles are summarized in Table 7.1. All bis- $\mu$-sulfido $\left[\mathrm{Fe}_{2} \mathrm{~S}_{2}\left\{\mathrm{~N}_{2}\right\}_{2}\right]^{2-}$ cores of $\mathbf{2 9 a - 2 9 c}$ contain two fourcoordinate iron atoms in a distorted tetrahedral coordination environment, with the two $\left\{\mathrm{FeN}_{2} \mathrm{~S}_{2}\right\}$-tetrahedra sharing one edge. The $\left[\mathrm{Fe}_{2} \mathrm{~S}_{2}\left\{\mathrm{~N}_{2}\right\}_{2}\right]^{2-}$ moieties of $\mathbf{2 9 b}$ and $29 \mathbf{c}$ have crystallographically imposed inversion symmetry with a half molecule in the asymmetric unit - therefore their rhomb-like $\mathrm{Fe}_{2} \mathrm{~S}_{2}$-rings are perfectly planar. The $\mathrm{Fe}_{2} \mathrm{~S}_{2}$-ring of $\mathbf{2 9 a}$, however, deviates from planarity, which causes the S-Fe-S planes to intersect at $17.48(3)^{\circ}$. Distances Fe-S (2.202(1) - 2.227(2) A) and Fe-N (1.966(1) - 1.988(6) $\AA$ ) as well as the S-Fe-S angles $\left(\simeq 104^{\circ}\right)$ are comparable to those reported for the parent bis- $\mu$-sulfido complex 4 (FeS $2.18 \AA$, Fe-N 1.96 and $2.09 \AA$ A, S-Fe-S 104.3 ${ }^{\circ} .{ }^{[141,142]}$ Interestingly, complex 4 is the only other crystallographically characterized $[2 \mathrm{Fe}-2 \mathrm{~S}]$ cluster with four terminal $\{\mathrm{N}\}$-donors reported in literature. As expected, the N-Fe-N angle for the monodentate pyrrolato ligands in $4\left(110.6^{\circ}\right)$ differs somewhat from the values determined for the less flexible chelate ligands in 29a- 29c $\left(92.2(1)-94.8(3)^{\circ}\right)$, whereas the Fe $\cdots$ Fe distance is slightly shorter in $4(2.677 \AA)$ compared to $\mathbf{2 9 a}$ - 29c $(2.692(1)$ - 2.724(1) A). Rhombs of [2Fe-2S] with terminal thiolato ligands, $\left[\mathrm{Fe}_{2} \mathrm{~S}_{2}(\mathrm{SR})_{4}\right]^{2-}$, usually feature similar metal-metal separations in the narrow range $2.69-2.72 \AA,{ }^{[129]}$ i.e. replacement of the thiolates by anionic $\{\mathrm{N}\}$-ligands does not cause any major geometric changes in the central core. 

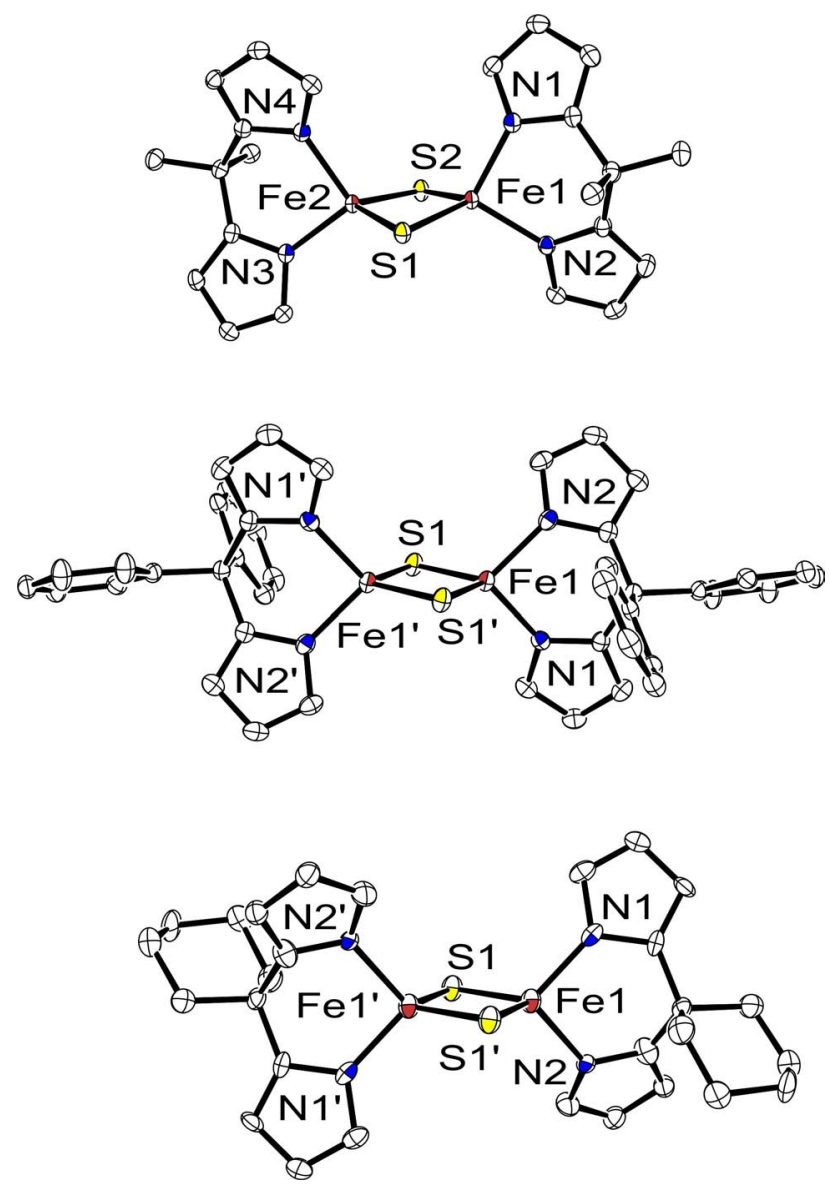

Figure 7.1: ORTEP plots (50\% probability thermal ellipsoids) of the molecular structures of $29 \boldsymbol{a}$ (top), $29 \boldsymbol{b}$ (middle) and 29c (bottom). For the sake of clarity all hydrogen atoms and $\mathrm{NEt}_{4}^{+}$counter ions have been omitted.

Table 7.1: Selected structural parameters for clusters 29a-29c. Interatomic distances are given in $[\AA]$ and angles in $\left[{ }^{\circ}\right]$.

\begin{tabular}{cccc}
\hline compound & $\mathbf{2 9 a}$ & $\mathbf{2 9 b}$ & $\mathbf{2 9 c}$ \\
\hline $\mathrm{Fe} \cdot \mathrm{Fe}$ & $2.6916(9)$ & $2.7235(6)$ & $2.722(2)$ \\
& $1.984(1) / 1.966(1)$ & $1.981(2)$ & $1.970(7)$ \\
$\mathrm{Fe}-\mathrm{N}$ & $1.970(1) / 1.987(1)$ & $1.972(2)$ & $1.988(6)$ \\
& $2.2193(7) / 2.2149(7)$ & $2.2021(6)$ & $2.215(3)$ \\
$\mathrm{Fe}-(\mu-\mathrm{S})$ & $2.2121(7) / 2.2151(7)$ & $2.2215(7)$ & $2.227(2)$ \\
$(\mu-\mathrm{S})-\mathrm{Fe}-(\mu-\mathrm{S})$ & $104.13(3) / 104.17(3)$ & $104.00(2)$ & $104.40(8)$ \\
$\mathrm{N}-\mathrm{Fe}-\mathrm{N}$ & $93.97(6) / 94.40(6)$ & $92.19(7)$ & $94.8(3)$ \\
\hline
\end{tabular}




\subsubsection{Spectroscopy in the Solid State}

Mössbauer spectra of the three new synthetic clusters were recorded at $80 \mathrm{~K}$ and the spectrum of $\mathbf{2 9 b}$ is shown in Figure 7.2 as an example. Spectral fits to the data are obtained by using Lorentzian line doublets with isomer shifts $\delta$ and quadrupole splittings $\Delta E_{\mathrm{Q}}$ summarized in Table 7.2. The empirical correlation $\delta=1.43-0.40 s$ between oxidation state $s$ and isomer shift $\delta$ was found for tetrahedral $\left\{\mathrm{S}_{4}\right\}$-ligated iron sites at $77 \mathrm{~K}$ by linear regression analysis. ${ }^{[167,227]}$ Applying the experimental $\delta$ values for the $\left\{\mathrm{FeN}_{2} \mathrm{~S}_{2}\right\}$ systems 29a-29c to this equation reveals the expected oxidation state of $s=3$, suggesting that the above correlation is valid not only for all-sulfur sites. Quadrupole splittings for $29 \mathbf{a}-\mathbf{2 9 c}$ are somewhat larger than for the parent complex 4 (Table 7.2), which is in accordance with greater distortion of the tetrahedral coordination environment that is imposed by the chelating ligands. It should also be noted that Mössbauer spectra of the oxidized Rieske centre isolated from Thermus thermophilus recorded at $4.2 \mathrm{~K}$ show values $\delta\left(\Delta E_{\mathrm{Q}}\right)$ of 0.24 (0.52) and $0.32(0.91) \mathrm{mm} / \mathrm{s}$ at $\mathrm{pH} \mathrm{10.}{ }^{[4]}$

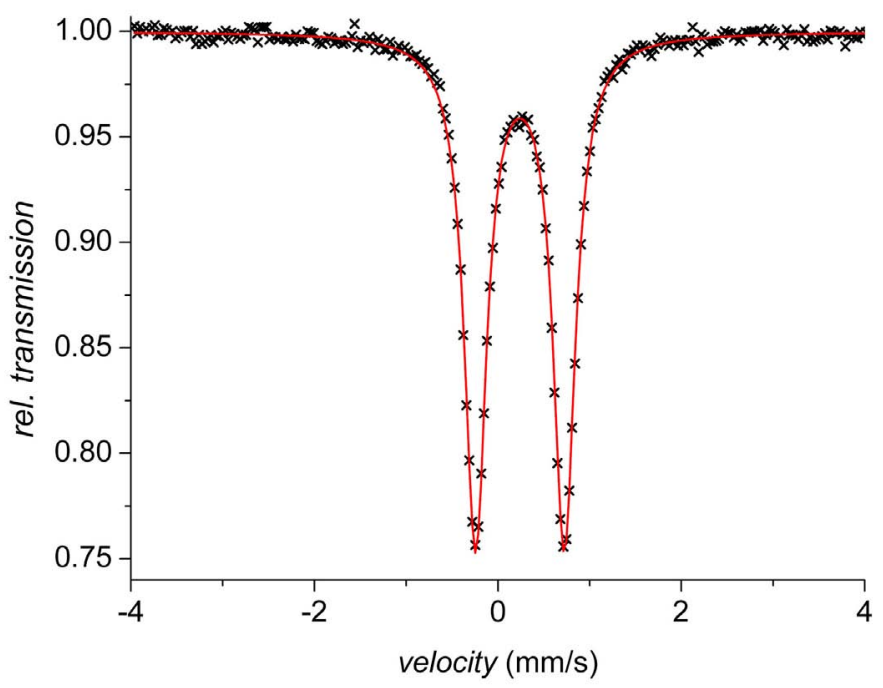

Figure 7.2: Zero-field Mössbauer spectrum of $29 \mathbf{b}$ at $80 \mathrm{~K}$. The solid line is a Lorentzian doublet fitted to the experimental values (crosses).

Magnetic susceptibility measurements for complexes 29a-29c were carried out at $1 \mathrm{~T}$ from $2 \mathrm{~K}$ to $290 \mathrm{~K}$. Magnetic moments $\mu_{\mathrm{eff}}$ at room temperature are in the range $1.8-2.4 \mu_{\mathrm{B}}$, i.e. much lower than the values expected for two uncoupled iron(III) ions, indicating significant antiferromagnetic coupling as is commonly observed for the $\mathrm{Fe}_{2} \mathrm{~S}_{2}$ core. This is confirmed by the temperature dependence of $\mu_{\text {eff }}$ for 29c shown in Figure 7.3. Upon cooling $\mu_{\text {eff }}$ decreases and tends towards zero at very low temperatures, in accordance with an $S=0$ ground state. Coupling constants $J$ were determined to $\simeq-167 \mathrm{~cm}^{-1}$ (Table 7.2) using a fitting procedure to the appropriate Heisenberg spin Hamiltonian for isotropic exchange coupling (detailed information is provided in Chapter 9.6). Magnetic data have been determined for only few synthetic $[2 \mathrm{Fe}-2 \mathrm{~S}]$ clusters in their diferric form, ${ }^{[129]}$ and the magnitude of the antiferromagnetic coupling in 29a-29c is at the upper end of what has previously been reported (e.g. $J \simeq-150 \mathrm{~cm}^{-1}$ for $\mathbf{1}^{[134]}$, see Chapter 9.6). 
Table 7.2: Analytical data for complexes 29a-29c.

\begin{tabular}{|c|c|c|c|c|}
\hline compound & $\delta\left(\Delta E_{\mathrm{Q}}\right)[\mathrm{mm} / \mathrm{s}]^{\mathrm{a}}$ & $\lambda_{\max }[\mathrm{nm}]\left(\varepsilon\left[\mathrm{M}^{-1} \mathrm{~cm}^{-1}\right]\right)^{\mathrm{b}}$ & $J\left[\mathrm{~cm}^{-1}\right]^{\mathrm{c}}$ & $E_{1 / 2}[\mathrm{~V}]^{\mathrm{d}}$ \\
\hline $29 a$ & $0.22(0.90)$ & $\begin{array}{c}335(s h, 5000), 393(9500) \\
522(4600)\end{array}$ & -165 & -1.19 \\
\hline $29 \mathrm{~b}$ & $0.24(0.97)$ & $\begin{array}{c}330(s h, 6100), 388(10500) \\
523(5800)\end{array}$ & -167 & -1.25 \\
\hline $29 c$ & $0.23(0.89)$ & $\begin{array}{c}335(s h, 6100), 397(10400) \\
526(5500)\end{array}$ & -168 & -1.31 \\
\hline $4^{[141,142]}$ & $0.26(0.49)^{\mathrm{e}}$ & $\begin{array}{c}370(8330), 490(s h, 3290) \\
550(s h, 2480)\end{array}$ & not reported & $-1.30^{\mathrm{f}}$ \\
\hline
\end{tabular}

(a) ${ }^{57} \mathrm{Fe}$ Mössbauer parameters at $80 \mathrm{~K}$. (b) Recorded in MeCN solution at room temperature. (c) Values obtained from fits to SQUID data, see Chapter 9.6. (d) Potentials in DMF/0.1 M $\mathrm{NBu}_{4} \mathrm{PF}_{6}$ at a scan rate of $100 \mathrm{mV} / \mathrm{s}$ vs. the $\mathrm{Cp}_{2}^{*} \mathrm{Fe} / \mathrm{Cp}_{2}^{*} \mathrm{Fe}^{+}$couple. (e) ${ }^{57} \mathrm{Fe}$ Mössbauer parameters at $77 \mathrm{~K}$. (f) Cathodic peak potential of the irreversible process in $\mathrm{DMF} / 0.1 \mathrm{M} \mathrm{NBu}_{4} \mathrm{ClO}_{4}$ at a scan rate of $200 \mathrm{mV} / \mathrm{s}$ vs. SCE is $-1.28 \mathrm{~V}$, corresponding to $-1.30 \mathrm{~V}$ vs. the $\mathrm{Cp}_{2}^{*} \mathrm{Fe} / \mathrm{Cp}_{2}^{*} \mathrm{Fe}^{+}$couple. ${ }^{[145,146]}$

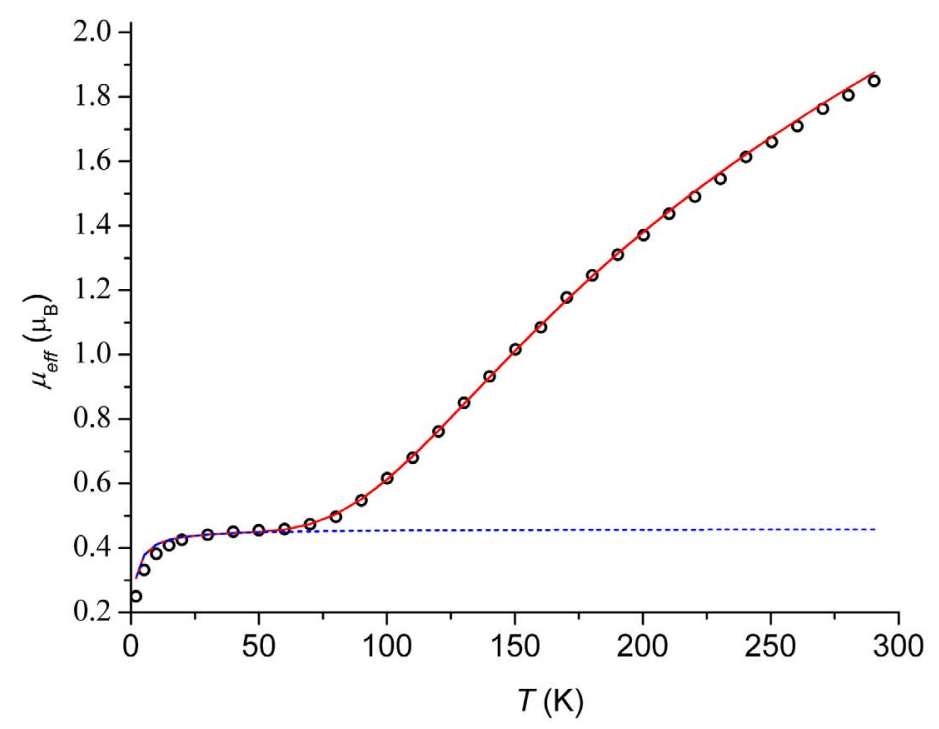

Figure 7.3: Plot of $\mu_{\text {eff }}\left(\mu_{B}\right)$ versus temperature for 29c at a magnetic field $B=1$ T. The solid line represents the best fit to the experimental values (circles).

\subsubsection{Spectroscopic and Electrochemical Characterization in So- lution}

The stability of compounds $\mathbf{2 9 a - 2 9 c}$ was also confirmed in solution. Proton NMR spectroscopy established that $\left[\mathrm{Fe}_{2} \mathrm{~S}_{2}\left\{\mathrm{~N}_{2}\right\}_{2}\right]^{2-}$ clusters are intact in DMSO- $\mathrm{d}_{6}$. The resulting spectra are reasonably well-resolved due to the strong antiferromagnetic coupling between the two ferric ions. Protons of the pyrrole moieties give rise to broadened resonances in a 
range between 9 and $11 \mathrm{ppm}$. Signals of $\alpha$-pyrrolic protons are not detected for any of the three compounds. The spectrum of $\mathbf{2 9 b}$ is shown in Figure 7.4 as an example.

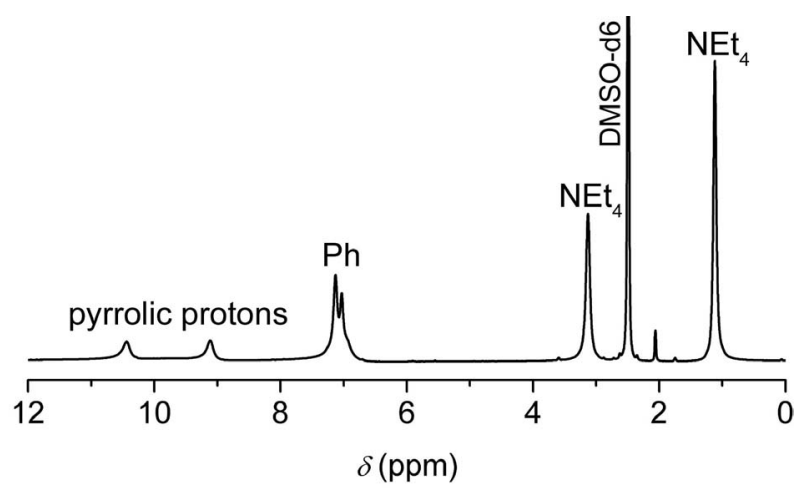

Figure 7.4: ${ }^{1} \mathrm{H} N \mathrm{NM}\left(500 \mathrm{MHz}, 25^{\circ} \mathrm{C}\right)$ of $\mathbf{2 9 b}$ recorded in DMSO-d .

All three complexes exhibit similar electronic spectra with prominent bands at $\simeq 525 \mathrm{~nm}$ $\left(\varepsilon \simeq 5000 \mathrm{M}^{-1} \mathrm{~cm}^{-1}\right)$ and $\simeq 390 \mathrm{~nm}\left(\varepsilon \simeq 10000 \mathrm{M}^{-1} \mathrm{~cm}^{-1}\right)$. Based on previously reported data for complexes $4^{[141]}$ and $\left(\mathrm{NEt}_{4}\right)_{2}\left[\mathrm{Fe}_{2} \mathrm{~S}_{2}\left(\left\{\mathrm{~N}_{2}\right\} \text {-bbzimp }\right)_{2}\right] \mathbf{3 0}^{[222]}\left(\left\{\mathrm{N}_{2}\right\}\right.$-bbzimp = dianion of 2,2-bis-(benzimidazolyl)-propane, structural drawing depicted on page 193), the former absorption is assigned, at least in part, to charge transfer transitions within the [2Fe-2S] core, while the latter is attributed to terminal ligand-to-metal charge transfer transitions. The oxidized Rieske iron sulfur protein from Thermus thermophilus shows electronic absorption bands at $560 \mathrm{~nm}\left(\mathrm{sh}, \varepsilon \simeq 3000 \mathrm{M}^{-1} \mathrm{~cm}^{-1}\right), 458 \mathrm{~nm}\left(\varepsilon \simeq 6000 \pm 200 \mathrm{M}^{-1} \mathrm{~cm}^{-1}\right)$ and $325 \mathrm{~nm}\left(\varepsilon \simeq 11500 \mathrm{M}^{-1} \mathrm{~cm}^{-1}\right) .{ }^{[4]}$ Redox properties of $29 \mathrm{a}-\mathbf{2 9 c}$ have been examined by cyclic voltammetry in $\mathrm{DMF} / 0.1 \mathrm{M} \mathrm{NBu}_{4} \mathrm{PF}_{6}$ at room temperature. All clusters exhibit a quasi-reversible reduction process in the range -1.19 to $-1.31 \mathrm{~V}$ vs. the $\mathrm{Cp}_{2}^{*} \mathrm{Fe} / \mathrm{Cp}_{2}^{*} \mathrm{Fe}^{+}$ couple (Figure 7.5, Table 7.2) assigned to the formation of the mixed-valent $\mathrm{Fe}^{\mathrm{II}} \mathrm{Fe}^{\mathrm{III}}$ species, followed by an irreversible second process at much lower potentials. These values (corresponding to around -1.38 to $-1.50 \mathrm{~V}$ vs. $\mathrm{SCE}^{[145,146]}$ ) are similar to the value reported for the parent pyrrolato complex $4^{[141]}$ (irreversible reduction with $E_{\mathrm{p}}^{\mathrm{c}}=-1.28 \mathrm{~V}$ vs. $\mathrm{SCE}$ in $\mathrm{DMF} / 0.1 \mathrm{M} \mathrm{NBu}_{4} \mathrm{ClO}_{4}$ at $200 \mathrm{mV} / \mathrm{s}$ ) and are even more negative than typical reductions for $[2 \mathrm{Fe}-2 \mathrm{~S}]$ clusters with terminal thiophenolate ligands. ${ }^{[129]}$ It is interesting to note that complex 29c that incorporates backbone cyclohexyl substituents is closest to approaching reversibility of the electrochemical process.

Electrochemical bulk reduction using constant potential coulometry at $-25^{\circ} \mathrm{C}$ however failed to generate the corresponding one-electron reduced species $\mathbf{2 9} \mathbf{a}^{\text {red }}-\mathbf{2 9} \mathbf{c}^{\text {red }}$, indicating that those $[2 \mathrm{Fe}-2 \mathrm{~S}]^{+}$compounds are stable on the timescale of the cyclic voltammetry experiment $(\simeq 20 \mathrm{~s})$ but not on the timescale of the coulometry experiment ( $\simeq 15$ minutes). Thus, $\mathbf{2 9} \mathrm{a}^{\text {red }}-\mathbf{2 9} \mathbf{c}^{\text {red }}$ are certainly not isolable as solids that could then be recrystallized for $\mathrm{X}$-ray diffraction. In the search for such isolable $[2 \mathrm{Fe}-2 \mathrm{~S}]^{+}$systems, the exploration of $\{\mathrm{N}\}$ ligated $[2 \mathrm{Fe}-2 \mathrm{~S}]$ clusters was extended to 1,2-bisbenzimidazole-benzene ligands (forming 7 -membered instead of 6-membered chelate rings) as described in the following section. 


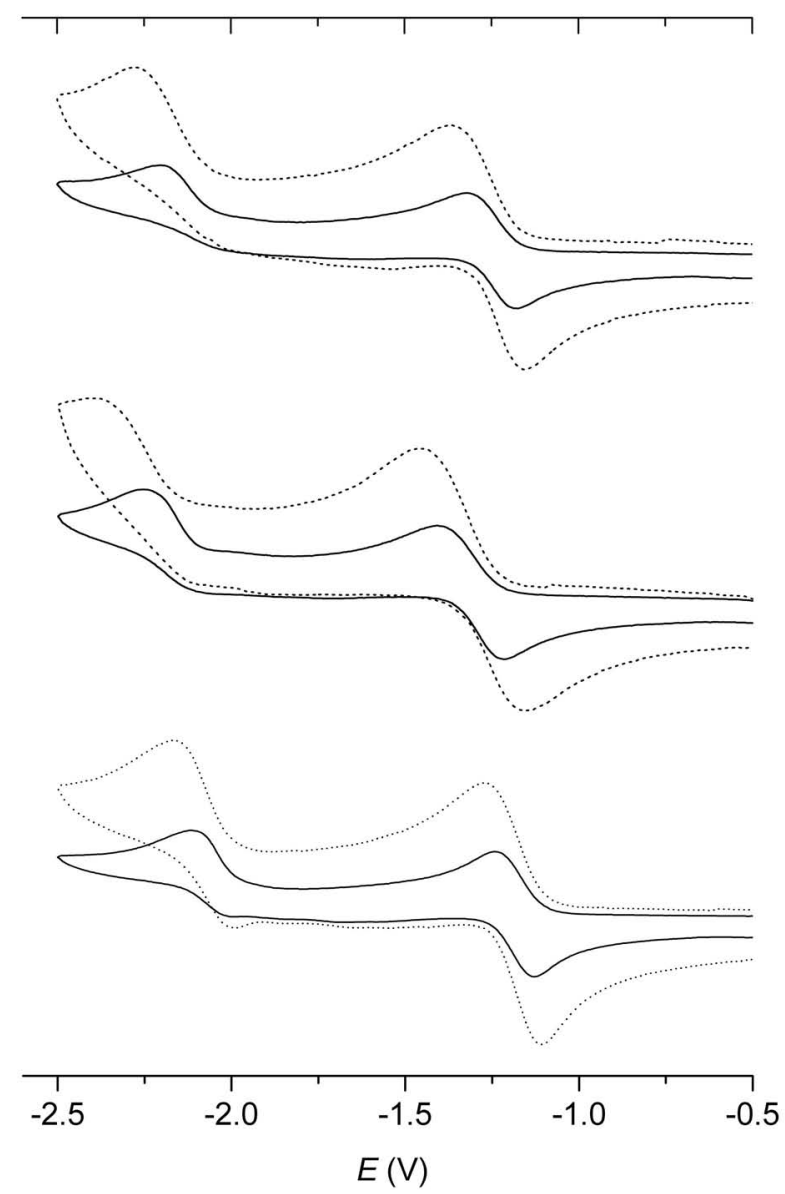

Figure 7.5: Cyclic voltammograms of 29a (bottom), 29b (middle), and 29c (top) in $D M F$ at scan rates of $100 \mathrm{mV} / \mathrm{s}$ (solid line) and $500 \mathrm{mV} / \mathrm{s}$ (dashed line). Potentials are given in volts vs. the $C p_{2}^{*} \mathrm{Fe} / \mathrm{Cp}_{2}^{*} \mathrm{Fe} e^{+}$couple.

\section{3 [2Fe-2S] Clusters Coordinated by 1,2-Benzene- Bis-Benzimidazolate}

\subsubsection{Synthesis and Structural Characterization}

Both 1,2-benzene-bis-benzimidazolate coordinated [2Fe-2S] cluster compounds $\mathbf{3 1 a}$ and 31b (Scheme 7.4) were synthesized using a standard salt metathesis reaction, starting from $\left(\mathrm{NEt}_{4}\right)_{2}\left[\mathrm{Fe}_{2} \mathrm{~S}_{2} \mathrm{Cl}_{4}\right] \mathbf{2}^{[138]}$. The corresponding ligands were deprotonated with potassium hydride in THF and the cluster precursor $\mathbf{2}$ added subsequently. The required free ligands XXIa and XXIb (Scheme 7.2) were obtained by condensation of phthalic acid and tetrafluor-phthalic acid with excess of 1,2-phenylenediamine according to literature methods. ${ }^{[226]}$ After purification, the target cluster 31a was crystallized for X-ray diffraction by slow diffusion of $\mathrm{Et}_{2} \mathrm{O}$ into a $\mathrm{MeCN}$ solution of the compound (Figure 7.6). Unfortunately, single crystals of $\mathbf{3 1 b}$ could not be obtained in sufficient quality. However, several 
crystallization attempts from $\mathrm{DMF} / \mathrm{Et}_{2} \mathrm{O}, \mathrm{MeCN} / \mathrm{Et}_{2} \mathrm{O}$ and saturated MeCN solutions afforded some crystalline material that in one attempt gave a picture of the molecular structure, although refinement was impossible (ORTEP plot depicted in Figure 7.7). Even if ligand orientation and therefore cluster symmetry apparently is different in $\mathbf{3 1 a}$ and $\mathbf{3 1} \mathbf{b}$, only the appropriately refined molecular structure of $\mathbf{3 1 a}$ can be discussed here. Compound 31a crystallizes in black blocks in the triclinic space group $P \overline{1}$ with a single molecule per unit cell. Due to an inversion centre in the middle of the central $\mathrm{Fe}_{2} \mathrm{~S}_{2}$-diamond, both irons are in geometrically equal coordination environments, with all intra-core parameters found in the typical range for synthetic [2Fe-2S] clusters. The overall symmetry is reduced from $C_{2 \mathrm{~h}}$, with the horizontal mirror plane through $\mathrm{Fe} 1, \mathrm{Fe} 1^{\prime}, \mathrm{S} 2$ and $\mathrm{S} 2^{\prime}$ and a perpendicular $C_{2}$ axis through the centroid of the $\mathrm{Fe}_{2} \mathrm{~S}_{2}$-core.

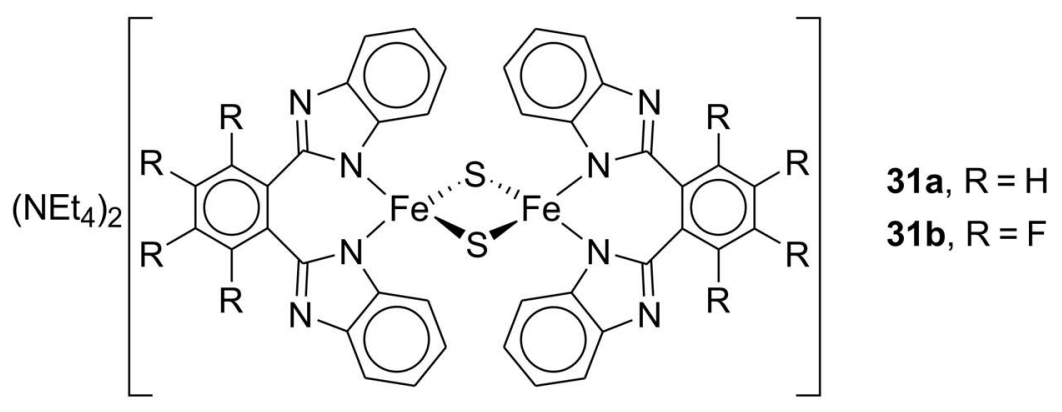

Scheme 7.4: Synthetic [2Fe-2S] clusters coordinated by 1,2-benzene-bis-benzimidazolate derivatives.

\subsubsection{Spectroscopy in Solution}

Solutions of both new clusters were characterized by positive and negative ESI mass spectrometry, ${ }^{1} \mathrm{H}$ NMR (and ${ }^{19} \mathrm{~F}$ NMR for $31 \mathbf{b}$ ) spectroscopy and UV-Vis spectroscopy. Especially ESI mass analysis is a powerful tool in the examination of initial crude reaction mixtures, since prominent peaks for $\left[\mathrm{M}+\mathrm{NEt}_{4}\right]^{+}$and $\left[\mathrm{M}-\mathrm{NEt}_{4}\right]^{-}$can be detected, indicating a successful cluster formation. Identity of the compounds is substantiated by an excellent agreement between the experimental and the simulated isotopic distribution patterns, and was further proven by high resolution $\mathrm{ESI}(+)$ mass spectrometry. Proton NMR spectra of the compounds could be recorded, due to the strong antiferromagnetic coupling of the iron atoms. However, magnetic moments at room temperature differ from zero and cause modest signal broadening of all resonances (even for the $\mathrm{NEt}_{4}^{+}$counter ions). A detailed signal assignment was not performed, integrals from aromatic resonances and signals from the counter ions, however, were found in the expected ratio (overall four benzimidazole protons - pointing towards the $[2 \mathrm{Fe}-2 \mathrm{~S}]$ core - are obscured in 31a, as previously observed for the related dipyrromethane-ligated clusters 29a-29c). Electronic absorption spectra for both clusters show a similar curvature in DMF solution, with a dominant band at $\simeq 290 \mathrm{~nm}$. Visible - less intensive - transitions in the range of $400 \mathrm{~nm}-550 \mathrm{~nm}$ are present in each case as well. The latter transitions are particularly valuable in reaction monitoring, as demonstrated by tracking an electrochemical reduction of $\mathbf{3 1 a}$ (see below). 


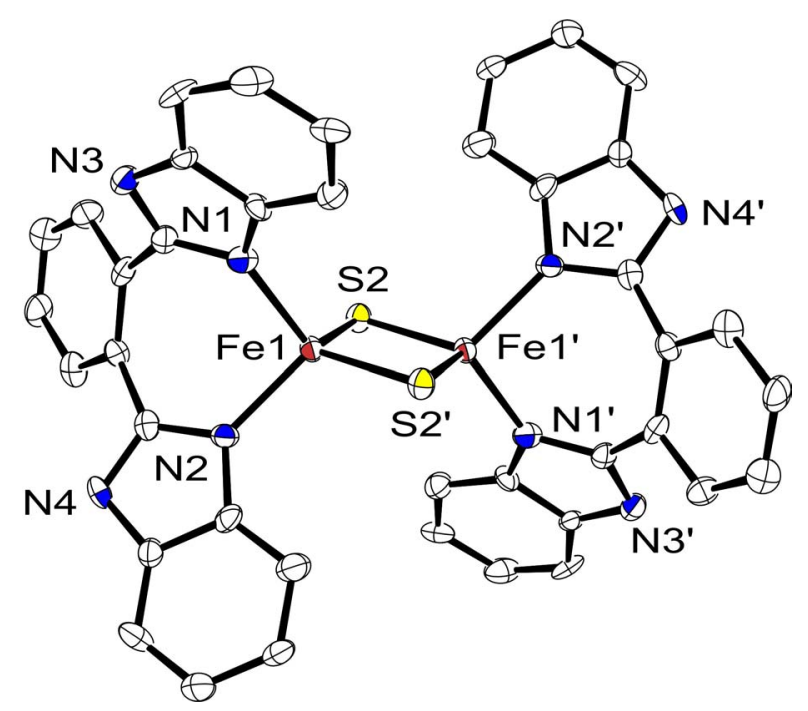

Figure 7.6: ORTEP plot (50\% probability thermal ellipsoids) of the molecular structure of 31a. The NEt $t_{4}^{+}$counter ions and all hydrogen atoms have been omitted for clarity. Selected interatomic distances [A] and angles [०]: Fe1 $\cdots F e 1$ 2.683(2), Fe1-N1 1.992(6), Fe1-N2 2.004(6), Fe1-S2 2.190(2), Fe1-S2' 2.199(2), N1-Fe1-N2 96.9(2), N1-Fe1-S2 113.8(2), N1Fe1 S2' 113.68(18), N2-Fe1-S2 113.22(19), N2-Fe1-S2' 114.98(18), S2-Fe1-S2' 104.64(8), Fe1-S2-Fe1' 75.36(8).

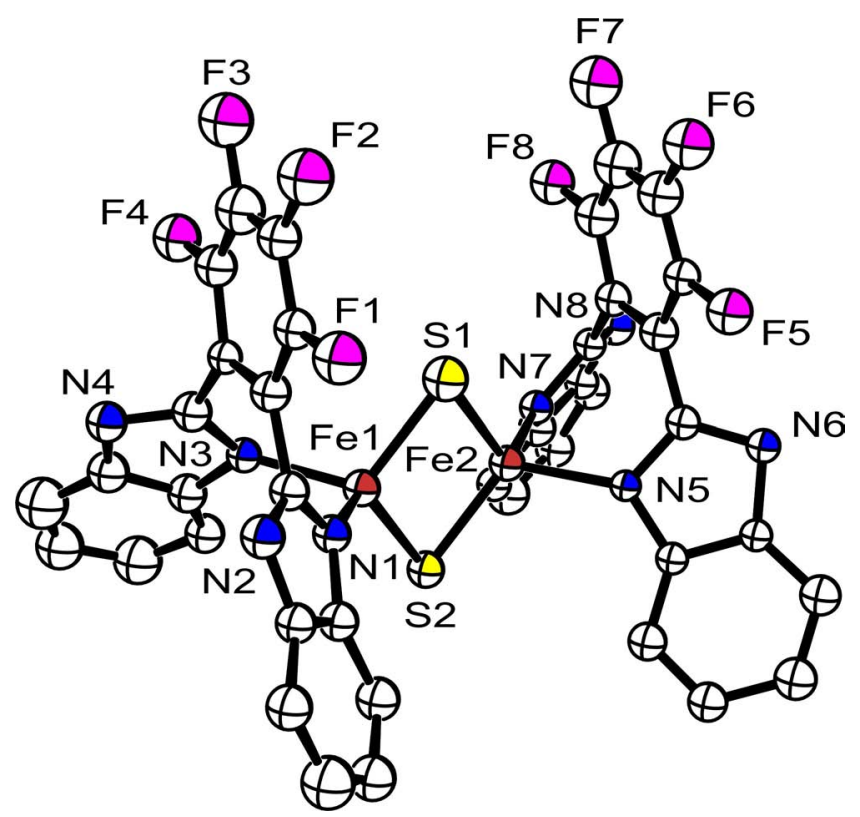

Figure 7.7: ORTEP plot of the molecular structure of 31b. The NEt $t_{4}^{+}$counter ions and all hydrogen atoms have been omitted for clarity. Note: Quality of single crystals and collected crystallographic data were insufficient for refinement. Therefore only a picture is shown as an "educated guess" for the molecular structure of $\mathbf{3 1 b}$. Further interpretation or analysis (e.g. distances, angles etc.) is inappropriate. 


\subsubsection{Spectroscopy in the Solid State}

Both new complexes 31a and 31b were further examined in the solid state by Mössbauer spectroscopy at $80 \mathrm{~K}$. Spectral fits to the data are obtained by using Lorentzian line doublets with isomer shifts $\delta$ and quadrupole splittings $\Delta E_{\mathrm{Q}}$ summarized in Table 7.3. Surprisingly, neither isomer shifts nor quadrupole splittings are significantly different, although the eight fluorine atoms present in $\mathbf{3 1 b}$ were expected to considerably decrease the d-electron density at the iron cores in $\mathbf{3 1 b}$ and therefore lower its isomer shift compared to 31a. As previously shown, ${ }^{[170]}$ the empirical correlation $\delta=1.43-0.40 s^{[167,227]}$ between oxidation state $s$ and isomer shift $\delta$ is not only valid for tetrahedral $\left\{\mathrm{S}_{4}\right\}$-coordinate $\mathrm{Fe}$ sites, but also for $\left\{\mathrm{FeN}_{2} \mathrm{~S}_{2}\right\}$-coordinate complexes. Applying the experimental $\delta$ values to the latter equation reveals the expected oxidation states $s \simeq 3$ for both all-ferric clusters with $s(\mathbf{3 1 a})=2.90$ and $s(\mathbf{3 1} \mathbf{b})=2.93$. Comparing the ligand orientation in both compounds (comparison of the overall structure including ligand-to-core alignment is reasonable, despite the insufficient X-ray refinement of the molecular structure of $\mathbf{3 1 b}$ ) a noteworthy dissimilarity in quadrupole splittings would intuitively be expected. Interestingly, values for both clusters were found in the range of $\Delta E_{\mathrm{Q}}=0.83 \pm 0.01 \mathrm{~mm} / \mathrm{s}$, thus positioned in between those values obtained for the monodentate homoleptic $\{\mathrm{N}\}$-ligated cluster $4^{[141,142]}\left(\Delta E_{\mathrm{Q}}=0.49-0.61 \mathrm{~mm} / \mathrm{s}\right)$ and the $\{\mathrm{N}\}$-homoleptic diyprromethane-chelated clusters 29a-29c ${ }^{[170]}\left(\Delta E_{\mathrm{Q}}=0.89-0.97 \mathrm{~mm} / \mathrm{s}\right)$. A closer inspection of all the structurally characterized all- $\{\mathrm{N}\}$-ligated clusters points towards the existence of a correlation between ligand bite angles $\mathrm{N}-\mathrm{Fe}-\mathrm{N}(\varphi)$ and corresponding quadrupole splittings $\left(\Delta E_{\mathrm{Q}}\right)$. Even though a plot of the available data couples $\Delta E_{Q} / \varphi$ spontaneously suggests a linear relationship (see Figure 7.8), additional data and evaluating theoretical studies are definitely required in order to confirm the empirically equation obtained by linear regression analysis: $\Delta E_{\mathrm{Q}}=3.31\left( \pm 8.4 \cdot 10^{-2}\right)-0.0255\left( \pm 8.6 \cdot 10^{-4}\right) \cdot \varphi$.

Reliable magnetic susceptibility data were only obtained for 31a. Paramagnetic impurities present in samples of $\mathbf{3 1 b}$ significantly perturbed the fitting procedure of the SQUID data and consequently gave incorrect magnetic coupling constants $J$ for this cluster. The magnetic moment $\mu_{\text {eff }}$ for 31a was found to rapidly decrease upon lowering the temperature from $300 \mathrm{~K}\left(1.9 \mu_{\mathrm{B}}\right)$ to $5 \mathrm{~K}\left(\simeq 0.8 \mu_{\mathrm{B}}\right)$. This behavior is in accordance with an antiferromagnetic coupling between the two ferric ions to give an $S=0$ ground state, as commonly observed for [2Fe-2S] clusters. A coupling constant $J=-199 \mathrm{~cm}^{-1}$ was determined by using a fitting procedure to the appropriate Heisenberg spin Hamiltonian for isotropic exchange coupling and Zeeman interaction:

$$
\mathcal{H}=-2 J \overrightarrow{S_{1}} \cdot \overrightarrow{S_{2}}+g \mu_{B}\left(\overrightarrow{S_{1}}+\overrightarrow{S_{2}}\right) \cdot \vec{B}
$$

This value indicates an amplified antiferromagnetic coupling compared to the related all$\{\mathrm{N}\}$-ligated clusters 29a-29c (see Table 7.2). The most negative coupling constant for synthetic $[2 \mathrm{Fe}-2 \mathrm{~S}]$ systems, however, was detected for an all- $\{\mathrm{S}\}$-ligated system, namely for compound $\mathbf{2 6}^{\mathbf{C}}$ (see Chapter 5, compare to Table 9.5).[134,143,170] 


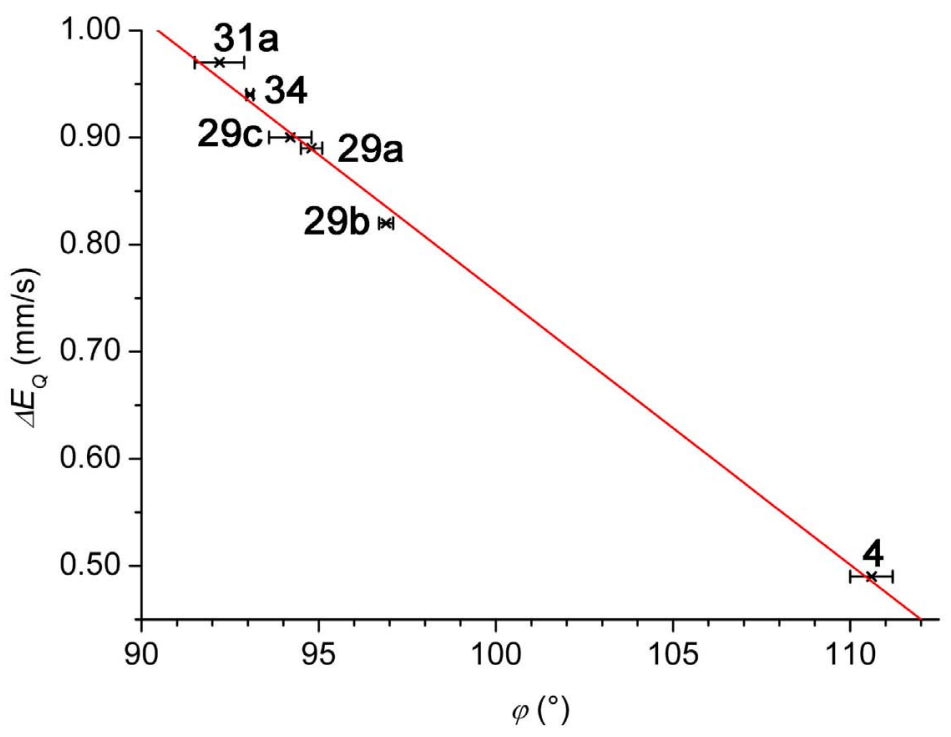

Figure 7.8: Possible correlation between ligand bite angles $N-F e-N(\varphi)$ and quadrupole splittings $\left(\Delta E_{Q}\right)$ for homoleptic $\{N\}$-coordinate [2Fe-2S] cluster compounds. Error bars in $x$-direction are adopted from X-ray diffraction error-values. Compound 34 is described in Chapter 8.

Table 7.3: Spectroscopic and electrochemical data for complexes $\mathbf{3 1 a}$ and $\mathbf{3 1 b .}$

\begin{tabular}{ccccc}
\hline compound & $\begin{array}{c}\delta\left(\Delta E_{\mathrm{Q}}\right) \\
{[\mathrm{mm} / \mathrm{s}]^{\mathrm{a}}}\end{array}$ & $\lambda_{\max }[\mathrm{nm}]\left(\varepsilon\left[\mathrm{M}^{-1} \mathrm{~cm}^{-1}\right]\right)^{\mathrm{b}}$ & $J\left[\mathrm{~cm}^{-1}\right]^{\mathrm{c}}$ & $E_{1 / 2}[\mathrm{~V}]^{\mathrm{d}}$ \\
\hline \multirow{3}{*}{ 31a } & $0.27(0.82)$ & $\begin{array}{c}290(20500), \simeq 406(s h, \simeq 3200), \\
482(2600), \simeq 550(s h, \simeq 1200)\end{array}$ & -199 & -0.84 \\
& & $289(13000), 408(2000)$, & & \\
31b $^{\mathrm{e}}$ & $0.26(0.84)$ & $468(2000), \simeq 550(s h, \simeq 1200)$ & $\mathrm{n} / \mathrm{a}$ & -0.73 \\
\hline
\end{tabular}

(a) ${ }^{57} \mathrm{Fe}$ Mössbauer parameters at $80 \mathrm{~K}$, relative to iron metal at room temperature. (b) Recorded in MeCN solution at room temperature. (c) Values obtained from fits to SQUID data, see Chapter 9.6. (d) Half-wave potentials of the (quasi)-reversible process in $\mathrm{DMF} / 0.1 \mathrm{M} \mathrm{NBu} \mathrm{PF}_{6}$ at a scan rate of $100 \mathrm{mV} / \mathrm{s}$ vs. the $\mathrm{Cp}_{2}^{*} \mathrm{Fe} / \mathrm{Cp}_{2}^{*} \mathrm{Fe}^{+}$couple (recorded at room temperature vs. the $\mathrm{Cp}_{2} \mathrm{Fe} / \mathrm{Cp}_{2} \mathrm{Fe}^{+}$couple and recalibrated to the $\mathrm{Cp}_{2}^{*} \mathrm{Fe} / \mathrm{Cp}_{2}^{*} \mathrm{Fe}^{+}$couple). ${ }^{[145,146]}$ (e) Complex purity determined by Mössbauer spectroscopy is $92 \%$ only. Isomer shift of the $8 \%$ impurity (not detectable in the proton and fluorine NMR) suggests this specie to correspond to a ferric salt (chloride, sulfide or oxide). Extensive purification affords were unsuccessful, due to the unpleasant crystallization properties and the low yielding synthesis of the cluster. Magnetic susceptibility measurements were not performed for this reason.

\subsubsection{Electrochemistry and Constant Potential Coulometry}

Redox properties of both clusters $31 \mathbf{a}$ and $\mathbf{3 1 b}$ were studied by cyclic voltammetry in DMF solution at room temperature. A quasi-reversible one-electron reduction process to the corresponding mixed-valent species $\mathbf{3 1} \mathrm{a}^{\text {red }}$ and $\mathbf{3 1} \mathbf{b}^{\text {red }}$ was found in addition to an 
irreversible reduction to the all-ferrous state of each cluster at much lower potential. As anticipated, the redox potential for the $\mathbf{3 1} \mathbf{b} / \mathbf{3} \mathbf{b} \mathbf{b}^{\text {red }}$ couple $\left(E_{1 / 2}=-0.84 \mathrm{~V}\right)$ is somewhat less negative than the potential for the $\mathbf{3 1 a} / \mathbf{3 1} \mathbf{a}^{\text {red }}$ couple $\left(E_{1 / 2}=-0.73 \mathrm{~V}\right)$, due to the electron withdrawing effect of the overall eight fluorine substituents in $\mathbf{3 1 b}$ (Table 7.3).

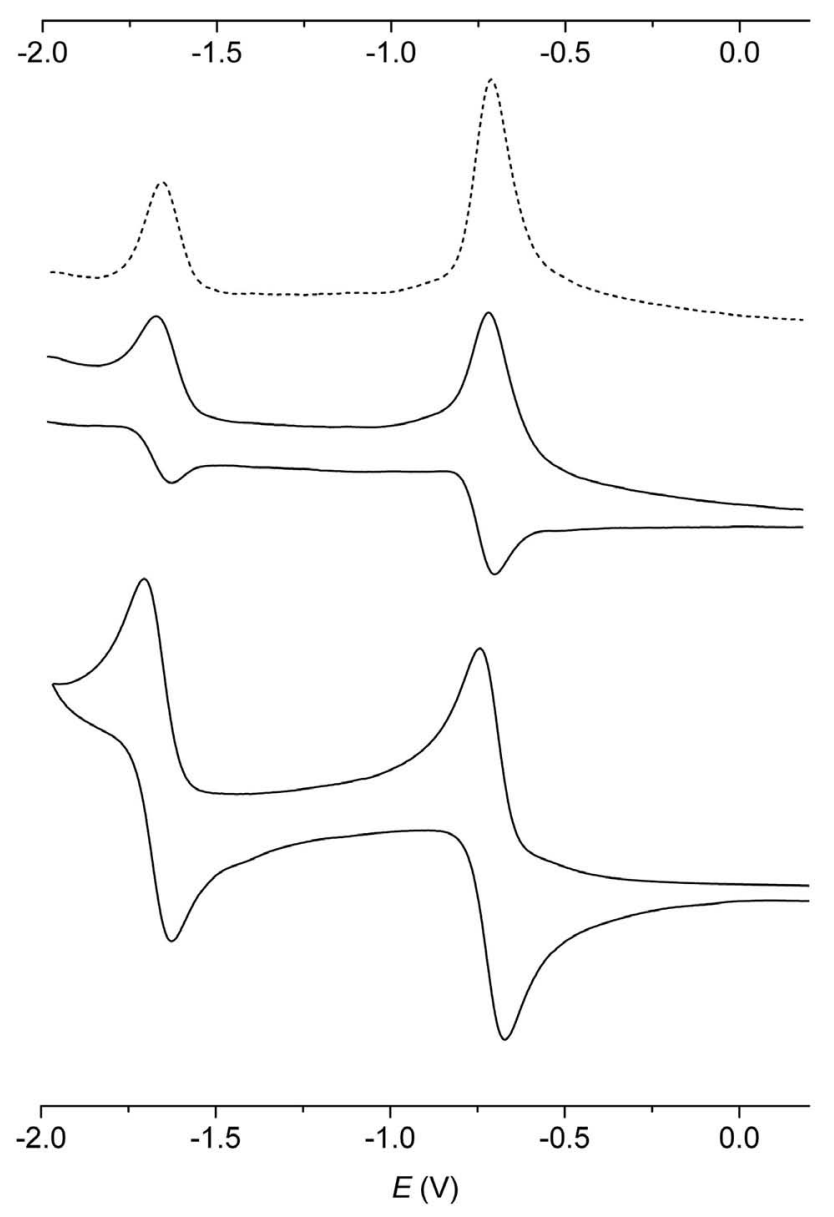

Figure 7.9: Cyclic voltammogram (bottom) and square wave plot (middle) of 31a in $\mathrm{MeCN} / 0.2 \mathrm{M} \mathrm{NBu} u_{4} \mathrm{PF}_{6}$ at scan rate of $100 \mathrm{mV} / \mathrm{s}$ at $-25^{\circ} \mathrm{C}$ vs. the $\mathrm{Cp}_{2}^{*} \mathrm{Fe} / \mathrm{Cp}_{2}^{*} \mathrm{Fe}^{+}$couple (recorded vs. the $\mathrm{Cp}_{2} \mathrm{Fe} / \mathrm{Cp}_{2} \mathrm{Fe}^{+}$couple and recalibrated to the $\mathrm{Cp} \mathrm{p}_{2}^{*} \mathrm{Fe} / \mathrm{Cp}_{2}^{*} \mathrm{Fe} e^{+}$couple). The dashed line (top) arises from a summation of the square wave data for the forward and the reverse scan.

For practical reasons however, compound 31a was chosen for further reduction studies since its synthesis could be carried out in $30 \%$ yield, while $\mathbf{3 1 b}$ was obtained in $7 \%$ yield and $92 \%$ purity only. Thus, only 31a was examined with respect to an electrochemical reduction by constant potential coulometry in MeCN solution at $-25^{\circ} \mathrm{C}$. The corresponding cyclic and square wave voltammograms recorded for $\mathbf{3 1 a}$ at the latter conditions are depicted in Figure 7.9. Compared to room temperature measurements, both reduction waves are closer to reversibility at $-25^{\circ} \mathrm{C}$. However, only the first electron transfer process to the mixed-valent system was suitable for coulometric bulk reduction of 31a. In a representative experiment, the electrochemical cell was charged with $8 \mathrm{ml}$ of a $1.13 \cdot 10^{-4} \mathrm{M}$ solution of $\mathbf{3 1 a}(0.9 \mu \mathrm{mol})$ in 
$\mathrm{MeCN} / 0.2 \mathrm{M} \mathrm{NBu}_{4} \mathrm{PF}_{6}$ under an argon atmosphere and voltage impressed on the sample at a potential of $-0.89 \mathrm{~V}$ vs. the $\mathrm{Cp}_{2}^{*} \mathrm{Fe} / \mathrm{Cp}_{2}^{*} \mathrm{Fe}^{+}$couple (corresponding to $-1.4 \mathrm{~V}$ vs. the $\mathrm{Cp}_{2} \mathrm{Fe} / \mathrm{Cp}_{2} \mathrm{Fe}^{+}$couple) $)^{[145,146]}$.

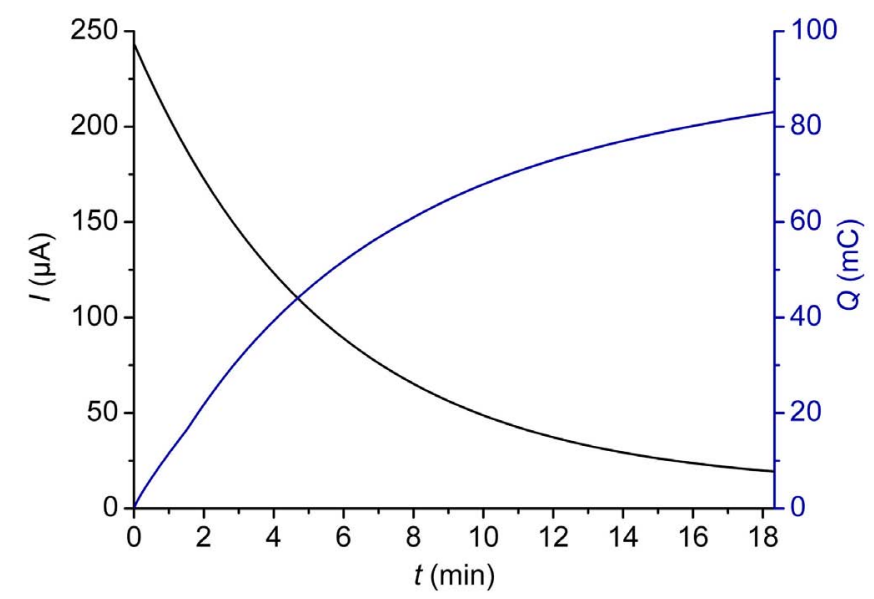

Figure 7.10: Plot of current I (black line/ordinate) and charge Q (blue line/ordinate) consumed during the bulk reduction of 31 a versus time $t$.

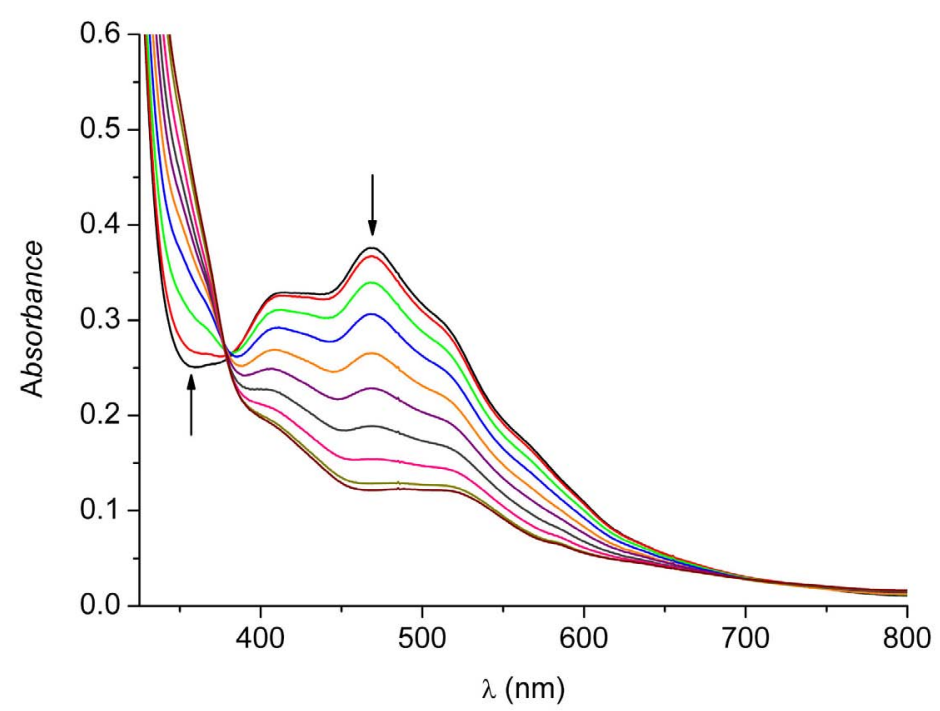

Figure 7.11: $U V$-Vis spectra during constant potential coulometry of $31 \boldsymbol{a}$ at $-25^{\circ} \mathrm{C}$, $-1.4 \mathrm{~V}$, black line: $t_{0}=0$ minutes, colored lines: $t_{0}+n \cdot \Delta t, \Delta t=2$ minutes, $n=1-9$, $c=1.13 \cdot 10^{-4} \mathrm{M}$ in $\mathrm{MeCN} / 0.2 \mathrm{M} \mathrm{NBu_{4 }} \mathrm{PF}_{6}$.

The course of the reduction was monitored by UV-Vis spectroscopy, the decreasing current $I$ consumed by the sample and the charge $Q$ taken up during the time (integral of current $I$ over time $t$, see Figure 7.10). A decreasing intensity of all bands between $\simeq 400 \mathrm{~nm}$ and $\simeq 650 \mathrm{~nm}$ and an increasing intensity of the shoulder at $\simeq 360 \mathrm{~nm}$ was observed in the electronic absorption spectra (recorded every 2 minutes), with two isosbestic points (at $\simeq 380 \mathrm{~nm}$ and $730 \mathrm{~nm}$ ) present in the superimposed plot of the individual spectra (Figure 
7.11). After 18 minutes, essentially no further changes are noticeable. At this point, an electrical charge close to the expected one for one-electron reduction was consumed by the sample $\left(Q_{\text {exp. }}=83 \mathrm{mC}, Q_{\text {calcd. }}=87 \mathrm{mC}\right)$. Therefore, the coulometric reduction was terminated at this stage, keeping the solution of $\mathbf{3 1} \mathbf{a}^{\text {red }}$ cooled and under an argon atmosphere.

\subsubsection{Spectroscopy on the Mixed-Valent $[2 \mathrm{Fe}-2 \mathrm{~S}]^{+}$Cluster}

Directly after completed reduction a sample of $\mathbf{3 1} \mathbf{a}^{\text {red }}$ was transferred to an EPR tube under an argon atmosphere and rapidly frozen in liquid dinitrogen affording a vitreous solvent matrix. The obtained rhombic EPR spectrum (spectrometer settings: $8.1 \mathrm{~K}, 1.0 \mathrm{~mW}$, $23 \mathrm{~dB}, 9.4467 \mathrm{GHz}, 25.0 \mathrm{G}$ modulation amplitude) was fitted with $g_{1}=2.019, g_{2}=1.917$ and $g_{3}=1.821$ (Figure 7.12 ).

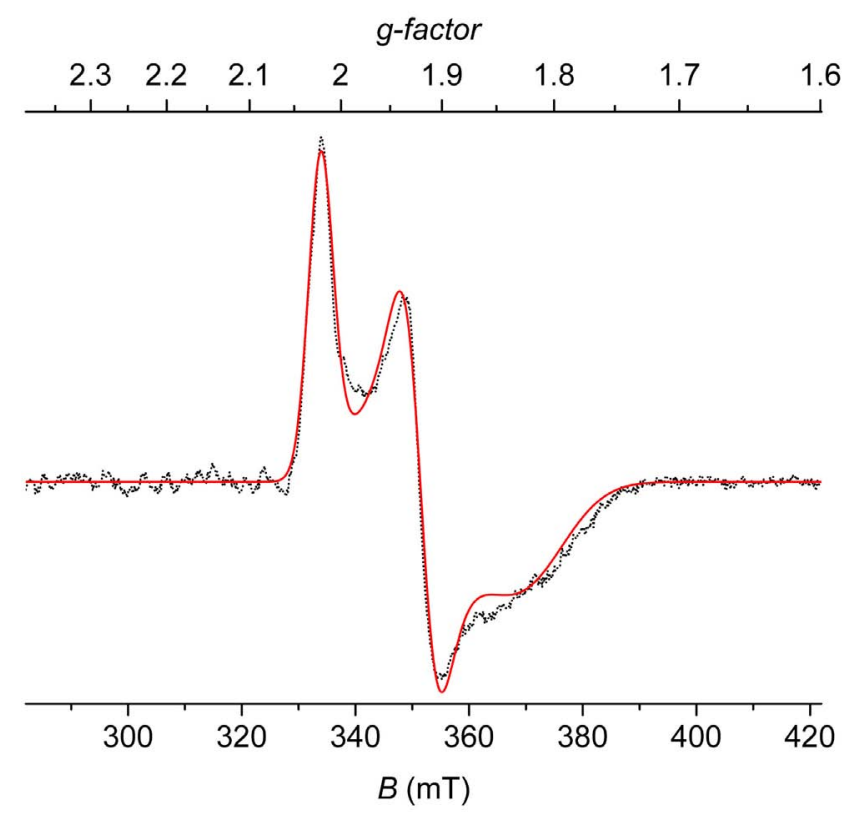

Figure 7.12: EPR spectrum of $31 \boldsymbol{a}^{\text {red }}$ in frozen $M e C N$ solution $\left(c=1.13 \cdot 10^{-4} \mathrm{M}\right)$ recorded at $8.1 \mathrm{~K}(1.0 \mathrm{~mW}, 23 \mathrm{~dB}, 9.4467 \mathrm{GHz}, 25.0 \mathrm{G}$ modulation amplitude). The solid red line is fitted to the experimental values (dotted black line) with $g_{1}=2.019, g_{2}=1.917, g_{3}=1.821$.

As expected, the averaged $g_{\mathrm{av}}$-value $=\frac{1}{3} \cdot\left(g_{1}+g_{2}+g_{3}\right)$ for $\mathbf{3 1} \mathbf{a}^{\text {red }}\left(g_{\mathrm{av}}=1.919\right)$ is significantly lower than the values usually obtained for reduced ferredoxins $\left(g_{\mathrm{av}} \simeq 1.96\right)$, due to the orthorhombic $C_{2 \mathrm{v}}$ distortion at the iron sites. A ligand field model with a detailed analysis of the possible distortions at the EPR-active ferrous site, based on a correlation of the main components of the $g$-tensor $\left(g_{1}, g_{2}, g_{3}\right)$ with the $\left(g_{2}-g_{3}\right)$-function was reported earlier for the $g_{\mathrm{av}} \simeq 1.91$ class of $[2 \mathrm{Fe}-2 \mathrm{~S}]^{+}$species. ${ }^{[222,228]}$ Assuming the absence of vitrification and solvent dependencies, as previously concluded for $[2 \mathrm{Fe}-2 \mathrm{~S}]^{+}$clusters coordinated by chelating capping ligands, ${ }^{[139]}$ the determined $g$-factors for $\mathbf{3 1} \mathbf{a}^{\text {red }}$ are somewhat lower than those reported earlier for the reduced homoleptic $\{\mathrm{N}\}$-ligated cluster $\left[\mathrm{Fe}_{2} \mathrm{~S}_{2}\left(\left\{\mathrm{~N}_{2}\right\}\right.\right.$ bbzimp $\left.)_{2}\right]^{3-} \mathbf{3 0}^{\text {red }} .^{[222]} \mathrm{EPR}$ data for selected biological $[2 \mathrm{Fe}-2 \mathrm{~S}]^{+}$sites are listed in Table 
7.4 together with the data for the reduced synthetic clusters $\mathbf{3 0}{ }^{\text {red }}, \mathbf{1}^{\text {red }}$ and $\mathbf{3 1} \mathbf{a}^{\text {red }}$. Interestingly, $\mathbf{3 1} \mathbf{a}^{\text {red }}$ exhibits a $g_{\text {av }}$-value in the range of those values observed for the natural Rieske sites, whereas $\mathbf{3 0} \mathbf{r e d}^{\text {red }}$ and $\mathbf{1}^{\text {red }}$ display $g_{\mathrm{av}}$-values comparable to reduced Fd sites.

Table 7.4: Previously reported g values for $[2 \mathrm{Fe}-2 \mathrm{~S}]^{+}$clusters compared to $31 \mathrm{a}^{\text {red }}$.

\begin{tabular}{|c|c|c|c|c|}
\hline$[2 \mathrm{Fe}-2 \mathrm{~S}]^{+}$cluster in & $g_{1}$ & $g_{2}$ & $g_{3}$ & $g_{\mathrm{av}}$ \\
\hline parsley $(\mathrm{Fd} \text { site })^{[229]}$ & 2.049 & 1.954 & 1.897 & 1.967 \\
\hline spinach $(\mathrm{Fd} \text { site })^{[230]}$ & 2.045 & 1.947 & 1.881 & 1.958 \\
\hline Spirulina maxima (Fd site) ${ }^{[231]}$ & 2.051 & 1.958 & 1.887 & 1.965 \\
\hline yeast (Rieske site) ${ }^{[4]}$ & 2.025 & 1.890 & 1.810 & 1.908 \\
\hline Thermus thermophilus (Rieske site) ${ }^{[132]}$ & 2.020 & 1.900 & 1.800 & 1.907 \\
\hline chloroplasts (Rieske site) ${ }^{[232]}$ & 2.020 & 1.890 & 1.780 & 1.897 \\
\hline $\mathbf{1}^{\text {red }}\left(\left\{\mathrm{S}_{4}\right\} \text {-coordinate }\right)^{\mathrm{a},[136]}$ & 2.010 & 1.940 & 1.930 & 1.960 \\
\hline $\mathbf{3 0}^{\text {red }}\left(\left\{\mathrm{N}_{4}\right\} \text {-coordinate }\right)^{\mathrm{b},[222]}$ & 2.012 & 1.933 & 1.875 & 1.940 \\
\hline $31 \mathbf{a}^{\text {red }}\left(\left\{\mathrm{N}_{4}\right\} \text {-coordinate }\right)^{\mathrm{c}}$ & 2.019 & 1.917 & 1.821 & 1.919 \\
\hline
\end{tabular}

(a) Recorded in DMF / $0.1 \mathrm{M} \mathrm{NBu}_{4} \mathrm{ClO}_{4}$ at $100 \mathrm{~K}$. (b) Recorded in $\mathrm{MeCN} / \mathrm{HMPA}(7 / 3)$ at $80 \mathrm{~K}$. (c) Recorded in $\mathrm{MeCN} / 0.2 \mathrm{M} \mathrm{NBu}_{4} \mathrm{PF}_{6}$ at $8 \mathrm{~K}$.

\subsection{Conclusions}

As series of new $[2 \mathrm{Fe}-2 \mathrm{~S}]^{2+}$ clusters with bidentate $\left\{\mathrm{N}_{2}\right\}$-ligands was prepared and fully characterized. Their structural, spectral and electrochemical properties are similar to those few related $[2 \mathrm{Fe}-2 \mathrm{~S}]$ complexes with $\{\mathrm{N}\}$-donor ligands reported previously. Considerable distinctions resulting from the distortion of the tetrahedral coordination sphere (imposed by the chelating caps) cause a significant increase of their quadrupole splittings. Complexes 29a-29c, 31a and 31b exhibit very negative reduction potentials, while their stability is enhanced due to the chelating nature of the terminal ligands. Generation and examination of the corresponding reduced specie in solution could be carried out for 31a. EPR characteristics of the $[2 \mathrm{Fe}-2 \mathrm{~S}]^{+}$system are in agreement with previous studies on mixed-valent synthetic and biological $[2 \mathrm{Fe}-2 \mathrm{~S}]$ clusters featuring terminal $\{\mathrm{N}\}$-ligation. 


\section{Chapter 8}

\section{A Synthetic Analogue of Rieske-Type $[2 \mathrm{Fe}-2 \mathrm{~S}]$ Clusters}

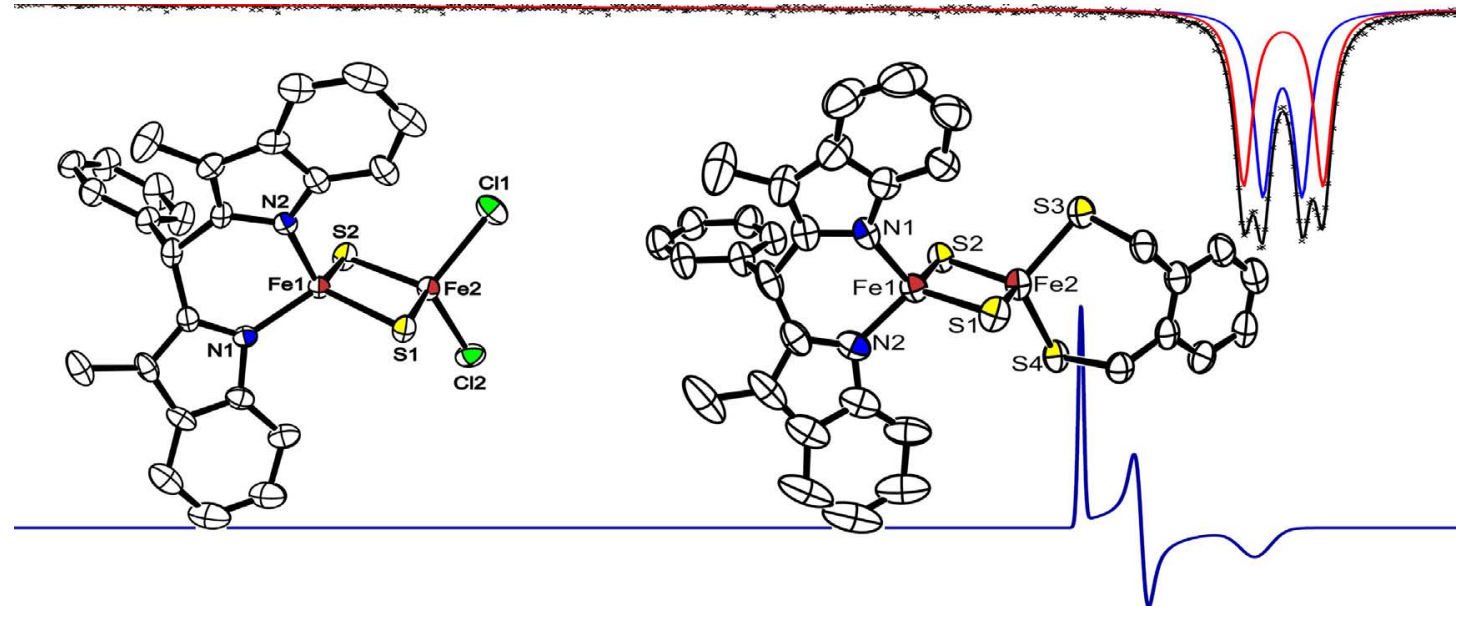

\section{Abstract}

An accurate synthetic model for Rieske-type [2Fe-2S] cluster has been prepared that emulates structural and spectroscopic features of the natural protein sites, including the characteristic low $g_{\text {av }}$ value in the EPR spectra of the reduced $[2 \mathrm{Fe}-2 \mathrm{~S}]^{+}$species. A dichlorosubstituted intermediate and a homoleptic $\{\mathrm{N}\}$-coordinate side-product were isolated and characterized. DFT calculations on the Rieske-type model compound are in agreement with the experimental findings. 


\subsection{Introduction}

In 1964 Rieske-type [2Fe-2S] clusters were discovered in biological systems and identified as variants of $[2 \mathrm{Fe}-2 \mathrm{~S}]$ ferredoxins. ${ }^{[109,233]}$ Structurally they differ form the parent ferredoxins by an asymmetrical terminal ligation at the $[2 \mathrm{Fe}-2 \mathrm{~S}]$ core with only one iron coordinated by two cysteinyl thiolates but the other coordinated by two histidine nitrogen donors (see Chapter 1). ${ }^{[110,115,119,234]}$ Spectroscopic (e.g. EPR and Mössbauer) and functional characteristics (namely the electrochemical potential) of Rieske-type [2Fe-2S] clusters are distinct, because of this unique coordination environment. ${ }^{[4,114]}$ The investigation of synthetic model complexes has provided valuable insight into the properties and electronic structures of iron-sulfur cofactors. ${ }^{[129]}$ While several biomimetic $[2 \mathrm{Fe}-2 \mathrm{~S}]$ clusters with all- $\{\mathrm{S}\}$ or all- $\{\mathrm{N}\}$ environment have been obtained over the last decades, ${ }^{[129,142,170]}$ no asymmetrically ligated cluster that emulates the particular situation of the Rieske ironsulfur proteins could be synthesized so far (few symmetrical [2Fe-2S] clusters with mixed $\{\mathrm{NS}\}$-ligand set at each iron have been reported ${ }^{[34,222]}$. This chapter describes the synthesis and spectroscopic as well as crystallographic characterisation of the first accurate structural model compound 32 for Rieske-type [2Fe-2S] clusters (Scheme 8.1).
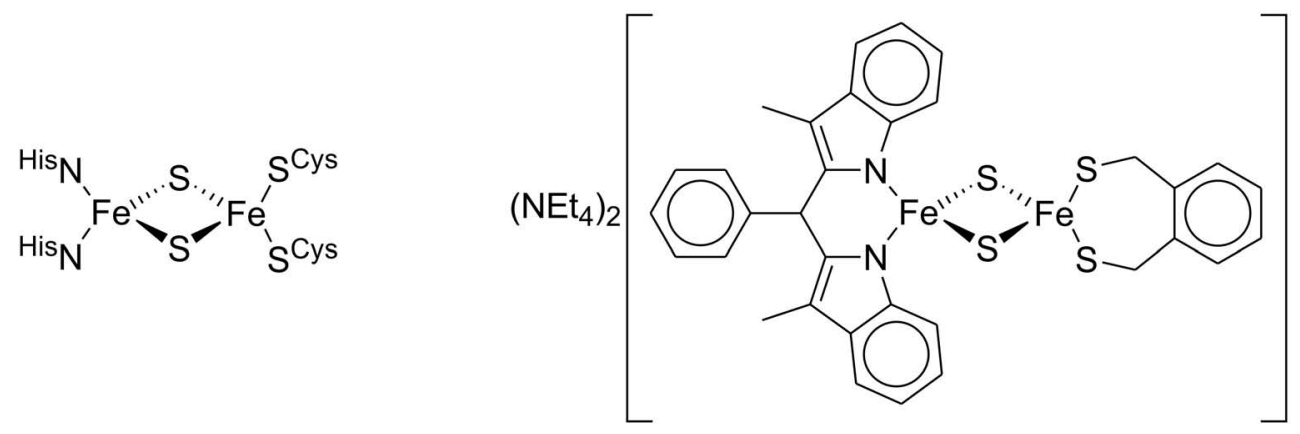

32

Scheme 8.1: Structure of the natural Rieske center and the synthetic analogue 32.

\subsection{Cluster Synthesis and Structural Characterization}

A stepwise ligand exchange strategy starting from $\left(\mathrm{NEt}_{4}\right)_{2}\left[\mathrm{Fe}_{2} \mathrm{~S}_{2} \mathrm{Cl}_{4}\right] \mathbf{2}^{[137,138]}$ seemed to be a convenient and simple approach towards a synthetic analogue of Rieske-type [2Fe-2S] sites. Closer inspection of this reaction by screening a variety of chelating $\left\{\mathrm{N}_{2}\right\}$ - and $\left\{\mathrm{S}_{2}\right\}$-donor ligands in different combinations revealed challenging difficulties, however, such as the preferred formation of the homoleptic $\{\mathrm{N}\}$ - and $\{\mathrm{S}\}$-coordinate compounds which where usually observed as the only products. In some cases, the sought-after asymmetrically $\left\{\mathrm{N}_{2} \mathrm{~S}_{2}\right\}$-ligated species were detected by ESI mass spectrometry, but rapid equilibria with the corresponding homoleptic clusters prevented successful isolation of the target material. An exception was finally discovered when using a backbone phenyl-substituted chelating diskatylmethane $\mathbf{X X I I}{ }^{[235]}$ capping ligand as a mimic for the natural histidine residues. In order to suppress cluster decomposition, the lithium salt of XXII was first 
added to a cooled solution of $\left(\mathrm{NEt}_{4}\right)_{2}\left[\mathrm{Fe}_{2} \mathrm{~S}_{2} \mathrm{Cl}_{4}\right] \mathbf{2}$ (Scheme 8.2). During optimization studies it was found advantageous to use $1.5 \mathrm{eq}$ of this particular $\left\{\mathrm{N}_{2}\right\}$-cap to ensure complete consumption of the cluster starting material, because some degradation to give monomeric $\{\mathrm{N}\}$-coordinate iron complexes could not be prevented even at $-40^{\circ} \mathrm{C}$ (fortunately, thess monomeric species are readily extracted during workup). This led to the isolation of the first asymmetrically ligated $[2 \mathrm{Fe}-2 \mathrm{~S}]$ cluster $\left(\mathrm{NEt}_{4}\right)_{2}\left[\left\{\mathrm{~N}_{2}\right\} \mathrm{Fe}_{2} \mathrm{~S}_{2} \mathrm{Cl}_{2}\right]$ 33. Minor amounts of the $\{\mathrm{N}\}$-homoleptic cluster $\left(\mathrm{NEt}_{4}\right)_{2}\left[\left\{\mathrm{~N}_{2}\right\} \mathrm{Fe}_{2} \mathrm{~S}_{2}\left\{\mathrm{~N}_{2}\right\}\right] \mathbf{3 4}$ were formed as a side-product and identified by X-ray diffraction (Figure 8.1). Compound 33 was crystallized from $\mathrm{DMF} / \mathrm{Et}_{2} \mathrm{O}$, affording crystals suitable for X-ray diffraction and refinement (Figure 8.2). Prominent intra-core distances and angles, as well as distances and angles to the terminal coordinating atoms are in agreement with the corresponding values determined for the related homoleptic $\left\{\mathrm{N}_{4}\right\}$ - or $\mathrm{Cl}_{4}$-ligated ${ }^{[236]}$ synthetic $[2 \mathrm{Fe}-2 \mathrm{~S}]$ clusters (selected interatomic distances and angles are collected in Table 8.3).
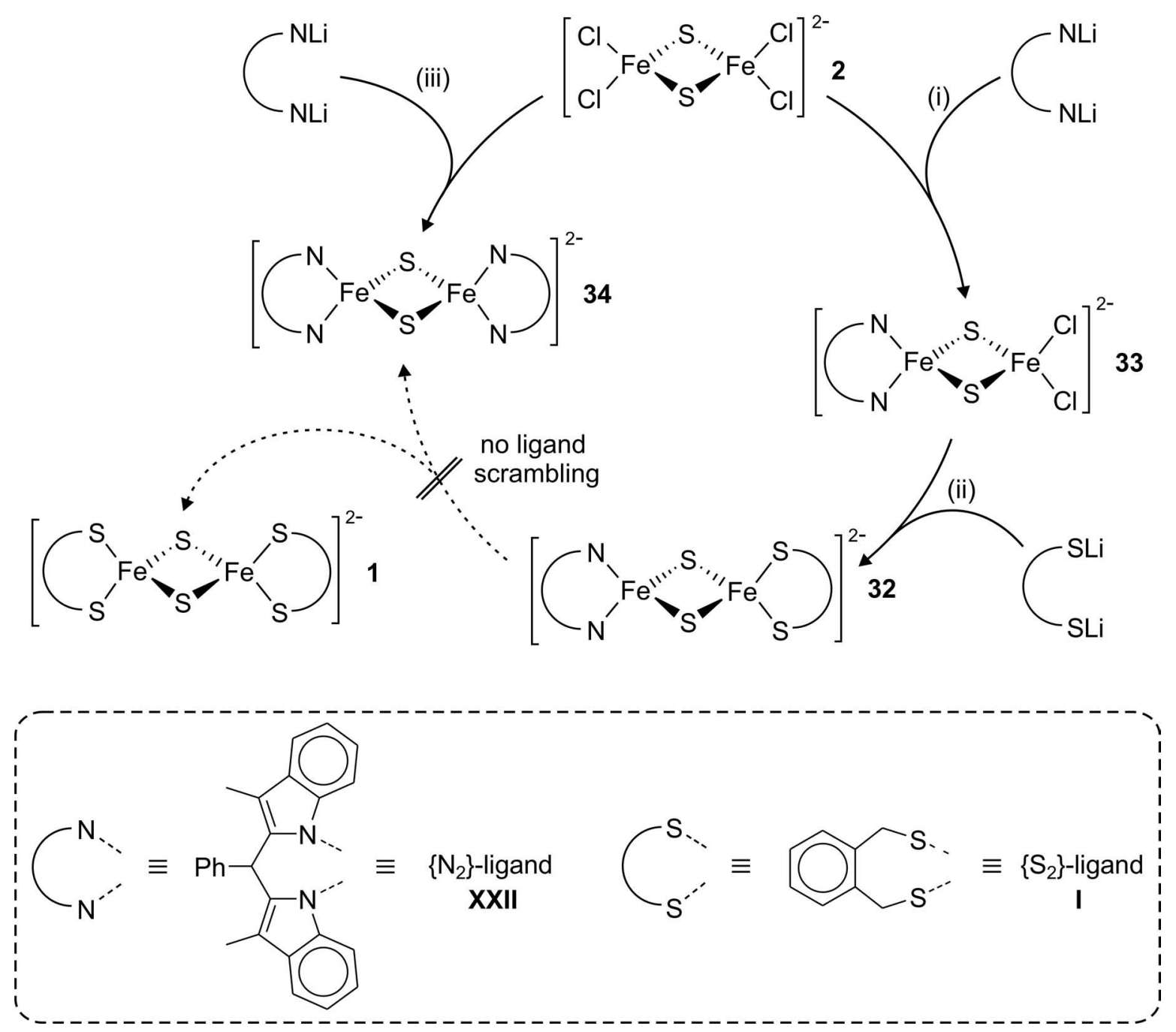

Scheme 8.2: Synthesis of 32, 33 and 34. Conditions: (i) 1.5 eq $\mathrm{Li}_{2}\left\{\mathrm{~N}_{2}\right\}, \mathrm{THF}, \mathrm{MeCN}$, $-40^{\circ} \mathrm{C}, 10$ minutes; (ii) $1.0 \mathrm{eq} L i_{2}\left\{S_{2}\right\}, 1 \mathrm{~h},-40^{\circ} \mathrm{C}$ to room temperature; (iii) $1.9 \mathrm{eq}$ $L i_{2}\left\{N_{2}\right\}, T H F, M e C N, 1 h,-40^{\circ} \mathrm{C}$ to room temperature. 


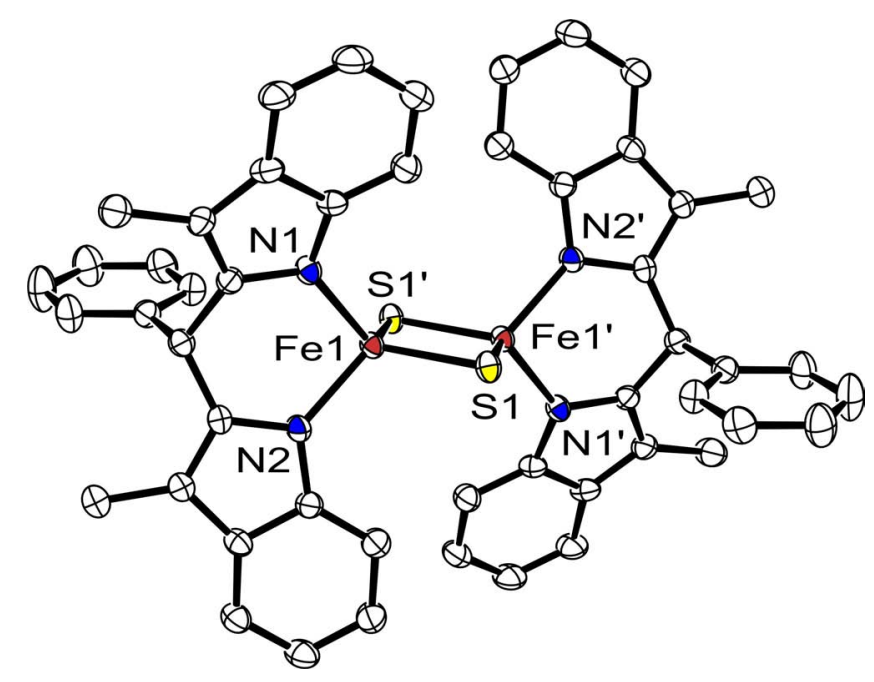

Figure 8.1: ORTEP plot (50\% probability thermal ellipsoids) of the molecular structure of 34. The NEt counter ions and all hydrogen atoms have been omitted for clarity. Selected atom distances $[\AA]$ ] and angles [o]:Fe1 $\cdots F e 1^{\prime}$ 2.7562(8), Fe1-N1 1.984(3), Fe1-N2 1.975(2), Fe1-S1 2.2078(10), Fe1-S1' 2.2301(9), N1-Fe1-N2 93.06(10), N1-Fe1-S1 116.09(8), N2-Fe1-S1 115.16(9), N1-Fe1-S1' 116.02(9), N2-Fe1-S1' 113.88(8), S1-Fe1-S1' 103.22(3), Fe1-S1-Fe1' 76.78(3).

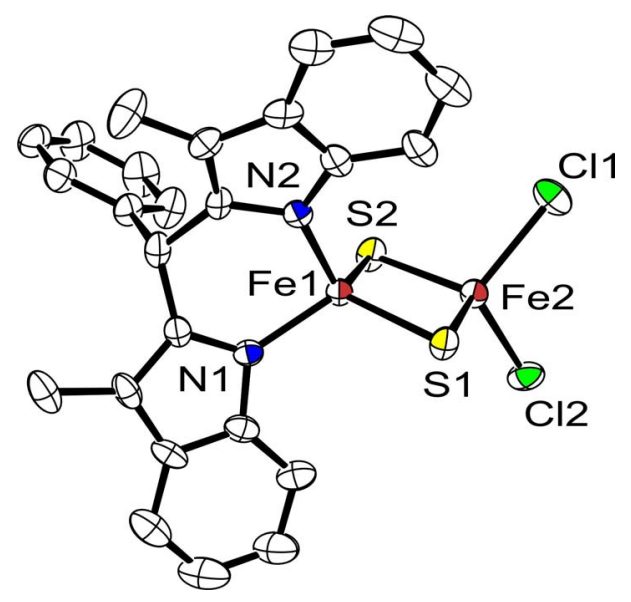

Figure 8.2: ORTEP plot (50\% probability thermal ellipsoids) of the molecular structure of 33. For the sake of clarity all hydrogen atoms and NEt $t_{4}^{+}$counter ions have

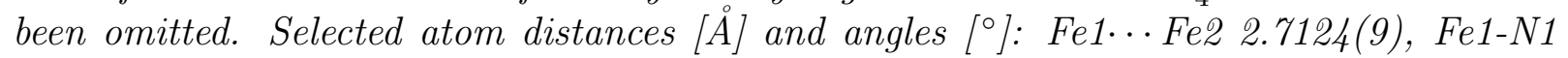
1.965(5), Fe1-N2 1.975(4), Fe1-S1 2.2037(13), Fe1-S2 2.2129(13), Fe2-S2 2.2088(14), Fe2-S1 2.2147(12), Fe2-Cl2 2.2490(15), Fe2-Cl1 2.2730(16), N1-Fe1-N2 93.78(17), N1Fe1-S1 117.18(12), N2-Fe1-S1 110.58(12), N1-Fe1-S2 115.01(12), N2-Fe1-S2 116.48(10), S1-Fe1-S2 104.19(5), S2-Fe2-S1 103.97(5), S2-Fe2-Cl2 113.81(6), S1-Fe2-Cl2 109.80(5), S2-Fe2-Cl1 108.60(6), S1-Fe2-Cl1 112.49(6), Cl2-Fe2-Cl1 108.22(6), Fe1-S1-Fe2 75.74(4), $\mathrm{Fe} 1-\mathrm{S} 2-\mathrm{Fe} 2 \mathrm{75.68(4).}$ 
With the key-intermediate 33 in hands, the intended synthetic route via subsequent exchange of the remaining two chlorine substituents proved successful. o-Xylene- $\alpha, \alpha^{\prime}$-dithiol I as $\left\{\mathrm{S}_{2}\right\}$-ligand ${ }^{[132]}$ was selected for mimicking the biological cysteinyl thiolates since this ligand had already been applied successfully in synthetic iron-sulfur chemistry. ${ }^{[129]}$ Finally, cluster 32 was most conveniently obtained in a one-pot synthesis at $-40^{\circ} \mathrm{C}$ by sequential addition of the deprotonated $\left\{\mathrm{N}_{2}\right\}$-ligand prior to addition of the $\operatorname{Li}_{2}\left\{\mathrm{~S}_{2}\right\}$-ligand salt (Scheme 8.2). After extraction of the monomeric by-products (e.g. $\left(\mathrm{NEt}_{4}\right)\left[\mathrm{Fe}\left\{\mathrm{N}_{2}\right\}_{2}\right]$ 35 - also observed in the synthesis of intermediate 33, structural drawing depicted on page 194), the pure target material was obtained after a single re-crystallisation from $\mathrm{DMF} / \mathrm{Et}_{2} \mathrm{O}$. Once isolated, solid 32 is stable at room temperature under an atmosphere of dry dinitrogen and can even be handled in air for short periods (ca. 30 minutes) without decomposition. In the absence of protic solvents, solutions of $\mathbf{3 2}$ can be stored for weeks at room temperature under an atmosphere of dry dinitrogen. Black plates suitable for X-ray diffraction were obtained by slow diffusion of $\mathrm{Et}_{2} \mathrm{O}$ into a concentrated solution of 32 in DMSO (Figure 8.3). The cluster crystallizes in the monoclinic space group $C 2 / \mathrm{c}$ with eight molecules per unit cell. Geometric parameters at both metal ions Fe1 and Fe2 are similar to the corresponding values observed for the homoleptic $\left\{\mathrm{N}_{4}\right\}$ - and $\left\{\mathrm{S}_{4}\right\}$-ligated ${ }^{[132]}$ compounds 34 and 1 (Table 8.1). Compared with the Rieske proteins, only the Fe-N distances and the N-Fe-N angles in $\mathbf{3 2}$ differ slightly - these differences most likely result from the protonated state of the histidine moieties in the proteins compared to the dianionic $\left\{\mathrm{N}_{2}\right\}$-ligand in the model complex. Other geometric parameters perfectly agree with those found for the natural systems (see Table 8.3). ${ }^{[110]}$

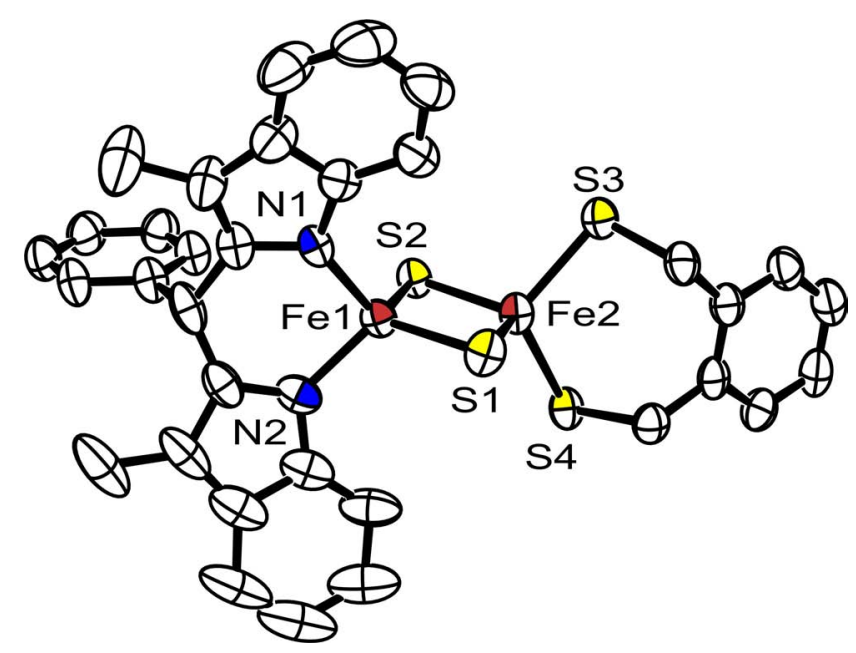

Figure 8.3: ORTEP plot (50\% probability thermal ellipsoids) of the molecular structure of 32. For the sake of clarity all hydrogen atoms and $\mathrm{NEt}_{4}^{+}$counter ions have been omitted. Selected atom distances [A] and angles []: Fe1 . F Fe2 2.7027(8), Fe1-N1 1.953(4), Fe1-N2 1.975(4), Fe1-S1 2.2012(14), Fe1-S2 2.2204(12), Fe2-S1 2.2228(13), Fe2-S2 2.1995(13), Fe2-S3 2.2969(14), Fe2-S4 2.2912(14), N1-Fe1-N2 94.14(16), N1-Fe1-S1 113.78(11), N2Fe1-S1 116.82(13), N1-Fe1-S2 114.75(12), N2-Fe1-S2 113.06(11), S1-Fe1-S2 104.64(5), S2-Fe2-S1 104.61(5), S2-Fe2-S4 112.57(5), S1-Fe2-S4 108.06(5), S2-Fe2-S3 110.17(5), S1-Fe2-S3 113.21(5), S4-Fe2-S3 108.25(5), Fe1-S1-Fe2 75.31(4), Fe1-S2-Fe2 75.39(4). 


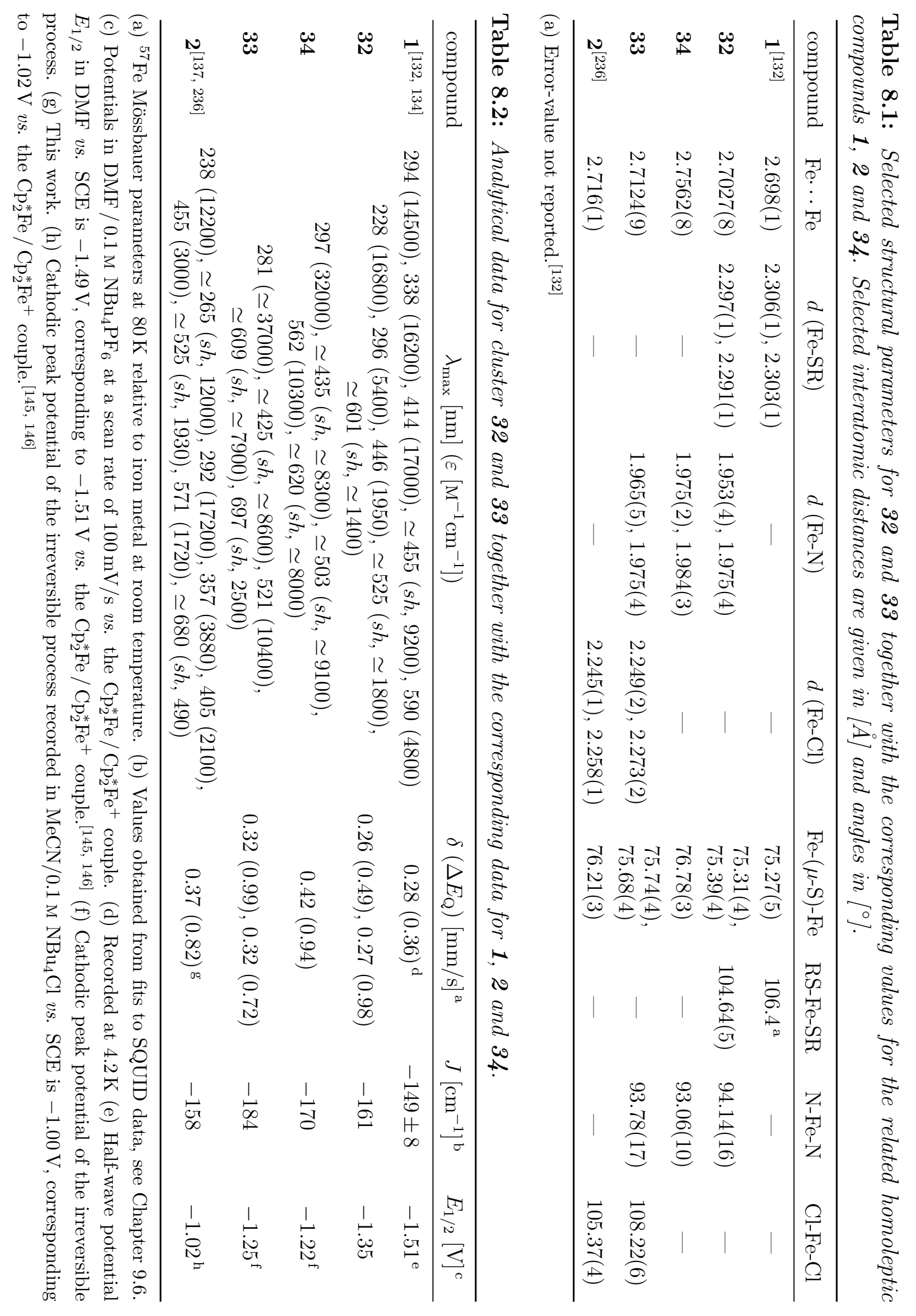


Table 8.3: Geometric parameters of representative Rieske [2Fe-2S] proteins compared to 32. ${ }^{[110]}$ Selected interatomic distances are given in $[\AA]$ and angles in $\left[{ }^{\circ}\right]$.

\begin{tabular}{ccccc}
\hline & SOXF $^{\mathrm{a}}$ & RIE $^{\mathrm{b}}$ & RFS $^{\mathrm{c}}$ & $\mathbf{3 2}$ \\
\hline $\mathrm{Fe} \cdots \mathrm{Fe}$ & 2.72 & 2.71 & 2.72 & 2.70 \\
$\mathrm{Fe}-\mathrm{SR}$ & $2.35 / 2.33$ & $2.29 / 2.22$ & $2.31 / 2.24$ & $2.30 / 2.29$ \\
$\mathrm{Fe}-\mathrm{N}$ & $2.10 / 2.08$ & $2.16 / 2.13$ & $2.19 / 2.23$ & $1.95 / 1.98$ \\
$\mathrm{Fe}-(\mu-\mathrm{S})-\mathrm{Fe}$ & $73.86 / 73.94$ & $74.66 / 74.04$ & $71.67 / 71.39$ & $75.31 / 75.39$ \\
$\mathrm{RS}-\mathrm{Fe}-\mathrm{SR}$ & 109.73 & 105.61 & 110.19 & 108.25 \\
$\mathrm{~N}-\mathrm{Fe}-\mathrm{N}$ & 92.12 & 90.78 & 90.52 & 94.14 \\
\hline
\end{tabular}

(a) Rieske protein II (soxF) from Sulfolobus acidocaldarius. (b) Soluble domain of Rieske protein from bovine mitochondrial $b c_{1}$ complex. (c) Soluble domain of Rieske protein from spinach chloroplast $b_{6} f$ complex.

\subsection{Spectroscopy in the Solid State}

In addition to X-ray diffraction, characterization in the solid state was completed by Mössbauer spectroscopy and magnetic susceptibility measurements. The zero-field Mössbauer spectrum of 32 is shown in Figure 8.4 (data summarized in Table 8.2). Two distinct quadrupole doublets are observed for 32 , with isomer shifts $(0.26 \mathrm{~mm} / \mathrm{s}$ and $0.27 \mathrm{~mm} / \mathrm{s})$ and quadrupole splittings $(0.49 \mathrm{~mm} / \mathrm{s}$ and $0.98 \mathrm{~mm} / \mathrm{s})$ that are in the same range as observed for the natural Rieske proteins (see Table 8.4).

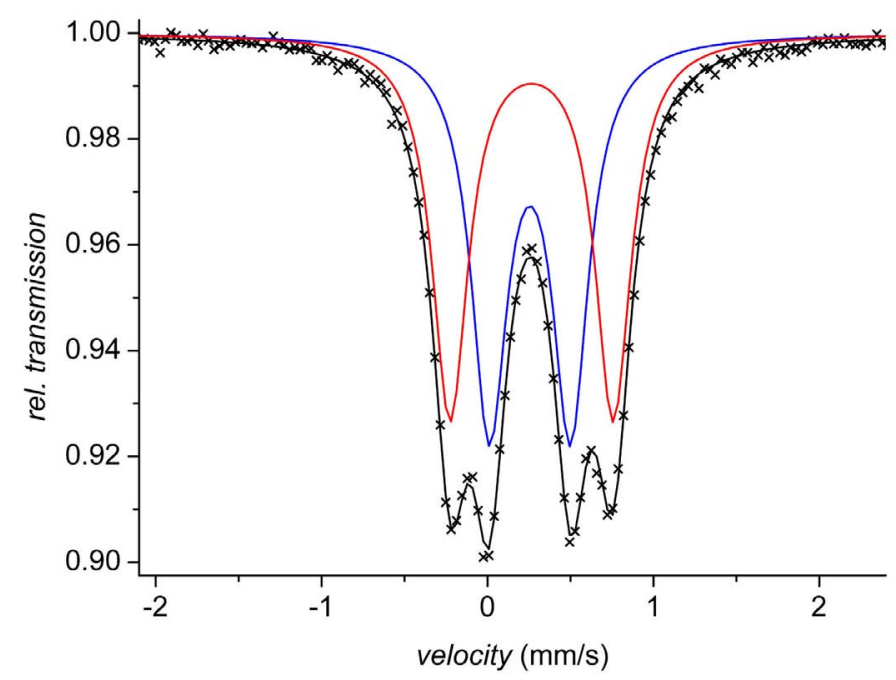

Figure 8.4: Zero-field Mössbauer spectrum of 32 recorded at $80 \mathrm{~K}$, relative to ${ }^{57} \mathrm{Fe}$ at room temperature. Isomer shifts and quadrupole splittings are summarized in Table 8.2. 
Table 8.4: ${ }^{57} \mathrm{Fe}$ Mössbauer parameters for oxidized [2Fe-2S] proteins compared to 32.

\begin{tabular}{ccc}
\hline protein / compound & $\delta[\mathrm{mm} / \mathrm{s}]$ & $\Delta E_{\mathrm{Q}}[\mathrm{mm} / \mathrm{s}]$ \\
\hline ferredoxins, data from different temperatures ${ }^{[140]}$ & $0.2-0.3$ & $0.6-0.8$ \\
Rieske ( Thermus thermophilus), pH 7.8, 4.2 $\mathrm{K}^{[4]}$ & $0.24,0.32$ & $0.52,0.91$ \\
Rieske (Thermus thermophilus), pH 10.4, 4.2 $\mathrm{K}^{[237]}$ & $0.24,0.28$ & $0.44,0.70$ \\
Rieske (Pseudomonas putida), 77 K ${ }^{[238]}$ & $0.23,0.33$ & $0.45,1.03$ \\
$\mathbf{3 2}, 80 \mathrm{~K}$ & $0.26,0.27$ & $0.49,0.98$ \\
\hline
\end{tabular}

As intuitively expected, previously reported for the biological systems ${ }^{[4,237,238]}$ and apparent from comparison with $\mathbf{1}$ and $\mathbf{3 4}$, the larger quadrupole doublet reflects the $\left\{\mathrm{N}_{2}\right\}$ coordinate $\mathrm{Fe} 1$ and the smaller one reflects the $\left\{\mathrm{S}_{2}\right\}$-coordinate $\mathrm{Fe} 2$. Essentially the same considerations apply to cluster compound 33, also ligated in an asymmetrical fashion (see Table 8.2, Figure 8.5). However, magnitudes of quadrupole splittings are closer to each other in this case, resulting in a broad combined doublet. The subsequently performed fitting procedure revealed the two expected subspectra in a 1:1 ratio.

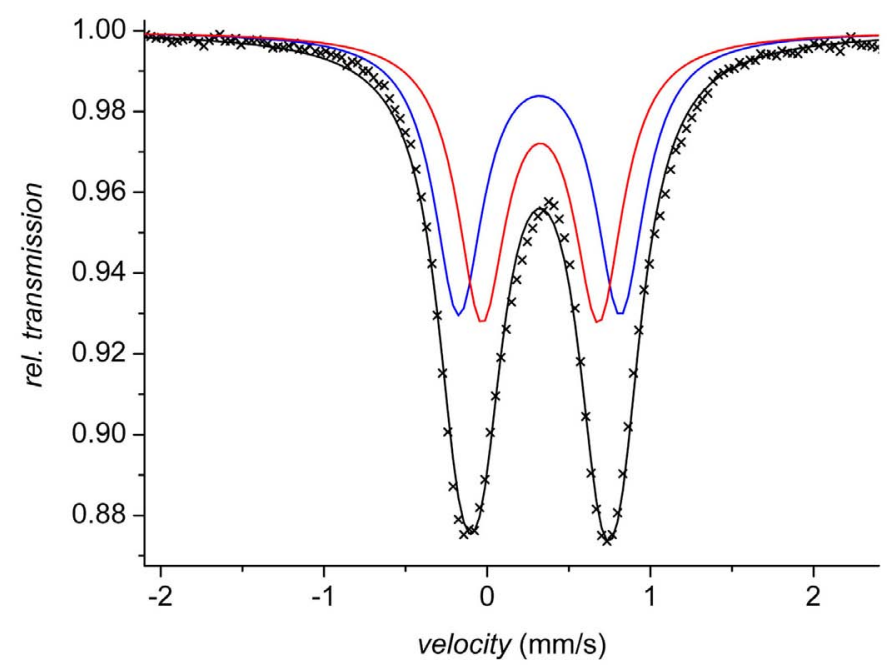

Figure 8.5: Zero-field Mössbauer spectrum of 33 recorded at $80 \mathrm{~K}$, relative to ${ }^{57} \mathrm{Fe}$ at room temperature. The blue and red subspectra are fitted to the experimental values (crosses) with isomer shifts and quadrupole splittings as summarized in Table 8.2. Fitting the experimental curve with a single quadrupole doublet (black line) is possible, but causes increased line width parameters $(\Gamma \simeq 0.6 \mathrm{~mm} / \mathrm{s})$ and is physically not reasonable.

Magnetic susceptibility measurements for both new complexes were carried out at a magnetic field $B=0.5 \mathrm{~T}$ from $2 \mathrm{~K}$ to $295 \mathrm{~K}$. Magnetic moments $\mu_{\mathrm{eff}}$ were found in the range $0.8-2.3 \mu_{\text {B }}$, i.e., much lower than expected for two uncoupled ferric $(S=5 / 2)$ ions, and they rapidly decrease upon lowering the temperature (Figure 8.6). This behavior is in accordance with strong antiferromagnetic coupling between the two ferric ions to give an $S=0$ 
ground state, as is usually observed for [2Fe-2S] clusters and proven for 32 by magnetic Mössbauer spectroscopy (Figure 8.7). Coupling constants $J$ (Table 8.2) were determined by using a fitting procedure to the appropriate Heisenberg spin Hamiltonian for isotropic exchange coupling and Zeeman interaction (see Chapter: 9.6). Interestingly, $J$ values for both asymmetrically coordinated compounds $\mathbf{3 2}\left(J=-161 \mathrm{~cm}^{-1}\right)$ and $\mathbf{3 3}\left(J=-184 \mathrm{~cm}^{-1}\right)$ indicate an increased antiferromagnetic coupling compared to the related homoleptic $\left\{\mathrm{S}_{4}\right\}$ or $\mathrm{Cl}_{4}$-ligated clusters $1\left(J=-149 \pm 8 \mathrm{~cm}^{-1}\right)$ or $2\left(J=-158 \mathrm{~cm}^{-1}\right)$.

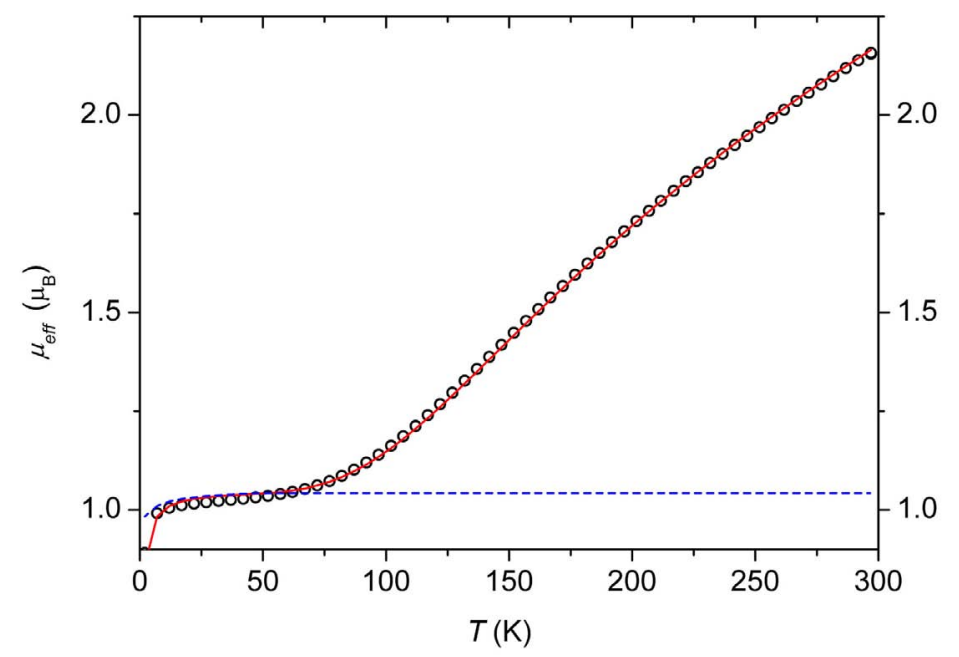

Figure 8.6: Plot of $\mu_{\mathrm{eff}}\left(\mu_{B}\right)$ vs. temperature for 32 with applied field $B=0.5$ T. The red solid line is fitted to the experimental values (circles), see Chapter 9.6.

\subsection{Spectroscopy in Solution}

All new $[2 \mathrm{Fe}-2 \mathrm{~S}]$ cluster compounds were additionally characterized in solution by UV-Vis, ${ }^{1} \mathrm{H}$ NMR spectroscopy and ESI mass spectrometry. Assignment of the partially overlapping electronic absorption bands remains somewhat speculative, and more detailed analyses will be required to locate the different charge-transfer transitions. Similar curvatures of the UV-Vis spectra for all the clusters containing the diskatyl- $\left\{\mathrm{N}_{2}\right\}$-ligand however indicate that prominent bands result form electronic transitions between this ligand and the cluster core. Reasonably well resolved ${ }^{1} \mathrm{H}$ NMR spectra could be recorded because of the strong antiferromagnetic coupling, and signal sets for the $\left\{\mathrm{N}_{2}\right\}$-ligand and the $\left\{\mathrm{S}_{2}\right\}$-ligand can be clearly distinguished. Overall six resonances of the $\left\{\mathrm{N}_{2}\right\}$-ligand are present in the spectra of 32, 33 and 34, with a characteristic signal at $\simeq 5 \mathrm{ppm}$ (assumingly the resonance of the meso-proton). Two additional signals of the xylyl- $\left\{\mathrm{S}_{2}\right\}$-ligand at $4.1 \mathrm{ppm}$ and $7.5 \mathrm{ppm}$ were detetced for 32. Positive and negative ion ESI mass spectra for all cluster compounds show dominant signals for $\left[\mathrm{M}+\mathrm{NEt}_{4}\right]^{+}$and $\left[\mathrm{M}-\mathrm{NEt}_{4}\right]^{-}$, respectively (as an example, the positive ESI mass spectra of $\mathbf{3 2}$ is shown in Figure 8.8). The expected isotopic patterns were observed in these spectra and in the corresponding HRMS spectra. 


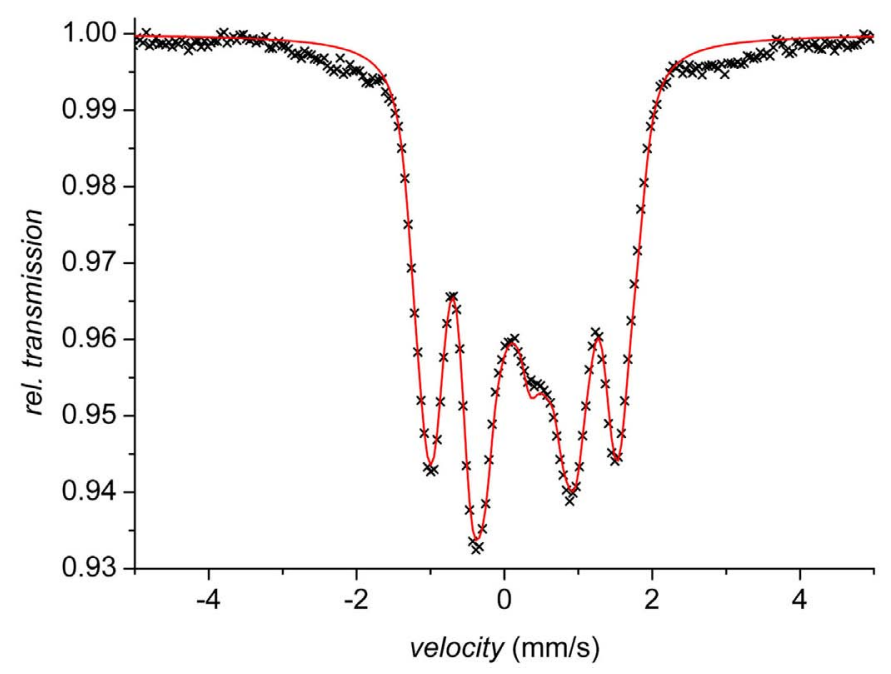

Figure 8.7: Magnetic Mössbauer spectrum of 32 recorded at $4.2 \mathrm{~K}$ with an applied field $B=7 \mathrm{~T}$. Velocities are referenced to ${ }^{57} \mathrm{Fe}$ at room temperature. The red solid line is a simulation for two subspectra as found from the zero-field measurement with parameters as given in Table 8.2, and with spin $S=0$. The electric field gradient tensors are found to be positive, but with large asymmetry parameters, $\eta(1)=1$ and $\eta(2)=0.75$. The satisfying overlap with the experimental data indicates the absence of antisymmetric exchange and confirms the $S=0$ ground state for compound 32 .

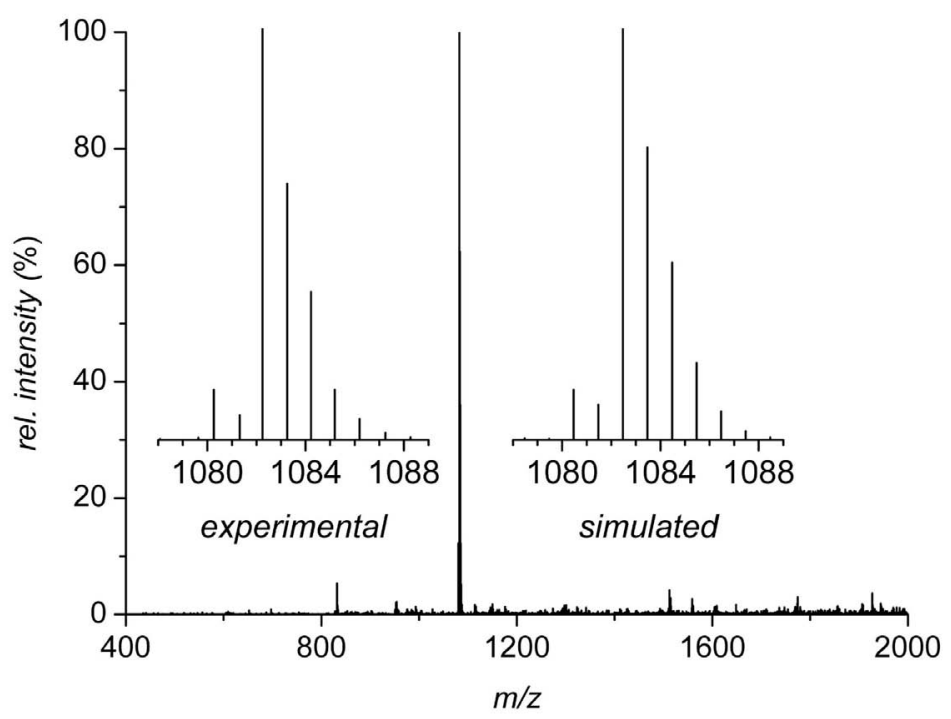

Figure 8.8: Positive ESI-MS spectrum of 32 in MeCN solution. The insets show the experimental and expected isotopic distribution pattern for $\left[M+N E t_{4}\right]^{+}$. 


\subsection{Electrochemistry and Generation of the Reduced Rieske Analogue}

Redox properties of all new complexes were studied by cyclic voltammetry in DMF / $0.1 \mathrm{M}$ $\mathrm{NBu}_{4} \mathrm{PF}_{6}$ solution at room temperature. The Rieske-type cluster 32 exhibits a reversible one-electron reduction at $-1.35 \mathrm{~V} v$ s. decamethylferrocene and a second irreversible reduction wave at approximately $-2.0 \mathrm{~V}$ corresponding to formation of the all-ferrous species. Thus, the half-wave potential corresponding to the $[2 \mathrm{Fe}-2 \mathrm{~S}]^{+} /[2 \mathrm{Fe}-2 \mathrm{~S}]^{2+}$ pair of $\mathbf{3 2}$ is shifted slightly positive compared to the one-electron reduction wave observed for the homoleptic analogue $\mathbf{1}(-1.51 \mathrm{~V}) .{ }^{[137,236]}$ As expected, the unusual high redox potentials of the biological Rieske sites are not reflected by the model cluster 32 , due to the dianionic character of the coordinated $\left\{\mathrm{N}_{2}\right\}$-ligand compared to the protonated neutral histidine residues. Since this first-generation synthetic system cannot undergo the same protonation-assisted electron transfer as the natural counterpart, which relies on the peripheral histidine- $\mathrm{N}$ as protonation sites, ${ }^{[62,120,123-126,239]}$ a dependence of the redox potential on the presence of proton sources is ruled out. Reduction of $\mathbf{3 3}$ is irreversible on the timescale of the cyclic voltammetry, as also observed for the homoleptic $\mathrm{Cl}_{4}$-ligated cluster $\mathbf{2}^{[137,236]}$ (electrochemical data are summarized in Table 8.2, the cyclic voltammogram of $\mathbf{3 2}$ is shown in Figure 8.9). The one-electron reduced mixed-valent species of 32 was generated in MeCN solution by constant potential coulometry $(\mathrm{CPC})$ at $-25^{\circ} \mathrm{C}$. Reduction was carried out at $-1.9 \mathrm{~V}$ vs. the $\mathrm{Cp}_{2} \mathrm{Fe} / \mathrm{Cp}_{2} \mathrm{Fe}^{+}$couple, corresponding to $-1.39 \mathrm{~V}$ vs. $\mathrm{Cp}_{2}^{*} \mathrm{Fe} / \mathrm{Cp}_{2}^{*} \mathrm{Fe}^{+}$. The progress was followed by UV-Vis spectroscopy (Figure 8.10), recorded every 1.5 minutes directly in the coulometric cell and stopped after a charge consumption of approximately $300 \mathrm{mC}$ (calculated for one-electron reduction: $304 \mathrm{mC}$ ). Over the time of the coulometric experiment (overall $\simeq 13.5$ minutes), intensities of the main visible bands decreased with two isosbestic points present. Cyclic voltammograms before and after coulometry were nearly identical in terms of peak potentials, intensities and the overall line shapes, indicating that the redox process is reversible on the voltammetry and the coulometry timescale.

Samples for EPR spectroscopy were taken after $\simeq 50 \%$ reduction (Figure 8.11) and after $100 \%$ reduction, and were immediately frozen in liquid dinitrogen. A characteristic low $g_{3}$ value, as detected for the reduced $[2 \mathrm{Fe}-2 \mathrm{~S}]^{+}$cluster in Rieske proteins ${ }^{[117,127]}$ $\left(g_{3} \simeq 1.78-1.81\right)$ was observed for the $50 \%$ reduced sample by fitting the experimental EPR data with $g_{1}=2.014, g_{2}=1.936$ and $g_{3}=1.804$. Nevertheless, $g_{1}$ is slightly lower and $g_{2}$ somewhat higher than the corresponding values found for Rieske proteins $\left(g_{1} \simeq 2.02\right.$ $\left.2.03, g_{2} \simeq 1.89-1.90\right) .{ }^{[4,117]}$ The low averaged $g_{\mathrm{av}}=1.918$ for 32 (compare $g_{\mathrm{av}}=1.90-1.91$ for Rieske proteins ${ }^{[4,232,240-243]}$ and $g_{\text {av }}=1.95-1.97$ for ferredoxins, ${ }^{[229-231,244-246]} g$ values of selected proteins are collected in Table 8.5) and the wide anisotropy of the main components of the $g$ tensor (mainly a result of the low $g_{3}$ value) suggest that reduction took place at the $\{\mathrm{N}\}$-ligated iron atom of $\mathbf{3 2}$. This lowering of $g_{3}$ and $g_{\mathrm{av}}$ in Rieske-type $[2 \mathrm{Fe}-2 \mathrm{~S}]^{+}$ species was previously attributed to a more pronounced orthorhombic $C_{2 \mathrm{v}}$ distortion at the $\left\{\mathrm{N}_{2} \mathrm{~S}_{2}\right\}$-surrounded tetrahedral ferrous iron. ${ }^{[228]}$ An improved overlap of the experimental values with the fit curve is observed for the $100 \%$ reduced sample with virtually identical $g$ values measured for the target material $\left(g_{1}=2.015, g_{2}=1.936, g_{3}=1.803\right)$. However, a 


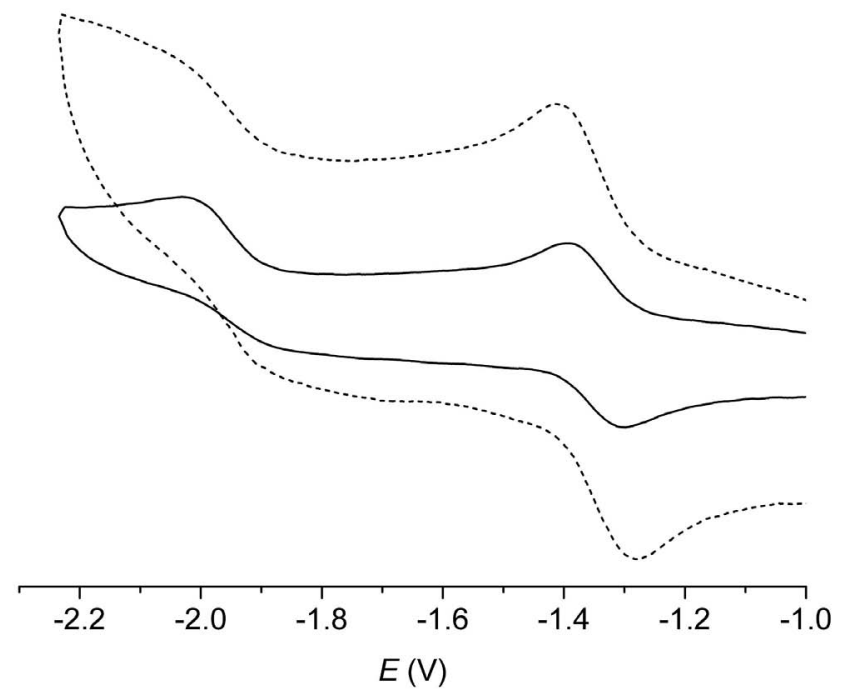

Figure 8.9: Cyclic voltammogram of 32 in DMF/0.1 M NBu $\mathrm{PF}_{6}$ solution at room temperature at a scan rate of $100 \mathrm{mV} / \mathrm{s}$ (solid line) and $500 \mathrm{mV} / \mathrm{s}$ (dashed line) vs. the $\mathrm{Cp}_{2}^{*} \mathrm{Fe} / \mathrm{Cp}_{2}^{*} \mathrm{Fe} e^{+}$couple.

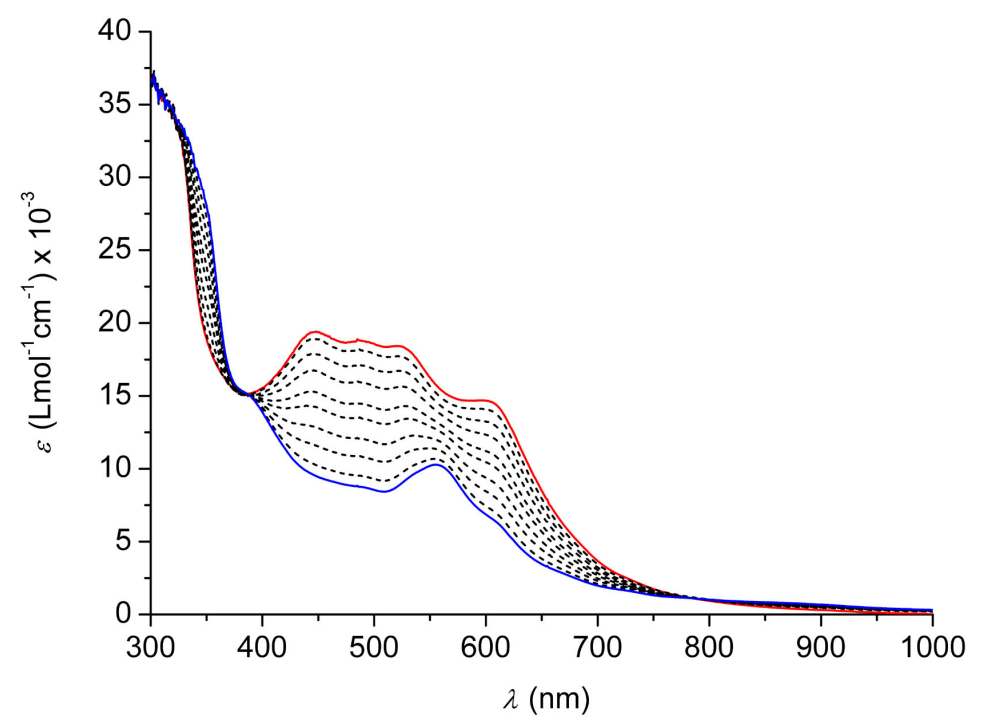

Figure 8.10: $U V$-Vis spectra during constant potential coulometry of 32 at $-25^{\circ} \mathrm{C}$, $-1.9 \mathrm{~V}$ vs. $\mathrm{Cp}_{2} \mathrm{Fe} / \mathrm{Cp}_{2} \mathrm{Fe}^{+}$, red line: $t_{0}=0$ minutes, dotted lines and blue line (after $\simeq 13.5$ minutes $): t_{0}+n \Delta t, \Delta t=1.5$ minutes, $n=1-9, c=3.94 \cdot 10^{-4} \mathrm{M}$ in MeCN $/ 0.2 \mathrm{M}$ $N B u_{4} P F_{6}$.

second, as yet unknown species $\left(\simeq 12 \%\right.$, delocalized $S=1 / 2$ radical, fitted with $g_{1}=2.096$, $g_{2}=2.021$ and $\left.g_{3}=1.906\right)$ formed during the $100 \% \mathrm{CPC}$, probably due to some overreduction. The reduced $[2 \mathrm{Fe}-2 \mathrm{~S}]^{+}$species seems to be slightly unstable, also indicated by an increasing UV-Vis absorption after completed coulometry (measured 3.5 minutes after $100 \% \mathrm{CPC}$, no electrical current applied to the sample, but kept under argon at $-25^{\circ} \mathrm{C}$ ). 
Interestingly, a recently reported novel EPR spectrum of a reduced Rieske site (Thermus thermophilus) at $\mathrm{pH} 14$ with $g_{\mathrm{av}} \simeq 1.97$ demonstrated that the usual $g_{\mathrm{av}} \simeq 1.91$ signal (observed at $\mathrm{pH} 7$ ) is not constant over the $\mathrm{pH}$ range. Especially $g_{1}$ is unusually large at $\mathrm{pH} 14$ (and $g_{2}$ is found in the range of ferredoxin-type proteins): $g_{1}=2.14, g_{2}=1.94, g_{3}=1.81$. It is stated that an antisymmetric (Dzyaloshinskii-Moriya) exchange interaction between the high-spin $(S=5 / 2) \mathrm{Fe}(\mathrm{III})$ - and the high-spin $(S=2) \mathrm{Fe}(\mathrm{II})$-site might be involved. ${ }^{[243]}$ 


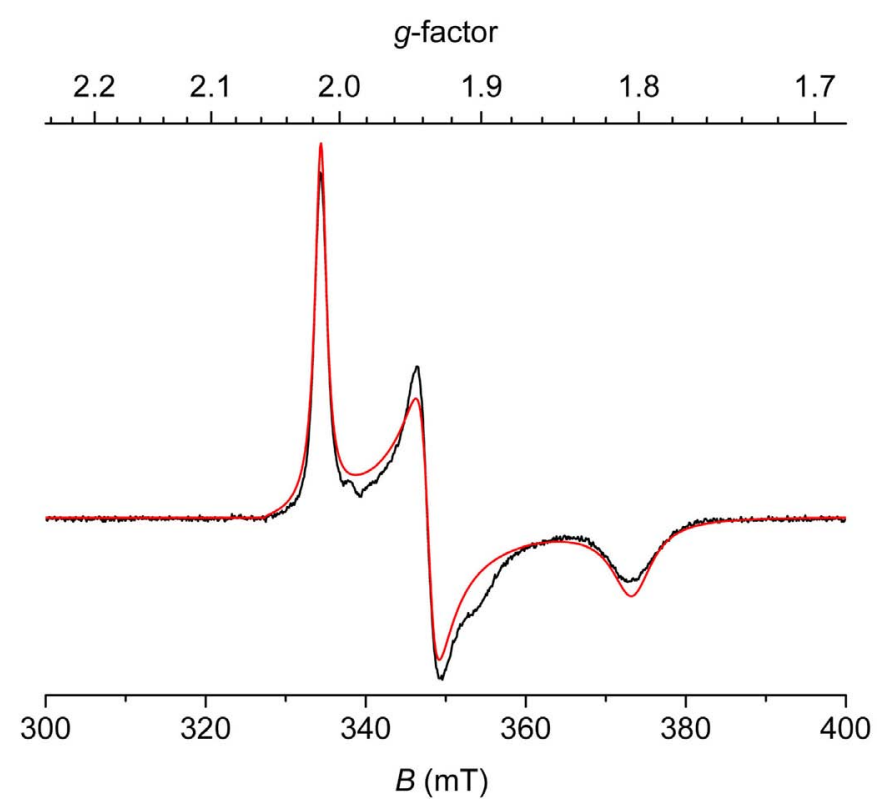

Figure 8.11: EPR spectrum of the one-electron reduced 32 (generated via $C P C$ at $-25^{\circ} \mathrm{C}$, $-1.9 \mathrm{~V}$ vs. $\mathrm{Cp}_{2} \mathrm{Fe} / \mathrm{Cp}_{2} \mathrm{Fe}^{+}$, sample taken after $\simeq 50 \%$ reduction), recorded at $20 \mathrm{~K}$ in fro-

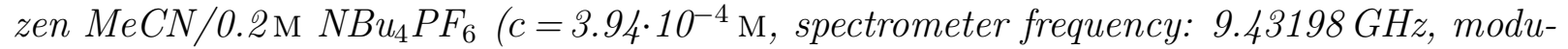
lation amplitude: $25 \mathrm{~mW}$ ). The red line is fitted to the experimental values (black line) with $g_{1}=2.014, g_{2}=1.936$ and $g_{3}=1.804$.

Table 8.5: Previously reported $g$ values for the $[2 \mathrm{Fe}-2 \mathrm{~S}]^{+}$clusters in Rieske proteins compared to 32 .

\begin{tabular}{|c|c|c|c|c|}
\hline$[2 \mathrm{Fe}-2 \mathrm{~S}]^{+}-$Rieske site in & $g_{1}$ & $g_{2}$ & $g_{3}$ & $g_{\mathrm{av}}$ \\
\hline yeast $^{[232]}$ & 2.025 & 1.890 & 1.810 & 1.908 \\
\hline $\mathrm{QH}_{2}$-cytochrome $c$ oxido-reductase (bovine heart) ${ }^{[240]}$ & 2.019 & 1.891 & 1.805 & 1.905 \\
\hline Rhodopseudomonas sphaeroides ${ }^{[241]}$ & 2.030 & 1.900 & 1.810 & 1.913 \\
\hline Thermus thermophilus ${ }^{[132]}$ & 2.020 & 1.900 & 1.800 & 1.907 \\
\hline chloroplasts $^{[242]}$ & 2.020 & 1.890 & 1.780 & 1.897 \\
\hline succinate-cytochrome $c$ reductase complex ${ }^{[229]}$ & 2.030 & 1.900 & 1.780 & 1.903 \\
\hline $32(100 \%$ reduction $)$ & 2.015 & 1.936 & 1.803 & 1.918 \\
\hline 32 (50\% reduction) & 2.014 & 1.936 & 1.804 & 1.918 \\
\hline
\end{tabular}

\subsection{DFT Calculations}

In order to corroborate conclusions from the EPR findings, DFT calculations were carried out, using the pure BP86 functional and the def2-SVP basis set (technical details are provided in Chapter 9.4). Both, the oxidized and reduced form of $\mathbf{3 2}$ were studied in the 
antiferromagnetically $(A F)$ coupled spin state. In accordance with the experimental findings, the $\mathrm{BP} 86$ results confirm that the $A F$ state of $\mathbf{3 2}$ (on crystal structure coordinates) is lower in energy by $117 \mathrm{~kJ} / \mathrm{mol}$ in vacuum or $111 \mathrm{~kJ} / \mathrm{mol}$ in a simulated MeCN environment (the COSMO module was utilized for the simulation of solvent effects, see Table 8.6).

Table 8.6: Selected DFT details for calculations on crystal structure coordinates of 32 in either the ferromagnetic $(F)$ or antiferromagnetic $(A F)$ spin state.

\begin{tabular}{lcccc}
\hline & \multicolumn{2}{c}{ SCF-energy (a.u.) } & \multicolumn{2}{c}{$E(A F)-E(F)[\mathrm{kJ} / \mathrm{mol}]$} \\
& vacuum & COSMO $^{\text {a }}$ & vacuum & COSMO $^{\text {a }}$ \\
\hline $\mathbf{3 2}\left({ }^{11} X=F\right)$ & -5502.575379057 & -5502.792540809 & \multirow{2}{*}{117} & 111 \\
$\mathbf{3 2}\left({ }^{1} X=A F\right)$ & -5502.619797812 & -5502.834836458 & & \\
\hline
\end{tabular}

(a) A dielectric constant $\varepsilon=37.5$ for MeCN was used in this calculations.

Calculated quadrupole splittings for the all-ferric form of $\mathbf{3 2}$ validate the assignment of the experimental Mössbauer signals (details are described in Chapter 9.4). Qualitatively identical results have been obtained, when all calculations were performed on optimized geometries. Analysis of the molecular orbitals revealed a localization of the LUMO in oxidized 32 at the $\{\mathrm{N}\}$-coordinate Fe atom. Accordingly, the HOMO in reduced $\mathbf{3 2}$ (Figure 8.12 ) is located at this unique iron, as previously concluded from DFT calculations on a fictive mixed-valent Rieske-type model system. ${ }^{247]}$

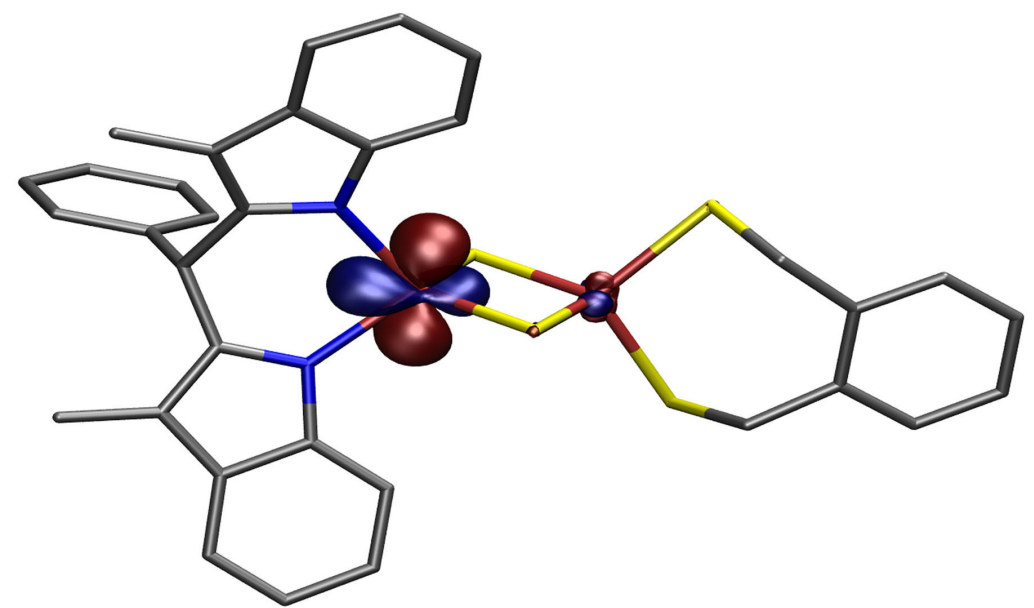

Figure 8.12: Illustration of the highest occupied molecular orbital (HOMO, contour value $=0.06)$ of 32 in the one-electron reduced mixed-valent state. Hydrogen atoms are omitted for clarity. 


\subsection{Conclusions}

In conclusion, the first exact synthetic model for [2Fe-2S] Rieske sites reported here adequately emulates structural, Mössbauer and EPR parameters of the analogous proteinbound clusters. Future efforts will focus on the incorporation of additional nitrogen atoms into the $\left\{\mathrm{N}_{2}\right\}$-ligand backbone, in order to provide potential protonation sites that would allow to more closely mimic the electrochemical properties of the natural enzymes and to support the role of the iron-ligated histidines in the $\mathrm{pH}$-dependence of the reduction potential. 


\section{Chapter 9}

\section{Experimental Section}

\subsection{General Considerations}

All manipulations were carried out under an anaerobic and anhydrous atmosphere of dry dinitrogen by employing standard Schlenk techniques or in a glovebox, unless mentioned otherwise. $\mathrm{Et}_{2} \mathrm{O}$ and pentane were dried over sodium benzophenone ketyl; THF, benzene, toluene and hexanes over potassium benzophenone ketyl; $\mathrm{CS}_{2}, \mathrm{DMF}, \mathrm{DMSO}, \mathrm{MeCN}$ and EtCN over $\mathrm{CaH}_{2} ; \mathrm{CH}_{2} \mathrm{Cl}_{2}, \mathrm{CHCl}_{3}, 1,1,1$-trichloroethane and $\mathrm{MeNO}_{2}$ over $\mathrm{P}_{4} \mathrm{O}_{10}$; $\mathrm{MeOH}$ and $\mathrm{EtOH}$ over $\mathrm{Mg}$; and distilled prior to use. Deuterated solvents were dried and distilled according to the undeuterated analogues. Glassware was dried at $120^{\circ} \mathrm{C}$ overnight. Elevated reaction temperatures $\left(>250^{\circ} \mathrm{C}\right)$ were kept constant using a calibrated controllable resistance. ${ }^{1} \mathrm{H}$ NMR and ${ }^{13} \mathrm{C}$ NMR spectra were recorded on a Bruker Avance $200 \mathrm{MHz}, 300 \mathrm{MHz}$ or $500 \mathrm{MHz}$ spectrometer. ${ }^{19} \mathrm{~F} \mathrm{NMR}$ (relative to $\mathrm{CFCl}_{3}$ ) and ${ }^{31} \mathrm{P}$ NMR (relative to $\mathrm{H}_{3} \mathrm{PO}_{4}$ ) spectra were recorded on a Bruker Avance $200 \mathrm{MHz}$ spectrometer. ${ }^{1} \mathrm{H}$ and ${ }^{13} \mathrm{C}$ chemical shifts are reported in ppm relative to residual solvent signals of $\mathrm{CDCl}_{3}(7.24 \mathrm{ppm}$ and $77.1 \mathrm{ppm}), \mathrm{MeCN} \mathrm{d}_{3}(1.94 \mathrm{ppm}$ and $118.3 \mathrm{ppm}), \mathrm{C}_{6} \mathrm{D}_{6}(7.15 \mathrm{ppm}$ and $128.0 \mathrm{ppm}) \mathrm{MeOH}-\mathrm{d}_{3}(3.31 \mathrm{ppm}$ and $49 . \mathrm{ppm})$ or DMSO-d $\mathrm{D}_{6}(2.46 \mathrm{ppm}$ and 29.9$) .{ }^{[248]} \mathrm{UV}-\mathrm{Vis}$ spectra were recorded with an Analytik Jena Specord S 100 using Schlenk quartz cuvettes. Microanalyses were performed by the "Analytical Laboratory of the Institute for Inorganic Chemistry at the University of Göttingen". Infrared spectra were recorded on a Digilab Excalibur FTS3000 spectrometer. Melting points were determined using a SRS OptiMelt apparatus. EI mass spectra were measured on a Finnigan MAT 8200, ESI mass spectra on a Thermo Finnigan Trace LCQ spectrometer and ESI HRMS spectra on a Bruker FTICR APEX IV instrument. Mössbauer experiments are described in Chapter 9.5. Temperaturedependent magnetic susceptibility measurements are described in Chapter 9.6. Electrochemical measurements are described in Chapter 9.7. X-band EPR derivative spectra were recorded on a Bruker ELEXSYS E500 spectrometer equipped with the Bruker standard cavity (ER4102ST) and a helium flow cryostat (Oxford Instruments ESR 910). Microwave frequencies were calibrated with a Hewlett-Packard frequency counter (HP5352B), and the field control was calibrated with a Bruker NMR field probe (ER035M). The spectra were simulated with the program GFIT for the calculation of powder spectra with effec- 
tive $g$ values and anisotropic line widths (Gaussian line shapes were used). $\mathrm{NaH}$ and $\mathrm{KH}$ was purchased as dispersion in mineral oil, washed repetitively with hexanes and dried in vacuum. Compounds o-xylene- $\alpha, \alpha^{\prime}$-dithiol $\mathbf{I},{ }^{[131,132]} 3,3^{\prime}, 5,5^{\prime}$-tetrachloro-2, $2^{\prime}$-dihydroxy1, $1^{\prime}$-biphenyl IIa, ${ }^{[160]} 3,3^{\prime}, 5,5^{\prime}$-tetrakis-tert-butyl-1, $1^{\prime}$-biphenyl IIc ${ }^{[161]} 2,2^{\prime}$-bis- $(N, N$-dimethylthiocarbamoyloxy)-1, $1^{\prime}$-biphenyl IIIb ${ }^{[162]} 2,2^{\prime}$-bis-( $N, N$-dimethylcarbamoylthio)1, $1^{\prime}$-biphenyl IVb, ${ }^{[162]} 2,2^{\prime}$-dithio-1, $1^{\prime}$-biphenyl $\mathbf{V b},{ }^{[162]}$ bis- $N, N^{\prime}$-(2, 6-di-iso-propylphenyl)-2, 4-diketiminopentane $\mathbf{X I},{ }^{[192]}$ 5-pentafluorophenyldipyrrine $\mathbf{X I I},{ }^{[193,194]}$ 2-(methylthio)-benzenethiol $\mathbf{X I I I}^{\mathbf{S}}{ }^{, 202]}$ 2, $2^{\prime}$-(methylamino)-dibenzenethiol $\mathbf{X I V}^{\mathbf{N}},{ }^{[210]} 2,2^{\prime}$-oxydibenzenethiol $\mathbf{X I V}^{\mathbf{O}},{ }^{[203]}$ 2, $2^{\prime}$-(phenylphosphino)-dibenzenethiol $\mathbf{X I V}^{\mathbf{P}},{ }^{[211]} 2,2^{\prime}$-thiodibenzenethiol XIV ${ }^{\mathbf{S}},{ }^{[204]} 5,5^{\prime}$-dimethyldipyrromethane XXa, ${ }^{[223]} 5,5^{\prime}$-diphenyldipyrromethane $\mathbf{X X b},{ }^{[194,224]} \quad 1,1^{\prime}$-dipyrrocyclohexane $\mathbf{X X c},{ }^{[225]} \quad 1,2$-bis-(benzimidazol-2-yl)-benzene $\mathbf{X X I a},{ }^{[226]}$ 1, 2-bis-(benzimidazol-2-yl)-tetrafluorobenzene XXIb, ${ }^{[226]} 2,2^{\prime}$-(phenylmethylene)-bis-(3-methyl- $1 H$-indole) $\mathbf{X X I I},{ }^{[235]}$ bis-(tetrabutylammonium)-bis- $\left[\left(o\right.\right.$-xylyl- $\alpha, \alpha^{\prime}$-dithiolato)-( $\mu$-sulfido)-ferrate(III)] $\mathbf{1}^{[132,135]}$ bis-(tetraethylammonium)-bis-[(dichloro)- $(\mu$ sulfido)-ferrate(III)] $\quad \mathbf{2},{ }^{[138]} \quad$ bis-(tetraethylammonium)-bis-[(dipyrrolato)-( $\mu$-sulfido)-ferrate(III)] $\mathbf{4}^{[141]}$ tetrakis-(hexamethyldisilamido)-diiron(II) $\mathbf{1 5},{ }^{[186]}$ tri-(iodo)-(thiourea)$\operatorname{iron}(\mathrm{III}) \mathbf{1 6},{ }^{[188]} \quad$ [bis- $N, N^{\prime}$-(2, 6-di-iso-propylphenyl)-pentyl-2, 4-diketiminato]-(dichloro)iron(III) 21, ${ }^{[189]}$ [bis- $N, N^{\prime}$-(2, 6-di-iso-propylphenyl)-pentyl-2, 4-diketiminato]-(hexamethyldisilamido)-iron(II) $\mathbf{1 8}^{[189]}$ and tetraethylammonium tetrachloroferrate(III) $\mathbf{2 8}^{[215]}$ were synthesized according to published procedures. Compounds bis- $N, N-(2$-chloroethyl)- $N$ methylamine VII, ${ }^{[178,179]} 6,6^{\prime}$-methylenebis-(2, 4-di-tert-butylphenol) $\mathbf{X V I}{ }^{[205]}$ and $2,2^{\prime}$ methylenediphenol XVII ${ }^{[206,207]}$ were synthesized by modifications of previously reported procedures. All other chemicals were used as purchased. 


\subsection{Synthesis of Ligands and Ligand Precursors}

\section{$2,2^{\prime}$ - Bis- $\left(N, N\right.$-dimethylthiocarbamoyloxy)- $3,3^{\prime}, 5,5^{\prime}$ - tetrachloro- 1, 1'-biphenyl (IIIa).}

To a vigorously stirred solution of 3, 3',5, $5^{\prime}$-tetrachloro-1, $1^{\prime}$-biphenyl IIa $(20.0 \mathrm{~g}, 62 \mathrm{mmol})$ in DMF $(160 \mathrm{ml})$ at $0{ }^{\circ} \mathrm{C}$ was added $\mathrm{NaH}(3.20 \mathrm{~g}, 133 \mathrm{mmol})$ in portions during 5 minutes. After completed addition the reaction mixture was stirred for $3 \mathrm{~h}$ at room temperature. Subsequently dimethylcarbamothioic chloride $(26.8 \mathrm{~g}, 218 \mathrm{mmol})$ was added in portions and the resulting mixture was stirred at $105^{\circ} \mathrm{C}$ for $18 \mathrm{~h}$. After cooling to room temperature, the reaction was quenched by addition of water $(650 \mathrm{ml})$ and acidified with aqueous $\mathrm{HCl}$ $(37 \%, 15 \mathrm{ml})$ to precipitate a brownish sticky solid. The mixture was left standing at $4{ }^{\circ} \mathrm{C}$ for $1 \mathrm{~h}$ in order to complete the precipitation (work-up procedure carried out in air). The supernatant was decanted off and the residual solid dried in vacuum with gentle heating. The obtained brownish solid was stirred in a mixture of hexanes $(240 \mathrm{ml})$ and chloroform $(35 \mathrm{ml})$ at $70^{\circ} \mathrm{C}$ for $6 \mathrm{~h}$ and cooled to room temperature again. The grey finely powdered undissolved solid was filtered off and dried in vacuum to afford the crude product $(12 \mathrm{~g}$, pure in ${ }^{1} \mathrm{H}$ NMR, but not suitable for Miyazaki-Newman-Kwart rearrangement). Final purification was achieved by column chromatography (silica, hexanes / EtOAc $=10 / 1$, TLCs recorded in hexanes $\left./ \mathrm{EtOAc}=5 / 2, R_{\mathrm{f}} \simeq 0.5\right)$ to obtain the pure product as a white powder $(10.0 \mathrm{~g}, 20 \mathrm{mmol}, 32 \%)$. Mp (uncorrected) $212{ }^{\circ} \mathrm{C}$. ${ }^{1} \mathrm{H} \mathrm{NMR}\left(300 \mathrm{MHz}, \mathrm{CDCl}_{3}\right)$ : $\delta=3.15\left(\mathrm{~s}, 6 \mathrm{H}, \mathrm{NMe}_{2}\right), 3.26\left(\mathrm{~s}, 3 \mathrm{H}, \mathrm{NMe}_{2}\right), 3.32\left(\mathrm{~s}, 3 \mathrm{H}, \mathrm{NMe}_{2}\right), 7.31\left(\mathrm{~d}, 1 \mathrm{H},{ }^{4} J_{\mathrm{H}, \mathrm{H}}=2.4 \mathrm{~Hz}\right.$, Ar-H), $7.45\left(\mathrm{dd}, 2 \mathrm{H},{ }^{4} J_{\mathrm{H}, \mathrm{H}}=2.1 \mathrm{~Hz},{ }^{4} J_{\mathrm{H}, \mathrm{H}}=2.4 \mathrm{~Hz}, \mathrm{Ar}-\mathrm{H}\right), 7.51\left(\mathrm{~s}_{\mathrm{br}}, 1 \mathrm{H}, \mathrm{Ar}-\mathrm{H}\right) .{ }^{13} \mathrm{C} \mathrm{NMR}$ $\left(125 \mathrm{MHz}, \mathrm{CDCl}_{3}\right): \delta=38.4\left(\mathrm{NMe}_{2}\right), 38.9\left(\mathrm{NMe}_{2}\right), 43.4\left(\mathrm{NMe}_{2}\right), 43.5\left(\mathrm{NMe}_{2}\right), 128.9(\mathrm{Ar}-$ C), 129.3 (Ar-C), 130.0 (Ar-C), 130.0 (Ar-C), 130.0 (Ar-C), 130.1 (Ar-C), 130.8 (Ar-C), 131.6 (Ar-C), 132.1 (Ar-C), 132.9 (Ar-C), 146.1 (Ar-C), 146.4 (Ar-C), 184.7 (CO), 185.0 $(\mathrm{CO})$. MS $(\mathrm{EI}+): m / z(\%)=499(2)[\mathrm{M}]^{+}, 463(38)[\mathrm{M}-\mathrm{Cl}]^{+}, 427(5)[\mathrm{M}-2 \mathrm{Cl}]^{+}, 88$ (100) $\left[\mathrm{Me}_{2} \mathrm{NC}(\mathrm{S})\right]^{+}, 72(39)\left[\mathrm{Me}_{2} \mathrm{NC}(\mathrm{O})\right]^{+}$. IR (KBr): $\widetilde{\nu}\left(\mathrm{cm}^{-1}\right)=1549$ (CS, s). Elemental analysis: Calcd. (\%) for $\mathrm{C}_{18} \mathrm{H}_{16} \mathrm{Cl}_{4} \mathrm{~N}_{2} \mathrm{O}_{2} \mathrm{~S}_{2}$ : C 43.39, $\mathrm{H}$ 3.24, N 5.62. Found: $\mathrm{C} 43.72, \mathrm{H}$ 3.37, N 5.71.

\section{$2,2^{\prime}$-Bis- $\left(N, N\right.$-dimethylthiocarbamoyloxy)-3, $3^{\prime}, 5,5^{\prime}$-tetrakis-tert- butyl-1, 1'-biphenyl (IIIc).}

To a vigorously stirred solution of $3,3^{\prime}, 5,5^{\prime}$-tetrakis-tert-butyl-1, $1^{\prime}$-biphenyl IIc $(8.5 \mathrm{~g}$, $21 \mathrm{mmol})$ in $\mathrm{DMF}(70 \mathrm{ml})$ at $0{ }^{\circ} \mathrm{C}$ was added $\mathrm{NaH}(1.44 \mathrm{~g}, 60 \mathrm{mmol})$ in portions during 10 minutes. After completed addition, HMPA $(5 \mathrm{ml})$ was added and the reaction mixture stirred for $12 \mathrm{~h}$ at room temperature. Then dimethylcarbamothioic chloride $(10.4 \mathrm{~g}$, $84 \mathrm{mmol}$ ) was added in portions and the resulting mixture was stirred for $3 \mathrm{~d}$ at $100^{\circ} \mathrm{C}$. After cooling to room temperature, the reaction was quenched by addition of aqueous $\mathrm{KOH}$ solution (3 g KOH in $300 \mathrm{ml}$ water, work-up procedure carried out in air). The precipitated brown solid was filtered off, dissolved in dichloromethane $(150 \mathrm{ml})$ and washed with water $(3 \times 100 \mathrm{ml})$. The organic phase was dried over $\mathrm{Na}_{2} \mathrm{SO}_{4}$, filtered and evaporated to dryness. 
The brown oily residue was filtered over a silica plug (hexanes / EtOAc $=10 / 1$ ), eluting all spots with $R_{\mathrm{f}}>0.5$ (TLCs recorded in hexanes / EtOAc $=5 / 1$; spots of mono-substituted compound, product and one minor unidentified compound are rather close to each other and were separated only in small scale by elution with hexanes / EtOAc $=20 / 1$ ). The obtained solid pale yellow crude product (containing $\simeq 5 \%$ mono-substituted compound) was recrystallized from chloroform / hexanes $\left(30 \mathrm{ml} / 3 \mathrm{ml}\right.$, cooling from reflux to $\left.-20^{\circ} \mathrm{C}\right)$ to obtain the pure product as a white crystalline powder $(8.0 \mathrm{~g}, 14 \mathrm{mmol}, 65 \%)$. Mp (uncorrected) $170{ }^{\circ} \mathrm{C} .{ }^{1} \mathrm{H} \mathrm{NMR}\left(300 \mathrm{MHz}, \mathrm{CDCl}_{3}\right): \delta=1.30\left(\mathrm{~s}, 18 \mathrm{H}, 3,3^{\prime}{ }^{t} \mathrm{Bu}\right), 1.37(\mathrm{~s}, 18 \mathrm{H}$, $\left.5,5^{\prime}{ }^{t} \mathrm{Bu}\right), 2.98\left(\mathrm{~s}, 6 \mathrm{H}, \mathrm{NMe}_{2}\right), 3.03\left(\mathrm{~s}, 6 \mathrm{H}, \mathrm{NMe}_{2}\right), 7.25\left(\mathrm{~d}, 2 \mathrm{H},{ }^{4} J_{\mathrm{H}, \mathrm{H}}=2.7 \mathrm{~Hz}, 6,6^{\prime}-\mathrm{Ar}-\mathrm{H}\right)$, $7.37\left(\mathrm{~d}, 2 \mathrm{H},{ }^{4} J_{\mathrm{H}, \mathrm{H}}=2.4 \mathrm{~Hz}, 4,4^{\prime}-\mathrm{Ar}-\mathrm{H}\right) .{ }^{13} \mathrm{C} \mathrm{NMR}\left(75 \mathrm{MHz}, \mathrm{CDCl}_{3}\right): \delta=31.4\left(3,3^{\prime}{ }^{-}{ }^{t} \mathrm{Bu}\right)$, $32.1\left(5,5^{\prime}-{ }^{t} \mathrm{Bu}\right), 34.7\left(5,5^{\prime}-\mathrm{CMe}_{3}\right), 35.5\left(3,3^{\prime}-\mathrm{CMe}_{3}\right), 38.2\left(\mathrm{NMe}_{2}\right), 42.8\left(\mathrm{NMe}_{2}\right), 124.3\left(4,4^{\prime}-\right.$ Ar-C), 129.3 (6, 6'-Ar-C), 132.2 (1,1'-Ar-C), 139.3 (3, 3'-Ar-C), 146.8 (5, 5'-Ar-C), 147.2 $\left(2,2^{\prime}-\mathrm{Ar}-\mathrm{C}\right), 187.1$ (CO). MS (EI+): $m / z(\%)=584(11)[\mathrm{M}]^{+}, 528(33)\left[\mathrm{M}-{ }^{t} \mathrm{Bu}\right]^{+}, 88$ (100) $\left[\mathrm{Me}_{2} \mathrm{NC}(\mathrm{S})\right]^{+}, 72(73)\left[\mathrm{Me}_{2} \mathrm{NC}(\mathrm{O})\right]^{+}$. IR $(\mathrm{KBr}): \widetilde{\nu}\left(\mathrm{cm}^{-1}\right)=1531$ (CS, m). Elemental analysis: Calcd. (\%) for $\mathrm{C}_{34} \mathrm{H}_{52} \mathrm{~N}_{2} \mathrm{O}_{2} \mathrm{~S}_{2}$ : C 69.82, H 8.96, N 4.79, S 10.96. Found: $\mathrm{C}$ 69.57, H 9.02, N 4.84, S 10.97.

\section{$2,2^{\prime}$ - Bis - ( $N, N$ - dimethylcarbamoylthio) - $3,3^{\prime}, 5,5^{\prime}$ - tetrachloro - 1, 1'-biphenyl (IVa).}

A Schlenk flask was charged with 2, 2'-bis- $N, N$-dimethylthiocarbamoyloxy)-3, $3^{\prime}, 5,5^{\prime}$-tetrachloro-1, 1'-biphenyl IIIa $(7.0 \mathrm{~g}, 14 \mathrm{mmol})$ and heated to $320^{\circ} \mathrm{C}$ without agitation for 10 minutes. The resulting yellow oil solidified at room temperature and was recrystallized (no protective atmosphere applied during work-up procedure) from chloroform / hexanes $\left(100 \mathrm{ml} / 10 \mathrm{ml}\right.$, cooling from reflux to $\left.4^{\circ} \mathrm{C}\right)$ to obtain the product as a white crystalline powder $(6.0 \mathrm{~g}, 12 \mathrm{mmol}, 85 \%)$. Samples for elemental analysis were additionally purified by repeated crystallization from chloroform / hexanes or column chromatography (silica, hexanes $\left./ \mathrm{EtOAc}=5 / 2, R_{\mathrm{f}} \simeq 0.3\right) . \mathrm{Mp}$ (uncorrected) $217^{\circ} \mathrm{C} .{ }^{1} \mathrm{H} \mathrm{NMR}\left(300 \mathrm{MHz}, \mathrm{CDCl}_{3}\right)$ : $\delta=2.91\left(\mathrm{~s}, 12 \mathrm{H}, \mathrm{NMe}_{2}\right), 7.19\left(\mathrm{~d}, 2 \mathrm{H},{ }^{4} J_{\mathrm{H}, \mathrm{H}}=2.4 \mathrm{~Hz}, 4,4^{\prime}-\mathrm{Ar}-\mathrm{H}\right), 7.54\left(\mathrm{~d}, 2 \mathrm{H},{ }^{4} J_{\mathrm{H}, \mathrm{H}}=2.1 \mathrm{~Hz}\right.$, $\left.6,6^{\prime}-\mathrm{Ar}-\mathrm{H}\right) .{ }^{13} \mathrm{C} \mathrm{NMR}\left(75 \mathrm{MHz}, \mathrm{CDCl}_{3}\right): \delta=37.1\left(\mathrm{~s}_{\mathrm{br}}, \mathrm{NMe}_{2}\right), 127.0\left(2,2^{\prime}-\mathrm{Ar}-\mathrm{C}\right), 128.8$ (4, 4'-Ar-C), 129.7 (6, 6'-Ar-C), 135.7 (1, 1'-Ar-C), 141.5 (5, 5'-Ar-C), 148.2 (3, 3'-Ar-C), $163.9(\mathrm{CO})$. MS (EI+): $m / z(\%)=498(0.1)[\mathrm{M}]^{+}, 463(4)[\mathrm{M}-\mathrm{Cl}]^{+}, 88(4)\left[\mathrm{Me}_{2} \mathrm{NC}(\mathrm{S})\right]^{+}$, $72(100)\left[\mathrm{Me}_{2} \mathrm{NC}(\mathrm{O})\right]^{+}$. IR $(\mathrm{KBr}): \widetilde{\nu}\left(\mathrm{cm}^{-1}\right)=1668(\mathrm{CO}, \mathrm{s})$. Elemental analysis: Calcd. (\%) for $\mathrm{C}_{18} \mathrm{H}_{16} \mathrm{Cl}_{4} \mathrm{~N}_{2} \mathrm{O}_{2} \mathrm{~S}_{2}$ : C 43.39, $\mathrm{H}$ 3.24, N 5.62. Found: C 43.18, H 3.35, N 5.64.

\section{$2,2^{\prime}$ - Bis - ( $N, N$ - dimethylcarbamoylthio) - $3,3^{\prime}, 5,5^{\prime}$ - tetrakis - tert - butyl-1, 1'-biphenyl (IVc).}

A Schlenk flask was charged with 2, 2'-bis-( $N, N$-dimethylthiocarbamoyloxy)-3, $3^{\prime}, 5,5^{\prime}$-tetrakis-tert-butyl-1, 1'-biphenyl IIIc $(7.0 \mathrm{~g}, 12 \mathrm{mmol})$ and heated to $320^{\circ} \mathrm{C}$ without agitation for $4 \mathrm{~h}$. The resulting dark orange oil was dissolved in a minimum amount of dichloromethane (no protective atmosphere applied during work-up procedure) and purified by column chromatography (silica, hexanes $/$ EtOAc $=10 / 1$, TLCs recorded in hexanes $/$ EtOAc $=$ 
$\left.5 / 1, R_{\mathrm{f}} \simeq 0.5\right)$ to obtain the product as a white powder $(5.0 \mathrm{~g}, 8.5 \mathrm{mmol}, 71 \%)$. Mp (uncorrected) $206{ }^{\circ} \mathrm{C} .{ }^{1} \mathrm{H} \mathrm{NMR}\left(500 \mathrm{MHz}, \mathrm{CDCl}_{3}\right): \delta=1.26\left(\mathrm{~s}, 18 \mathrm{H}, 3,3^{\prime}{ }^{-}{ }^{\mathrm{B}} \mathrm{Bu}\right), 1.50(\mathrm{~s}, 18 \mathrm{H}$, $\left.5,5^{\prime}-{ }^{t} \mathrm{Bu}\right), 2.65\left(\mathrm{~s}_{\mathrm{br}}, 12 \mathrm{H}, \mathrm{NMe}_{2}\right), 3.03\left(\mathrm{~s}, 6 \mathrm{H}, \mathrm{NMe}_{2}\right), 7.12\left(\mathrm{~s}_{\mathrm{br}}, 2 \mathrm{H}, 6,6^{\prime}-\mathrm{Ar}-\mathrm{H}\right), 7.44(\mathrm{~d}, 2 \mathrm{H}$, $\left.{ }^{4} J_{\mathrm{H}, \mathrm{H}}=2.5 \mathrm{~Hz}, 4,44^{\prime}-\mathrm{Ar}-\mathrm{H}\right) .{ }^{13} \mathrm{C} \mathrm{NMR}\left(125 \mathrm{MHz}, \mathrm{CDCl}_{3}\right): \delta=31.3\left({ }^{t} \mathrm{Bu}\right), 31.5\left({ }^{t} \mathrm{Bu}\right), 34.9$ $\left(\mathrm{CMe}_{3}\right), 36.8\left(\mathrm{~s}_{\mathrm{br}}, \mathrm{NMe}_{2}\right) 37.1\left(\mathrm{CMe}_{3}\right), 122.7$ (4, 4'-Ar-C), 123.3 (6, 6'-Ar-C), 126.3 (1, 1'-ArC), 150.6 (3, 3'-Ar-C), 151.1 (5, 5'-Ar-C), 152.2 (2, 2'-Ar-C), 167.1 (CO). MS (EI+): $\mathrm{m} / z$ $(\%)=584(12)[\mathrm{M}]^{+}, 528(32)\left[\mathrm{M}-{ }^{t} \mathrm{Bu}\right]^{+}, 88(100)\left[\mathrm{Me}_{2} \mathrm{NC}(\mathrm{S})\right]^{+}, 72(63)\left[\mathrm{Me}_{2} \mathrm{NC}(\mathrm{O})\right]^{+}$. IR $(\mathrm{KBr}): \widetilde{\nu}\left(\mathrm{cm}^{-1}\right)=1669(\mathrm{CO}, \mathrm{m})$. Elemental analysis: Calcd. (\%) for $\mathrm{C}_{34} \mathrm{H}_{52} \mathrm{~N}_{2} \mathrm{O}_{2} \mathrm{~S}_{2}$ : C 69.82, H 8.96, N 4.79, S 10.96. Found: C 69.85, H 9.02, N 4.70, S 11.00.

\section{2, 2'-Dithio-3, 3', 5, 5'-tetrachloro-1, 1'-biphenyl (Va).}

Solid, finely powdered 2, 2'-bis-( $N, N$-dimethylcarbamoylthio)-3, $3^{\prime}, 5,5^{\prime}$-tetrachloro-1, $1^{\prime}$-biphenyl IIIa $(2.0 \mathrm{~g}, 4 \mathrm{mmol})$ was added in one portion to a stirred suspension of $\mathrm{LiAlH}_{4}$ $(1.0 \mathrm{~g}, 26 \mathrm{mmol})$ in $\mathrm{THF}(90 \mathrm{ml})$ at room temperature and the resulting reaction mixture refluxed for $12 \mathrm{~h}$. Excess $\mathrm{LiAlH}_{4}$ was destroyed at $0{ }^{\circ} \mathrm{C}$ by dropwise addition of degassed water. After hydrogen evolution decreased, additional degassed water $(30 \mathrm{ml})$ was added and the resulting mixture was acidified to $\mathrm{pH}=1$ with aqueous $\mathrm{HCl}(37 \%)$. Subsequently, all volatiles were removed at $60^{\circ} \mathrm{C}$ in vacuum and the obtained pale yellow residue was extracted with $\mathrm{Et}_{2} \mathrm{O}(3 \times 75 \mathrm{ml})$ under an atmosphere of dry dinitrogen. The combined organic phases were dried over $\mathrm{Na}_{2} \mathrm{SO}_{4}$, filtered and evaporated to dryness. The crude product was stirred in boiling EtOH $(25 \mathrm{ml})$ for 20 minutes and left standing over night at $-20^{\circ} \mathrm{C}$. The precipitate was filtered off and dried in vacuum over night to afford the product as a colorless microcrystalline powder $(0.7 \mathrm{~g}, 2 \mathrm{mmol}, 50 \%)$. Mp (uncorrected) $154{ }^{\circ} \mathrm{C}$. ${ }^{1} \mathrm{H} \mathrm{NMR}$ $\left(500 \mathrm{MHz}, \mathrm{CDCl}_{3}\right): \delta=3.82(\mathrm{~s}, 2 \mathrm{H}, \mathrm{SH}), 7.06\left(\mathrm{~d}, 2 \mathrm{H},{ }^{4} J_{\mathrm{H}, \mathrm{H}}=1.2 \mathrm{~Hz}, 4,4^{\prime}-\mathrm{Ar}-\mathrm{H}\right), 7.47(\mathrm{~d}$, $\left.2 \mathrm{H},{ }^{4} J_{\mathrm{H}, \mathrm{H}}=1.2 \mathrm{~Hz}, 6,6{ }^{\prime}-\mathrm{Ar}-\mathrm{H}\right) .{ }^{13} \mathrm{C} \mathrm{NMR}\left(125 \mathrm{MHz}, \mathrm{CDCl}_{3}\right): \delta=128.4$ (Ar-C), 129.7 (ArC), 131.2 (Ar-C), 131.4 (Ar-C), 133.1 (Ar-C), 139.4 (Ar-C). MS (EI+): $m / z(\%)=354$ (33) $[\mathrm{M}]^{+}, 322(100)[\mathrm{M}-\mathrm{S}]^{+}, 286(72)[\mathrm{M}-\mathrm{S}-\mathrm{Cl}]^{+}$. IR $(\mathrm{KBr}): \widetilde{\nu}\left(\mathrm{cm}^{-1}\right)=2577(\mathrm{SH}$, m). HRMS (EI+): Calcd. $(\mathrm{m} / z)$ for $\mathrm{C}_{12} \mathrm{H}_{6} \mathrm{Cl}_{4} \mathrm{~S}_{2}$ : 353.8665. Found: 353.8635 .

\section{2, 2'-Dithio-3,3', 5, 5'-tetrakis-tert-butyl-1, 1'-biphenyl (Vc).}

A solution of 2, 2'-bis-( $N, N$-dimethylcarbamoylthio)-3, $3^{\prime}, 5,5^{\prime}$-tetrakis-tert-butyl-1, $1^{\prime}$-biphenyl IVc $(2.0 \mathrm{~g}, 3.4 \mathrm{mmol})$ in THF $(50 \mathrm{ml})$ was added dropwise during $1 \mathrm{~h}$ to a suspension of $\mathrm{LiAlH}_{4}(0.65 \mathrm{~g}, 17 \mathrm{mmol})$ in THF $(30 \mathrm{ml})$ at $0{ }^{\circ} \mathrm{C}$ and then refluxed for $3 \mathrm{~h}$. The resulting reaction mixture was stirred over night at room temperature, cooled to $0{ }^{\circ} \mathrm{C}$ and carefully quenched by dropwise addition of degassed water. After hydrogen evolution decreased, additional degassed water $(30 \mathrm{ml})$ was added and the resulting mixture was acidified to $\mathrm{pH}=1$ with diluted aqueous $\mathrm{HCl}(\simeq 12 \%)$. The reaction mixture was extracted with $\mathrm{Et}_{2} \mathrm{O}$ $(3 \times 100 \mathrm{ml})$ under an atmosphere of dry dinitrogen. The combined organic phases were dried over $\mathrm{Na}_{2} \mathrm{SO}_{4}$, filtered and evaporated to dryness. The crude product was stirred in boiling EtOH $(30 \mathrm{ml})$ for 20 minutes and left standing over night at $4{ }^{\circ} \mathrm{C}$. The precipitate was filtered off and dried in vacuum over night to afford the product as a white powder 
$(0.8 \mathrm{~g}, 1.8 \mathrm{mmol}, 52 \%) . \mathrm{Mp}$ (uncorrected) $173{ }^{\circ} \mathrm{C} .{ }^{1} \mathrm{H}$ NMR $\left(500 \mathrm{MHz}, \mathrm{CDCl}_{3}\right): \delta=1.31$ $\left(\mathrm{s}, 18 \mathrm{H}, 3,3^{\prime}{ }^{t} \mathrm{Bu}\right), 1.55\left(\mathrm{~s}, 18 \mathrm{H}, 5,5^{\prime}{ }^{t} \mathrm{Bu}\right), 3.47(\mathrm{~s}, 2 \mathrm{H}, \mathrm{SH}), 7.06\left(\mathrm{~d}, 2 \mathrm{H},{ }^{4} J_{\mathrm{H}, \mathrm{H}}=2.5 \mathrm{~Hz}, 2 \mathrm{H}\right.$, $\left.6,6^{\prime}-\mathrm{Ar}-\mathrm{H}\right), 7.48\left(\mathrm{~d}, 2 \mathrm{H},{ }^{4} J_{\mathrm{H}, \mathrm{H}}=2.0 \mathrm{~Hz}, 4,4^{\prime}-\mathrm{Ar}-\mathrm{H}\right) .{ }^{13} \mathrm{C} \mathrm{NMR}\left(125 \mathrm{MHz}, \mathrm{CDCl}_{3}\right): \delta=30.1$ $\left(3,3^{\prime}{ }^{t} \mathrm{Bu}\right), 31.4\left(5,5^{\prime}{ }^{t} \mathrm{Bu}\right), 34.7\left(5,5^{\prime}-\mathrm{CMe}_{3}\right), 36.8\left(3,3^{\prime}-\mathrm{CMe}_{3}\right), 123.6$ (4, $\left.4^{\prime}-\mathrm{Ar}-\mathrm{C}\right), 125.2$ (6, 6'-Ar-C), 127.8 (1, 1'-Ar-C), 142.2 (3, 3'-Ar-C), 147.2 (5, 5'-Ar-C), 148.0 (2, 2'-Ar-C). $\mathrm{MS}(\mathrm{EI}+): m / z(\%)=442(3)[\mathrm{M}]^{+}, 409(16)[\mathrm{M}-\mathrm{S}]^{+}, 393(10)[\mathrm{M}-\mathrm{SMe}]^{+}, 353(9)$ $\left[\mathrm{M}-\mathrm{S}^{t} \mathrm{Bu}\right]^{+}, 57(100)\left[{ }^{t} \mathrm{Bu}\right]^{+}$. IR $(\mathrm{KBr}): \widetilde{\nu}\left(\mathrm{cm}^{-1}\right)=2569(\mathrm{SH}, \mathrm{m})$. HRMS (EI+): Calcd. $(\mathrm{m} / \mathrm{z})$ for $\mathrm{C}_{28} \mathrm{H}_{42} \mathrm{~S}_{2}$ : 442.2728. Found: 442.2722 .

\section{Bis- $N, N$-(2-chloroethyl)- $N$-methylamine (VII).}

A solution of bis- $N, N$-(2-hyroxyethyl)- $N$-methylamine VI $(60 \mathrm{~g}, 57 \mathrm{ml}, 0.50 \mathrm{~mol})$ in 1,1,1trichloroethane (degassed, $45 \mathrm{ml}$ ) was added dropwise over $\simeq 2 \mathrm{~h}$ to a solution of thionylchloride $(138 \mathrm{~g}, 85 \mathrm{ml}, 1.16 \mathrm{~mol})$ in 1,1,1-trichlorethane (degassed, $90 \mathrm{ml}$ ) at $60^{\circ} \mathrm{C}$ (Note: A reflux condenser with oil bubbler on top is required, due to vigorous $\mathrm{SO}_{2}$ and $\mathrm{HCl}$ gas formation. Utilization of a dropping funnel without pressure compensation to the flask is recommended to avoid reaction of $\mathrm{HCl}$ gas with the starting material). After completed addition, the reaction mixture was stirred for $1 \mathrm{~h}$ at $60^{\circ} \mathrm{C}$ and then refluxed for $2 \mathrm{~h}$ at $80^{\circ} \mathrm{C}$. Subsequently, the reaction was carefully quenched with water $(600 \mathrm{ml})$ at $0{ }^{\circ} \mathrm{C}$. The aqueous phase was separated and the organic phase extracted with water $(2 \times 100 \mathrm{ml})$. In order to liberate the free amine, the combined aqueous solutions of the amine-hydrochloride were brought to $\mathrm{pH}=12$ by addition of a saturated aqueous $\mathrm{NaOH}$ (Caution: At this point, the crude product should be handled with glove-protection only, due to its venomous properties upon skin contact. Fortunately, this compound - known as N-Lost - is not as volatile and therefore not as harmful as the sulfur analogue S-Lost). Extraction with chloroform $(4 \times 150 \mathrm{ml})$, drying over $\mathrm{Na}_{2} \mathrm{SO}_{4}$ and evaporation of the solvent afforded the product as a colorless oil (55.0 g, $0.35 \mathrm{~mol}, 30 \%$ ) pure in ${ }^{1} \mathrm{H}$ NMR. The thus obtained compound should be directly used in the next step since storage is forbidden by the "Kriegswaffenkontrollgesetz" and preparation only allowed for academic purposes. ${ }^{1} \mathrm{H}$ NMR $\left(200 \mathrm{MHz}, \mathrm{CDCl}_{3}\right)$ : $\delta=2.31(\mathrm{~s}, 3 \mathrm{H}, \mathrm{Me}), 2.76\left(\mathrm{t}, 4 \mathrm{H},{ }^{3} J_{\mathrm{H}, \mathrm{H}}=6.8 \mathrm{~Hz}, \mathrm{CH}_{2}\right), 3.50\left(\mathrm{t}, 4 \mathrm{H},{ }^{3} J_{\mathrm{H}, \mathrm{H}}=7.0 \mathrm{~Hz}, \mathrm{CH}_{2}\right)$.

\section{Bis - $N, N$ - (2 - acetylthioethyl) - $N, N$ - dimethylammonium iodide (VIII).}

Solid potassium-thioacetate $(100 \mathrm{~g}, 0.88 \mathrm{~mol})$ was added in portions to a stirred solution of bis- $N, N$-(2-chlorethyl)- $N$-methylamine VII $(55 \mathrm{~g}, 0.35 \mathrm{~mol})$ in DMF $(300 \mathrm{ml})$ at room temperature. The reaction mixture was then heated to $90^{\circ} \mathrm{C}$ for $2 \mathrm{~h}$, cooled to $0{ }^{\circ} \mathrm{C}$ and filtered ( $\mathrm{KCl}$ precipitates during the course of the reaction as a white powder). The filtrate was evaporated (work-up procedure carried out in air) and the residual dark orange oil taken up in $\mathrm{CH}_{2} \mathrm{Cl}_{2} /$ water $(1 \mathrm{l} / 3 \mathrm{l})$. The organic layer was separated and the aqueous one extracted with $\mathrm{CH}_{2} \mathrm{Cl}_{2}(3 \times 500 \mathrm{ml})$. The combined organic phases were dried over $\mathrm{Na}_{2} \mathrm{SO}_{4}$ and the solvent evaporated to afford the crude bis- $N, N$-(2-acetylthioethyl)- $N$-methylamine $(70.0 \mathrm{~g}, 0.30 \mathrm{~mol}, 85 \%)$ as a sticky orange solid. ${ }^{1} \mathrm{H} \mathrm{NMR}\left(200 \mathrm{MHz}, \mathrm{CDCl}_{3}\right): \delta=2.28\left(\mathrm{~s}_{\mathrm{br}}\right.$, 
$9 \mathrm{H}, \mathrm{NMe}$ overlapping with Ac), $2.55\left(\mathrm{t}, 4 \mathrm{H},{ }^{3} \mathrm{~J}_{\mathrm{H}, \mathrm{H}}=7.6 \mathrm{~Hz}, \mathrm{CH}_{2}\right), 2.94\left(\mathrm{t}, 4 \mathrm{H},{ }^{3} \mathrm{~J}_{\mathrm{H}, \mathrm{H}}=7.0 \mathrm{~Hz}\right.$, $\left.\mathrm{CH}_{2}\right)$. This material was taken up in toluene $(2 \mathrm{l})$ and reacted with methyliodide $(113 \mathrm{~g}$, $50 \mathrm{ml}, 0.80 \mathrm{~mol}$ ), added in one portion at room temperature. The reaction mixture was heated to $70^{\circ} \mathrm{C}$ for $1 \mathrm{~h}$, slowly cooled to room temperature and finally to $0^{\circ} \mathrm{C}$. Colorless crystals of the target material precipitated. Filtration, rinsing with $\mathrm{Et}_{2} \mathrm{O}(2 \times 250 \mathrm{ml})$ and drying in vacuum afforded the pure product $(92.0 \mathrm{~g}, 0.24 \mathrm{~mol}, 81 \%$ with respect to the intermediate thioacetyl-substituted amine, $69 \%$ with respect to the chlorine-substituted starting material). ${ }^{1} \mathrm{H}$ NMR $\left(200 \mathrm{MHz}, \mathrm{DMSO}_{\mathrm{d}}\right): \delta=2.41(\mathrm{~s}, 6 \mathrm{H}, \mathrm{Ac}), 3.14(\mathrm{~s}, 6 \mathrm{H}$, $\left.\mathrm{NMe}_{2}\right), 3.2-3.3\left(\mathrm{~m}, 4 \mathrm{H}, \mathrm{CH}_{2}\right), 3.4-3.5\left(\mathrm{~m}, 4 \mathrm{H}, \mathrm{CH}_{2}\right) . \mathrm{MS}(\mathrm{ESI}+): m / z(\%)=250(100)$ $[\mathrm{M}-\mathrm{I}]^{+}$. Elemental Analysis: Calcd.(\%) for $\mathrm{C}_{10} \mathrm{H}_{20} \mathrm{INO}_{2} \mathrm{~S}_{2}: \mathrm{C} 31.83, \mathrm{H}$ 5.34, N 3.71, S 17.00. Found: C 31.93, H 5.27, N 3.74, 17.18.

\section{Bis- $N, N$-(2-mercaptoethyl)- $N, N$-dimethylammonium hexafluoro- phosphate (IX).}

To a cooled solution of bis- $N, N$-(2-acetylthioethyl)- $N, N$-dimethylammonium iodide VIII $(16.0 \mathrm{~g}, 42.4 \mathrm{mmol})$ in water (degassed, $400 \mathrm{ml})$ was added aqueous hydrobromic acid $(48 \%$, $200 \mathrm{ml}$ ) and the resulting reaction mixture heated to $90^{\circ} \mathrm{C}$ for $1 \mathrm{~h}$, then refluxed for 20 minutes and cooled to room temperature. Subsequently, aqueous hexafluorophosphoric acid (tech., $65-68 \%, 200 \mathrm{ml}$ ) was added in portions and the mixture left standing at $0{ }^{\circ} \mathrm{C}$ for $\simeq 4 \mathrm{~h}$. The precipitated crystalline solid was filtered off and dried in vacuum over $24 \mathrm{~h}$ (complete removal of water is crucial in order to prevent redissolution of the material during the washing procedure). Rinsing with $\mathrm{Et}_{2} \mathrm{O}(400 \mathrm{ml})$, THF $(400 \mathrm{ml})$ and another portion of $\mathrm{Et}_{2} \mathrm{O}(400 \mathrm{ml})$ and subsequent drying in vacuum for $2 \mathrm{~h}$ afforded the product $(6.20 \mathrm{~g}$, $19.9 \mathrm{mmol}, 47 \%)$ as a white solid. ${ }^{1} \mathrm{H}$ NMR $\left(200 \mathrm{MHz}\right.$, DMSO- $\left.\mathrm{d}_{6}\right): \delta=2.77-2.91(\mathrm{~m}$, $6 \mathrm{H}, \mathrm{CH}_{2}$ overlapping with $\left.\mathrm{SH}\right), 3.03(\mathrm{~s}, 6 \mathrm{H}, \mathrm{Me}), 3.38-3.46\left(\mathrm{~m}, 4 \mathrm{H}, \mathrm{CH}_{2}\right) .{ }^{1} \mathrm{H} \mathrm{NMR}$ $\left(200 \mathrm{MHz}, \mathrm{MeOH}-\mathrm{d}_{4}\right): \delta=2.8-3.0\left(\mathrm{~m}_{\mathrm{br}}, 4 \mathrm{H}, \mathrm{CH}_{2}\right), 3.12\left(\mathrm{~s}_{\mathrm{br}}, 6 \mathrm{H}, \mathrm{Me}\right), 3.4-3.6\left(\mathrm{~m}_{\mathrm{br}}\right.$, $\left.4 \mathrm{H}, \mathrm{CH}_{2}\right) .{ }^{19} \mathrm{~F}$ NMR $\left(188 \mathrm{MHz}, \mathrm{DMSO}-\mathrm{d}_{6}\right): \delta=91.7\left(\mathrm{~d},{ }^{1} J_{\mathrm{F}, \mathrm{P}}=705 \mathrm{~Hz}, \mathrm{PF}_{6}\right) .{ }^{31} \mathrm{P} \mathrm{NMR}$ $\left(81 \mathrm{MHz}, \mathrm{DMSO}-\mathrm{d}_{6}\right): \delta=-143.8\left(\right.$ sept, $\left.{ }^{1} J_{\mathrm{F}, \mathrm{P}}=705 \mathrm{~Hz}, \mathrm{PF}_{6}\right)$. MS (ESI+): $m / z(\%)=166$ (100) $\left[\mathrm{M}-\mathrm{PF}_{6}\right]^{+}$. Elemental Analysis: Calcd.(\%) for $\mathrm{C}_{6} \mathrm{H}_{16} \mathrm{~F}_{6} \mathrm{NPS}_{2}$ : C 23.15, H 5.18, $\mathrm{N}$ 4.50, S 20.60. Found: C 23.13, H 5.28, N 4.54, 20.98.

\section{2, 2'-Methylenediphenol (XVII).}

To a solution of 2, 4-di-tert-butylphenol XV (500 g, $2.42 \mathrm{~mol}$ ) and para-formaldehyde (36 g, $1.20 \mathrm{~mol}$ ) in xylene ( $260 \mathrm{~g}$, mixture of isomers) was added aqueous $\mathrm{HCl}(37 \%, 40 \mathrm{ml})$ and the resulting reaction mixture was stirred for $4 \mathrm{~d}$ at $100^{\circ} \mathrm{C}$. All volatiles were distilled off, the red-brown residue was suspended in hexanes (1l) and vigorously stirred over night. The undissolved white solid was filtered off and washed with hexanes $(2 \times 450 \mathrm{ml})$ to afford the off-white 6,6'-methylenebis-(2,4-di-tert-butylphenol) XVI (350 g). The combined filtrates and washings were condensed to a volume of 11 . The precipitate formed during condensation was filtered off and washed with hexanes $(2 \times 150 \mathrm{ml})$ to afford a second crop of XVI (100 g). Total yield of 6,6'-methylenebis-(2, 4-di-tert-butylphenol) XVI: $450 \mathrm{~g}, 1.06 \mathrm{~mol}$, 
$87 \%$. Analytical data were identical to those previously reported: ${ }^{[205]}{ }^{1} \mathrm{H}$ NMR $(500 \mathrm{MHz}$, $\left.\mathrm{CDCl}_{3}\right): \delta=1.27\left(\mathrm{~s}, 18 \mathrm{H}, 2,2^{\prime}{ }^{t} \mathrm{Bu}\right), 1.40\left(\mathrm{~s}, 18 \mathrm{H}, 4,4^{\prime}{ }^{t} \mathrm{Bu}\right), 3.92\left(\mathrm{~s}, 2 \mathrm{H}, \mathrm{CH}_{2}\right), 4.20-6.20$ $\left(\mathrm{s}_{\mathrm{br}}, 2 \mathrm{H}, \mathrm{OH}\right), 7.14\left(\mathrm{~d}, 2 \mathrm{H},{ }^{4} J_{\mathrm{H}, \mathrm{H}}=2.4 \mathrm{~Hz}, 5,5^{\prime}-\mathrm{Ar}-\mathrm{H}\right), 7.18\left(\mathrm{~d}, 2 \mathrm{H},{ }^{4} J_{\mathrm{H}, \mathrm{H}}=2.4 \mathrm{~Hz}, 3,3^{\prime}-\mathrm{Ar}-\right.$ H). ${ }^{13} \mathrm{C} \mathrm{NMR}\left(125 \mathrm{MHz}, \mathrm{CDCl}_{3}\right): \delta=30.0\left({ }^{t} \mathrm{Bu}\right), 31.6\left({ }^{t} \mathrm{Bu}\right), 32.5\left(\mathrm{CMe}_{3}\right), 34.3\left(\mathrm{CMe}_{3}\right)$, $34.6\left(\mathrm{CH}_{2}\right), 122.5$ (Ar-C), 125.2 (Ar-C), 126.1 (Ar-C), 135.5 (Ar-C), 143.0 (Ar-C), 149.9 $\left(\right.$ Ar-C). MS $(\mathrm{EI}+): m / z(\%)=424(100)[\mathrm{M}]^{+}$.

To a stirred solution of XVI $(42.4 \mathrm{~g}, 0.10 \mathrm{~mol})$ in toluene $(500 \mathrm{ml})$ at $0{ }^{\circ} \mathrm{C}$ was added a solution of anhydrous $\mathrm{AlCl}_{3}(26.6 \mathrm{~g}, 0.20 \mathrm{~mol})$ in toluene $(400 \mathrm{ml})$ and nitromethane $(60 \mathrm{ml})$. The reaction mixture was allowed to warm to room temperature over night and quenched with diluted aqueous $\mathrm{HCl}$ (11, prepared from $500 \mathrm{ml}$ water and $500 \mathrm{ml} 37 \%$ aqueous $\mathrm{HCl}$ ). No protective atmosphere was applied during the work-up procedure. The resulting mixture was stirred at room temperature until two clear phases were obtained. The organic layer was separated and the aqueous phase was extracted with $\mathrm{Et}_{2} \mathrm{O}(3 \times 400 \mathrm{ml})$. The combined organic phases were dried over $\mathrm{Na}_{2} \mathrm{SO}_{4}$, filtered and evaporated to dryness. The byproduct (tert-butyltoluene) was distilled off at $70^{\circ} \mathrm{C} / 10^{-3}$ mbar to obtain the intermediate crude 2, 2'-methylenebis-(4-tert-butylphenol) (30 g). ${ }^{[206]}{ }^{1} \mathrm{H} \mathrm{NMR}\left(200 \mathrm{MHz}, \mathrm{CDCl}_{3}\right)$ : $\delta=1.25\left(\mathrm{~s}, 18 \mathrm{H},{ }^{t} \mathrm{Bu}\right), 3.89\left(\mathrm{~s}, 2 \mathrm{H}, \mathrm{CH}_{2}\right), 6.72\left(\mathrm{~d}, 2 \mathrm{H},{ }^{3} J_{\mathrm{H}, \mathrm{H}}=8.4 \mathrm{~Hz}, 6,6^{\prime}-\mathrm{Ar}-\mathrm{H}\right), 7.10$ $\left(\mathrm{dd}, 2 \mathrm{H},{ }^{3} J_{\mathrm{H}, \mathrm{H}}=8.2 \mathrm{~Hz},{ }^{4} J_{\mathrm{H}, \mathrm{H}}=2.4 \mathrm{~Hz}, 5,5^{\prime}-\mathrm{Ar}-\mathrm{H}\right), 7.28\left(\mathrm{~d}, 2 \mathrm{H},{ }^{4} J_{\mathrm{H}, \mathrm{H}}=2.4 \mathrm{~Hz}, 3,3^{\prime}-\mathrm{Ar}-\mathrm{H}\right)$ ). The crude 2, $2^{\prime}$-methylenebis-(4-tert-butylphenol) was redissolved in dry toluene (300 ml) and the solution added dropwise during 10 minutes to a stirred suspension of anhydrous $\mathrm{AlCl}_{3}(32.2 \mathrm{~g}, 0.24 \mathrm{~mol})$ in toluene $(150 \mathrm{ml})$. The resulting reaction mixture was stirred at $50^{\circ} \mathrm{C}$ for $18 \mathrm{~h}$, cooled to room temperature and quenched with diluted aqueous $\mathrm{HCl}$ $(600 \mathrm{ml}, 10 \%)$. No protective atmosphere was applied during the work-up procedure. The organic layer was separated and the aqueous layer was extracted with $\mathrm{Et}_{2} \mathrm{O}(2 \times 300 \mathrm{ml})$. The combined organic phases were washed with water and the solvent evaporated. The byproduct (4-tert-butyltoluene) was removed together with remaining amounts of water by distillation $\left(70^{\circ} \mathrm{C} / 10^{-3} \mathrm{mbar}\right)$. Distillation in high vacuum $\left(170^{\circ} \mathrm{C} / 10^{-6} \mathrm{mbar}\right)$ afforded a yellow-grey sticky crude product. It was suspended in hexanes $(400 \mathrm{ml})$ and vigorously stirred for $4 \mathrm{~h}$ at $50^{\circ} \mathrm{C}$, cooled to room temperature, filtered off and dried in vacuum to afford the off-white product XVII as a fine powder $(15.0 \mathrm{~g}, 0.75 \mathrm{~mol}, 78 \%)$. Analytical data were identical to those previously reported: ${ }^{[207]}{ }^{1} \mathrm{H} \mathrm{NMR}\left(500 \mathrm{MHz}, \mathrm{CDCl}_{3}\right): \delta=3.92$ $\left(\mathrm{s}, 2 \mathrm{H}, \mathrm{CH}_{2}\right), 5.20-6.30\left(\mathrm{~s}_{\mathrm{br}}, 2 \mathrm{H}, \mathrm{OH}\right), 6.80(\mathrm{~m}, 2 \mathrm{H}, \mathrm{Ar}-\mathrm{H}), 6.88(\mathrm{~m}, 2 \mathrm{H}, \mathrm{Ar}-\mathrm{H}), 7.07$ (m, $2 \mathrm{H}, \mathrm{Ar}-\mathrm{H}), 7.25(\mathrm{~m}, 2 \mathrm{H}, \mathrm{Ar}-\mathrm{H}) .{ }^{13} \mathrm{C} \mathrm{NMR}\left(125 \mathrm{MHz}, \mathrm{CDCl}_{3}\right): \delta=30.8\left(\mathrm{CH}_{2}\right), 115.7(\mathrm{Ar}-$ C), 121.5 (Ar-C), 126.8 (Ar-C), 128.0 (Ar-C), 130.8 (Ar-C), 152.5 (Ar-C). MS (EI+): m/z $(\%)=200(80)[\mathrm{M}]^{+}, 107(100)\left[\mathrm{M}-\mathrm{C}_{6} \mathrm{H}_{5} \mathrm{OH}\right]^{+}$.

\section{2,2'-Methylenebis-(2,1-phenylene)-bis- $O, O^{\prime}$ - (dimethylcarbamo- thioate) (XVIII).}

To a stirred solution of 2, 2'-methylenediphenol XVII (12.5 g, $62 \mathrm{mmol})$ in DMF (175 ml) was added $\mathrm{NaH}(4.25 \mathrm{~g}, 177 \mathrm{mmol})$ in portions during 15 minutes. HMPA (25 ml) was then added and the reaction mixture stirred at room temperature for $2.5 \mathrm{~h}$. Dimethylcarbamothioic chloride $(27.0 \mathrm{~g}, 220 \mathrm{mmol})$ was then added in one portion and the resulting yellow suspension stirred at $80-85^{\circ} \mathrm{C}$ for $36 \mathrm{~h}$. The resulting reaction mixture was 
cooled to room temperature, quenched with water (1l) and extracted with a mixture of chloroform / pentane $(4 / 1,4 \times 200 \mathrm{ml})$. No protective atmosphere was applied during the work-up procedure. The combined organic phases were washed with aqueous $\mathrm{NaOH}(10 \%$, $2 \times 100 \mathrm{ml})$ and brine $(200 \mathrm{ml})$, dried over $\mathrm{MgSO}_{4}$, filtered and evaporated to dryness. The resulting brown liquid was treated with methanol $(250 \mathrm{ml})$, causing the crude product to precipitate as a yellow powder. It was filtered off and recrystallized from methanol $\left(200 \mathrm{ml}\right.$, cooling from reflux to $\left.+4{ }^{\circ} \mathrm{C}\right)$ to afford the product as a white crystalline powder (17.1 g, $46 \mathrm{mmol}, 74 \%)$. Mp (uncorrected) $163^{\circ} \mathrm{C} .{ }^{1} \mathrm{H}$ NMR $\left(300 \mathrm{MHz}, \mathrm{CDCl}_{3}\right)$ : $\delta=3.08\left(\mathrm{~s}, 6 \mathrm{H}, \mathrm{NMe}_{2}\right), 3.39\left(\mathrm{~s}, 6 \mathrm{H}, \mathrm{NMe}_{2}\right), 3.85\left(\mathrm{~s}, 2 \mathrm{H}, \mathrm{CH}_{2}\right), 7.01\left(\mathrm{dd}, 2 \mathrm{H},{ }^{3} J_{\mathrm{H}, \mathrm{H}}=\right.$ $\left.7.9 \mathrm{~Hz},{ }^{4} J_{\mathrm{H}, \mathrm{H}}=1.3 \mathrm{~Hz}, 6,6^{\prime}-\mathrm{Ar}-\mathrm{H}\right), 7.08\left(\mathrm{dd}, 2 \mathrm{H},{ }^{3} J_{\mathrm{H}, \mathrm{H}}=7.4 \mathrm{~Hz},{ }^{4} J_{\mathrm{H}, \mathrm{H}}=1.9 \mathrm{~Hz}, 3,3^{\prime}-\mathrm{Ar}-\right.$ $\mathrm{H}), 7.15\left(\mathrm{dt}, 2 \mathrm{H},{ }^{3} J_{\mathrm{H}, \mathrm{H}}=7.4 \mathrm{~Hz},{ }^{4} J_{\mathrm{H}, \mathrm{H}}=1.3 \mathrm{~Hz}, 4,4^{\prime}-\mathrm{Ar}-\mathrm{H}\right), 7.26\left(\mathrm{dt}, 2 \mathrm{H},{ }^{3} J_{\mathrm{H}, \mathrm{H}}=7.9 \mathrm{~Hz}\right.$, $\left.{ }^{4} J_{\mathrm{H}, \mathrm{H}}=1.9 \mathrm{~Hz}, 5,5^{\prime}-\mathrm{Ar}-\mathrm{H}\right) .{ }^{13} \mathrm{C} \mathrm{NMR}\left(75 \mathrm{MHz}, \mathrm{CDCl}_{3}\right): \delta=31.4\left(\mathrm{NMe}_{2}\right), 38.5\left(\mathrm{CH}_{2}\right), 43.2$ $\left(\mathrm{NMe}_{2}\right.$ ), 123.3 (Ar-C), 126.1 (Ar-C), 127.4 (Ar-C), 130.5 (Ar-C), 132.5 (Ar-C), 152.3 (ArC), 186.7 (CS). MS (EI+): $m / z(\%)=374(10)[\mathrm{M}]^{+}, 270(25)\left[\mathrm{M}-\mathrm{Me}_{2} \mathrm{NC}(\mathrm{O}) \mathrm{S}\right]^{+}, 197(20)$ $\left[\mathrm{M}-\mathrm{Me}_{2} \mathrm{NC}(\mathrm{O}) \mathrm{SC}(\mathrm{O}) \mathrm{NMe}_{2}\right]^{+}, 88(100)\left[\mathrm{Me}_{2} \mathrm{NCS}\right]^{+}, 72(40)\left[\mathrm{Me}_{2} \mathrm{NC}(\mathrm{O})\right]^{+}$. IR (KBr): $\widetilde{\nu}$ $\left(\mathrm{cm}^{-1}\right)=1535$ (CS, s). Elemental analysis: Calcd. (\%) for $\mathrm{C}_{19} \mathrm{H}_{22} \mathrm{~N}_{2} \mathrm{O}_{2} \mathrm{~S}_{2}$ : C 60.93, H 5.92, N 7.48, S 17.12. Found: C 60.68, H 5.88, N 7.34, S 16.87.

\section{$2,2^{\prime}$ - Methylenebis- (2,1 - phenylene) - bis - $S, S^{\prime}$ - (dimethylcarbamo- thioate (XIX).}

A $250 \mathrm{ml}$ Schlenk flask was charged with XVIII $(8.0 \mathrm{~g}, 21 \mathrm{mmol})$ and heated to $310^{\circ} \mathrm{C}$ without agitation for 45 minutes. The resulting yellow oil was cooled to room temperature and purified by column chromatography in air (silica, $\mathrm{CH}_{2} \mathrm{Cl}_{2} / \mathrm{EtOAc}=20 / 1$, $\mathrm{R}_{\mathrm{f}} \simeq 0.25$ ). The pale yellow product was dried at $120^{\circ} \mathrm{C} / 10^{-3} \mathrm{mbar}$ for $4 \mathrm{~h}$ in order to remove remaining EtOAc. The product solidified after standing for several days at room temperature and was ground in a mortar to afford a fine yellow powder $(5.2 \mathrm{~g}, 14 \mathrm{mmol}$, $65 \%)$. Mp (uncorrected) $76{ }^{\circ} \mathrm{C} .{ }^{1} \mathrm{H} \mathrm{NMR}\left(300 \mathrm{MHz}, \mathrm{CDCl}_{3}\right): \delta=3.00\left(\mathrm{~s}_{\mathrm{br}}, 12 \mathrm{H}, \mathrm{NMe}_{2}\right)$, $4.31\left(\mathrm{~s}, 2 \mathrm{H}, \mathrm{CH}_{2}\right), 7.05\left(\mathrm{dd}, 2 \mathrm{H},{ }^{3} J_{\mathrm{H}, \mathrm{H}}=7.5 \mathrm{~Hz},{ }^{4} J_{\mathrm{H}, \mathrm{H}}=1.7 \mathrm{~Hz}, 3,3^{\prime}-\mathrm{Ar}-\mathrm{H}\right), 7.20$ (dt, $2 \mathrm{H}$, $\left.{ }^{3} J_{\mathrm{H}, \mathrm{H}}=7.5 \mathrm{~Hz},{ }^{4} J_{\mathrm{H}, \mathrm{H}}=1.7 \mathrm{~Hz}, 4,4^{\prime}-\mathrm{Ar}-\mathrm{H}\right), 7.27\left(\mathrm{dt}, 2 \mathrm{H},{ }^{3} J_{\mathrm{H}, \mathrm{H}}=7.5 \mathrm{~Hz},{ }^{4} J_{\mathrm{H}, \mathrm{H}}=1.7 \mathrm{~Hz}, 5,5^{\prime}-\right.$ Ar-H), $7.50\left(\mathrm{dd}, 2 \mathrm{H},{ }^{3} J_{\mathrm{H}, \mathrm{H}}=7.5 \mathrm{~Hz},{ }^{4} J_{\mathrm{H}, \mathrm{H}}=1.7 \mathrm{~Hz}, 6,66^{\prime}-\mathrm{Ar}-\mathrm{H}\right) .{ }^{13} \mathrm{C} \mathrm{NMR}\left(75 \mathrm{MHz}, \mathrm{CDCl}_{3}\right)$ : $\delta=36.9\left(\mathrm{NMe}_{2}\right), 38.3\left(\mathrm{CH}_{2}\right), 126.8$ (Ar-C), 128.4 (Ar-C), 130.0 (Ar-C), 130.6 (Ar-C), 137.5 (Ar-C), 145.3 (Ar-C), 166.5 (CO). MS (EI+): $m / z(\%)=374(15)[\mathrm{M}]^{+}, 270(25)$ $\left[\mathrm{M}-\mathrm{Me}_{2} \mathrm{NC}(\mathrm{O}) \mathrm{S}\right]^{+}, 197(35)\left[\mathrm{M}-\mathrm{Me}_{2} \mathrm{NC}(\mathrm{O}) \mathrm{SC}(\mathrm{O}) \mathrm{NMe}_{2}\right]^{+}, 72(100)\left[\mathrm{Me}_{2} \mathrm{NC}(\mathrm{O})\right]^{+}$. IR $(\mathrm{KBr}): \widetilde{\nu}\left(\mathrm{cm}^{-1}\right)=1670(\mathrm{CO}, \mathrm{s})$. Elemental analysis: Calcd. (\%) for $\mathrm{C}_{19} \mathrm{H}_{22} \mathrm{~N}_{2} \mathrm{O}_{2} \mathrm{~S}_{2}$ : C 60.93, H 5.92, N 7.48, S 17.12. Found: C 60.62, H 5.76, N 7.42, S 16.98.

\section{2, 2'-Methylenedibenzenethiol $\left(\mathrm{XIV}^{\mathrm{C}}\right)$.}

A solution of XIX $(4.8 \mathrm{~g}, 13 \mathrm{mmol})$ in THF $(60 \mathrm{ml})$ was slowly added to a suspension of $\mathrm{LiAlH}_{4}(2.4 \mathrm{~g}, 63 \mathrm{mmol})$ in THF $(120 \mathrm{ml})$ and heated to reflux for $24 \mathrm{~h}$. The resulting reaction mixture was cooled to $0{ }^{\circ} \mathrm{C}$ and excess $\mathrm{LiAlH}_{4}$ was destroyed by dropwise addition of degassed water. After hydrogen evolution decreased additional degassed water $(180 \mathrm{ml})$ 
was added and the resulting mixture was acidified to $\mathrm{pH}=1$ with aqueous $\mathrm{HCl}(37 \%)$. The reaction mixture was extracted with $\mathrm{Et}_{2} \mathrm{O}(4 \times 120 \mathrm{ml})$ under an atmosphere of dry dinitrogen. The combined organic phases were dried over $\mathrm{MgSO}_{4}$, filtered and evaporated to dryness. The crude product was taken up in boiling hexanes $(30 \mathrm{ml})$ and filtered through dry celite. After washing the celite pad with hot hexanes $(30 \mathrm{ml})$ all volatiles were removed in vacuum to afford the product as a colorless microcrystalline powder $(2.0 \mathrm{~g}, 8.6 \mathrm{mmol}$, $66 \%$ ). $\mathrm{Mp}$ (uncorrected) $57^{\circ} \mathrm{C} .{ }^{1} \mathrm{H}$ NMR $\left(500 \mathrm{MHz}, \mathrm{CDCl}_{3}\right): \delta=3.33(\mathrm{~s}, 2 \mathrm{H}, \mathrm{SH}), 4.05$ $\left(\mathrm{s}, 2 \mathrm{H}, \mathrm{CH}_{2}\right), 6.93\left(\mathrm{dd}, 2 \mathrm{H},{ }^{3} J_{\mathrm{H}, \mathrm{H}}=7.9 \mathrm{~Hz},{ }^{4} J_{\mathrm{H}, \mathrm{H}}=1.9 \mathrm{~Hz}, 3,3^{\prime}-\mathrm{Ar}-\mathrm{H}\right), 7.09-7.12(\mathrm{~m}, 4 \mathrm{H}$, $\left.4,4^{\prime}-\mathrm{Ar}-\mathrm{H}, 5,5^{\prime}-\mathrm{Ar}-\mathrm{H}\right), 7.35\left(\mathrm{dd}, 2 \mathrm{H},{ }^{3} J_{\mathrm{H}, \mathrm{H}}=7.1 \mathrm{~Hz},{ }^{4} J_{\mathrm{H}, \mathrm{H}}=1.9 \mathrm{~Hz}, 6,6{ }^{\prime}-\mathrm{Ar}-\mathrm{H}\right) .{ }^{13} \mathrm{C} \mathrm{NMR}$ $\left(125 \mathrm{MHz}, \mathrm{CDCl}_{3}\right): \delta=39.0\left(\mathrm{CH}_{2}\right), 126.3$ (Ar-C), 127.2 (Ar-C), 129.9 (Ar-C), 130.9 (Ar-C), $131.2\left(\right.$ Ar-C), $137.4\left(\right.$ Ar-C). MS (EI+): $m / z(\%)=232(55)[\mathrm{M}]^{+}, 197(100)\left[\mathrm{M}-\mathrm{H}_{2} \mathrm{~S}\right]^{+}$. $\mathrm{IR}(\mathrm{KBr}): \widetilde{\nu}\left(\mathrm{cm}^{-1}\right)=2563(\mathrm{SH}, \mathrm{m})$. HRMS (EI+): Calcd. $(\mathrm{m} / z)$ for $\mathrm{C}_{13} \mathrm{H}_{12} \mathrm{~S}_{2}: 232.0380$. Found: 232.0379. 


\title{
9.3 Synthesis of Iron Complexes and Cluster Com- pounds
}

\author{
Bis-(tetraethylammonium)-bis-[di-(indolato)-( $\mu$-sulfido)-ferrate- \\ (III)] (5).
}

To a solution of indole $(4.70 \mathrm{~g}, 40.1 \mathrm{mmol})$ in THF $(50 \mathrm{ml})$ at $0{ }^{\circ} \mathrm{C}$ was added dropwise $n$-BuLi $(13.6 \mathrm{ml}, 3.0 \mathrm{M}$ solution in hexane, $40.8 \mathrm{mmol})$ and the resulting yellow solution was stirred at $0{ }^{\circ} \mathrm{C}$ for 10 minutes. The stirring bar was then removed and a solution of $\left(\mathrm{NEt}_{4}\right)_{2}\left[\mathrm{Fe}_{2} \mathrm{~S}_{2} \mathrm{Cl}_{4}\right] 2(5.78 \mathrm{~g}, 10.0 \mathrm{mmol})$ in $\mathrm{MeCN}(200 \mathrm{ml})$ added in one portion via cannula. The flask was sealed, turned around once and left standing at room temperature for $\simeq 6 \mathrm{~h}$. Initial product precipitation occurred and was completed by storage of the reaction mixture at $-20{ }^{\circ} \mathrm{C}$ over night. Filtration, rinsing with $\mathrm{Et}_{2} \mathrm{O}$ and drying in vacuum afforded a black powder of the product $(4.50 \mathrm{~g}, 5.00 \mathrm{mmol}, 50 \%)$. ${ }^{1} \mathrm{H}$ NMR $\left(500 \mathrm{MHz}, \mathrm{DMSO}-\mathrm{d}_{6}\right): \delta=1.02$ $\left(\mathrm{s}_{\mathrm{br}}, 24 \mathrm{H}, \mathrm{NEt}_{4}\right), 2.98\left(\mathrm{~s}_{\mathrm{br}}, 16 \mathrm{H}, \mathrm{NEt}_{4}\right), 4.57\left(\mathrm{~s}_{\mathrm{br}}, 4 \mathrm{H}, \mathrm{Ar}-\mathrm{H}\right), 5.24\left(\mathrm{~s}_{\mathrm{br}}, 4 \mathrm{H}, \mathrm{Ar}-\mathrm{H}\right), 6.64\left(\mathrm{~s}_{\mathrm{br}}\right.$, $8 \mathrm{H}, \mathrm{Ar}-\mathrm{H}), 10.13\left(\mathrm{~s}_{\mathrm{br}}, 4 \mathrm{H}, \mathrm{Ar}-\mathrm{H}\right)$. MS (ESI+): $m / z(\%)=857$ (100) $[\mathrm{M}-\mathrm{Fe}-\text { indole }]^{+}$, 1030 (67) $\left[\mathrm{M}+\mathrm{NEt}_{4}\right]^{+}$. MS (ESI-): $m / z(\%)=770(100)\left[\mathrm{M}_{-} \mathrm{NEt}_{4}\right]^{-}$. UV-Vis (DMF solution): $\lambda_{\max }[\mathrm{nm}]\left(\varepsilon\left[\mathrm{M}^{-1} \mathrm{~cm}^{-1}\right]\right)=281(\simeq 50000), 288$ ( 250000$), 410(\mathrm{sh}, 12225), 520$ (16200). In several attempts no satisfactory elemental analysis was obtained with carbon values found significantly too low in all cases, e.g.: Calcd.(\%) for $\mathrm{C}_{48} \mathrm{H}_{64} \mathrm{Fe}_{2} \mathrm{~N}_{6} \mathrm{~S}_{2}$ : C 63.99, H 7.16, N 9.33, S 7.12. Found: C 61.38, H 7.01, N 8.93, S 7.68. HRMS (ESI+): Calcd. $(m / z)$ for $\mathrm{C}_{56} \mathrm{H}_{84} \mathrm{Fe}_{2} \mathrm{~N}_{7} \mathrm{~S}_{2}$ : 1030.4926. Found: 1030.4894 .

\section{Bis-(tetraethylammonium)-bis-[di-(carbazolato)-( $\mu$-sulfido $)$-fer- $\operatorname{rate}(\mathrm{III})](6)$.}

To a solution of carbazole $(1.00 \mathrm{~g}, 97 \%$ purity by $\mathrm{GC}, 5.99 \mathrm{mmol})$ in $\mathrm{THF}(30 \mathrm{ml})$ at $0{ }^{\circ} \mathrm{C}$ was added dropwise $n$-BuLi $(3.75 \mathrm{ml}, 1.6 \mathrm{~m}$ solution in hexane, $6.00 \mathrm{mmol})$ and the resulting opaque orange solution stirred at $0{ }^{\circ} \mathrm{C}$ for 30 minutes. Subsequently MeCN (10 ml) was added affording a clear orange solution, followed by addition of solid $\left(\mathrm{NEt}_{4}\right)_{2}\left[\mathrm{Fe}_{2} \mathrm{~S}_{2} \mathrm{Cl}_{4}\right] \mathbf{2}$ $(0.87 \mathrm{~g}, 1.50 \mathrm{mmol})$ and further $\mathrm{MeCN}(30 \mathrm{ml})$. The resulting purple-blue reaction mixture was stirred for $1 \mathrm{~h}$ at $0^{\circ} \mathrm{C}$ with gradual precipitation of the crude product observable during the course of the reaction. The black precipitate was filtered off, washed with $\mathrm{Et}_{2} \mathrm{O}(20 \mathrm{ml})$ and dried in vacuum for 20 minutes. The obtained solid was extracted with DMF $(5 \times 20 \mathrm{ml})$ under vigorous stirring. Subsequently, the combined DMF fractions were diluted with $\mathrm{Et}_{2} \mathrm{O}(50 \mathrm{ml})$ and left standing at $-20^{\circ} \mathrm{C}$ for $4 \mathrm{~d}$. The deep purple to black homogeneous precipitate formed was filtered off, washed with $\mathrm{Et}_{2} \mathrm{O}(2 \times 20 \mathrm{ml})$ and dried in vacuum over night to afford the powdered product $(0.53 \mathrm{~g}, 0.48 \mathrm{mmol}, 32 \%) .{ }^{1} \mathrm{H} \mathrm{NMR}$ $\left(500 \mathrm{MHz}, \mathrm{DMSO}-\mathrm{d}_{6}\right): \delta=0.99\left(\mathrm{~s}_{\mathrm{br}}, 24 \mathrm{H}, \mathrm{NEt}_{4}\right), 2.97\left(\mathrm{~s}_{\mathrm{br}}, 16 \mathrm{H}, \mathrm{NEt}_{4}\right), 3.81\left(\mathrm{~s}_{\mathrm{br}}, 8 \mathrm{H}\right.$, Ar-H), $7.69\left(\mathrm{~s}_{\mathrm{br}}, 8 \mathrm{H}, \mathrm{Ar}-\mathrm{H}\right), 11.11\left(\mathrm{~s}_{\mathrm{br}}, 8 \mathrm{H}, \mathrm{Ar}-\mathrm{H}\right) . \mathrm{MS}(\mathrm{ESI}+): m / z(\%)=1230(100)$ $\left[\mathrm{M}+\mathrm{NEt}_{4}\right]^{+}$. MS (ESI-): $m / z(\%)=970(100)\left[\mathrm{M}-\mathrm{NEt}_{4}\right]^{-}$. UV-Vis (DMF solution): $\lambda_{\max }[\mathrm{nm}]\left(\varepsilon\left[\mathrm{M}^{-1} \mathrm{~cm}^{-1}\right]\right)=293(\simeq 35000), 324$ (11000), 337 (10900), 360 (7700), 548 (9800). In several attempts no satisfactory elemental analysis was obtained with carbon values 
found significantly too low in all cases, e.g.: Calcd.(\%) for $\mathrm{C}_{64} \mathrm{H}_{72} \mathrm{Fe}_{2} \mathrm{~N}_{6} \mathrm{~S}_{2}$ : C $69.81, \mathrm{H}$ 6.59, N 7.63, S 5.82. Found: C 67.82, H 6.59, N 7.45, S 5.86. HRMS (ESI+): Calcd. $(m / z)$ for $\mathrm{C}_{72} \mathrm{H}_{92} \mathrm{Fe}_{2} \mathrm{~N}_{7} \mathrm{~S}_{2}$ : 1230.5553. Found: 1230.5515 .

\section{General experimental procedure for the ligand exchange reactions starting from $\left.\left(\mathrm{NEt}_{4}\right)_{2}\left[\mathrm{Fe}_{2} \mathrm{~S}_{2} \text { (indolate) }\right)_{4}\right] 5$.}

To a suspension of $\left.\left(\mathrm{NEt}_{4}\right)_{2}\left[\mathrm{Fe}_{2} \mathrm{~S}_{2} \text { (indolate }\right)_{4}\right] \mathbf{5}(0.90 \mathrm{~g}, 1.00 \mathrm{mmol}, 1 \mathrm{eq})$ in $\mathrm{MeCN}(50 \mathrm{ml})$ was added the neat thiophenol/phenol (4.4 eq for monodentate ligands, 2.2 eq for bidentante ligands) in one portion (solid ligands as well as liquid ones) at room temperature. The resulting reaction mixture was stirred for $24 \mathrm{~h}$ at room temperature, causing the initial reddish purple suspension to turn clear and deep red during the course of the conversion. Removal of all volatiles, extraction of the free indole by-product with THF / $\mathrm{Et}_{2} \mathrm{O}(1 / 3$, $\simeq 60 \mathrm{ml}$ of the mixture of solvents) and drying of the residual powder in vacuum afforded the target material pure in ${ }^{1} \mathrm{H}$ NMR. A single crystallization from $\mathrm{MeCN}$ or $\mathrm{MeCN} / \mathrm{Et}_{2} \mathrm{O}$ afforded homogeneous crystalline powders in all cases.

\section{Bis-(tetraethylammonium)-bis-(dithiophenolato)-( $\mu$-sulfido)-fer- rate(III)] (7a).}

Compound preparation was carried out following the general experimental procedure for ligand exchange reactions. Yield: $0.57 \mathrm{~g}, 0.65 \mathrm{mmol}, 65 \%$. ${ }^{1} \mathrm{H} \mathrm{NMR}(300 \mathrm{MHz}, \mathrm{MeCN}-$ $\left.\mathrm{d}_{3}\right): \delta=1.17\left(\mathrm{~s}_{\mathrm{br}}, 24 \mathrm{H}, \mathrm{NEt}_{4}\right), 3.09\left(\mathrm{~s}_{\mathrm{br}}, 16 \mathrm{H}, \mathrm{NEt}_{4}\right), 3.42\left(\mathrm{~s}_{\mathrm{br}}, 4 \mathrm{H}, \mathrm{Ar}-\mathrm{H}\right), 4.90\left(\mathrm{~s}_{\mathrm{br}}\right.$, $8 \mathrm{H}, \mathrm{Ar}-\mathrm{H}), 9.31\left(\mathrm{~s}_{\mathrm{br}}, 8 \mathrm{H}, \mathrm{Ar}-\mathrm{H}\right)$. MS (ESI+) $m / z(\%): 893(25)\left[\mathrm{M}+\mathrm{NEt}_{4}-\mathrm{L}\right]^{+} 1002$ (100) $\left[\mathrm{M}+\mathrm{NEt}_{4}\right]^{+}$. MS (ESI-): $m / z(\%)=394(100)\left[\mathrm{M}-2 \mathrm{NEt}_{4}-2 \mathrm{~L}\right]^{-}, 503(60)$ $\left[\mathrm{M}-2 \mathrm{NEt}_{4}-\mathrm{L}\right]^{-}, 7422(15)\left[\mathrm{M}-\mathrm{NEt}_{4}\right]^{-}$. UV-Vis (MeCN solution): $\lambda_{\max }[\mathrm{nm}](\varepsilon$ $\left.\left[\mathrm{M}^{-1} \mathrm{~cm}^{-1}\right]\right)=265(\simeq 41500), 330$ (21300), 481 (12000). In several attempts no satisfactory elemental analysis was obtained with carbon values found significantly too low in all cases, e.g.: Calcd.(\%) for $\mathrm{C}_{40} \mathrm{H}_{60} \mathrm{Fe}_{2} \mathrm{~N}_{2} \mathrm{~S}_{6}$ : C 55.03, H 6.93, N 3.21, S 22.04. Found: C 54.23, $\mathrm{H}$ 7.27, N 3.23, S 21.62. HRMS (ESI+): Calcd. $(\mathrm{m} / z)$ for $\mathrm{C}_{48} \mathrm{H}_{80} \mathrm{Fe}_{2} \mathrm{~N}_{3} \mathrm{~S}_{6}$ : 1002.3372 . Found: 1002.3326 .

\section{Bis- (tetraethylammonium) - bis - [di- (4-methylthiophenolato) - ( $\mu$ - sulfido)-ferrate(III)] (7b).}

Compound preparation was carried out following the general experimental procedure for ligand exchange reactions. Yield: $0.66 \mathrm{~g}, 0.71 \mathrm{mmol}, 71 \%$. ${ }^{1} \mathrm{H}$ NMR $\left(300 \mathrm{MHz}, \mathrm{MeCN}-\mathrm{d}_{3}\right)$ : $\delta=1.16\left(\mathrm{~s}_{\mathrm{br}}, 24 \mathrm{H}, \mathrm{NEt}_{4}\right), 3.08\left(\mathrm{~s}_{\mathrm{br}}, 16 \mathrm{H}, \mathrm{NEt}_{4}\right), 9.66\left(\mathrm{~s}_{\mathrm{br}}, 8 \mathrm{H}, \mathrm{Ar}-\mathrm{H}\right), 5.98\left(\mathrm{~s}_{\mathrm{br}}, 12 \mathrm{H}, \mathrm{Me}\right)$, $9.18\left(\mathrm{~s}_{\mathrm{br}}, 8 \mathrm{H}, \mathrm{Ar}-\mathrm{H}\right)$. MS (ESI+): $m / z(\%)=1058(100)\left[\mathrm{M}+\mathrm{NEt}_{4}\right]^{+}$. MS (ESI-): $m / z$ $(\%)=422(100)\left[\mathrm{M}-2 \mathrm{NEt}_{4}-2 \mathrm{~L}\right]^{-}, 545(40)\left[\mathrm{M}-2 \mathrm{NEt}_{4}-\mathrm{L}\right]^{-}, 798(10)\left[\mathrm{M}-\mathrm{NEt}_{4}\right]^{-}$. UV-Vis (MeCN solution): $\lambda_{\max }[\mathrm{nm}]\left(\varepsilon\left[\mathrm{M}^{-1} \mathrm{~cm}^{-1}\right]\right)=265(\simeq 42000), 335$ (20400), 488 (12000). Elemental analysis: Calcd.(\%) for $\mathrm{C}_{44} \mathrm{H}_{68} \mathrm{Fe}_{2} \mathrm{~N}_{2} \mathrm{~S}_{6}$ : C 56.88, H 7.38, N 3.02, S 
20.71. Found: C 56.41, H 7.47, N 3.00, S 20.81. HRMS (ESI+): Calcd. $(\mathrm{m} / \mathrm{z})$ for $\mathrm{C}_{52} \mathrm{H}_{88} \mathrm{Fe}_{2} \mathrm{~N}_{3} \mathrm{~S}_{6}$ : 1058.3999. Found: 1058.3987.

\section{Bis-(tetraethylammonium)-bis-[di-(2,6-dimethylthiophenolato)- ( $\mu$-sulfido)-ferrate(III)] (7c).}

Compound preparation was carried out following the general experimental procedure for ligand exchange reactions, but stirring was continued for $48 \mathrm{~h}$. Yield: $0.74 \mathrm{~g}, 0.75 \mathrm{mmol}, 75 \%$. ${ }^{1} \mathrm{H}$ NMR $\left(300 \mathrm{MHz}, \mathrm{MeCN}-\mathrm{d}_{3}\right): \delta=1.18\left(\mathrm{~s}_{\mathrm{br}}, 24 \mathrm{H}, \mathrm{NEt}_{4}\right), 2.85\left(\mathrm{~s}_{\mathrm{br}}, 4 \mathrm{H}, \mathrm{Ar}-\mathrm{H}\right), 3.09\left(\mathrm{~s}_{\mathrm{br}}\right.$, $\left.16 \mathrm{H}, \mathrm{NEt}_{4}\right), 5.76\left(\mathrm{~s}_{\mathrm{br}}, 24 \mathrm{H}, \mathrm{Me}\right), 9.76\left(\mathrm{~s}_{\mathrm{br}}, 8 \mathrm{H}, \mathrm{Ar}-\mathrm{H}\right)$. MS (ESI+): $m / z(\%)=977(45)[\mathrm{M}+$ $\left.\mathrm{NEt}_{4}-\mathrm{L}\right]^{+}, 1114(100)\left[\mathrm{M}+\mathrm{NEt}_{4}\right]^{+} . \mathrm{MS}(\mathrm{ESI}-): m / z(\%)=450(100)\left[\mathrm{M}-2 \mathrm{NEt}_{4}-2 \mathrm{~L}\right]^{-}$, $587(55)\left[\mathrm{M}-2 \mathrm{NEt}_{4}-\mathrm{L}\right]^{-}, 854(15)\left[\mathrm{M}-\mathrm{NEt}_{4}\right]^{-}$. UV-Vis (MeCN solution): $\lambda_{\max }[\mathrm{nm}](\varepsilon$ $\left.\left[\mathrm{M}^{-1} \mathrm{~cm}^{-1}\right]\right)=265(\simeq 40200), 287$ (32000), 333 (13000), 418 (10800). In several attempts no satisfactory elemental analysis was obtained with carbon values found significantly too low in all cases, e.g.: Calcd.(\%) for $\mathrm{C}_{48} \mathrm{H}_{76} \mathrm{Fe}_{2} \mathrm{~N}_{2} \mathrm{~S}_{6}$ : C 58.52, H 7.78, N 2.84, S 19.53. Found: $\mathrm{C} 57.03, \mathrm{H}$ 8.08, N 2.82, $\mathrm{S}$ 18.95. HRMS (ESI+): Calcd. $(\mathrm{m} / z)$ for $\mathrm{C}_{56} \mathrm{H}_{96} \mathrm{Fe}_{2} \mathrm{~N}_{3} \mathrm{~S}_{6}$ : 1114.4625. Found: 1114.4585.

\section{Bis-(tetraethylammonium)-bis- [di-(3,5-dimethylthiophenolato)- ( $\mu$-sulfido)-ferrate(III)] (7d).}

Compound preparation was carried out following the general experimental procedure for ligand exchange reactions. Yield: $0.80 \mathrm{~g}, 0.81 \mathrm{mmol}, 81 \%$. ${ }^{1} \mathrm{H}$ NMR $(300 \mathrm{MHz}, \mathrm{MeCN}-$ $\left.\mathrm{d}_{3}\right): \delta=1.17\left(\mathrm{~s}_{\mathrm{br}}, 24 \mathrm{H}, \mathrm{NEt}_{4}\right), 1.74\left(\mathrm{~s}_{\mathrm{br}}, \mathrm{Me}\right), 2.53\left(\mathrm{~s}_{\mathrm{br}}, \mathrm{Me}\right), 3.09\left(\mathrm{~s}_{\mathrm{br}}, 16 \mathrm{H}, \mathrm{NEt}_{4}\right), 4.60$ $\left(\mathrm{s}_{\mathrm{br}}, \mathrm{Ar}-\mathrm{H}\right), 4.98\left(\mathrm{~s}_{\mathrm{br}}, \mathrm{Ar}-\mathrm{H}\right), 5.79\left(\mathrm{~s}_{\mathrm{br}}, \mathrm{Me}\right), 6.92\left(\mathrm{~s}_{\mathrm{br}}, \mathrm{Ar}-\mathrm{H}\right), 7.13\left(\mathrm{~s}_{\mathrm{br}}, \mathrm{Ar}-\mathrm{H}\right) . \mathrm{MS}$ (ESI+): $m / z(\%)=1114(100)\left[\mathrm{M}+\mathrm{NEt}_{4}\right]^{+} . \mathrm{MS}(\mathrm{ESI}-): m / z(\%)=450(100)\left[\mathrm{M}-2 \mathrm{NEt}_{4}-2 \mathrm{~L}\right]^{-}$, $587(25)\left[\mathrm{M}-2 \mathrm{NEt}_{4}-\mathrm{L}\right]^{-}, 854(3)\left[\mathrm{M}-\mathrm{NEt}_{4}\right]^{-}$. UV-Vis (MeCN solution): $\lambda_{\max }[\mathrm{nm}](\varepsilon$ $\left.\left[\mathrm{M}^{-1} \mathrm{~cm}^{-1}\right]\right)=263(\simeq 40000), 332(20300), 473$ (7800). In several attempts no satisfactory elemental analysis was obtained with carbon values found significantly too low in all cases, e.g.: Calcd.(\%) for $\mathrm{C}_{48} \mathrm{H}_{76} \mathrm{Fe}_{2} \mathrm{~N}_{2} \mathrm{~S}_{6}$ : C 58.52, $\mathrm{H}$ 7.78, N 2.84, S 19.53. Found: C 55.25, $\mathrm{H}$ 7.46, N 2.81, S 18.74. HRMS (ESI+): Calcd. $(\mathrm{m} / z)$ for $\mathrm{C}_{56} \mathrm{H}_{96} \mathrm{Fe}_{2} \mathrm{~N}_{3} \mathrm{~S}_{6}$ : 1114.4625 . Found: 1114.4618.

\section{Bis-(tetraethylammonium)-bis-[di-(2,4-dimethylthiophenolato)- ( $\mu$-sulfido)-ferrate(III)] (7e).}

Compound preparation was carried out following the general experimental procedure for ligand exchange reactions. Yield: $0.65 \mathrm{~g}, 0.66 \mathrm{mmol}, 66 \%$. ${ }^{1} \mathrm{H}$ NMR $\left(300 \mathrm{MHz}, \mathrm{MeCN}-\mathrm{d}_{3}\right)$ : $\delta=1.16\left(\mathrm{~s}_{\mathrm{br}}, 24 \mathrm{H}, \mathrm{NEt}_{4}\right), 3.06\left(\mathrm{~s}_{\mathrm{br}}, 16 \mathrm{H}, \mathrm{NEt}_{4}\right), 5.38\left(\mathrm{~s}_{\mathrm{br}}, 12 \mathrm{H}, \mathrm{Me}\right), 6.05\left(\mathrm{~s}_{\mathrm{br}}, 12 \mathrm{H}, \mathrm{Me}\right)$, $9.18\left(\mathrm{~s}_{\mathrm{br}}, 4 \mathrm{H}, \mathrm{Ar}-\mathrm{H}\right), 10.18\left(\mathrm{~s}_{\mathrm{br}}, 4 \mathrm{H}, \mathrm{Ar}-\mathrm{H}\right)$. MS (ESI+): $m / z(\%)=1114(100)\left[\mathrm{M}^{2} \mathrm{NEt}_{4}\right]^{+}$. MS (ESI-): $m / z(\%)=450(100)\left[\mathrm{M}-2 \mathrm{NEt}_{4}-2 \mathrm{~L}\right]^{-}, 587(15)\left[\mathrm{M}-2 \mathrm{NEt}_{4}-\mathrm{L}\right]^{-}, 854$ (3) $\left[\mathrm{M}-\mathrm{NEt}_{4}\right]^{-}$. UV-Vis (MeCN solution): $\lambda_{\max }[\mathrm{nm}]\left(\varepsilon\left[\mathrm{M}^{-1} \mathrm{~cm}^{-1}\right]\right)=262(\simeq 44700)$, 
340 (21600), 432 (13100). In several attempts no satisfactory elemental analysis was obtained since carbon values were found significantly too low in all cases, e.g.: Calcd.(\%) for $\mathrm{C}_{48} \mathrm{H}_{76} \mathrm{Fe}_{2} \mathrm{~N}_{2} \mathrm{~S}_{6}$ : C 58.52, H 7.78, N 2.84, S 19.53. Found: C 56.92, H 7.66, N 2.78, S 19.17. HRMS (ESI+): Calcd. $(m / z)$ for $\mathrm{C}_{56} \mathrm{H}_{96} \mathrm{Fe}_{2} \mathrm{~N}_{3} \mathrm{~S}_{6}$ : 1114.4625. Found: 1114.4622 .

\section{Bis-(tetraethylammonium)-bis-[di-(4-fluorothiophenolato)-( $\mu$-sul- fido)-ferrate(III)] (7f).}

Compound preparation was carried out following the general experimental procedure for ligand exchange reactions. Yield: $0.53 \mathrm{~g}, 0.56 \mathrm{mmol}, 56 \% .{ }^{1} \mathrm{H} \mathrm{NMR}\left(300 \mathrm{MHz}, \mathrm{MeCN}-\mathrm{d}_{3}\right)$ : $\delta=1.18\left(\mathrm{~s}_{\mathrm{br}}, 24 \mathrm{H}, \mathrm{NEt}_{4}\right), 3.10\left(\mathrm{~s}_{\mathrm{br}}, 16 \mathrm{H}, \mathrm{NEt}_{4}\right), 4.71\left(\mathrm{~s}_{\mathrm{br}}, 8 \mathrm{H}, \mathrm{Ar}-\mathrm{H}\right), 8.94\left(\mathrm{~s}_{\mathrm{br}}, 8 \mathrm{H}, \mathrm{Ar}-\right.$ H). ${ }^{19} \mathrm{~F} \mathrm{NMR}\left(188 \mathrm{MHz}, \mathrm{MeCN}_{3}\right): \delta=-115.6\left(\mathrm{~s}_{\mathrm{br}}\right)$. MS (ESI+): $m / z(\%)=1030(80)$ $\left[\mathrm{M}+\mathrm{NEt}_{4}-\mathrm{C}_{2} \mathrm{H}_{6} \mathrm{~N}\right.$ (fragment of $\left.\left.\mathrm{NEt}_{4}\right)\right]^{+}, 1074(100)\left[\mathrm{M}+\mathrm{NEt}_{4}\right]^{+}$. MS (ESI-): $\mathrm{m} / z$ $(\%)=430(100)\left[\mathrm{M}-2 \mathrm{NEt}_{4}-2 \mathrm{~L}\right]^{-}, 557(35)\left[\mathrm{M}-2 \mathrm{NEt}_{4}-\mathrm{L}\right]^{-}, 814(10)\left[\mathrm{M}-\mathrm{NEt}_{4}\right]^{-}$. UV-Vis (MeCN solution): $\lambda_{\max }[\mathrm{nm}]\left(\varepsilon\left[\mathrm{M}^{-1} \mathrm{~cm}^{-1}\right]\right)=257(\mathrm{sh}, \simeq 44200), 310(22400), 460$ (sh, $\simeq 10400)$. Elemental analysis: Calcd.(\%) for $\mathrm{C}_{40} \mathrm{H}_{56} \mathrm{~F}_{4} \mathrm{Fe}_{2} \mathrm{~N}_{2} \mathrm{~S}_{6}$ : C 50.84, H 5.97, N 2.96, S 20.36. Found: C 50.61, H 5.71, N 3.41, S 19.40. HRMS (ESI+): Calcd. $(\mathrm{m} / \mathrm{z}$ ) for $\mathrm{C}_{48} \mathrm{H}_{76} \mathrm{~F}_{4} \mathrm{Fe}_{2} \mathrm{~N}_{3} \mathrm{~S}_{6}$ : 1074.2995. Found: 1074.2981.

\section{Bis-(tetraethylammonium)-bis- $\left[\left(3,3^{\prime}, 5,5^{\prime}\right.\right.$-tetrachloro-1, $1^{\prime}$-biphen- yl-2, $2^{\prime}$-diolato)-( $\mu$-sulfido)-ferrate(III)] (8a).}

Compound preparation was carried out following the general experimental procedure for ligand exchange reactions with a slightly modified work-up method: After complete conversion was observed (by color change), the MeCN solution was filtered via cannula leaving a small amount $(\simeq 25 \mathrm{mg}$ ) of an insoluble impurity behind. The filtrate was condensed to dryness and the residual crude product crystallized from THF $(20 \mathrm{ml}$, no complete dissolution of the product takes place - cooling from room temperature to $-20{ }^{\circ} \mathrm{C}$ causes precipitation of the product) to afford the product as a fine powder. Yield: $0.60 \mathrm{~g}, 0.55 \mathrm{mmol}$, $55 \% .{ }^{1} \mathrm{H}$ NMR $\left(500 \mathrm{MHz}, \mathrm{MeCN}_{-} \mathrm{d}_{3}\right): \delta=1.15\left(\mathrm{~s}_{\mathrm{br}}, 24 \mathrm{H}, \mathrm{NEt}_{4}\right), 3.10\left(\mathrm{~s}_{\mathrm{br}}, 16 \mathrm{H}, \mathrm{NEt}_{4}\right)$, $9.22\left(\mathrm{~s}_{\mathrm{br}}, 4 \mathrm{H}, \mathrm{Ar}-\mathrm{H}\right), 9.84(\mathrm{sbr}, 4 \mathrm{H}, \mathrm{Ar}-\mathrm{H})$. MS (ESI+): $m / z(\%)=1210(100)\left[\mathrm{M}+\mathrm{NEt}_{4}\right]^{+}$. MS (ESI-) m/z (\%): 950 (100) $\left[\mathrm{M}-\mathrm{NEt}_{4}\right]^{-}$. UV-Vis (MeCN solution): $\lambda_{\max }[\mathrm{nm}](\varepsilon$ $\left.\left[\mathrm{M}^{-1} \mathrm{~cm}^{-1}\right]\right)=278(\simeq 26000), 315(\simeq 27000), 413$ (9700), 492 (sh, 4500), 552 (sh, 2900). In several attempts no satisfactory elemental analysis was obtained since carbon values were found significantly too low in all cases, e.g.: Calcd.(\%) for $\mathrm{C}_{40} \mathrm{H}_{48} \mathrm{Cl}_{8} \mathrm{Fe}_{2} \mathrm{~N}_{2} \mathrm{O}_{4} \mathrm{~S}_{2}$ : C 44.47, H 4.48, N 2.95. Found: C 43.22, H 4.97, N 2.95. HRMS (ESI+): Calcd. $(\mathrm{m} / z)$ for $\mathrm{C}_{48} \mathrm{H}_{68} \mathrm{Cl}_{8} \mathrm{Fe}_{2} \mathrm{~N}_{3} \mathrm{O}_{4} \mathrm{~S}_{2}$ : 1206.0859. Found: 1206.0842 .

Compound $\mathbf{8} \mathbf{a}$ is also accesible via salt metathesis reaction. The experimental procedure is provided here for completeness and comparison reasons: To a solution of $3,3^{\prime}, 5,5^{\prime}-$ tetrachlor-2, 2'-dihydroxy-1, $1^{\prime}$-biphenyl IIa $(0.68 \mathrm{~g}, 2.08 \mathrm{mmol})$ in THF $(20 \mathrm{ml})$ at $0{ }^{\circ} \mathrm{C}$ was added $\mathrm{KH}(0.17 \mathrm{~g}, 4.18 \mathrm{mmol})$ in portions and the resulting reaction mixture stirred at room temperature for $1.5 \mathrm{~h}$. The slightly yellow solution formed during this time was diluted with MeCN $(60 \mathrm{ml})$ and treated with $\left(\mathrm{NEt}_{4}\right)_{2}\left[\mathrm{Fe}_{2} \mathrm{~S}_{2} \mathrm{Cl}_{4}\right] \mathbf{2}(0.60 \mathrm{~g}, 1.04 \mathrm{mmol})$. The dark brown 
reaction mixture was stirred for $24 \mathrm{~h}$ at room temperature and condensed to dryness. The crude product was extracted with THF $(40 \mathrm{ml})$ with vigorous stirring for $1 \mathrm{~h}$ prior to filtration (no product is left in the residual black solid as indicated by ${ }^{1} \mathrm{H}$ NMR spectroscopy in $\left.\mathrm{MeCN}-\mathrm{d}_{3}\right)$. The deep red-brown THF filtrate was left standing at $-80^{\circ} \mathrm{C}$ for $24 \mathrm{~h}$ and filtered (sometimes $\simeq 50 \mathrm{mg}$ of a bright brown unidentified material precipitates at $-80^{\circ} \mathrm{C}$, sometimes not - filtration is recommended). The obtained filtrate is diluted with hexane $(10 \mathrm{ml})$ and then layered with further hexane $(20 \mathrm{ml})$. After diffusion at room temperature, crystallization was completed at $-20^{\circ} \mathrm{C}$ over night. The crystalline powder was filtered off, washed with pentane $(10 \mathrm{ml})$ and dried in vacuum to afford the target material $(0.22 \mathrm{~g}$, $0.20 \mathrm{mmol}, 20 \%$ ) with identical spectroscopic characteristics as given above for synthesis via ligand exchange reaction.

\section{Bis-(tetraethylammonium)-bis-[di-(thiophene-2-thiolato)-( $\mu$-sul- fido)-ferrate(III)] (10).}

Compound preparation was carried out following the general experimental procedure for ligand exchange reactions with minor modifications: Similar to the starting material, solubility of the target material is restricted in MeCN. Therefore the product was filtered off after $24 \mathrm{~h}$ and recrystallized from $\mathrm{DMF} / \mathrm{Et}_{2} \mathrm{O}(1 / 2$, total volume $\simeq 150 \mathrm{ml})$. Yield: $0.58 \mathrm{~g}, 0.65 \mathrm{mmol}, 65 \%$. ${ }^{1} \mathrm{H}$ NMR $\left(500 \mathrm{MHz}, \mathrm{DMSO}-\mathrm{d}_{6}\right): \delta=1.14\left(\mathrm{~s}_{\mathrm{br}}, 24 \mathrm{H}, \mathrm{NEt}_{4}\right), 1.76$ $\left(\mathrm{s}_{\mathrm{br}}, \mathrm{Ar}-\mathrm{H}\right), 3.14\left(\mathrm{~s}_{\mathrm{br}}, 16 \mathrm{H}, \mathrm{NEt}_{4}\right), 5.75\left(\mathrm{~s}_{\mathrm{br}}, 4 \mathrm{H}, \mathrm{Ar}-\mathrm{H}\right), 9.05\left(\mathrm{~s}_{\mathrm{br}}, 4 \mathrm{H}, \mathrm{Ar}-\mathrm{H}\right)$. MS (ESI+): $m / z(\%)=1026(100)\left[\mathrm{M}+\mathrm{NEt}_{4}\right]^{+} . \mathrm{MS}(\mathrm{ESI}-): m / z(\%)=406(100)\left[\mathrm{M}-2 \mathrm{~L}-2 \mathrm{NEt}_{4}\right]^{-}$, 520 (15) $\left[\mathrm{M}-\mathrm{L}-2 \mathrm{NEt}_{4}\right]^{-}, 766(5)\left[\mathrm{M}^{-} \mathrm{NEt}_{4}\right]^{-}$. UV-Vis (DMF solution): $\lambda_{\max }[\mathrm{nm}](\varepsilon$ $\left.\left[\mathrm{M}^{-1} \mathrm{~cm}^{-1}\right]\right)=287(\simeq 27000), 333(\simeq 35000), 453$ (8700), 468 (9300), 697 (sh, 2500). Elemental Analysis: Calcd.(\%) for $\mathrm{C}_{32} \mathrm{H}_{52} \mathrm{Fe}_{2} \mathrm{~N}_{2} \mathrm{~S}_{10}$ : C 42.84, H 5.84, N 3.12. Found: C 42.16, $\mathrm{H}$ 5.56, N 3.16. HRMS (ESI+): Calcd. $(\mathrm{m} / z)$ for $\mathrm{C}_{40} \mathrm{H}_{72} \mathrm{Fe}_{2} \mathrm{~N}_{3} \mathrm{~S}_{10}$ : 1026.1628. Found: 1026.1592 .

Compound 10 was additionally synthesized via salt metathesis reaction starting from 2 . This procedure is straight forward too since $\mathrm{LiCl}$ remains dissolved in the reaction medium with the target material precipitating during the course of the conversion: To a solution of 2-mercaptothiophene $(0.89 \mathrm{~g}, 7.67 \mathrm{mmol})$ in THF $(20 \mathrm{ml})$ at $0{ }^{\circ} \mathrm{C}$ was added dropwise $n$ $\mathrm{BuLi}(4.80 \mathrm{ml}, 1.6 \mathrm{M}$ solution in hexane, $7.67 \mathrm{mmol})$ and the resulting solution stirred at $0{ }^{\circ} \mathrm{C}$ for 30 minutes. Subsequently, MeCN (5 ml), solid $\left(\mathrm{NEt}_{4}\right)_{2}\left[\mathrm{Fe}_{2} \mathrm{~S}_{2} \mathrm{Cl}_{4}\right] \mathbf{2}(1.11 \mathrm{~g}, 1.92 \mathrm{mmol})$ and a further portion of MeCN $(25 \mathrm{ml})$ was added in this order. Stirring was continued for $2.5 \mathrm{~h}$ at $0{ }^{\circ} \mathrm{C}, \mathrm{Et}_{2} \mathrm{O}(20 \mathrm{ml})$ was added and the reaction mixture kept at $-20^{\circ} \mathrm{C}$ for $24 \mathrm{~h}$. The black precipitate was filtered off, washed with $\mathrm{Et}_{2} \mathrm{O}(20 \mathrm{ml})$ and dried in vacuum to afford the product $(0.72 \mathrm{~g}, 0.80 \mathrm{mmol}, 42 \%)$ pure in ${ }^{1} \mathrm{H}$ NMR. Analytical pure samples with identical spectroscopic data (as given above for synthesis via ligand exchange reaction) were obtained by recrystallization from DMF / $\mathrm{Et}_{2} \mathrm{O}(1 / 2)$. 


\section{Bis - (tetraethylammonium) - bis - $\left[\left(3,3^{\prime}, 5,5^{\prime}\right.\right.$ - tetrachloro - $2,2^{\prime}$ - di - thiolato-1, 1'-biphenyl)-( $\mu$-sulfido)-ferrate(III)] (11a).}

To a solution of $3,3^{\prime}, 5,5^{\prime}$-tetrachloro- $2,2^{\prime}$-dithio-1, $1^{\prime}$-biphenyl Va $(0.74 \mathrm{~g}, 2.08 \mathrm{mmol})$ in THF $(20 \mathrm{ml})$ was added solid $\mathrm{KH}(0.17 \mathrm{~g}, 4.18 \mathrm{mmol})$ at room temperature and the resulting mixture stirred for $1 \mathrm{~h}$ (evolution of hydrogen was observed during the first 10 minutes after addition). Subsequently, the opaque yellow reaction phase was diluted with MeCN (60 ml) and solid $\left(\mathrm{NEt}_{4}\right)_{2}\left[\mathrm{Fe}_{2} \mathrm{~S}_{2} \mathrm{Cl}_{4}\right] \mathbf{2}(0.60 \mathrm{~g}, 1.04 \mathrm{mmol})$ was added in one portion in a positive stream of dinitrogen. After stirring the dark brown reaction mixture for $24 \mathrm{~h}$ at room temperature, all volatiles were removed under reduced pressure and the obtained solid residue briefly dried in vacuum. The product was extracted with MeCN (60 ml, vigorously stirred for $4 \mathrm{~h}$ at room temperature) and the supernatant filtered to a $250 \mathrm{ml}$ Schlenk flask via cannula. The dark red filtrate was diluted with $\mathrm{Et}_{2} \mathrm{O}(60 \mathrm{ml})$ and left standing at $-20^{\circ} \mathrm{C}$ for $3 \mathrm{~d}$. Initial product crystallization was observed during that time and completed by addition of further $\mathrm{Et}_{2} \mathrm{O}(20 \mathrm{ml})$ and storage at $-20^{\circ} \mathrm{C}$ for another $3 \mathrm{~d}$. The crystalline powder was isolated by filtration, washed with $\mathrm{Et}_{2} \mathrm{O}(10 \mathrm{ml})$ and dried in vacuum to obtain the pure dark red target material $(0.60 \mathrm{~g}, 0.53 \mathrm{mmol}, 51 \%) .{ }^{1} \mathrm{H} \mathrm{NMR}(500 \mathrm{MHz}, \mathrm{MeCN}-$ $\left.\mathrm{d}_{3}\right): \delta=1.31\left(\mathrm{~s}_{\mathrm{br}}, 24 \mathrm{H}, \mathrm{NEt}_{4}\right), 3.24\left(\mathrm{~s}_{\mathrm{br}}, 16 \mathrm{H}, \mathrm{NEt}_{4}\right), 9.11\left(\mathrm{~s}_{\mathrm{br}}, 4 \mathrm{H}, \mathrm{Ar}-\mathrm{H}\right), 9.81\left(\mathrm{~s}_{\mathrm{br}}, 4 \mathrm{H}\right.$, Ar-H). MS (ESI+) $m / z(\%): 1273(100)\left[\mathrm{M}+\mathrm{NEt}_{4}\right]^{+}$. MS (ESI-): $m / z(\%)=1014(100)$ $\left[\mathrm{M}-\mathrm{NEt}_{4}\right]^{-}$.UV-Vis $\left(\mathrm{MeCN}\right.$ solution): $\lambda_{\max }[\mathrm{nm}]\left(\varepsilon\left[\mathrm{M}^{-1} \mathrm{~cm}^{-1}\right]\right)=260(\mathrm{sh}, \simeq 34000), 350$ ( $\simeq 25000), 424$ (17800), 523 (6050). Elemental Analysis: Calcd.(\%) for $\mathrm{C}_{40} \mathrm{H}_{48} \mathrm{Cl}_{8} \mathrm{Fe}_{2} \mathrm{~N}_{2} \mathrm{~S}_{6}$ : C 41.98, H 4.23, N 2.45. Found: C 42.79, H 4.66, N 2.69. HRMS (ESI+): Calcd. (m/z) for $\mathrm{C}_{48} \mathrm{H}_{68} \mathrm{Cl}_{8} \mathrm{Fe}_{2} \mathrm{~N}_{3} \mathrm{~S}_{6}$ : 1269.9946. Found: 1269.9936 .

Compound 11a is also accesible following the general experimental procedure for ligand exchange reactions, with minor modifications: A solution of $3,3^{\prime}, 5,5^{\prime}$-tetrachloro- $2,2^{\prime}$-dithio-1, 1'-biphenyl $\mathbf{~ V a ~}(0.30 \mathrm{~g}, 0.85 \mathrm{mmol})$ in THF $(10 \mathrm{ml})$ was added to a stirred suspension of $\left.\left(\mathrm{NEt}_{4}\right)_{2}\left[\mathrm{Fe}_{2} \mathrm{~S}_{2} \text { (indolate }\right)_{4}\right] \mathbf{5}(0.30 \mathrm{~g}, 0.33 \mathrm{mmol})$ in $\mathrm{MeCN}(40 \mathrm{ml})$ at room temperature and the resulting reaction mixture stirred for $6 \mathrm{~h}$. The reaction volume was concentrated to $\simeq 20 \mathrm{ml}$ and the crude product solution layered with $\mathrm{Et}_{2} \mathrm{O}(30 \mathrm{ml})$. After diffusion at room temperature (during $\simeq 2 \mathrm{~d}$ ) and initial product crystallization, the mixture was kept at $-20^{\circ} \mathrm{C}$ over night. Additional $\mathrm{Et}_{2} \mathrm{O}(10 \mathrm{ml})$ was added to the Schlenk flask and left standing at $-20^{\circ} \mathrm{C}$ for another $24 \mathrm{~h}$. Beautiful large deep red crystals were filtered off, washed with $\mathrm{Et}_{2} \mathrm{O}(10 \mathrm{ml})$ and dried for $\simeq 6 \mathrm{~h}$ in vacuum to afford the pure product $(0.25$ $-0.35 \mathrm{~g}, 0.22-0.26 \mathrm{mmol}, 66-80 \%)$. Analytical data were identical with those reported above utilizing the salt metathesis route.

\section{Bis-(tetraethylammonium)-bis- $\left[\left(2,2^{\prime}\right.\right.$-dithiolato-1,1'-biphenyl)-( $\mu$ - sulfido)-ferrate(III)] (11b).}

This compound was synthesized following the general experimental procedure for ligand exchange reactions, with minor modifications: A $100 \mathrm{ml}$ Schlenk flask was charged with a stirring bar, $\left.\left(\mathrm{NEt}_{4}\right)_{2}\left[\mathrm{Fe}_{2} \mathrm{~S}_{2} \text { (indolate) }\right)_{4}\right] \mathbf{5}(0.99 \mathrm{~g}, 1.00 \mathrm{mmol})$ and 2, 2'-dithio-1, 1'-biphenyl $\mathbf{V b}(0.48 \mathrm{~g}, 2.20 \mathrm{mmol})$ in a glove box, prior to addition of MeCN $(50 \mathrm{ml})$. The resulting 
reddish purple suspension was stirred at room temperature for $20 \mathrm{~h}$ affording a deep red solution (indication for complete conversion). Removal of the solvent and extraction of free

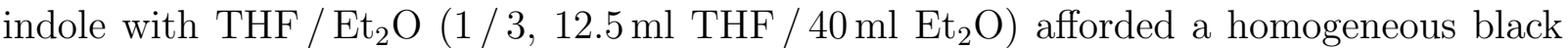
powder. This crude product was washed with a further amount of $\mathrm{Et}_{2} \mathrm{O}(20 \mathrm{ml})$, stirred for 30 minutes in MeCN $(40 \mathrm{ml})$ causing complete dissolution and left standing at $-20^{\circ} \mathrm{C}$ over night. $\mathrm{Et}_{2} \mathrm{O}(10 \mathrm{ml})$ was added to the $\mathrm{MeCN}$ solution of the cluster and the mixture kept at $-20{ }^{\circ} \mathrm{C}$ for $24 \mathrm{~h}$. Filtration, rinsing with $\mathrm{Et}_{2} \mathrm{O}(20 \mathrm{ml})$ and removal of residual solvents in vacuum over night afforded the product as a crystalline powder $(0.60 \mathrm{~g}, 0.69 \mathrm{mmol}$, $69 \%) .{ }^{1} \mathrm{H}$ NMR $\left(500 \mathrm{MHz}, \mathrm{MeCN}_{-} \mathrm{d}_{3}\right): \delta=1.18\left(\mathrm{~s}_{\mathrm{br}}, 24 \mathrm{H}, \mathrm{NEt}_{4}\right), 2.71\left(\mathrm{~d}_{\mathrm{br}}, 4 \mathrm{H}, \mathrm{Ar}-\mathrm{H}\right)$, $3.09\left(\mathrm{~s}_{\mathrm{br}}, 16 \mathrm{H}, \mathrm{NEt}_{4}\right), 4.07\left(\mathrm{~d}_{\mathrm{br}}, 4 \mathrm{H}, \mathrm{Ar}-\mathrm{H}\right), 9.85\left(\mathrm{~s}_{\mathrm{br}}, 4 \mathrm{H}, \mathrm{Ar}-\mathrm{H}\right), 9.05\left(\mathrm{~d}_{\mathrm{br}}, 4 \mathrm{H}, \mathrm{Ar}-\mathrm{H}\right)$. MS $(\mathrm{ESI}+) \mathrm{m} / z(\%): 998(100)\left[\mathrm{M}+\mathrm{NEt}_{4}\right]^{+}$. MS (ESI-): $m / z(\%)=488(60)\left[\mathrm{L}_{2} \mathrm{Fe}\right]^{-}, 637$ (100) $\left[\mathrm{M}-\mathrm{NEt}_{4}-\mathrm{NEt}_{3}\right]^{-}, 738(30)\left[\mathrm{M}-\mathrm{NEt}_{4}\right]^{-}$. UV-Vis (MeCN solution): $\lambda_{\max }[\mathrm{nm}](\varepsilon$ $\left.\left[\mathrm{M}^{-1} \mathrm{~cm}^{-1}\right]\right)=257(\simeq 47500), 336(\simeq 33400), 425$ (29500), 520 (13250), 547 (13500). HRMS (ESI+): Calcd. $(\mathrm{m} / z)$ for $\mathrm{C}_{48} \mathrm{H}_{76} \mathrm{Fe}_{2} \mathrm{~N}_{3} \mathrm{~S}_{6}$ : 996.3104. Found: 996.3101.

\section{Bis - (tetraethylammonium) - bis - $\left[\left(3,3^{\prime}, 5,5^{\prime}\right.\right.$ - tetrakis - tert - butyl - 2, 2'-dithiolato-1, 1'-biphenyl)-( $\mu$-sulfido)-ferrate(III)] (11c).}

To a colorless solution of $3,3^{\prime}, 5,5^{\prime}$-tetrakis-tert-butyl-2,2'-dithio-1,1'-biphenyl Vc $(1.20 \mathrm{~g}$, $2.71 \mathrm{mmol})$ in THF $(40 \mathrm{ml})$ at $0{ }^{\circ} \mathrm{C}$ was added dropwise $n$-BuLi $(1.80 \mathrm{ml}, 3.0 \mathrm{M}$ solution in hexanes, $5.42 \mathrm{~mol}$ ) and the resulting slightly yellow solution stirred for $1 \mathrm{~h}$ at $0^{\circ} \mathrm{C}$. The obtained solution of the deprotonated ligand was cooled to $-40^{\circ} \mathrm{C}$ prior to rapid addition of $\left(\mathrm{NEt}_{4}\right)_{2}\left[\mathrm{Fe}_{2} \mathrm{~S}_{2} \mathrm{Cl}_{4}\right] \mathbf{2}(0.75 \mathrm{~g}, 1.30 \mathrm{mmol})$ dissolved in $\mathrm{MeCN}\left(100 \mathrm{ml}\right.$, pre-cooled to $\left.0^{\circ} \mathrm{C}\right)$. The resulting reaction mixture was stirred for $1 \mathrm{~h}$, raising the temperature slowly to $-30^{\circ} \mathrm{C}$, and finally allowed to warm to room temperature. All volatiles were removed in vacuum and the residual black solid crude product extracted with THF $(200 \mathrm{ml}$, vigorously stirred for 30 minutes prior to filtration). The reddish brown THF filtrate was condensed to a volume of $\simeq 80 \mathrm{ml}, \mathrm{Et}_{2} \mathrm{O}(100 \mathrm{ml})$ was added and the mixture left standing at $-20^{\circ} \mathrm{C}$ over night. Small amounts $(\simeq 50 \mathrm{mg}$ ) of an unidentified precipitate were filtered off and discarded prior to addition of further $\mathrm{Et}_{2} \mathrm{O}(200 \mathrm{ml})$ and pentane $(100 \mathrm{ml})$. Subsequent cooling to $-80^{\circ} \mathrm{C}$ for $3 \mathrm{~d}$ caused product precipitation. Filtration, rinsing with $\mathrm{Et}_{2} \mathrm{O}(40 \mathrm{ml})$ and drying over night in vacuum afforded the product $(0.40 \mathrm{~g}, 0.30 \mathrm{mmol}, 23 \%)$ as a red-brown powder. ${ }^{1} \mathrm{H} \mathrm{NMR}\left(500 \mathrm{MHz}, \mathrm{MeCN}_{-} \mathrm{d}_{3}\right): \delta=1.18\left(\mathrm{~s}_{\mathrm{br}}, 24 \mathrm{H}, \mathrm{NEt}_{4}\right), 1.48\left(\mathrm{~s}_{\mathrm{br}}, 36 \mathrm{H}, 5,5^{\prime}{ }^{t} \mathrm{Bu}\right), \simeq 2.0$ ( $\mathrm{s}_{\mathrm{br}}, 3,3^{\prime}{ }^{t} \mathrm{Bu}$, overlapping with residual $\mathrm{MeCN}-\mathrm{d}_{3}$ resonances), 3.11 ( $\left.\mathrm{s}_{\mathrm{br}}, 16 \mathrm{H}, \mathrm{NEt}_{4}\right), 8.95$ $\left(\mathrm{s}_{\mathrm{br}}, 4 \mathrm{H}, \mathrm{Ar}-\mathrm{H}\right), 9.91\left(\mathrm{~s}_{\mathrm{br}}, 4 \mathrm{H}, \mathrm{Ar}-\mathrm{H}\right)$. MS (ESI+): $m / z(\%)=1345(100)\left[\mathrm{M}-\mathrm{C}_{2} \mathrm{H}_{6}\right]^{+}$, $1446(75)\left[\mathrm{M}+\mathrm{NEt}_{4}\right]^{+}$. MS (ESI-): $m / z(\%)=937(100)\left[\mathrm{L}_{2} \mathrm{Fe}\right]^{-}, 1186(30)\left[\mathrm{M}-\mathrm{NEt}_{4}\right]^{-}$. UV-Vis (MeCN solution), $\lambda_{\max }[\mathrm{nm}]\left(\varepsilon\left[\mathrm{M}^{-1} \mathrm{~cm}^{-1}\right]\right)=260(\mathrm{sh}, \simeq 45000), 345$ (26500), 383 (23600), 443 (22000), 550 (sh, 12000). HRMS (ESI+): Calcd. $(m / z)$ for $\mathrm{C}_{72} \mathrm{H}_{120} \mathrm{Fe}_{2} \mathrm{~N}_{2} \mathrm{~S}_{6}$ : 1317.6552. Found: 1317.6550. 


\section{Bis - tetraphenylphosphonium - tetrakis - [(1,2 - diphenyl-ethylene - 1, 2-dithiolato)-( $\mu_{3}$-sulfido)-ferrate(III)] (13).}

A suspension of diphenylacetylene $(4.0 \mathrm{~g}, 22.5 \mathrm{mmol})$, elemental sulfur $(5.0 \mathrm{~g}, 156 \mathrm{mmol})$ and $\mathrm{Fe}_{3}(\mathrm{CO})_{12}(5.0 \mathrm{~g}, 9.90 \mathrm{mmol})$ in toluene $(30 \mathrm{ml})$ was heated to $80^{\circ} \mathrm{C}$ under an atmosphere of dry dinitrogen, causing initial formation of $\mathrm{CO}$ gas. The temperature was then slowly increased to $135{ }^{\circ} \mathrm{C}$ over a period of $\simeq 2 \mathrm{~h}$ (vigorous gas evolution) and the reaction mixture kept at this temperature for $24 \mathrm{~h}$. Subsequent cooling to room temperature and removal of the solvent afforded a brown solid that was transferred to a $100 \mathrm{ml}$ soxhlet extraction thimble under a positive flow of dinitrogen gas. Soxhlet extraction with hexane/pentane $(150 \mathrm{ml} / 150 \mathrm{ml})$ was carried out over $4 \mathrm{~d}$ in order to remove residual sulfur and tolane starting materials. After disposal of the latter phase, the extraction apparatus was charged with $\mathrm{CS}_{2}(350 \mathrm{ml})$ and the product extracted over $5 \mathrm{~d}$ (oil bath temperature kept at $80^{\circ} \mathrm{C}$ ). Caution: The material left over in the extraction thimble is pyrophoric. Thus residual $\mathrm{CS}_{2}$ was removed in vacuum and the dry material added in portion to water. Cooling the extract to room temperature, filtration and drying in vacuum afforded the neutral intermediate as a finely powdered blue solid $(2.0 \mathrm{~g}, 1.5 \mathrm{mmol}, 15 \%)$. A solution of hydrazine (1 $\mathrm{M}$ in THF, $30 \mathrm{ml}, 30 \mathrm{mmol}$ ) was added dropwise to a vigorously stirred suspension of the thus obtained material in methanol $(25 \mathrm{ml})$ during 10 minutes. To the clear purple solution obtained after stirring for 30 minutes at room temperature was subsequently added a solution of tetraphenylphosphonium bromide $(4.7 \mathrm{~g}, 11 \mathrm{mmol})$ in methanol $(30 \mathrm{ml})$ via cannula. Stirring was continued for another 30 minutes and the precipitate formed in the meantime filtered off. Rinsing with methanol $(3 \times 20 \mathrm{ml})$ and drying in vacuum afforded the product $(2.2 \mathrm{~g}, 1.1 \mathrm{mmol}, 73 \%$ with respect to the neutral intermediate, $11 \%$ with respect to $\mathrm{Fe}_{3}(\mathrm{CO})_{12}$ starting material) as black powder. ${ }^{1} \mathrm{H}$ NMR $\left(200 \mathrm{MHz}, \mathrm{DMSO}-\mathrm{d}_{6}\right)$ : $\delta=7.2-7.8\left(\mathrm{~m}_{\mathrm{br}}, \mathrm{Ar}-\mathrm{H}\right) .{ }^{31} \mathrm{P} \mathrm{NMR}\left(81 \mathrm{MHz}, \mathrm{DMSO}_{6}\right): \delta=22.4\left(\mathrm{PPh}_{4}\right) . \mathrm{MS}(\mathrm{ESI}+): \mathrm{m} / z$ $(\%)=339(100)\left[\mathrm{PPh}_{4}\right]^{+}$, signal for $\left[\mathrm{M}+\mathrm{PPh}_{4}\right]^{+}$not observed due to limited range of the spectrometer. MS (ESI-): $m / z(\%)=375(100)\left[\mathrm{PPh}_{4} \mathrm{Cl}\right]^{-}, 1320(80)\left[\mathrm{M}+\mathrm{H}-2 \mathrm{PPh}_{4}\right]^{-}$.

\section{Bis-(5-pentafluorophenyl-dipyrrinato)-iron(II) (23).}

The following procedure afforded the title compound in adequate yields. Neither alteration in reaction stochiometry nor in reaction conditions (e.g. $-30^{\circ} \mathrm{C}$ instead of room temperature) furnished the semi-substituted intermediate 22: A colorless solution of 5pentafluorophenyldipyrrine XII $(0.32 \mathrm{~g}, 1.04 \mathrm{mmol})$ in toluene $(10 \mathrm{ml})$ was added dropwise to a stirred slightly greenish solution of $\left\{\mathrm{Fe}\left[\mathrm{N}\left(\mathrm{SiMe}_{3}\right)_{2}\right]_{2}\right\}_{2} \mathbf{1 5}(0.20 \mathrm{~g}, 0.26 \mathrm{mmol})$ in toluene $(20 \mathrm{ml})$ at room temperature. The obtained deep red solution was stirred for $1 \mathrm{~h}$ at room temperature and the volume of solvent reduced to $\simeq 1 \mathrm{ml}$ in an oil pump vacuum with gentle heating. The flask was brought into a glovebox and the toluene phase layered with pentane $(3 \mathrm{ml})$. Storage at $-30^{\circ} \mathrm{C}$ over $2 \mathrm{~d}$, filtration and rinsing with cold pentane $(2 \mathrm{ml}$, pre-cooled to $-30{ }^{\circ} \mathrm{C}$ ) afforded green crystals of the product $(0.25 \mathrm{~g}, 0.37 \mathrm{mmol}, 71 \%)$. ${ }^{1} \mathrm{H}$ NMR $\left(200 \mathrm{MHz}, \mathrm{C}_{6} \mathrm{D}_{6}\right): \delta=4.4\left(\mathrm{~s}_{\mathrm{br}}, 4 \mathrm{H}\right), 47.5\left(\mathrm{~s}_{\mathrm{br}}, 4 \mathrm{H}\right), 52.3\left(\mathrm{~s}_{\mathrm{br}}, 4 \mathrm{H}\right) .{ }^{19} \mathrm{~F} \mathrm{NMR}$ $\left(188 \mathrm{MHz}, \mathrm{C}_{6} \mathrm{D}_{6}\right): \delta=1.66(\mathrm{~m}, 4 \mathrm{~F}, m-\mathrm{Ar}-\mathrm{F}), 10.25\left(\mathrm{t}, 2 \mathrm{~F},{ }^{3} J_{\mathrm{F}, \mathrm{F}}=21 \mathrm{~Hz}, p-\mathrm{Ar}-\mathrm{F}\right), 21.33(\mathrm{~d}$, $\left.4 \mathrm{~F},{ }^{3} J_{\mathrm{F}, \mathrm{F}}=17 \mathrm{~Hz}, o-\mathrm{Ar}-\mathrm{F}\right)$. UV-Vis (cyclohexane solution): $\lambda_{\max }[\mathrm{nm}]\left(\varepsilon\left[\mathrm{M}^{-1} \mathrm{~cm}^{-1}\right]\right)=287$ 
(8000), 425 (16000), 477 (28000), 500 (28000). Free 5-pentafluorophenyldipyrrine ligand: ${ }^{1} \mathrm{H}$ NMR $\left(200 \mathrm{MHz}, \mathrm{CDCl}_{3}\right): \delta=6.40(\mathrm{~m}, 4 \mathrm{H}, \mathrm{Ar}-\mathrm{H}), 7.65(\mathrm{~s}, 2 \mathrm{H}, \mathrm{Ar}-\mathrm{H}), 11.8\left(\mathrm{~s}_{\mathrm{br}}, 1 \mathrm{H}\right.$, $\mathrm{NH}) \cdot{ }^{19} \mathrm{~F} \mathrm{NMR}\left(188 \mathrm{MHz}, \mathrm{CDCl}_{3}\right): \delta=0.90(\mathrm{~m}, 2 \mathrm{~F}, m-\mathrm{Ar}-\mathrm{F}), 9.50\left(\mathrm{t}, 1 \mathrm{~F},{ }^{3} J_{\mathrm{F}, \mathrm{F}}=21 \mathrm{~Hz}\right.$, $p$-Ar-F), 23.48 (dd, $\left.2 \mathrm{~F},{ }^{3} J_{\mathrm{F}, \mathrm{F}}=21 \mathrm{~Hz},{ }^{4} J_{\mathrm{F}, \mathrm{F}}=7.5 \mathrm{~Hz}, o-\mathrm{Ar}-\mathrm{F}\right)$. Oxidized tris-(5-pentafluorophenyl-dipyrrinato)-iron(III): ${ }^{1} \mathrm{H}$ NMR $\left(200 \mathrm{MHz}, \mathrm{C}_{6} \mathrm{D}_{6}\right): \delta=-28.8\left(\mathrm{~s}_{\mathrm{br}}, 6 \mathrm{H}\right),-8.2\left(\mathrm{~s}_{\mathrm{br}}\right.$, $6 \mathrm{H}),-6.8\left(\mathrm{~s}_{\mathrm{br}}, 6 \mathrm{H}\right) .{ }^{19} \mathrm{~F}$ NMR $\left(188 \mathrm{MHz}, \mathrm{C}_{6} \mathrm{D}_{6}\right): \delta=1.84(\mathrm{~m}, 6 \mathrm{~F}, m-\mathrm{Ar}-\mathrm{F}), 10.78(\mathrm{t}, 2 \mathrm{~F}$, $\left.{ }^{3} J_{\mathrm{F}, \mathrm{F}}=21 \mathrm{~Hz}, p-\mathrm{Ar}-\mathrm{F}\right), 25.37\left(\mathrm{~d}, 4 \mathrm{~F},{ }^{3} J_{\mathrm{F}, \mathrm{F}}=16 \mathrm{~Hz}, o-\mathrm{Ar}-\mathrm{F}\right)$.

\section{Bis-(tetraethylammonium)-bis-[di-(2-ethylthiophenolato)-( $\mu$-sul- fido)-ferrate(III)] $\left(25^{\mathrm{C}}\right)$.}

To a solution of 2-ethylbenzenethiol ( $1 \mathrm{~g}$, tech. grade $90 \%, 6.5 \mathrm{mmol})$ in THF $(20 \mathrm{ml})$ at $0{ }^{\circ} \mathrm{C}$ was added dropwise $n$-BuLi $(4.1 \mathrm{ml}, 1.6 \mathrm{M}$ solution in hexanes, $6.5 \mathrm{mmol})$ and the resulting yellow solution was stirred at $0{ }^{\circ} \mathrm{C}$ for 30 minutes. Then MeCN $(10 \mathrm{ml})$, powdered $\left(\mathrm{NEt}_{4}\right)_{2}\left[\mathrm{Fe}_{2} \mathrm{~S}_{2} \mathrm{Cl}_{4}\right] \mathbf{2}(0.94 \mathrm{~g}, 1.63 \mathrm{mmol})$ and additional MeCN $(20 \mathrm{ml})$ were added in this order. The reaction mixture was stirred for 20 minutes at $0{ }^{\circ} \mathrm{C}$ and then for $1 \mathrm{~h}$ at room temperature. THF $(40 \mathrm{ml})$ and $\mathrm{Et}_{2} \mathrm{O}(40 \mathrm{ml})$ were added with agitation and the reaction mixture was left standing at $-20{ }^{\circ} \mathrm{C}$ for $2 \mathrm{~d}$. The precipitate was filtered off, washed with $\mathrm{Et}_{2} \mathrm{O}(20 \mathrm{ml})$ and dried in vacuum for $1 \mathrm{~h}$. The obtained crude product was vigorously stirred in $\mathrm{MeCN}(30 \mathrm{ml})$ at room temperature for $3 \mathrm{~h}$ and insoluble byproducts were filtered off successively. The deep red filtrate was kept at $-20^{\circ} \mathrm{C}$ for $4 \mathrm{~d}$. The resulting black crystals were collected by filtration, washed with $\mathrm{Et}_{2} \mathrm{O}$ and dried in vacuum over night to afford the pure product $(0.50 \mathrm{~g}, 0.51 \mathrm{mmol}, 31 \%) .{ }^{1} \mathrm{H}$ NMR $\left(500 \mathrm{MHz}\right.$, DMSO- $\left.\mathrm{d}_{6}\right)$ : $\delta=1.09\left(\mathrm{~s}_{\mathrm{br}}, 24 \mathrm{H}, \mathrm{NEt}_{4}\right), 1.29\left(\mathrm{~s}_{\mathrm{br}}, 12 \mathrm{H}, \mathrm{CH}_{3}\right), 3.09\left(\mathrm{~s}_{\mathrm{br}}, 16 \mathrm{H}, \mathrm{NEt}_{4}, 4 \mathrm{H}, \mathrm{Ar}-\mathrm{H}\right), 4.68$ $\left(\mathrm{s}_{\mathrm{br}}, 8 \mathrm{H}, \mathrm{CH}_{2}\right), 9.18\left(\mathrm{~s}_{\mathrm{br}}, 4 \mathrm{H}, \mathrm{Ar}-\mathrm{H}\right), 10.19\left(\mathrm{~s}_{\mathrm{br}}, 4 \mathrm{H}, \mathrm{Ar}-\mathrm{H}\right)$. MS (ESI+): $m / z(\%)=1114$ (100) $\left[\mathrm{M}+\mathrm{NEt}_{4}\right]^{+}$. UV-Vis (DMF solution): $\lambda_{\max }[\mathrm{nm}]\left(\varepsilon\left[\mathrm{M}^{-1} \mathrm{~cm}^{-1}\right]\right)=331(29000), 476$ (15000). Elemental Analysis: Calcd.(\%) for $\mathrm{C}_{48} \mathrm{H}_{76} \mathrm{Fe}_{2} \mathrm{~N}_{2} \mathrm{~S}_{6}$ : C 58.52, H 7.78, N 2.84, S 19.53. Found: C 57.97, H 7.75, N 2.83, S 19.07.

\section{Bis - (tetraethylammonium) - bis - [di - [2 - methoxy - thiophenolato] - ( $\mu$-sulfido)-ferrate(III)] $\left(25^{\mathrm{O}}\right)$.}

To a solution of 2-(methoxy)-benzenethiol $(1.0 \mathrm{ml}, 1.15 \mathrm{~g}, 8.23 \mathrm{mmol})$ in THF $(20 \mathrm{ml})$ at $0{ }^{\circ} \mathrm{C}$ was added dropwise $n$-BuLi $(4.1 \mathrm{ml}, 2.0 \mathrm{M}$ solution in hexanes, $8.23 \mathrm{mmol})$ and the reaction mixture was stirred for $1.5 \mathrm{~h}$ at room temperature. Then MeCN $(20 \mathrm{ml})$, powdered $\left(\mathrm{NEt}_{4}\right)_{2}\left[\mathrm{Fe}_{2} \mathrm{~S}_{2} \mathrm{Cl}_{4}\right] 2(1.19 \mathrm{~g}, 2.05 \mathrm{mmol})$ and additional MeCN $(20 \mathrm{ml})$ were added in this order. The resulting dark violet reaction mixture was stirred for $1 \mathrm{~h}$. The precipitate formed in the course of the reaction was filtered off, washed with THF $(20 \mathrm{ml})$ and $\mathrm{Et}_{2} \mathrm{O}$ $(20 \mathrm{ml})$ and dried in vacuum over night to afford the product as a fine black powder $(0.80 \mathrm{~g}$, $0.81 \mathrm{mmol}, 39 \%$ ). Crystals were obtained by diffusion of $\mathrm{Et}_{2} \mathrm{O}$ into deep violet solutions of the complex in DMF. ${ }^{1} \mathrm{H}$ NMR $\left(500 \mathrm{MHz}, \mathrm{DMSO}_{-}\right): \delta=1.13\left(\mathrm{~s}_{\mathrm{br}}, 24 \mathrm{H}, \mathrm{NEt}_{4}\right), 3.12$ $\left(\mathrm{s}_{\mathrm{br}}, 16 \mathrm{H}, \mathrm{NEt}_{4}, 4 \mathrm{H}, \mathrm{Ar}-\mathrm{H}\right), 3.88$ ( $\left.\mathrm{s}_{\mathrm{br}}, 12 \mathrm{H}, \mathrm{OMe}\right), 9.00$ ( $\left.\mathrm{s}_{\mathrm{br}}, 4 \mathrm{H}, \mathrm{Ar}-\mathrm{H}\right), 10.19$ ( $\mathrm{s}_{\mathrm{br}}, 4 \mathrm{H}$, Ar-H). UV-Vis (DMF solution): $\lambda_{\max }[\mathrm{nm}]\left(\varepsilon\left[\mathrm{M}^{-1} \mathrm{~cm}^{-1}\right]\right)=296(s h, 58000), 336$ (33000), 
509 (15000). Elemental Analysis: Calcd.(\%) for $\mathrm{C}_{44} \mathrm{H}_{68} \mathrm{Fe}_{2} \mathrm{~N}_{2} \mathrm{O}_{4} \mathrm{~S}_{6}$ : C 53.21, H 6.90, N 2.82, S 18.96. Found: C 52.78, H 6.84, N 3.01, S 18.96.

\section{Bis-(tetraethylammonium) - bis- $\{$ di - [2 - (methylthio)-thiophenol- ato]-( $\mu$-sulfido)-ferrate(III) $\}\left(25^{\mathrm{S}}\right)$.}

To a solution of 2-(methylthio)-benzenethiol $(1.27 \mathrm{~g}, 8.14 \mathrm{mmol})$ in THF $(20 \mathrm{ml})$ at $0{ }^{\circ} \mathrm{C}$ was added dropwise $n$-BuLi $(5.1 \mathrm{ml}, 2.0 \mathrm{M}$ solution in hexanes, $8.14 \mathrm{mmol})$ and the resulting yellow solution was stirred at room temperature for $1.5 \mathrm{~h}$. MeCN $(10 \mathrm{ml}),\left(\mathrm{NEt}_{4}\right)_{2}\left[\mathrm{Fe}_{2} \mathrm{~S}_{2} \mathrm{Cl}_{4}\right]$ $\mathbf{2}(1.18 \mathrm{~g}, 2.03 \mathrm{mmol})$ and an additional portion of $\mathrm{MeCN}(20 \mathrm{ml})$ were added to the reaction mixture. After stirring for $1 \mathrm{~h}$ the precipitate was filtered off, washed with a mixture of THF and MeCN $(20 \mathrm{ml}, 1 / 1)$ and dried in vacuum. The crude product was dissolved in a minimum amount of DMF and layered with $\mathrm{Et}_{2} \mathrm{O}\left(\mathrm{DMF} / \mathrm{Et}_{2} \mathrm{O}=7 / 4\right)$. After completed diffusion, black crystals of the product $(0.80 \mathrm{~g}, 0.76 \mathrm{mmol}, 37 \%)$ were separated by filtration and dried in vacuum. ${ }^{1} \mathrm{H}$ NMR $\left(500 \mathrm{MHz}, \mathrm{DMSO}-\mathrm{d}_{6}\right): \delta=1.13\left(\mathrm{~s}_{\mathrm{br}}, 24 \mathrm{H}, \mathrm{NEt}_{4}\right), 2.37\left(\mathrm{~s}_{\mathrm{br}}\right.$, $12 \mathrm{H}, \mathrm{SMe}), 3.12\left(\mathrm{~s}_{\mathrm{br}}, 16 \mathrm{H}, \mathrm{NEt}_{4}\right), 3.29\left(\mathrm{~s}_{\mathrm{br}}, 4 \mathrm{H}, \mathrm{Ar}-\mathrm{H}\right), 9.18\left(\mathrm{~s}_{\mathrm{br}}, 4 \mathrm{H}, \mathrm{Ar}-\mathrm{H}\right), 10.06\left(\mathrm{~s}_{\mathrm{br}}, 4 \mathrm{H}\right.$, Ar-H). UV-Vis (DMF solution): $\lambda_{\max }[\mathrm{nm}]\left(\varepsilon\left[\mathrm{M}^{-1} \mathrm{~cm}^{-1}\right]\right)=307$ (50000), 350 (sh, 27000), 491 (12000). Elemental Analysis: Calcd.(\%) for $\mathrm{C}_{44} \mathrm{H}_{68} \mathrm{Fe}_{2} \mathrm{~N}_{2} \mathrm{~S}_{10}$ : C 49.98, H 6.48, N 2.65. Found: C 49.68, H 6.63, N 2.62.

\section{Bis - (tetraethylammonium) - bis - $\left[\left(2,2^{\prime}\right.\right.$ - methylenedibenzenethiol - ato)-( $\mu$-sulfido)-ferrate(III)] $\left(26^{\mathrm{C}}\right)$.}

To a solution of $2,2^{\prime}$-methylenedibenzenethiol $\mathbf{X I V}^{\mathbf{C}}(0.72 \mathrm{~g}, 3.10 \mathrm{mmol})$ in THF $(20 \mathrm{ml})$ at $0{ }^{\circ} \mathrm{C}$ was added dropwise $n$-BuLi $(3.90 \mathrm{ml}, 1.6 \mathrm{M}$ solution in hexanes, $6.20 \mathrm{mmol})$. After stirring for 30 minutes at $0{ }^{\circ} \mathrm{C}, \mathrm{MeCN}(10 \mathrm{ml})$, solid $\left(\mathrm{NEt}_{4}\right)_{2}\left[\mathrm{Fe}_{2} \mathrm{~S}_{2} \mathrm{Cl}_{4}\right] 2(0.90 \mathrm{~g}, 1.55 \mathrm{mmol})$ and further $\mathrm{MeCN}(20 \mathrm{ml})$ were added. The resulting reaction mixture was stirred for 20 minutes at $0{ }^{\circ} \mathrm{C}$ and then 30 minutes at room temperature. The precipitate formed in the course of the reaction was filtered off and washed with THF $(2 \times 20 \mathrm{ml})$. The obtained brown solid was extracted with $\mathrm{MeCN}(6 \times 40 \mathrm{ml})$. The combined extracts were condensed to a volume of $120 \mathrm{ml}$ and layered with $\mathrm{Et}_{2} \mathrm{O}(120 \mathrm{ml})$. Diffusion at room temperature led to formation of small black crystals. Cooling the mixture to $-20^{\circ} \mathrm{C}$ for $3 \mathrm{~d}$ completed the crystallization process. The product $(0.49 \mathrm{~g}, 0.55 \mathrm{mmol}, 35 \%)$ was filtered off, washed with $\mathrm{Et}_{2} \mathrm{O}(2 \times 20 \mathrm{ml})$ and dried in vacuum. ${ }^{1} \mathrm{H}$ NMR $\left(500 \mathrm{MHz}, \mathrm{DMSO}-\mathrm{d}_{6}\right): \delta=1.14\left(\mathrm{~s}_{\mathrm{br}}, 24 \mathrm{H}\right.$, $\left.\mathrm{NEt}_{4}\right), 2.68\left(\mathrm{~s}_{\mathrm{br}}, 4 \mathrm{H}, \mathrm{Ar}-\mathrm{H}\right), 3.12\left(\mathrm{~s}_{\mathrm{br}}, 16 \mathrm{H}, \mathrm{NEt}_{4}\right), 3.29\left(\mathrm{~s}_{\mathrm{br}}, 4 \mathrm{H}, \mathrm{CH}_{2}\right), 5.44\left(\mathrm{~s}_{\mathrm{br}}, 4 \mathrm{H}, \mathrm{Ar}-\mathrm{H}\right)$, $8.98\left(\mathrm{~s}_{\mathrm{br}}, 4 \mathrm{H}, \mathrm{Ar}-\mathrm{H}\right), 9.63\left(\mathrm{~s}_{\mathrm{br}}, 4 \mathrm{H}, \mathrm{Ar}-\mathrm{H}\right)$. MS (ESI+): $m / z(\%)=1026(100)\left[\mathrm{M}+\mathrm{NEt}_{4}\right]^{+}$. UV-Vis (DMF solution): $\lambda_{\max }[\mathrm{nm}]\left(\varepsilon\left[\mathrm{M}^{-1} \mathrm{~cm}^{-1}\right]\right)=291(16000), 352(20500), 444(10000)$, 547 (10500), 616 (6500). HRMS (ESI+): Calcd. $(m / z)$ for $\mathrm{C}_{50} \mathrm{H}_{80} \mathrm{Fe}_{2} \mathrm{~N}_{3} \mathrm{~S}_{6}: 1026.33723$. Found: 1026.33675. 


\section{Bis - (tetraethylammonium) - bis - $\left[\left(2,2^{\prime}\right.\right.$ - oxydibenzenethiolato $)$ - $(\mu$ - sulfido-)ferrate(III)] $\left(26^{\mathrm{O}}\right)$.}

To a solution of $2,2^{\prime}$-oxydibenzenethiol $\mathbf{X I V}^{\mathbf{O}}(1.38 \mathrm{~g}, 5.88 \mathrm{mmol})$ in THF $(30 \mathrm{ml})$ at $0{ }^{\circ} \mathrm{C}$ was added dropwise $n$-BuLi $(5.90 \mathrm{ml}, 2.0 \mathrm{M}$ solution in hexanes, $11.80 \mathrm{mmol})$. After stirring for 20 minutes at $0{ }^{\circ} \mathrm{C}, \mathrm{MeCN}(15 \mathrm{ml})$, solid $\left(\mathrm{NEt}_{4}\right)_{2}\left[\mathrm{Fe}_{2} \mathrm{~S}_{2} \mathrm{Cl}_{4}\right] \mathbf{2}(1.70 \mathrm{~g}, 2.95 \mathrm{mmol})$ and additional MeCN $(35 \mathrm{ml})$ were added. The resulting dark reaction mixture was allowed to warm to room temperature over night. The precipitate was separated by filtration, washed with $\mathrm{MeCN}(2 \times 20 \mathrm{ml})$ and dried in vacuum for $2 \mathrm{~h}$. The crude product was dissolved in DMF $(200 \mathrm{ml}), \mathrm{Et}_{2} \mathrm{O}(160 \mathrm{ml})$ was added and the solution was left standing at $-20^{\circ} \mathrm{C}$ for $2 \mathrm{~d}$. Crystallization was completed by addition of further $\mathrm{Et}_{2} \mathrm{O}(80 \mathrm{ml})$. After $1 \mathrm{~d}$ at $-20^{\circ} \mathrm{C}$ black crystals of the product $(0.80 \mathrm{~g}, 0.89 \mathrm{mmol}, 30 \%)$ were filtered off, washed with $\mathrm{Et}_{2} \mathrm{O}(2 \times 20 \mathrm{ml})$ and dried in vacuum. ${ }^{1} \mathrm{H} \mathrm{NMR}\left(500 \mathrm{MHz}, \mathrm{DMSO}-\mathrm{d}_{6}\right): \delta=1.15\left(\mathrm{~s}_{\mathrm{br}}\right.$, $\left.24 \mathrm{H}, \mathrm{NEt}_{4}\right), 3.15\left(\mathrm{~s}_{\mathrm{br}}, 16 \mathrm{H}, \mathrm{NEt}_{4}\right), 3.59\left(\mathrm{~s}_{\mathrm{br}}, 4 \mathrm{H}, \mathrm{Ar}-\mathrm{H}\right), 5.76\left(\mathrm{~s}_{\mathrm{br}}, 4 \mathrm{H}, \mathrm{Ar}-\mathrm{H}\right), 8.41\left(\mathrm{~s}_{\mathrm{br}}\right.$, $4 \mathrm{H}, \mathrm{Ar}-\mathrm{H}), 9.60\left(\mathrm{~s}_{\mathrm{br}}, 4 \mathrm{H}, \mathrm{Ar}-\mathrm{H}\right) . \mathrm{UV}-\mathrm{Vis}$ (DMF solution): $\lambda_{\max }[\mathrm{nm}]\left(\varepsilon\left[\mathrm{M}^{-1} \mathrm{~cm}^{-1}\right]\right)=329$ (19000), 486 (9000). Elemental Analysis: Calcd.(\%) for $\mathrm{C}_{40} \mathrm{H}_{56} \mathrm{Fe}_{2} \mathrm{~N}_{2} \mathrm{O}_{2} \mathrm{~S}_{6}$ : C 53.32, $\mathrm{H}$ 6.26, N 3.11, S 21.35. Found: C 52.13, H 6.21, N 3.46, S 20.70.

\section{Bis- (tetraethylammonium) - bis- $\left[\left(2,2^{\prime}\right.\right.$ - thiodibenzenethiolato $)$ - $(\mu$ - sulfido)-ferrate(III)] $\left(26^{\mathrm{S}}\right)$.}

To a solution of $2,2^{\prime}$-thiodibenzenethiol $\mathbf{X I V}^{\mathbf{S}}(0.90 \mathrm{~g}, 3.60 \mathrm{mmol})$ in $\mathrm{THF}(20 \mathrm{ml})$ at $0{ }^{\circ} \mathrm{C}$ was added dropwise $n$-BuLi $(3.60 \mathrm{ml}, 2.0 \mathrm{M}$ solution in hexanes, $7.20 \mathrm{mmol})$. After stirring for 20 minutes at $0^{\circ} \mathrm{C}, \mathrm{MeCN}(10 \mathrm{ml})$ was added and the reaction mixture cooled to $-20^{\circ} \mathrm{C}$. Then powdered $\left(\mathrm{NEt}_{4}\right)_{2}\left[\mathrm{Fe}_{2} \mathrm{~S}_{2} \mathrm{Cl}_{4}\right] \mathbf{2}(1.04 \mathrm{~g}, 1.80 \mathrm{mmol})$ and additional MeCN (20 ml) were added. The resulting dark reaction mixture was slowly allowed to warm to room temperature over a period of $4 \mathrm{~h}$. The black precipitate formed was separated by filtration, washed with MeCN $(2 \times 20 \mathrm{ml})$ and dried in vacuum for $1 \mathrm{~h}$. The crude product was extracted with $\mathrm{DMF}(5 \times 20 \mathrm{ml})$ yielding a deep purple solution, and $\mathrm{Et}_{2} \mathrm{O}(80 \mathrm{ml})$ was added with agitation. The mixture was left standing at room temperature for $3 \mathrm{~h}$ causing initial crystal formation. After $4 \mathrm{~d}$ at $-20{ }^{\circ} \mathrm{C}$ crystallization was completed. The precipitate was filtered off, washed with $\mathrm{Et}_{2} \mathrm{O}(30 \mathrm{ml})$ and dried in vacuum to afford black crystals of the product (0.25 g, $0.27 \mathrm{mmol}, 15 \%) .{ }^{1} \mathrm{H}$ NMR $\left(500 \mathrm{MHz}, \mathrm{DMSO}-\mathrm{d}_{6}\right): \delta=1.15\left(\mathrm{~s}_{\mathrm{br}}, 24 \mathrm{H}, \mathrm{NEt}_{4}\right), 3.10$ $\left(\mathrm{s}_{\mathrm{br}}, 16 \mathrm{H}, \mathrm{NEt}_{4}\right), 3.57\left(\mathrm{~s}_{\mathrm{br}}, 4 \mathrm{H}, \mathrm{Ar}-\mathrm{H}\right), 9.00\left(\mathrm{~s}_{\mathrm{br}}, 4 \mathrm{H}, \mathrm{Ar}-\mathrm{H}\right), 9.13\left(\mathrm{~s}_{\mathrm{br}}, 4 \mathrm{H}, \mathrm{Ar}-\mathrm{H}\right), 10.29\left(\mathrm{~s}_{\mathrm{br}}\right.$, $4 \mathrm{H}, \mathrm{Ar}-\mathrm{H})$. UV-Vis (DMF solution), $\lambda_{\max }[\mathrm{nm}]\left(\varepsilon\left[\mathrm{M}^{-1} \mathrm{~cm}^{-1}\right]\right)=288(59000), 322$ (37000, sh), 475 (11000). Elemental Analysis: Calcd.(\%) for $\mathrm{C}_{40} \mathrm{H}_{56} \mathrm{Fe}_{2} \mathrm{~N}_{2} \mathrm{~S}_{8}$ : C 51.49, H 6.05, N 3.00. Found: C 50.50, H 6.42, N 3.34.

\section{General Synthetic Procedure for the Preparation of Type-27 Com- plexes.}

To a solution of the free ligand $(2 \mathrm{eq})$ in $\mathrm{THF}(30 \mathrm{ml})$ at $0{ }^{\circ} \mathrm{C}$ was added dropwise $n$-BuLi (1.6 $\mathrm{M}$ solution in hexanes, $4 \mathrm{eq}$ ) and the resulting yellow to deep orange solution was stirred 
at $0{ }^{\circ} \mathrm{C}$ for 20 minutes. Subsequently, a solution of $\left(\mathrm{NEt}_{4}\right)\left[\mathrm{FeCl}_{4}\right] \mathbf{2 8}(0.2 \mathrm{M}$ in THF, $1 \mathrm{eq})$ was added and stirring continued for further 30 minutes at $0^{\circ} \mathrm{C}$. The crude product precipitated during the course of the reaction and was filtered off, washed with $\mathrm{Et}_{2} \mathrm{O}(20 \mathrm{ml})$ and dried in vacuum. Analytically pure products were obtained after crystallization (cooling from room temperature to $-20^{\circ} \mathrm{C}$ ) from DMF $/ \mathrm{Et}_{2} \mathrm{O}$ (for $\mathbf{2 7 ^ { \mathbf { P } }}$ and $\mathbf{2 7 ^ { \mathbf { S } }}$ ) or $\mathrm{MeCN} / \mathrm{Et}_{2} \mathrm{O}$ (for $27^{\mathrm{N}}$ and $27^{\mathrm{O}}$ ).

\section{Tetraethylammonium - bis - $\left[2,2^{\prime}\right.$ - (methylamino) - dibenzenethiol - ato]-ferrate(III) $\left(27^{\mathrm{N}}\right)$.}

Synthesis was carried out according to the general procedure, using 2, 2'-(methylamino)dibenzenethiol $\mathbf{X I V}^{\mathbf{N}}(0.84 \mathrm{~g}, 3.4 \mathrm{mmol}), n$-BuLi $(4.3 \mathrm{ml}, 6.8 \mathrm{mmol})$ and the $\left(\mathrm{NEt}_{4}\right)\left[\mathrm{FeCl}_{4}\right]$ 28 solution $(8.5 \mathrm{ml}, 1.7 \mathrm{mmol})$ yielding $0.52 \mathrm{~g}$ of the product $(0.77 \mathrm{mmol}, 45 \%)$. IR (KBr): $\widetilde{\nu}\left(\mathrm{cm}^{-1}\right)=1455($ Ar-C-H, s). MS (ESI +$): m / z(\%)=806(100)\left[\mathrm{M}+\mathrm{NEt}_{4}\right]^{+}$. MS (ESI-): $m / z(\%)=546(100)\left[\mathrm{M}-\mathrm{NEt}_{4}\right]^{-}$. UV-Vis (DMF solution): $\lambda_{\max }[\mathrm{nm}]\left(\varepsilon\left[\mathrm{M}^{-1} \mathrm{~cm}^{-1}\right]\right)=340$ (sh, 15000), 580 (5150). Elemental Analysis: In numerous attempts irreproducible results for elemental composition were obtained with the following best values: Calcd.(\%) for $\mathrm{C}_{34} \mathrm{H}_{42} \mathrm{FeN}_{3} \mathrm{~S}_{4}$ : C 60.34, H 6.25, N 6.21, S 18.95. Found: C 58.24, H 6.50, N 6.46, S 18.55. HRMS (ESI+): Calcd. $(m / z)$ for $\mathrm{C}_{42} \mathrm{H}_{62} \mathrm{FeN}_{4} \mathrm{~S}_{4}$ : 806.3202. Found: 806.3201.

\section{Tetraethylammonium-bis-[2,2'-oxydibenzenethiolato]-ferrate(III) $\left(27^{\mathrm{O}}\right)$.}

Synthesis was carried out according to the general procedure, using $2,2^{\prime}$-oxydibenzenethiol $\mathbf{X I V}^{\mathbf{O}}(0.84 \mathrm{~g}, 3.4 \mathrm{mmol}), n$-BuLi $(4.3 \mathrm{ml}, 6.8 \mathrm{mmol})$ and the $\left(\mathrm{NEt}_{4}\right)\left[\mathrm{FeCl}_{4}\right] \mathbf{2 8}$ solution $(8.5 \mathrm{ml}, 1.7 \mathrm{mmol})$ yielding $1.05 \mathrm{~g}$ of the product $(1.6 \mathrm{mmol}, 56 \%)$. IR $(\mathrm{KBr}): \widetilde{\nu}$ $\left(\mathrm{cm}^{-1}\right)=1555\left(\right.$ Ar-C-H, s). MS (ESI+): $m / z(\%)=780(100)\left[\mathrm{M}+\mathrm{NEt}_{4}\right]^{+}$. MS (ESI-): $m / z(\%)=520(100)\left[\mathrm{M}-\mathrm{NEt}_{4}\right]^{-}$. UV-Vis (DMF solution): $\lambda_{\max }[\mathrm{nm}]\left(\varepsilon\left[\mathrm{M}^{-1} \mathrm{~cm}^{-1}\right]\right)=359$ (12500), 603 (7920). Elemental Analysis: In numerous attempts irreproducible results for elemental composition were obtained with the following best values: Calcd.(\%) for $\mathrm{C}_{32} \mathrm{H}_{36} \mathrm{FeNO}_{2} \mathrm{~S}_{4}$ : C 59.06, H 5.58, N 2.15, S 19.71. Found: C 59.54, H 5.68, N 2.86, S 18.05. HRMS (ESI+): Calcd. $(m / z)$ for $\mathrm{C}_{40} \mathrm{H}_{56} \mathrm{FeN}_{2} \mathrm{O}_{2} \mathrm{~S}_{4}$ : 780.2569. Found: 780.2559 .

\section{Tetraethylammonium - bis - $\left[2,2^{\prime}\right.$ - (phenylphosphino) - dibenzene - thiolato]-ferrate(III) $\left(27^{\mathrm{P}}\right)$.}

Synthesis was carried out according to the general procedure, using 2, 2 '-(phenylphosphino)dibenzenethiol XIV $\mathbf{P}^{\mathbf{P}}(0.84 \mathrm{~g}, 3.4 \mathrm{mmol}), n$-BuLi $(4.3 \mathrm{ml}, 6.8 \mathrm{mmol})$ and the $\left(\mathrm{NEt}_{4}\right)\left[\mathrm{FeCl}_{4}\right]$ 28 solution $(8.5 \mathrm{ml}, 1.7 \mathrm{mmol})$ yielding $0.97 \mathrm{~g}$ of the product $(1.20 \mathrm{mmol}, 73 \%) .{ }^{1} \mathrm{H} \mathrm{NMR}$ $\left(500 \mathrm{MHz}, \mathrm{DMSO}-\mathrm{d}_{6}\right): \delta=-10.0\left(\mathrm{~s}_{\mathrm{br}}, \mathrm{Ar}-\mathrm{H}\right),-6.2\left(\mathrm{~s}_{\mathrm{br}}, \mathrm{Ar}-\mathrm{H}\right),-1.1\left(\mathrm{~s}_{\mathrm{br}}, \mathrm{Ar}-\mathrm{H}\right), 0.0\left(\mathrm{~s}_{\mathrm{br}}\right.$, Ar-H), $1.1\left(\mathrm{~s}_{\mathrm{br}}, \mathrm{NEt}_{4}\right), 3.2\left(\mathrm{~s}_{\mathrm{br}}, \mathrm{NEt}_{4}\right), 4.6\left(\mathrm{~s}_{\mathrm{br}}, \mathrm{Ar}-\mathrm{H}\right), 5.2\left(\mathrm{~s}_{\mathrm{br}}, \mathrm{Ar}-\mathrm{H}\right), 5.9\left(\mathrm{~s}_{\mathrm{br}}, \mathrm{Ar}-\mathrm{H}\right)$, $7.9\left(\mathrm{~s}_{\mathrm{br}}, \mathrm{PPh}-\mathrm{H}\right), 10.1\left(\mathrm{~s}_{\mathrm{br}}, \mathrm{Ar}-\mathrm{H}\right) . \mathrm{IR}(\mathrm{KBr}): \widetilde{\nu}\left(\mathrm{cm}^{-1}\right)=1420$ (Ar-C-H, s), 1438 (Ar-C-H, 
s). $\operatorname{MS}(\mathrm{ESI}+): m / z(\%)=964(100)\left[\mathrm{M}+\mathrm{NEt}_{4}\right]^{+} \cdot \mathrm{MS}(\mathrm{ESI}-): m / z(\%)=704(100)$ $\left[\mathrm{M}-\mathrm{NEt}_{4}\right]^{-}$. UV-Vis (DMF solution): $\lambda_{\max }[\mathrm{nm}]\left(\varepsilon\left[\mathrm{M}^{-1} \mathrm{~cm}^{-1}\right]\right)=579$ (1730), 753 (1550). Elemental Analysis: In numerous attempts irreproducible results for elemental composition were obtained with the following best values: Calcd.(\%) for $\mathrm{C}_{44} \mathrm{H}_{46} \mathrm{FeNP}_{2} \mathrm{~S}_{4}$ : C 63.30, $\mathrm{H}$ 5.55, N 1.68, S 15.36. Found: C 61.59, H 5.54, N 1.95, S 14.86. HRMS (ESI+): Calcd. $(\mathrm{m} / z)$ for $\mathrm{C}_{52} \mathrm{H}_{66} \mathrm{FeN}_{2} \mathrm{P}_{2} \mathrm{~S}_{4}$ : 696.2929. Found: 696.2933.

\section{Tetraethylammonium-bis-(2,2'-thiodibenzenethiolato)-ferrate(III) $\left(27^{\mathrm{S}}\right)$.}

Synthesis was carried out according to the general procedure, using 2, 2'-thiodibenzenethiol $\mathbf{X I V}^{\mathbf{S}}(0.84 \mathrm{~g}, 3.4 \mathrm{mmol}), n$-BuLi $(4.3 \mathrm{ml}, 6.8 \mathrm{mmol})$ and the $\left(\mathrm{NEt}_{4}\right)\left[\mathrm{FeCl}_{4}\right] 28$ solution $(8.5 \mathrm{ml}, 1.7 \mathrm{mmol})$ yielding $1.1 \mathrm{~g}$ of the product $(1.6 \mathrm{mmol}, 74 \%) .{ }^{1} \mathrm{H} \mathrm{NMR}(500 \mathrm{MHz}$, DMSO- $\left.\mathrm{d}_{6}\right): \delta=-9.72\left(\mathrm{~s}_{\mathrm{br}}, \mathrm{Ar}-\mathrm{H}\right),-9.44\left(\mathrm{~s}_{\mathrm{br}}, \mathrm{Ar}-\mathrm{H}\right),-7.14\left(\mathrm{~s}_{\mathrm{br}}, \mathrm{Ar}-\mathrm{H}\right), 1.13\left(\mathrm{~s}_{\mathrm{br}}, \mathrm{NEt}_{4}\right)$, $2.05\left(\mathrm{~s}_{\mathrm{br}}, \mathrm{Ar}-\mathrm{H}\right), 3.15\left(\mathrm{~s}_{\mathrm{br}}, \mathrm{NEt}_{4}\right), 4.32\left(\mathrm{~s}_{\mathrm{br}}, \mathrm{Ar}-\mathrm{H}\right), 5.96\left(\mathrm{~s}_{\mathrm{br}}, \mathrm{Ar}-\mathrm{H}\right), 6.42\left(\mathrm{~s}_{\mathrm{br}}, \mathrm{Ar}-\mathrm{H}\right), 8.25$ $\left(\mathrm{s}_{\mathrm{br}}, \mathrm{Ar}-\mathrm{H}\right)$. IR $(\mathrm{KBr}): \widetilde{\nu}\left(\mathrm{cm}^{-1}\right)=1418(\mathrm{Ar}-\mathrm{C}-\mathrm{H}, \mathrm{s}), 1437(\mathrm{Ar}-\mathrm{C}-\mathrm{H}, \mathrm{s})$. MS (ESI+): $m / z$ $(\%)=812(100)\left[\mathrm{M}+\mathrm{NEt}_{4}\right]^{+}, 1494(57)\left[2 \mathrm{M}+\mathrm{NEt}_{4}\right]^{+}$. MS (ESI-): $m / z(\%)=336(75)$ $[\mathrm{L}+\mathrm{Fe}+\mathrm{S}]^{-}, 552(100)\left[\mathrm{M}-\mathrm{NEt}_{4}\right]^{-}$. UV-Vis (DMF solution): $\lambda_{\max }[\mathrm{nm}]\left(\varepsilon\left[\mathrm{M}^{-1} \mathrm{~cm}^{-1}\right]\right)=$ 504 (1580), 842 (940). Elemental Analysis: In numerous attempts irreproducible results for elemental composition were obtained with samples measured twice right after each other giving significantly different results. HRMS (ESI+): Calcd. $(m / z)$ for $\mathrm{C}_{40} \mathrm{H}_{56} \mathrm{FeN}_{2} \mathrm{~S}_{6}$ : 812.2113. Found: 812.2106.

\section{Bis - (tetraethylammonium) - bis - [(5, $5^{\prime}$ - dimethyldipyrromethan - ato)-( $\mu$-sulfido)-ferrate(III)] (29a).}

To a solution of $5,5^{\prime}$-dimethyldipyrromethane XXa $(0.77 \mathrm{~g}, 4.42 \mathrm{mmol})$ in THF $(50 \mathrm{ml})$ at $0{ }^{\circ} \mathrm{C}$ was added $n$-BuLi $(2.5 \mathrm{M}$ solution in hexanes, $3.54 \mathrm{ml}, 8.85 \mathrm{mmol})$. The resulting solution was stirred for $3 \mathrm{~h}$ at room temperature and a solution of $\left(\mathrm{NEt}_{4}\right)_{2}\left[\mathrm{Fe}_{2} \mathrm{~S}_{2} \mathrm{Cl}_{4}\right] \mathbf{2}$ $(1.27 \mathrm{~g}, 2.21 \mathrm{mmol})$ in $\mathrm{MeCN}(50 \mathrm{ml})$ was added dropwise via syringe. The mixture was stirred for $2 \mathrm{~d}$ at room temperature. The precipitate was then filtered off and washed with THF $(20 \mathrm{ml})$ followed by MeCN $(20 \mathrm{ml})$. The brown solid residue was extracted with DMF $(10 \times 20 \mathrm{ml})$ to give a deep red solution, which was concentrated to $100 \mathrm{ml}$ and left standing at $-20^{\circ} \mathrm{C}$. After $2 \mathrm{~d}$ the precipitate was filtered off and washed with $\mathrm{Et}_{2} \mathrm{O}(20 \mathrm{ml})$ to afford the product as black crystals $(0.25 \mathrm{~g}, 0.32 \mathrm{mmol}, 14 \%) .{ }^{1} \mathrm{H}$ NMR $\left(500 \mathrm{MHz}, \mathrm{DMSO}-\mathrm{d}_{6}\right)$ : $\delta=1.1\left(\mathrm{~s}_{\mathrm{br}}, 24 \mathrm{H}, \mathrm{NEt}_{4}\right), 2.2-3.0\left(\mathrm{~s}_{\mathrm{br}}, 12 \mathrm{H}, \mathrm{Me}\right), 3.2\left(\mathrm{~s}_{\mathrm{br}}, 16 \mathrm{H}, \mathrm{NEt}_{4}\right), 8.9\left(\mathrm{~s}_{\mathrm{br}}, 4 \mathrm{H}\right.$ pyrrolic protons), $11.5\left(\mathrm{~s}_{\mathrm{br}}, 4 \mathrm{H}\right.$, pyrrolic protons). MS (ESI +$): m / z(\%)=911(100)\left[\mathrm{M}+\mathrm{NEt}_{4}\right]^{+}$, 1690 (46) $\left[2 \mathrm{M}+\mathrm{NEt}_{4}\right]^{+}$. UV-Vis (MeCN solution): $\lambda_{\max }[\mathrm{nm}]\left(\varepsilon\left[\mathrm{M}^{-1} \mathrm{~cm}^{-1}\right]\right)=335(\mathrm{sh}$, 5000), 393 (9500), 522 (4600). Elemental analysis: Calcd. (\%) for $\mathrm{C}_{38} \mathrm{H}_{64} \mathrm{~N}_{6} \mathrm{~S}_{2} \mathrm{Fe}_{2}$ : C 58.46, H 8.26, N 10.76. Found: C 58.02, H 8.11, N 10.71. 


\section{Bis-(tetraethylammonium)-bis-[( $5,5^{\prime}$-diphenyldipyrromethanato)- ( $\mu$-sulfido)-ferrate(III)] (29b).}

To a solution of 5,5-diphenyldipyrromethane $\mathbf{X X b}(0.93 \mathrm{~g}, 3.13 \mathrm{mmol})$ in $\mathrm{THF}(50 \mathrm{ml})$ at room temperature was added $\mathrm{KH}(0.25 \mathrm{~g}, 6.26 \mathrm{mmol})$ and the reaction mixture was stirred for $3 \mathrm{~h}$. Then $\left(\mathrm{NEt}_{4}\right)_{2}\left[\mathrm{Fe}_{2} \mathrm{~S}_{2} \mathrm{Cl}_{4}\right] 2(0.88 \mathrm{~g}, 1.52 \mathrm{mmol})$ was added and the resulting suspension was stirred for further $2 \mathrm{~d}$ at room temperature. The precipitate was then filtered off and washed with THF $(20 \mathrm{ml})$. The brown residue was extracted with MeCN $(8 \times 20 \mathrm{ml})$ to give a deep red solution, which was concentrated to $80 \mathrm{ml}$ and left standing at $-20^{\circ} \mathrm{C}$. After $2 \mathrm{~d}$ the precipitate was filtered off and washed with $\mathrm{Et}_{2} \mathrm{O}(20 \mathrm{ml})$ to afford the product as black crystals $(0.45 \mathrm{~g}, 0.44 \mathrm{mmol}, 28 \%) .{ }^{1} \mathrm{H}$ NMR $(500 \mathrm{MHz}$, DMSO$\left.\mathrm{d}_{6}\right): \delta=1.1\left(\mathrm{~s}_{\mathrm{br}}, 24 \mathrm{H}, \mathrm{NEt}_{4}\right), 3.1\left(\mathrm{~s}_{\mathrm{br}}, 16 \mathrm{H}, \mathrm{NEt}_{4}\right), 6.6-7.4\left(\mathrm{~m}_{\mathrm{br}}, 10 \mathrm{H}, \mathrm{Ar}-\mathrm{H}\right), 9.1\left(\mathrm{~s}_{\mathrm{br}}\right.$, $4 \mathrm{H}$, pyrrolic protons), 10.4 ( $\mathrm{s}_{\mathrm{br}}, 4 \mathrm{H}$, pyrrolic protons). MS (ESI+): $m / z(\%)=1028(100)$ $[\mathrm{M}]^{+}, 1158(70)\left[\mathrm{M}+\mathrm{NEt}_{4}\right]^{+}$. UV-Vis (MeCN solution), $\lambda_{\max }[\mathrm{nm}]\left(\varepsilon\left[\mathrm{M}^{-1} \mathrm{~cm}^{-1}\right]\right)=330$ (sh, 6100), 388 (10500), 523 (5800). Elemental analysis: Calcd. (\%) for $\mathrm{C}_{58} \mathrm{H}_{72} \mathrm{~N}_{6} \mathrm{~S}_{2} \mathrm{Fe}_{2}: \mathrm{C}$ 67.70, H 7.05, N 8.17. Found: C 67.54, H 7.06, N 8.59.

\section{Bis- (tetraethylammonium) - bis- [(5,5' - cyclohexenylendimethyldi- pyrromethanato)-( $\mu$-sulfido)-ferrate(III)] (29c).}

To a solution of $1,1^{\prime}$-dipyrrocyclohexane $\mathbf{X X c}(0.67 \mathrm{~g}, 3.13 \mathrm{mmol})$ in $\mathrm{THF}(50 \mathrm{ml})$ at $0{ }^{\circ} \mathrm{C}$ was added $n$-BuLi (1.6 M solution in hexanes, $3.90 \mathrm{ml}, 6.25 \mathrm{mmol})$ and the reaction mixture stirred at room temperature for $3 \mathrm{~h}$. A solution of $\left(\mathrm{NEt}_{4}\right)_{2}\left[\mathrm{Fe}_{2} \mathrm{~S}_{2} \mathrm{Cl}_{4}\right] 2(0.90 \mathrm{~g}, 1.56 \mathrm{mmol})$ in $\mathrm{MeCN}(40 \mathrm{ml})$ was then added and the resulting suspension stirred for further $2 \mathrm{~d}$ at room temperature. The precipitate was filtered off and washed with THF $(20 \mathrm{ml})$ followed by $\mathrm{MeCN}(20 \mathrm{ml})$. The brown solid residue was extracted with DMF $(9 \times 20 \mathrm{ml})$ to give a deep red solution, which was concentrated to $100 \mathrm{ml}$ and left standing at $-20^{\circ} \mathrm{C}$. After $5 \mathrm{~d}$ the precipitate was filtered off and washed with $\mathrm{Et}_{2} \mathrm{O}(10 \mathrm{ml})$ to afford the product as black crystals $(0.15 \mathrm{~g}, 0.17 \mathrm{mmol}, 11 \%) .{ }^{1} \mathrm{H}$ NMR $\left(500 \mathrm{MHz}, \mathrm{DMSO}-\mathrm{d}_{6}\right): \delta=1.1\left(\mathrm{~s}_{\mathrm{br}}\right.$, $\left.24 \mathrm{H}, \mathrm{NEt}_{4}\right), 1.5-1.9\left(\mathrm{~m}_{\mathrm{br}}, 20 \mathrm{H}, \mathrm{CH}_{2}\right), 3.1\left(\mathrm{~s}_{\mathrm{br}}, 16 \mathrm{H}, \mathrm{NEt}_{4}\right), 8.9$ ( $\mathrm{s}_{\mathrm{br}}, 4 \mathrm{H}$, pyrrolic protons), $11.8\left(\mathrm{~s}_{\mathrm{br}}, 4 \mathrm{H}\right.$, pyrrolic protons). MS (ESI+): $m / z(\%)=991(100)\left[\mathrm{M}+\mathrm{NEt}_{4}\right]^{+}, 1850(37)$ $\left[2 \mathrm{M}+\mathrm{NEt}_{4}\right]^{+}$. UV-Vis (MeCN solution): $\lambda_{\max }[\mathrm{nm}]\left(\varepsilon\left[\mathrm{M}^{-1} \mathrm{~cm}^{-1}\right]\right)=335(s h, 6100), 397$

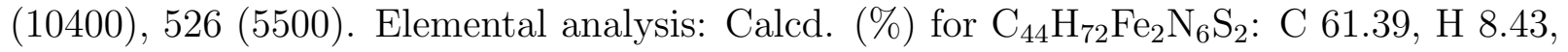
N 9.76. Found: C 60.51, H 8.24, N 9.78.

\section{Bis-(tetraethylammonium)-bis- $\{$ [benzene-1,2-bis-(2-benzimidazol- ato)]-( $\mu$-sulfido)-ferrate(III) $\}$ (31a).}

To a vigorously stirred suspension of benzene-1,2-bis-(2-benzimidazolyl) XXIa (1.55 g, $5.00 \mathrm{mmol})$ in THF $(80 \mathrm{ml})$ was added solid potassium hydride $(0.40 \mathrm{~g}, 0.01 \mathrm{~mol})$ in small portions at room temperature. Deprotonation was completed after stirring the reaction mixture for $12 \mathrm{~h}$ at room temperature. At this stage, the potassium salt of the ligand forms a fine yellowish suspension in THF. Subsequently, powdered $\left(\mathrm{NEt}_{4}\right)_{2}\left[\mathrm{Fe}_{2} \mathrm{~S}_{2} \mathrm{Cl}_{4}\right] 2$ 
(1.44 g, $2.50 \mathrm{mmol})$ was added in one portion, followed by the addition of MeCN $(60 \mathrm{ml})$ after 10 minutes. Stirring at room temperature was continued for $24 \mathrm{~h}$ prior to filtration. The thus collected precipitate was extracted with DMF $(2 \times 60 \mathrm{ml})$ and the combined filtrates condensed to half of their volume. The obtained deep-red solution of the crude product was layered with $\mathrm{Et}_{2} \mathrm{O}(120 \mathrm{ml})$. Diffusion at room temperature and subsequent cooling to $-20^{\circ} \mathrm{C}$ caused product precipitation. Filtration, rinsing with $\mathrm{Et}_{2} \mathrm{O}$ (10 ml) and drying in vacuum over night afforded the pure product (residual DMF signals present in the ${ }^{1} \mathrm{H}$ NMR). Yield: $0.80 \mathrm{~g}, 0.76 \mathrm{mmol}, 30 \%$. ${ }^{1} \mathrm{H}$ NMR $\left(500 \mathrm{MHz}, \mathrm{DMSO}-\mathrm{d}_{6}\right): \delta=1.24$ $\left(\mathrm{s}_{\mathrm{br}}, 24 \mathrm{H}, \mathrm{NEt}_{4}\right), 3.08\left(\mathrm{~s}_{\mathrm{br}}, 16 \mathrm{H}, \mathrm{NEt}_{4}\right), 5.59\left(\mathrm{~s}_{\mathrm{br}}, 4 \mathrm{H}, \mathrm{BzIm}-\mathrm{Ar}-\mathrm{H}\right), 7.40\left(\mathrm{~s}_{\mathrm{br}}, 8 \mathrm{H}\right.$, Phth$\mathrm{Ar}-\mathrm{H}), \simeq 7.85\left(\mathrm{~s}_{\mathrm{br}}, \simeq 4 \mathrm{H}\right.$, BzIm-Ar-H, overlapping with aldehyde resonance of residual DMF), $10.41\left(\mathrm{~s}_{\mathrm{br}}, 4 \mathrm{H}, \mathrm{BzIm}-\mathrm{Ar}-\mathrm{H}\right)$. MS (ESI+): $m / z(\%)=1182(100)\left[\mathrm{M}+\mathrm{NEt}_{4}\right]^{+}$. MS $(\mathrm{ESI}-): m / z(\%)=793(75)\left[\mathrm{M}-2 \mathrm{NEt}_{4}+\mathrm{H}\right]^{-}, 922(100)\left[\mathrm{M}-\mathrm{NEt}_{4}\right]^{-}$. UV-Vis $(\mathrm{DMF}$ solution): $\lambda_{\max }[\mathrm{nm}]\left(\varepsilon\left[\mathrm{M}^{-1} \mathrm{~cm}^{-1}\right]\right)=290(20500), \simeq 406(\mathrm{sh}, \simeq 3200), 482(2600), \simeq 550$ (sh, $\simeq 1200$ ). Elemental Analysis: Calcd.(\%) for $\mathrm{C}_{48} \mathrm{H}_{76} \mathrm{Fe}_{2} \mathrm{~N}_{2} \mathrm{~S}_{6} \times$ DMF: C 62.93, H 6.36, N 13.68. Found: C 62.74, H 6.14, N 13.83. HRMS (ESI+): Calcd. $(m / z)$ for $\mathrm{C}_{64} \mathrm{H}_{84} \mathrm{Fe}_{2} \mathrm{~N}_{11} \mathrm{~S}_{2}$ : 1182.5050. Found: 1182.5048.

\section{Bis-(tetraethylammonium)-bis- $\{[3,4,5,6$-tetrafluorobenzene-1,2- bis-(2-benzimidazolato)]-( $\mu$-sulfido)-ferrate(III) $\}$ (31b).}

To a vigorously stirred suspension of 3,4,5, 6-tetrafluorobenzene-1, 2-bis-(2-benzimidazolyl) XXIb $(1.10 \mathrm{~g}, 2.88 \mathrm{mmol})$ in THF $(50 \mathrm{ml})$ was added solid potassium hydride $(0.23 \mathrm{~g}$, $5.76 \mathrm{mmol}$ ) in small portions at room temperature. Deprotonation was completed after stirring the reaction mixture for $12 \mathrm{~h}$ at room temperature. At this stage, the potassium salt of the ligand forms a turbid orange solution/suspension in THF. Subsequently, powdered $\left(\mathrm{NEt}_{4}\right)_{2}\left[\mathrm{Fe}_{2} \mathrm{~S}_{2} \mathrm{Cl}_{4}\right] 2(0.83 \mathrm{~g}, 1.44 \mathrm{mmol})$ was added in one portion, directly followed by the addition of MeCN $(40 \mathrm{ml})$. Stirring was continued over night $(\simeq 16 \mathrm{~h})$ prior to filtration of the deep red-brown reaction mixture. Residual product in the collected brown solid was extracted with $\mathrm{MeCN}(2 \times 20 \mathrm{ml})$ and the combined filtrates and washings were condensed to dryness (the remaining unidentified bright brown solid on the frit only shows $\mathrm{NEt}_{4}^{+}$resonances in the proton NMR and was discarded). The black solid obtained from the dried filtrate was taken up in $\mathrm{MeCN}$ and stirred for 20 minutes at room temperature. Filtration via filter cannula affords a deep red filtrate (with some amounts of the bright brown unidentified solid remaining again), that was subsequently layered with $\mathrm{Et}_{2} \mathrm{O}$ $(100 \mathrm{ml})$. Diffusion at room temperature and subsequent cooling to $-20^{\circ} \mathrm{C}$ for $2 \mathrm{~d}$ caused the product precipitation. Filtration, rinsing with $\mathrm{Et}_{2} \mathrm{O}(10 \mathrm{ml})$ and drying in vacuum afforded the product pure in the ${ }^{1} \mathrm{H}$ and ${ }^{19} \mathrm{~F}$ NMR. Yield: $0.12 \mathrm{~g}, 0.10 \mathrm{mmol}, 7 \%$ (in $92 \%$ purity according to Mössbauer spectroscopy). ${ }^{1} \mathrm{H}$ NMR $\left(500 \mathrm{MHz}, \mathrm{MeCN}_{3} \mathrm{~d}_{3}\right): \delta=1.11$ $\left(\mathrm{s}_{\mathrm{br}}, 24 \mathrm{H}, \mathrm{NEt}_{4}\right), 3.16\left(\mathrm{~s}_{\mathrm{br}}, 16 \mathrm{H}, \mathrm{NEt}_{4}\right), \simeq 5.9$ (two overlapping broad singlets, $4 \mathrm{H}, \mathrm{Ar}-$ $\mathrm{H}), \simeq 7.4$ (three partially overlapping broad singlets, 8H, Ar-H), 10.07 ( $\left.\mathrm{s}_{\mathrm{br}}, 4 \mathrm{H}, \mathrm{Ar}-\mathrm{H}\right)$. ${ }^{19} \mathrm{~F}$ NMR $\left(188 \mathrm{MHz}, \mathrm{MeCN}-\mathrm{d}_{3}\right): \delta=4.6(\mathrm{~s}, 4 \mathrm{~F}), 22.3(\mathrm{~s}, 4 \mathrm{~F})$. MS (ESI+) $: m / z(\%)=939$ (100) $\left[\mathrm{M}-2 \mathrm{NEt}_{4}+3 \mathrm{H}\right]^{+}$. MS (ESI-): $m / z(\%)=937(100)\left[\mathrm{M}-2 \mathrm{NEt}_{4}+\mathrm{H}\right]^{-}, 1066$ (50) $\left[\mathrm{M}-\mathrm{NEt}_{4}\right]^{-}$. UV-Vis (DMF solution): $\lambda_{\max }[\mathrm{nm}]\left(\varepsilon\left[\mathrm{M}^{-1} \mathrm{~cm}^{-1}\right]\right)=289(13000), 408$ (2000), $468(2000), \simeq 550$ (sh, $\simeq 1200)$. Due to the present impurity (8\%), no satisfactory 
elemental analyses were obtained. HRMS (ESI+): Calcd. $(m / z)$ for $\mathrm{C}_{64} \mathrm{H}_{76} \mathrm{~F}_{8} \mathrm{Fe}_{2} \mathrm{~N}_{11} \mathrm{~S}_{2}$ : 1326.4296. Found: 1326.4286.

\section{Bis-(tetraethylammonium)- $\left\{\left(\alpha, \alpha^{\prime}\right.\right.$-xylyl-dithiolato)-(meso-phenyl- diskatylato)-bis-( $\mu$-sulfido)-bis-[ferrate(III)]\} (32).}

A solution of $n$-BuLi $(3.0 \mathrm{M}$ in hexanes, $2.13 \mathrm{ml}, 6.39 \mathrm{mmol})$ was added dropwise to a stirred solution of 2, $2^{\prime}$-(phenylmethylene)-bis-(3-methyl- $1 H$-indole) XXII $(1.11 \mathrm{~g}, 3.17 \mathrm{mmol})$ in THF $(40 \mathrm{ml})$ at $0{ }^{\circ} \mathrm{C}$ and the resulting mixture was stirred for $1 \mathrm{~h}$ at room temperature to form an orange solution (solution A). In the meanwhile a solution of $n$-BuLi $(3.0 \mathrm{M}$ in hexanes, $1.53 \mathrm{ml}, 4.60 \mathrm{mmol}$ ) was added dropwise to a stirred solution of 1,2-phenylenedimethanethiol $\mathbf{I}(0.39 \mathrm{~g}, 2.30 \mathrm{mmol})$ in $\mathrm{THF}(7.5 \mathrm{ml})$ at $0{ }^{\circ} \mathrm{C}$ and the resulting mixture stirred for $1 \mathrm{~h}$ at $0^{\circ} \mathrm{C}$ to form a pale yellow solution (solution B). Solution A was added dropwise during 10 minutes to a solution of $\left(\mathrm{NEt}_{4}\right)_{2}\left[\mathrm{Fe}_{2} \mathrm{~S}_{2} \mathrm{Cl}_{4}\right] \mathbf{2}(1.21 \mathrm{~g}, 2.09 \mathrm{mmol})$ in $\mathrm{MeCN}(150 \mathrm{ml})$ at $-40^{\circ} \mathrm{C}$. The reaction mixture was stirred for 10 minutes at $-40{ }^{\circ} \mathrm{C}$ and solution $\mathrm{B}$ was added dropwise during 5 minutes. The resulting reaction mixture was stirred for $1 \mathrm{~h}$ at $-35^{\circ} \mathrm{C}$. Subsequently all volatiles were removed in vacuum at room temperature. The black solid residue was washed with THF $(60 \mathrm{ml})$ to remove monomeric byproducts and further washed with $\mathrm{MeCN}(60 \mathrm{ml})$ dissolving minor amounts of the product together with small amounts of the partially substituted compound $\left(\mathrm{NEt}_{4}\right)_{2}\left[\left\{\mathrm{~N}_{2}\right\} \mathrm{Fe}_{2} \mathrm{~S}_{2} \mathrm{Cl}_{2}\right]$ 33. The residual solid was extracted with DMF $(3 \times 20 \mathrm{ml}$ and $1 \times 10 \mathrm{ml})$ and the combined DMF extracts diluted with $\mathrm{Et}_{2} \mathrm{O}(60 \mathrm{ml})$. After $1 \mathrm{~d}$ at $-20^{\circ} \mathrm{C}$ an additional amount of $\mathrm{Et}_{2} \mathrm{O}$ $(20 \mathrm{ml})$ was added and the mixture left standing for $3 \mathrm{~d}$ at $-20^{\circ} \mathrm{C}$ to complete product precipitation. The black microcrystalline powder was filtered off, washed with $\mathrm{Et}_{2} \mathrm{O}(20 \mathrm{ml})$ and dried over night in vacuum to afford the product $(0.25 \mathrm{~g}, 0.26 \mathrm{mmol}, 12 \%) .{ }^{1} \mathrm{H} \mathrm{NMR}$ $\left(500 \mathrm{MHz}, \mathrm{DMSO}-\mathrm{d}_{6}\right): \delta=1.05\left(\mathrm{~s}_{\mathrm{br}}, \mathrm{NEt}_{4}\right), 3.06\left(\mathrm{~s}_{\mathrm{br}}, \mathrm{NEt}_{4}\right), 4.10\left(\mathrm{~s}_{\mathrm{br}},\left\{\mathrm{S}_{2}\right\}\right.$-ligand $), 4.89$ ( $\mathrm{s}_{\mathrm{br}},\left\{\mathrm{N}_{2}\right\}$-ligand), 6.44 ( $\mathrm{s}_{\mathrm{br}},\left\{\mathrm{N}_{2}\right\}$-ligand), $6.94\left(\mathrm{~s}_{\mathrm{br}},\left\{\mathrm{N}_{2}\right\}\right.$-ligand), 7.07 ( $\mathrm{s}_{\mathrm{br}},\left\{\mathrm{N}_{2}\right\}$-ligand), 7.50 ( $\mathrm{s}_{\mathrm{br}},\left\{\mathrm{S}_{2}\right\}$-ligand), $9.61\left(\mathrm{~s}_{\mathrm{br}},\left\{\mathrm{N}_{2}\right\}\right.$-ligand), $10.20\left(\mathrm{~s}_{\mathrm{br}},\left\{\mathrm{N}_{2}\right\}\right.$-ligand). MS (ESI+): $\mathrm{m} / z$ $(\%)=1082(100)\left[\mathrm{M}+\mathrm{NEt}_{4}\right]^{+}$. MS (ESI-): $m / z(\%)=822(100)\left[\mathrm{M}-\mathrm{NEt}_{4}\right]^{-}$. UVVis (MeCN solution): $\lambda_{\max }[\mathrm{nm}]\left(\varepsilon\left[\mathrm{M}^{-1} \mathrm{~cm}^{-1}\right]\right)=228$ (16800), 296 (5400), 446 (1950), $\simeq 525(s h, \simeq 1800), \simeq 601(s h, \simeq 1400)$. No satisfactory elemental analysis was obtained in several attempts, probably due to variable DMF contents in the samples, e.g.: Calcd.(\%) for $\mathrm{C}_{49} \mathrm{H}_{68} \mathrm{Fe}_{2} \mathrm{~N}_{4} \mathrm{~S}_{4} \times$ DMF: C 60.86, $\mathrm{H}$ 7.37, N 6.82, $\mathrm{S}$ 12.50. Found: $\mathrm{C}$ 59.61, H 7.30, $\mathrm{N}$ 6.34, S 11.76. HRMS (ESI+): Calcd. $(\mathrm{m} / z)$ for $\mathrm{C}_{57} \mathrm{H}_{88} \mathrm{Fe}_{2} \mathrm{~N}_{5} \mathrm{~S}_{4}$ : 1082.46190. Found: 1082.46148 .

\section{Bis - (tetraethylammonium) - $\{$ (dichloro) - (meso - phenyl - diskatyl - ato)-bis-( $\mu$-sulfido)-bis-[ferrate(III)] $\}$ (33).}

A solution of $n$-BuLi (3.0 M in hexanes, $2.13 \mathrm{ml}, 6.39 \mathrm{mmol})$ was added dropwise to a stirred solution of 2, 2'-(phenylmethylene)-bis-(3-methyl-1H-indole) XXII $(1.11 \mathrm{~g}, 3.17 \mathrm{mmol})$ in THF $(40 \mathrm{ml})$ at $0{ }^{\circ} \mathrm{C}$ and the resulting mixture was stirred for $1 \mathrm{~h}$ at room temperature to form an orange solution. The thus obtained solution of the deprotonated ligand was 
precooled to $0{ }^{\circ} \mathrm{C}$ and added dropwise during 10 minutes to a solution of $\left(\mathrm{NEt}_{4}\right)_{2}\left[\mathrm{Fe}_{2} \mathrm{~S}_{2} \mathrm{Cl}_{4}\right]$ $2(1.21 \mathrm{~g}, 2.09 \mathrm{mmol})$ in $\mathrm{MeCN} / \mathrm{EtCN}(40 \mathrm{ml} / 80 \mathrm{ml})$ at $-35^{\circ} \mathrm{C}$. The resulting reaction mixture was stirred for $2 \mathrm{~h}$ and gradually warmed up from $-35^{\circ} \mathrm{C}$ to $\simeq 10^{\circ} \mathrm{C}$ during that time. Subsequently all volatiles were removed in vacuum at room temperature. The black solid residue was washed with THF $(60 \mathrm{ml})$ to remove monomeric byproducts and extracted with MeCN $(2 \times 50 \mathrm{ml})$. The volume of the combined MeCN extracts was reduced to $\sim 40 \mathrm{ml}$ and stored over night at $-20^{\circ} \mathrm{C}$. The black microcrystalline precipitate formed, was filtered off, washed with $\mathrm{Et}_{2} \mathrm{O}(10 \mathrm{ml})$ and dried over night in vacuum to afford the product $(0.20 \mathrm{~g}, 0.23 \mathrm{mmol}, 11 \%)$. ${ }^{1} \mathrm{H} \mathrm{NMR}\left(500 \mathrm{MHz}, \mathrm{DMSO}-\mathrm{d}_{6}\right): \delta=1.04\left(\mathrm{~s}_{\mathrm{br}}, \mathrm{NEt}_{4}\right)$, 3.10 ( $\left.\mathrm{s}_{\mathrm{br}}, \mathrm{NEt}_{4}\right), 4.75$ ( $\mathrm{s}_{\mathrm{br}},\left\{\mathrm{N}_{2}\right\}$-ligand), 6.50 ( $\mathrm{s}_{\mathrm{br}},\left\{\mathrm{N}_{2}\right\}$-ligand), 7.02 ( $\mathrm{s}_{\mathrm{br}},\left\{\mathrm{N}_{2}\right\}$-ligand), $7.74\left(\mathrm{~s}_{\mathrm{br}},\left\{\mathrm{N}_{2}\right\}\right.$-ligand), 10.17 ( $\mathrm{s}_{\mathrm{br}}$ with shoulder, $\left\{\mathrm{N}_{2}\right\}$-ligand). MS (ESI+): $m / z(\%)=985$ (100) $\left[\mathrm{M}+\mathrm{NEt}_{4}\right]^{+}, 1841(75)\left[2 \mathrm{M}+\mathrm{NEt}_{4}\right]^{+}$. MS (ESI-): $m / z(\%)=559$ (62) $[\mathrm{M}-2$ $\left.\mathrm{NEt}_{4}-\mathrm{Cl}\right]^{-}, 724(30)\left[\mathrm{M}-\mathrm{NEt}_{4}\right]^{-}, 1002(100)\left[\mathrm{M}-\mathrm{NEt}_{4}-2 \mathrm{Cl}+\left\{\mathrm{N}_{2}\right\} \text {-ligand }\right]^{-}$. UV-Vis (DMF solution): $\lambda_{\max }[\mathrm{nm}]\left(\varepsilon\left[\mathrm{M}^{-1} \mathrm{~cm}^{-1}\right]\right)=281(\simeq 37000), \simeq 425(\mathrm{sh}, \simeq 8600), 521$ (10400), $\simeq 609(s h, \simeq 7900), 697(s h, 2500)$. HRMS (ESI+): Calcd. $(m / z)$ for $\mathrm{C}_{49} \mathrm{H}_{80} \mathrm{Cl}_{2} \mathrm{Fe}_{2} \mathrm{~N}_{5} \mathrm{~S}_{2}$ : 985.3957. Found: 985.3931.

\section{Tetraethylammonium)-[Bis-(meso-phenyl-diskatylato)-ferrate(III)] (35) and Bis- (tetraethylammonium)-bis- [(meso-phenyl-diskatyl- ato)-( $\mu$-sulfido)-ferrate(III)] (34).}

A solution of $n$-BuLi (3.0 M in hexanes, $2.13 \mathrm{ml}, 6.39 \mathrm{mmol})$ was added dropwise to a stirred solution of 2, 2'-(phenylmethylene)-bis-(3-methyl- $H$-indole) XXII $(1.11 \mathrm{~g}, 3.17 \mathrm{mmol})$ in THF $(40 \mathrm{ml})$ at $0{ }^{\circ} \mathrm{C}$ and the resulting mixture was stirred for $1 \mathrm{~h}$ at room temperature to form an orange solution. The thus obtained solution of the deprotonated ligand was precooled to $0^{\circ} \mathrm{C}$ and added dropwise during 10 minutes to a solution of $\left(\mathrm{NEt}_{4}\right)_{2}\left[\mathrm{Fe}_{2} \mathrm{~S}_{2} \mathrm{Cl}_{4}\right]$ $2(0.96 \mathrm{~g}, 1.66 \mathrm{mmol})$ in $\mathrm{MeCN}(80 \mathrm{ml})$ at $-40^{\circ} \mathrm{C}$. The resulting reaction mixture was gradually warmed up from $-40^{\circ} \mathrm{C}$ to room temperature during $\sim 3 \mathrm{~h}$ and stirring continued over night at room temperature. Subsequently all volatiles were removed in vacuum at room temperature. The black solid residue was extracted with THF $(60 \mathrm{ml})$ to separate the monomeric complex $\left(\mathrm{NEt}_{4}\right)\left[\mathrm{Fe}\left\{\mathrm{N}_{2}\right\}_{2}\right]$ 35. The THF filtrate was concentrated to dryness and the residual crude complex recrystallized from $\mathrm{MeCN} / \mathrm{Et}_{2} \mathrm{O}(20 \mathrm{ml} / 60 \mathrm{ml}$, the MeCN solution of the complex was layered with $\mathrm{Et}_{2} \mathrm{O}$, diffusion at room temperature; subsequent cooling to $-20{ }^{\circ} \mathrm{C}$ completes product precipitation) to afford the monomeric product. Yield: $1.00 \mathrm{~g}, 1.13 \mathrm{mmol}, 68 \%$ (if one Fe per cluster core is reactive in the formation of this species; the yield is $34 \%$ if both irons are convertible to the monomeric iron complex). ${ }^{1} \mathrm{H}$ NMR $\left(200 \mathrm{MHz}, \mathrm{DMSO}_{6}\right): \delta=1.02\left(\mathrm{~s}_{\mathrm{br}}, 24 \mathrm{H}, \mathrm{NEt}_{4}\right), 3.10\left(\mathrm{~s}_{\mathrm{br}}, 16 \mathrm{H}, \mathrm{NEt}_{4}\right), 5.97\left(\mathrm{~s}_{\mathrm{br}}\right.$, $1 \mathrm{H},\left\{\mathrm{N}_{2}\right\}$-ligand), $6.80-7.35$ (four partially overlapping broad singlets, $11 \mathrm{H},\left\{\mathrm{N}_{2}\right\}$-ligand), $10.43\left(\mathrm{~s}_{\mathrm{br}}, 2 \mathrm{H},\left\{\mathrm{N}_{2}\right\}\right.$-ligand). MS (ESI+): $m / z(\%)=1012(100)\left[\mathrm{M}+\mathrm{NEt}_{4}\right]^{+}, 1048(75)$ $\left[\mathrm{M}+\mathrm{NEt}_{4}+\mathrm{Cl}\right]^{+}$. MS (ESI-) $m / z(\%): 752(100)\left[\mathrm{M}-\mathrm{NEt}_{4}\right]^{-}$. UV-Vis (MeCN solution): $\lambda_{\max }[\mathrm{nm}]\left(\varepsilon\left[\mathrm{M}^{-1} \mathrm{~cm}^{-1}\right]\right)=225(\simeq 78000), 286$ (24500), $292(24000), \simeq 498($ broad,$\simeq 4000)$, $\simeq 650$ (broad, $\sim 3500)$. HRMS (ESI+): Calcd. $(m / z)$ for $\mathrm{C}_{66} \mathrm{H}_{80} \mathrm{FeN}_{6}$ : 1012.5790. Found: 1012.5785 . 
The residual solid (left over after THF extraction) was stirred for $3 \mathrm{~h}$ in a mixture of DMF $(30 \mathrm{ml})$ and $\mathrm{MeCN}(30 \mathrm{ml})$ and filtered. The filtrate was kept at $-30^{\circ} \mathrm{C}$ over $2 \mathrm{~d}$, causing product precipitation. Filtrating, rinsing with $\mathrm{Et}_{2} \mathrm{O}$ and drying in vacuum afford the analytically pure $\left(\mathrm{NEt}_{4}\right)_{2}\left[\left\{\mathrm{~N}_{2}\right\} \mathrm{Fe}_{2} \mathrm{~S}_{2}\{\mathrm{~N}\}_{2}\right]$ cluster 34 . Yield: $75 \mathrm{mg}, 66 \mu \mathrm{mol}, 4 \%$. The DMF / MeCN filtrate does not contain further amounts of the product, but minor amounts of $\left(\mathrm{NEt}_{4}\right)_{2}\left[\left\{\mathrm{~N}_{2}\right\} \mathrm{Fe}_{2} \mathrm{~S}_{2} \mathrm{Cl}_{2}\right]$ 33, some $\mathrm{NEt}_{4} \mathrm{Cl}$ and the tetraethylammonium salt of the $\left\{\mathrm{N}_{2}\right\}$-ligand. ${ }^{1} \mathrm{H}$ NMR $\left(500 \mathrm{MHz}, \mathrm{DMSO}-\mathrm{d}_{6}\right): \delta=1.04\left(\mathrm{~s}_{\mathrm{br}}, \mathrm{NEt}_{4}\right), 3.10\left(\mathrm{~s}_{\mathrm{br}}, \mathrm{NEt}_{4}\right)$, $4.96\left(\mathrm{~s}_{\mathrm{br}},\left\{\mathrm{N}_{2}\right\}\right.$-ligand), $6.23\left(\mathrm{~s}_{\mathrm{br}},\left\{\mathrm{N}_{2}\right\}\right.$-ligand), $7.09\left(\mathrm{~s}_{\mathrm{br}},\left\{\mathrm{N}_{2}\right\}\right.$-ligand), $7.74\left(\mathrm{~s}_{\mathrm{br}},\left\{\mathrm{N}_{2}\right\}-\right.$ ligand), 10.15 ( $\mathrm{sbr}_{\mathrm{br}},\left\{\mathrm{N}_{2}\right\}$-ligand), $10.50\left(\mathrm{~s}_{\mathrm{br}},\left\{\mathrm{N}_{2}\right\}\right.$-ligand). MS (ESI+) $m / z(\%): 1062(25)$ $\left[\mathrm{M}+\mathrm{NEt}_{4}\right]^{+}, 1514(100)\left[\mathrm{M}-2 \mathrm{NEt}_{4}+\left\{\mathrm{N}_{2}\right\} \text {-ligand }+\mathrm{S}\right]^{+}$. MS (ESI-): $\mathrm{m} / z(\%)=1002$ (100) $\left[\mathrm{M}-\mathrm{NEt}_{4}\right]^{-}, 1052(65)\left[\mathrm{M}-2 \mathrm{NEt}_{4}+\left\{\mathrm{N}_{2}\right\} \text {-ligand }+\mathrm{S}\right]^{-}$. UV-Vis (DMF solution): $\lambda_{\max }[\mathrm{nm}]\left(\varepsilon\left[\mathrm{M}^{-1} \mathrm{~cm}^{-1}\right]\right)=297(32000), \simeq 435(s h, \simeq 8300), \simeq 503(s h, \simeq 9100), 562$ (10300), $\simeq 620(s h, \simeq 8000)$. HRMS (ESI+): Calcd. $(m / z)$ for $\mathrm{C}_{74} \mathrm{H}_{100} \mathrm{Fe}_{2} \mathrm{~N}_{7} \mathrm{~S}_{2}: 1262.6180$. Found: 1262.6146 . 


\subsection{DFT Calculations}

DFT calculations were performed with the Turbomole 5.9 software ${ }^{[249]}$ using the BeckePerdew-1986 functional (BP86) ${ }^{[250,251]}$ and the def2-SVP basis set ${ }^{[252]}$. Electric field gradients were calculated with the same method. Atoms-in-molecules (AIM) analysis ${ }^{[253,254]}$ was performed with the Gaussian-03 software, ${ }^{[255]}$ with the B3LYP method ${ }^{[256,257]}$ and the DZpdf basis set ${ }^{[258]}$ for Fe and the $6-31 G^{*}$ basis set ${ }^{[259]}$ for all the other atoms . For technical reasons, the latter calculations were performed only for the ferromagnetically coupled state. Atom coordinates were obtained from the crystal structure data, geometry optimization was carried out with the Turbomole RELAX module. The ${ }^{11} X$ state of the all-ferric 32 readily converged in SCF energy on the crystal coordinates and was subsequently optimized in geometry. The thus obtained optimized atom coordinates were then used in a spin-unrestricted RI-DFT energy optimization affording the ${ }^{1} X$ state of $\mathbf{3 2}$. The alpha and beta files of the latter calculation could then be used as an initial guess for the calculation of the ${ }^{1} X$ state on the original crystal structure coordinates of 32 . SCF energy convergence of the ${ }^{2} X$ state of the one-electron reduced 1 proceeded without complications. Solvent effects were simulated utilizing the COSMO module. In order to verify DFT calculation results, electrical field gradients at the positions of the iron nucleii were calculated for 32 and $32^{\text {red }}$. Theoretical quadrupole splittings show the correct trend compared to the experimental $\Delta E_{\mathrm{Q}}$ values, but differ by a constant factor. Selected DFT data for 32 and $32^{\text {red }}$ are summarized in Tables 9.1-9.3. Illustrations of spin densities and molecular orbitals were prepared using the VMD 1.8.6 software $^{[260]}$ or the Molden software ${ }^{[261]}$ and ray-traced using the POV-Ray 3.6 software $^{[262]}$ package.

Table 9.1: Selected DFT details for calculations on optimized coordinates of 32 and $32^{\text {red }}$ in either the ferromagnetic $(F)$ or antiferromagnetic $(A F)$ spin state.

\begin{tabular}{lcccc}
\hline & \multicolumn{2}{c}{ SCF-energy (a.u.) } & \multicolumn{2}{c}{$E(A F)-E(F)[\mathrm{kJ} / \mathrm{mol}]$} \\
& vacuum & COSMO $^{\text {a }}$ & vacuum & COSMO $^{\text {a }}$ \\
\hline $\mathbf{3 2}\left({ }^{11} X=F\right)$ & -5503.243231303 & -5503.454338313 & & \multirow{2}{*}{8} \\
$\mathbf{3 2}\left({ }^{1} X=A F\right)$ & -5503.277923875 & -5503.487475281 & & 199 \\
$\mathbf{3 2}^{\text {red }}\left({ }^{12} X=F\right)$ & -5503.075949623 & -5503.489832890 & \multirow{2}{*}{137} & \\
$\mathbf{3 2}^{\text {red }}\left({ }^{2} X=A F\right)$ & -5503.128306866 & -5503.565749476 & & \\
\hline
\end{tabular}

(a) A dielectric constant $\varepsilon=37.5$ for MeCN was used in this calculations. 
Table 9.2: Details for calculations of EFG-tensors of 32 on crystal structure coordinates.

\begin{tabular}{cccccc}
\hline & & \multicolumn{3}{c}{ eigenvalues of EFG-tensor } & calcd. $\Delta E_{Q}$ \\
& $\mathrm{Fe}^{\mathrm{a}}$ & $V_{x x}$ & $V_{y y}$ & $V_{z z}$ & {$[\mathrm{~mm} / \mathrm{s}]^{\mathrm{c}}$} \\
\hline \multirow{2}{*}{$\mathbf{3 2}\left({ }^{11} X=F\right)$} & $\mathrm{Fe} 1$ & $1.078 \cdot 10^{-1}$ & $1.648 \cdot 10^{-1}$ & $-2.726 \cdot 10^{-1}$ & 0.44 \\
& $\mathrm{Fe} 2$ & $2.461 \cdot 10^{-2}$ & $7.383 \cdot 10^{-2}$ & $-9.844 \cdot 10^{-2}$ & 0.17 \\
\multirow{3}{*}{$\mathbf{3 2}\left({ }^{1} X=A F\right)$} & $\mathrm{Fe} 1$ & $1.179 \cdot 10^{-1}$ & $2.770 \cdot 10^{-1}$ & $-3.949 \cdot 10^{-1}$ & 0.66 \\
& $\mathrm{Fe} 2$ & $1.403 \cdot 10^{-3}$ & $1.049 \cdot 10^{-1}$ & $-1.063 \cdot 10^{-1}$ & 0.20 \\
\hline
\end{tabular}

(a) $\mathrm{Fe} 1=\{\mathrm{N}\}$-coordinate, $\mathrm{Fe} 2=\{\mathrm{S}\}$-coordinate. (b) The three eigenvalues of the electrical field gradient tensor (EFG tensor) are given in atomic units $\left(1\right.$ a.u. $\left.=9 \cdot 72 \cdot 10^{21} \mathrm{~V} / \mathrm{m}^{2}\right)$. (c) Theoretical quadrupole splittings were calculated according to $\Delta E_{Q}=\frac{1}{2} e Q V_{z z} \cdot\left(1+\eta^{2} / 3\right)^{1 / 2}$, where the quadrupole moment $Q$ is 0.16 barn $\left(0.16 \cdot 10^{-28} \mathrm{~m}^{2}\right)$ for ${ }^{57} \mathrm{Fe}, V_{z z}$ is the main value of the EFG, $\eta=\left(V_{x x}-V_{y y}\right) / V_{z z}$ (with $\left.\left|V_{x x}\right|<\left|V_{y y}\right|<\left|V_{z z}\right|\right)$ and $1 \mathrm{~mm} / \mathrm{s}=4.8075 \cdot 10^{-8} \mathrm{eV}$.

Table 9.3: Details for calculations of EFG-tensors of 32 and 32 ${ }^{\text {red }}$ on optimized coordinates.

\begin{tabular}{lccccc}
\hline & & \multicolumn{3}{c}{ eigenvalues of EFG-tensor } & calcd. $\Delta E_{Q}$ \\
& $\mathrm{Fe}^{\mathrm{b}}$ & $V_{x x}$ & $V_{y y}$ & $V_{z z}$ & {$[\mathrm{~mm} / \mathrm{s}]^{\mathrm{c}}$} \\
\hline \multirow{2}{*}{$\mathbf{3 2}\left({ }^{11} X=F\right)$} & $\mathrm{Fe} 1$ & $1.585 \cdot 10^{-1}$ & $1.956 \cdot 10^{-1}$ & $-3.541 \cdot 10^{-1}$ & 0.57 \\
& $\mathrm{Fe} 2$ & $1.024 \cdot 10^{-1}$ & $1.420 \cdot 10^{-1}$ & $-2.444 \cdot 10^{-1}$ & 0.40 \\
$\mathbf{3 2}\left({ }^{1} X=A F\right)$ & $\mathrm{Fe} 1$ & $2.491 \cdot 10^{-1}$ & $2.629 \cdot 10^{-1}$ & $-5.120 \cdot 10^{-1}$ & 0.83 \\
& $\mathrm{Fe} 2$ & $-1.373 \cdot 10^{-2}$ & $-1.260 \cdot 10^{-1}$ & $1.398 \cdot 10^{-1}$ & 0.25 \\
$\mathbf{3 2}^{\text {red }}\left({ }^{12} X=F\right)$ & $\mathrm{Fe} 1$ & $1.608 \cdot 10^{-1}$ & $2.162 \cdot 10^{-1}$ & $-3.770 \cdot 10^{-1}$ & 0.61 \\
\multirow{3}{*}{$\mathbf{3 2}^{\text {red }}\left({ }^{2} X=A F\right)$} & $\mathrm{Fe} 2$ & $8.449 \cdot 10^{-2}$ & $2.010 \cdot 10^{-1}$ & $-2.855 \cdot 10^{-1}$ & 0.47 \\
& $\mathrm{Fe} 1$ & $1.717 \cdot 10^{-1}$ & $6.847 \cdot 10^{-1}$ & $-8.563 \cdot 10^{-1}$ & 1.47 \\
& $\mathrm{Fe} 2$ & $1.378 \cdot 10^{-1}$ & $5.601 \cdot 10^{-1}$ & $-6.979 \cdot 10^{-1}$ & 1.20 \\
\hline
\end{tabular}

(a) $\mathrm{Fe} 1=\{\mathrm{N}\}$-coordinate, $\mathrm{Fe} 2=\{\mathrm{S}\}$-coordinate. (b) The three eigenvalues of the electrical field gradient tensor (EFG tensor) are given in atomic units $\left(1 \mathrm{a} \cdot \mathrm{u} .=9 \cdot 72 \cdot 10^{21} \mathrm{~V} / \mathrm{m}^{2}\right)$. (c) Theoretical quadrupole splittings were calculated according to $\Delta E_{Q}=\frac{1}{2} e Q V_{z z} \cdot\left(1+\eta^{2} / 3\right)^{1 / 2}$, where the quadrupole moment $Q$ is 0.16 barn $\left(0.16 \cdot 10^{-28} \mathrm{~m}^{2}\right)$ for ${ }^{57} \mathrm{Fe}, V_{z z}$ is the main value of the EFG, $\eta=\left(V_{x x}-V_{y y}\right) / V_{z z}$ (with $\left.\left|V_{x x}\right|<\left|V_{y y}\right|<\left|V_{z z}\right|\right)$ and $1 \mathrm{~mm} / \mathrm{s}=4.8075 \cdot 10^{-8} \mathrm{eV}$. 


\subsection{Mössbauer Experiments}

Mössbauer spectra were recorded on an alternating constant-acceleration spectrometer (WissEl). Isomer shifts are given relative to iron metal at RT. The experimental data were fitted with Lorentzian line shapes using the program MFit (by E. Bill).

Table 9.4: Best fit parameters for Mössbauer data presented in this work together with data for selected literature-known [2Fe-2S] clusters.

\begin{tabular}{|c|c|c|c|}
\hline compound & $\delta[\mathrm{mm} / \mathrm{s}]$ & $\Delta E_{\mathrm{Q}}[\mathrm{mm} / \mathrm{s}]$ & $\Gamma[\mathrm{mm} / \mathrm{s}]$ \\
\hline $\mathbf{1}^{[134], \mathrm{a}}$ & 0.28 & 0.36 & 0.26 \\
\hline 2 & 0.37 & 0.82 & 0.34 \\
\hline $4^{[141], \mathrm{b}}$ & 0.26 & 0.49 & $\mathrm{n} / \mathrm{a}$ \\
\hline 5 & 0.27 & 0.61 & 0.32 \\
\hline 6 & 0.30 & 0.60 & 0.43 \\
\hline $7 \mathbf{a}^{[134], \mathrm{b}}$ & 0.28 & 0.32 & 0.26 \\
\hline $8 \mathbf{a}$ & 0.33 & 1.15 & 0.32 \\
\hline $8 \mathbf{b}^{[141], \mathrm{b}}$ & 0.35 & 1.02 & $\mathrm{n} / \mathrm{a}$ \\
\hline 10 & 0.30 & 0.40 & 0.28 \\
\hline $11 \mathrm{a}$ & 0.28 & 0.77 & 0.32 \\
\hline $11 b$ & 0.28 & 0.86 & 0.27 \\
\hline $11 c$ & 0.29 & 0.83 & 0.41 \\
\hline $25^{\mathrm{C}}$ & 0.30 & 0.44 & 0.36 \\
\hline $25^{\mathrm{O}}$ & 0.29 & 0.42 & 0.33 \\
\hline $25^{\mathrm{S}}$ & 0.29 & 0.44 & 0.32 \\
\hline $26^{\mathrm{C}}$ & 0.30 & 0.56 & 0.44 \\
\hline $26^{\mathrm{O}}$ & 0.32 & 0.56 & 0.36 \\
\hline $26^{\mathrm{S}}$ & 0.39 & 0.61 & 0.37 \\
\hline $29 a$ & 0.22 & 0.90 & 0.28 \\
\hline $29 b$ & 0.24 & 0.97 & 0.30 \\
\hline $29 c$ & 0.23 & 0.89 & 0.34 \\
\hline $30^{[222], \mathrm{b}}$ & 0.25 & 0.90 & 0.24 \\
\hline $31 \mathrm{a}$ & 0.27 & 0.82 & 0.32 \\
\hline $31 b$ & 0.26 & 0.84 & 0.36 \\
\hline 32 & $0.26 / 0.27$ & $0.49 / 0.98$ & $0.26 / 0.26$ \\
\hline 33 & $0.32 / 0.32$ & $0.99 / 0.72$ & $0.36 / 0.36$ \\
\hline 34 & 0.42 & 0.94 & 0.63 \\
\hline
\end{tabular}

(a) Recorded at $4.2 \mathrm{~K}$. (b) Recorded at $77 \mathrm{~K}$. 


\subsection{Magnetic Susceptibility Measurements}

Temperature-dependent magnetic susceptibilities of powdered samples were measured by using a SQUID magnetometer (Quantum Design MPMS-5S or MPMS-7) at 0.5 T or 1.0 T. Simulation of the experimental magnetic data with a full-matrix diagonalization of exchange coupling and Zeeman splitting was performed with the julX program (by E. Bill): $\mathcal{H}=-2 J \overrightarrow{S_{1}} \cdot \overrightarrow{S_{2}}+g \mu_{\mathrm{B}}\left(\overrightarrow{S_{1}}+\overrightarrow{S_{2}}\right) \cdot \vec{B}$. Before simulation the experimental data were corrected for the underlying diamagnetism by using tabulated Pascal constants (incremental method) and for temperature-independent paramagnetism (TIP). A Curie-Weiss-behaved paramagnetic impurity $(P I)$ with spin $S=5 / 2$ was included: $\chi=(1-P I) \cdot \chi+P I \cdot \chi_{\text {mono }}$.

Table 9.5: Best fit parameters for magnetic susceptibility measurements.

\begin{tabular}{cccccc}
\hline compound & $J\left[\mathrm{~cm}^{-1}\right]^{\mathrm{a}}$ & $P I[\%]$ & $\begin{array}{c}\chi(T I P) \\
{\left[10^{-6} \mathrm{~cm}^{3} \mathrm{~mol}^{-1}\right]}\end{array}$ & $\theta_{\text {mono }}[\mathrm{K}]$ & $B$-field $[\mathrm{T}]$ \\
\hline $\mathbf{5}$ & -159 & 2.6 & 7.3 & -10.0 & 0.5 \\
$\mathbf{6}$ & -199 & 1.2 & 760.0 & -3.0 & 1.0 \\
$\mathbf{8 a}$ & -188 & 2.5 & 350.0 & -3.0 & 1.0 \\
$\mathbf{1 0}$ & -176 & 2.8 & 104.1 & -10.0 & 0.5 \\
$\mathbf{1 1 a}$ & -179 & 1.7 & 35.3 & -1.5 & 0.5 \\
$\mathbf{1 1 b}$ & -158 & 0.9 & 10.1 & -5.0 & 0.5 \\
$\mathbf{1 1 c}$ & -141 & 9.0 & 20.1 & -1.5 & 0.5 \\
$\mathbf{2 5}$ & -197 & 1.7 & 100.0 & -3.0 & 1.0 \\
$\mathbf{2 5}$ & -180 & 0.5 & 405.0 & -2.0 & 1.0 \\
$\mathbf{2 5}$ & -181 & 0.8 & 300.0 & -2.5 & 1.0 \\
$\mathbf{2 6}$ & -204 & 1.6 & 100.0 & -3.0 & 1.0 \\
$\mathbf{2 6}$ & -158 & 2.3 & 500.0 & -2.0 & 1.0 \\
$\mathbf{2 6}$ & -126 & 5.8 & 0.0 & -1.0 & 1.0 \\
$\mathbf{2 9 a}$ & -165 & 0.9 & 756.5 & -7.5 & 1.0 \\
$\mathbf{2 9 b}$ & -167 & 2.2 & 459.9 & -7.5 & 1.0 \\
$\mathbf{2 9 c}$ & -168 & 0.8 & 780.4 & -7.5 & 1.0 \\
$\mathbf{3 1 a}$ & -199 & 3.4 & 32.0 & -7.5 & 1.0 \\
$\mathbf{3 2}$ & -161 & 3.1 & 28.9 & -1.0 & 0.5 \\
$\mathbf{3 3}$ & -184 & 3.0 & 29.6 & -5.0 & 0.5 \\
$\mathbf{3 4}$ & -172 & 3.9 & 40.3 & -5.0 & 0.5 \\
\hline
\end{tabular}

(a) $g$-values fixed to 2.000, unless noted otherwise. (b) $g$-value fitted to 1.898 . (c) $g$-value fitted to 1.855 . 


\subsection{Electrochemical Measurements}

Cyclic voltammetry at room temperature was performed with a potentiostat/galvanostat Perkin-Elmer Model 263A with glassy carbon working electrode and platinum reference and counter electrodes in $\mathrm{DMF} / 0.1 \mathrm{M} \mathrm{NBu}_{4} \mathrm{PF}_{6}$ or $\mathrm{MeCN} / 0.1 \mathrm{M} \mathrm{NBu}_{4} \mathrm{PF}_{6}$. Ferrocene or decamethylferrocene was used as internal standard (added after the measurements) and all potentials are referenced relative to the $\mathrm{Cp}_{2}^{*} \mathrm{Fe} / \mathrm{Cp}_{2}^{*} \mathrm{Fe}^{+}$couple. ${ }^{[145,146]}$ Cyclic voltammetry and coulometric experiments at $-25^{\circ} \mathrm{C}$ were performed with an EG\&G 273A potentiostat / galvanostat. Voltammograms were recorded by using a three electrode arrangement with a glassy carbon working electrode (2 mm diameter), a $\mathrm{Ag} / 0.01 \mathrm{M} \mathrm{AgNO}_{3}$ reference electrode and a $\mathrm{Pt}$ wire counter electrode. Ferrocene was added as an internal standard after the measurements and all potentials are referenced relative to the $\mathrm{Cp}_{2}^{*} \mathrm{Fe} / \mathrm{Cp}_{2}^{*} \mathrm{Fe}^{+}$ couple. ${ }^{[145,146]}$ All coulometric experiments were performed under an argon atmosphere. Electronic spectra during coulometric measurements were recorded with an HP 8452A diode array spectrometer (range: 190 - $1100 \mathrm{~nm}$ ).

Table 9.6: Electrochemical data for all cluster compounds presented in this work together with the corresponding data for selected literature-known examples.

\begin{tabular}{|c|c|c|c|}
\hline compound & solvent $^{\mathrm{a}}$ & $E_{1 / 2}[\mathrm{~V}]^{\mathrm{b}}$ & $E_{\mathrm{p}}^{\mathrm{c}} / E_{\mathrm{p}}^{\mathrm{a}}[\mathrm{V}]^{\mathrm{b}}$ \\
\hline $\mathbf{1}^{[132,134], \mathrm{c}}$ & $\mathrm{DMF}$ & -1.51 & $-1.57 /-1.45$ \\
\hline $2^{[137,236], \mathrm{c}, \mathrm{d}}$ & $\mathrm{MeCN}$ & irrev. & $-1.02 /-0.77$ \\
\hline $3^{[34], \mathrm{e}, \mathrm{f}}$ & THF & irrev. & $-0.92 /-$ \\
\hline $4^{[141], c, d, g}$ & $\mathrm{DMF}$ & irrev. & $-1.30 /-$ \\
\hline $5^{g}$ & $\mathrm{DMF}$ & irrev. & $-1.24 /-$ \\
\hline $6^{\mathrm{g}}$ & $\mathrm{DMF}$ & irrev. & $-1.19 / \simeq-1.10$ \\
\hline $7 \mathbf{a}^{[134], \mathrm{h}}$ & $\mathrm{MeCN}$ & -1.11 & $-1.22 /-1.00$ \\
\hline $7 \mathbf{b}^{[132], \mathrm{h}}$ & $\mathrm{MeCN}$ & -1.09 & $-1.22 /-0.96$ \\
\hline $7 \mathrm{c}$ & $\mathrm{MeCN}$ & irrev. & $-1.35 /-$ \\
\hline $7 d$ & $\mathrm{MeCN}$ & -1.01 & $-1.08 /-0.93$ \\
\hline $7 \mathrm{e}$ & $\mathrm{MeCN}$ & -0.93 & $-0.99 /-0.87$ \\
\hline $\mathbf{7 f} \mathbf{f}^{[151], \mathrm{h}}$ & $\mathrm{MeCN}$ & -1.13 & $-1.25 /-1.00$ \\
\hline $8 \mathbf{a}$ & $\mathrm{MeCN}$ & -0.99 & $-1.12 /-0.87$ \\
\hline $8 \mathbf{b}^{[141], c, d}$ & $\mathrm{MeCN}$ & irrev. & $-1.22 /-$ \\
\hline 10 & $\mathrm{DMF}$ & irrev. & $-1.05 /-$ \\
\hline $11 \mathrm{a}$ & $\mathrm{MeCN}$ & -0.96 & $-1.01 /-0.90$ \\
\hline $11 b$ & $\mathrm{MeCN}$ & -1.14 & $-1.19 /-1.09$ \\
\hline $11 c^{e}$ & $\mathrm{MeCN}$ & -1.43 & $-1.54 /-1.32$ \\
\hline $25^{\mathrm{C}}$ & DMF & irrev. & $-1.24 /-$ \\
\hline $25^{\mathrm{O}}$ & DMF & irrev. & $-1.32 /-0.35$ \\
\hline
\end{tabular}




\begin{tabular}{|c|c|c|c|}
\hline $25^{\mathrm{S}}$ & $\mathrm{DMF}$ & irrev. & $-1.14 /-0.54$ \\
\hline $26^{\mathrm{C}}$ & $\mathrm{DMF}$ & irrev. & $-1.24 /-$ \\
\hline $26^{\mathrm{O}}$ & $\mathrm{DMF}$ & irrev. & $-1.48 / \simeq-1.05$ \\
\hline $26^{\mathrm{S}}$ & $\mathrm{DMF}$ & irrev. & $-0.99 / \simeq-0.81$ \\
\hline $29 a$ & $\mathrm{DMF}$ & -1.19 & $-1.25 /-1.13$ \\
\hline $29 \mathrm{~b}$ & $\mathrm{DMF}$ & -1.25 & $-1.35 /-1.14$ \\
\hline $29 \mathrm{c}$ & $\mathrm{DMF}$ & -1.31 & $-1.44 /-1.18$ \\
\hline $\mathbf{3 0}^{[222], \mathrm{i}}$ & $\mathrm{DMF}$ & -0.82 & not reported \\
\hline $31 a^{e}$ & $\mathrm{DMF}$ & -0.84 & $-0.93 /-0.76$ \\
\hline $31 b^{e}$ & $\mathrm{DMF}$ & -0.73 & $-0.83 /-0.63$ \\
\hline 32 & $\mathrm{DMF}$ & -1.35 & $-1.40 /-1.30$ \\
\hline 33 & $\mathrm{DMF}$ & irrev. & $-1.25 /-$ \\
\hline 34 & $\mathrm{DMF}$ & irrev. & $-1.22 /-$ \\
\hline
\end{tabular}

(a) Recorded at RT in presence of $\mathrm{NBu}_{4} \mathrm{PF}_{6}(0.2 \mathrm{M})$ at a scan rate of $100 \mathrm{mV} / \mathrm{s}$. (b) Decamethylferrocene was used as internal standard unless noted otherwise. All potentials are referenced or recalibrated to the $\mathrm{Cp}_{2}^{*} \mathrm{Fe} / \mathrm{Cp}_{2}^{*} \mathrm{Fe}^{+}$couple. Listed potentials correspond to the $[2 \mathrm{Fe}-2 \mathrm{~S}]^{2+} /[2 \mathrm{Fe}-2 \mathrm{~S}]^{+}$couple. Irreversible (at $\mathrm{RT}$ ) redox waves corresponding to the $[2 \mathrm{Fe}-2 \mathrm{~S}]^{+} /[2 \mathrm{Fe}-2 \mathrm{~S}]^{0}$ couple were observed in some cases at lower potentials. (c) Recorded vs. SCE and recalibrated to the $\mathrm{Cp}_{2}^{*} \mathrm{Fe} / \mathrm{Cp}_{2}^{*} \mathrm{Fe}^{+}$couple. (d) Recorded in presence of $\mathrm{NBu}_{4} \mathrm{ClO}_{4}(0.1 \mathrm{M})$. (e) Ferrocene was used as internal standard. (f) Detail not reported. (g) Recorded at a scan rate of $200 \mathrm{mV} / \mathrm{s}$. (h) Values are identical or - in case of different experimental conditions - in agreement with those reported in literature. (i) A mercury dropping electrode was used. 


\section{Chapter 10}

\section{Crystallography}

The crystal data and details of the data collections are collected in Tables 10.1-10.10. Xray data were collected on a Bruker SMART 6000 4K CCD diffractometer (monochromated $\mathrm{Cu}-\mathrm{K}_{\alpha}$ radiation, $\lambda=1.54178 \AA$, $\omega$ scans) at $-173^{\circ} \mathrm{C}$, on a STOE IPDS II diffractometer (graphite monochromated Mo-K $\mathrm{K}_{\alpha}$ radiation, $\lambda=0.71073 \AA$, $\omega$ scans) at $-140^{\circ} \mathrm{C}$ or on a four-circle diffractometer (QUATERMAS) with CCD detector (graphite-monochromated Mo- $\mathrm{K}_{\alpha}$ radiation, $\lambda=0.71073 \AA, \varphi$ and $\omega$ scans) at $-140^{\circ} \mathrm{C}$. The structures were solved by direct methods and refined on $F^{2}$ using all reflections with SHELX-97 or SHELXL97. ${ }^{[263-265]}$ Most non-hydrogen atoms were refined anisotropically. Hydrogen atoms were placed in calculated positions and assigned to an isotropic displacement parameter of $0.08 \AA^{2}$. Face-indexed absorption corrections for $\mathbf{7 f}, \mathbf{8 a}, \mathbf{1 1} \mathbf{a}^{\prime}, \mathbf{1 1 a}, \mathbf{1 1} \mathbf{b}, \mathbf{1 1} \mathbf{c}, \mathbf{2 5 ^ { \mathrm { C } }}, \mathbf{2 5 ^ { \mathrm { O } }}$, $25^{\mathrm{S}}, \mathbf{2 6 ^ { \mathrm { C } }}, \mathbf{2 6 ^ { \mathrm { O } }}, \mathbf{2 9 \mathrm { b }}$ and 32 were performed numerically with the program X-RED. ${ }^{[266]}$

The cationic $\mathrm{NEt}_{4}^{+}$moieties in $\mathbf{7} \mathbf{d}$ and $\mathbf{7 e}$ are disordered about two positions. The respective occupancy factors were refined to $0.639(6) / 0.361(6)$ and $0.725(15) / 0.275(15)$. The absolute structure parameter for $\mathbf{7 f}$ was determined according to the method of Flack with SHELXL-97 and refined to 0.617(6). ${ }^{[267]}$ SADABS was used to perform area-detector scaling and absorption corrections for $\mathbf{7 e} .^{[268]}$ One cationic $\mathrm{NEt}_{4}^{+}$moiety in $\mathbf{1 1} \mathbf{a}^{\prime}$ and one MeCN solvent molecule in $\mathbf{1 1} \mathbf{c}$ are disordered about two positions. The respective occupancy factors were refined to $\left.0.719(5) / 0.281(5)\left(\mathbf{1 1}^{\prime}\right)\right)$ and $0.657(10) / 0.343(10)(\mathbf{1 1} \mathbf{c})$. For

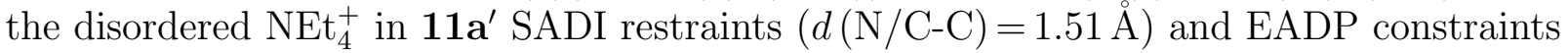
were used to model the disorder. Atoms of the disordered parts of $\mathbf{2 5}^{\mathrm{C}}$ were refined isotropically. The ethyl groups of $\mathbf{2 5}^{\mathrm{C}}$ and the acetonitrile solvent molecule were found to be disordered about two positions ((occupancy factors of $0.562(16) / 0.438(16)$ (C8), $0.681(16) / 0.319(16)(\mathrm{C} 16)$, and 0.64(3)/0.36(3) (N3, C33, C34)). Additionally two $\mathrm{NEt}_{4}^{+}$ cations in $25^{\mathrm{C}}$ are disordered about special positions and were refined with fixed occupancy factors of 0.5. DFIX restraints (Ph-Et: $d(\mathrm{C}-\mathrm{C})=1.51 \AA$; MeCN: $d(\mathrm{C}-\mathrm{C})=1.47 \AA$, $\left.d(\mathrm{C} \equiv \mathrm{N})=1.14 \AA ; \mathrm{NEt}_{4}^{+}: d(\mathrm{C}-\mathrm{C} / \mathrm{N})=1.51 \AA\right)$ and EADP constraints $(\mathrm{C} 16 \mathrm{~A} / \mathrm{B})$ were used to model the disorder. Crystals of $\mathbf{2 6}^{\mathrm{S}}$ are non-merohedrally twinned (ratio of the two twin components approximately $70: 30$, twinlaw $1,0,0 / 0,-1,0 /-0.35,0,-1)$ and the reflection data for refinement were prepared using the program X-AREA. ${ }^{[269]}$ The absolute structure parameter of $\mathbf{2 6}^{\mathrm{S}}(x=-0.01(4))$ was determined according to Flack with 
SHELX-97. ${ }^{[267]}$ SADABS was used to perform area-detector scaling and absorption corrections for 29a. ${ }^{[268]}$ One cationic $\mathrm{NEt}_{4}^{+}$moiety in $\mathbf{2 9 a}$ is disordered about two positions. The respective occupancy factors were refined to 0.8956(16)/0.1044(16). In 32 and 34 some of the $\mathrm{NEt}_{4}^{+}$moieties were found to be disordered. In case of $\mathbf{3 2}$ one $\mathrm{NEt}_{4}^{+}$is disordered about a 2-fold rotation axis and additionally about two positions and was refined with a fixed occupancy factor of 0.25 for each position. DFIX restraints $(d(\mathrm{~N}-\mathrm{C})=1.51 \AA$, $d(\mathrm{C}-\mathrm{C})=1.51 \AA$ ) and EADP constraints were used to model the disorder. For a second disordered $\mathrm{NEt}_{4}^{+}$in $32 \mathrm{SADI}$ restraints $(d(\mathrm{C}-\mathrm{C})=1.51 \AA)$ and EADP constraints were used to model the disorder. EADP constraints were also applied for the disordered $\mathrm{NEt}_{4}^{+}$in 34. The unit cell of $\mathbf{3 2}$ contains disordered DMF solvent molecules that occupy an area of $577.6 \AA^{3}$ (5.7). No satisfactory model for the disorder could be found, and for further refinement the contribution of the missing solvent molecule (total electron count 137) was subtracted from the reflection data by the SQUEEZE ${ }^{[270]}$ routine of the PLATON ${ }^{[271]}$ program. Crystals of $\mathbf{3 3}$ are non-merohedrally twinned (ratio of the two twin components $0.548(1): 0.452(1)$, twinlaw $-1,-0.03,0 /-0.03,1,0 / 0,0.02,-1)$ and the reflection data for refinement were prepared using the program X-AREA. ${ }^{[269]}$

Supplementary crystallographic data can be obtained free of charge from the Cambridge Crystallographic Data Centre via www.ccdc.cam.ac.uk/data_request/cif: CCDC-689045 (Va), CCDC-273911 (29a), CCDC-273912 (29b), CCDC-273913 (29c), CCDC-692367 (32), CCDC-692368 (33) and CCDC-692369 (34). 
Table 10.1: Crystal data and refinement details.

\begin{tabular}{|c|c|c|c|}
\hline compound & $\mathrm{Va}$ & $7 \mathrm{c}$ & $7 d$ \\
\hline empirical formula & $\mathrm{C}_{12} \mathrm{H}_{6} \mathrm{Cl}_{4} \mathrm{~S}_{2}$ & $\begin{array}{l}{\left[\mathrm{C}_{32} \mathrm{H}_{36} \mathrm{Fe}_{2} \mathrm{~S}_{6}\right]^{2-}} \\
2\left(\mathrm{C}_{8} \mathrm{H}_{20} \mathrm{~N}^{+}\right)\end{array}$ & $\begin{array}{l}{\left[\mathrm{C}_{32} \mathrm{H}_{36} \mathrm{Fe}_{2} \mathrm{~S}_{6}\right]^{2-}} \\
2\left(\mathrm{C}_{8} \mathrm{H}_{20} \mathrm{~N}^{+}\right)\end{array}$ \\
\hline formula weight & 356.09 & 985.17 & 985.17 \\
\hline$T[\mathrm{~K}]$ & $133(2)$ & $100(2)$ & $100(2)$ \\
\hline crystal size $[\mathrm{mm}]$ & $0.50 \times 0.50 \times 0.42$ & $0.20 \times 0.20 \times 0.20$ & $0.20 \times 0.20 \times 0.20$ \\
\hline crystal system & monoclinic & monoclinic & monoclinic \\
\hline space group & $P 2_{1} / c$ (No. 14$)$ & $P 2_{1} / n($ No. 14$)$ & $P 2_{1} / n($ No. 14$)$ \\
\hline$a[\AA]$ & $13.4957(6)$ & $11.86(11)$ & $10.060(1)$ \\
\hline$b[\AA]$ & $13.4293(5)$ & $16.79(17)$ & $16.140(1)$ \\
\hline$c[\AA]$ & $7.6701(3)$ & $13.23(7)$ & $15.700(1)$ \\
\hline$\alpha\left[^{\circ}\right]$ & 90 & 90 & 90 \\
\hline$\beta\left[^{\circ}\right]$ & $99.860(3)$ & $106.1(3)$ & $97.94(2)$ \\
\hline$\gamma\left[^{\circ}\right]$ & 90 & 90 & 90 \\
\hline$V\left[\AA^{3}\right]$ & $1369.58(10)$ & $2532(37)$ & $2524.7(5)$ \\
\hline$\rho_{\text {calcd. }}\left[\mathrm{g} \mathrm{cm}^{-3}\right]$ & 1.727 & 1.292 & 1.293 \\
\hline$Z$ & 4 & 2 & 2 \\
\hline$F(000)$ & 712 & 1052 & 1048 \\
\hline$\mu\left[\mathrm{mm}^{-1}\right]$ & $1.144\left(\mathrm{Mo}^{-\mathrm{K}_{\alpha}}\right)$ & $7.151\left(\mathrm{Cu}-\mathrm{K}_{\alpha}\right)$ & $7.171\left(\mathrm{Cu}-\mathrm{K}_{\alpha}\right)$ \\
\hline$T_{\max } / T_{\min }$ & $0.6920 / 0.4990$ & $-/-$ & $-1-$ \\
\hline$h k l$ range & $\pm 17,-17-16,-9-7$ & $\pm 13, \pm 18, \pm 14$ & $\pm 10, \pm 16, \pm 16$ \\
\hline$\theta$ range $\left[{ }^{\circ}\right]$ & $2.16-26.96$ & $4.36-59.19$ & $3.95-54.19$ \\
\hline measured refl. & 13164 & 32320 & 29576 \\
\hline unique refl. $\left[R_{\text {int }}\right]$ & $2896[0.0319]$ & $3646[0.0427]$ & $3083[0.0587]$ \\
\hline observed refl. $I>2 \sigma(I)$ & 2767 & 3403 & 2738 \\
\hline Refined parameters & 169 & 264 & 342 \\
\hline restraints & 0 & 212 & 414 \\
\hline goodness-of-fit & 1.077 & 1.034 & 1.045 \\
\hline$R 1, w R 2(I>2 \sigma(I))$ & $0.0260,0.0658$ & $0.0304,0.0795$ & $0.0369,0.0865$ \\
\hline$R 1, w R 2$ (all data) & $0.0278,0.0668$ & $0.0332,0.0814$ & $0.0442,0.0907$ \\
\hline resid. el. dens. $\left[\mathrm{e} \AA^{-3}\right]$ & $0.624 /-0.437$ & $0.228 /-0.282$ & $0.537 /-0.293$ \\
\hline
\end{tabular}


Table 10.2: Crystal data and refinement details.

\begin{tabular}{|c|c|c|c|}
\hline compound & $7 \mathrm{e}$ & $7 f$ & $8 \mathbf{a}$ \\
\hline empirical formula & $\begin{array}{l}{\left[\mathrm{C}_{32} \mathrm{H}_{36} \mathrm{Fe}_{2} \mathrm{~S}_{6}\right]^{2-}} \\
2\left(\mathrm{C}_{8} \mathrm{H}_{20} \mathrm{~N}^{+}\right)\end{array}$ & $\begin{array}{l}{\left[\mathrm{C}_{24} \mathrm{H}_{16} \mathrm{~F}_{4} \mathrm{Fe}_{2} \mathrm{~S}_{6}\right]^{2-}} \\
2\left(\mathrm{C}_{8} \mathrm{H}_{20} \mathrm{~N}^{+}\right)\end{array}$ & $\begin{array}{l}{\left[\mathrm{C}_{24} \mathrm{H}_{8} \mathrm{O}_{4} \mathrm{~S}_{2} \mathrm{Fe}_{2}\right]^{2-},} \\
2\left(\mathrm{C}_{8} \mathrm{H}_{20} \mathrm{~N}^{+}\right), \\
3 \mathrm{THF}\end{array}$ \\
\hline formula weight & 985.17 & 944.93 & 1296.54 \\
\hline$T[\mathrm{~K}]$ & $133(2)$ & $133(2)$ & $133(2)$ \\
\hline crystal size $[\mathrm{mm}]$ & $0.80 \times 0.40 \times 0.10$ & $0.50 \times 0.41 \times 0.05$ & $0.50 \times 0.33 \times 0.25$ \\
\hline crystal system & monoclinic & triclinic & triclinic \\
\hline space group & $P 2_{1} / c$ (No. 14$)$ & $P 1$ (No. 1) & $P \overline{1}($ No. 2$)$ \\
\hline$a[\AA]$ & $17.958(4)$ & $8.7961(2)$ & $9.1684(5)$ \\
\hline$b[\AA]$ & $18.794(4)$ & $15.7706(3)$ & $16.8066(8)$ \\
\hline$c[\AA]$ & $16.390(3)$ & $16.0927(3)$ & $20.4441(10)$ \\
\hline$\alpha\left[^{\circ}\right]$ & 90 & $86.266(2)$ & $79.572(4)$ \\
\hline$\beta\left[^{\circ}\right]$ & $111.11(3)$ & $87.872(2)$ & $81.551(4)$ \\
\hline$\gamma\left[{ }^{\circ}\right]$ & 90 & $80.465(2)$ & $77.079(4)$ \\
\hline$V\left[\AA^{3}\right]$ & $5160.4(18)$ & $2196.07(8)$ & $3001.1(3)$ \\
\hline$\rho_{\text {calcd. }}\left[\mathrm{g} \mathrm{cm}^{-3}\right]$ & 1.268 & 1.429 & 1.435 \\
\hline$Z$ & 4 & 2 & 2 \\
\hline$F(000)$ & 2104 & 988 & 1348 \\
\hline$\mu\left[\mathrm{mm}^{-1}\right]$ & $0.838\left(\mathrm{Mo}-\mathrm{K}_{\alpha}\right)$ & $0.994\left(\mathrm{Mo}-\mathrm{K}_{\alpha}\right)$ & $0.958\left(\mathrm{Mo}^{-\mathrm{K}_{\alpha}}\right)$ \\
\hline$T_{\max } / T_{\min }$ & $0.9209 / 0.5537$ & $0.9687 / 0.5999$ & $0.7767 / 0.4952$ \\
\hline$h k l$ range & $-24-19, \pm 25, \pm 22$ & $-11-10, \pm 20, \pm 20$ & $\pm 10, \pm 19, \pm 24$ \\
\hline$\theta$ range $\left[^{\circ}\right]$ & $2.17-29.53$ & $1.77-26.96$ & $1.49-24.79$ \\
\hline measured refl. & 41559 & 68964 & 55140 \\
\hline unique refl. $\left[R_{\text {int }}\right]$ & $13443[0.0260]$ & $18276[0.0427]$ & $10268[0.0918]$ \\
\hline observed refl. $I>2 \sigma(I)$ & 11327 & 17248 & 8180 \\
\hline Refined parameters & 617 & 990 & 666 \\
\hline restraints & 408 & 3 & 0 \\
\hline goodness-of-fit & 1.048 & 1.005 & 1.018 \\
\hline$R 1, w R 2(I>2 \sigma(I))$ & $0.0298,0.0657$ & $0.0252,0.0587$ & $0.0375,0.0950$ \\
\hline$R 1, w R 2$ (all data) & $0.0410,0.0697$ & $0.0277,0.0593$ & $0.0486,0.0983$ \\
\hline resid. el. dens. $\left[\mathrm{e} \AA^{-3}\right]$ & $0.328 /-0.290$ & $0.258 /-0.446$ & $0.622 /-0.595$ \\
\hline
\end{tabular}


Table 10.3: Crystal data and refinement details.

\begin{tabular}{|c|c|c|c|}
\hline compound & $11 \mathrm{a}$ & $11 a^{\prime}$ & $11 b$ \\
\hline empirical formula & $\begin{array}{l}{\left[\mathrm{C}_{24} \mathrm{H}_{8} \mathrm{Cl}_{8} \mathrm{~S}_{6} \mathrm{Fe}_{2}\right]^{2-},} \\
2\left(\mathrm{C}_{8} \mathrm{H}_{20} \mathrm{~N}^{+}\right), \\
2 \mathrm{MeCN}\end{array}$ & $\begin{array}{l}{\left[\mathrm{C}_{24} \mathrm{H}_{8} \mathrm{Cl}_{8} \mathrm{~S}_{6} \mathrm{Fe}_{2}\right]^{2-},} \\
\left(\mathrm{C}_{8} \mathrm{H}_{20} \mathrm{~N}^{+}\right) \\
\left(\mathrm{C}_{10} \mathrm{H}_{10} \mathrm{Co}^{+}\right) \\
\text {MeCN }\end{array}$ & $\begin{array}{l}{\left[\mathrm{C}_{24} \mathrm{H}_{16} \mathrm{~S}_{6} \mathrm{Fe}_{2}\right]^{2-},} \\
2\left(\mathrm{C}_{8} \mathrm{H}_{20} \mathrm{~N}^{+}\right)\end{array}$ \\
\hline formula weight & 1226.57 & 1244.38 & 868.93 \\
\hline$T[\mathrm{~K}]$ & $133(2)$ & $133(2)$ & $133(2)$ \\
\hline crystal size $[\mathrm{mm}]$ & $0.50 \times 0.39 \times 0.21$ & $0.50 \times 0.41 \times 0.33$ & $0.50 \times 0.36 \times 0.04$ \\
\hline crystal system & monoclinic & triclinic & monoclinic \\
\hline space group & $P 2_{1} / n($ No. 14$)$ & $P \overline{1}($ No. 2$)$ & $C 2 / c($ No. 15$)$ \\
\hline$a[\AA]$ & $11.656(2)$ & $14.1135(8)$ & $15.9479(4)$ \\
\hline$b[\AA]$ & $25.766(5)$ & $19.3727(9)$ & $10.8406(4)$ \\
\hline$c[\AA]$ & $18.583(4)$ & $21.7784(11)$ & $24.9594(6)$ \\
\hline$\alpha\left[^{\circ}\right]$ & 90 & $110.516(4)$ & 90 \\
\hline$\beta\left[^{\circ}\right]$ & $102.56(3)$ & $91.699(4)$ & $90.894(2)$ \\
\hline$\gamma\left[^{\circ}\right]$ & 90 & $110.553(4)$ & 90 \\
\hline$V\left[\AA^{3}\right]$ & $5447.8(19)$ & $5142.2(5)$ & $4314.6(2)$ \\
\hline$\rho_{\text {calcd. }}\left[\mathrm{g} \mathrm{cm}^{-3}\right]$ & 1.495 & 1.607 & 1.338 \\
\hline$Z$ & 4 & 4 & 4 \\
\hline$F(000)$ & 2520 & 2520 & 1832 \\
\hline$\mu\left[\mathrm{mm}^{-1}\right]$ & $1.191\left(\mathrm{Mo}-\mathrm{K}_{\alpha}\right)$ & $1.570\left(\mathrm{Mo}-\mathrm{K}_{\alpha}\right)$ & $0.993\left(\mathrm{Mo}^{-\mathrm{K}_{\alpha}}\right)$ \\
\hline$T_{\max } / T_{\min }$ & $0.7467 / 0.4728$ & $0.6872 / 0.3840$ & $0.9856 / 0.6610$ \\
\hline$h k l$ range & $\pm 13, \pm 30, \pm 21$ & $\pm 16,-21-22, \pm 25$ & $-20-18, \pm 13, \pm 31$ \\
\hline$\theta$ range $\left[{ }^{\circ}\right]$ & $1.37-24.85$ & $1.22-24.81$ & $1.63-26.72$ \\
\hline measured refl. & 102247 & 30132 & 24789 \\
\hline unique refl. $\left[R_{i n t}\right]$ & $9395[0.0841]$ & $9684[0.0429]$ & $4569[0.0470]$ \\
\hline observed refl. $I>2 \sigma(I)$ & 7655 & 6645 & 4003 \\
\hline Refined parameters & 587 & 1125 & 230 \\
\hline restraints & 0 & 34 & 0 \\
\hline goodness-of-fit & 1.009 & 1.012 & 1.041 \\
\hline$R 1, w R 2(I>2 \sigma(I))$ & $0.0254,0.0617$ & $0.0475,0.1261$ & $0.0278,0.0701$ \\
\hline$R 1, w R 2$ (all data) & $0.0344,0.0634$ & $0.0717,0.1353$ & $0.0334,0.0721$ \\
\hline resid. el. dens. $\left[\mathrm{e} \AA^{-3}\right]$ & $0.344 /-0.418$ & $0.792 /-0.761$ & $0.366 /-0.383$ \\
\hline
\end{tabular}


Table 10.4: Crystal data and refinement details.

\begin{tabular}{|c|c|c|c|}
\hline compound & 11c & 13 & 23 \\
\hline empirical formula & $\begin{array}{l}{\left[\mathrm{C}_{56} \mathrm{H}_{80} \mathrm{~S}_{6} \mathrm{Fe}_{2}\right]^{2-},} \\
2\left(\mathrm{C}_{8} \mathrm{H}_{20} \mathrm{~N}^{+}\right), \\
4 \mathrm{MeCN}\end{array}$ & $\begin{array}{l}{\left[\mathrm{C}_{56} \mathrm{H}_{40} \mathrm{~S}_{12} \mathrm{Fe}_{4}\right]^{2-},} \\
2\left(\mathrm{C}_{24} \mathrm{H}_{20} \mathrm{P}^{+}\right), \\
\text {DMF }\end{array}$ & $\begin{array}{l}{\left[\mathrm{C}_{30} \mathrm{H}_{12} \mathrm{~F}_{10} \mathrm{~N}_{4} \mathrm{Fe}\right]} \\
\mathrm{C}_{6} \mathrm{H}_{6}\end{array}$ \\
\hline formula weight & 1481.98 & 2072.84 & 752.39 \\
\hline$T[\mathrm{~K}]$ & $133(2)$ & $133(2)$ & $133(2)$ \\
\hline crystal size $[\mathrm{mm}]$ & $0.48 \times 0.41 \times 0.33$ & $0.49 \times 0.20 \times 0.05$ & $0.45 \times 0.19 \times 0.11$ \\
\hline crystal system & triclinic & monoclinic & orthorhombic \\
\hline space group & $P \overline{1}($ No. 2$)$ & $P 2 / c$ (No. 13$)$ & $P 2_{1} 2_{1} 2_{1}$ (No. 19$)$ \\
\hline$a[\AA]$ & $12.4568(7)$ & $13.8619(4)$ & $7.9696(2)$ \\
\hline$b[\AA]$ & $13.0536(7)$ & $11.6418(2)$ & $15.6532(4)$ \\
\hline$c[\AA]$ & $15.6085(9)$ & $29.9323(8)$ & $25.3709(8)$ \\
\hline$\alpha\left[^{\circ}\right]$ & $90.433(4)$ & 90 & 90 \\
\hline$\beta\left[^{\circ}\right]$ & $107.152(4)$ & $101.204(2)$ & 90 \\
\hline$\gamma\left[^{\circ}\right]$ & $117.565(4)$ & 90 & 90 \\
\hline$V\left[\AA^{3}\right]$ & $2118.6(2)$ & $4738.3(2)$ & $3165.01(15)$ \\
\hline$\rho_{\text {calcd. }}\left[\mathrm{g} \mathrm{cm}^{-3}\right]$ & 1.162 & 1.453 & 1.579 \\
\hline$Z$ & 1 & 2 & 4 \\
\hline$F(000)$ & 802 & 2140 & 1512 \\
\hline$\mu\left[\mathrm{mm}^{-1}\right]$ & $0.533\left(\mathrm{Mo}^{-\mathrm{K}_{\alpha}}\right)$ & $0.950\left(\mathrm{Mo}^{-\mathrm{K}_{\alpha}}\right)$ & $0.570\left(\mathrm{Mo}-\mathrm{K}_{\alpha}\right)$ \\
\hline$T_{\max } / T_{\min }$ & $0.8675 / 0.7570$ & $0.9591 / 0.7443$ & $0.9553 / 0.8289$ \\
\hline$h k l$ range & $\pm 14,-15-14, \pm 18$ & $\begin{array}{l} \pm 17,-12-14 \\
-37-36\end{array}$ & $-10-9, \pm 19, \pm 31$ \\
\hline$\theta$ range $\left[^{\circ}\right]$ & $1.79-24.80$ & $1.39-26.60$ & $1.53-26.57$ \\
\hline measured refl. & 30968 & 38943 & 30017 \\
\hline unique refl. $\left[R_{\text {int }}\right]$ & $7260[0.0325]$ & $9636[0.0488]$ & $6579[0.0295]$ \\
\hline observed refl. $I>2 \sigma(I)$ & 6288 & 7837 & 6194 \\
\hline Refined parameters & 460 & 570 & 491 \\
\hline restraints & 0 & 6 & 0 \\
\hline goodness-of-fit & 1.054 & 1.032 & 1.031 \\
\hline$R 1, w R 2(I>2 \sigma(I))$ & $0.0283,0.0751$ & $0.0344,0.0721$ & $0.0232,0.0568$ \\
\hline$R 1, w R 2$ (all data) & $0.0341,0.0769$ & $0.0485,0.0758$ & $0.0258,0.0575$ \\
\hline resid. el. dens. $\left[\mathrm{e} \AA^{-3}\right]$ & $0.375 /-0.232$ & $0.510 /-0.691$ & $0.188 /-0.249$ \\
\hline
\end{tabular}


Table 10.5: Crystal data and refinement details.

\begin{tabular}{|c|c|c|c|}
\hline compound & 23-b & $25^{\mathrm{C}}$ & $25^{\mathrm{O}}$ \\
\hline empirical formula & $\begin{array}{l}{\left[\mathrm{C}_{30} \mathrm{H}_{12} \mathrm{~F}_{10} \mathrm{~N}_{4} \mathrm{Fe}\right]} \\
\mathrm{C}_{7} \mathrm{H}_{8}\end{array}$ & $\begin{array}{l}{\left[\mathrm{C}_{32} \mathrm{H}_{36} \mathrm{Fe}_{2} \mathrm{~S}_{6}\right]^{2-},} \\
2\left(\mathrm{C}_{8} \mathrm{H}_{20} \mathrm{~N}^{+}\right) \\
2 \mathrm{MeCN}\end{array}$ & $\begin{array}{l}{\left[\mathrm{C}_{28} \mathrm{H}_{28} \mathrm{Fe}_{2} \mathrm{O}_{4} \mathrm{~S}_{6}\right]^{2-},} \\
2\left(\mathrm{C}_{8} \mathrm{H}_{20} \mathrm{~N}^{+}\right)\end{array}$ \\
\hline formula weight & 766.42 & 1067.28 & 993.06 \\
\hline$T[\mathrm{~K}]$ & $133(2)$ & $133(2)$ & $133(2)$ \\
\hline crystal size $[\mathrm{mm}]$ & $0.51 \times 0.48 \times 0.42$ & $0.50 \times 0.47 \times 0.45$ & $0.46 \times 0.38 \times 0.15$ \\
\hline crystal system & orthorhombic & monoclinic & monoclinic \\
\hline space group & $P 2_{1} 2_{1} 2_{1}$ (No. 19$)$ & $C 2 / c($ No. 15$)$ & $P 2_{1} / c($ No. 14$)$ \\
\hline$a[\AA]$ & $7.8587(2)$ & $17.7778(8)$ & $9.9311(4)$ \\
\hline$b[\AA]$ & $15.9633(4)$ & $14.5054(5)$ & $21.7363(7)$ \\
\hline$c[\AA]$ & $25.7229(8)$ & $22.5604(11)$ & $22.5717(10)$ \\
\hline$\left.\alpha{ }^{\circ}\right]$ & 90 & 90 & 90 \\
\hline$\beta\left[{ }^{\circ}\right]$ & 90 & $97.015(4)$ & $94.367(3)$ \\
\hline$\gamma\left[^{\circ}\right]$ & 90 & 90 & 90 \\
\hline$V\left[\AA^{3}\right]$ & $3226.96(15)$ & $5774.2(4)$ & $4858.3(3)$ \\
\hline$\rho_{\text {calcd. }}\left[\mathrm{g} \mathrm{cm}^{-3}\right]$ & 1.578 & 1.228 & 1.358 \\
\hline$Z$ & 4 & 4 & 4 \\
\hline$F(000)$ & 1544 & 2280 & 2104 \\
\hline$\mu\left[\mathrm{mm}^{-1}\right]$ & $0.560\left(\mathrm{Mo}^{-\mathrm{K}_{\alpha}}\right)$ & $0.755\left(\mathrm{Mo}^{-\mathrm{K}_{\alpha}}\right)$ & $0.897\left(\mathrm{Mo}^{-\mathrm{K}_{\alpha}}\right)$ \\
\hline$T_{\max } / T_{\min }$ & $0.7875 / 0.7148$ & $0.7364 / 0.6709$ & $0.8490 / 0.6252$ \\
\hline$h k l$ range & $\pm 10, \pm 20, \pm 33$ & $\pm 20, \pm 17, \pm 26$ & $-11-10, \pm 25, \pm 26$ \\
\hline$\theta$ range $\left[^{\circ}\right]$ & $1.58-27.35$ & $1.82-24.81$ & $1.30-24.85$ \\
\hline measured refl. & 79538 & 41296 & 67941 \\
\hline unique refl. $\left[R_{\text {int }}\right]$ & $7261[0.0352]$ & $4967[0.0297]$ & $8378[0.0660]$ \\
\hline observed refl. $I>2 \sigma(I)$ & 6781 & 4604 & 6504 \\
\hline Refined parameters & 470 & 292 & 535 \\
\hline restraints & 0 & 24 & 0 \\
\hline goodness-of-fit & 1.086 & 1.119 & 1.017 \\
\hline$R 1, w R 2(I>2 \sigma(I))$ & $0.0250,0.0646$ & $0.0800,0.1919$ & $0.0273,0.0588$ \\
\hline$R 1, w R 2$ (all data) & $0.0274,0.0653$ & $0.0837,0.1934$ & $0.0416,0.0610$ \\
\hline resid. el. dens. $\left[\mathrm{e} \AA^{-3}\right]$ & $0.217 /-0.274$ & $1.057 /-0.650$ & $0.314 /-0.214$ \\
\hline
\end{tabular}


Table 10.6: Crystal data and refinement details.

\begin{tabular}{|c|c|c|c|}
\hline compound & $25^{\mathrm{S}}$ & $26^{\mathrm{C}}$ & $26^{\mathrm{O}}$ \\
\hline empirical formula & $\begin{array}{l}{\left[\mathrm{C}_{28} \mathrm{H}_{28} \mathrm{Fe}_{2} \mathrm{~S}_{10}\right]^{2-}} \\
2\left(\mathrm{C}_{8} \mathrm{H}_{20} \mathrm{~N}^{+}\right)\end{array}$ & $\begin{array}{l}{\left[\mathrm{C}_{26} \mathrm{H}_{20} \mathrm{Fe}_{2} \mathrm{~S}_{6}\right]^{2-}} \\
2\left(\mathrm{C}_{8} \mathrm{H}_{20} \mathrm{~N}^{+}\right)\end{array}$ & $\begin{array}{l}{\left[\mathrm{C}_{24} \mathrm{H}_{16} \mathrm{Fe}_{2} \mathrm{O}_{2} \mathrm{~S}_{6}\right]^{2-},} \\
2\left(\mathrm{C}_{8} \mathrm{H}_{20} \mathrm{~N}^{+}\right)\end{array}$ \\
\hline formula weight & 1057.30 & 896.98 & 900.93 \\
\hline$T[\mathrm{~K}]$ & $133(2)$ & $133(2)$ & $133(2)$ \\
\hline crystal size $[\mathrm{mm}]$ & $0.43 \times 0.37 \times 0.25$ & $0.38 \times 0.32 \times 0.26$ & $0.40 \times 0.36 \times 0.32$ \\
\hline crystal system & monoclinic & monoclinic & monoclinic \\
\hline space group & $P 2_{1} / n($ No. 14$)$ & $P 2_{1} / n($ No. 14$)$ & $P 2_{1} / c$ (No. 14$)$ \\
\hline$a[\AA]$ & $10.7835(4)$ & $11.4189(6)$ & $16.1247(6)$ \\
\hline$b[\AA]$ & $17.5216(6)$ & $11.5647(4)$ & $13.3743(4)$ \\
\hline$c[\AA]$ & $14.4262(6)$ & $16.5968(9)$ & $19.7471(7)$ \\
\hline$\alpha\left[^{\circ}\right]$ & 90 & 90 & 90 \\
\hline$\beta\left[^{\circ}\right]$ & $109.692(3)$ & $95.162(4)$ & $90.234(3)$ \\
\hline$\gamma\left[^{\circ}\right]$ & 90 & 90 & 90 \\
\hline$V\left[\AA^{3}\right]$ & $2566.34(17)$ & $2182.82(18)$ & $4258.6(3)$ \\
\hline$\rho_{\text {calcd. }}\left[\mathrm{g} \mathrm{cm}^{-3}\right]$ & 1.368 & 1.365 & 1.405 \\
\hline$Z$ & 2 & 2 & 4 \\
\hline$F(000)$ & 1116 & 948 & 1896 \\
\hline$\mu\left[\mathrm{mm}^{-1}\right]$ & $1.004\left(\mathrm{Mo}-\mathrm{K}_{\alpha}\right)$ & $0.983\left(\mathrm{Mo}^{-\mathrm{K}_{\alpha}}\right)$ & $1.012\left(\mathrm{Mo} \mathrm{K}_{\alpha}\right)$ \\
\hline$T_{\max } / T_{\min }$ & $0.8188 / 0.6693$ & $0.8154 / 0.6726$ & $0.7843 / 0.6765$ \\
\hline$h k l$ range & $\begin{array}{l}-13-12, \pm 21 \\
\pm 17\end{array}$ & $\begin{array}{l}-12-13, \pm 13 \\
\pm 19\end{array}$ & $\pm 20, \pm 17, \pm 25$ \\
\hline$\theta$ range $\left[^{\circ}\right]$ & $1.90-25.90$ & $2.08-24.78$ & $1.84-27.38$ \\
\hline measured refl. & 43925 & 29971 & 82478 \\
\hline unique refl. $\left[R_{i n t}\right]$ & $4969[0.0500]$ & $3750[0.0449]$ & $9602[0.0446]$ \\
\hline observed refl. $I>2 \sigma(I)$ & 4464 & 3336 & 7608 \\
\hline Refined parameters & 268 & 239 & 47 \\
\hline restraints & 0 & 0 & 0 \\
\hline goodness-of-fit & 1.043 & 1.039 & 1.038 \\
\hline$R 1, w R 2(I>2 \sigma(I))$ & $0.0247,0.0653$ & $0.0227,0.0595$ & $0.0279,0.0654$ \\
\hline$R 1, w R 2$ (all data) & $0.0289,0.0667$ & $0.0269,0.0606$ & $0.0410,0.0683$ \\
\hline resid. el. dens. $\left[\mathrm{e} \AA^{-3}\right]$ & $0.300 /-0.197$ & $0.294 /-0.206$ & $0.329 /-0.272$ \\
\hline
\end{tabular}


Table 10.7: Crystal data and refinement details.

\begin{tabular}{|c|c|c|c|}
\hline compound & $26^{\mathrm{S}}$ & $27^{\mathrm{N}}$ & $27^{\mathrm{O}}$ \\
\hline empirical formula & $\begin{array}{l}{\left[\mathrm{C}_{24} \mathrm{H}_{16} \mathrm{Fe}_{2} \mathrm{~S}_{8}\right]^{2-}} \\
2\left(\mathrm{C}_{8} \mathrm{H}_{20} \mathrm{~N}^{+}\right)\end{array}$ & $\begin{array}{l}{\left[\mathrm{C}_{26} \mathrm{H}_{22} \mathrm{~S}_{4} \mathrm{~N}_{2} \mathrm{Fe}\right]^{-},} \\
\left(\mathrm{C}_{8} \mathrm{H}_{20} \mathrm{~N}^{+}\right)\end{array}$ & $\begin{array}{l}{\left[\mathrm{C}_{24} \mathrm{H}_{16} \mathrm{O}_{2} \mathrm{~S}_{4} \mathrm{Fe}\right]^{-}} \\
\left(\mathrm{C}_{8} \mathrm{H}_{20} \mathrm{~N}^{+}\right), \mathrm{Et}_{2} \mathrm{O}\end{array}$ \\
\hline formula weight & 933.05 & 676.80 & 724.83 \\
\hline$T[\mathrm{~K}]$ & $133(2)$ & $133(2)$ & $133(2)$ \\
\hline crystal size $[\mathrm{mm}]$ & $0.32 \times 0.07 \times 0.06$ & $0.50 \times 0.42 \times 0.36$ & $0.50 \times 0.41 \times 0.31$ \\
\hline crystal system & monoclinic & monoclinic & orthorombic \\
\hline space group & $P 2_{1}($ No. 14$)$ & $P 2_{1} / c$ (No. 14$)$ & $P$ c c $n($ No. 56$)$ \\
\hline$a[\AA]$ & $9.4152(7)$ & $27.3422(10)$ & $16.2962(4)$ \\
\hline$b[\AA]$ & 13.1074(11) & $14.6841(3)$ & $32.5862(10)$ \\
\hline$c[\AA]$ & $17.7976(16)$ & $16.5464(6)$ & $14.7324(4)$ \\
\hline$\alpha\left[^{\circ}\right]$ & 90 & 90 & 90 \\
\hline$\beta\left[^{\circ}\right]$ & $95.289(6)$ & $101.474(3)$ & 90 \\
\hline$\gamma\left[^{\circ}\right]$ & 90 & 90 & 90 \\
\hline$V\left[\AA^{3}\right]$ & $2187.0(3)$ & $6510.5(4)$ & $7823.4(4)$ \\
\hline$\rho_{\text {calcd. }}\left[\mathrm{g} \mathrm{cm}^{-3}\right]$ & 1.417 & 1.381 & 1.231 \\
\hline$Z$ & 2 & 8 & 8 \\
\hline$F(000)$ & 980 & 2856 & 3064 \\
\hline$\mu\left[\mathrm{mm}^{-1}\right]$ & $1.076\left(\mathrm{Mo}-\mathrm{K}_{\alpha}\right)$ & $0.749\left(\mathrm{Mo}^{-\mathrm{K}_{\alpha}}\right)$ & $0.632\left(\mathrm{Mo}^{-\mathrm{K}_{\alpha}}\right)$ \\
\hline$T_{\max } / T_{\min }$ & $-/-$ & $0.8210 / 0.6427$ & $0.8718 / 0.7183$ \\
\hline$h k l$ range & $\begin{array}{l}-10-11, \pm 15 \\
-21-20\end{array}$ & $\pm 32,-17-15, \pm 19$ & $-17-19, \pm 38, \pm 17$ \\
\hline$\theta$ range $\left[^{\circ}\right]$ & $2.17-24.85$ & $1.52-24.75$ & $1.25-24.85$ \\
\hline measured refl. & 10276 & 88554 & 95454 \\
\hline unique refl. $\left[R_{\text {int }}\right]$ & $4981[0.1106]$ & 11099 [0.0614] & $6765[0.0519]$ \\
\hline observed refl. $I>2 \sigma(I)$ & 3047 & 8557 & 5884 \\
\hline Refined parameters & 477 & 769 & 446 \\
\hline restraints & 1 & 0 & 37 \\
\hline goodness-of-fit & 1.006 & 1.000 & 1.210 \\
\hline$R 1, w R 2(I>2 \sigma(I))$ & $0.0598,0.0976$ & $0.0315,0.0753$ & $0.0757,0.2030$ \\
\hline$R 1, w R 2$ (all data) & $0.1064,0.1085$ & $0.0477,0.0795$ & $0.0845,0.2068$ \\
\hline resid. el. dens. $\left[\mathrm{e}^{\circ}{ }^{-3}\right]$ & $0.457 /-0.330$ & $0.300 /-0.233$ & $1.369 /-0.530$ \\
\hline
\end{tabular}


Table 10.8: Crystal data and refinement details.

\begin{tabular}{|c|c|c|c|}
\hline compound & $27^{\mathrm{P}}$ & $27^{\mathrm{S}}$ & $29 a$ \\
\hline empirical formula & $\begin{array}{l}{\left[\mathrm{C}_{36} \mathrm{H}_{26} \mathrm{~S}_{4} \mathrm{P}_{2} \mathrm{Fe}\right]^{-}} \\
\left(\mathrm{C}_{8} \mathrm{H}_{20} \mathrm{~N}^{+}\right)\end{array}$ & $\begin{array}{l}{\left[\mathrm{C}_{24} \mathrm{H}_{16} \mathrm{~S}_{6} \mathrm{Fe}\right]^{-},} \\
\left(\mathrm{C}_{8} \mathrm{H}_{20} \mathrm{~N}^{+}\right), \\
2 \mathrm{DMF}\end{array}$ & $\begin{array}{l}{\left[\mathrm{C}_{22} \mathrm{H}_{24} \mathrm{Fe}_{2} \mathrm{~N}_{4} \mathrm{~S}_{2}\right]^{2-},} \\
2\left(\mathrm{C}_{8} \mathrm{H}_{20} \mathrm{~N}^{+}\right)\end{array}$ \\
\hline formula weight & 834.85 & 829.02 & 780.77 \\
\hline$T[\mathrm{~K}]$ & $133(2)$ & $133(2)$ & $100(2)$ \\
\hline crystal size $[\mathrm{mm}]$ & $0.50 \times 0.45 \times 0.38$ & $0.50 \times 0.26 \times 0.05$ & $0.10 \times 0.10 \times 0.10$ \\
\hline crystal system & monoclinic & monoclinic & monoclinic \\
\hline space group & $P 2_{1}$ (No. 14$)$ & $P 2_{1} / c($ No. 14$)$ & $P 2_{1} / n($ No. 14$)$ \\
\hline$a[\AA]$ & $16.4815(5)$ & $11.0087(7)$ & $17.555(4)$ \\
\hline$b[\AA]$ & $13.7138(6)$ & $14.9498(6)$ & $10.078(2)$ \\
\hline$c[\AA]$ & $17.8088(5)$ & $24.3049(16)$ & $22.965(5)$ \\
\hline$\alpha\left[^{\circ}\right]$ & 90 & 90 & 90 \\
\hline$\beta\left[^{\circ}\right]$ & $90.479(2)$ & $100.147(5)$ & $98.38(3)$ \\
\hline$\gamma\left[{ }^{\circ}\right]$ & 90 & 90 & 90 \\
\hline$V\left[\AA^{3}\right]$ & $4025.1(2)$ & $3937.5(4)$ & $4019.6(15)$ \\
\hline$\rho_{\text {calcd. }}\left[\mathrm{g} \mathrm{cm}^{-3}\right]$ & 1.378 & 1.398 & 1.290 \\
\hline$Z$ & 4 & 4 & 4 \\
\hline$F(000)$ & 1748 & 1748 & 1672 \\
\hline$\mu\left[\mathrm{mm}^{-1}\right]$ & $0.695\left(\mathrm{Mo}-\mathrm{K}_{\alpha}\right)$ & $0.739\left(\mathrm{Mo}^{-\mathrm{K}_{\alpha}}\right)$ & $7.009\left(\mathrm{Cu}-\mathrm{K}_{\alpha}\right)$ \\
\hline$T_{\max } / T_{\min }$ & $0.8136 / 0.7110$ & $0.8970 / 0.7674$ & $0.5408 / 0.5408$ \\
\hline$h k l$ range & $\begin{array}{l} \pm 21, \pm 17 \\
-22-23\end{array}$ & $\begin{array}{l} \pm 12,-15-17 \\
\pm 28\end{array}$ & $\pm 19, \pm 11, \pm 25$ \\
\hline$\theta$ range $\left[^{\circ}\right]$ & $1.87-27.35$ & $1.61-24.76$ & $2.97-60.05$ \\
\hline measured refl. & 59144 & 27278 & 17656 \\
\hline unique refl. $\left[R_{\text {int }}\right]$ & $9061[0.0398]$ & $6730[0.0656]$ & $5896[0.0212]$ \\
\hline observed refl. $I>2 \sigma(I)$ & 7592 & 5095 & 5116 \\
\hline Refined parameters & 473 & 459 & 527 \\
\hline restraints & 0 & 0 & 525 \\
\hline goodness-of-fit & 1.062 & 1.018 & 1.043 \\
\hline$R 1, w R 2(I>2 \sigma(I))$ & $0.0263,0.0691$ & $0.0417,0.0846$ & $0.0240,0.0618$ \\
\hline$R 1, w R 2$ (all data) & $0.0344,0.0709$ & $0.0653,0.0928$ & $0.0303,0.0650$ \\
\hline resid. el. dens. $\left[\mathrm{e}^{-3}\right]$ & $0.333 /-0.227$ & $0.327 /-0.345$ & $0.202 /-0.255$ \\
\hline
\end{tabular}


Table 10.9: Crystal data and refinement details.

\begin{tabular}{|c|c|c|c|}
\hline compound & $29 \mathrm{~b}$ & $29 c$ & $31 \mathrm{a}$ \\
\hline empirical formula & $\begin{array}{l}{\left[\mathrm{C}_{42} \mathrm{H}_{32} \mathrm{Fe}_{2} \mathrm{~N}_{4} \mathrm{~S}_{2}\right]^{2-},} \\
2\left(\mathrm{C}_{8} \mathrm{H}_{20} \mathrm{~N}^{+}\right)\end{array}$ & $\begin{array}{l}{\left[\mathrm{C}_{28} \mathrm{H}_{32} \mathrm{Fe}_{2} \mathrm{~N}_{4} \mathrm{~S}_{2}\right]^{2-},} \\
2\left(\mathrm{C}_{8} \mathrm{H}_{20} \mathrm{~N}^{+}\right), \\
2 \mathrm{MeCN}\end{array}$ & $\begin{array}{l}{\left[\mathrm{C}_{40} \mathrm{H}_{24} \mathrm{Fe}_{2} \mathrm{~S}_{2} \mathrm{~N}_{8}\right]^{2-},} \\
2\left(\mathrm{C}_{8} \mathrm{H}_{20} \mathrm{~N}^{+}\right), \\
2 \mathrm{MeCN}\end{array}$ \\
\hline formula weight & 1029.04 & 943.00 & 1135.10 \\
\hline$T[\mathrm{~K}]$ & $133(2)$ & $133(2)$ & $133(2)$ \\
\hline crystal size $[\mathrm{mm}]$ & $0.32 \times 0.29 \times 0.14$ & $0.12 \times 0.11 \times 0.10$ & $0.22 \times 0.19 \times 0.17$ \\
\hline crystal system & monoclinic & monoclinic & triclinic \\
\hline space group & $P 2_{1} / n$ (No. 14$)$ & $P 2_{1} / n$ (No. 14$)$ & $P \overline{1}($ No. 2$)$ \\
\hline$a[\AA]$ & $16.4410(9)$ & $12.215(2)$ & $9.9057(14)$ \\
\hline$b[\AA]$ & $10.0988(6)$ & $13.489(2)$ & $12.4802(17)$ \\
\hline$c[\AA]$ & $17.3492(11)$ & $15.714(2)$ & $13.4656(19)$ \\
\hline$\alpha\left[^{\circ}\right]$ & 90 & 90 & $65.575(10)$ \\
\hline$\beta\left[^{\circ}\right]$ & $115.351(4)$ & $102.709(12)$ & $73.563(11)$ \\
\hline$\gamma\left[^{\circ}\right]$ & 90 & 90 & $76.717(11)$ \\
\hline$V\left[\AA^{3}\right]$ & $2603.2(3)$ & $2525.7(7)$ & $1441.6(3)$ \\
\hline$\rho_{\text {calcd. }}\left[\mathrm{g} \mathrm{cm}^{-3}\right]$ & 1.313 & 1.240 & 1.308 \\
\hline$Z$ & 2 & 2 & 1 \\
\hline$F(000)$ & 1092 & 1012 & 598 \\
\hline$\mu\left[\mathrm{mm}^{-1}\right]$ & $0.682\left(\mathrm{Mo}-\mathrm{K}_{\alpha}\right)$ & $0.697\left(\mathrm{Mo}-\mathrm{K}_{\alpha}\right)$ & $0.625\left(\mathrm{Mo}^{-\mathrm{K}_{\alpha}}\right)$ \\
\hline$T_{\max } / T_{\min }$ & $0.9547 / 0.8694$ & $-/-$ & $0.9479 / 0.8152$ \\
\hline$h k l$ range & $\begin{array}{l}-19-18, \pm 11 \\
\pm 20\end{array}$ & $\begin{array}{l}-14-13,-15-13 \\
0-18\end{array}$ & $\begin{array}{l}-10-11, \pm 14 \\
\pm 15\end{array}$ \\
\hline$\theta$ range $\left[^{\circ}\right]$ & $2.26-24.83$ & $2.01-24.51$ & $1.70-24.50$ \\
\hline measured refl. & 18671 & 6593 & 7557 \\
\hline unique refl. $\left[R_{i n t}\right]$ & $4471[0.0413]$ & $4032[0.1416]$ & $4663[0.089]$ \\
\hline observed refl. $I>2 \sigma(I)$ & 3765 & 1890 & 2236 \\
\hline Refined parameters & 311 & 276 & 348 \\
\hline restraints & 0 & 0 & 0 \\
\hline goodness-of-fit & 1.017 & 0.929 & 0.914 \\
\hline$R 1, w R 2(I>2 \sigma(I))$ & $0.0337,0.0772$ & $0.0787,0.1283$ & $0.0793,0.1050$ \\
\hline$R 1, w R 2$ (all data) & $0.0443,0.0811$ & $0.1773,0.1579$ & $0.1748,0.1302$ \\
\hline resid. el. dens. $\left[\mathrm{e}^{-3}\right]$ & $0.335 /-0.293$ & $0.649 /-0.466$ & $0.466 /-0.383$ \\
\hline
\end{tabular}


Table 10.10: Crystal data and refinement details.

\begin{tabular}{|c|c|c|c|}
\hline compound & 32 & 33 & 34 \\
\hline empirical formula & $\begin{array}{l}{\left[\mathrm{C}_{33} \mathrm{H}_{28} \mathrm{Fe}_{2} \mathrm{~S}_{4} \mathrm{~N}_{2}\right]^{2-},} \\
2\left(\mathrm{C}_{8} \mathrm{H}_{20} \mathrm{~N}^{+}\right)\end{array}$ & $\begin{array}{l}{\left[\mathrm{C}_{25} \mathrm{H}_{20} \mathrm{Fe}_{2} \mathrm{~S}_{2} \mathrm{~N}_{2} \mathrm{Cl}_{2}\right]^{2-},} \\
2\left(\mathrm{C}_{8} \mathrm{H}_{20} \mathrm{~N}^{+}\right)\end{array}$ & $\begin{array}{l}{\left[\mathrm{C}_{50} \mathrm{H}_{40} \mathrm{Fe}_{2} \mathrm{~S}_{2} \mathrm{~N}_{4}\right]^{2-},} \\
2\left(\mathrm{C}_{8} \mathrm{H}_{20} \mathrm{~N}^{+}\right), \\
2 \mathrm{MeCN}\end{array}$ \\
\hline formula weight & 953.01 & 855.65 & 1215.29 \\
\hline$T[\mathrm{~K}]$ & $133(2)$ & $133(2)$ & $133(2)$ \\
\hline crystal size $[\mathrm{mm}]$ & $0.40 \times 0.18 \times 0.05$ & $0.30 \times 0.27 \times 0.25$ & $0.48 \times 0.06 \times 0.04$ \\
\hline crystal system & monoclinic & monoclinic & monoclinic \\
\hline space group & $C 2 / c($ No. 15$)$ & $\operatorname{Pn}($ No. 7$)$ & $P 2_{1} / n$ (No. 14$)$ \\
\hline$a[\AA]$ & $18.2105(6)$ & $11.4793(6)$ & $9.2493(4)$ \\
\hline$b[\AA]$ & $22.1315(10)$ & $11.9175(10)$ & $23.6080(13)$ \\
\hline$c[\AA]$ & $25.6251(9)$ & $16.5061(9)$ & $14.5653(6)$ \\
\hline$\alpha\left[^{\circ}\right]$ & 90 & 90 & 90 \\
\hline$\beta\left[^{\circ}\right]$ & $100.945(3)$ & $106.295(4)$ & $98.659(3)$ \\
\hline$\gamma\left[^{\circ}\right]$ & 90 & 90 & 90 \\
\hline$V\left[\AA^{3}\right]$ & 10139.7(7) & $2167.4(2)$ & $3144.2(3)$ \\
\hline$\rho_{\text {calcd. }}\left[\mathrm{g} \mathrm{cm}^{-3}\right]$ & 1.249 & 1.311 & 1.284 \\
\hline$Z$ & 8 & 2 & 2 \\
\hline$F(000)$ & 4048 & 904 & 1292 \\
\hline$\mu\left[\mathrm{mm}^{-1}\right]$ & $0.773\left(\mathrm{Mo}_{\mathrm{N}} \mathrm{K}_{\alpha}\right)$ & $0.922\left(\mathrm{Mo}-\mathrm{K}_{\alpha}\right)$ & $0.576\left(\mathrm{Mo}-\mathrm{K}_{\alpha}\right)$ \\
\hline$T_{\max } / T_{\min }$ & $0.8811 / 0.6432$ & $-/-$ & $-/-$ \\
\hline$h k l$ range & $\begin{array}{l}-23-22,-28-27 \\
-31-32\end{array}$ & $\pm 13, \pm 14, \pm 20$ & $\pm 11, \pm 29, \pm 18$ \\
\hline$\theta$ range $\left[^{\circ}\right]$ & $1.46-26.78$ & $1.71-25.60$ & $1.66-26.78$ \\
\hline measured refl. & 43914 & 29289 & 28180 \\
\hline unique refl. $\left[R_{i n t}\right]$ & $10757[0.1103]$ & 29289 [0.0000] & $6678[0.1283]$ \\
\hline observed refl. $I>2 \sigma(I)$ & 6399 & 21215 & 4560 \\
\hline Refined parameters & 565 & 471 & 442 \\
\hline restraints & 9 & 2 & 0 \\
\hline goodness-of-fit & 1.011 & 1.022 & 1.040 \\
\hline$R 1, w R 2(I>2 \sigma(I))$ & $0.0667,0.1306$ & $0.0838,0.2108$ & $0.0584,0.1062$ \\
\hline$R 1, w R 2$ (all data) & $0.1230,0.1522$ & $0.1073,0.2259$ & $0.1007,0.1203$ \\
\hline resid. el. dens. $\left[\mathrm{e} \AA^{-3}\right]$ & $0.473 /-0.620$ & $1.064 /-0.979$ & $0.449 /-0.653$ \\
\hline
\end{tabular}




\section{Appendix A}

Table A.1: Selected interatomic distances $[\stackrel{\circ}{\not}]$ and angles $\left[{ }^{\circ}\right]$ for $\mathbf{1}, \mathbf{7 a}$ and $\mathbf{7 b}$.

\begin{tabular}{cccc}
\hline compound & $\mathbf{1}^{[132]}$ & $\mathbf{7 a}^{[152]}$ & $\mathbf{7 b}^{[132]}$ \\
\hline $\mathrm{Fe} \cdot \mathrm{Fe}$ & $2.698(1)$ & $2.691(2)$ & $2.691(1)$ \\
$\mathrm{Fe}-\mathrm{SR}$ & $2.306(1), 2.303(1)$ & $2.296(3), 2.306(3)$ & $2.312(1), 2.312(1)$ \\
$\mathrm{Fe}-(\mu-\mathrm{S})$ & $2.185(2), 2.232(1)$ & $2.197(3), 2.197(3)$ & $2.200(1), 2.202(1)$ \\
$\mathrm{RS}-\mathrm{Fe}-\mathrm{SR}$ & $106.4^{\mathrm{a}}$ & $110.0(2)$ & $111.20(4)$ \\
$\mathrm{Fe}-(\mu-\mathrm{S})-\mathrm{Fe}$ & $75.27(5)$ & $75.5(2)$ & $75.39(4)$ \\
$(\mu-\mathrm{S})-\mathrm{Fe}-(\mu-\mathrm{S})$ & $104.73(5)$ & $104.5(2)$ & $104.61(4)$ \\
$\tau_{4}$ & b $^{\mathrm{b}}$ & 0.951 & 0.920 \\
\hline
\end{tabular}

(a) Error deviation not reported. (b) two angles S-Fe-S not reported.

Table A.2: Selected interatomic distances $[\AA]$ ] and angles $\left[{ }^{\circ}\right]$ for $\mathbf{7 g}, \boldsymbol{7 h}$ and $\boldsymbol{7 \boldsymbol { i }}$.

\begin{tabular}{cccc}
\hline compound & $\mathbf{7 g}^{[153]}$ & $\mathbf{7 h}$ & $\mathbf{7 i}^{[155], \mathrm{a}}$ \\
\hline $\mathrm{Fe} \cdot \mathrm{Fe}$ & $2.703(2)$ & $2.698(5)$ & $2.671(6)$ \\
$\mathrm{Fe}-\mathrm{SR}$ & $2.296(3), 2.309(3)$ & $2.299(8), 2.318(6)$ & $2.308(8), 2.328(7)$ \\
$\mathrm{Fe}-(\mu-\mathrm{S})$ & $2.196(3), 2.198(3)$ & $2.195(6), 2.204(6)$ & $2.198(7), 2.203(6)$ \\
$\mathrm{RS}-\mathrm{Fe}-\mathrm{SR}$ & $100.3(2)$ & $107.9(3)$ & $107.7(3)$ \\
$\mathrm{Fe}-(\mu-\mathrm{S})-\mathrm{Fe}$ & $75.92(9)$ & $75.62(20)$ & $74.8(2)$ \\
$(\mu-\mathrm{S})-\mathrm{Fe}-(\mu-\mathrm{S})$ & $104.08(9)$ & $104.4(3)$ & $104.1(2)$ \\
$\tau_{4}$ & 0.916 & 0.935 & 0.940 \\
\hline
\end{tabular}

(a) Cluster core deviates from planarity by a dihedral angle of $\mathrm{Fe}-(\mu-\mathrm{S})-\mathrm{Fe}-(\mu-\mathrm{S})=4.20^{\circ}$. 
Table A.3: Selected interatomic distances $[\AA]$ ] and angles $\left[{ }^{\circ}\right]$ for 3, 4 and 14.

\begin{tabular}{cccc}
\hline compound & $\mathbf{3}^{[34]}$ & $\mathbf{4}^{[141], \mathrm{b}}$ & $\mathbf{1 4}^{[169], \mathrm{c}}$ \\
\hline $\mathrm{Fe} \cdots \mathrm{Fe}$ & $2.783(2)$ & $2.677(3)$ & $2.701(3)$ \\
$\mathrm{Fe}-\mathrm{SR}$ & $2.421(2)$ & - & $2.323(3), 2.318(3)^{\mathrm{d}}$ \\
$\mathrm{Fe}-\mathrm{NR}_{2}$ & $1.930(5)$ & $1.872(24)-2.092(26)$ & - \\
$\mathrm{Fe}-(\mu-\mathrm{S})$ & $2.2112(2)$ & $2.078(8)-2.316(8)$ & $2.187(3), 2.197(3)$ \\
$\left(\mathrm{RS} / \mathrm{R}_{2} \mathrm{~N}\right)-\mathrm{Fe}-\left(\mathrm{SR} / \mathrm{NR}_{2}\right)$ & a $^{\mathrm{a}}$ & $99.5(7)-110.6(6)$ & — $^{\mathrm{a}}$ \\
$\mathrm{Fe}-(\mu-\mathrm{S})-\mathrm{Fe}$ & $78.11(6)$ & $74.5(2)-75.7(2)$ & $76.1(1)$ \\
$(\mu-\mathrm{S})-\mathrm{Fe}-(\mu-\mathrm{S})$ & $101.89(6)$ & $104.3(4)-105.5(4)$ & $104.0(2)$ \\
$\tau_{4}$ & a $^{\mathrm{a}}$ & $0.917-0.948$ & a $^{\mathrm{a}}$ \\
\hline
\end{tabular}

(a) Some angles $(\mathrm{N} / \mathrm{S})-\mathrm{Fe}-(\mathrm{N} / \mathrm{S})$ not reported. (b) One pyrrolate at each iron is disordered about two positions and both sulfides are disordered, each about two positions. (c) $\mathrm{PPh}_{4}$ salt. (d) In this case, $\mathrm{R}$ stands for the $\mathrm{S}_{3}$-backbone of the chelating $\mathrm{S}_{5}^{2-}$ ligand instead of an arbitrary organic residue.

Table A.4: Selected interatomic distances $[\AA]$ ] and angles $\left[{ }^{\circ}\right]$ for $\mathbf{8 a}, \boldsymbol{8 b}$ and $\boldsymbol{9}$.

\begin{tabular}{cccc}
\hline compound & $\mathbf{8 a}^{\mathrm{a}}$ & $\mathbf{8 b}^{[141]}$ & $\mathbf{9}^{[141], \mathrm{a}, \mathrm{b}}$ \\
\hline $\mathrm{Fe} \cdot \mathrm{Fe}$ & $2.6688(8), 2.7146(7)$ & $2.699(1)$ & $2.725(5), 2.772(5)$ \\
$\mathrm{Fe}-\mathrm{OR}$ & $1.8976(18)-1.9112(19)$ & $1.892(2), 1.895(2)$ & $1.855(9)-1.879(11)$ \\
$\mathrm{Fe}-(\mu-\mathrm{S})$ & $2.2073(8)-2.2207(7)$ & $2.209(1), 2.220(1)$ & $2.219(5)-2.229(5)$ \\
$\mathrm{RO}-\mathrm{Fe}-\mathrm{OR}$ & $95.29(7), 96.31(8)$ & $96.1(1)$ & $93.1(5), 97.2(6)$ \\
$\mathrm{Fe}-(\mu-\mathrm{S})-\mathrm{Fe}$ & $74.28(3), 75.41(2)$ & $75.1(1)$ & $77.0(2), 75.7(2)$ \\
$(\mu-\mathrm{S})-\mathrm{Fe}-(\mu-\mathrm{S})$ & $104.59(2), 105.72(3)$ & $104.9(1)$ & $103.0(3), 104.3(3)$ \\
$\tau_{4}$ & $0.889,0.904$ & 0.899 & $0.900,0.911$ \\
\hline
\end{tabular}

(a) Two independent molecules found in the asymmetric unit. (b) $\mathrm{PPh}_{4}$ salt. 


\section{Appendix B}

Exploration of the general coordination chemistry of ligands $\mathrm{Va}, \mathrm{Vc}$ and $\mathrm{XIV}^{\mathrm{C}}$. In order to probe the general coordination chemistry of the free ligands $\mathbf{V a}, \mathbf{V c}$ and $\mathbf{X I V} \mathbf{V}^{\mathbf{C}}$, zinc was thought as a suitable transition metal for several reasons: Inter alia, diamagnetic zinc allows useful investigations by NMR spectroscopy, and $\{\mathrm{S}\}$-coordination is prominent in various biological zinc sites. In view of the relevance of $\left\{\mathrm{S}_{2} \mathrm{~N}_{2}\right\}$-ligated zinc species as biomimetic model compounds for the most common zinc finger motive, ${ }^{[272]} 4,4^{\prime}$-di-tertbutylbipyridine was utilized as coligand. Synthesis of the complexes $\mathbf{3 6 a}, \mathbf{3 6} \mathbf{b}$ and $\mathbf{3 6 c}$ was carried out in a two step procedure according to Scheme B.1. Reaction of dimethylzinc with the free ligands results in the formation of polymeric thiophenolate-zinc species, probably because of the bridging tendency of thiophenolates. These sparingly soluble intermediates were filtered off and subsequently reacted with $4,4^{\prime}$-di-tert-butylbipyridine in benzene at reflux temperature, causing the polymers to break up (a similar synthetic methodology was previously applied in the synthesis of some related $\left\{\mathrm{O}_{2} \mathrm{~N}_{2}\right\}$-coordinate zinc and cadmium complexes $\left.{ }^{[273]}\right)$. Thus, $\mathbf{3 6 a}, \mathbf{3 6} \mathbf{b}$ and $\mathbf{3 6 c}$ were isolated in 54 to $62 \%$ yield.

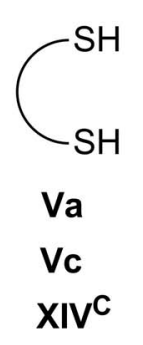

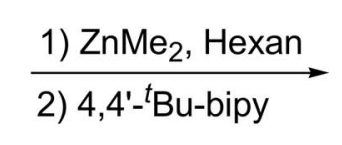

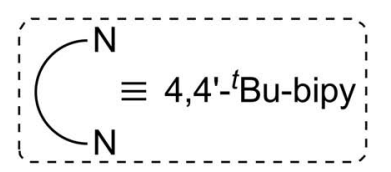

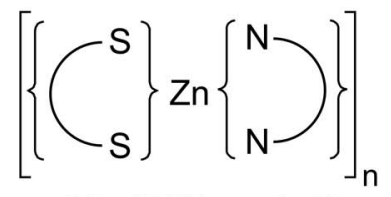

$36 \mathbf{a}(54 \%, n=1,2)$

36b $(57 \%, n=1)$

36 c $(62 \%, n=2)$

Scheme B.1: Synthesis of zinc complexes 36a, 36b and 36c.

It was previously stated that combining chelating aromatic thiols with nitrogen donor coligands on a zinc centre in a $\left\{\mathrm{S}_{2} \mathrm{~N}_{2}\right\}$-Zn stoichiometry generally results in the formation of mononuclear complexes. ${ }^{[274]}$ In contrast to this, however, a ligand dependent nuclearity for the $\left\{\mathrm{S}_{2} \mathrm{~N}_{2}\right\}-\mathrm{Zn}$ complexes $\mathbf{3 6 a}, \mathbf{3 6} \mathbf{b}$ and $\mathbf{3 6} \mathbf{c}$ was observed. In the solid state $\mathbf{3 6} \mathbf{b}$ is found to be monomeric, most likely due to the sterically demanding tert-butyl substituents, whereas 36a and 36c are found to be dimeric (see Figures B.1- B.3). The coordination environment in $\mathbf{3 6} \mathrm{b}$ is strongly distorted from tetrahedral $\left(\tau_{4}=0.86\right)^{[166]}$ because of the acute angle N1-Zn1-N2 $\left(78.84(10)^{\circ}\right)$ imposed by the rigid bipyridine. Aryl rings of the dithiolate are nearly perpendicular with respect to each other (twisted by $88.48(9)^{\circ}$ ). The zinc atoms 
in dimerized 36a ligated by the sterically less hindered ligand $\mathbf{V a}$ are five-coordinated, as one of the thiolate sulfurs from each ligand adopts a bridging position. The Zn $\cdots$ Zn separation in the resulting $\mathrm{Zn}_{2} \mathrm{~S}_{2}$ diamond core is $3.75 \AA$. The coordination polyhedra of both crystallographically equivalent zinc atoms are best described as distorted square pyramids $\left(\tau_{5}=0.25\right)^{[275]}$ with the basal planes through the nitrogen and the bridging sulfur atoms. Compared to the free ligand $\mathbf{V a}$, a reduced twist angle between both aryl ring planes of the dithiobiphenolate is observed $\left(60.19(8)^{\circ}\right)$ for 36a. Interestingly, the zinc atoms in 36c are tetrahedral (distorted, $\left.\tau_{4}=0.83\right)^{[166]}$ with both ligands bridging the two zinc atoms to form a 16-membered metalla-macrocycle. Most likely, the large chelate ring size disfavors a chelate situation, while the higher flexibility of the methylene-extended system $\mathbf{X I V}^{\mathbf{C}}$ (compared to $\mathbf{V a}-\mathbf{V c}$ ) permits the formation of this unusual macrocyclic motif. It should be noted, however, that bidentate ligation of $\mathbf{X I V}^{\mathbf{C}}$ to a single metal is possible too, as this ligand can be successfully applied in the coordination to a [2Fe-2S] cluster core affording tetrahedral iron incorporated in an eight-membered chelate ring. ${ }^{[143]}$ High flexibility of the complete metalla-macrocycle is apparent from comparison of the molecular structures found for $\mathbf{3 6 c}$ and $\mathbf{3 6} \mathbf{c}^{\prime}$ (the latter containing two molecules of non-coordinated $\mathrm{CH}_{2} \mathrm{Cl}_{2}$ per $\mathrm{Zn}_{2}$-molecule). All related angles at the zinc atoms differ to some extent (even so an almost identical $\tau_{4}=0.84$ is observed), just as the Zn-S and Zn-N bond distances (less pronounced effect), causing a significant difference in the measured Zn $\cdots \mathrm{Zn}$ separation $\left(\mathrm{Zn} 1 \cdots \mathrm{Zn} 1^{\prime} \simeq 5.7 \AA\right.$ in $\mathbf{3 6 c}$ versus $\mathrm{Zn} 1 \cdots \mathrm{Zn} 1^{\prime} \simeq 6.0 \AA$ in $\left.\mathbf{3 6 \mathbf { c } ^ { \prime }}\right)$. It should be mentioned that compounds $\mathbf{3 6 a}$ and $\mathbf{3 6} \mathbf{b}$ represent the first structurally characterized $2,2^{\prime}$-dithiobiphenolate $\left\{\mathrm{S}_{2} \mathrm{~N}_{2}\right\}$-coordinated zinc complexes and $\mathbf{3 6 c} / \mathbf{3 6} \mathbf{c}^{\prime}$ the only crystallographically characterized zinc complex ligated by 2,2'-methylenedibezenethiolate (derivatives). Comparison with structures for the related mononuclear $(\mathrm{PhS})_{2} \mathrm{Zn}\left(\right.$ bipy) analogues ${ }^{[276]}$ with non-chelating thiophenolates reveals that Zn-S and Zn-N distances for $\mathbf{3 6 b}$ and $\mathbf{3 6 c} / \mathbf{3 6} \mathbf{c}^{\prime}$ are in the usual range $(\mathrm{Zn}-\mathrm{S} \simeq 2.3 \AA$, $\mathrm{Zn}-\mathrm{N} \simeq 2.1 \AA$ ), whereas $\mathrm{Zn}-\mathrm{S}$ bond lengths involving the bridging sulfur atoms in $\mathbf{3 6 a}$ are somewhat elongated (Zn1-S1 2.5721(7) A, Zn1-S1' 2.3959(6) A). Zn-N distances in $\mathbf{3 6 a}$ are similar to those observed in $\mathbf{3 6} \mathbf{b}$ and $\mathbf{3 6} \mathbf{c} / \mathbf{3 6} \mathbf{c}-$ b), and angles N-Zn-N are very similar for all type $\mathbf{3 6}$ complexes and in accordance with those observed for the $(\mathrm{PhS})_{2} \mathrm{Zn}$ (bipy) analogues. As expected, however, angles S-Zn-S in the $(\mathrm{PhS})_{2} \mathrm{Zn}$ (bipy) complexes are significantly different $\left(\simeq 125^{\circ}\right)$ from those reported herein because of the less bendable chelating dithio ligands in the type $\mathbf{3 6}$ compounds.

The zinc complexes $\mathbf{3 6 a}, \mathbf{3 6} \mathbf{b}$ and $\mathbf{3 6 c}$ were also investigated by variable temperature $\mathrm{NMR}$ in $\mathrm{CD}_{2} \mathrm{Cl}_{2}$. At room temperature, all three complexes show a single set of ${ }^{1} \mathrm{H}$ and ${ }^{13} \mathrm{C}$ signals. Strong crosspeaks between the $2,2^{\prime}$ protons of bipyridine and the $3,3^{\prime}$-tert-butyl and $\mathrm{CH}_{2}$ group in NOESY spectra of $36 \mathrm{~b}$ and $\mathbf{3 6 c}$, respectively, verify that both ligands are simultaneously coordinated to Zn (crosspeaks between the aromatic dithiolate-ligand resonances and the former bipyridine signals are observed for all three complexes). Upon cooling, the ${ }^{1} \mathrm{H}$ signals of $\mathbf{3 6 a}$ and $\mathbf{3 6}$ broaden somewhat, but otherwise remain unaffected down to $-75^{\circ} \mathrm{C}$, indicating in both cases the presence of a single stable species in solution. In contrast, the ${ }^{1} \mathrm{H}$ signals of $36 \mathrm{c}$ are already very broad at $-25^{\circ} \mathrm{C}$ and split at about $-50{ }^{\circ} \mathrm{C}$ into two distinct sets in a $2: 1$ ratio that become reasonably resolved at $-75^{\circ} \mathrm{C}$. At this temperature, the $\mathrm{CH}_{2}$ group of the major species still appears as a singlet, while that of the minutesor species is split into an AB spin system. Again, both species show 


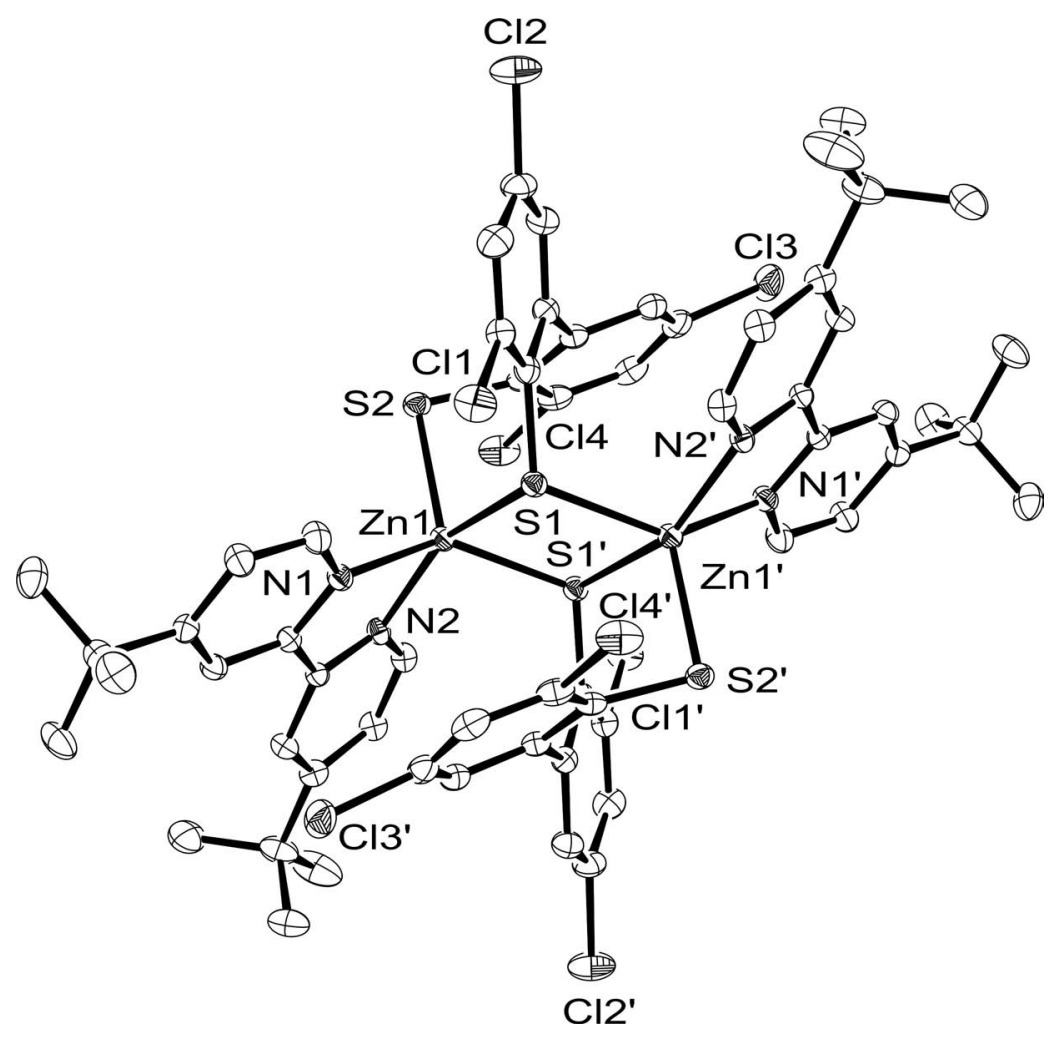

Figure B.1: ORTEP plot (50\% probability thermal ellipsoids) of the molecular structure of 36a. Selected atom distances $[A]$ and angles [०]: Zn1-S1 2.5721(7), Zn1-S2 2.3071(6),

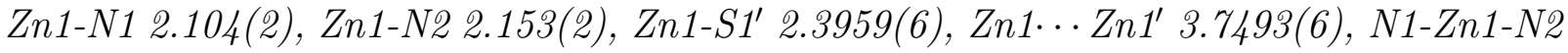
76.44(8), N1-Zn1-S2 108.66(6), N2-Zn1-S2 112.66(6), N1-Zn1-S1' 139.57(6), N2-Zn1-S1' 91.48(5), S2-Zn1-S1' 111.59(2), N1-Zn1-S1 92.64(6), N2-Zn1-S1 154.64(6), S2-Zn1-S1 92.43(2), S1-Zn1-S1' 82.07(2), Zn1-S1-Zn1' 97.93(2).

the characteristic NOESY crosspeak between the $\mathrm{CH}_{2}$ protons and the respective bipy-2,2' protons, underlining the strong preference for mixed $\left\{\mathrm{S}_{2} \mathrm{~N}_{2}\right\}$ coordination. ${ }^{[274]}$ In order to gain further insight into the aggregation / nuclearity of the three zinc complexes in solution, DOSY NMR techniques were used to measure diffusion coefficients of the complexes and the corresponding free ligands $\mathbf{V a}, \mathbf{V} \mathbf{c}$ and $\mathbf{X I V} \mathbf{C}$. Then the molecular radii obtained via the Stokes-Einstein relation were compared to the radii calculated from crystallographic volumes, where the volumes of the dithiols $\mathbf{V} \mathbf{c}$ and $\mathbf{X I V} \mathbf{V}^{\mathbf{C}}$ were approximated by that of the previously characterized corresponding diols $\mathbf{I I}{ }^{[277]}$ and $\mathbf{X V I I}{ }^{[278]}$. The solution ratio $R_{\text {complex }} / R_{\text {ligand }}$ of 1.4 for $\mathbf{3 6 a}$ (calcd. from X-ray for monomeric species: 1.3 , calcd. for dimeric species: 1.7) and 1.4 for $36 \mathrm{~b}$ (calcd. for monomeric species: 1.5 calcd. for dimeric species: 1.9), together with the variable temperature data, indicates both complexes are momomeric in solution. Thus, the dimeric solid state structure determinutesed for 36a by X-ray diffraction is broken up to the corresponding monomeric complexes upon dissolution. In contrast, the molecular structure of $\mathbf{3 6 b}$ is monomeric in both states of aggregation. In the case of $\mathbf{3 6 c}$, the results from the DOSY NMR spectra are less clear: Although the ratio $R_{\text {complex }} / R_{\text {ligand }}$ of 1.8 (calcd. from X-ray for monomeric species: 1.4, 


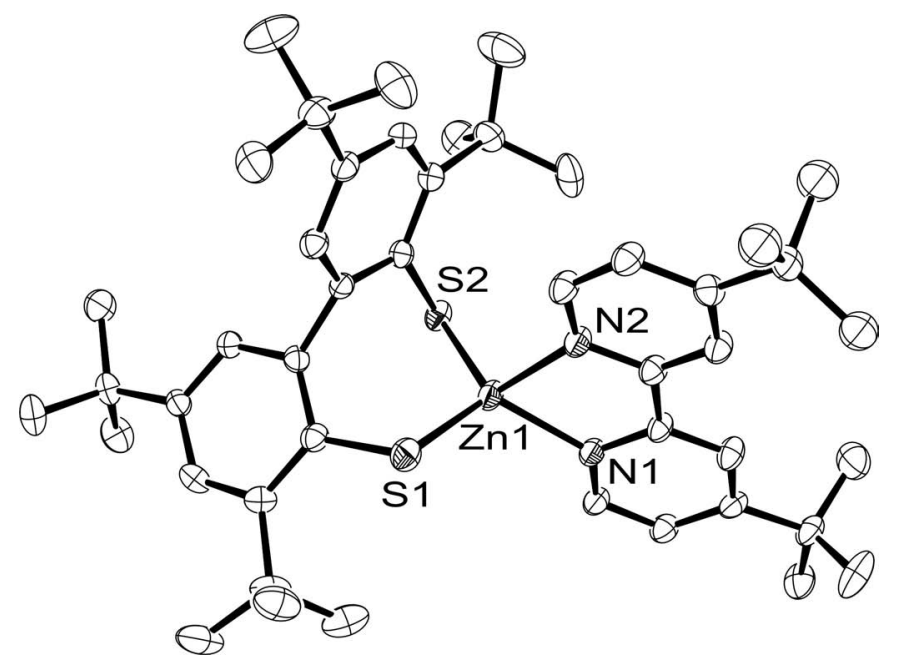

Figure B.2: ORTEP plot (50\% probability thermal ellipsoids) of the molecular structure of 36b. Selected atom distances $[\AA]$ ] and angles [०]: Zn1-S1 2.2592(9), Zn1-S2 2.2653(9), Zn1-N1 2.101(3), Zn1-N2 2.091(3), N2-Zn1-N1 78.84(10), N2-Zn1-S1 102.75(8), N1-Zn1S1 120.87(8), N2-Zn1-S2 114.61(8), N1-Zn1-S2 114.66(8), S1-Zn1-S2 117.31(3).

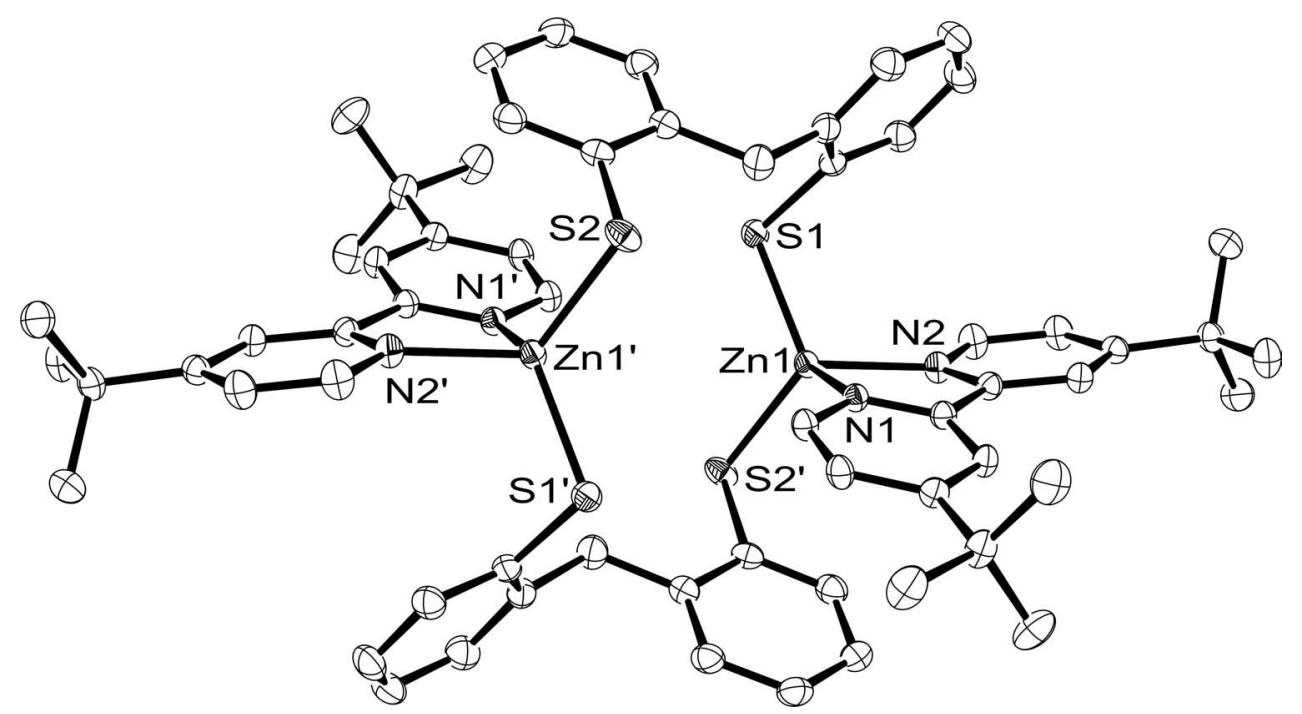

Figure B.3: ORTEP plot (50\% probability thermal ellipsoids) of the molecular structure of 36c. Selected atom distances [A] and angles [०]: Zn1-S1 2.2463(5), Zn1-S2 2.2681(4),

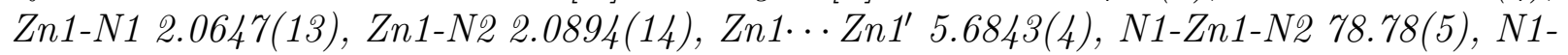
Zn1-S1 121.54(4), N2-Zn1-S1 110.06(4), N1-Zn1-S2' 106.29(4), N2-Zn1-S2' 121.03(4), S1-Zn1-S2' 114.877(18).

calcd. for dimeric species: 1.8) would suggest a dimeric species in solution, the measured absolute value $R_{\text {complex }}$ of $5.0 \AA$ agrees better with that calculated for a monomeric species $(5.6 \AA)$ than with that calculated for a dimeric species $(7.0 \AA)$. Repeat of the DOSY experiment at $-95^{\circ} \mathrm{C}$ (at higher temperatures the experiment is still affected by exchange during the diffusion period) yielded different diffusion coefficients for the two species that 
can be translated into relative molecular radii of 5:4 for the major to minutesor species. From this it was concluded that in solution 36c exists as a monomer-dimer equilibrium, possibly with the dimer assuminutesg the macrocyclic structure observed in the solid state, and the momomer acuminates a structure similar to that of $\mathbf{3 6 b}$. This conclusion is further supported by the observation that the equilibrium is shifted towards the monomeric species upon dilution and increase of temperature (entropy effect). According to this model, the interconversion between the momomeric and dimeric forms of 36c requires breaking and reformation of two Zn-S bonds, a process for which an energetic barrier of about $10 \mathrm{kcal} / \mathrm{mol}$ or $42 \mathrm{~kJ} / \mathrm{mol}$ was calculated from the coalescence temperature. Conformational rearrangements within the dimeric structure, such as a boat-boat ring-flip of the metalla-macrocycle are still fast at $-95^{\circ} \mathrm{C}$, possibly due to the high flexibility of the methylene-bridged ligand $\mathbf{X I V}^{\mathbf{C}}$. Interestingly, two duplets for the methylene-linkage in the monomeric structure of 36c are detected at the latter temperature, indicating a slower ring-flip isomerization of the anticipated eight-membered chelate-ring in this case.

Experimental Section. All reactions were carried out under an anaerobic and anhydrous atmosphere of dry dinitrogen by employing standard Schlenk techniques. Solvents were dried and distilled prior to use as described in Chapter 9. NOESY and DOSY NMR spectra were recorded on Bruker Avance $500 \mathrm{MHz}$ spectrometer at $25^{\circ} \mathrm{C}$. The mixing period used in room temperature NOESY spectra $(500 \mathrm{~ms})$ was reduced to $100 \mathrm{~ms}$ at $-75^{\circ} \mathrm{C}$ to prevent spin diffusion. DOSY spectra were recorded with a $z$-Gradient ramped linearly from 1 to $53 \mathrm{G} / \mathrm{cm}$, bipolar gradient pulses of $2 \mathrm{~ms}$, and a diffusion delay of $70 \mathrm{~ms}$ (reduced to $30 \mathrm{~ms}$ to prevent exchange). Chemical shifts are reported in ppm relative to residual ${ }^{1} \mathrm{H}$ and ${ }^{13} \mathrm{C}$ signals of $\mathrm{CD}_{2} \mathrm{Cl}_{2}$ at $5.32 \mathrm{ppm}$ and $54.0 \mathrm{ppm}$ or $\mathrm{C}_{6} \mathrm{D}_{6}$ at $7.15 \mathrm{ppm}$ and $128.0 \mathrm{ppm}$, respectively. ESI mass spectrometry, HRMS spectrometry, IR spectroscopy, melting point measurements and elemental analyses were conducted as described in Chapter 9.

[(2,2'-Dithio-3, $3^{\prime}, 5,5^{\prime}$-tetrachlorobiphenyl)-(4,4'-di-tert-butylbipyridine)-zinc $]_{1,2}$ (36a). A solution of dimethylzinc $(2.0 \mathrm{M}$ in toluene, $1.86 \mathrm{ml}, 3.73 \mathrm{mmol})$ was added dropwise to a vigorously stirred suspension of $2,2^{\prime}$-dithio- $3,3^{\prime}, 5,5^{\prime}$-tetrachlorobiphenyl (Va) $(1.32 \mathrm{~g}, 3.73 \mathrm{mmol})$ in hexanes $(20 \mathrm{ml})$ at room temperature. The reaction mixture was heated to reflux for 20 minutes in order to complete methane formation. After cooling to room temperature the white polymeric thiophenolate-zinc species were filtered off, washed with hexanes $(2 \times 10 \mathrm{ml})$ and briefly dried in vacuo. This polymeric intermediate was transferred to a schlenk flask, previously charged with 4,4'-di-tert-butylbipyridine $(1.0 \mathrm{~g}, 3.7 \mathrm{mmol})$ and suspended in benzene $(30 \mathrm{ml})$. The reaction mixture was heated to reflux (oil bath temperature $100^{\circ} \mathrm{C}$ ) for $1 \mathrm{~h}$, cooled to room temperature and filtered. The yellowish powder was washed with hexanes $(2 \times 10 \mathrm{ml})$ and $\mathrm{Et}_{2} \mathrm{O}(3 \times 20 \mathrm{ml})$ and subsequently dried in vacuo to afford the pure product $(1.4 \mathrm{~g}, 2.0 \mathrm{mmol}, 54 \%)$. Mp (uncorrected) $303^{\circ} \mathrm{C} .{ }^{1} \mathrm{H}$ NMR $\left(300 \mathrm{MHz}, \mathrm{CD}_{2} \mathrm{Cl}_{2}\right): \delta=1.45\left(\mathrm{~s}, 18 \mathrm{H}, 4,4^{\prime}{ }^{t} \mathrm{Bu}\right), 7.05$ $\left(\mathrm{d}, 2 \mathrm{H},{ }^{4} J_{\mathrm{H}, \mathrm{H}}=2.4 \mathrm{~Hz}, 4,4^{\prime}-\mathrm{Ar}-\mathrm{H}\right), 7.47\left(\mathrm{~d}, 2 \mathrm{H},{ }^{4} J_{\mathrm{H}, \mathrm{H}}=2.4 \mathrm{~Hz}, 6,66^{\prime}-\mathrm{Ar}-\mathrm{H}\right), 7.68$ (dd, $2 \mathrm{H}$, ${ }^{3} J_{\mathrm{H}, \mathrm{H}}=5.6 \mathrm{~Hz},{ }^{4} J_{\mathrm{H}, \mathrm{H}}=1.8 \mathrm{~Hz}, 5,5^{\prime}-$ bipy-H$), 8.20\left(\mathrm{dd}, 2 \mathrm{H},{ }^{4} J_{\mathrm{H}, \mathrm{H}}=1.8 \mathrm{~Hz},{ }^{5} J_{\mathrm{H}, \mathrm{H}}=0.5 \mathrm{~Hz}, 3,3^{\prime}-\right.$ bipy-H), $8.40\left(\mathrm{~d}, 2 \mathrm{H},{ }^{3} J_{\mathrm{H}, \mathrm{H}}=5.6 \mathrm{~Hz}, 6,6^{\prime}\right.$-bipy-H). ${ }^{13} \mathrm{C} \mathrm{NMR}\left(75 \mathrm{MHz}, \mathrm{CD}_{2} \mathrm{Cl}_{2}\right): \delta=30.4$ ( $\left.{ }^{t} \mathrm{Bu}\right), 36.1$ ( $\left.\mathrm{CMe}_{3}\right), 118.9$ (3,3'-bipy-C), 124.7 (5,5'-bipy-C), 127.8 (6,6'-Ar-C), 127.9 (4,4'Ar-C), 129.1 (Ar-C), 139.4 (Ar-C), 139.9 (Ar-C), 149.2 (6,6'-bipy-C), 149.7 (Ar-C), 150.2 (2,2'-bipy-C), 166.9 (4,4'-bipy-C). MS (ESI+): $m / z(\%)=367(95)[(\text { bipy }) \mathrm{Zn}+\mathrm{Cl}]^{+}, 639$ 
(100) $\left[(\text { bipy })_{2} \mathrm{Zn}+\mathrm{Cl}\right]^{+}, 1055(22)\left[(\mathrm{L})(\text { bipy })_{2} \mathrm{Zn}_{2}+\mathrm{Cl}\right]^{+}$. MS (ESI-): $m / z(\%)=455$ (100) $[(\mathrm{L}) \mathrm{Zn}+\mathrm{Cl}]^{-}$. IR $(\mathrm{KBr}): \widetilde{\nu}\left(\mathrm{cm}^{-1}\right)=2964(\mathrm{~m}), 1615(\mathrm{~s}), 1550(\mathrm{~m}), 1412(\mathrm{~s}), 1368$ (s). Elemental analysis: Calcd. (\%) for $\mathrm{C}_{60} \mathrm{H}_{56} \mathrm{Cl}_{8} \mathrm{~N}_{4} \mathrm{~S}_{4} \mathrm{Zn}_{2}$ : $\mathrm{C}$ 52.38, H 4.10, N 4.07, S 9.32. Found: C 52.31, H 4.21, N 4.29, S 7.90. HRMS (ESI+): Calcd. $(m / z)$ for $\mathrm{C}_{30} \mathrm{H}_{29} \mathrm{Cl}_{4} \mathrm{~N}_{2} \mathrm{~S}_{2} \mathrm{Zn}$ $\left([\mathrm{M}+\mathrm{H}]^{+}\right)$: 684.9812. Found: 684.9811, Calcd. $(\mathrm{m} / z)$ for $\mathrm{C}_{60} \mathrm{H}_{56} \mathrm{Cl}_{8} \mathrm{~N}_{4} \mathrm{~S}_{4} \mathrm{Zn}_{2}: 1374.9482$. Found: 1374.9474.

(2,2'-Dithio-3,3' $, 5,5^{\prime}$-tetrakis-tert-butylbiphenyl)-(4,4'-di-tert-butylbipyridine)zinc (36b). A solution of dimethylzinc $(2.0 \mathrm{M}$ in toluene, $0.62 \mathrm{ml}, 1.24 \mathrm{mmol})$ was added dropwise to a stirred solution of 2,2'-dithio-3,3',5,5'-tetrakis-tert-butylbiphenyl (Vc) ( $0.55 \mathrm{~g}$, $1.24 \mathrm{mmol})$ in hexanes $(10 \mathrm{ml})$ at room temperature and stirring was continued for $1 \mathrm{~h}$. The turbid viscous reaction mixture was condensed to dryness and re-dissolved in toluene $(15 \mathrm{ml})$. Solid 4,4'-di-tert-butylbipyridine $(0.33 \mathrm{~g}, 1.24 \mathrm{mmol})$ was added and the reaction mixture stirred for 10 minutes to afford a clear orange solution. The solvent was reduced to half of its volume and pentane $(10 \mathrm{ml})$ was added to precipitate a fine yellow powder. It was filtered off, washed with pentane $(2 \times 10 \mathrm{ml})$ and dried in vacuo over night to afford the yellowish product $(0.55 \mathrm{~g}, 0.71 \mathrm{mmol}, 57 \%)$ largely free of residual solvents. Mp (uncorrected) $281{ }^{\circ} \mathrm{C} .{ }^{1} \mathrm{H}$ NMR $\left(500 \mathrm{MHz}, \mathrm{C}_{6} \mathrm{D}_{6}\right): \delta=0.86\left(\mathrm{~s}, 18 \mathrm{H}, 3,3^{\prime}{ }^{t} \mathrm{Bu}\right), 1.45\left(\mathrm{~s}, 18 \mathrm{H}, 4,4^{\prime}{ }^{t}{ }^{t} \mathrm{Bu}\right), 2.03$ $\left(\mathrm{s}, 18 \mathrm{H}, 5,5^{\prime}{ }^{t} \mathrm{Bu}\right), 6.10\left(\mathrm{dd}, 2 \mathrm{H},{ }^{3} J_{\mathrm{H}, \mathrm{H}}=5.5 \mathrm{~Hz},{ }^{4} J_{\mathrm{H}, \mathrm{H}}=1.5 \mathrm{~Hz}, 5,5^{\prime}\right.$-bipy-H), 7.15 (d, approximately $2 \mathrm{H}$ - overlapping with $\left.\mathrm{C}_{6} \mathrm{D}_{6},{ }^{4} J_{\mathrm{H}, \mathrm{H}}=2.5 \mathrm{~Hz}, 6,6^{\prime}-\mathrm{Ar}-\mathrm{H}\right), 7.34\left(\mathrm{~d}, 2 \mathrm{H},{ }^{4} J_{\mathrm{H}, \mathrm{H}}=1.5 \mathrm{~Hz}\right.$, $3,3^{\prime}$-bipy-H), 7.57 (d, 2H, $\left.{ }^{4} J_{\mathrm{H}, \mathrm{H}}=2.5 \mathrm{~Hz}, 4,4^{\prime}-\mathrm{Ar}-\mathrm{H}\right), 7.84\left(\mathrm{~d}, 2 \mathrm{H},{ }^{3} J_{\mathrm{H}, \mathrm{H}}=6.0 \mathrm{~Hz}, 6,6^{\prime}\right.$-bipyH). ${ }^{13} \mathrm{C}$ NMR $\left(125 \mathrm{MHz}, \mathrm{C}_{6} \mathrm{D}_{6}\right): \delta=29.8\left(3,3^{\prime}{ }^{t} \mathrm{Bu}\right), 31.3\left(5,5^{\prime}{ }^{t} \mathrm{Bu}\right), 32.0\left(4,4^{\prime}{ }^{t} \mathrm{Bu}\right), 34.8$

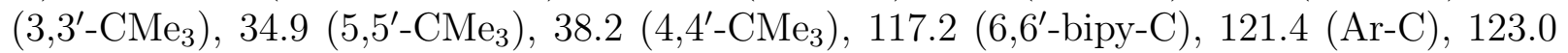
(3,3'-bipy-C), 126.2 (6,6'-Ar-C), 128.3 (5,5'-bipy-C), 137.3 (Ar-C), 145.1 (Ar-C), 149.3 (ArC), 149.7 (4,4'-Ar-C), 154.2 (2,2'-bipy-C), 166.9 (4,4'-bipy-C). MS (ESI+): $\mathrm{m} / z(\%)=773$ (100) $[\mathrm{M}+\mathrm{Na}]^{+}, 1571(35)\left[\mathrm{M}_{2}+\mathrm{Na}\right]^{+}$. IR $(\mathrm{KBr}): \widetilde{\nu}\left(\mathrm{cm}^{-1}\right)=2961(\mathrm{~s}), 2905(\mathrm{~m}), 1615(\mathrm{~s})$, $1550(\mathrm{w}), 1409(\mathrm{~m})$. Elemental analysis: Calcd. (\%) for $\mathrm{C}_{64} \mathrm{H}_{64} \mathrm{~N}_{2} \mathrm{~S}_{2} \mathrm{Zn}$ : C 71.38, H 8.33, N 3.62, S 8.28. Found: C 70.43, H 8.23, N 3.30, S 7.35. HRMS (ESI+): Calcd. $(\mathrm{m} / \mathrm{z})$ for $\mathrm{C}_{64} \mathrm{H}_{65} \mathrm{~N}_{2} \mathrm{~S}_{2} \mathrm{Zn}\left([\mathrm{M}+\mathrm{H}]^{+}\right)$: 773.3875. Found: 773.3870 .

Bis-[(2,2'-methylenedibenzenethio)-(4,4'-di-tert-butylbipyridine)-zinc] (36c). A solution of dimethylzinc $(2.0 \mathrm{M}$ in toluene, $1.0 \mathrm{ml}, 2.0 \mathrm{mmol})$ was added dropwise to a vigorously stirred suspension of 2,2'-methylenedibenzenethiol $\left(\mathbf{X I V}^{\mathbf{C}}\right)(0.46 \mathrm{~g}, 2.0 \mathrm{mmol})$ in hexanes $(10 \mathrm{ml})$ at room temperature. The reaction mixture was heated to reflux for 20 minutes in order to complete methane formation. After cooling to room temperature the white polymeric thiophenolate-zinc species were filtered off, washed with hexanes $(2 \times 5 \mathrm{ml})$ and briefly dried in vacuo. This polymeric intermediate was transferred to a schlenk flask, previously charged with 4,4'-di-tert-butylbipyridine $(0.54 \mathrm{~g}, 2.0 \mathrm{mmol})$ and suspended in benzene $(15 \mathrm{ml})$. The reaction mixture was heated to reflux (oil bath temperature $100^{\circ} \mathrm{C}$ ) for $1 \mathrm{~h}$, cooled to approximately $45^{\circ} \mathrm{C}$ and filtered. The yellowish powder was washed with benzene $(2 \times 5 \mathrm{ml})$ and dried in vacuo to afford the pure product $(0.70 \mathrm{~g}, 1.2 \mathrm{mmol}$, $62 \%$ ). Mp (uncorrected) $292{ }^{\circ} \mathrm{C} .{ }^{1} \mathrm{H}$ NMR $\left(500 \mathrm{MHz}, \mathrm{CD}_{2} \mathrm{Cl}_{2}\right): \delta=1.44$ (s, 36H, 4, $\left.4^{\prime}{ }^{t} \mathrm{Bu}\right)$, 4.47 (s, 4H, $\left.\mathrm{CH}_{2}\right), 6.74\left(\mathrm{~m}, 4 \mathrm{H}, 4,4^{\prime}-\mathrm{Ar}-\mathrm{H}\right), 6.80$ (m, 4H, 5, $\left.5^{\prime}-\mathrm{Ar}-\mathrm{H}\right), 7.87$ (m, 4H, 3,3'Ar-H), $7.22\left(\mathrm{~d}, 4 \mathrm{H},{ }^{3} J_{\mathrm{H}, \mathrm{H}}=7.5 \mathrm{~Hz}, 6,66^{\prime}-\mathrm{Ar}-\mathrm{H}\right), 7.60\left(\mathrm{dd}, 4 \mathrm{H},{ }^{3} J_{\mathrm{H}, \mathrm{H}}=5.5 \mathrm{~Hz},{ }^{4} J_{\mathrm{H}, \mathrm{H}}=1.5 \mathrm{~Hz}\right.$, $5,5^{\prime}$-bipy-H), 8.06 (s, 4H, 3,3'-bipy-H), 8.69 (d, 4H, ${ }^{3} J_{\mathrm{H}, \mathrm{H}}=5.5 \mathrm{~Hz}, 6,6^{\prime}$-bipy-H). ${ }^{13} \mathrm{C}$ NMR $\left(125 \mathrm{MHz}, \mathrm{CD}_{2} \mathrm{Cl}_{2}\right): \delta=30.5\left({ }^{t} \mathrm{Bu}\right), 35.9\left(\mathrm{CMe}_{3}\right), 41.9\left(\mathrm{CH}_{2}\right), 118.4\left(3,3^{\prime}\right.$-bipy-C), 123.4 
(5,5'-Ar-C), 124.4 (5,5'-bipy-C), 125.5 (4,4'-Ar-C), 129.8 (3,3'-Ar-C), 134.9 (6,6'-Ar-C), 142.1 (Ar-C), 142.3 (Ar-C), 149.5 (6,6'-bipy-C), 149.7 (2,2'-bipy-C), 165.7 (4,4'-bipy-C). MS (ESI+): $m / z(\%)=291(100)[\mathrm{bipy}+\mathrm{Na}]^{+}, 1151(9)[\mathrm{M}+\mathrm{Na}]^{+}$. MS $(\mathrm{ESI}-): m / z$ $(\%)=331(100)[(\mathrm{L}) \mathrm{Zn}+\mathrm{Cl}]^{-}$. IR $(\mathrm{KBr}): \widetilde{\nu}\left(\mathrm{cm}^{-1}\right)=2961(\mathrm{~s}), 1615(\mathrm{~s}), 1410(\mathrm{~m}), 1391$ (m). Elemental analysis: Calcd. (\%) for $\mathrm{C}_{62} \mathrm{H}_{68} \mathrm{~N}_{4} \mathrm{~S}_{4} \mathrm{Zn}_{2}$ : C 66.00, H 6.07, N 4.97, S 11.37. Found: C 64.95, H 5.89, N 4.87, S 11.22. HRMS (ESI+): Calcd. $(m / z)$ for $\mathrm{C}_{62} \mathrm{H}_{69} \mathrm{~N}_{4} \mathrm{~S}_{4} \mathrm{Zn}_{2}$ $\left([\mathrm{M}+\mathrm{H}]^{+}\right):$1125.29825. Found: 1125.29754.

Table B.1: Crystal data and refinement details.

\begin{tabular}{|c|c|c|c|c|}
\hline compound & $36 a$ & $36 \mathrm{~b}$ & $36 \mathrm{c}$ & $36 c^{\prime}$ \\
\hline empirical formula & $\mathrm{C}_{62} \mathrm{H}_{60} \mathrm{Cl}_{12} \mathrm{~N}_{4} \mathrm{~S}_{4} \mathrm{Zn}_{2}$ & $\begin{array}{l}\mathrm{C}_{46} \mathrm{H}_{64} \mathrm{~N}_{2} \mathrm{~S}_{2} \mathrm{Zn}, \\
\text { hexane }\end{array}$ & $\mathrm{C}_{62} \mathrm{H}_{68} \mathrm{~N}_{4} \mathrm{~S}_{4} \mathrm{Zn}_{2}$ & $\begin{array}{l}\mathrm{C}_{62} \mathrm{H}_{68} \mathrm{~N}_{4} \mathrm{~S}_{4} \mathrm{Zn}_{2} \\
2 \mathrm{CH}_{2} \mathrm{Cl}_{2}\end{array}$ \\
\hline formula weight & 1545.52 & 860.65 & 1128.18 & 1298.04 \\
\hline crystal size $[\mathrm{mm}]$ & $0.19 \times 0.18 \times 0.05$ & $0.32 \times 0.14 \times 0.07$ & $0.29 \times 0.28 \times 0.13$ & $0.22 \times 0.20 \times 0.12$ \\
\hline crystal system & monoclinic & orthorhombic & triclinic & monoclinic \\
\hline space group & $P 2_{1} / \mathrm{n}($ No. 14$)$ & $\mathrm{P} 2_{1} 2_{1} 2_{1}$ (No. 19$)$ & P'̄ (No. 2) & $P 2_{1} / \mathrm{n}($ No. 14$)$ \\
\hline$a[\AA]$ & $17.7471(6)$ & $13.5908(3)$ & $11.0651(5)$ & $11.5284(4)$ \\
\hline$b[\AA]$ & $11.0026(2)$ & $14.5658(5)$ & $11.7647(5)$ & $20.6049(6)$ \\
\hline$c[\AA]$ & $18.2732(7)$ & $25.4526(6)$ & $12.4259(6)$ & $13.0810(5)$ \\
\hline$\alpha\left[^{\circ}\right]$ & 90 & 90 & $66.503(3)$ & 90 \\
\hline$\beta\left[^{\circ}\right]$ & $112.274(3)$ & 90 & $85.194(4)$ & $101.024(3)$ \\
\hline$\gamma\left[{ }^{\circ}\right]$ & 90 & 90 & $74.564(4)$ & 90 \\
\hline$V\left[\AA^{3}\right]$ & $3301.86(18)$ & $5038.6(2)$ & $1429.48(11)$ & $3049.94(18)$ \\
\hline$\rho_{\text {calcd. }}\left[\mathrm{g} \mathrm{cm}^{-3}\right]$ & 1.555 & 1.135 & 1.311 & 1.413 \\
\hline$Z$ & 2 & 4 & 1 & 2 \\
\hline$F(000)$ & 1576 & 1864 & 592 & 1352 \\
\hline$\mu\left[\mathrm{mm}^{-1}\right]$ & 1.382 & 0.604 & 1.027 & 1.142 \\
\hline$T_{\max } / T_{\min }$ & $0.8933 / 0.7558$ & $0.9427 / 0.7820$ & $0.8639 / 0.7231$ & $0.9028 / 0.7871$ \\
\hline$h k l$ range & $\pm 22,-11-14, \pm 23$ & $\begin{array}{l}-14-17, \pm 18 \\
-30-32\end{array}$ & $\pm 14, \pm 14, \pm 15$ & $\begin{array}{l} \pm 14,-25-26 \\
\pm 16\end{array}$ \\
\hline$\theta$ range $\left[^{\circ}\right]$ & $2.03-26.99$ & $1.61-26.74$ & $1.91-26.95$ & $1.87-26.97$ \\
\hline measured refl. & 39436 & 27050 & 18124 & 20413 \\
\hline unique refl. $\left[R_{\text {int }}\right]$ & $7170[0.0617]$ & $10670[0.0523]$ & $6187[0.0312]$ & $6606[0.0522]$ \\
\hline observed refl. $I>2 \sigma(I)$ & 5722 & 8878 & 5370 & 5325 \\
\hline Refined parameters & 392 & 516 & 338 & 358 \\
\hline restraints & 30 & 19 & 30 & 0 \\
\hline goodness-of-fit & 1.041 & 1.024 & 1.037 & 1.037 \\
\hline$R 1, w R 2(I>2 \sigma(I))$ & $0.0373,0.0784$ & $0.0483,0.1051$ & $0.0269,0.0676$ & $0.0382,0.0867$ \\
\hline$R 1, w R 2$ (all data) & $0.0547,0.0831$ & $0.0617,0.1098$ & $0.0336,0.0696$ & $0.0529,0.0912$ \\
\hline resid. el. dens. $\left[\mathrm{e}^{-3}\right]$ & $0.461 /-0.469$ & $0.694 /-0.431$ & $0.350 /-0.334$ & $0.432 /-0.562$ \\
\hline
\end{tabular}

The crystal data and details of the data collections for $36 \mathrm{a}, 36 \mathrm{~b}, \mathbf{3 6 c}$ and $\mathbf{3 6} \mathbf{c}^{\prime}$ (per molecule of 36c , one molecule of $\mathrm{CH}_{2} \mathrm{Cl}_{2}$ is present) are given in Table B.1. X-ray data were 
collected on a STOE IPDS II diffractometer (graphite monochromated Mo-K $\mathrm{K}_{\alpha}$ radiation, $\lambda=0.71073 \AA$ ) by use of $\omega$ scans at $-140^{\circ} \mathrm{C}$. The structures were solved by direct methods and refined on $F^{2}$ using all reflections with SHELX-97. ${ }^{[263-265]}$ Most non-hydrogen atoms were refined anisotropically. Hydrogen atoms were placed in calculated positions and assigned to an isotropic displacement parameter of $0.08 \AA^{2}$. One tert-butyl group of the 4,4'-di-tert-butyl-2,2'-dipyridyl ligands in $\mathbf{3 6 a}, \mathbf{3 6} \mathbf{b}$, and $\mathbf{3 6} \mathbf{c}$ was found to be disordered and was refined using SADI restraints $(d(\mathrm{C}-\mathrm{C})$ and $d(\mathrm{C} \cdots \mathrm{C})$ and EADP constraints in case of $\mathbf{3 6 a}$ and $\mathbf{3 6 c}$. Furthermore the hexane solvent molecule in $\mathbf{3 6 b i s}$ disordered and was refined using DFIX restraints $(d(\mathrm{C}-\mathrm{C})=1.51$ and $1.52 \AA)$ and EADP constraints. Faceindexed absorption corrections were performed numerically with the program X-RED. ${ }^{[266]}$ Supplementary crystallographic data can be obtained free of charge from the Cambridge Crystallographic Data Centre via www.ccdc.cam.ac.uk/data_request/cif: CCDC-689046 (36a), CCDC-689047 (36b), CCDC-689048 (36c) and CCDC-689049 (36c' ${ }^{\prime}$. 


\section{Appendix C}
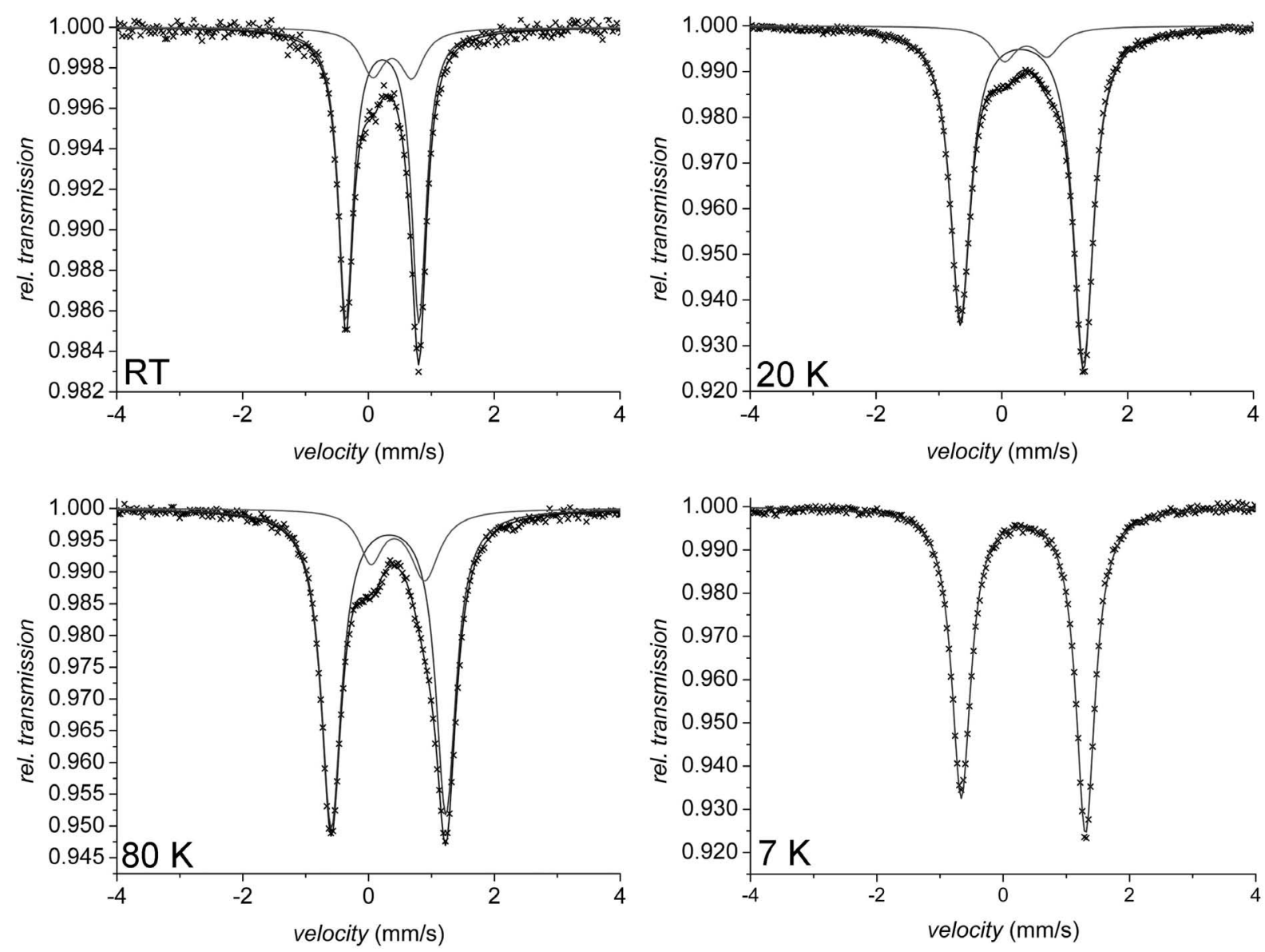

Figure C.1: Zero-field Mössbauer spectra of $\mathfrak{2}^{\boldsymbol{Y}}$ recorded at $R T, 80 \mathrm{~K}, 20 \mathrm{~K}$ and $7 \mathrm{~K}$. 

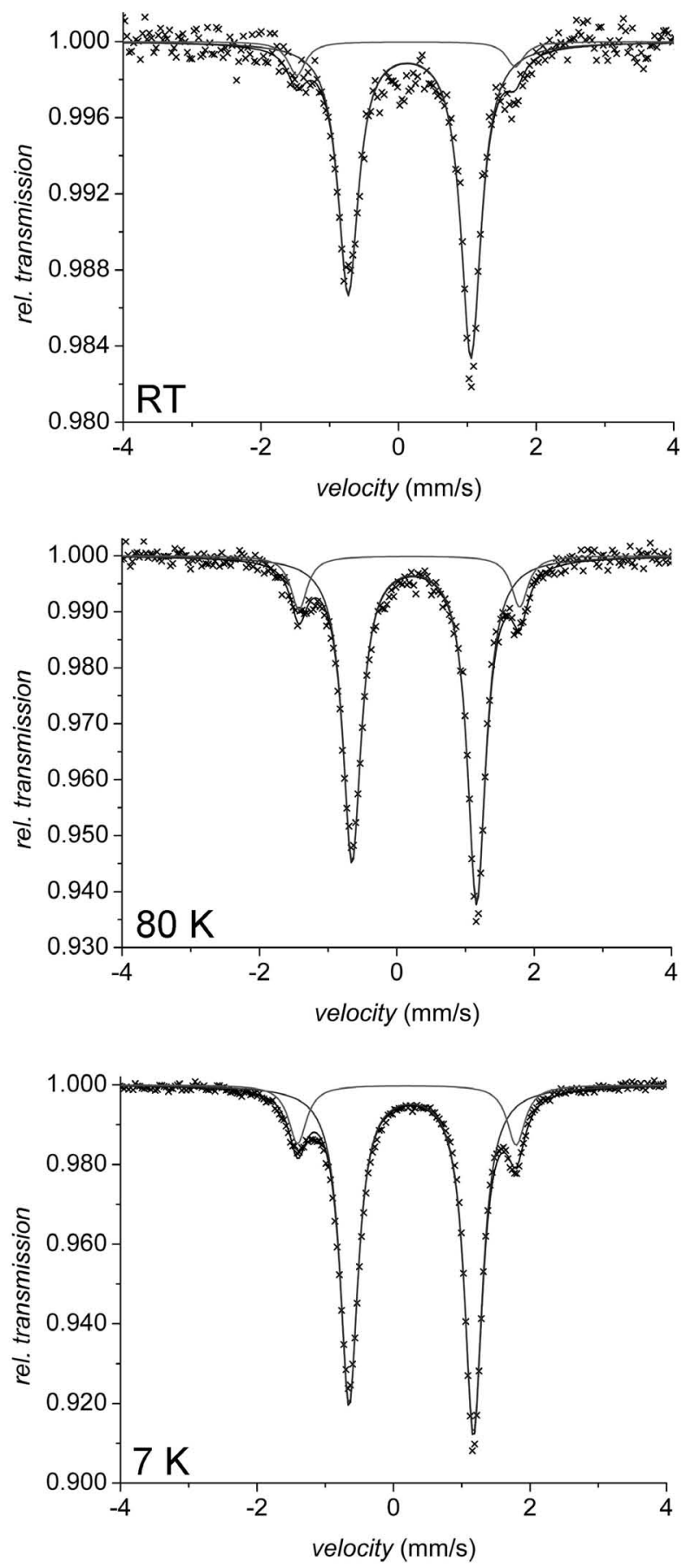

Figure C.2: Zero-field Mössbauer spectra of $\mathbf{2}^{\boldsymbol{P}}$ recorded at $R T, 80 \mathrm{~K}$ and $7 \mathrm{~K}$. 

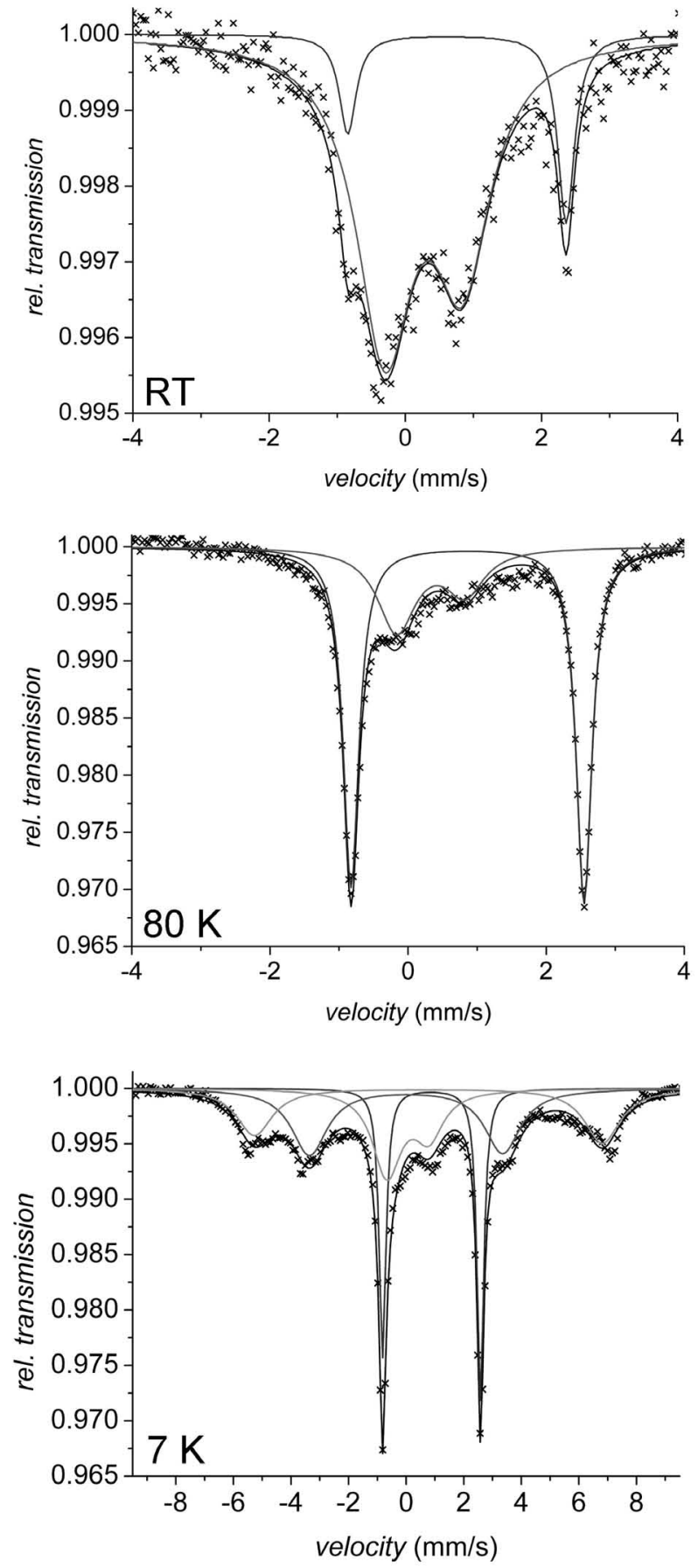

Figure C.3: Zero-field Mössbauer spectra of $\mathbf{2 7}^{\mathrm{O}}$ recorded at $R T, 80 \mathrm{~K}$ and $7 \mathrm{~K}$. 


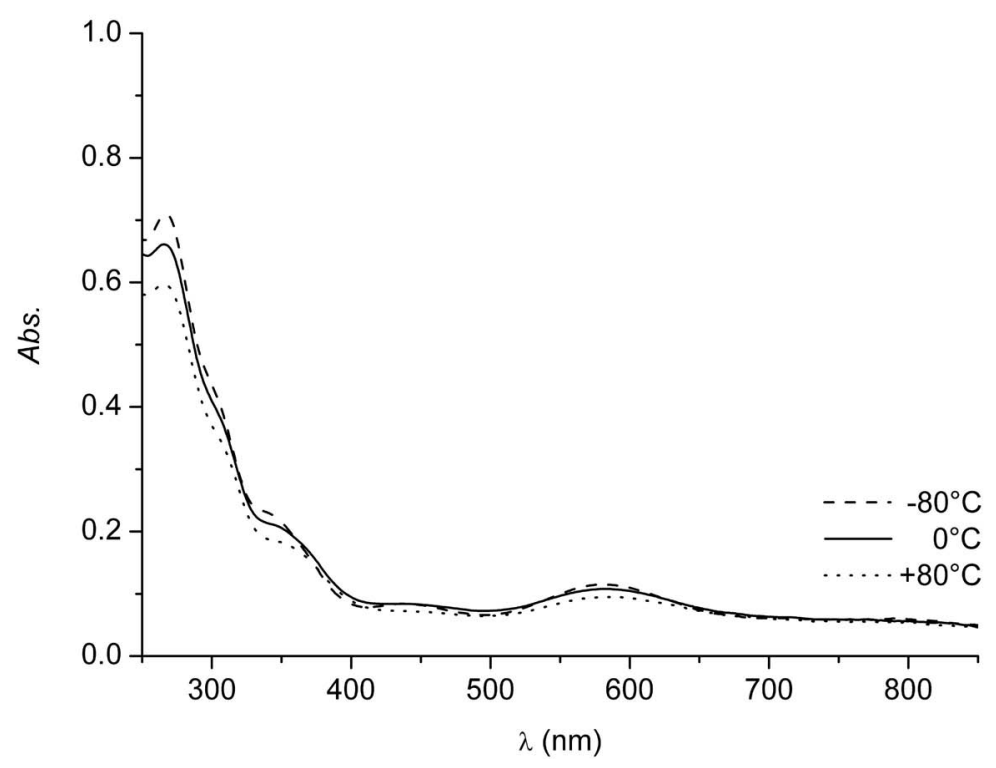

Figure C.4: Electronic absorption spectra of $2^{\gamma^{N}}$ in EtCN solution at $-80^{\circ} \mathrm{C}$ (dashed line), $0^{\circ} \mathrm{C}$ (solid line) and $+80^{\circ} \mathrm{C}$ (dotted line).

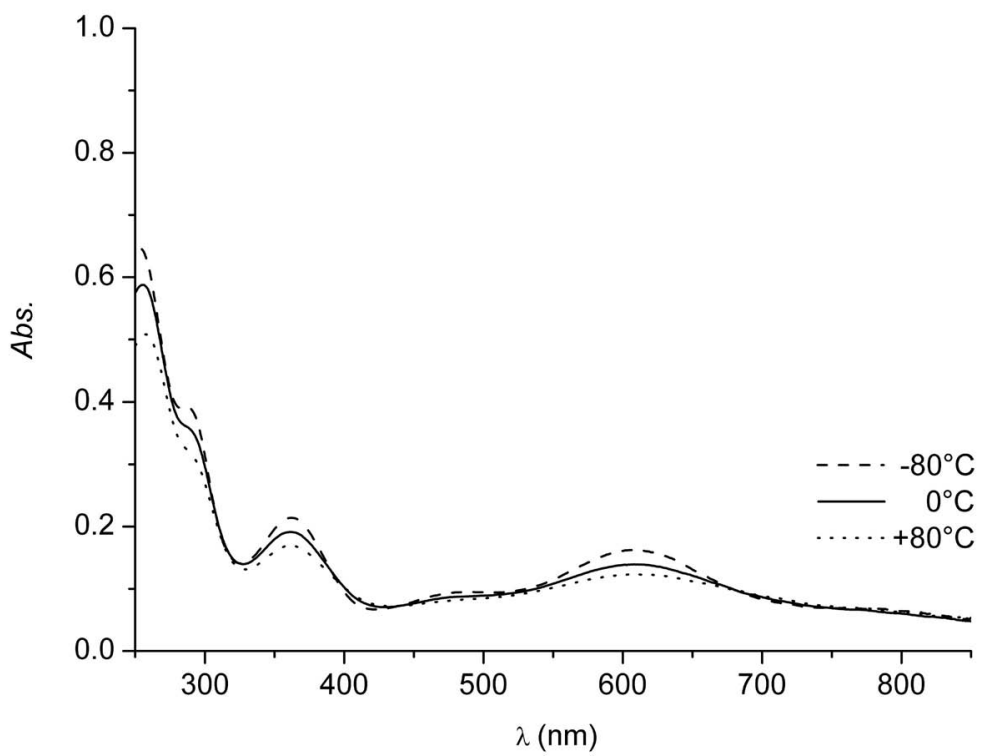

Figure C.5: Electronic absorption spectra of $2^{2} 7^{\circ}$ in EtCN solution at $-80^{\circ} \mathrm{C}$ (dashed line), $0^{\circ} \mathrm{C}$ (solid line) and $+80^{\circ} \mathrm{C}$ (dotted line). 


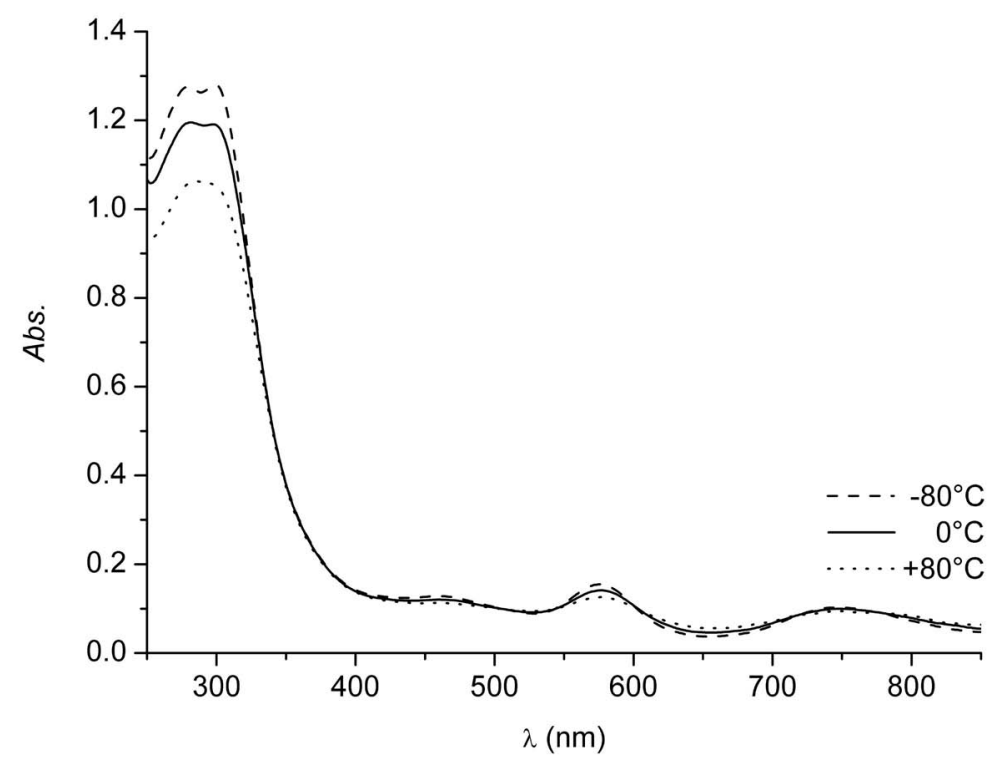

Figure C.6: Electronic absorption spectra of $\mathbf{2 7}^{\boldsymbol{P}}$ in EtCN solution at $-80^{\circ} \mathrm{C}$ (dashed line), $0^{\circ} \mathrm{C}$ (solid line) and $+80^{\circ} \mathrm{C}$ (dotted line).

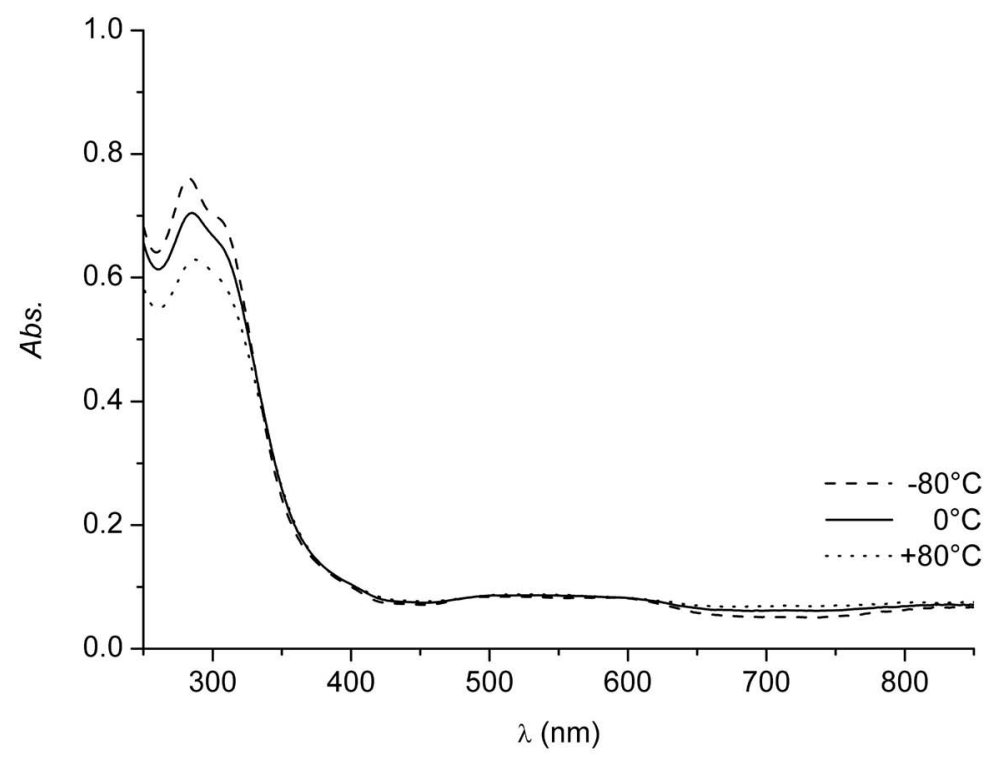

Figure C.7: Electronic absorption spectra of $27^{S}$ in EtCN solution at $-80^{\circ} \mathrm{C}$ (dashed line), $0^{\circ} \mathrm{C}$ (solid line) and $+80^{\circ} \mathrm{C}$ (dotted line). 


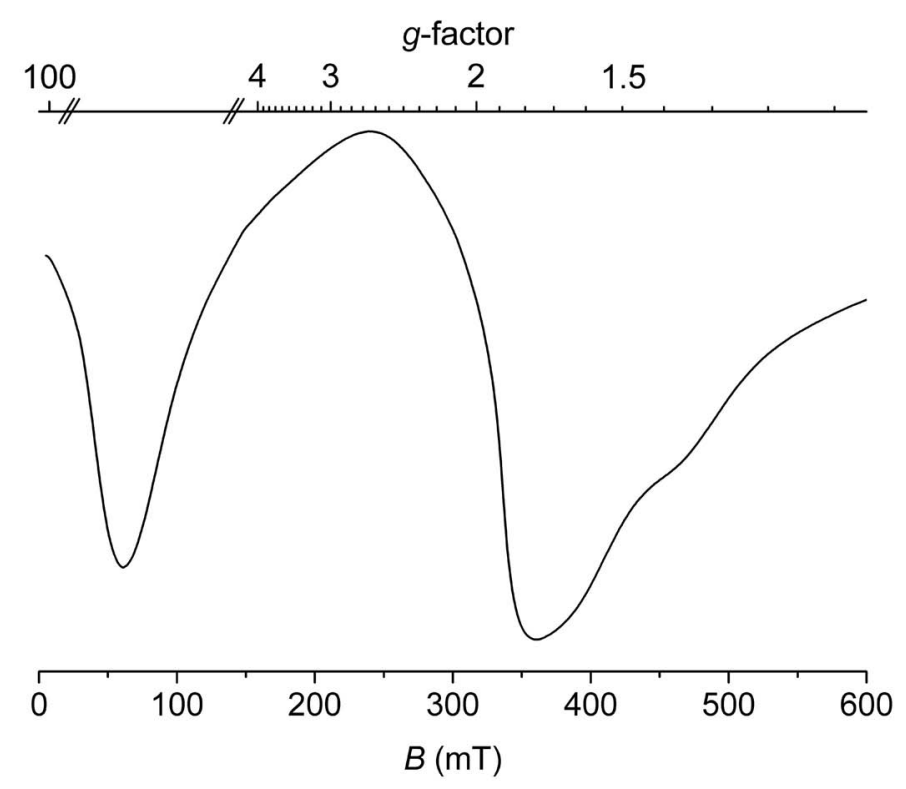

Figure C.8: Solid state EPR spectrum of $2^{r N}$ at $4.4 \mathrm{~K}$.

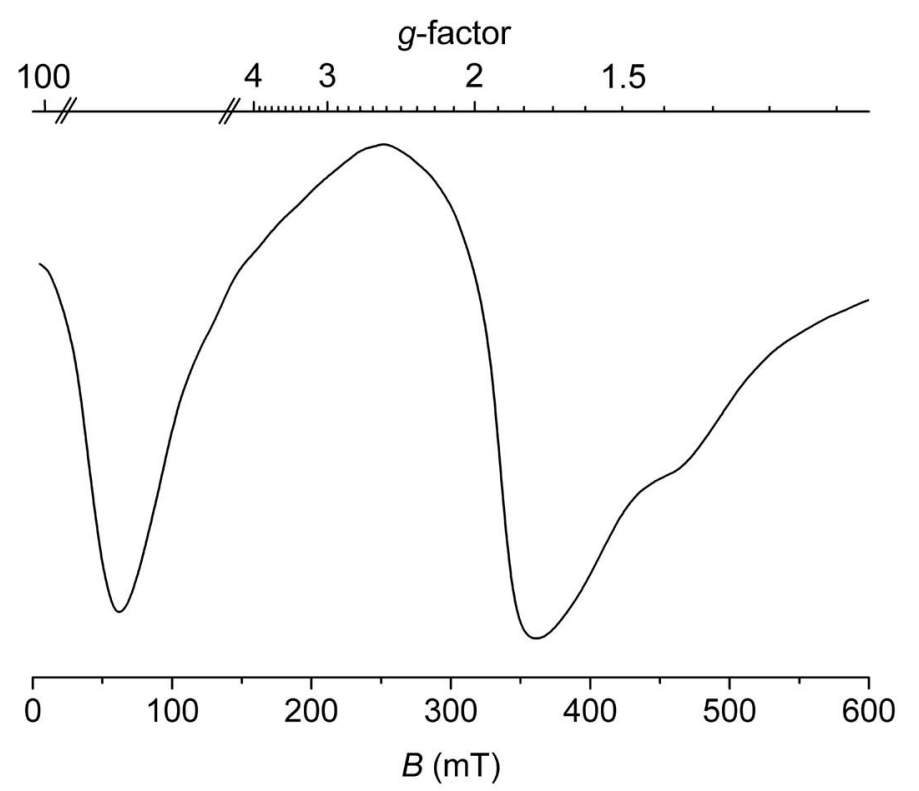

Figure C.9: Solid state EPR spectrum of $2^{r O}$ at $4.2 \mathrm{~K}$. 


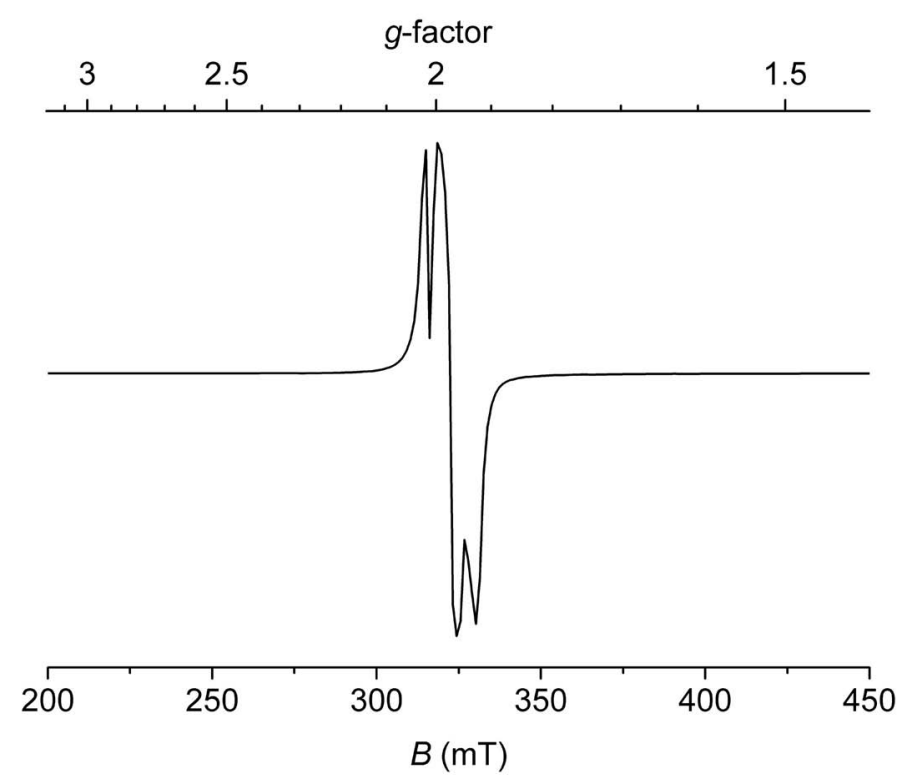

Figure C.10: Solid state EPR spectrum of $\mathfrak{2}^{\boldsymbol{P}}$ at $4.4 \mathrm{~K}$.

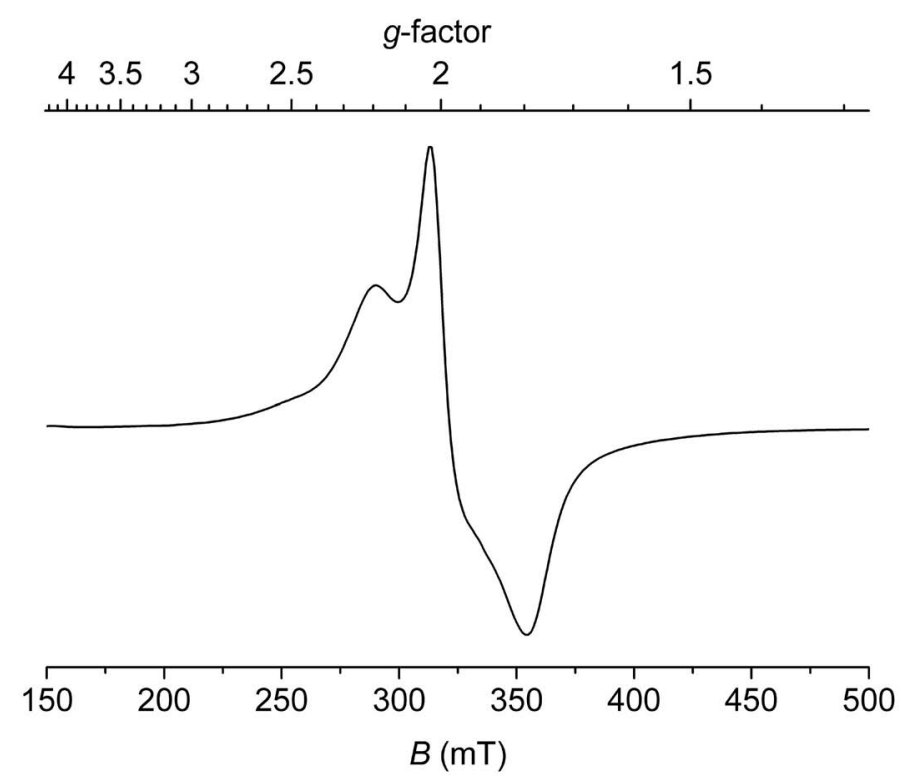

Figure C.11: Solid state EPR spectrum of $\mathfrak{2}^{\boldsymbol{T}}$ at $4.4 \mathrm{~K}$. 


\section{Bibliography}

[1] H. Beinert, R. H. Sands, Biochem. Biophys. Res. Commun. 1960, 3, 41-46.

[2] K. Tagawa, D. I. Arnon, Nature 1962, 195, 537-543.

[3] L. E. Mortenson, R. C. Valentine, J. E. Carnahan, Biochem. Biophys. Res. Commun. 1962, $7,448-452$.

[4] J. A. Fee, K. L. Findling, T. Yoshida, R. Hille, G. E. Tarr, D. O. Hearshen, W. R. Dunham, E. P. Day, T. A. Kent, E. Münck, J. Biol. Chem. 1984, 259, 124-133.

[5] R. Malkin, J. C. Rabinowitz, Biochem. Biophys. Res. Commun. 1966, 23, 822-827.

[6] T. G. Spiro, Iron-Sulfur Proteins, Wiley-Interscience, New York, 1982, 1-434.

[7] R. Cammack, Adv. Inorg. Chem. 1992, 38, 281-322.

[8] M. Fontecave, Nature Chem. Biol. 2006, 2, 171-174.

[9] H. Beinert, R. H. Holm, E. Münck, Science 1997, 277, 653-659.

[10] C. Huber, G. Wächtershäuser, Science 1998, 281, 670-672.

[11] J. L. Kirschvink, Engineering \&S Science 2005, 4, 10-20.

[12] S. P. Cramer, K. O. Hodgson, W. O. Gillum, L. E. Mortenson, J. Am. Chem. Soc. 1978, 100, 3814-3819.

[13] O. Einsle, F. A. Tezcan, S. L. Andrade, B. Schmid, M. Yoshida, J. B. Howard, D. C. Rees, Science 2002, 297, 1696-1700.

[14] A. Volbeda, M. H. Charon, C. Piras, E. C. Hatchikian, M. Frey, J. C. FontecillaCamps, Nature 1995, 373, 580-587.

[15] H. Dobbek, V. Svetlitchnyi, L. Gremer, R. Huber, O. Meyer, Science 2001, 293, 1281-1285.

[16] M. Frey, ChemBioChem 2002, 3, 153-160.

[17] H. Beinert, J. Biol. Inorg. Chem. 2000, 5, 2-15.

[18] D. C. Rees, H. B. James, Science 2003, 300, 929-931. 
[19] D. C. Johnson, D. R. Dean, A. D. Smith, M. K. Johnson, Annu. Rev. Biochem. 2005, $74,247-281$.

[20] J. M. Chan, J. Christiansen, D. R. Dean. L. C. Seefeldt, Biochemistry 1999, 38, 5779-5785.

[21] J. Meyer, J.-M. Moulis in Handbook of Metalloproteins, Vol. 1, (Eds.: A. Messerschmidt, R. Huber, T. Poulos, K. Wieghardt), John Wiley \& Sons, Chichester, 2001, 505-542.

[22] K. S. Hagen, A. D. Watson, R. H. Holm, J. Am. Chem. Soc. 1983, 105, 3905-3913.

[23] G. Zanetti, C. Binda, A. Aliverti in Handbook of Metalloproteins, Vol. 1, (Eds.: A. Messerschmidt, R. Huber, T. Poulos, K. Wieghardt), John Wiley \& Sons, Chichester, 2001, 532-542.

[24] M. F. J. M. Verhagen, T. A. Link, W. R. Hagen, FEBS Lett. 1995, 361,75-78.

[25] J. J. G. Moura, A. L. Macedó, B. L. Goodfellow, I. Moura in Handbook of Metalloproteins, Vol. 1, (Eds.: A. Messerschmidt, R. Huber, T. Poulos, K. Wieghardt), John Wiley \& Sons, Chichester, 2001, 553-559.

[26] M. C. Kennedy, T. A. Kent, M. Emptage, H. Merkle, H. Beinert, E. Münck, J. Biol. Chem. 1984, 259, 14463-14471.

[27] H. Beinert, M. C. Kennedy, Eur. J. Biochem. 1989, 186, 5-15.

[28] D. W. Plank, M. C. Kennedy, H. Beinert, J. B. Howard, J. Biol. Chem. 1989, 264, 20385-20493.

[29] J. Zhou, R. H. Holm, J. Am. Chem. Soc. 1995, 117, 11353-11354.

[30] J. Zhou, Z. Hu, E. Münck, R. H. Holm, J. Am. Chem. Soc. 1996, 118, 1966-1980.

[31] T. D. P. Stack, R. H. Holm, J. Am. Chem. Soc. 1988, 110, 2484-2494.

[32] T. D. P. Stack, J. A. Weigel, R. H. Holm, Inorg. Chem. 1990, 29, 3745-3760.

[33] K. Fukuyama, Handbook of Metalloproteins, Vol. 1, (Eds.: A. Messerschmidt, R. Huber, T. Poulos, K. Wieghardt), John Wiley \& Sons, Chichester, 2001, 543-552.

[34] Y. Ohki, Y. Sunada, K. Tatsumi, Chem. Lett. 2005, 34, 172-173.

[35] C. W. Charter Jr., Handbook of Metalloproteins, Vol. 1, (Eds.: A. Messerschmidt, R. Huber, T. Poulos, K. Wieghardt), John Wiley \& Sons, Chichester, 2001, 602-609.

[36] B. K. Burgess, D. J. Lowe, Chem. Rev. 1996, 96, 2983-3011.

[37] J. Rabinowitz, Methods Enzymol. 1972, 24, 431-446. 
[38] S. E. Mulholland, B. R. Gibney, F. Rabanal, P. L. Dutton, J. Am. Chem. Soc. 1998, 120, 10296-10302.

[39] J. B. Broderick, Nature Chem. Biol. 2007, 3, 243-244.

[40] D. J. A. Netz, A. J. Pierik, M. Stümpfig, U. Mülhenhoff, R. Lill, Nature Chem. Biol. 2007, 3, 278-286.

[41] R. Lill, U. Mülhenhoff, Trends Biochem. Sci. 2005, 30, 133-141.

[42] L. Zheng, V. L. Cash, D. H. Flint, D. R. Dean, J. Biol. Chem. 1998, 273, 1326413272.

[43] T. Land, T. A. Rouault, Mol. Cell. 1998, 2, 807-815.

[44] H. Lange, G. Kispal, A. Kaut, R. Lill, Proc. Natl. Acad. Sci. USA 2000, 97, 10501055.

[45] J. Gerber, U. Mühlenhoff, R. Lill, EMBO Rep. 2003, 4, 906-911.

[46] K. Chandramouli, M. K. Johnson, Biochemistry 2006, 45, 11087-11095.

[47] M.-P. Golinell, L. A. Akin, B. R. Crouse, M. K. Johnson, J. Meyer, Biochemistry 1996, 35, 8995-9002.

[48] J. J. G. Moura, I. Moura, T. A. Kent, J. D. Lipscomb, B. H. Huynh, J. LeGall, A. V. Xavier, E. Münck, J. Biol. Chem. 1982, 257, 6259-6267.

[49] B. H. Huynh, J. J. G. Moura, I. Moura, T. A. Kent, J. LeGall, A. V. Xavier, E. Münck, Proc. Natl. Acad. Sci. USA 1982, 79, 1096-1100.

[50] J. Meyer, J. Gagnon, J. Gaillard, M. Lutz, C. Achim, E. Münck, Y. Pétillot, C. M. Colangelo, R. A. Scott, Biochemistry 1997, 36, 13374-13380.

[51] J. C. Crack, J. Green, M. R. Cheesman, N. E. LeBrun, A. J. Thomson, Proc. Natl. Acad. Sci. USA 2007, 104, 2092-2097.

[52] J. Cambray, R. W. Lane, A. G. Wedd, R. W. Johnson, R. H. Holm, Inorg. Chem. 1977, 16, 2565-2571.

[53] K. S. Hagen, J. G. Reynolds, R. H. Holm, J. Am. Chem. Soc. 1981, 103, 4054-4063.

[54] V. R. Sutton, A. Stubna, T. Patschkowski, E. Münck, H. Beinert, P. J. Kiley, Biochemistry 2004, 43, 791-798.

[55] S. E. J. Fawcett, D. Davis, J. L. Breton, A. J. Thomson, F. A. Armstrong, Biochem. J. 1998, 335, 357-368.

[56] M. K. Johnson in Encyclopedia of Inorganic Chemistry, (Ed.: R. B. King), John Wiley \& Sons, Chichester, 1994, 1896-1915. 
[57] L. A. Sazanov, P. Hinchliffe, Science 2006, 311, 1430-1436.

[58] L. C. Sieker, E. T. Adam in Handbook of Metalloproteins, Vol. 1, (Eds.: A. Messerschmidt, R. Huber, T. Poulos, K. Wieghardt), John Wiley \& Sons, Chichester, 2001, 574-592.

[59] C. D. Stout in Handbook of Metalloproteins, Vol. 1, (Eds.: A. Messerschmidt, R. Huber, T. Poulos, K. Wieghardt), John Wiley \& Sons, Chichester, 2001, 560-573.

[60] S. Bailey, S. J. Cooper, W. F. Hagen, A. F. Arendsen, P. F. Lindley in Handbook of Metalloproteins, Vol. 1, (Eds.: A. Messerschmidt, R. Huber, T. Poulos, K. Wieghardt), John Wiley \& Sons, Chichester, 2001, 593-601.

[61] F. Capozzi, S. Ciurli, C. Luchinat, Struct. Bonding 1998, 127-160.

[62] D. J. Kolling, J. S. Brunzelle, S. Lhee, A. R. Crofts, S. K. Nair, Structure 2007, 15, 29-38.

[63] G. Sainz, J. Jakoncic, L. C. Sieker, V. Stojanoff, N. Sanishvili, M. Asso, P. Bertrand, J. Armengaud, Y. Jouanneau, J. Biol. Inorg. Chem. 2006, 11, 235-246.

[64] L. Banci, I. Bertini, S. Ciurli, S. Ferretti, C. Luchinat, M. Piccioli, Biochemistry 1993, 32, 9387-9397.

[65] E. L. Bominaar, Z. Hu, E. Münck, J. J. Girerd, S. A. Borshch, J. Am. Chem. Soc. 1995, 117, 6975-6989.

[66] F. Malatesta, F. Nicoletti, V. Zickermann, B. Ludwig, M. Brunori, FEBS Lett. 1998, 434, 322-324.

[67] E. L. Bominaar, C. Achim, S. A. Borshch, J. J. Girerd, E. Münck, Inorg. Chem. 1997, 36, 3689-3701.

[68] H. Beinert, M. C. Kennedy, C. D. Stout, Chem. Rev. 1996, 96, 2335-2374.

[69] D. H. Flint, R. M. Allen, Chem. Rev. 1996, 96, 2315-2334.

[70] H. Ding, B. Demple, Proc. Natl. Acad. Sci. USA 1997, 94, 8445-8449.

[71] P. Gaudu, B. Weiss, Proc. Natl. Acad. Sci. USA 1996, 93, 10094-10098.

[72] E. Hidalgo, J. M. Bollinger Jr., T. M. Bradley, C. T. Walsh, B. Demple, J. Biol. Chem. 1995, 270, 20908-20914.

[73] M. Hentze, L. C. Kühn, Proc. Natl. Acad. Sci. USA 1996, 93, 8175-8182.

[74] J. Rudolf, V. Makrantoni, W. J. Ingledew, M. J. R. Stark, M. F. White, Mol. Cell. 2006, 23, 801-808.

[75] E. J. Merino, A. K. Boal, J. K. Barton, Curr. Opin. Chem. Biol. 2008, 12, 229-237. 
[76] M. Lotierzo, B. T. S. Bui, D. Florenti, F. Escalettes, A. Marquet, Biochem. Soc. Trans. 2005, 33, 820-823.

[77] R. W. Busby, J. P. M. Schelvis, D. S. Yu, G. T. Babcock, M. A. Marletta, J. Am. Chem. Soc. 1999, 121, 4706-4707.

[78] J. Meyer, J. Biol. Inorg. Chem. 2008, 13, 157-170.

[79] I. Bertini C. Luchinat, A. Provenzani, A. Rosato, P. R. Vasos, Proteins 2002, 46, 110-127.

[80] Y. Takahashi, T. Hase, K. Wada, H. Matsubara, Plant Cell. Physiol. 1983, 24, 189198.

[81] D. B. Knaff in Oxygenic Photosynthesis, Vol. 4, (Eds.: D. R. Ort, C. F. Yocum), Kluwer, Dordrecht, 1996, 333-361.

[82] H. Sticht, P. Rösch, Prog. Biophys. Mol. Biol. 1998, 70, 95-136.

[83] Y. I. Shethna, P. W. Wilson, R. E. Hansen, H. Beinert, Proc. Natl. Acad. Sci. USA 1964, 52, 1263-1271.

[84] A. P. Yeh, C. Chatelet, S. M. Soltis, P. Kuhn, J. Meyer, D. C. Rees, J. Mol. Biol. 2000, 300, 587-595.

[85] I. Hanukoglu, C. R. Jefcoate, J. Biol. Chem. 1980, 255, 3057-3061.

[86] A. V. Grinberg, F. Hannemann, B. Schiffler, J. Müller, U. Heinemann, R. Bernhardt, Proteins 2000, 40, 590-612.

[87] J. R. Mason, R. Cammack, Ann. Rev. Microbiol. 1992, 46, 277-305.

[88] R. Morales, M.-H. Chron, G. Hudry-Clergeon, Y. Pétillot, S. Norager, M. Medina, M. Frey, Biochemistry 1999, 38, 15764-15773.

[89] N. Rouhier, H. Unno, S. Bandyopadhyay, L. Masip, S.-K. Kim, M. Hirasawa, J. M. Gualberto, V. Lattard, M. Kusunoki, D. B. Knaff, G. Georgiou, T. Hase, M. K. Johnson, J.-P. Jacquot, Proc. Natl. Acad. Sci. USA 2007, 104, 7379-7384.

[90] A. P. Fernandes, A. Holmgren, Antioxid. Redox Signal. 2004, 6, 63-74.

[91] C. H. Lillig, C. Berndt, O. Vergnolle, M. E. Löhn, C. Hudemann, E. Bill, A. Holmgren, Proc. Natl. Acad. Sci. USA 2005, 102, 8168-8173.

[92] S. Achebach, Q. H. Tran, A. Vlamis-Gardikas, M. Mullner, A. Holmgren, G. Unden, FEBS Lett. 2004, 565, 203-206.

[93] W. R. Hagen, P. J. Silva, M. A. Amorim, P.-L. Hagedoorn, H. Wassink, H. Haaker, F. T. Robb, J. Biol. Inorg. Chem. 2000, 5, 527-534. 
[94] A. P. Yeh, X. I. Ambroggio, S. L. A. Andrade, O. Einsle, C. Chatelet, J. Meyer, D. C. Rees, J. Biol. Chem. 2002, 277, 24499-34507.

[95] B. R. Crouse, J. Meyer, M. K. Johnson, J. Am. Chem. Soc. 1995, 117, 9612-9613.

[96] C. Achim, M. P. Golinell, E. L. Bominaar, J. Meyer, E. Münck, J. Am. Chem. Soc. 1996, 118, 8168-8169.

[97] F. Berkovitch, Y. Nicolet, J. T. Wan, J. T. Jarrett, C. L. Drennan, Science 2004, 303, 76-79.

[98] N. B. Ugulava, B. R. Gibney, J. T. Jarrett, Biochemistry 2001, 40, 8343-8351.

[99] J. T. Jarrett, Arch. Biochem. Biophys. 2005, 433, 312-321.

[100] N. B. Ugulava, K. K. Surerus, J. T. Jarrett, J. Am. Chem. Soc. 2002, 124, 9050-9051.

[101] B. T. S. Bui, T. A. Mattioli, D. Florentin, G. Bolbach, A. Marquet, Biochemistry 2006, 45, 3824-3834.

[102] N. B. Ugulava, C. J. Sacanell, J. T. Jarrett, Biochemistry 2001, 40, 8352-8358.

[103] J. Lin, T. Zhou, J. Wang, Proc. Natl. Acad. Sci. USA 2007, 104, 14640-14645.

[104] J. R. Colca, W. G. McDonald, D. J. Waldon, J. W. Leone, J. M. Lull, C. A. Bannow, E. T. Lund, W. R. Mattews, Am. J. Physiol. 2004, 286, 252-260.

[105] S. E. Wiley, A. N. Murphy, S. A. Ross, P. van der Geer, J. E. Dixon, Proc. Natl. Acad. Sci. USA 2007, 104, 5318-5323.

[106] S. E. Wiley, M. L. Paddock, E. C. Abresch, L. Gross, P. van der Geer, R. Nechushtai, A. N. Murphy, P. A. Jennings, J. E. Dixon, J. Biol. Chem. 2007, 282, 23745-23749.

[107] T. A. Link in Handbook of Metalloproteins, Vol. 1, (Eds.: A. Messerschmidt, R. Huber, T. Poulos, K. Wieghardt), John Wiley \& Sons, Chichester, 2001, 518-531.

[108] T. A. Link, Adv. Inorg. Chem. 1999, 47, 83-157.

[109] J. S. Rieske, D. H. MacLennan, R. Coleman, Biochem. Biophys. Res. Commun. 1964, 15, 338-344.

[110] H. Bönisch, C. L. Schmidt, G. Schäfer, R. Ladenstein, J. Mol. Biol. 2002, 319, 791-805.

[111] D. Xia, C.-A. Yu, H. Kim, J.-Z. Xia, A. M. Kachurin, L. Zhang, L. Yu, J. Deisenhofer, Science 1997, 277, 60-66.

[112] C. J. Carrell, H. Zhang, W. A. Cramer, J. L. Smith, Structure 1997, 5, 1613-1625.

[113] K. Lee, B. Kauppi, R. E. Parales, D. T. Gibson, S. Ramaswamy, Biochem. Biophys. Res. Commun. 1997, 241, 553-557. 
[114] D. J. Ferraro, L. Gakhar, S. Ramaswamy, Biochem. Biophys. Res. Commun. 2005, 338, 175-190.

[115] H. Schägger, T. Hagen, B. Roth, U. Brandt, T. A. Link, G. von Jagow, Eur. J. Biochem. 1989, 190, 123-130.

[116] M. Brugna, S. Rodgers, A. Schricker, G. Montoya, K. Kazmeier, W. Nitschke, I. Sinning, Proc. Natl. Acad. Sci. USA 2000, 97, 2069-2074.

[117] M. K. Bowman, E. A. Berry, A. G. Roberts, D. M. Kramer, Biochemistry 2004, 43, 430-436.

[118] T. Schröter, O. M. Hatzheld, S. Gemeinhardt, M. Korn, T. Friedrich, B. Ludwig, T. A. Link, Eur. J. Biochem. 1998, 255, 100-106.

[119] T. A. Link, O. M. Hatzfeld, M. Saynovits in Bioinorganic Chemistry, (Ed.: A. X. Trautwein), Wiley-VCH, Weinheim, 1997, 312-325.

[120] Y. Zu, M. M.-J. Couture, D. R. J. Kolling, A. R. Crofts, L. D. Eltis, J. A. Fee, J. Hirst, Biochemistry 2003, 42, 12400-12408.

[121] S. Ramaswamy in Handbook of Metalloproteins, Vol. 1, (Eds.: A. Messerschmidt, R. Huber, T. Poulos, K. Wieghardt), John Wiley \& Sons, Chichester, 2001, 613-620.

[122] R. E. Parales, J. V. Parales, D. T. Gibson, J. Bacteriol. 1999, 181, 1831-1837.

[123] E. J. Leggate, J. Hirst, Biochemistry 2005, 44, 7048-7058.

[124] A. R. Klingen, G. M. Ullmann, Biochemistry 2004, 43, 12383-12389.

[125] I.-J. Lin, Y. Chen, J. A. Fee, J. Song, W. M. Westler, J. L. Markley, J. Am. Chem. Soc. 2006, 128, 10672-10673.

[126] N. J. Cosper, D. M. Eby, A. Kounosu, N. Kurosawa, E. L. Neidle, D. M. Kurtz Jr., T. Iwasaki, R. A. Scott, Protein Sci. 2002, 11, 2969-2973.

[127] M. Rampp, E. Kellner, A. Müller, A. Riedel in Bioinorganic Chemisrty, (Ed.: A. X. Trautwein), Wiley-VCH, Weinheim, 1997, 295-301.

[128] S. C. Lee, R. H. Holm, Chem. Rev. 2004, 104, 1135-1157.

[129] P. V. Rao, R. H. Holm, Chem. Rev. 2004, 104, 527-559.

[130] W. Autenrieth R. Hennings, Chem. Ber. 1901, 34, 1772-1778.

[131] J. J. Mayerle, R. B. Frankel, R. H. Holm, J. A. Ibers, W. D. Phillips, J. F. Weiher, Proc. Natl. Acad. Sci. USA 1973, 70, 2429-2433.

[132] J. J. Mayerle, S. E. Denmark, B. V. DePamphilis, J. A. Ibers, R. H. Holm, J. Am. Chem. Soc. 1975, 97, 1032-1045. 
[133] B. K. Teo, R. G. Shulman, G. S. Brown, A. E. Meixner, J. Am. Chem. Soc. 1979, 101, 5624-5631.

[134] W. O. Gillum, R. B. Frankel, S. Foner, R. H. Holm, Inorg. Chem. 1976, 15, 10951100 .

[135] J. G. Reynolds, R. H. Holm, Inorg. Chem. 1980, 19, 3257-3260.

[136] P. K. Mascharak, G. C. Papaefthymiou, R. B. Frankel, R. H. Holm, J. Am. Chem. Soc. 1981, 103, 6110-6116.

[137] G. B. Wong, M. A. Bobrik, R. H. Holm, Inorg. Chem. 1978, 17, 578-584.

[138] Y. Do, E. D. Simhon, R. H. Holm, Inorg. Chem. 1983, 22, 3809-3812.

[139] P. Beardwood, J. F. Gibson, J. Chem. Soc., Dalton Trans. 1983, 737-748.

[140] P. Beardwood, J. F. Gibson, C. E. Johnson, J. D. Rush, J. Chem. Soc., Dalton Trans. 1982, 2015-2020.

[141] A. Salifoglou, A. Simopoulos, A. Kostikas, R. W. Dunham, M. G. Kanatzidis, D. Coucouvanis, Inorg. Chem. 1988, 27, 3394-3406.

[142] D. Coucouvanis, A. Salifoglou, M. G. Kanatzidis, A. Simopoulos, V. Papaefthymiou, J. Am. Chem. Soc. 1984, 106, 6081-6082.

[143] J. Ballmann, S. Dechert, E. Bill, U. Ryde, F. Meyer, Inorg. Chem. 2008, 47, 15861598.

[144] R. E. Johnson, G. C. Papaefthymiou, R. B. Frankel, R. H. Holm, J. Am. Chem. Soc. 1983, 105, 7280-7287.

[145] N. G. Connelly, W. E. Geiger, Chem. Rev. 1996, 96, 877-910.

[146] J. R. Aranzaes, M.-C. Daniel, D. Astuc, Can. J. Chem. 2006, 84, 288-299.

[147] R. H. Holm, Acc. Chem. Res. 1977, 10, 427-434.

[148] S. Ueno, N. Ueyama, A. Nakamura, T. Tukihara, Inorg. Chem. 1986, 25, 1000-1005.

[149] R. H. Holm in Comprehensive Coordination Chemistry, Vol. 8, (Eds.: J. A. McCleverty, T. J. Meyer), Elsevier, New York, 2003, 61-90.

[150] L. E. Maelia, S. A. Koch, Inorg. Chem. 1986, 25, 1896-1904.

[151] P. K. Mascharak, M. C. Smith, W. H. Armstrong, B. K. Burgess, R. H. Holm, Proc. Natl. Acad. Sci. USA 1982, 79, 7056-7060.

[152] C. Jinhua, C. H. Changneng, Jiegou Huaxue (J. Struct. Chem.) 1985, 4, 199-202.

[153] C. Jinhua, C. H. Changneng, Jiegou Huaxue (J. Struct. Chem.) 1988, 7, 43-46. 
[154] N. Ueyama, S. Ueno, T. Sugawara, K. Tatsumi, A. Nakamura, N. Yasuoka, J. Chem. Soc., Dalton Trans. 1991, 2723-2727.

[155] N. Ueyama, Y. Yamada, T. Okamura, S. Kimura, A. Nakamura, Inorg. Chem. 1996, 35, 6473-6484.

[156] G. B. Wong, D. M. Kurtz Jr., R. H. Holm, L. E. Mortenson, R. G. Upchurch J. Am. Chem. Soc 1979, 101, 3078-3090.

[157] S. Cossu, O. DeLucchi, D. Fabbri, G. Valle, G. F. Painter, R. A. J. Smith, Tetrahedron 1997, 53, 6073-6084.

[158] K. Miyazaki, $18^{\text {th }}$ Annual Meeting of the Chemical Society of Japan, Ref-Nr. 9505, Osaka, 1965, 175-180.

[159] C. Zonta, O. DeLucchi, R. Volpicelli, L. Cotarca, Top. Curr. Chem. 2007, 275, 131-161.

[160] A. Alexakis, D. Polet, S. Rosset, S. March, J. Org. Chem. 2004, 69, 5660-5667.

[161] T. Yamato, K. Hawagawa, Y. Saruwatari, L. K. Doamekpor, Chem. Ber. 1993, 126, 1435-1439.

[162] T. N. Sorrell, E. H. Cheesman, Synth. Commun. 1981, 11, 909-912.

[163] R. M. Pontes, E. A. Basso, F. P. dos Santos, J. Org. Chem. 2007, 72, 1901-1911.

[164] A. E. Lemire, J. C. Thomson, Can. J. Chem. 1970, 48, 824-829.

[165] Q. Zhu-Ohlbach, R. Gleiter, F. Rohminger, H.-L. Schmidt, T. Reda, Eur. J. Org. Chem. 1998, 11, 2409-2416.

[166] L. Yang, D. R. Powell, R. P. Houser, Dalton Trans. 2007, 955-964.

[167] J. T. Hoggins, H. Steinfink, Inorg. Chem. 1976, 15, 1682-1685.

[168] S. Han, R. S. Czernuszewicz, T. G. Spiro, Inorg. Chem. 1986, 25, 2276-2277.

[169] D. Coucouvanis, D. Swenson, P. Stremple, N. C. Baenziger, J. Am. Chem. Soc. 1979, 101, 3392-3394.

[170] J. Ballmann, X. Sun, S. Dechert, E. Bill, F. Meyer, J. Inorg. Biochem. 2007, 101, 305-312.

[171] P. Beardwood, J. F. Gibson, J. Chem. Soc., Chem. Commun. 1986, 490-492.

[172] P. Beardwood, J. F. Gibson, J. Chem. Soc., Dalton Trans. 1985, 1345-1347.

[173] P. Beardwood, J. F. Gibson, J. Chem. Soc., Dalton Trans. 1985, 102-104.

[174] W. E. Cleland, B. A. Averill, Inorg. Chem. 1984, 23, 4192-4197. 
[175] W. Hieber, J. Gruber, Z. Anorg. Allg. Chem. 1958, 296, 91-103.

[176] P. F. Brandt, D. A. Lesch, P. R. Stafford, T. B. Rauchfuss, Inorg. Synth. 1997, 31, 112-116.

[177] A. Shaver, P. J. Fitzpatrick, K. Steliou, I. S. Butler, J. Am. Chem. Soc. 1979, 101, 1313-1315.

[178] L. G. A. Van de Water, F. ten Hoonte, W. L. Driesen, J. Reedijk, D. J. Sherrington, Inorg. Chim. Acta 2000, 303, 77-85.

[179] Y. Jin, I. Yoon, J. Seo, J.-E. Lee, S.-T. Moon, J. Kim, S. W. Han, K.-M. Park, L. F. Lindoy, S. S. Lee, Dalton Trans. 2005, 788-796.

[180] G. N. Schrauzer, V. P. Mayweg, H. W. Flick, W. Heinrich, J. Am. Chem. Soc. 1966, 88, 4604-4609.

[181] A. L. Balch, J. Am. Chem. Soc. 1969, 91, 6962-6967.

[182] T. H. Lemmen, J. A. Kocal, F. J.-K. Lo, M. W. Chen, L. F. Dahl, J. Am .Chem. Soc. 1981, 103, 1932-1941.

[183] H. Ogino, S. Inomata, H. Tobita, Chem. Rev. 1998, 98, 2093-2121.

[184] A. L. Balch, Inorg. Chem. 1971, 10, 276-281.

[185] N. Ueyama, S. Ueno, A. Nakamura, Bull. Chem. Soc., Jpn. 1987, 60, 283-287.

[186] R. A. Andersen, K. Faegri Jr., J. C. Green, A. Haaland, M. F. Lappert, W.-P. Leung, K. Rypdal, Inorg. Chem. 1988, 27, 1782-1786.

[187] M. M. Olmstead, P. P. Power, S. C. Shoner, Inorg. Chem. 1991, 30, 2547-2551.

[188] S. Pohl, U. Bierbach, W. Saak, Angew. Chem. Int. Ed. Engl. 1989, 28, 776-777.

[189] A. Panda, M. Stender, R. J. Wright, M. M. Olmstead, P. Klavins, P. P. Power, Inorg. Chem. 2002, 41, 3909-3916.

[190] J. Vela, S. Stoian, C. J. Flaschenriem, E. Münck, P. L. Holland, J. Am. Chem. Soc. 2004, 126, 4522-4523.

[191] J. M. Smith, R. J. Lachicotte, K. A. Pittard, T. R. Cundari, G. Lukat-Rodgers, K. R. Rodgers, P. L. Holland, J. Am. Chem. Soc. 2001, 123, 9222-9223.

[192] M. Stender, R. J. Wright, B. E. Eichler, J. Prust, M. M. Olmstead, H. W. Roesky, P. P. Power, J. Chem. Soc., Dalton Trans. 2001, 3465-3469.

[193] L. Yu, K. Muthukumaran, I. V. Sazanovich, C. Kirmaier, E. Hindin, J. R. Diers, P. D. Boyle, D. F. Bocian, D. Holten, J. S. Lindsey, Inorg. Chem. 2003, 42, 6629-6647. 
[194] B. J. Littler, M. A. Miller, C.-H. Hung, R. W. Wagner, D. F. O'Shea, P. D. Boyle, L. S. Lindsey, J. Org. Chem. 1999, 64, 1391-1396.

[195] J.-Y. Shin, D. Dolphin, B. O. Patrick, Crystal Growth $\&$ Design 2004, 4, 659-661.

[196] R. B. Broach, J. T. Jarrett, J. Biochem. 2006, 45, 14166-14171.

[197] L. Di Costanzo Jr., D. W. Christianson, Proteins 2006, 65, 637-642.

[198] M. A. Walters, C. L. Roche, A. L. Rheingold, S. W. Kassel, Inorg. Chem. 2005, 44, 3777-3779.

[199] G. N. L. Jameson, E. M. Walters, W. Manieri, P. Schürmann, M. K. Johnson, B. H. Huynh, J. Am. Chem. Soc. 2003, 125, 1146-1147.

[200] J. Degani, R. Fochi, Synthesis 1976, 6, 471-472.

[201] D. Sellmann, P. Frank, Angew. Chem. Int. Ed. Engl. 1986, 25, 1107-1108.

[202] D. Sellmann, P. Frank, F. Knoch, J. Organomet. Chem. 1988, 339, 345-356.

[203] J. G. Alvarado-Rogríguez, N. Andrade-López, S. González-Montiel, G. Merino, A. Vela, Eur. J. Inorg. Chem. 2003, 3554-3562.

[204] D. Sellmann, D. Häußinger,F. W. Heinemann, Eur. J. Inorg. Chem. 1999, 1715-1725.

[205] G. Casiragi, G. Casnati, A. Pochini, A. Puglia, R. Ungaro, G. Sartori, Synthesis 1981, 2, 143-146.

[206] G. Sartori, F. Bigi, R. Maggi, C. Porta, Tetrahedron Lett. 1994, 35, 7073-7076.

[207] V. Böhmer, D. Rathay, H. Kämmerer, Org. Prep. Proced. Int. 1978, 10, 113-121.

[208] W. Hiller, W. Rundel, Acta Cryst. 1993, C49, 1127-1128.

[209] J. Vela, J. Cirera, J. M. Smith, R. J. Lachicotte, C. J. Flaschenriem, S. Alvarez, P. L. Holland, Inorg. Chem. 2007, 46, 60-71.

[210] R. Hahn, A. Nakamura, K. Tanaka, Y. Nakayama, Inorg. Chem. 1995, 34, 6562-6564.

[211] E. Block, G. Ofori-Okai, J. Zubieta, J. Am. Chem. Soc. 1989, 111, 2327-2329.

[212] M. T. Bes, E. Parisini, L. A. Inda, L. M. Saraiva, M. L. Peleato, G. M. Sheldrick, Structure 1999, 7, 1201-1211.

[213] M. Wollenberg, C. Berndt, E. Bill, J. D. Schwenn, A. Seidler, Eur. J. Biochem. 2003, 270, 1662-1671.

[214] G. Palmer, W. R. Dunham, J. A. Fee, R. H. Sands, T. Iizuka, T. Yonetani, Biochim. Biophys. Acta 1971, 245, 201-207. 
[215] N. S. Gill, F. B. Taylor, Inorg. Synth. 1967, 9, 136-142.

[216] M. K. Johnson, Curr. Opin. Chem. Biol. 1998, 2, 173-181.

[217] M. K. Johnson, C. R. Staples, E. C. Duin, M. E. Lafferty, R. E. Duderstadt, Pure Appl. Chem. 1998, 70, 939-946.

[218] J. Ceek, J. B. Broderick, J. Biol. Inorg. Chem. 2001, 6, 209-226.

[219] C. L. Drennan, J. W. Peters, Curr. Opin, Struct. Biol. 2003, 13, 220-226.

[220] J. T. Jarrett, Curr. Opin. Chem. Biol. 2003, 7, 174-182.

[221] N. B. Ugulava, B. R. Gibney, J. T. Jarrett, J. Am. Chem. Soc. 2002, 124, 9050-9051.

[222] P. Beardwood, J. F. Gibson, J. Chem. Soc., Dalton Trans. 1992, 2457-2466.

[223] W. H. Brown, W. N. Frensch, Can. J. Chem. 1958, 36, 371-377.

[224] B. Tuner, A. Shterenberg, M. Kapson, K. Suwinska, Y. Eichen, Chem. Commun. 2001, 13-14.

[225] D. M. M. Freckmann, T. Dubé, C. D. Bérubé, S. Gambarotta, G. P. A. Yap, Organometallics 2002, 21, 1240-1246.

[226] W. Wu, A. C. Grimsdale, K. Müllen, Chem. Commun. 2003, 1044-1045.

[227] G. Cristou, P. K. Mascharak, W. H. Armstrong, G. C. Papaefthymiou, R. B. Frankel, R. H. Holm, J. Am. Chem. Soc. 1982, 104, 2820-2831.

[228] P. Bertrand, B. Guigliarelli, J.-P. Gayda, P. Beardwood, J. F. Gibson, Biochim. Biophys. Acta 1985, 831, 261-266.

[229] B. L. Trumpower, C. A. Edwards, T. Ohnishi, J. Biol. Chem. 1980, 92, 209-223.

[230] J. Fritz, R. Anderson, J. Fee, G. Palmer, R. H. Sands, J. C. M. Tsibris, I. C. Gunsalus, W. H. Orme-Johnson, H. Beinert, Biochim. Biophys. Acta 1971, 253, 110-133.

[231] D. V. Devartanian, Y. I. Shethna, H. Beinert, Biochim. Biophys. Acta 1969, 194, 548-563.

[232] J. N. Siedow, S. Power, F. F. De la Rosa, G. Palmer, J. Biol. Chem. 1978, 263, 2392-2399.

[233] J. S. Rieske, J. Biol. Chem. 1968, 239, 3017-3022.

[234] S. Iwata, M. Saynovits, T. A. Link, H. Michel, Structure 1996, 4, 567-579.

[235] K. Dittmann, U. Pindur, Arch. Pharm. 1985, 318, 340-350.

[236] M. A. Bobrik, K. O. Hodgson, R. H. Holm, Inorg. Chem. 1977, 16, 1851-1858. 
[237] D. Kuila, J. A. Fee, J. Biol. Chem. 1986, 261, 2768-2771.

[238] P. J. Geary, D. P. E. Dickson, Biochem. J. 1981, 195, 199-203.

[239] T. Iwasaki, A. Kounosu, D. R. J. Kolling, A. R. Crofts, S. A. Dikanov, A. Jin, T. Imai, A. Urushiyama, J. Am. Chem. Soc. 2004, 126, 4788-4789.

[240] S. De Vries, S. P. J. Albracht, F. I. Leeuwerik, Biochim. Biophys. Acta 1979, 546, 316-333.

[241] R. C. Prince, Biochim. Biophys. Acta 1983, 723, 133-138.

[242] R. Malkin, A. J. Bearden, Biochim. Biophys. Acta 1978, 505, 147-181.

[243] F. T. de Oliveira, E. L. Bominaar, J. Hirst, J. A. Fee, E. Münck, J. Am. Chem. Soc. 2004, 126, 5338-5339.

[244] J. A. Fee, G. Palmer, Biochim. Biophys. Acta 1971, 245, 175-195.

[245] K. Mukai, T. Kimura, J. Helbert, L. Kevan, Biochim. Biophys. Acta 1973, 295, 49-56.

[246] P. Bertrand, J.-P. Gayda, Biochim. Biophys. Acta 1979, 579, 107-121.

[247] M. Shoji, K. Koizumi, Y. Kitagawa, S. Yamanaka, M. Okumura, K. Yamaguchi, Int. J. Quantum Chem. 2007, 10\%, 609-627.

[248] H. E. Gottlieb, V. Kotlyar, A. Nudelman, J. Org. Chem. 1997, 62, 7512-7515.

[249] R. Ahlrichs, M. Bär, M. Häser, H. Horn, C. Kölmel, Chem. Phys. Lett. 1989, 162, 165-169.

[250] A. D. Becke, Phys. Rev. A 1988, 38, 3098-3100.

[251] J. P. Perdew, Phys. Rev. B 1986, 33, 8822-8824.

[252] F. Weigend, R. Ahlrichs, Phys. Chem. Chem. Phys. 2005, 7, 3297-3305.

[253] R. F. W. Bader, Atoms in Molecules: A Quantum Theory, Oxford University Press, Oxford, 2005, 169-240.

[254] J. Cioslowski, J. Chem. Phys. Lett. 1992, 194, 73-78.

[255] M. J. Frisch, G. W. Trucks, H. B. Schlegel, G. E. Scuseria, M. A. Robb, J. R. Cheeseman, J. A. Montgomery Jr., T. Vreven, K. N. Kudin, J. C. Burant, J. M. Millam, S. S. Iyengar, J. Tomasi, V. Barone, B. Mennucci, M. Cossi, G. Scalmani, N. Rega, G. A. Petersson, H. Nakatsuji, M. Hada, M. Ehara, K. Toyota, R. Fukuda, J. Hasegawa, M. Ishida, T. Nakajima, Y. Honda, O. Kitao, H. Nakai, M. Klene, X. Li, J. E. Knox, H. P. Hratchian, J. B. Cross, V. Bakken, C. Adamo, J. Jaramillo, R. Gomperts, R. E. Stratmann, O. Yazyev, A. J. Austin, R. Cammi, C. Pomelli, J. W. 
Ochterski, P. Y. Ayala, K. Morokuma, G. A. Voth, P. Salvador, J. J. Dannenberg, V. G. Zakrzewski, S. Dapprich, A. D. Daniels, M. C. Strain, O. Farkas, D. K. Malick, A. D. Rabuck, K. Raghavachari, J. B. Foresman, J. V. Ortiz, Q. Cui, A. G. Baboul, S. Clifford, J. Cioslowski, B. B. Stefanov, G. Liu, A. Liashenko, P. Piskorz, I. Komaromi, R. L. Martin, D. J. Fox, T. Keith, M. A. Al-Laham, C. Y. Peng, A. Nanayakkara, M. Challacombe, P. M. W. Gill, B. Johnson, W. Chen, M. W. Wong, C. Gonzalez, J. A. Pople, Gaussian 03, Revision C.02, Gaussian, Inc., Wallingford CT 2004.

[256] A. D. J. Becke, J. Chem. Phys. 1993, 98, 5648-5652.

[257] P. J. Stephens, F. J. Devlin, M. J. Frisch, C. F. Chabalowski, J. Phys. Chem. 1994, 98, 11623-11627.

[258] E. Sigfridsson, U. Ryde, J. Biol. Inorg. Chem. 1999, 4, 99-110.

[259] W. J. Hehre, L. Radom, P. v. R. Schleyer, J. A. Pople, Ab initio molecular orbital theory, Wiley-Interscience, New York, 1986.

[260] W. Humphrey, A. Dalke, K. Schulten, J. Molec. Graphics 1996, 14, 33-38.

[261] G. Schaftenaar, J. H. Noordik, J. Comput.-Aided Mol. Design 2000, 14, 123-134.

[262] Persistence of Vision Pty. Ltd. (Version 3.6) 2004, retrieved from http://www.povray.org.

[263] G. M. Sheldrick, SHELXL-97, Program for Crystal Structure Refinement, University of Göttingen, Göttingen, Germany, 1997.

[264] G. M. Sheldrick, SHELXS-97, Program for Crystal Structure Solution, University of Göttingen, Göttingen, Germany, 1997.

[265] G. M. Sheldrick, Acta Cryst. 2008, A64, 112-122.

[266] STOE \& CIE GmbH: Darmstadt, Germany, 2002.

[267] H. D. Flack, Acta Cryst. 1983, A39, 876-881.

[268] G.M. Sheldrick, SADABS, Program for Empirical Absorption Correction of Area Detector Data, University of Göttingen, Germany, 2002.

[269] STOE \& CIE GmbH, Darmstadt, Germany, 2002.

[270] P. van der Sluis, A. L. Spek, Acta Cryst. 1990, A46, 194-201.

[271] A. L. Speck, J. Appl. Crystallogr. 2003, 36, 7-13.

[272] D. W. Christianson, Adv. Protein Chem. 1991, 42, 281-355.

[273] K. Andrä, H.-R. Hoppe, Z. Chem. 1980, 20, 267-268.

[274] R. Burth, H. Vahrenkamp, Inorg. Chim. Acta 1998, 282, 193-199. 
[275] A. W. Addison, T. N. Rao, J. Reedijk, J. van Rijn, G. C. Verschoor, J. Chem. Soc., Dalton Trans. 1984, 1349-1356.

[276] K. S. Anjali, J. T. Sampanthar, J. J. Vittal, Inorg. Chim. Acta 1999, 295, 9-17.

[277] T. Thuéry, M. Nierlich, Z. Asfari, J. Vicens, J. Chem. Soc., Dalton Trans. 2000, 1297-1301.

[278] R. B. Redford, S. L. Hazelwood, M. E. Limmert, J. M. Brown, S. Ramdeehul, A. R. Cowley, S. J. Coles, M. B. Hursthouse, Organometallics 2003, 22, 1364-1371. 


\section{Structures of Free Ligands}<smiles>SCc1ccccc1CS</smiles>

I<smiles>CN(C)CCS</smiles>

$\mathbf{X}$<smiles>[X]c1ccccc1S</smiles>

$\mathbf{X I V}^{\mathbf{C}}, \mathrm{X}=\mathrm{CH}_{2}$ $X^{N} V^{N}, X=N M e$ $X^{\prime} V^{0}, X=O$ $\mathbf{X I V}^{\mathbf{P}}, \mathbf{X}=\mathbf{P P h}$ $X^{\prime} V^{S}, X=S$

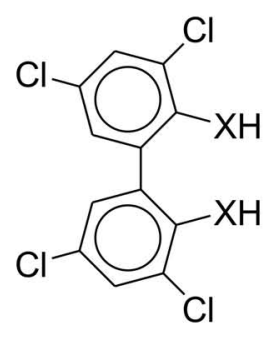

Ila, $X=0$

Va, $X=S$<smiles>C/C(=C/C(C)=N/Br)N[Al]</smiles>

$\mathbf{X I}$ $\mathrm{Ar}=$ 2,6-di-isopropylphenyl<smiles>[R]C(c1ccc[nH]1)c1ccc[nH]1</smiles>

$X X a, R=R^{\prime}=M e$

$\mathbf{X X b}, \mathrm{R}=\mathrm{R}^{\prime}=\mathrm{Ph}$

$\mathbf{X X c},-\left[R \mathrm{R}^{\prime}\right]-=-\left[\left(\mathrm{CH}_{2}\right)_{5}\right]-$

XXIb, $R=F$
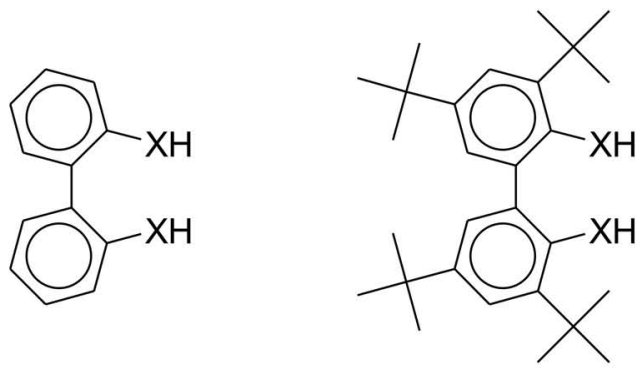

Ilb, $X=0$

Vb, $X=S$

Ilc, $X=0$

Vc, $X=S$<smiles>FC(F)(F)C(=C1C=CC=N1)c1ccc[nH]1</smiles>

XII

$X I I I I^{0}, X=0$

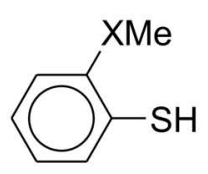

${ }^{X I I I}{ }^{C}, X=\mathrm{CH}_{2}$
XIII $^{S}, X=s$<smiles>Cc1c(C(c2ccccc2)c2[nH]c3ccccc3c2C)[nH]c2ccccc12</smiles>

XXIa, $\mathrm{R}=\mathrm{H}$<smiles>[R]c1c([R])c([R])c(-c2nc3ccccc3[nH]2)c(-c2nc3ccccc3[nH]2)c1[R]</smiles>

XXII 


\section{Structures of Complexes and Clusters}
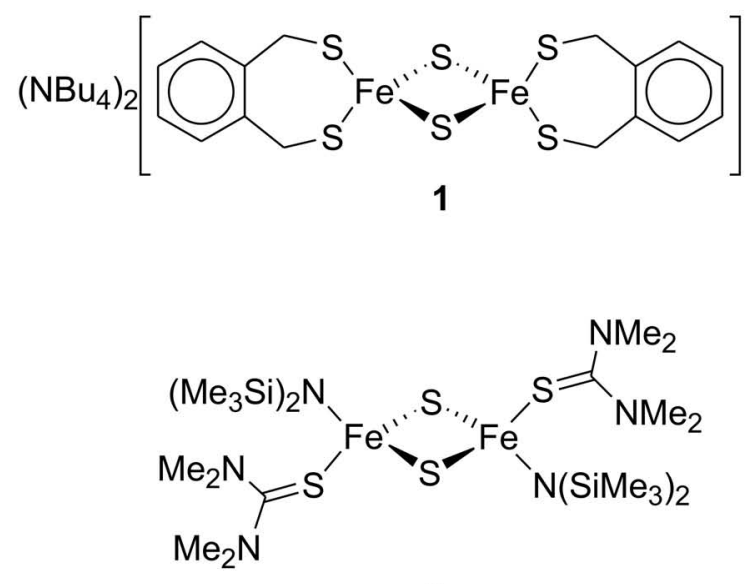

3

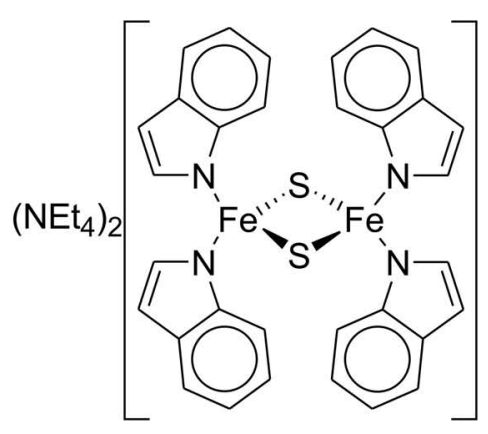

5

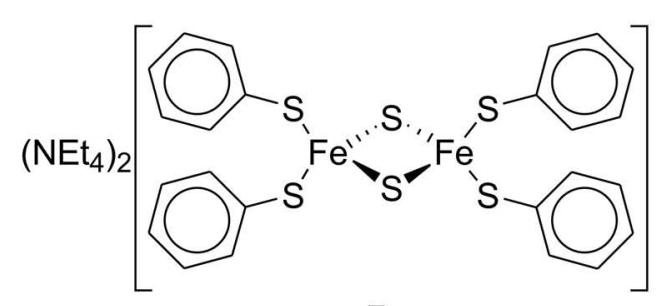

$7 a$
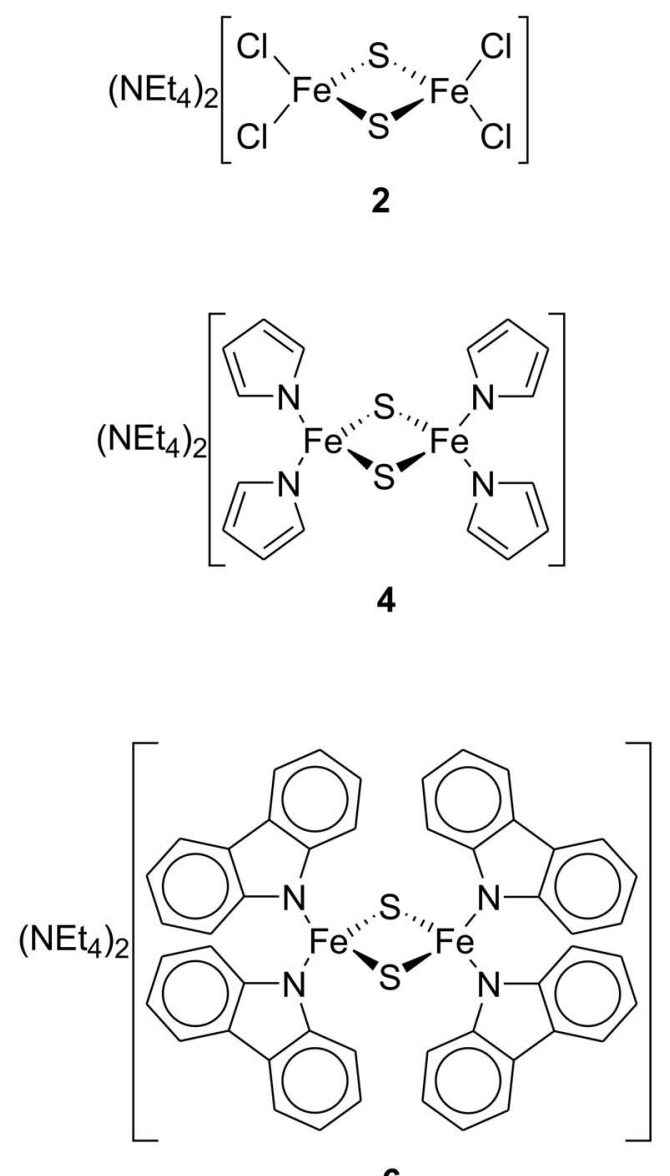

6

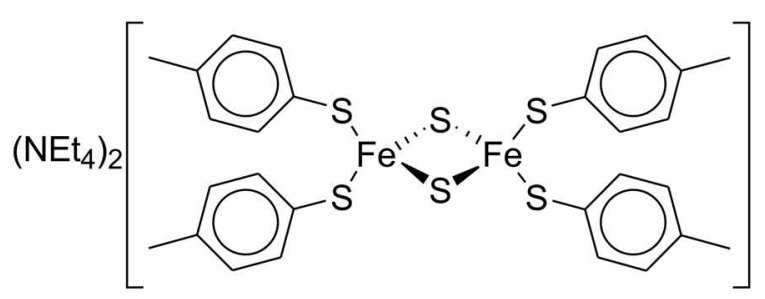

$7 b$ 

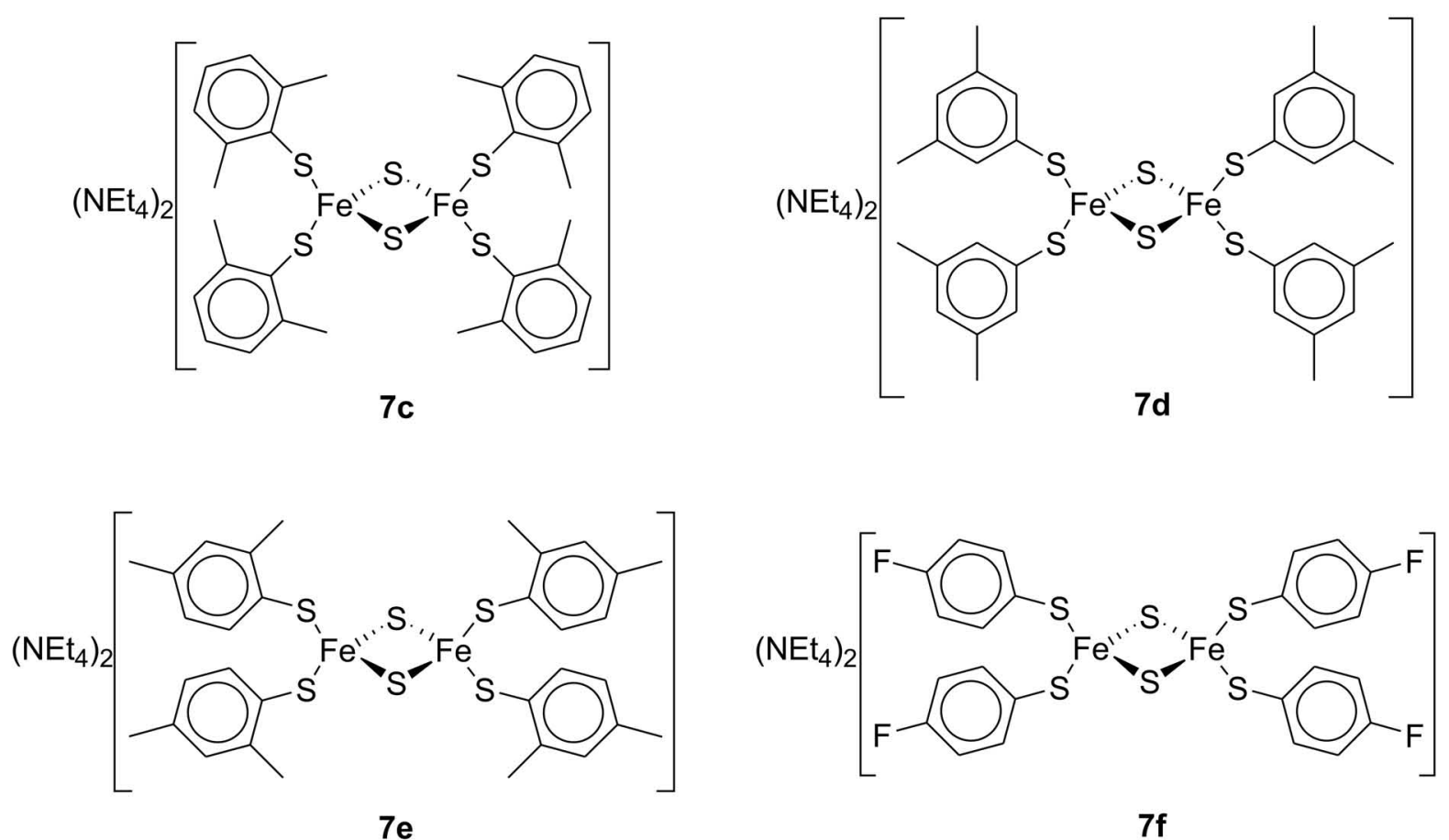

$7 f$
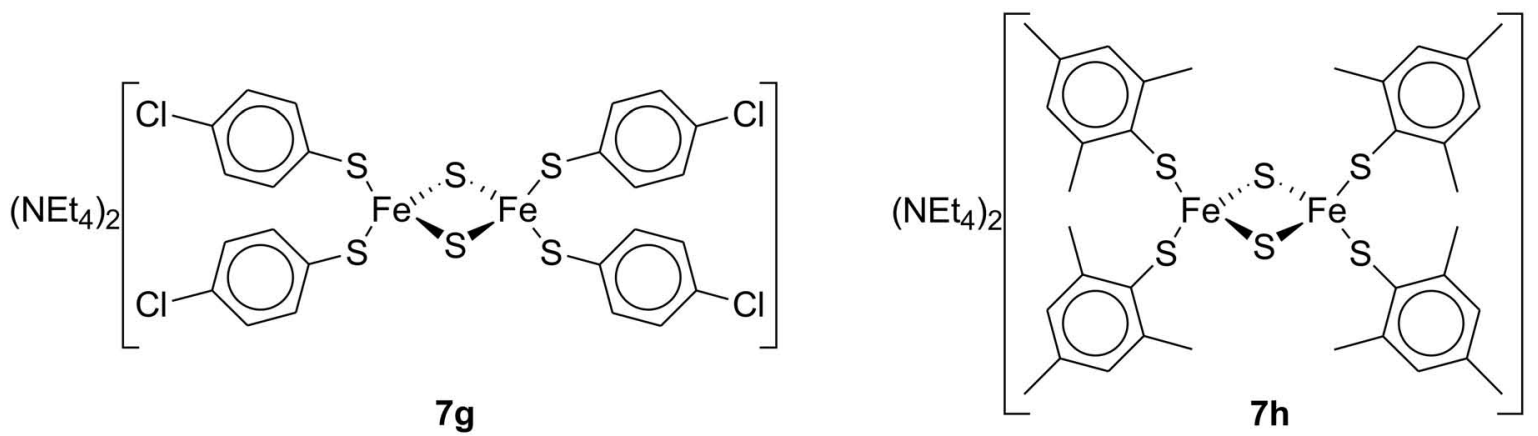

$7 g$

$7 \mathrm{~h}$

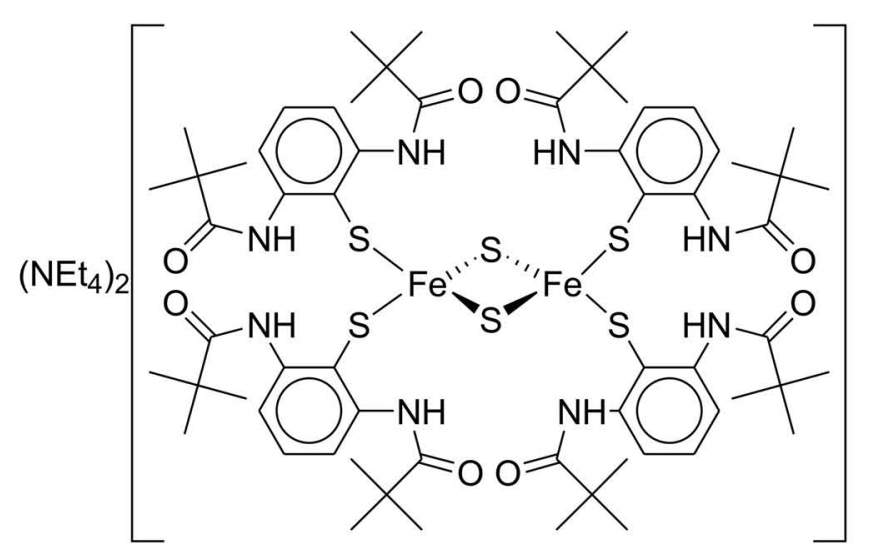




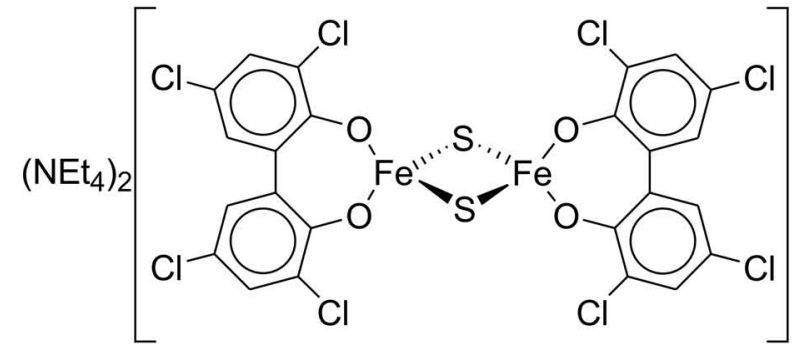

$8 a$

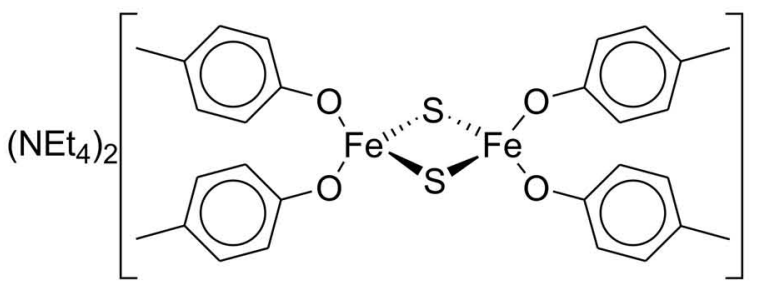

9

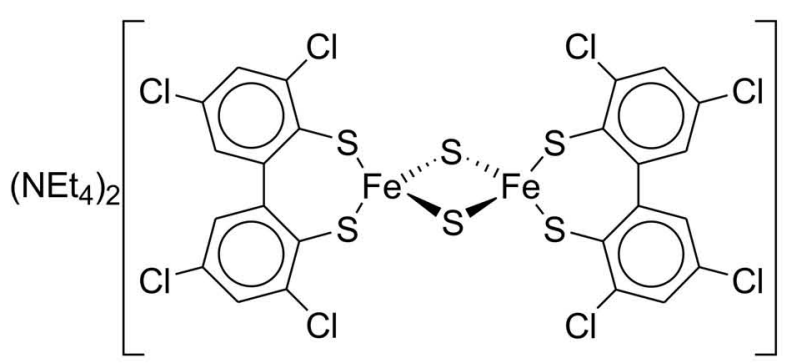

$11 \mathrm{a}$

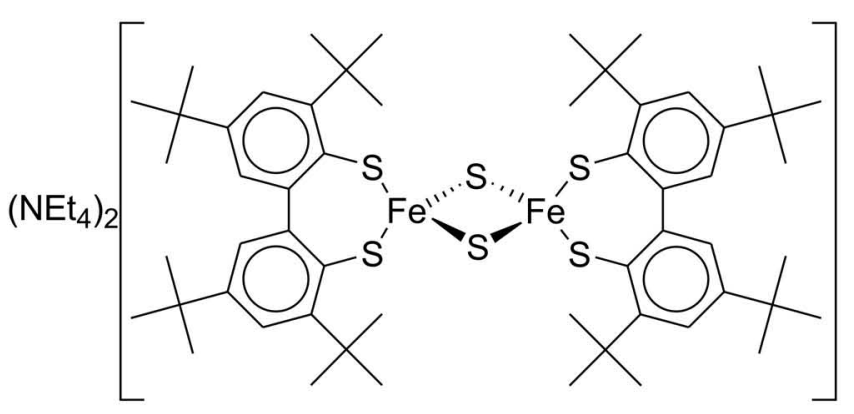

$11 \mathrm{c}$<smiles></smiles>

8b<smiles></smiles>

10<smiles></smiles>

$11 b$

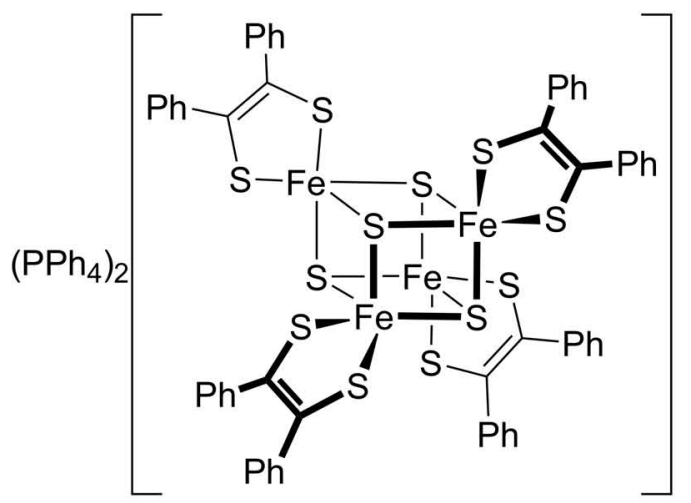

13

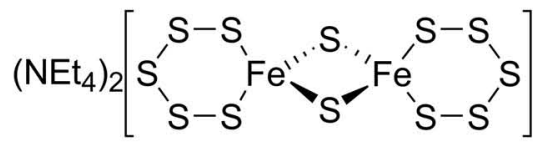

14

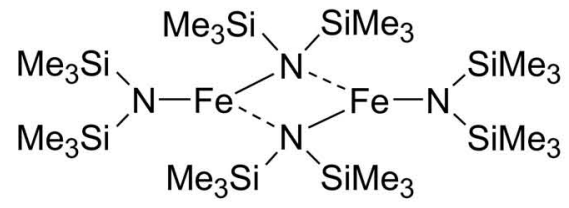

15 


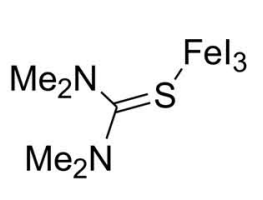

16

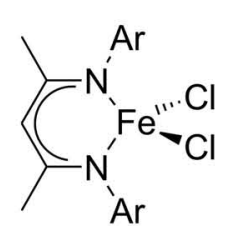

$21^{(*)}$

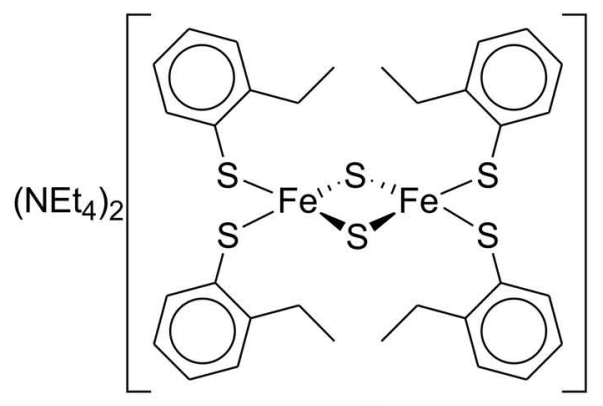

$25^{C}$

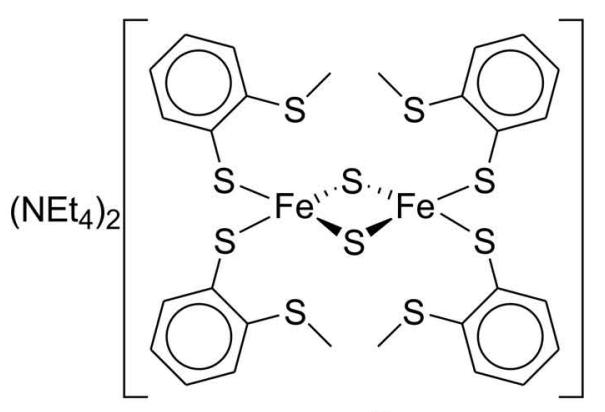

$25^{S}$

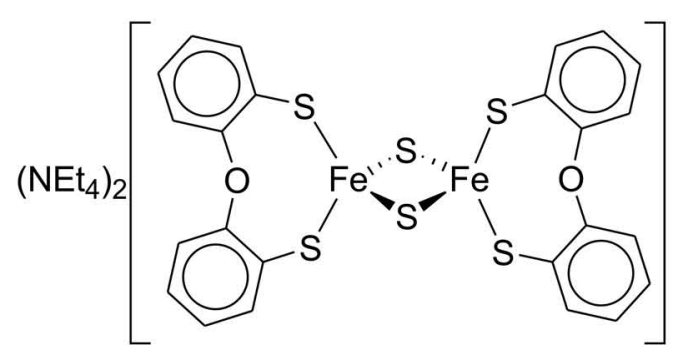

$26^{\circ}$

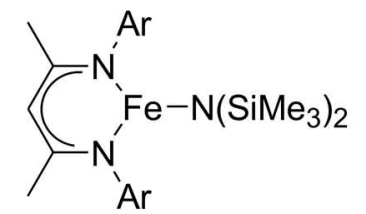

$18^{(*)}$<smiles></smiles>

23

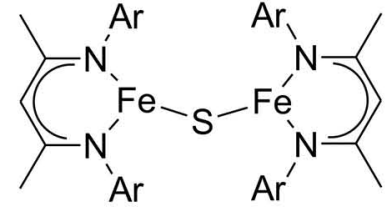

$19^{(*)}$

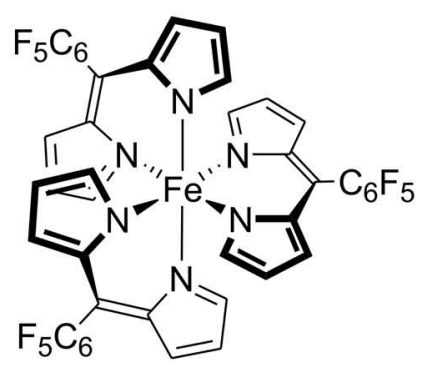

24

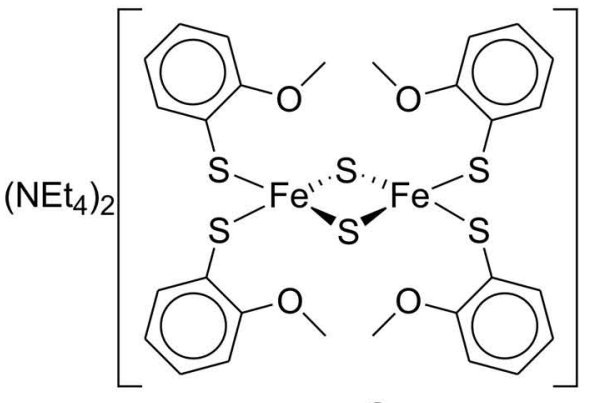

$25^{\circ}$

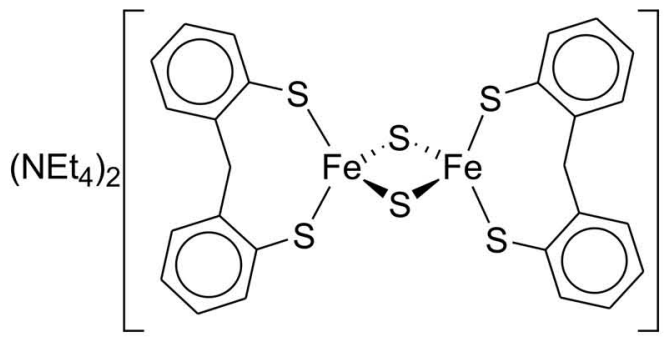

$26^{\mathrm{C}}$

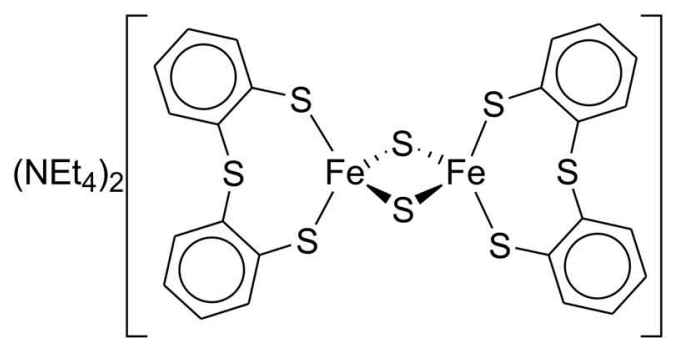

$26^{\mathrm{S}}$

${ }^{(*)} \mathrm{Ar}=2,6$-di-iso-propylphenyl 


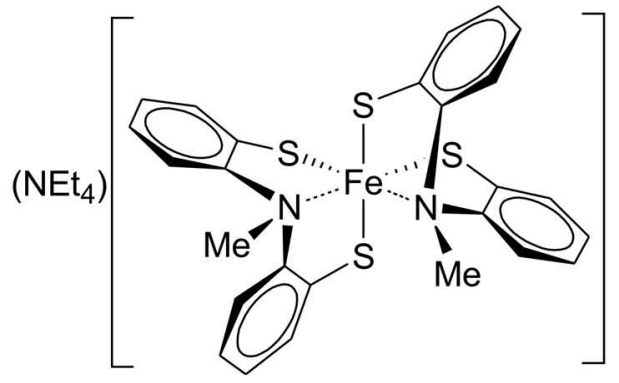

$27^{N}$

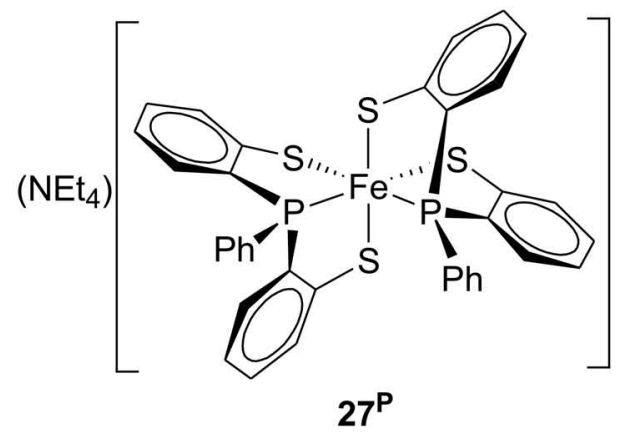

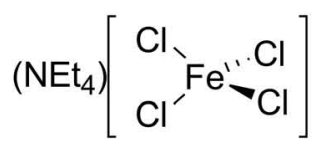

28

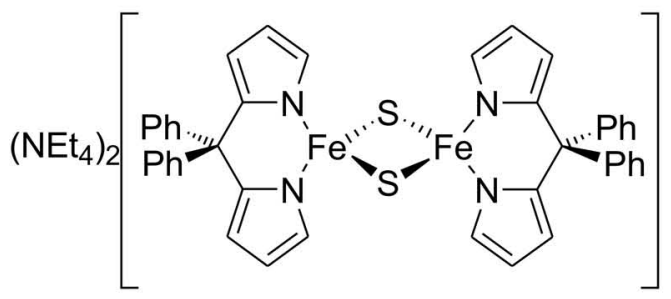

$29 b$

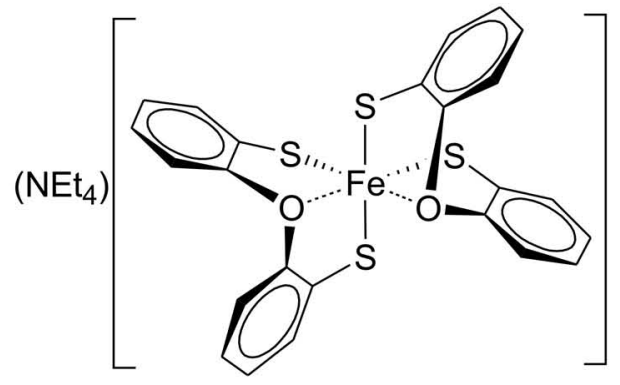

$27^{\circ}$

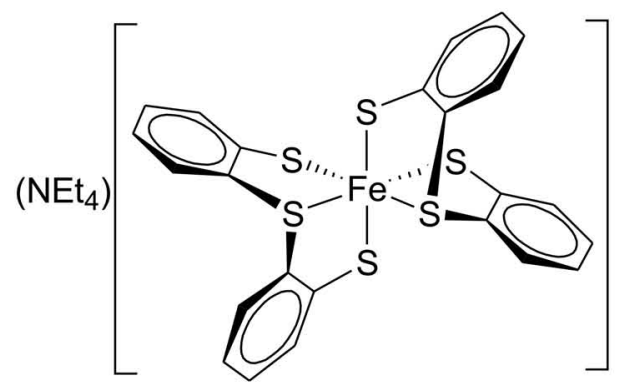

$27^{S}$

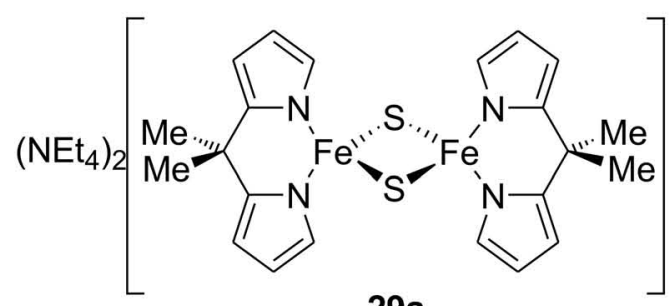

$29 a$

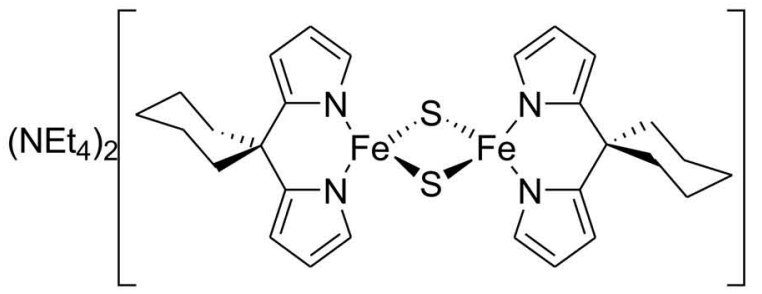

29c

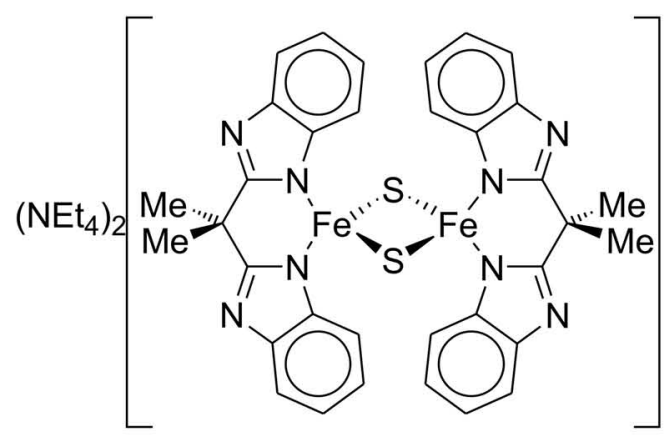




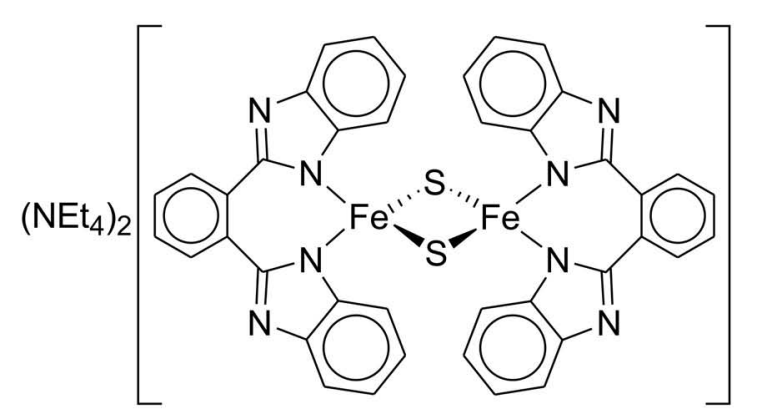

$31 a$

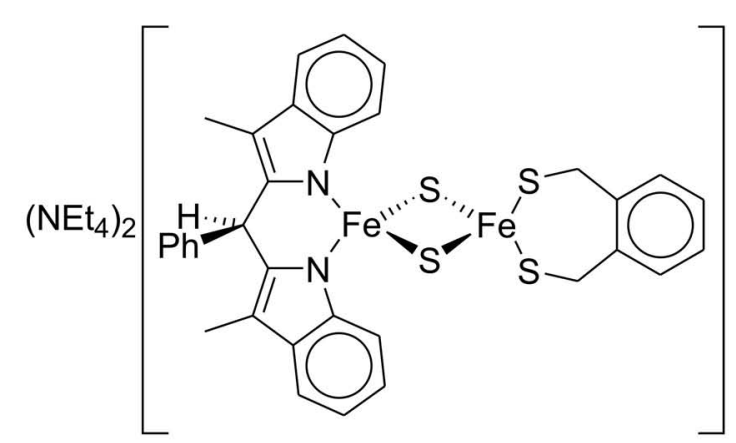

32

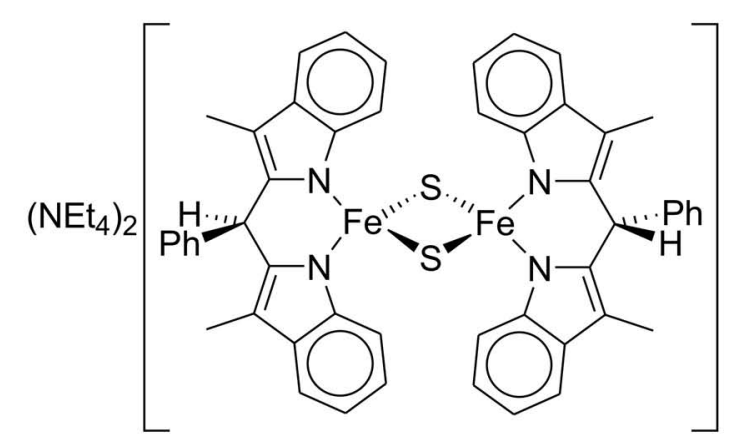

34

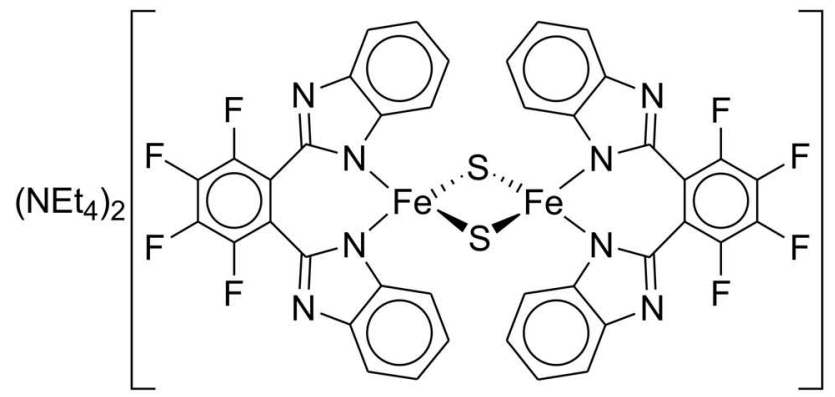

31b

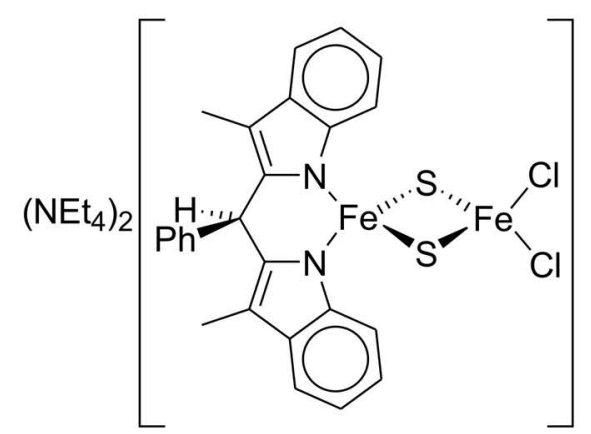

33

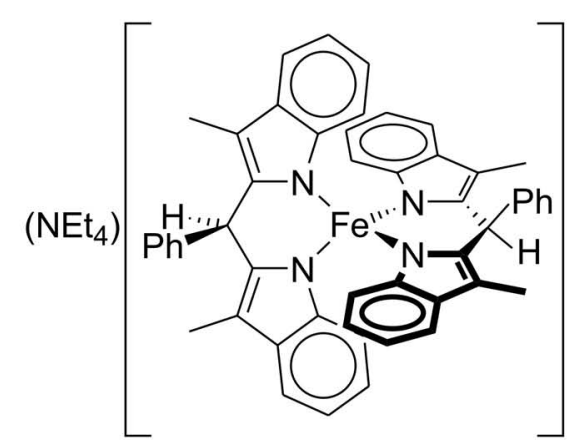

35 


\title{
List of Abbreviations
}

\author{
$A F$ antiferromagnetic \\ AIM atoms-in-molecules analysis (DFT calculation) \\ Ar aryl \\ B3LYP hybrid functional (DFT calculation) \\ bipy 2,2'-bipyridine \\ BP86 functional (DFT calculation) \\ br broad \\ ${ }^{(t)} \mathrm{Bu} \quad$ (tert-)butyl \\ $\mathrm{Cp}^{(*)}$ (pentamethyl-)cyclopentadienyl \\ CPC constant potetnial coloumetry \\ d doublet \\ $d$ distance \\ $\delta$ chemical shift (NMR), isomeric shift (Mössbauer) \\ $\Delta E_{\mathrm{Q}}$ quadropole splitting \\ DFT density functional theory \\ DMF $N, N$-dimethylformamide \\ DOSY diffusion ordered spectroscopy (NMR technique) \\ $E_{1 / 2}$ half-wave potential \\ $E_{\mathrm{p}}^{\mathrm{a}} / E_{\mathrm{p}}^{\mathrm{c}}$ anodic/ cathodic peak potential \\ EPR electron paramagnetic resonance \\ $\varepsilon$ extinction coefficient \\ $e q$ equivalents \\ ESI electron spray ionization \\ $\eta$ asymmetry parameters of EFG tensor \\ Et ethyl \\ $F$ ferromagnetic \\ $\mathrm{Fd}$ ferredoxin \\ $\Gamma \quad$ line width parameter \\ HiPIP high potential iron-sulfur protein \\ HMPA hexamethylphosphoramide \\ HOMO highest occupied molecular orbital
}


HRMS high resolution mass spectrometry

$H S$ high spin

IR infra-red

$J$ magnetic or NMR coupling constant

$\mathrm{L}$ ligand

$\lambda$ wavelength

$L S$ low spin

LUMO lowest unoccupied molecular orbital

m multiplet (NMR), medium (IR)

M molar

Me methyl

$\mu_{\text {eff }}$ effective magnetic moment

MS mass spectrometry

$\mathrm{NAD}(\mathrm{P}) \mathrm{H}$ reduced nicotine-amide adenine dinucleotide (phosphate)

NHE normal hydrogen electrode

NMR nuclear magnetic resonance

NOESY nuclear OvERHAUSER effect spectroscopy (NMR technique)

$\widetilde{\nu}$ wavenumber

OAc acetate

$\mathrm{Ph}$ phenyl

$P I$ paramagnetic impurity

$Q$ electrical charge

$\mathrm{R}$ arbitrary organic sustituent

$R$ radius

RNA ribonucleic acid

RT room temperature

s singlet (NMR), strong (IR)

$S$ spin ground state

SAM sulfur bound adenosylmethionine

SCE saturated calomel electrode

sh shoulder

SQUID superconducting quantum interference device

SVP single-valence plus polarization

$\mathrm{t}$ triplet

THF tetrahydrofuran

TIP temperature independent paramagnetism

TLC thin-layer chromatography

UV-Vis ultraviolet-visible

w weak (IR) 


\title{
List of Scientific Contributions
}

\author{
PUBLICATIONS
}

M. Gaertner, J. Ballmann, C. Damm, F. W. Heinemann, H. Kisch, "Support-controlled chemoselective olefin-imine addition photocatalyzed by cadmium sulfide on a zinc sulfide carrier", Photochem. Photobiol. Sci. 2007, 6, 159-164.

J. Ballmann, X. Sun, S. Dechert, E. Bill, F. Meyer, "Relatively stable N-ligated $[2 \mathrm{Fe} 2 \mathrm{~S}]^{2+}$ clusters with dipyrromethane capping ligands "J. Inorg. Biochem. 2007, 101, 305-312.

J. Ballmann, S. Dechert, E. Bill, U. Ryde, F. Meyer, "Secondary Bonding Interactions in Biomimetic [2Fe-2S] Clusters ", Inorg. Chem. 2008, 47, 1586-1598.

J. Ballmann, F. Meyer, "New Biomimetic Analogues Functional Fe/S Clusters ", Proceedings of the $9^{\text {th }}$ European Biological Inorganic Chemistry Conference 2008, in print.

J. Ballmann, M. G. G. Fuchs, S. Dechert, M. John, F. Meyer, "Synthesis and Coordination Properties of Chelating Dithiophenolate Ligands ", Inorg. Chem. 2008, accepted.

J. Ballmann, A. Albers, S. Demeshko, S. Dechert, E. Bill, E. Bothe, U. Ryde, F. Meyer, "A Synthetic Analogue of Rieske-Type [2Fe-2S] Clusters", Angew. Chem. 2008, accepted.

\section{PRESENTATIONS AT CONFERENCES AND INTERNATIONAL WORKSHOPS}

J. Ballmann, F. Meyer, "Synthetic [2Fe-2S] Clusters", $1^{\text {st }}$ Workshop of the International Research Training Group GRK 1422 "Metal Sites in Biomolecules: Structures, Regulation and Mechanisms", Fuldatal-Simmershausen, Germany, January 2007.

J. Ballmann, F. Meyer, "New Perspectives in Biomimetic [2Fe-2S] Cluster Chemistry", $2^{\text {nd }}$ Workshop of the International Research Training Group GRK 1422 "Metal Sites in Biomolecules: Structures, Regulation and Mechanisms", Lund, Sweden, June 2007.

J. Ballmann, F. Meyer, "A Synthetic Analogue of Rieske-type [2Fe-2S] Clusters", 3rd Workshop of the International Research Training Group GRK 1422 "Metal Sites in Biomolecules: Structures, Regulation and Mechanisms", Mariaspring, Germany, April 2008.

J. Ballmann, F. Meyer, "Synthetische [2Fe-2S] Cluster des Rieske Typs: Eine strukturell exakte Modellverbindung", $2^{\text {nd }}$ Göttinger Chemieforum, Göttingen, Germany, July 2008. 
J. Ballmann, X. Sun, S. Dechert, E. Bill, F. Meyer, "[2Fe2S] Clusters with Chelating NDonor Capping Ligands: Synthesis, Structure and Reactivity", $8^{\text {th }}$ European Biological Inorganic Chemistry Conference (EuroBIC 8), Aveiro, Portugal, July 2006.

J. Ballmann, S. Dechert, E. Bill, F. Meyer, "Sekundäre Wechselwirkungen in synthetischen [2Fe2S] Clustern", Göttinger Tage der Chemie, Göttingen, Germany, Mai 2007.

J. Ballmann, S. Dechert, E. Bill, F. Meyer, "Effects of Secondary Bonding Interactions in Synthetic, Bioinspired [2Fe2S] Clusters", $13^{\text {th }}$ International Conference on Biological Inorganic Chemistry (ICBIC XIII), Vienna, Austria, July 2007.

J. Ballmann, S. Dechert, E. Bothe, E. Bill, F. Meyer, "An Accurate Synthetic Model for Rieske Type [2Fe-2S] Clusters", Gordon Research Conference on Iron-Sulfur Enzymes, New London, USA, June 2008. 


\section{Acknowledgments}

At first, I would like to thank Prof. Dr. Franc Meyer for the supervision of this thesis. I am truly grateful for an excellent working atmosphere, granting of academic freedom and his continuous motivation to tackle the veritable challenges in synthetic [2Fe- $2 \mathrm{~S}]$ chemistry - instead of varying a few substituents only. His confidence that things will turn to work in the end definitively encouraged me to overcome a couple of experimental difficulties and not to give up too early - fortunately.

Furthermore, I would like to address my sincere thanks to all colleagues and external collaborators that added a great deal to the completion of this thesis: Prof. Dr. Ulf Ryde for spending plenty of his time in order to introduce me to the Turbomole software package and for the accommodation in his group in Lund during that period. Dr. Sebastian Dechert for conducting the X-ray diffraction experiments and structure refinements. I am especially thankful for solving the data of the twinned crystal of the asymmetrically coordinated Rieske intermediate - without those substantial efforts I probably would have missed the entire Rieske project. Dr. Eckhard Bill at the Max-Planck Institute in Mülheim a. d. R. for Mössbauer, SQUID and EPR measurements as well as Dr. Eberhardt Bothe for the electrochemical and coulometric experiments at the same research facility. Dr. Andrea Stückl for EPR measurements, Dr. Serhiy Demeshko and Benjamin Schneider for Mössbauer and SQUID measurements and Jörg Teichgräber for cyclic voltammetry measurements at our institute. Dr. Michael John for the NOESY and DOSY experiments and his assistance in all NMR-related concerns. Dr. Holm Frauendorf for performing all HRMS experiments and for his instructions regarding the use of the ESI spectrometers. I would also like to acknowledge all research students that have been involved in the experimental work: André Nadler, Thomas Theis, Alexander Düfert, Alexander Döring, Karsten Meyenberg, Tanja Polupanow, Björn Springer, Julia Grottker, Frank Behrendt, Heinrich Prinzhorn, Sabrina Polk, Nadia Burkard, Steffen Meyer, Alexander Orth, Christian Brand, Joachim Morik, Christoph Saßen, Lina Frensch and especially Antonia Albers (three-months IRTG research student). Moreover, I want to thank Dr. Xian-Ru Sun and Dr. Marco Klingele, both working previously on the $[2 \mathrm{Fe}-2 \mathrm{~S}]$ project, inter alia for their exploratory studies that facilitated my start-up in the laboratory. At this point, I want to thank my labmate Michael Fuchs for the excellent laboratory atmosphere (including useful discussions) and for the way we 'shared' the topic - never as competitors, but always working hand in hand. Also for reading and correcting almost everything I 'produced', thanks a lot, Michael. Many thanks as well to all other colleagues that added suggestions, improvements and corrections to this document (Dr. Daniel Olsson, Benjamin Schneider and Dr. Ulrich Scheele). 
I would also like to acknowledge all employees of the analytical laboratory, of the NMR and MS facilities, of the library, of the electrical and machine crafts shop and of the glassblowing crafts shop (especially Hans Joachim Schlette). Deep thanks furthermore to all house-stewards and the cleaning service for maintaining the laboratory, to Matthias Hesse, Jörg Teichgräber and Andreas Schwarz for the distribution of chemicals and laboratory equipment, to Petra Unger and Dr. Andrea Stückl for handling all administrational issues and to Dr. Sebastian Dechert for assistance in all kinds of software problems.

Research fellowships (including financial support) from the DFG (International Research Training Group GRK 1422 "Metal Sites in Biomolecules: Structures, Regulation and Mechanisms") and the "Fonds der Chemischen Industrie" are gratefully acknowledged.

Finally, I would like to direct loving thanks to my girlfriend, parents and brothers for their reliable backup. In particular, I want to thank Stefan Ballmann for his assistance in DFT calculations and for his exceptional efforts while converting this document to $\mathrm{LT}_{\mathrm{E} X}$.
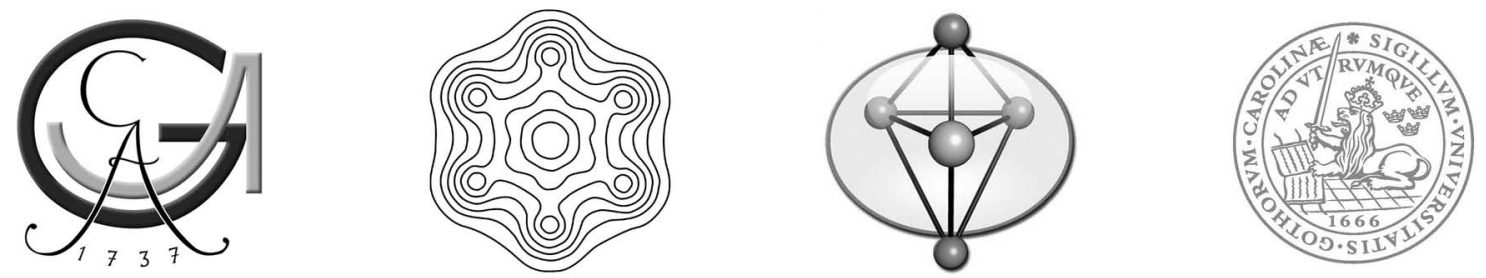


\title{
Curriculum Vitae
}

\author{
PERSONAL
}

Name: Hans Joachim Ballmann

Date of Birth: June $20^{\text {th }} 1979$

Place of Birth: Rothenburg o. d. T.

Nationality: German

Marital Status: unmarried

PRE-ACADEMIC EDUCATION

09/1985-07/1987 Grundschule Ohrenbach

09/1987-07/1989 Grund- und Volksschule Geslau

09/1989-06/1998 Reichsstadt-Gymnasium Rothenburg o. d. T.

Degree: Allgemeine Hochschulreife (Cumulative Grade: 1.5)

MILITARY SERVICE

07/1998-04/1999 Otto-Lilienthal-Kaserne Roth

ACADEMIC CAREER

10/1999-10/2001 Friedrich-Alexander-Universität Erlangen-Nürnberg

Undergraduate student at the Department of Chemistry

10/2001 Diplomvorprüfung (Cumulative Grade: 1.0)

10/2001-04/2004 Diploma student at the Department of Chemistry

04/2004-11/2004 Diploma Thesis "Cadmiumsulfid photokatalysierte Additionen zur Synthese von Homoallylaminen" at the Chair of Inorganic Chemistry, supervised by Prof. Dr. H. Kisch

(Cumulative Grade: 1.0 "mit Auszeichnung") 
since $02 / 2005$

Georg-August-Universität Göttingen

Ph.D. student at the Institute of Inorganic Chemistry, supervised by Prof. Dr. F. Meyer

Ph.D. Thesis "New Biomimetic Analogues of Functional [2Fe-2S] Proteins" 